\title{
Investigating the A-type stars using Kepler data
}

\author{
Simon J. Murphy
}

A THESIS SUBMITTED IN PARTIAL FULFILMENT OF THE REQUIREMENTS FOR THE DEGREE OF Doctor of PhILOSOPhy

\footnotetext{
Jeremiah Horrocks Institute for Astrophysics and Supercomputing University of Central Lancashire
} 


\section{Abstract}

Rotation is a key physical process operating in the A stars. We present a method by which rotation periods might be measured with Fourier transforms of Kepler data, potentially removing the requirement of spectroscopic observations to acquire similar information. Angular rotational velocities reach their maximum at about A5, but slowly rotating A stars are also seen and these tend to be chemically peculiar. For Ap stars, rotational braking is understood, but too few progenitors are observed. A review of a rare class of stars, the 'sn' stars, leads us to suggest these may contribute to the 'missing' Ap progenitors. For Am stars, we find the tidal braking mechanisms proposed in the literature induce mixing that is incompatible with observed abundance anomalies. At the other end of the scale are the $\lambda$ Boo stars, whose rotation velocities are above average. The two main theories for the origin of their metal underabundances are discussed and it is suggested that both of them imply the $\lambda$ Boo stars contain a high fraction of pulsators - a suggestion that is backed up by observations in the literature. Many $\lambda$ Boo stars also have circumstellar material, suggesting they are potential planet hosts. This, and the use of asteroseismology to study their interiors, are two excellent reasons to adopt them as prime targets for detailed investigation with Kepler.

Pulsation is a common phenomenon in A stars. The $\delta$ Sct stars receive wide attention but the fraction of stars that pulsate at the $50 \mu$ mag level is shown to be only 56 per cent. The non- $\delta$ Sct stars in the $\delta$ Sct instability strip receive far less attention. Some of these stars, without appreciable granulation or stellar winds, 
are probably the least variable objects on the HR diagram. It is shown that they have the potential to be among the most peculiar Am stars. Their investigation has led to two important conclusions: (1) the presence of $\gamma$ Dor pulsation in non- $\delta$ Sct stars in the $\delta$ Sct instability strip may inhibit the development of the expected Am peculiarities; and (2) chemically normal, non- $\delta$ Sct stars in the $\delta$ Sct instability strip do exist at the $\mu$ mag level.

Fourier transforms are invaluable tools in the field of stellar pulsation. The Kepler Space Telescope is providing data of exquisite precision, and thus more detail is seen in the Fourier transform than ever before. Truly understanding the properties of the data is fundamental to their successful utilisation. Through statistical analysis of noise levels in over 20000 stars, granulation is concluded to be visible in Fourier transforms of stars cooler than $7500 \mathrm{~K}$. Another property investigated is the Nyquist frequency. We found that periodically modulated sampling on board Kepler allows distinction between real pulsation frequencies and Nyquist aliases, even when those real frequencies exceed the Nyquist frequency of the data. This discovery opens up study of many hundreds of stars previously thought to have insurmountable Nyquist ambiguities in their data. 


\section{Contents}

Abstract

$\begin{array}{ll}\text { Acknowledgements } & \text { ix }\end{array}$

Prologue $x$

Context for advancement . . . . . . . . . . . . . . $\mathrm{x}$

Development of space photometry . . . . . . . . . . . . . . xi

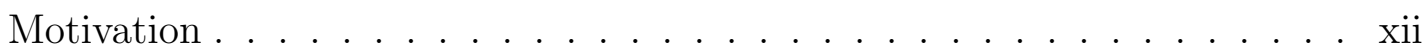

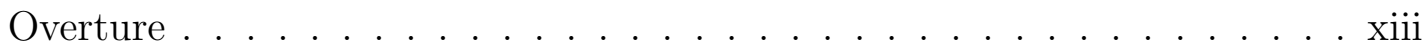

1 A Fourier view of Kepler data 1

1.1 An introduction to Kepler data . . . . . . . . . . . . . . . . 2

1.2 Characteristics of short- and long-cadence data . . . . . . . . 5

1.2.1 Sampling rate . . . . . . . . . . . . . . 5

1.2.2 Amplitude reduction . . . . . . . . . . . . . . 9

1.2.3 Precision . . . . . . . . . . . . . . . . . . . . 13

1.2.4 Noise and pipeline performance reviews . . . . . . . . . . . 14

1.2.5 Conclusions on data characteristics . . . . . . . . . . . 25

1.3 Characterising Kepler targets . . . . . . . . . . . . . . . 27

1.3.1 Noise amplitude as a proxy for data quality . . . . . . . . 28

1.3.2 Noise as a function of $T_{\text {eff }} \ldots \ldots \ldots$. . . . . . . . . 31 
1.3.3 Noise as a function of magnitude and mission quarter . . . . . 33

1.3.4 Discussion . . . . . . . . . . . . . . . . . 35

1.4 Gaps in the data . . . . . . . . . . . . . . . . . . . . 37

1.4.1 The non-gapped case . . . . . . . . . . . . . . . . . 38

1.4.2 Kepler is safe from cycle count ambiguities . . . . . . . . . 39

1.4.3 The failure of Module $3 \ldots$. . . . . . . . . . . . . 42

1.4.4 Overcoming aliasing in ground-based data . . . . . . . . . . 44

1.4.5 An aside: choosing a step-size . . . . . . . . . . . . . . 48

1.5 The Nyquist frequency and super-Nyquist asteroseismology with Kepler 48

1.5.1 MNRAS paper . . . . . . . . . . . . . . . . 49

1.5.2 The rescue of Module $3 \ldots \ldots 2$

1.6 Chapter summary . . . . . . . . . . . . . . . . . . . 65

1.A Published Content . . . . . . . . . . . . . . 66

1.A.1 An examination of some characteristics of Kepler short- and long-cadence data . . . . . . . . . . . . . . . 67

1.A.2 Kepler Fourier concepts: the performance of the Kepler data

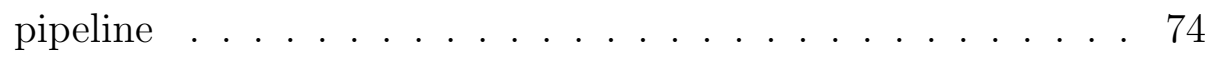

2 An observational review of rotation in A stars $\quad 78$

2.1 Introductory remarks . . . . . . . . . . . . . . . . . 79

2.2 Velocity distributions . . . . . . . . . . . . . . . . . 81

2.2.1 Is the distribution bimodal? . . . . . . . . . . . 81

2.2.2 Variations between observers . . . . . . . . . . . 83

2.2 .3 Evolution . . . . . . . . . . . . . . . . . 86

2.2.4 Inferring velocity distributions from Fourier transforms of Kepler data . . . . . . . . . . . . . . . . . . . 93

2.3 The magnetic Ap stars . . . . . . . . . . . . . . . . . . . . . . . . . 97

2.3.1 The oblique rotator model . . . . . . . . . . . . . 97 
2.3.2 Pre-main sequence evolution . . . . . . . . . . . . . . 99

2.3.3 Main sequence evolution . . . . . . . . . . . . . . . 101

2.4 Binaries . . . . . . . . . . . . . . . . . . . 102

2.4.1 Observational results . . . . . . . . . . . . . . 103

2.4.2 Short-range tidal braking . . . . . . . . . . . 105

2.4.3 Medium-range tidal braking . . . . . . . . . . . 105

2.5 Metallic-lined A stars (Am stars) . . . . . . . . . . . . 109

2.5.1 Presence in binaries . . . . . . . . . . . . . . . 110

2.5.2 Implications on mixing . . . . . . . . . . . . . . . . 112

2.6 The Kraft break . . . . . . . . . . . . . . . . . . . . . . . . . . . . 112

2.7 Chapter Summary . . . . . . . . . . . . . . . . . . . . . . 114

2.A Spectral type calibrations . . . . . . . . . . . . . 118

2.B Angular momentum transfer . . . . . . . . . . . . . 125

2.B.1 Orbital angular momentum . . . . . . . . . . . . 125

2.B.2 Rotational angular momentum . . . . . . . . . 126

2.B.3 Transfer of momentum . . . . . . . . . . . . . . . . 126

3 A selective review of spectral peculiarities in the A stars $\quad 129$

3.1 Introduction . . . . . . . . . . . . . . . . . . . 130

3.2 What is a normal A star? . . . . . . . . . . . . . . . . 132

3.2.1 The peculiar Sun . . . . . . . . . . . . . 136

3.2.2 The effect of rotation on spectral type assignment . . . . . . . 137

3.3 Am stars . . . . . . . . . . . . . . . . . . . 137

3.3.1 Abundance anomalies . . . . . . . . . . . . . . . . 139

3.3.2 Modelling Am stars . . . . . . . . . . . . . . . . . 143

3.3.3 Pulsating Am stars . . . . . . . . . . . . . . . 147

3.3.4 Non- $\delta$ Sct stars as Am star candidates . . . . . . . . . . . . 152

3.3.5 Microturbulence . . . . . . . . . . . . . . . . . . 159 
3.3.6 Other observed properties of Am stars . . . . . . . . . 161

$3.4 \rho$ Pup stars . . . . . . . . . . . . . . . . . . . 162

$3.5 \lambda$ Boo stars . . . . . . . . . . . . . . . . 166

3.6 'sn'stars . . . . . . . . . . . . . . . . . . . 172

3.7 Chapter summary . . . . . . . . . . . . . . . . . . . . . . . 174

3.A List of 'sn' stars . . . . . . . . . . . . . . . . . . . 177

4 A pulsation review of delta Scuti and related stars 179

4.1 Introduction . . . . . . . . . . . . . . . . . . . . . 179

4.2 Observational selection effects . . . . . . . . . . . . . . . . 181

4.3 Instability strip . . . . . . . . . . . . . . . . . . . 183

4.4 Mode selection and identification . . . . . . . . . . . . . . 188

4.4.1 Observability of modes . . . . . . . . . . . . . 188

4.4.2 Mode selection and frequency distribution . . . . . . . . . 191

4.4.3 Mode identification . . . . . . . . . . . . . . . . . 194

4.4 .4 Mode density . . . . . . . . . . . . . . . . . . . . 198

4.4.5 The amplitude detection limit . . . . . . . . . . . . 203

4.4.6 Combination frequencies and phases . . . . . . . . . 205

4.5 Related Variables . . . . . . . . . . . . . . . . . . . 208

4.5.1 HADS stars . . . . . . . . . . . . . . . . . 208

4.5.2 SX Phe stars . . . . . . . . . . . . . . . . 210

4.5.3 Gamma Doradus variables . . . . . . . . . . . . . . . . 211

4.6 Selected case studies _. . . . . . . . . . . . . . . . . . . . . 218

$4.6 .14 \mathrm{CVn} \ldots \ldots \ldots \ldots . \ldots \ldots \ldots$

$4.6 .2 \quad$ FGVir . . . . . . . . . . . . . . . . . . . . . . . . . . . 219

$4.6 .344 \mathrm{Tau} \ldots \ldots \ldots \ldots . \ldots \ldots . \ldots \ldots 21$

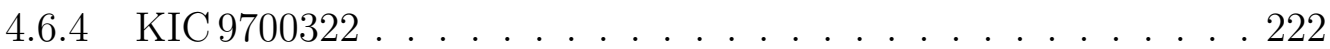

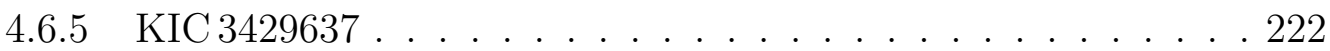




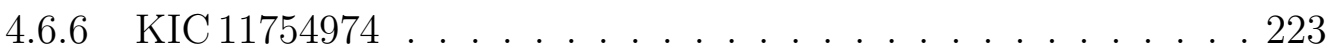

4.6 .7 KIC $10977859 \ldots \ldots \ldots . \ldots \ldots . \ldots \ldots$

4.7 Chapter Summary . . . . . . . . . . . . . . . . . . . . . . 224

$5 \quad$ Kepler catalogues and published case studies $\quad 227$

5.1 Description of Kepler catalogues . . . . . . . . . . . . . . . . . . 227

5.2 Kepler catalogues: an extract . . . . . . . . . . . . . . . . 229

5.3 Pulsational amplitude growth in the $\rho$ Pup star KIC 3429637 (HD 178875) in the context of Am and $\rho$ Pup stars . . . . . . . . . . . 233

5.4 Asteroseismology of KIC 11754974: a high-amplitude SX Phe pulsator in a 343-d binary system . . . . . . . . . . . . . . . . . . 244

6 Conclusions and Future Work 258

6.1 Conclusions . . . . . . . . . . . . . . . . . . . . . . 258

6.2 Future Work . . . . . . . . . . . . . . . . . . . . 261 


\section{Acknowledgements}

I have been fortunate.

This $\mathrm{PhD}$ commenced 18 months after the launch of the Kepler Space Telescope, which has provided data in abundance. Funding for the Kepler mission is provided by the NASA Science Mission directorate, and I'd like to thank them for that.

In Don Kurtz, I have had the best supervisor this project could have bestowed. Such a wealth of knowledge I could only aspire to achieve, and I look forward to many more years of us working together. If this thesis presents a valuable contribution to the field, it is only because his help and guidance have made it so. To Don, I owe a sincere debt of gratitude.

Of course, $\mathrm{PhD}$ programmes are not handed out indiscriminately. I would like to acknowledge the financial support of the STFC through the studentship programme, and to thank the JHI interview panel members Danielle Bewsher and Gordon Bromage for offering me a place.

I was lucky to grow up with a mother who always put her children first, and a brother with whom to compete intellectually. I wish him well for his PhD in particle physics. I dedicate this thesis to my father, and hope he would have been proud.

I am grateful to friends who have supported me over the last few years. In particular, I would like to thank Ben Langfeld for being a patient RUBY instructor, and for assistance with the coding that really got this $\mathrm{PhD}$ off the ground. To my fellow students, colleagues and friends in the astronomy community, too many to name, I am so glad to have met you and I continue to treasure your friendship.

I have been very fortunate. 


\section{Prologue}

\section{Context for advancement}

It is natural to look up at the sky and wonder about our place in the universe, about the nature of the stars, about the existence of life on other planets. It is a credit to human achievement that we set out to measure that upon which we look. Sometimes astronomy can seem so far removed from everyday life that astronomers are often asked in a malcontented tone "Why should we explore space?". This often reflects an absent sense of adventure, but occasionally the question is contextualised with how unexplored the ocean bottom remains here on Earth. The keen astronomer knows that the enjoyment factor is insufficient reason to the questioner, and that eventually the debate comes down to money. While it is true that the space industry contributes greatly to the economy and that many inventions we find so useful were originally designed for space missions (memory foam and cordless tools are the common examples, but dialysis machines might make a punchier argument), that doesn't really capture the spirit of scientific advancement. Though Tycho Brahe had no shortage of money, it was fascination with astronomy that drove him from the study of law to making the most accurate celestial measurements of his day, measurements that would later be used by Johannes Kepler to revolutionise planetary motion. The point is that pushing the boundaries of knowledge is good in its own right; those scientific advancements that don't occur through serendipity 
emerge through new methods of thinking, or the advancement of instrumentation, along with some downright hard work. Yet there's nothing to say those pathways to discovery have to be mutually exclusive.

\section{Development of space photometry}

Asteroseismology has entered a new era: one in which observations are made from space. NASA's Kepler mission has been designed to find Earth-like planets around solar-like stars, and the photometric precision required to fulfil that goal is unprecedented. Kepler is capable of micromagnitude-precision observations of $\sim 150000$ stars in a 115- $\operatorname{deg}^{2}$ field of view, and a few percent of those observations are dedicated to asteroseismology. Moreover, these observations are almost continuous gone are the days of ground-based observations with insurmountable time gaps and millimagnitude noise levels.

Irrespective of how carefully one takes photometric measurements from the ground, the weather and day-night cycle limit the observational duty cycle, and precision is limited by atmospheric scintillation. This is why the future of photometry is in space. But the Kepler Mission was not the first. After the failure of the primary mission of the Wide Field Infrared Explorer (WIRE) mission, the star-tracker provided a testbed for CCD observations in space (Buzasi, 2000). The first purpose-built asteroseismology satellite was MOST (Microvariability and Oscillations of STars; Walker et al. 2003), which after launch in June 2003 began capturing white-light CCD images of bright $(0.4 \leq V \leq 5.5)$ stars every 0.5 - 60 s for up to $60 \mathrm{~d}$, though for a maximum of only six stars simultaneously. More recently, the CoRoT (Convection, Rotation \& planetary Transits) mission has been making almost $\mu$ mag-precision observations from its polar orbit at either 512-s or 32-s cadences for up to $150 \mathrm{~d}$ continuously, for 12000 targets between magnitude 11 and 16 .

Ground-based observations are not obsolete, though. The Kepler field of view is 
fixed, and its photometry is white-light only. Multi-colour observations still require telescope time on the ground. Full abundance analyses, or even just classification spectra for so many targets require dedicated ground-based observing teams. The reductions, models and theory require further efforts. Entire networks of collaborators and consortia are proliferating under the auspices of this new wealth of high-quality space photometry.

\section{Motivation}

Whenever such vast increases in data quality and quantity become available, the discoveries just flow out of the data. Such was the case with Galileo and the moons of Jupiter. One doesn't necessarily know what one is looking for until one finds it. So many Kepler light curves are available that some completely take you by surprise. Something strange is happening and you cannot quite explain it. This was the case when I first presented Don Kurtz with a light curve of KIC 4150611 (HD 181469), which he immediately dubbed "The Thing" for lack of a sound morphological characterisation. This system consists of an eclipsing, eccentric binary pair of $\mathrm{G}$ stars that are a common proper motion pair with a $\delta$ Sct star orbited by a pair of K stars (Prsa et al., in prep.). The system became the test-subject of the seminal paper on using frequency modulation to infer orbital parameters of pulsating $\delta$ Sct stars (Shibahashi \& Kurtz, 2012). In this case, the theory quickly followed the observations, but this is not always so. With the vastness of the Kepler data we have scenarios where observations are outpacing theory (the pulsating Am stars), and scenarios where the theory has been waiting years for suitable observations like these to put it to the test (the "Heartbeat" stars; Kumar, Ao \& Quataert 1995; Thompson et al. 2012). The tone of the material in this thesis is thus set: with enough observations discoveries will come thick and fast; all one has to do is try to keep up! 


\section{Overture}

The structure of this thesis is perhaps unusual. Rather than beginning with an introduction that reviews the literature related to the thesis content, each chapter contains its own literature review, and the research herein presented is thus contextualised with immediacy. This begins in Chapter 1 with an introduction to Kepler data and themes related to the examination of those data with Fourier transforms. Chapter 2 is dedicated to rotation as a physical process of great importance in the A stars. Through its effect on diffusion and mixing mechanisms, the rotation velocity has a strong influence on many of the spectral peculiarities that are seen in A stars, which are discussed in Chapter 3. In turn, spectral peculiarities may determine whether a star pulsates, and if so, to which of the classes of pulsators the star belongs. Of the classes of pulsating stars, $\delta$ Sct stars are the most common at spectral type A, and many of the 'normal', i.e., non-peculiar A stars belong to this class. Hence a pulsational review of $\delta$ Sct and related stars is presented in Chapter 4 . Then, in Chapter 5 , two published case studies of $\delta$ Sct stars are included, before the conclusions and future directions of the thesis material are laid out in Chapter 6. 


\section{Chapter 1}

\section{A Fourier view of Kepler data}

\section{Abstract}

The Kepler data, with their regular sampling, very high duty cycle, micromagnitude precision and long observational time span are revolutionising asteroseismic studies. Understanding and disentangling instrumental artefacts is of paramount importance to fully utilise the data. In this chapter we examine the characteristics and differences between the Kepler short- and long-cadence data (as published in Murphy 2012a), investigate the quality of the pipelines, past and present, and discuss the validity of their use (based on Murphy 2012b). Those two supporting publications are provided in an appendix. One can seek understanding of the noise present in Fourier transforms of Kepler data; here we characterise the brightness and temperature distribution of Kepler targets and show how noise depends on these properties, concluding that granulation noise is present in Fourier transforms of stars up to $T_{\text {eff }}=7500 \mathrm{~K}$. We look at the effect of having gaps in the data and make a comparison to the ground-based case. Finally, we see how relative motion of the Kepler spacecraft around the Sun requires a barycentric time correction to the 


\section{CHAPTER 1}

otherwise regularly sampled data, how this periodically modulated sampling splits Nyquist aliases into multiplets for long data sets, and how real frequencies can now be identified in the Fourier transform without any Nyquist ambiguities, even when the real frequency is well above the Nyquist frequency (Murphy, Shibahashi \& Kurtz, 2013).

Any material herein presented that has been published elsewhere is owned by the author, and other sources have been properly acknowledged.

\subsection{An introduction to Kepler data}

The Kepler Space Telescope was launched on 2009 March 6. It is in a 372.5-d heliocentric Earth-trailing orbit, collecting white-light photometric data for around 150000 stars simultaneously in its $115 \mathrm{deg}^{2}$ field of view, and at near-micromagnitude precision. The core goal of the mission is detection of Earth-like planets orbiting Sun-like stars within the habitable zone. The details of the mission goals and design are described by Koch et al. (2010) and Borucki et al. (2010). An early review of the characteristics of planet candidates is given by Borucki et al. (2011).

Secondary to the main goal of the mission is asteroseismology, which can provide valuable information on the host stars that is important in planet characterisation. With transit depths, only the ratio of the planet and host star radii is available, but asteroseismology allows detailed analysis of the star's interior if the star pulsates, and for solar-like oscillators can yield the star's radius to better than 3 per cent (Stello et al., 2009). Through asteroseismology, Kepler continues to promise significant advances in stellar astrophysics. Initially, the Kepler Asteroseismic Science Consortium was given one year of proprietary access to $\sim 1$ per cent of the Kepler observations. All Kepler data are now made public once they have been processed by the Kepler Science Office. 


\section{CHAPTER 1}

Kepler data are available in two cadences, long (LC) and short (SC). Each cadence is composed of multiple 6.02-s exposures with associated 0.52-s readout times (Gilliland et al., 2010). The LC data integrate over 270 exposures to give 29.45-min observations, whereas the SC data contain nine exposures giving one data point every $58.85 \mathrm{~s}$. Both cadences are stored on-board and downlinked to Earth roughly every $32 \mathrm{~d}$, introducing gaps up to $\sim 24 \mathrm{~h}$ in length while the photometer is not collecting data. Kepler completes one quarter of its orbit after three downlinks, and must then perform a quarterly roll to keep its solar panels pointing towards the Sun, and its radiator pointing to deep space. Kepler data are therefore organized into quarters and thirds around those rolls and downlinks. LC data quarters are denoted by Qn, and SC quarters by Qn.m to indicate which third (or 'month') of that quarter the data correspond to. Although LC data are not divided into months, the downlink gaps can still be seen in the data. Kepler's hardware limits SC observing slots to 512 at any given time.

Kepler's 42 CCDs had four-fold symmetry, meaning that after each quarterly roll the satellite still covers the same area of sky. Unfortunately, Module 3, consisting of two CCDs, failed early in the mission, and a small fraction $\left(\sim \frac{4}{21}\right)$ of the field of view is only observable for three quarters of the year. The focal plane was chosen so that the brightest stars in the field fall in the gaps between CCDs. The brightest star that remains directly on silicon is $\theta \mathrm{Cyg}$, with $V=4.48$, but the observations of bright stars are generally restricted due to telemetry considerations. Indeed, although the mission was launched with sufficient propellant for $10 \mathrm{y}$ of operations, telemetry will become a limitation after 7-8y as the satellite drifts farther from Earth.

The Kepler data are available in FITs format at the Mikluski Archive for Space Telescopes $\left(\mathrm{MAST}^{1}\right)$. Members of the Kepler Asteroseismic Science Consortium (KASC) can download the same data in FITS format, or in ASCII format (including some processed data and data bundles) from the Kepler Asteroseismic Science

\footnotetext{
${ }^{1}$ http://archive.stsci.edu/kepler/data_search/search.php
} 


\section{CHAPTER 1}

Operations Centre $\left(\mathrm{KASOC}^{2}\right)$. In addition to these archival data, a Guest Observer program exists ${ }^{3}$, whereby anyone can propose to observe a target, and find the tools and documentation to make use of the data.

The data processing pipelines have come under some scrutiny, not least of which from this author (Murphy 2012a; Murphy 2012b). The information presented therein is also contained within this chapter. Pre-Q9, Kepler data were available in two forms: (i) Simple Aperture Photometry (SAP), for which basic calibration is performed distinguishing it from the truly raw flux, but for which the term 'raw flux' is still incorrectly used, and (ii) Pre-search Data Conditioned (PDC) 'corrected' flux. The PDC data were created as a step towards facilitating planetary transit searches, without the intention that they should be used for astrophysical analyses because some stellar variability in the light curves can be modified by the PDC pipeline. The pipeline as it was in data releases 11 and earlier is now called PDC-LS, denoting the least-squares algorithms used to remove some trends from the data, and to distinguish it from the newer pipeline, PDC-MAP. The latter utilises 'Maximum-APosteriori cotrending algorithms', which identify trends affecting the light curves of neighbouring stars and seeks to remove them (with limited success). All new quarters use the PDC-MAP pipeline, and the existing LC quarters were re-processed with this pipeline. At the time of the Q12 data release, SC data still used the PDCLS pipeline. For further reading on the pipelines, beyond that presented here in $\S 1.2 .4$, see the Kepler Data Characteristics Handbook and the articles by Stumpe et al. (2012) and Smith et al. (2012).

\footnotetext{
${ }^{2}$ http://kasoc.phys.au.dk/

${ }^{3}$ http://keplergo.arc.nasa.gov/
} 


\section{CHAPTER 1}

\subsection{Characteristics of short- and long-cadence data}

There are many advantages of SC data over LC data. Here the following advantages of SC data are discussed: increased sampling rate; higher Nyquist frequency; fewer low-frequency artefacts (resulting from differences in data pipelines); and reduced errors on frequency, amplitude and phase determinations in the Fourier spectrum. The difference in distribution of data points between SC and LC data is also discussed. Initial characteristics of the LC and SC data can be found in Jenkins et al. (2010) and Gilliland et al. (2010), respectively. For a detailed review of Kepler noise properties, see Gilliland et al. (2011) and references therein.

\subsubsection{Sampling rate}

There are 30 times more SC points than LC points in a quarter, arising from the integration of more exposures to make the LC data. Two well-known effects this has on time-series analysis are the time resolution available, and the associated Nyquist frequency.

\section{Time resolution}

The primary mission goal of planet detection requires the increased sampling rate to time transits more precisely; signs of gravitational perturbation seen as changes in transit duration (known as Transit Timing Variations, or TTVs) may lead to subsequent detections of other planets orbiting the same star. Holman \& Murray (2005) calculated that under the gravitational influence of other solar system bodies, Earth's apparent transit time for an observer viewing along the orbital plane would appear shorter by around $650 \mathrm{~s}$ for 2 in 10 of its orbits, depending on the relative position of each planet and the observer with respect to orbital phase. The effect is even greater for planets orbiting farther from their star (up to $~ 6000 \mathrm{~s}$ for Mars), and for planets orbiting less massive stars. The sensitivity required for such detections is 


\section{CHAPTER 1}

easily met by Kepler, where transit durations would vary by 11 and 101 SC points for the Earth and Mars cases respectively, even where errors from photon statistics on such a transit duration reach $\sim 500 \mathrm{~s}$, equivalent to $8 \mathrm{SC}$ points (Holman \& Murray, 2005). The first previously unknown planet to be detected using this technique, Kepler-19c, does not appear to transit its star, but was detected because of its influence on the planet Kepler-19b (Ballard et al., 2011).

The higher sampling rate is useful for astrophysical studies too, particularly for short-lived events like flares (see e.g. Balona 2012b). The star KIC 12406908 is one of the $\sim 15$ per cent of the Kepler Input Catalogue (KIC) stars that have no fundamental parameters listed, that is, no $T_{\text {eff }}$ or $\log g$ values are available. Fig. 1.1 shows a 7.2-h sample of the light curve of the largest flare on this star in Q3.1. The LC data have been plotted underneath the SC data for comparison, and a shift in magnitude has been created for demonstrative purposes only. The longer integration time in LC has the effect of averaging the SC points, and under-samples the 0.056mag flare. The shape of the flare, including its erratic nature as its luminosity output rises and falls numerous times across the event, is lost in the LC data. Only a rough approximation of its magnitude and duration would be determinable without the SC data. Events with such short time-scales can clearly only be studied in SC.

\section{An example of Nyquist aliasing}

A direct consequence of the different cadences available in Kepler data is their different Nyquist frequencies. Detailed discussion of the Nyquist frequency in those data will take place in $\S 1.5$. For now, we shall demonstrate the potential for confusion caused by Nyquist aliases for short Kepler data sets.

Many asteroseismic targets pulsate at frequencies higher than the Nyquist frequency of the LC data $\left(24.469 \mathrm{~d}^{-1} ; 283.21 \mu \mathrm{Hz}\right)$, which, until the recent investigation of Murphy, Shibahashi \& Kurtz (2013), made them difficult to study - one would 


\section{CHAPTER 1}

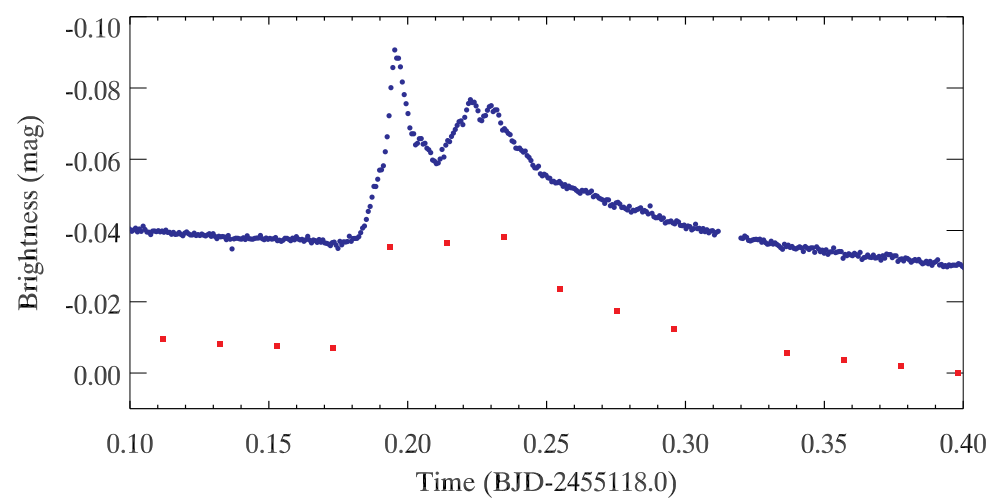

Figure 1.1: A flare on KIC 12406908. Simulated LC data (red squares) are plotted beneath the real SC data (blue circles) for comparison. The change in brightness is precise, but not accurate - the dimmest LC point was chosen as the zero point for the graph, and all SC points are offset for clarity. The SC data used are Q3.1 PDC-LS, and the LC data are simulated by binning 30 consecutive SC points.

need either SC data or to tolerate severe aliasing. Solar-like oscillations (in main sequence stars) and roAp star pulsations only occur at frequencies much higher than the LC Nyquist frequency. Straddling both sides of the LC Nyquist frequency are the $\delta$ Sct stars. These stars pulsate in low-order p modes (pressure modes) and typically have frequencies in the range $4-50 \mathrm{~d}^{-1}(46-579 \mu \mathrm{Hz}$; Breger 2000b), although the highest published frequency for a $\delta$ Sct star is currently $79.5 \mathrm{~d}^{-1}(920 \mu \mathrm{Hz}$; Amado et al. 2004).

The Nyquist frequency of equally spaced (regularly sampled) data is equal to half the sampling rate. Since LC has a point every $29.45 \mathrm{~min}$, there are 48.9 points per day, and the Nyquist frequency is therefore $24.5 \mathrm{~d}^{-1}(284 \mu \mathrm{Hz})$. Hence if in the Fourier spectrum a signal is detected with a frequency higher than the Nyquist frequency, it is not fully sampled, and an alias will be detected at $2 f_{\text {Nyquist }}-f_{\text {signal }}$. It is not always obvious that these detected frequencies are aliases or 'reflections' of frequencies higher than $f_{\text {Nyquist }}$, and can sometimes be interpreted as real pulsation frequencies.

One is naturally cautious when any frequencies are detected in LC near the 


\section{CHAPTER 1}

Nyquist frequency, as the star could have pulsation frequencies above the Nyquist frequency even if the detected frequencies are real. However, for much lower frequencies the possibility of aliasing seems more remote.

KIC 10977859 is a $\delta$ Sct star in which the SC data show only high-order p modes, and a lack of pulsations at frequencies below the LC Nyquist frequency (Fig. 1.2, upper panel). When only the LC data are considered (if SC data were not available, for instance), the spectrum looks entirely different. Nyquist frequency limitations

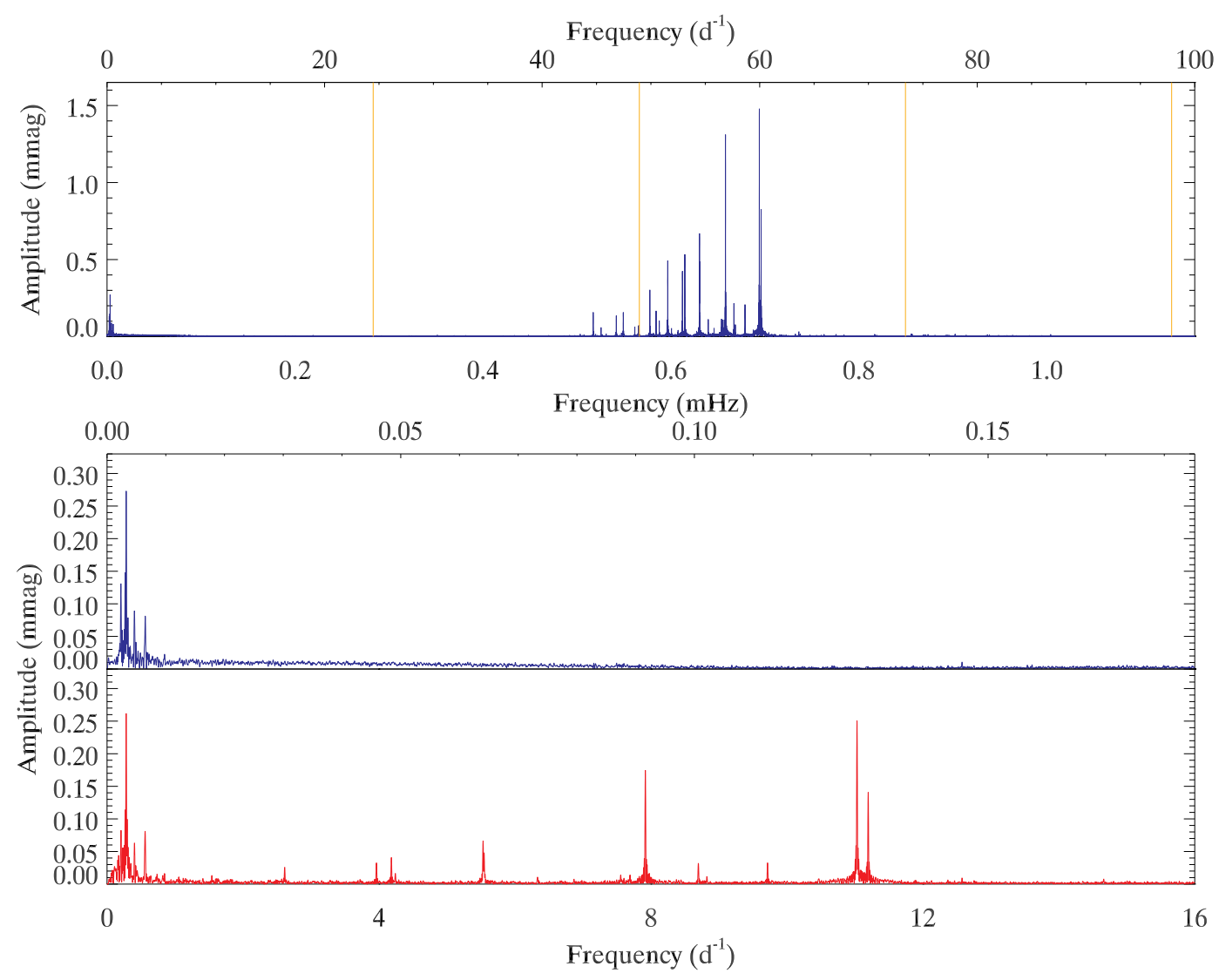

Figure 1.2: Upper panel: the Fourier spectrum of the $\delta$ Sct star KIC 10977859 for Q5, showing dominant high-order, high-frequency p modes. Multiples of the LC Nyquist frequency are plotted as vertical orange lines. Lower panels: A magnification of the spectrum between 0 and $16 \mathrm{~d}^{-1}$ for the same star, in SC (blue, middle) and LC (red, bottom). The amplitude of the real peaks in the top panel are around five times greater than corresponding peaks in the bottom panel, indicating a significant amplitude reduction in aliased peaks. To aid the eye, the y-axis scale in the lower panels is one fifth of that of the upper panel. 


\section{CHAPTER 1}

mean that in LC data the true frequencies of high-order p modes would not be discernible, but a number of peaks are visible below the LC Nyquist frequency instead (Fig. 1.2, lower panel), strongly implying the star pulsates in low-order p modes and maybe g modes (gravity modes), too. What is even more misleading in this case, and makes the situation problematic, is the absence of peaks in the periodogram of the LC data between 16 and $24.4 \mathrm{~d}^{-1}(185-282 \mu \mathrm{Hz})$, fooling the observer into believing these are low-order p modes with only a small likelihood of signals at higher frequencies - there is no warning of what lies beyond the LC Nyquist frequency.

For short data sets, one must exercise extreme caution when analysing LC data if there are no SC data to test for aliasing problems associated with the LC Nyquist frequency. However, for long data sets (at least four Kepler mission quarters), one can use the 'super-Nyquist asteroseismology' technique, which is developed in $\S 1.5$.

\subsubsection{Amplitude reduction}

The width of a peak in the Fourier spectrum of a data set of length $T$ can be approximated by $1 / T$ (the Rayleigh criterion). For data sets of equal length, the greater number of points in SC data has no effect on the width of the peak, but the amplitude of peaks is different. As mentioned in $\S 1.2 .1$, the longer integration time of the LC data causes an averaging effect that is more noticeable for shorterperiod events or pulsations. The same effect reduces the amplitude of peaks in the periodogram of LC data, as shown in Fig. 1.3. The percentage difference in amplitude between SC and LC increases with higher frequencies.

Even when photometric data are sampled frequently enough to avoid Nyquist aliasing, one observes an amplitude reduction of the pulsation modes that is a function of the number of data points per pulsation cycle. The following is a mathematical derivation of the effect. ${ }^{4}$

\footnotetext{
${ }^{4}$ Thanks to Darragh O'Donoghue for describing the derivation.
} 


\section{CHAPTER 1}
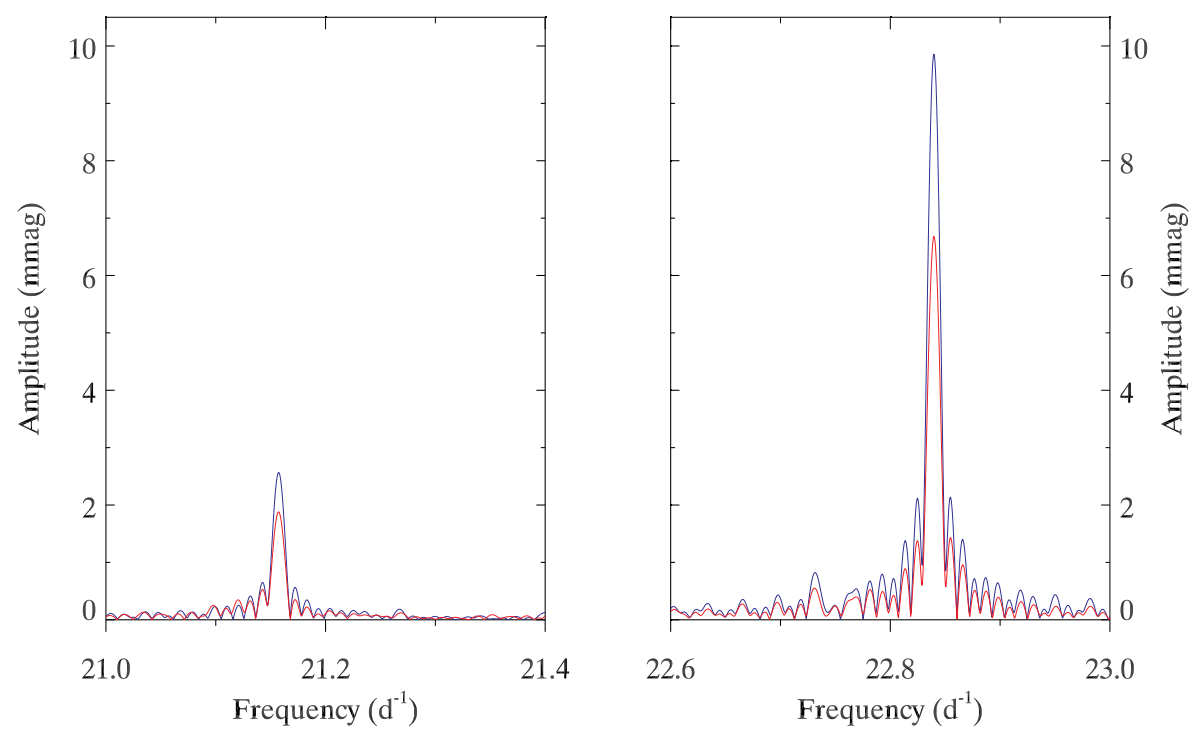

Figure 1.3: The Fourier transform of two peaks present in the data of KIC 5724810. The depicted LC (red) and SC (blue) Q5 data show that the SC data have higher amplitude peaks due to the shorter integration time and the averaging effect inherent within LC data.

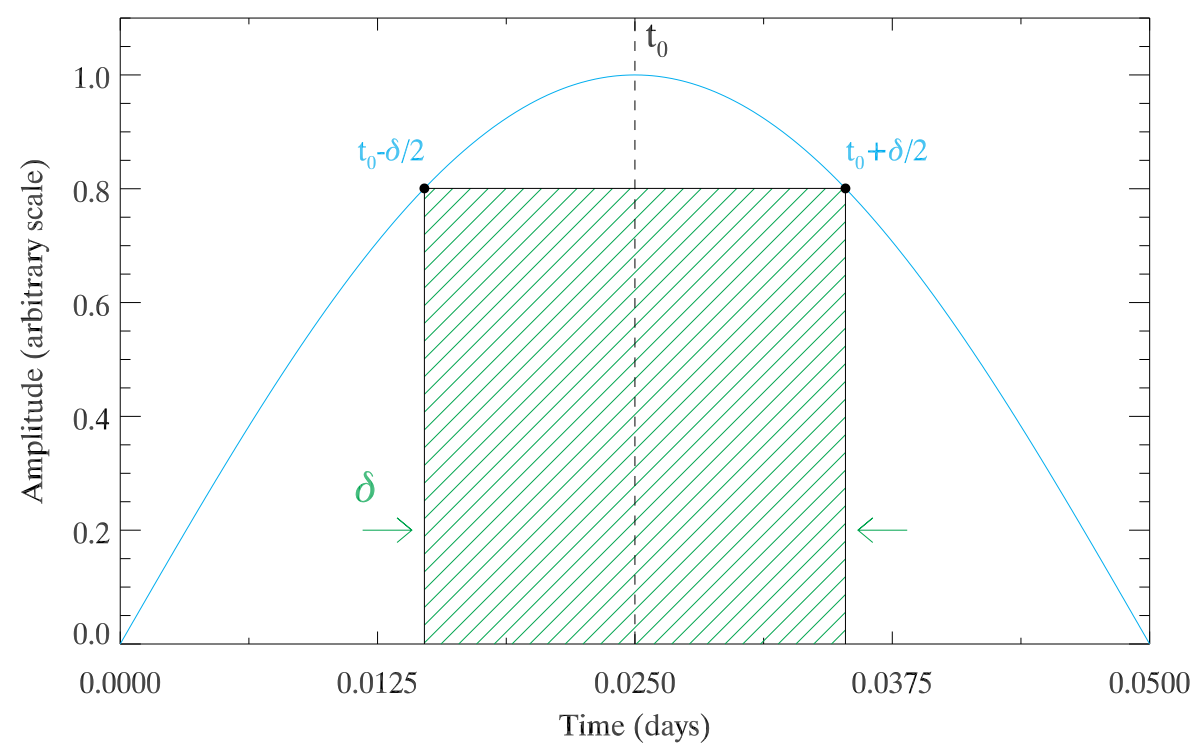

Figure 1.4: If we assume the amplitude of a pulsation mode is a sinusoidal function in time, with a frequency here of $10 \mathrm{~d}^{-1}$ and arbitrary amplitude of 1 , then undersampling leads to an underestimate in amplitude. The interval, $\delta$, between two consecutive points in this example is that of Kepler LC data. 


\section{CHAPTER 1}

The observed amplitude of the peak in Fig. 1.4, which is centred around a time $t_{0}$, and sampled by two points equidistant from its centre, is the height of the green rectangle of width $\delta$. That height is the quotient of the rectangle's area by its width. For a brightness function $\cos (\omega t)$, the height of the rectangle is given by the expression:

$$
\begin{aligned}
H & =\frac{1}{\delta} \int_{t_{0}-\frac{\delta}{2}}^{t_{0}+\frac{\delta}{2}} \cos (\omega t) d t \\
& =\frac{1}{\delta} \frac{1}{\omega}\left\{\sin \left[\omega\left(t_{0}+\frac{\delta}{2}\right)\right]-\sin \left[\omega\left(t_{0}-\frac{\delta}{2}\right)\right]\right\}
\end{aligned}
$$

Using the standard trigonometric identity $\sin (X+Y)=\sin X \cos Y+\cos X \sin Y$, this becomes

$$
\begin{aligned}
H=\frac{1}{\delta} \frac{1}{\omega} & {\left[\sin \left(\omega t_{0}\right) \cos \left(\frac{\omega \delta}{2}\right)+\cos \left(\omega t_{0}\right) \sin \left(\frac{\omega \delta}{2}\right)\right.} \\
& \left.-\sin \left(\omega t_{0}\right) \cos \left(\frac{\omega \delta}{2}\right)+\cos \left(\omega t_{0}\right) \sin \left(\frac{\omega \delta}{2}\right)\right] \\
=\frac{1}{\omega \delta} & {\left[2 \cos \left(\omega t_{0}\right) \sin \left(\frac{\omega \delta}{2}\right)\right] } \\
= & \cos \left(\omega t_{0}\right) \operatorname{sinc}\left(\frac{\omega \delta}{2}\right)
\end{aligned}
$$

So for an initial brightness function $\cos (\omega t)$, the reduction factor is of the form $\operatorname{sinc}\left(\frac{\omega \delta}{2}\right)$, which as $\omega=\frac{2 \pi}{P}$ is $\operatorname{sinc}\left(\frac{\pi \delta}{\mathrm{P}}\right)$, with $P$ being the period of the oscillation mode.

We can now use the fact that $\delta$, the time interval between points, is related to the number of points per cycle, $n$, by the expression $\delta=\frac{P}{n}$, to conclude that the true amplitude $\left(A_{0}\right)$ of a sinusoidal brightness variation is related to the observed amplitude $(A)$, by:

$$
A=\operatorname{sinc}\left(\frac{\pi}{n}\right) \mathrm{A}_{0}
$$

Using KIC 5724810 (from Fig. 1.3) as a numerical example, the ratio of the observed 


\section{CHAPTER 1}

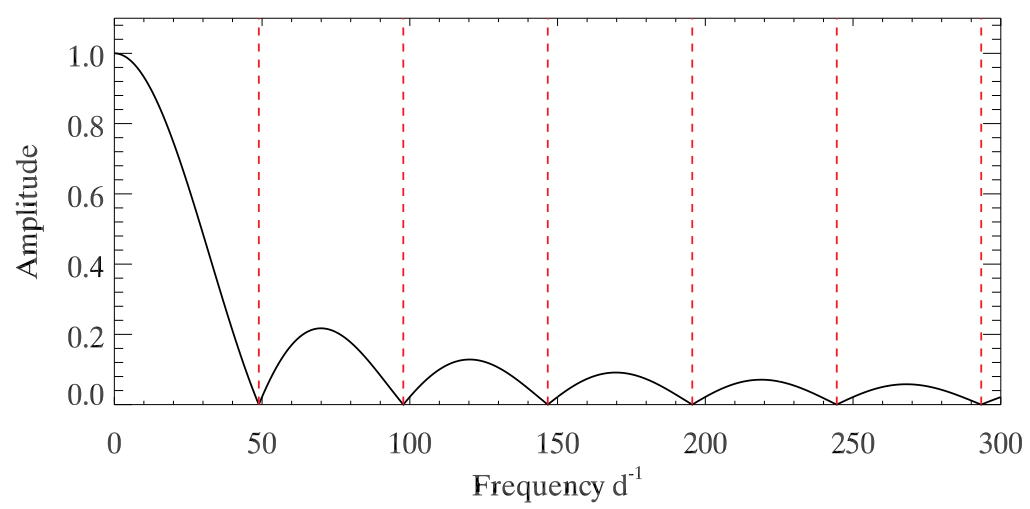

Figure 1.5: The observed amplitude (black line) of a peak whose intrinsic amplitude is 1.0, shown as a function of frequency for Kepler LC data. As a result of the decreasing number of data points per pulsation cycle, $n$, the amplitude is suppressed at higher frequencies. However, it is only completely suppressed at integer multiples of the sampling frequency (dashed red lines). Peaks well above the Nyquist frequency can still have significant amplitudes; their aliases will have this same amplitude.

amplitude in SC to that of LC, $A_{\mathrm{SC}} / A_{\mathrm{LC}}$, is 1.48 for the peak at $22.84 \mathrm{~d}^{-1}$, up from $A_{\mathrm{SC}} / A_{\mathrm{LC}}=1.38$ for the peak at $21.15 \mathrm{~d}^{-1}$.

KIC 10977859 (Fig. 1.2) demonstrates the same effect for high-frequency pulsations that are reflected in the Nyquist frequency. The highest peak in the bottom panel is located in frequency where expected from the reflection of the highest peak in the top panel (to well within one FWHM, at $-f_{\mathrm{S}}+f_{\mathrm{R}}$, where $f_{\mathrm{S}}$ and $f_{\mathrm{R}}$ are the sampling and real frequencies, respectively), and has an amplitude reduction in agreement with Eq.1.1 to within the least-squares errors. The nature of the amplitude reduction as a function of frequency is illustrated in Fig. 1.5.

One can therefore do asteroseismology on p modes above the LC Nyquist frequency using LC data, providing one has at least one month of SC data to overcome the aliasing problem. Alternatively, when one has a time span greater than 1 Kepler orbit (i.e. more than 4 quarters), one can distinguish aliases from real peaks using LC data alone, as we shall see in $\S 1.5$. 


\section{CHAPTER 1}

\subsubsection{Precision}

Having a greater number of points allows a more precise determination of pulsation frequencies, amplitudes and phases. To give a quantitative example, one month of LC data was compared to one month of SC data for the star KIC 3437940 and points were only removed to truncate the LC Q3 data set to exactly the same timespan as the SC Q3.2 data set. Since the nature of any frequency peaks in the Fourier transform depends greatly on the length of the data set, it is imperative to ensure that comparisons are performed on data sets of the same length. Regardless of cadence, each quarter is divided into thirds by Kepler's downlinking process, thereby the cadences corresponding to Q3.1 and Q3.3 in the LC data were easily removed. The errors on frequency, amplitude and phase for the different cadences are summarised in Table 1.1, and are on the order of 5 times greater for the LC data. The result applies to both SAP and PDC flux, indicating the precision difference is not pipeline-generated, and is robust beyond this single example. This is an important discovery pointing to greater quality of the SC data, and implies the data are not normally distributed. Degroote et al. (2009) found a similar result for CoRoT noise properties.

The scatter of points in SC data is greater than that of LC data. There are 30 times more points in SC data, so the scatter of points is expected to be $\sqrt{30}$

Table 1.1: The formal least-squares errors on frequency, amplitude, and phase in SC and LC data from Q3.2 for the star KIC 3437940, with the LC errors being greater in each case by factors of $\sim 5$. The least-squares errors were calculated with PERIOD04, on data processed with the PDC-LS pipeline.

\begin{tabular}{lccc}
\hline Cadence & $\begin{array}{c}\text { Frequency error } \\
\times 10^{-5} \mathrm{~d}^{-1}\end{array}$ & $\begin{array}{c}\text { Amplitude error } \\
\mu \mathrm{mag}\end{array}$ & $\begin{array}{c}\text { Phase error } \\
\times 10^{-3} \mathrm{rad}\end{array}$ \\
\hline SC PDC-LS & 4.9 & 14 & 0.4 \\
LC PDC-LS & 25.8 & 70 & 2.2 \\
SC SAP & 4.6 & 13 & 0.4 \\
LC SAP & 25.8 & 70 & 2.2 \\
\hline
\end{tabular}




\section{CHAPTER 1}

times greater if the noise is assumed to be white ${ }^{5}$. This is particularly noticeable in those stars that are approximately constant. In stars that pulsate, one has to be careful when discarding those points that appear to be outliers - there should be more outliers in $\mathrm{SC}$ because of the greater number of points, but sampling the brightness variations more often produces higher amplitude peaks in both the light curve and the Fourier transform. If one 'sigma clips' the data too closely from the beginning, the extrema of those peaks in the light curve might be lost. Clipping at $3 \sigma$ is too tight - if the data were normally distributed, one discards 1 in 200 points that naturally belong to the distribution in this manner; these points may lie further from the mean, but are not necessarily erroneous outliers. Outlier removal should be done alongside frequency fitting in an iterative fashion. A discussion of the validity of sigma clipping and other outlier removal procedures can be found in Hogg, Bovy \& Lang (2010).

\subsubsection{Noise and pipeline performance reviews}

\section{Very low frequency noise}

Another benefit of SC data over LC is the reduced number of high-amplitude peaks at low frequency. Such peaks can arise naturally from long time-scale processes such as differential velocity aberration, with stars moving across the CCD by up to 1.5 pixels, which results in a different flux fraction being captured by the CCD, and the amount of background contaminating light changing. García et al. (2011) cite CCD degradation as a cause of long time-scale drifts too, but CCD degradation is likely to arise from high-energy cosmic ray impacts and will often, as a result, be more of a step-function than a long-term trend. SAP data do contain strong instrumental trends that dominate at low frequency, rendering the difference in prevalence of low-frequency peaks between the two cadences insignificant in SAP. However, the

\footnotetext{
${ }^{5}$ The noise is not white, but this serves as a useful approximation.
} 


\section{CHAPTER 1}

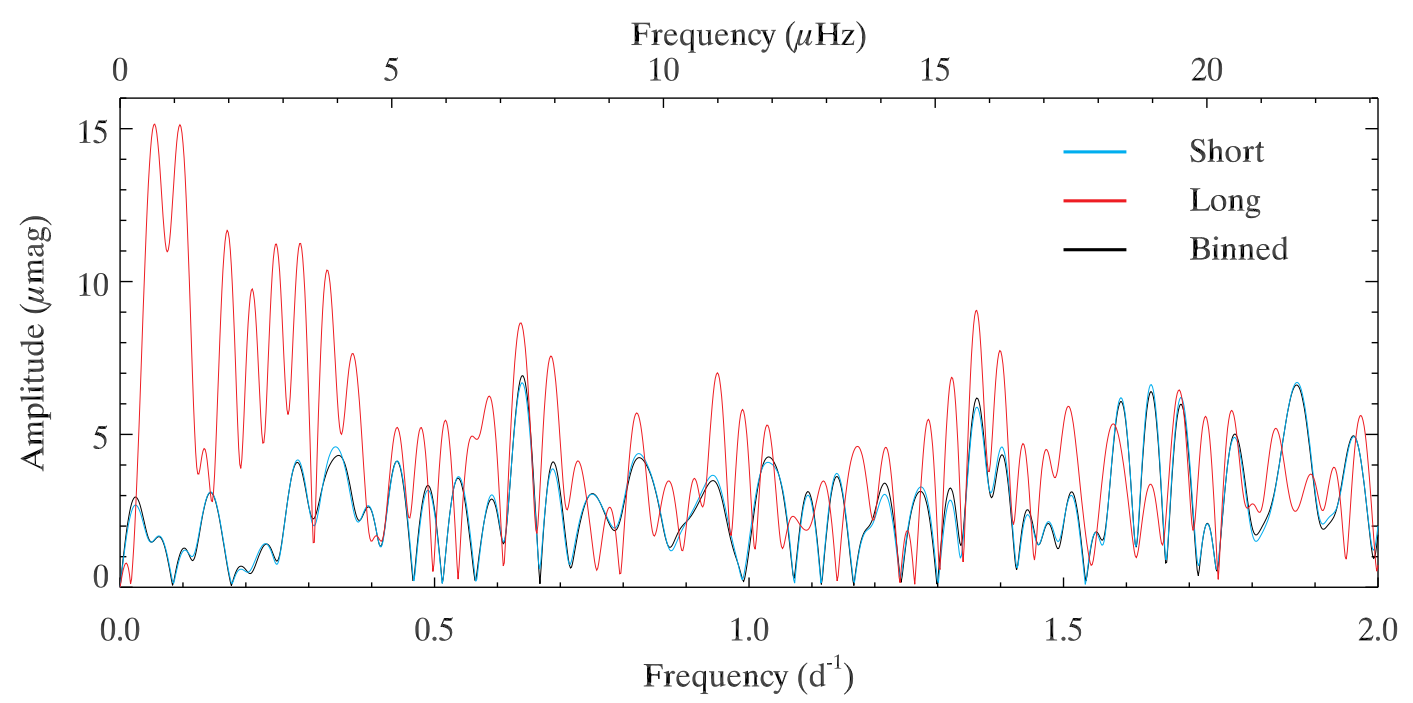

Figure 1.6: The PDC-LS SC data (blue) are plotted on top of the PDC-LS LC data (red) of Q2.2 for the near-constant star KIC 9390100 for frequencies 0-2 d ${ }^{-1}$. Plotted in black are simulated LC data created by binning the SC data. The blue and black lines almost completely overlap, but the displacement of the red line indicates the extent of artificial low-frequency peaks introduced by the PDC-LS pipeline.

LC PDC-LS data, from which instrumental trends had mostly been removed, still contained some relatively high-amplitude peaks at low frequency.

In order to demonstrate this phenomenon, a nearly constant star was selected to minimize the number of peaks seen as a result of pulsations. For this demonstration using KIC 9390100, it was necessary to truncate the length of the Q2 LC data to that of the SC Q2.2 data, using the same method from $\S 1.2 .3$ for KIC 3437940. No outliers were removed from either data set. A third data set was made for comparison by binning sets of 30 consecutive $\mathrm{SC}$ points into a single point; the fluxes of the SC points were added and their times averaged. This binning process creates points that are entirely concurrent with the LC data (for every LC point there is a binned-SC point at exactly the same time), and the binned and Kepler LC data sets shown in Fig. 1.6 contain exactly the same number of points. To achieve this, two more points had to be removed from the LC data set, at BJD $=2455042.3243$ and 2455051.4377, because undefined SC flux values in these bins did not allow accurate binned data 


\section{CHAPTER 1}

points to be created, but Kepler LC points existed at those times. The $29 \times 2 \mathrm{SC}$ data points corresponding to each of these LC points were also removed for a fair comparison in this PDC-LS data example.

At lower frequencies (below $2 \mathrm{~d}^{-1}$, and especially below $0.4 \mathrm{~d}^{-1}$ ) the SC and LC Fourier transforms are significantly different (Fig. 1.6). There are a few coherent peaks common to both cadences, but the LC data have many more high-amplitude ${ }^{6}$ peaks at those frequencies under $2 \mathrm{~d}^{-1}$. This difference between SC and LC Kepler data is often encountered in the constant stars.

At higher frequencies (above $2 \mathrm{~d}^{-1}$ ) the two amplitude spectra are almost the same, and can be seen converging in the figure. The binned data, which one expects to be similar to the LC data because they are created by integrating fluxes over the same cadence-numbers, mimic the SC data very well over the entire frequency range. Slight differences can be accounted for by considering that there are occasionally SC data points next to data gaps that do not get incorporated into binned data, because the binned data must be concurrent with the LC data for a direct comparison.

The Kepler LC data should not behave exactly like the binned data, because LC and SC data go through a different calibration process (involving such methods as dark/bias subtraction and flat-field removal), but the scale of the difference suggests that discrepancies do remain after the PDC correction procedure. These discrepancies are not seen to the same extent in the SAP data; the difference in peak heights in the PDC example in Fig. 1.6 is $12 \mu \mathrm{mag}$ (400 per cent) compared with $6 \mu$ mag in SAP (only 2 per cent of the peak height), noting that instrumental trends dominate in SAP data. That some discrepancies remain in the PDC data is not surprising, as they were designed to facilitate planet-finding, not asteroseismology. While the residual peaks contribute significantly to the noise on time-scales important to asteroseismology, planetary transit searches are not greatly affected. Gilliland et al.

\footnotetext{
${ }^{6}$ relatively speaking: amplitudes of $10^{-5} \mathrm{mag}$ are normally considered tiny in the analyses of $\delta$ Sct stars!
} 


\section{CHAPTER 1}

(2011) compared noise on 6.5-h time-scales, chosen to be representative of planet transit durations. This corresponds to a frequency of $3.7 \mathrm{~d}^{-1}$, but all three lines in Fig. 1.6 are already converging at $2 \mathrm{~d}^{-1}$. In fact, a planet orbiting a sun-like star and having a transit time of $1 \mathrm{~d}-\mathrm{a}$ time-scale where the discrepancies are an important source of excess noise - would have a semi-major axis of $3.4 \mathrm{AU}$ and a period of $6.3 \mathrm{y}$. The detection and confirmation of such a planet is pushing the boundaries of Kepler's design capabilities even if the mission continues to compete successfully for extensions.

In addition to contributions to the total noise level, the low-frequency artefacts can cause other problems, an example of which is in automatic frequency extraction procedures that select the highest amplitude peak or peaks in the Fourier spectrum to classify a star. If the spectrum is dominated by non-astrophysical low-frequency peaks, then false classification may occur and lead to incorrect statistics on both dominant frequencies and amplitudes. Due to the vast number of light curves produced by Kepler and indeed other missions, an increasing amount of effort is being put into developing such automated routines (e.g. Huber et al. 2009; Blomme et al. 2010).

The PDC-LS pipeline for LC data has now been superseded by the PDC-MAP pipeline. PDC-MAP sees a $\sim 10-20$ per cent improvement in signal-to-noise on the 6.5-h time-scales reported on in Gilliland et al. (2011) (Jon Jenkins, priv. comm.), and supports their conclusions that the major contributor to the observed excess noise still originates in the stars themselves. However, we shall see in the following that in the first year of its introduction, PDC-MAP has been substantially worse at treating low-frequency artefacts than PDC-LS. 


\section{CHAPTER 1}

\section{Pipeline data}

\section{Overview}

It is the job of the PDC pipeline, irrespective of version, to remove systematic trends from the data. The trends can be caused by differential velocity aberration, where the target moves across the CCD and as a result, a different amount of flux is captured by the pre-defined 'optimal' mask. The optimal mask is not designed to capture all of the light, but to maximise signal-to-noise. Trends are also caused by thermal effects, such as the change in temperatures encountered during monthly downlinking and occasional safe-mode events. In the SAP data, exponential returns to science operating temperature and focus can be seen following these gaps, which are discussed in $\S 1.4$. The PDC pipelines should remove these, but the PDC-LS pipeline did a much better job than PDC-MAP currently does. The LS pipeline used temperature (and other) information from the spacecraft to fit these trends rather well. The MAP pipeline currently incorporates effects common to many neighbouring stars, but since different stars fall on different locations on the CCD, and react differently to temperature fluctuations, this pipeline has struggled to mitigate such trends. Even so, both of the PDC pipelines are a marked improvement on the SAP data as far as trends are concerned.

\section{Instrumental effects in detail}

There are many instrumental effects present in Kepler light curves. Distinguishing these from genuine stellar variation or planetary transits is no easy task. The Data Characteristics Handbook is the most complete repository for such information, and also keeps track of lists of known spurious peaks belonging to the SC and LC data. Here, the most significant effects on Kepler light curves are laid out, with the qualification that this list is non-exhaustive. The effects are: 


\section{CHAPTER 1}

1. The aforementioned differential velocity aberration and quarter-to-quarter variations. Although differential velocity aberration describes the variation within one quarter, when the spacecraft performs a roll the target falls on a different detector. In addition to this, the amount of flux in the defined aperture changes from quarter to quarter and discontinuities in flux are seen as a result. The PDC pipelines correct for both: a) the amount of light contributed from the intended target and not contaminating stars - the median flux over a month or quarter is multiplied by $(1-$ contamination $)$ and subtracted from each cadence; and b) the fraction of flux not captured by the 'optimal aperture'. The SAP-flux data contain no such corrections.

2. Loss of fine pointing, which occurs during the regular 'reaction wheel angular momentum desaturation' events. These 'desats' are necessitated as solar radiation torque causes angular momentum to build up in the reaction wheels. Fine pointing control is not maintained for a few hours and the telescope goes into coarse point mode (Christiansen et al., 2012). Desats occur approximately every three days, and in the fits files obtained at MAST, these events are flagged and are then easily removed. ${ }^{7}$

3. Reaction wheel zero-crossings, during which the spacecraft shakes for a few hours (not corrected in PDC data, but are flagged). When the reaction wheels cross zero angular velocity, the wheel may rumble and degrade the pointing on timescales of minutes. Consequently, SC data are much more strongly and frequently affected than LC data.

4. Cosmic ray events, which cause a step-function change in the flux level, which decays exponentially back to $90-100$ per cent of the original level (corrected in PDC data and flagged).

\footnotetext{
${ }^{7}$ More information on flags can be found in the Kepler Archive Manual http://archive.stsci. edu/kepler/manuals/archive_manual .pdf, table 2-3. Accessed 2012/10/29.
} 


\section{CHAPTER 1}

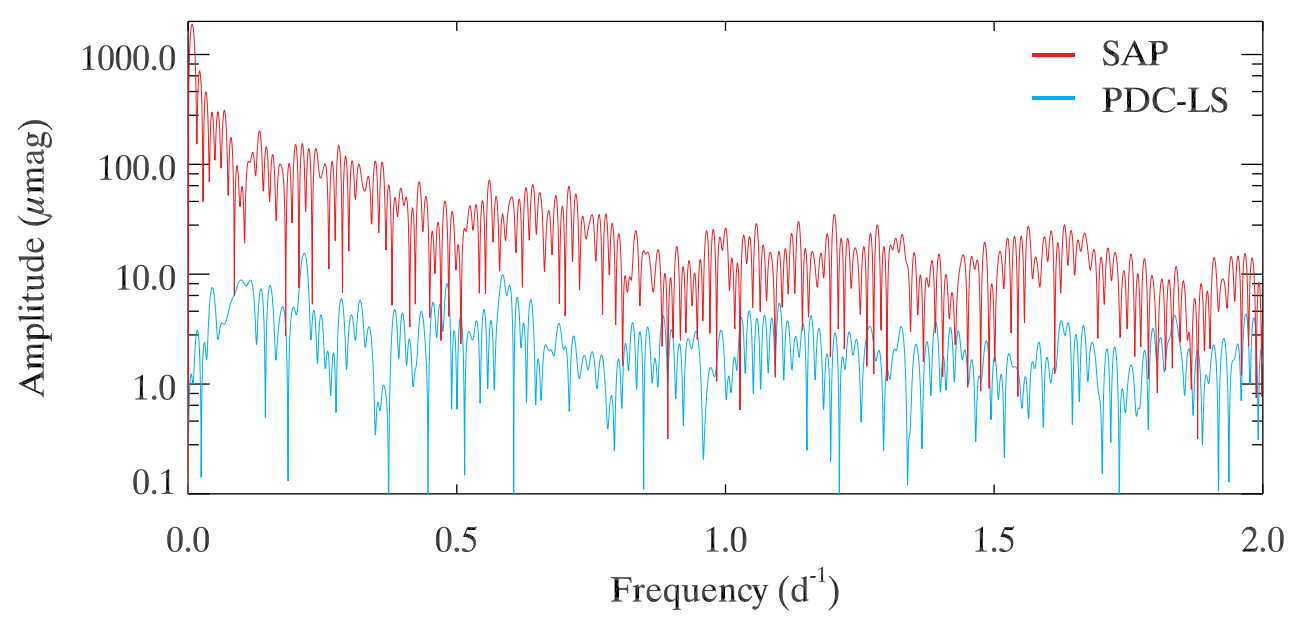

Figure 1.7: The PDC-LS data (blue) have been plotted over the SAP data (red) for the Kepler object KIC 7450391 during Q2. No points were removed from either data set. The low-frequency peaks in PDC LC data mentioned in $\S 1.2 .4$ are present but insignificant by comparison to the difference between PDC and SAP data.

5. Monthly Earth downlinks, identifiable by a gap in the data of up to $\sim 24 \mathrm{~h}$ followed by an exponential increase/decrease in flux level as the telescope returns to science operating focus and temperature (corrected in PDC data, but see 'Overview' $§ 1.2 .4)$.

6. Attitude tweaks, which have thus far occurred only twice in the entire mission, during science operations in Q2, and are no longer expected to be a problem (not fully corrected in PDC data).

The PDC-LS data were a marked improvement on SAP data in terms of mitigating noise as shown in Fig. 1.7. The PDC-LS pipeline was replaced by PDC-MAP because the former could remove some stellar signal, too, if that signal mimicked some of the instrumental effects described above. 


\section{CHAPTER 1}

\section{PDC-LS pipeline injecting noise}

In addition to the potential to remove long-term stellar variation, the PDC-LS data had another problem. Murphy (2012b) showed how the PDC-LS pipeline injected noise in the frequency range $0-24 \mathrm{~d}^{-1}$ (up to $\sim 277 \mu \mathrm{Hz}$ ) into $\sim 15$ per cent of lightcurves. The affected light curves show visible distortion (Fig. 1.8) provided pulsation amplitudes are not so large as to hide the extra noise, and the Fourier transform shows a related noise excess (Fig. 1.9). One of the characteristic features of the injected noise is the drop-off in power around $24 \mathrm{~d}^{-1}$. Specifically, in a logarithmic plot of the power spectrum an extremely rapid reduction in power is seen (Fig. 1.10). This was used to identify stars affected by the noise injection. The search focussed on stars with SC data available and KIC temperatures between 5500 and $9500 \mathrm{~K}$. The power-reduction was hard to detect in LC due to the low Nyquist frequency, but the LC data did show the injected noise.

Fig. 1.11 shows how for the star KIC 3429637 the PDC-LS pipeline injects noise into the SC Q7 data. The noise is restricted solely to PDC-LS data and affects Q7 but not Q8 for this star. The grass level (defined in Fig. 1.9 caption) is higher in PDC-LS Q7 LC than in SAP Q7 LC (after pre-whitening), so the effect is present
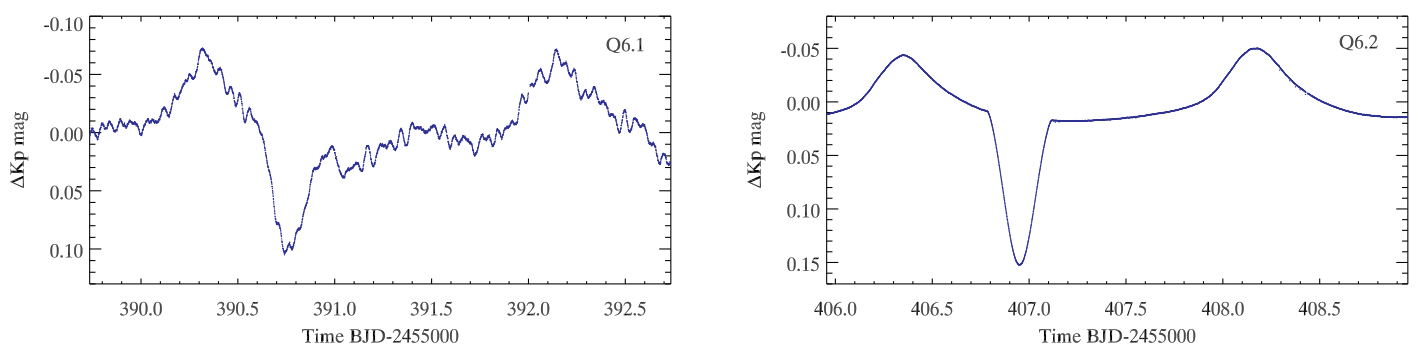

Figure 1.8: Three-day segments of the light curves for the detached eclipsing binary system KIC 11285625 in SC PDC flux. In Q6.1 the pipeline is injecting noise, distorting the light-curve, whereas the Q6.2 light-curve looks comparatively very clean. The corresponding Fourier transforms are shown in Fig. 1.9. It is well-known that PDC-LS does not treat binaries well, but the noise injection is not limited to eclipsing binary systems. 


\section{CHAPTER 1}

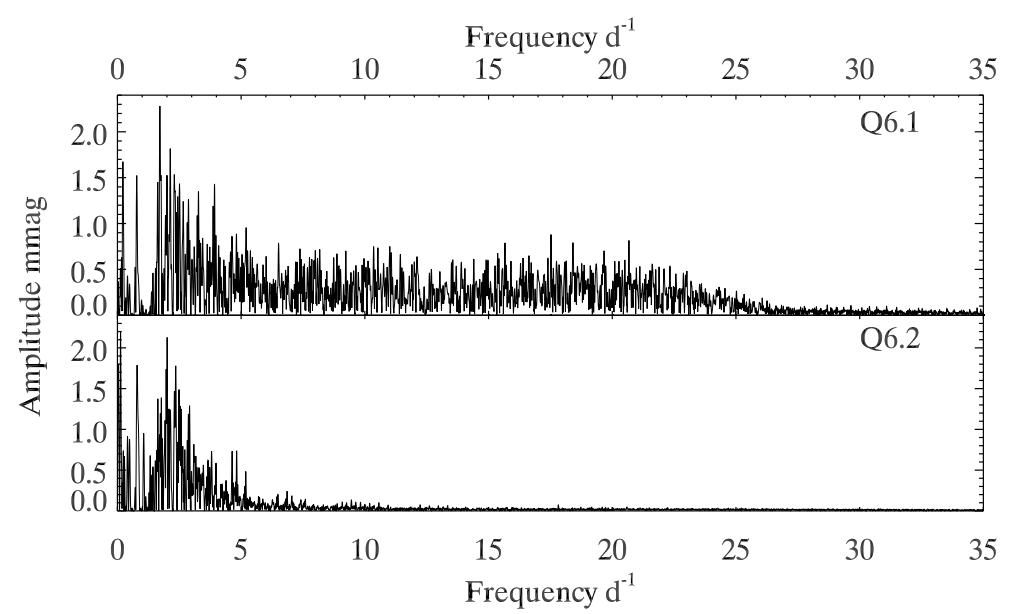

Figure 1.9: The prewhitened Fourier transforms for Q6.1 and Q6.2 corresponding to the light curves in Fig. 1.8, and typical for stars in which the noise injection is seen. Pulsations and many harmonics of the orbit have been prewhitened; the appearance of the Fourier transform below $\sim 5 \mathrm{~d}^{-1}$ is strongly affected by those harmonics that remain. Upper panel: in the affected Q6.1 data, there is an elevated 'grass level' the amplitude of the Fourier peaks that somewhat resemble mown grass - up to a frequency of $\sim 24 \mathrm{~d}^{-1}$ after which there is a sharp drop-off in power. Lower panel: the Q6.2 data do not suffer the noise injection. The sharp power-reduction is not noticeable in LC data (not shown) because of the low Nyquist frequency of $24.4 \mathrm{~d}^{-1}$.

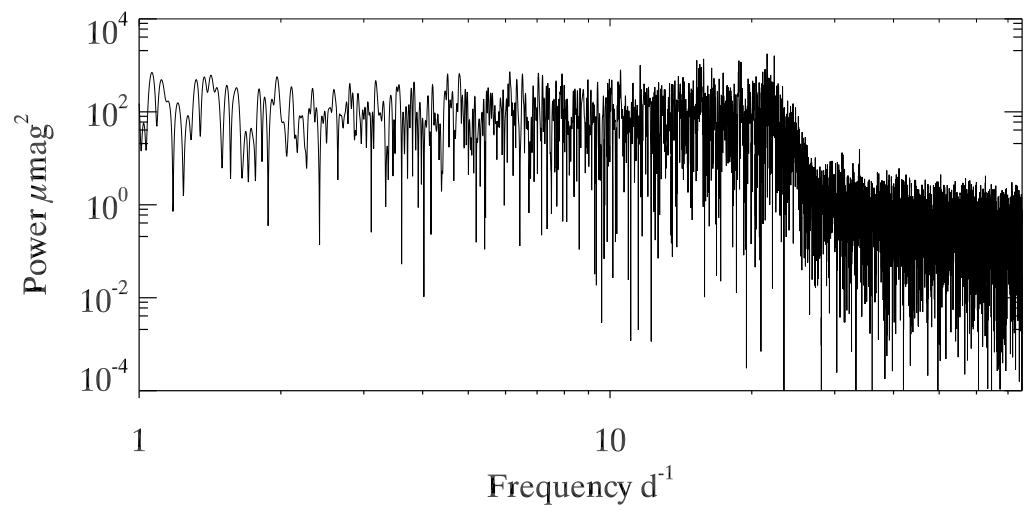

Figure 1.10: The injected noise is easily identified by a rapid power reduction at $24 \mathrm{~d}^{-1}$ in the Fourier transform, plotted here in log-log space. The example given is KIC 3429637 Q7 SC data, after pre-whitening, that is, fitting and removing the statistically significant (signal-to-noise $\geq 4$ ) sine curves from this $\delta$ Sct star. 


\section{CHAPTER 1}

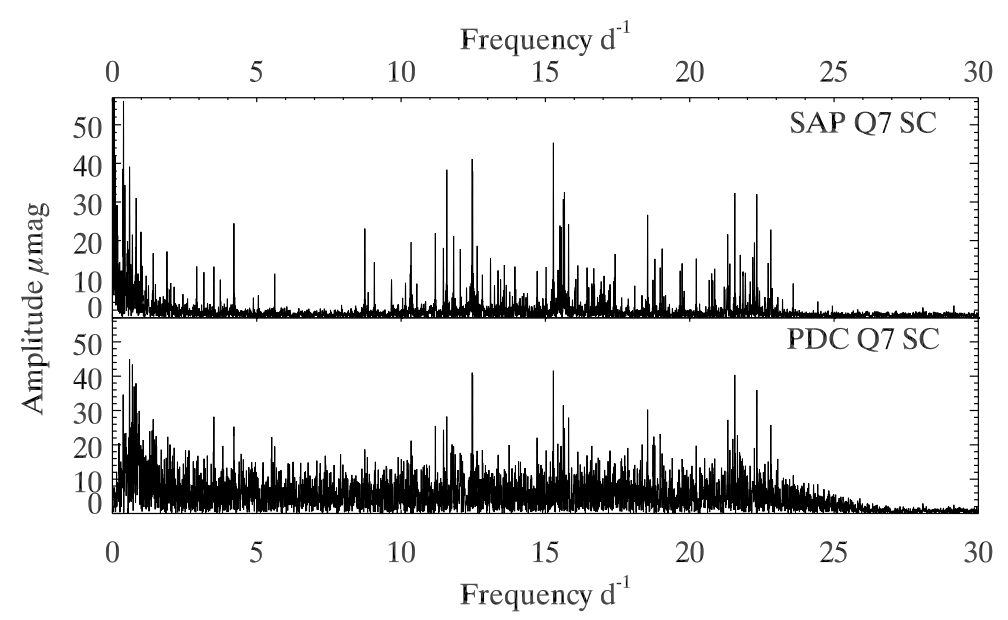

Figure 1.11: Linear Fourier transforms for KIC 3429637, showing the extent of the injected noise: the grass level is $\sim 15 \mu$ mag in the PDC-LS data, compared to just $2 \mu \mathrm{mag}$ in the SAP data. The decrease in power at $\sim 24 \mathrm{~d}^{-1}$ is clear. The effect is present in Q7 in both SC and LC PDC-LS data for this star, but not present at all in Q8.

in LC too, even if the low Nyquist frequency hides the power drop.

It is not known why some stars are affected while others are not, nor why, for the same star, only some quarters are affected and that these vary for different affected stars. No correlation in fraction of stars affected was seen with $T_{\text {eff }}$, nor was any correlation seen with sky/CCD position (Fig. 1.12).

Sometimes the pipeline fails to fit a light curve and passes the light curve through without modification. In the lower panel of Fig. 1.9, it can be seen that although noise is not injected, very low-frequency peaks, which are often caused by trends in the data, are not removed either. In this case, the light curve has perhaps emerged untouched by the pipeline.

\section{Performance of PDC-MAP}

At the time of writing, PDC-MAP has only recently become available for public data. In comparing PDC-MAP with the older PDC-LS and the SAP data (Fig. 1.13), it is clear that PDC-MAP has strong trends (over many data points) following the 


\section{CHAPTER 1}

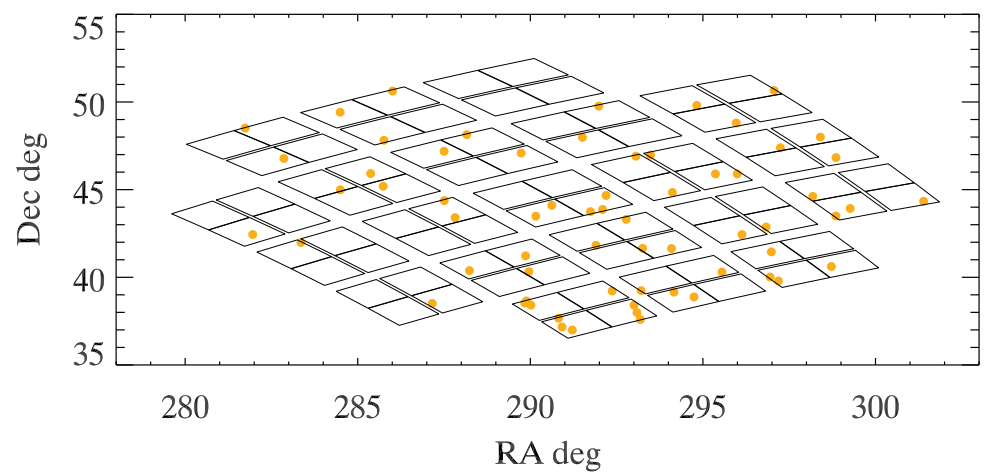

Figure 1.12: The spatial distribution of the affected stars (examples possessing SC data are plotted as orange circles) is the same as the spatial distribution of all stars across Kepler's CCDs, which is denser toward the galactic plane lying toward the lower-right of the figure.

gaps in the data that arise from the scheduled data downlinking events. In the older pipeline these trends were fixed quite well. Such teething problems might be expected with new software. PDC-MAP identifies systematic behaviour common to many stars to create a fit to subtract from the data, but there appears to be a great variation between the response of different pixels to the thermal effects that cause the trends, and this effect is convolved with differential velocity aberration, making the light-curves particularly hard to treat.

In $\S 1.2 .4$ we investigated the difference between SAP and PDC-LS data in Fourier space, noting that the noise in the latter was reduced by factors of 10-100 over the former. Here, we extend that comparison to also include the PDC-MAP data (Fig. 1.14). It appears that although PDC-MAP is noisier at very low frequency due to the remaining thermal and focussing effects, at higher frequency (above $\sim 2 \mathrm{~d}^{-1}$ ) it out-performs PDC-LS, and has the added reassurance that it preserves stellar variability more satisfactorily than PDC-LS. From Q15 onwards, a cubic fit has been calculated and subtracted from the data in a pipeline version called 'multi-scale' MAP. It is therein assumed that long-term trends on the scale of around one month are systematic and are thus removed. 


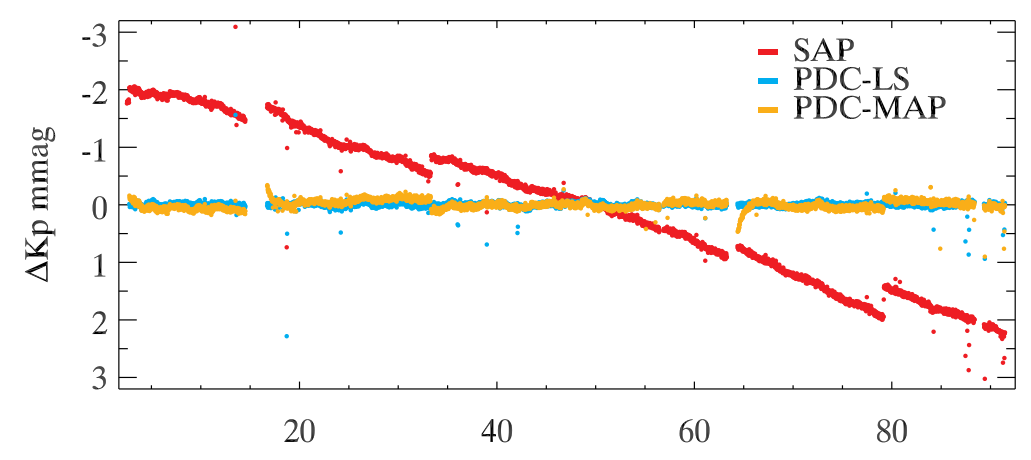

(Q2) Time BJD-2455000

Figure 1.13: Although PDC-MAP removes many of the long-term trends satisfactorily, it has initially struggled to mitigate thermal effects. For this reason, small exponential thermal recoveries are seen at the data downlink events in PDC-MAP, just as they are in the SAP data. The example shown is KIC 7450391 - a nonpulsating late-A star, for Kepler Quarter 2, noting that Q2 is the most extreme example and that in other Quarters PDC-MAP does better than this.

\subsubsection{Conclusions on data characteristics}

The SC data are almost always better than the LC data. The SC slot availability is the major limitation one faces when using and obtaining $\mathrm{SC}$ data. We have seen the necessity of the increased sampling rate of SC data for resolving short time-scale events such as flares, and for precise transit timing.

The Nyquist frequency of LC data can be problematic and certainly limiting in asteroseismic analysis of short data sets (longer data sets will be discussed in $\S 1.5)$. Pulsations detected near the Nyquist frequency can be indicative of higher frequency pulsations that require $\mathrm{SC}$ data, and one must consider that peaks in the periodogram may be aliases of frequencies above the Nyquist frequency. However, a lack of pulsation frequencies near the Nyquist frequency does rule out the presence of higher order p modes.

There is an amplitude difference between SC and LC data associated with the longer effective integration time of LC data. The result is that peaks in the periodogram have higher amplitudes in SC data, and the percentage difference between 


\section{CHAPTER 1}

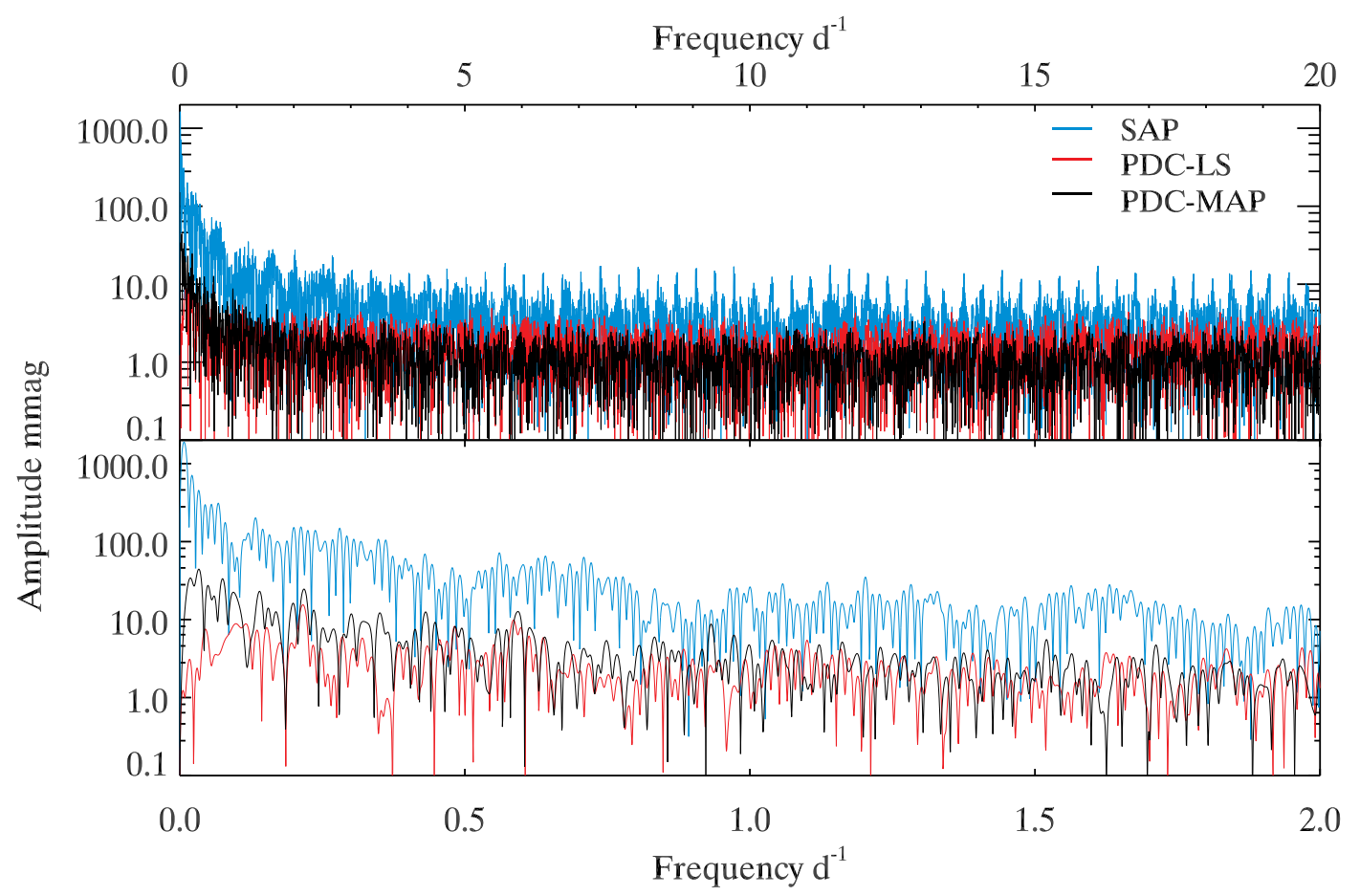

Figure 1.14: Overlaid Fourier plot for SAP, PDC-LS and PDC-MAP for KIC 7450391, Quarter 2, corresponding to the light-curves in Fig. 1.13. Upper panel: below $\sim 2 \mathrm{~d}^{-1}$ PDC-MAP is slightly noisier than PDC-LS, but has lower noise above that frequency. Lower panel: zoom-in on the region below $2 \mathrm{~d}^{-1}$. SAP is substantially noisier, and the convergence in performance of PDC-MAP and PDC-LS can be seen as low as $0.5 \mathrm{~d}^{-1}$. PDC-MAP can thus be said to be at least as good as PDC-LS on time-scales important to astrophysics.

the two cadences grows with increasing frequency.

Effects that are not directly concerned with the different sampling rates have also been observed. LC PDC-LS data often contain spurious peaks of non-astrophysical origin at very low frequency that are not always present with similar amplitudes in SC PDC-LS data, though the PDC-LS data still outperform the PDC-MAP data on these timescales. The low-frequency artefacts can affect studies of long-period brightness variations arising from such things as spots on slow rotators, though one can argue such signals can be falsely removed by PDC-LS in the most extreme cases. Automatic frequency extraction routines must consider these artefacts. 


\section{CHAPTER 1}

The greater number of points in SC data does not produce narrower peaks in the periodogram and cannot improve resolution of two closely spaced frequencies. Frequencies can be determined with greater precision though, as can their corresponding amplitudes and phases. For this to be true, the data cannot be normally distributed.

Noise in the periodogram can be vastly reduced by analysing the PDC data instead of SAP data. The PDC data have fewer drifts, jumps and outliers, generating cleaner light curves and Fourier spectra. The PDC-LS data treated these drifts and jumps more satisfactorily than the PDC-MAP data, but they may also modify astrophysical signals. Furthermore, the LC PDC-LS data, which are now reprocessed with the MAP pipeline, suffered from injected noise in the range $[0,24] \mathrm{d}^{-1}$. New (post-Q13) SC PDC data implement the MAP pipeline, but older quarters may still suffer from such noise injection. One is advised, whenever using PDC data, to check against SAP data that no artificial signal has been injected, or stellar signal removed. Using the data quality flags from the fits files on MAST may prove useful in reducing noise.

The PDC-LS pipeline left artificial peaks in the LC data. These peaks could be relatively high in amplitude but were observed in quiet stars. The PDC-MAP pipeline, with its poorer treatment of instrumental effects, actually leaves substantially higher peaks at low frequency. Pipeline improvement is ongoing.

\subsection{Characterising Kepler targets}

In the previous section, much was written about the characteristics of the data themselves. The data characteristics can depend on the targets observed, too. A detailed discussion of Kepler mission design, including star field selection, can be found in Koch et al. (2010). The field was chosen to look along the galactic plane, specifically the Orion arm of our galaxy, but with an inclination such that stars 


\section{CHAPTER 1}

more than a few kpc away are out of the plane and hence the number background giants is limited. The distance to a $V=12$, A5 dwarf, having absolute magnitude $M_{V}=+2.2$, is then about $1 \mathrm{kpc}$.

In this section, we shall look at the distribution of Kepler targets as a function of effective temperature and Kepler magnitude (Kp mag), and also investigate the quality of the Kepler data as a function of the observing season (Kepler mission Quarter). A more rigorous analysis was performed for solar-like stars by Gilliland et al. (2011) earlier in the mission, where they found noise levels 50 per cent higher than was budgeted for in telescope design, perhaps indicating that the Sun is an unusually quiet star. Here, we give special emphasis to the A stars and we shall see how the precision of the data varies with these three observables ( $T_{\text {eff }}, \mathrm{Kp}$ mag and season) by investigating the distribution of peaks in Fourier transforms of the light curves of the stars.

\subsubsection{Noise amplitude as a proxy for data quality}

Many approximations to the noise present in the Fourier transforms can be used. The grass level, the average amplitude over a frequency range, and the 'rms (root mean square) noise' are all approximations used that have varying utility when estimating the noise level for thousands of Fourier transforms. An estimate for the data quality might take the amplitude of the highest noise peaks as a good proxy, but at a certain point (usually signal-to-noise ratio of 4.0, where 'noise' in this context is the mean of the amplitude in the Fourier transform in a given frequency range) these peaks become 'significant' and are no longer classed as noise. An alternative would be the grass level, but the grass level is not precisely defined and its value is usually determined by eye. I coded an algorithm to agree with the empirically determined grass level of each Fourier transform.

Fourier transforms are calculated over a given frequency range, and amplitudes 


\section{CHAPTER 1}

are calculated at a 'step rate' or 'size', that is, a frequency interval normally relating to the time-span, $T$, of the data set. A step size of $1 /(20 T)$ is commonly used (step size is discussed further in $\S 1.4 .5$ ). The process I have adopted in the algorithmic determination of the noise is as follows: the Fourier transform, calculated as above, is divided into 25 equal segments in frequency, and within each segment the 95th percentile of the calculated amplitudes is chosen as corresponding to the grass level. Finally, the median of the collection of 95th percentiles is taken as being representative of the whole frequency range.

How valid is this method? The 95th percentile was chosen to empirically match the observed grass level in non-pulsating stars, noting that the algorithm will overestimate the noise for pulsating stars. The validity is illustrated in Fig. 1.15.

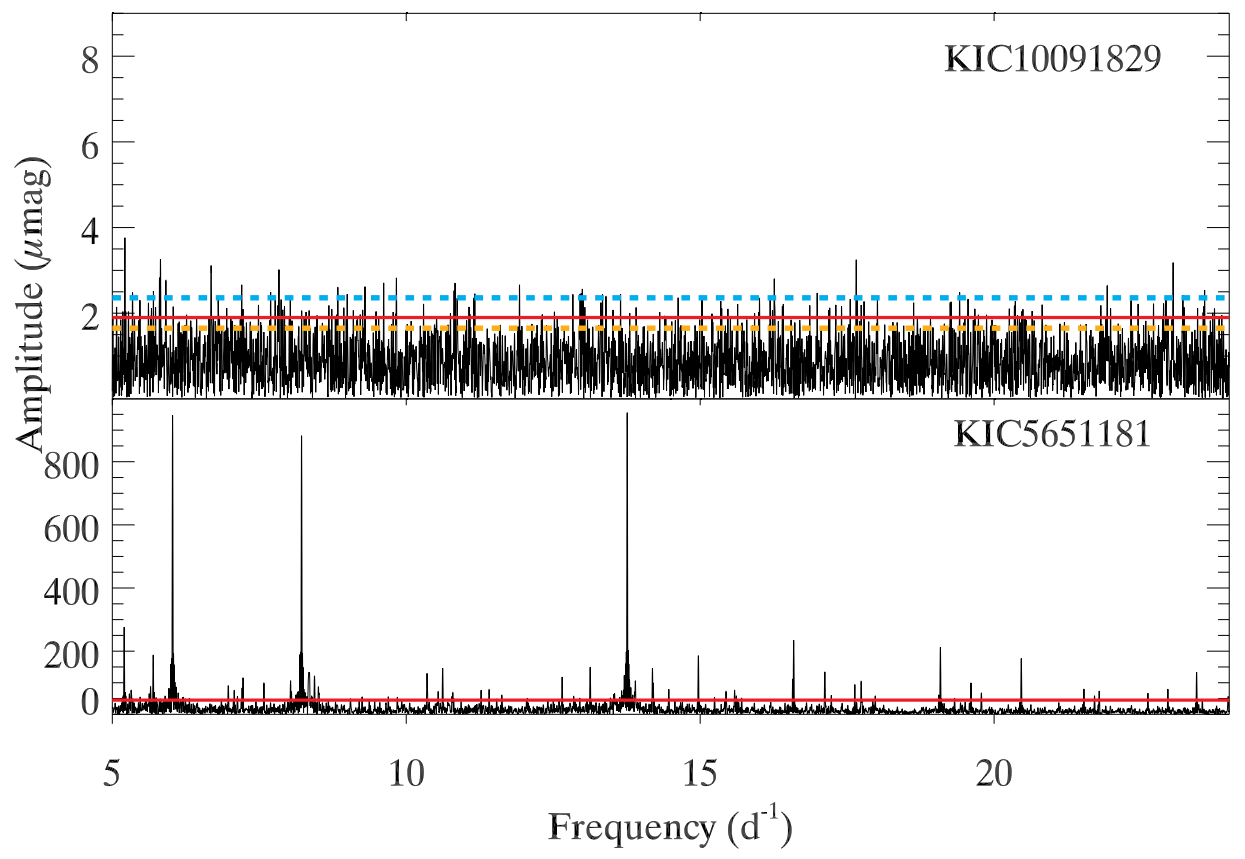

Figure 1.15: Upper panel: the algorithmic determination (red line) of the grass level for a randomly selected non-pulsating star; and lower panel: the determination for a randomly selected $\delta$ Sct star. The empirical match is good for the non-pulsating star, but overestimated for the $\delta$ Sct star because it includes all the variance in the data. For reference, the 90th and 99th percentiles are also plotted for the non-pulsating star, as the orange and blue lines, respectively. Data are PDC-MAP, Q3, LC. 


\section{CHAPTER 1}

In order not to bias the observations with pipeline-generated signals, the calculations were not performed from $0 \mathrm{~d}^{-1}$, but from $5 \mathrm{~d}^{-1}$ instead. Furthermore, PDC-MAP data were used because of claims that PDC-LS can remove astrophysical signal, and observations of it injecting noise (§1.2.4). Fig. 1.13 showed that although PDC-MAP does not perfectly remove trends of thermal effects from the light curve, it substantially out-performs SAP, and is less noisy at higher frequencies (Fig. 1.14).

It is necessary to check that the grass level can be reliably determined for stars of various magnitudes. Furthermore, since the grass level is overestimated in pulsating stars, we must define some boundary between stars that are pulsating and those that are not. Since faint stars can have noise levels of tens of $\mu$ mag without being pulsating stars, $50 \mu \mathrm{mag}$ was chosen as an appropriate limit on what constitutes a pulsating star; if the amplitude of the highest peak in the Fourier transform of the star's light curve is below $50 \mu \mathrm{mag}$, it is labelled as 'constant'. 50.0 per cent of stars in the KIC temperature range 7000 to $10000 \mathrm{~K}$ are 'constant' by this definition this number changes to 44.1 per cent for the range 7000 to $8500 \mathrm{~K}$, which covers the $\delta$ Sct instability strip (a $\delta$ Sct review is given in Chapter 4 ). Fig. 1.16 compares the accuracy of grass-level estimates for constant stars of three different magnitudes, with different empirical grass levels. The choice of the 95th percentile removes susceptibility to noise spikes and low amplitude pulsation. The grass level is not especially well-defined in the case of the 15th magnitude star presented, in that it could be drawn a few $\mu$ mag above or below where the 95 th percentile situates it, but the algorithmic estimation is appropriate. Thus, even when the noise peaks reach tens of $\mu \mathrm{mag}$, the algorithm performs satisfactorily. 


\section{CHAPTER 1}

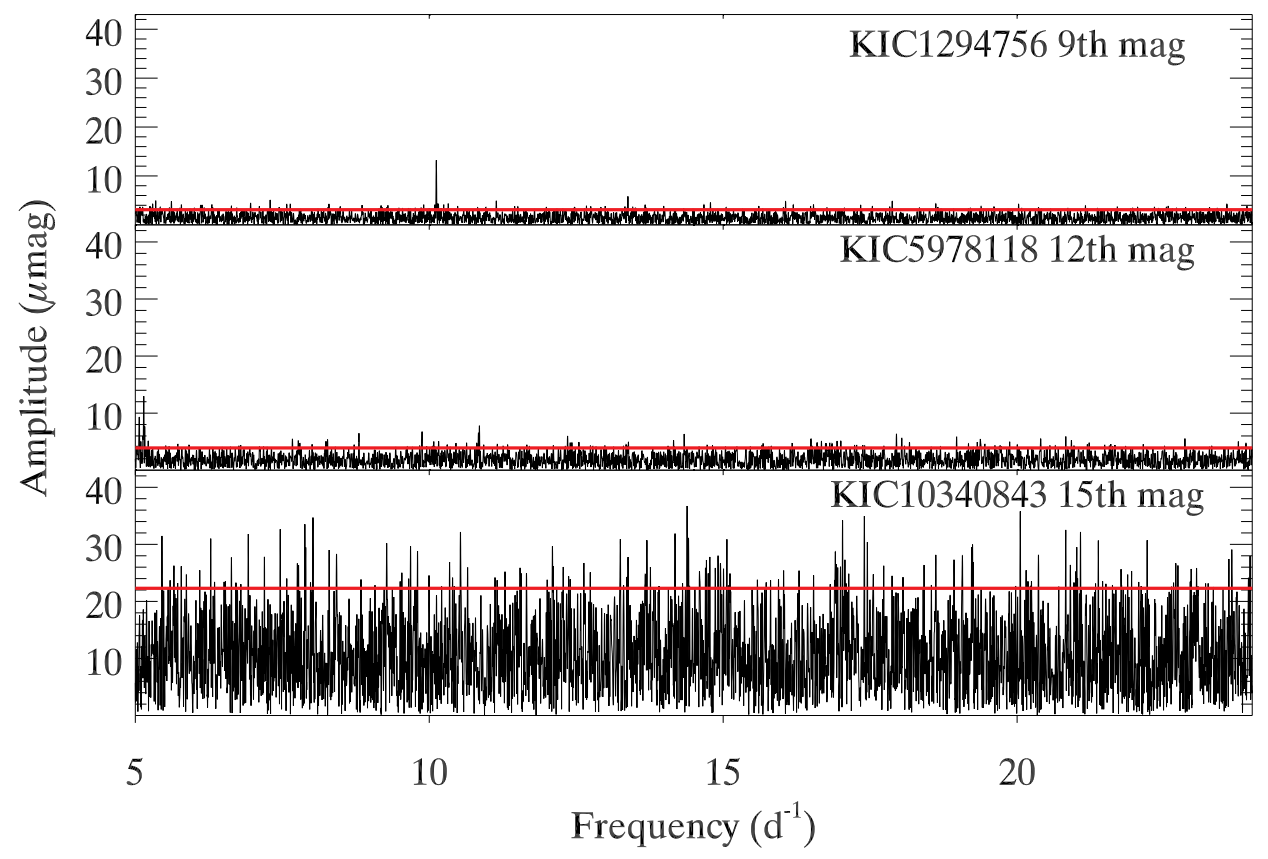

Figure 1.16: The algorithm determines an appropriate grass level in the presence of statistically significant signal (upper panel), as well as for cases of high noise levels as seen in fainter stars (bottom panel). The ambiguity of the grass level is illustrated in the bottom panel, where one can see the level could conceivably be drawn anywhere between about 21 and $30 \mu \mathrm{mag}$.

\subsubsection{Noise as a function of $T_{\text {eff }}$}

The algorithm was applied to the A stars to see how the noise changes with $T_{\text {eff }}$. Stars were binned by temperature in $250-\mathrm{K}$ bins. A byproduct of doing this is it demonstrates the distribution of stars in $T_{\text {eff }}$ (Fig. 1.17). The number of stars decreases towards higher $T_{\text {eff }}$, as one expects. The figure shows that the noise amplitude is decreasing towards stars of higher $T_{\text {eff }}$, where the effects of stellar activity and convection at the surface, especially granulation, are expected to diminish. However, when the pulsating stars are included (upper-left panel), issues arise with the algorithmic noise estimate: as indicated in Fig. 1.15, it is overestimated. Thus in the upper-right panel the same calculation was performed for the 'constant' stars only. In the bottom panel, the analysis is extended down to $6000 \mathrm{~K}$ using only constant 


\section{CHAPTER 1}
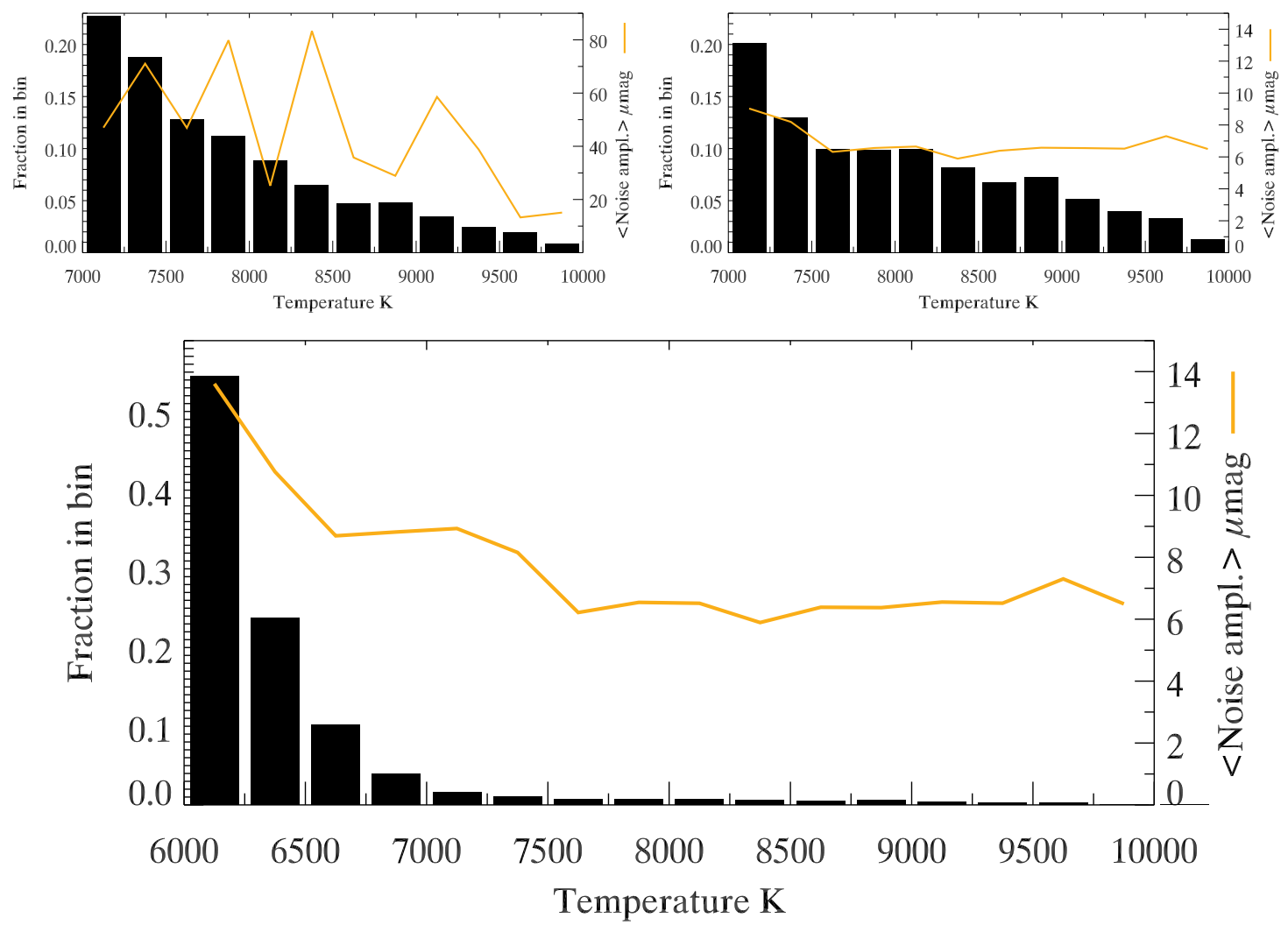

Figure 1.17: Upper-left panel: Histogram of the distribution in $T_{\text {eff }}$ of all A stars; upper-right panel: the same plot but including only those stars whose highest peak is below $50 \mu \mathrm{mag}$; bottom panel: as upper-right panel but extended down to $6000 \mathrm{~K}$. The orange line indicates the mean algorithmic noise amplitudes for stars in each bin (measured on the right y-axis; notice the change in scale between different panels). See the text for further explanation. Data are PDC-MAP, Q3, LC.

stars. One must also consider that these KIC temperatures are underestimated by a couple of hundred K (Pinsonneault et al., 2012).

The upper-left panel clearly demonstrates the issue of including pulsating stars, in that an unreliable noise level is determined; this is alleviated in the other panels. Comparison of bar heights between the upper-left and upper-right panels shows greater removal of stars between 7250 and $8500 \mathrm{~K}$ (i.e. stars in the $\delta$ Sct instability strip) in the upper-right panel.

The noise rises noticeably at the cooler end of Fig. 1.17, as one would expect as the surface convection zone is larger in cooler stars (on the order of 1 per cent in 


\section{CHAPTER 1}

radius for the cool A stars, compared to 30 per cent in the Sun). This rise in noise, which this author believes is attributable to granulation (that is, the surface feature resulting from turbulent convection), is particularly evident in the bottom panel.

\subsubsection{Noise as a function of magnitude and mission quarter}

The same method can be applied to find the number of stars in bins of different magnitude and Kepler mission quarter. Here, only the constant stars are used to avoid the unreliability associated with pulsating stars. Fig. 1.18 illustrates how the noise amplitude is a strong function of Kp mag, and that the magnitude distribution of the Kepler A stars peaks at magnitudes too faint for abundance analyses using 2-m class, ground-based telescopes, that limit being $\sim 10$ th magnitude for a signalto-noise ratio of 100 in about an hour's observing.

Up until now, comparisons have been made with Q3 data for consistency; using data of different quarters would affect the noise level. One expects the shorter Q0 and Q1 data to have higher noise levels, and it is known that Q2 was a 'bad' quarter, because of the safe-mode events that occurred, though those data have been reprocessed multiple times now. The constant stars make great diagnostic tools for the quality of different quarters, since they have no pulsations that might alter the

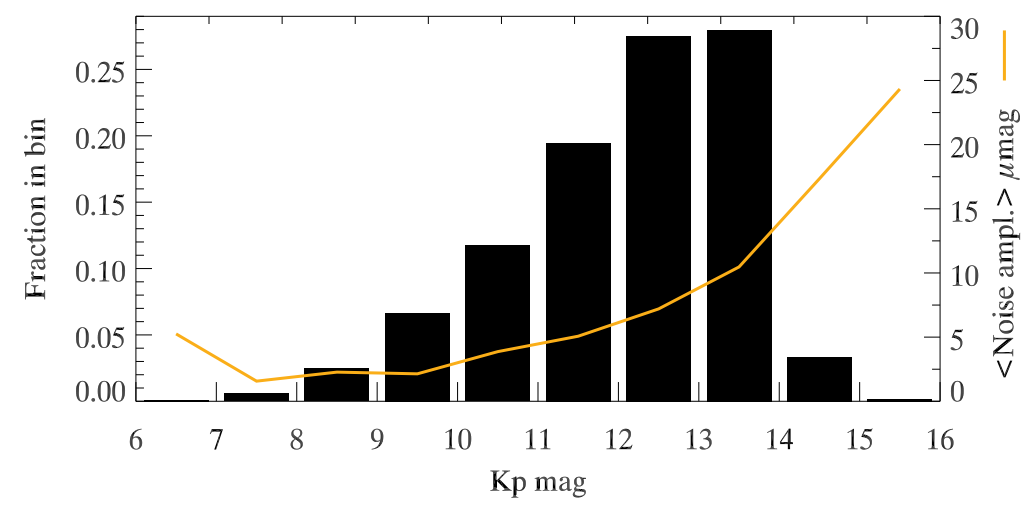

Figure 1.18: Histogram of the distribution in Kp mag of the Kepler A stars. The noise grows rapidly at fainter magnitudes. Data are PDC-MAP, Q3, LC. 


\section{CHAPTER 1}

measured 'noise' levels. As such, stars that were determined to be 'constant' from the Q3 data were used in the analysis, taking their PDC-MAP data for each mission quarter up to the latest release at the time of the investigation: Q13. There is no guarantee that a star observed in Q3 has been observed in every quarter. It could be missing observations from one in four quarters, through being on Module 3 (the full implications of which are described in more detail in $\S 1.4 .3$ ), or it could have been dropped as a target for some reason, possibly being reinstated later.

The number of stars included in the noise calculations for each quarter is given in Table 1.2, and aside from Q0, only varies by 5-10 per cent between one quarter and the next. The noise level in each quarter is presented in Fig. 1.19. The shorter quarters (Q0 and Q1) clearly have higher noise levels, but the PDC-MAP pipeline acts to smooth out differences between quarters. The noise level for Q3 is, of course, as one would expect from Fig. 1.18 if one multiplies the fraction of stars in each magnitude bin by the mean noise amplitude for that quarter, i.e., the mean noise is biased by the fainter stars whose grass levels are higher.

Since noise levels depend on the length of the data set, the analysis was extended to compare quarters as if they all had the same number of data points. Quarters were normalised against Q9 because it has the most data points, having started early following a safe-mode event at the end of Q8. Comparing the quarters in this way has no particular practical meaning, but as a proxy for the noise per data point, the red triangles in Fig. 1.19 provide a different view of the quarter quality.

Table 1.2: The number of stars in each quarter used for noise calculations for Fig. 1.19.

\begin{tabular}{cccccccc}
\hline Quarter: & 0 & 1 & 2 & 3 & 4 & 5 & 6 \\
\hline Number: & 1291 & 1633 & 1689 & 1703 & 1661 & 1640 & 1469 \\
\hline Quarter: & 7 & 8 & 9 & 10 & 11 & 12 & 13 \\
\hline Number: & 1517 & 1603 & 1582 & 1450 & 1499 & 1582 & 1596 \\
\hline
\end{tabular}




\section{CHAPTER 1}

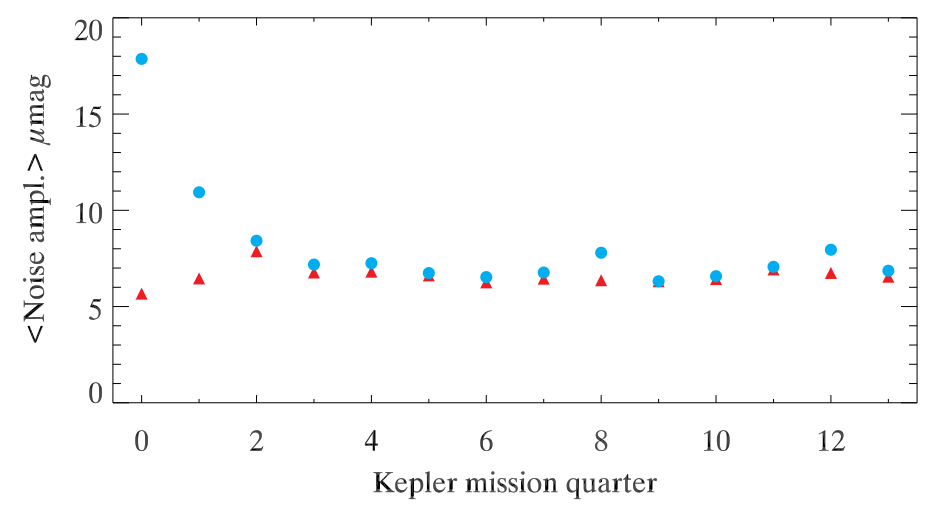

Figure 1.19: Mean grass level vs. Kepler mission quarter for the stars having no peak above $50 \mu \mathrm{mag}$ in Q3 (blue circles). The noise is higher in Q8 because that quarter has fewer data points than other post-Q2 quarters, owing to a safe-mode event. The red triangles are a proxy to the relative noise per data point (see text). Data are PDC-MAP.

\subsubsection{Discussion}

We have seen the shape of the $T_{\text {eff }}$ distribution for Kepler targets above $6000 \mathrm{~K}$. Using an algorithm that estimates the grass level in Fourier transforms, and pre-selecting constant stars, we have seen that the mean noise level in the Fourier transform grows as a function of Kp mag, is made constant by the pipeline for different quarters with the exception of differences arising from data set length, and noise grows towards decreasing temperature, which has been attributed to granulation (and activity, though the frequency range chosen avoids any contribution from light-variations induced by rotation and spots). The latter finding is not strictly new, but it is the first time it has been demonstrated in this manner. Balona (2011) also described the median noise of Kepler data, $\sigma_{\mathrm{M}}$, which, ignoring readout noise, is parametrized as

$$
\log \left(\sigma_{\mathrm{M}}\right)=a+\frac{1}{5} K p-\frac{1}{2} \log (\Delta T)
$$

where $\Delta T$ is the length of the data set (determining the number of data points), $K p$ is the Kepler magnitude and $a$ is a constant. Since we are using a constant time 


\section{CHAPTER 1}

interval (i.e. Q3), then $\log \left(\sigma_{\mathrm{M}}\right)$ should correlate linearly with the magnitude, with a constant. Balona fitted 175 of the 'least variable' A-F stars using SC data ${ }^{8}$, and found

$$
\log \left(\sigma_{\mathrm{M}}\right)=-1.33+0.21 K p-0.47 \log (\Delta T)
$$

Until now I have been algorithmically calculating the grass level, that being considered a more useful measure of the noise and being approximately 2.5 times the median noise level (Balona, 2011). In order to verify Balona's results, I made the same calculations of noise against Kp mag using the median. The performance of my algorithm for determining the grass level was already analysed in Figs 1.15 and 1.16, and deemed accurate. The median is a statistical value whose validation is not required, but I found that, on average for the 1691 constant stars fainter than $K p=8.0$ tested, the grass level is between 2.0 and 2.5 times the median level. Fitting the noise vs. Kp mag for these stars, I found

$$
\log \left(\sigma_{\mathrm{M}}\right)=-1.80+0.18 K p
$$

noting again that using the same time base for all stars eliminates the $\Delta T$ term. Thus for a twelfth magnitude star in Q3, our results differ by 25 per cent. The differences in results are easy to explain qualitatively: Balona used SAP data, whose noise level even at higher frequencies has been shown to be higher than the PDCMAP data used here; using the LC data offered a ten-fold increase in the number of stars to be used in the statistics, however, the top 175 were not cherry-picked from this sample, so perhaps Balona's analysis used quieter stars. That Balona's data were Q0 and Q1 was taken into account by his $\Delta T$ parameter, but using SC limited his sample, so that to pick 175 stars from my 1691 would create an equally uneven comparison.

\footnotetext{
${ }^{8}$ the exact selection criteria were not explicitly given
} 


\section{CHAPTER 1}

In agreement with findings presented here, Balona found that noise increases as temperature decreases. His calculations were based on a different frequency interval, though, from $20-80 \mathrm{~d}^{-1}$ rather than the $5-24 \mathrm{~d}^{-1}$ used here. His interval is, in theory, more suited to granulation studies according to his calculations of where the maximum power in granulation should be, but it is worth noting that there is no observed 'bump' in power at the supposed frequency of maximum power from granulation. In the absence of pulsation, the difference in noise amplitude between the two frequency intervals is not significant.

In conclusion, there is evidence for granulation among the cool A stars, but it has not been possible to determine whether this is linked to pulsation in any way. The ability to select the non-pulsating stars from the Kepler A stars has been very useful, and will continue to be so. Ideas on the significance of the constant stars will be developed in later chapters. For now, we shall continue to characterise the Kepler data, moving away from the targets and on to other properties of the data.

\subsection{Gaps in the data}

Kepler data, while nearly continuous, do suffer some gaps. Some of these are scheduled, such as those at the monthly downlinking events and quarterly rolls of the spacecraft, which see a break of up to $24 \mathrm{~h}$ in the observations. Some gaps, however, are not planned. These can be short: the data flagged as 'poor' due to 'desats' (see $§ 1.2 .4$ ), are such an example, as some users will delete these flagged points. The gaps can also be large, such as the occasional safe-mode event: the one at the start of Q8 resulted in nearly $18 \mathrm{~d}$ of data outage, counting from the end of Q7. In this section, we shall look at the effect on the Fourier transform of having gaps in the data, particularly for short runs, using simulated data. We will also investigate procedures to mitigate the aliases associated with gaps. First though, let us inspect the Fourier transform in the case of non-gapped data. 


\section{CHAPTER 1}

\subsubsection{The non-gapped case}

In Fig. 1.20, the upper panel corresponds to a Fourier transform taken for one quarter of simulated Kepler data, at LC and without any gaps. The lower panel is 2.5 orbits of the spacecraft around the Sun, hence ten times longer. The simulation mimics Kepler's heliocentric orbit with the same period and same observing cadence as the LC-mode, but without noise and the restriction of the mission's limited duration. The side-lobes and surrounding window pattern in each panel are the effect of having a finite observing span. Since the full-width at half-maximum of the peaks is determined by the observational time span, $T$, as $\sim 1 / T$, all peaks (central and window pattern) in the lower panel are 10 times narrower.

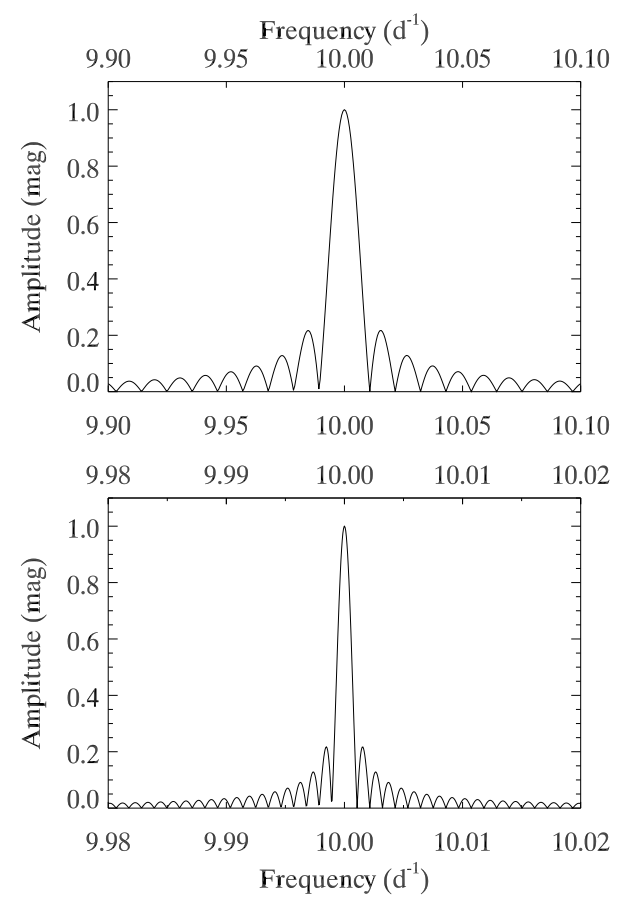

Figure 1.20: Upper panel: A pulsation frequency of $10 \mathrm{~d}^{-1}$ with an amplitude of $1 \mathrm{mag}$, in data covering one Kepler quarter in LC, without gaps. Lower panel: As upper panel, but for ten quarters. Notice the change in scale between the two panels. 


\section{CHAPTER 1}

\subsubsection{Kepler is safe from cycle count ambiguities}

Now that we know what to expect from non-gapped data, let us investigate the situation with large gaps, like those resulting from safe-mode events. In Fig. 1.21, a single gap occupies 25 per cent of the observing time, and its location within the data is indicated by the gaps in black bars in the upper right of each panel. The left panels correspond to one quarter, the right to ten quarters. Large gaps have two main consequences. The first is that a rare event might be missed, such as the transit of an Earth-like planet. The second is the loss of useful data for frequency determination. With a large gap in the middle of the data, it becomes harder to determine a pulsation frequency precisely because there is a certain ambiguity, known as the cycle count ambiguity, in counting the pulsation cycles that have passed over the duration of the gap. To illustrate the cycle count ambiguity, Fig. 1.22 plots two different frequency fits to the same simulated data. The upper panel fits the correct frequency $\left(1 \mathrm{~d}^{-1}\right)$ to the gapped LC data, whereas the lower panel incorporates one extra cycle over the gap. The observational time span was arbitrarily chosen to be 0.1 Kepler orbits (for both panels).

Having the gaps of the same absolute size but in a much longer observational time span would diminish the cycle count ambiguity - the data surrounding the gap then become sufficient to determine the frequency precisely. This is why gaps were kept the same relative size in Fig. 1.21. The only difference between the left and right panels is then the frequency resolution (and the zoom chosen). The gap introduces a cycle count ambiguity, meaning that for those data, within the frequency precision attainable, there could be one or more extra (or fewer) cycles within the gap, in which case the frequency would be slightly higher (or lower). The window pattern then looks much different from that in Fig. 1.20 as the central peak (known to us in the simulation as the real peak) now has much larger side-lobes, whose amplitude ratios to each other also change. The cycle count ambiguity is creating a scenario 


\section{CHAPTER 1}

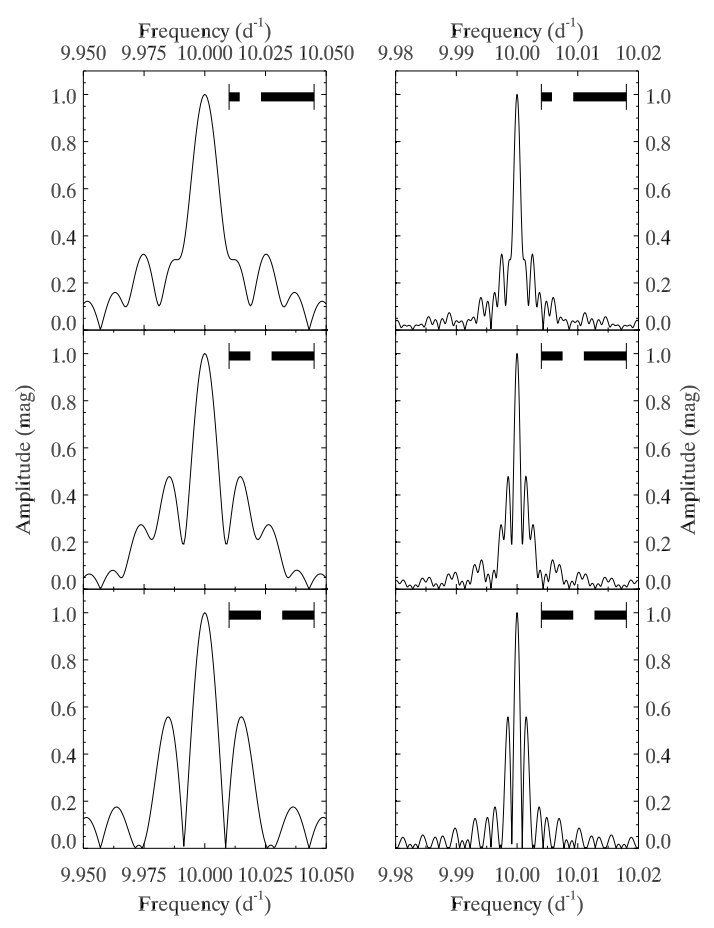

Figure 1.21: Fourier transforms of simulated data of one Kepler quarter (left) and ten quarters (right) in LC-mode. Gaps were simulated at different positions (relative coverage indicated by axis-independent black bars in the upper right of each panel), and comprise 25 per cent of the total observing time. Locations of gaps were shifted towards the centre by one eighth of the time span between vertically adjacent panels, starting at a gap location of $0.125-0.375$.

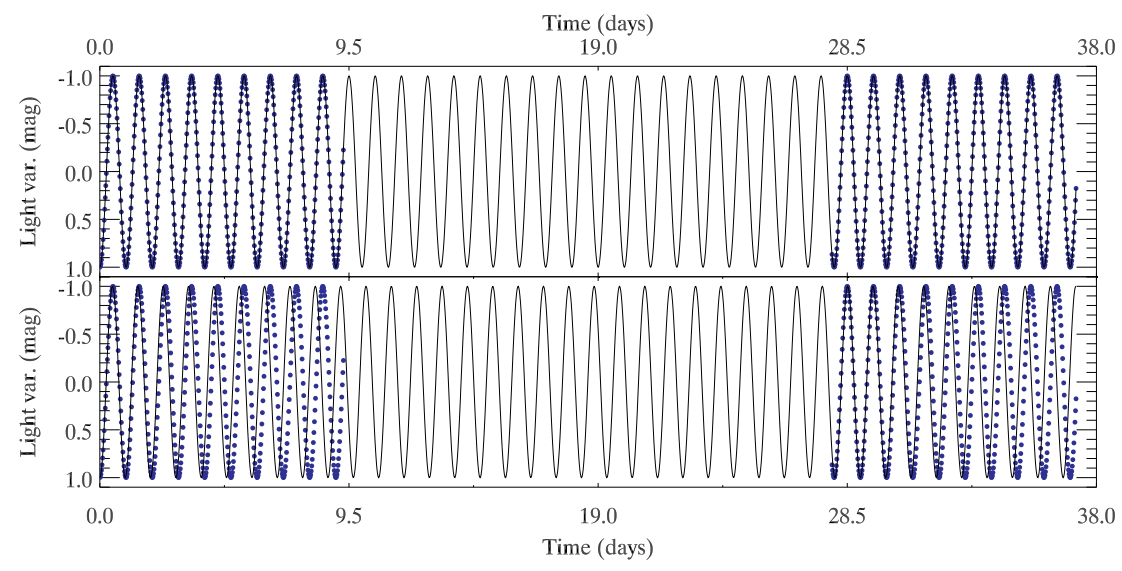

Figure 1.22: The same simulated LC data (blue dots) are plotted in both panels. The upper panel fits the true frequency of $1 \mathrm{~d}^{-1}$ (black line), but the fit in the lower panel has an extra 1.25 cycles across the whole light curve $\left(f=1.0346 \mathrm{~d}^{-1}\right)$. Since the phase difference is zero at the start (time $=0 \mathrm{~d}$ ), the times of greatest phase difference occur in the gap, such that the lower panel fit still matches the data... just not very well in these noise-free data. 


\section{CHAPTER 1}

where those side-lobes are becoming an increasingly good fit to the data, hence their increasing amplitudes. In reality, one can always select the correct peak if the difference in amplitude between the highest and second-highest peaks is greater than the grass level. Then one may safely assume no noise spike has boosted a peak arising from a cycle count ambiguity to a height that is greater than that of the real frequency.

This observation was used in the creation of Fig. 1.22, to choose the frequency fit in the lower panel that best matches the data without being the true frequency; the chosen fit corresponds to one of the two equal side-lobes of highest amplitude from the Fourier transform of those data (not shown, but comparable to the bottom panels of Fig. 1.21). Notice how in the lower panel the phase slowly drifts due to the extra cycle. That fit is precisely in phase at the start of the data set. At the start of the gap, the phase of the incorrect fit is only around 0.2 cycles different from the correct one (and the data), as it is at the end of the data set at $37.2 \mathrm{~d}$. In the centre of the gap, there would have been a much larger discrepancy - the phase difference would have grown from 0.2 to 0.5 , and continued to its value of almost unity (i.e. perceived to be in phase again) at the end of the gap.

Since here we have depicted a worst-case scenario, where a safe-mode event occurs in the centre of a short observing run (Kepler has not yet experienced safe-mode events in any two consecutive quarters), we can be confident that cycle count ambiguities are not significant with Kepler data, which normally provide observations in LC-mode for consecutive quarters for the same target, spanning several-hundred days. The presence of these 'large' gaps is then diminished, and the existence of 'medium' and 'small' gaps corresponding to monthly downlinking and momentum desaturations (if flagged points are deleted), respectively, are no longer of any importance whatsoever to the cycle count ambiguities. What is important with respect 


\section{CHAPTER 1}

to gaps of all sizes, as we shall see later $(\S 1.5)$, is that on-board the satellite, observations are always evenly sampled - the data taken at time $t_{n}$ are still represented by the formalism $t_{n}=t_{0}+n \Delta t$, for an arbitrary (fixed) starting time $t_{0}$, say, the very first observation made by the satellite, and an integer product $n$ of the constant sampling interval $\Delta t$. Missing any number of points does not deviate from this formalism..$^{9}$

\subsubsection{The failure of Module 3}

In $\S 1.1$ we mentioned the early failure of Module 3 and that this results in a small fraction $\left(\sim \frac{4}{21}\right)$ of the field of view being observable for only three quarters of the year. The Module 3 failure represents the largest gaps (one Quarter; $~ 93 \mathrm{~d}$ ) that we now anticipate in the data. Fig. 1.23 illustrates how the rotation of the satellite and failure of Module 3 render parts of the field unobservable.

To demonstrate the gaps introduced in the data, simulated data have been created with the Module 3 gaps, along with the $\sim 24$-h downlink gaps, for four satellite orbits. Their time-coverage is plotted in Fig. 1.24. The gaps in the data, falling at regular satellite orbital phase, are a longer time-scale analogy of the ground-based aliases on Earth that arise from our planet's rotation, which are discussed in $\S 1.4 .4$, below. We can expect cycle count ambiguities to result, and indeed these are present in the simulated data, as shown in Fig. 1.25. The sidelobes are separated from the central peak by the orbital frequency of the satellite, $\nu_{\mathrm{orb}}\left(\equiv 1 / P_{\mathrm{orb}}\right)$.

\footnotetext{
${ }^{9}$ It does mean that irrespective of the number of points, the Fourier transform of the data must be of the 'Discrete Fourier Transform (DFT) for unequally spaced data' type (with computational cost scaling as $\mathcal{O}\left(N^{2}\right)$ for number of data points $N$ ), as opposed to a Fast Fourier Transform (FFT) scaling as $\mathcal{O}\left(\mathrm{Nlog}_{2} N\right)$. The DFT is normally of the form in Deeming (1975), sometimes, as O'Donoghue \& Warner (1982) suggested, with the order of the computational loops swapped to increase computational speed. Furthermore, Townsend (2010) has shown how graphical processing units can be used to speed up periodogram calculations by an order of magnitude compared to single CPU cores.
} 


\section{CHAPTER 1}

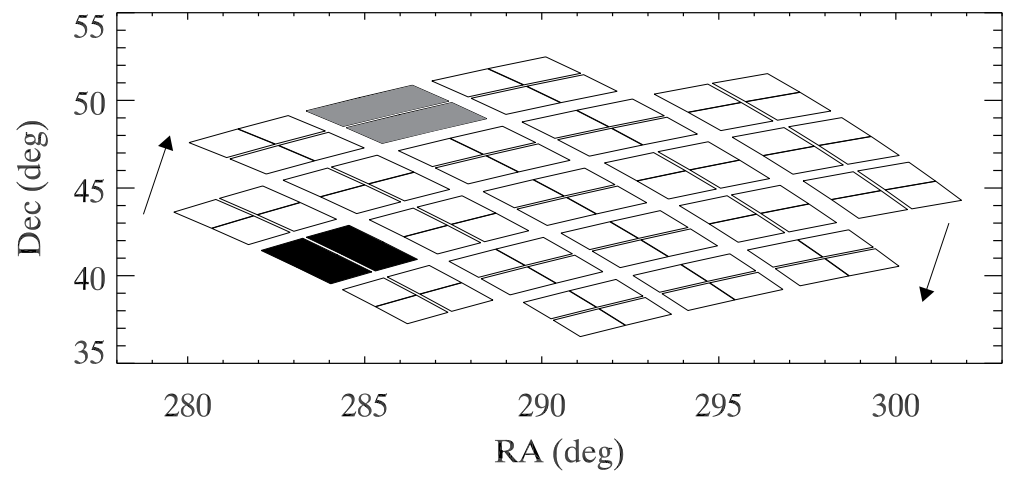

Figure 1.23: Kepler field showing the 21 modules (42 CCDs). The area corresponding to Module 3, for which no data will be gathered that quarter, is shaded black. In the following quarter, the grey area instead will receive no CCD coverage. Arrows indicate the direction of the quarterly roll.

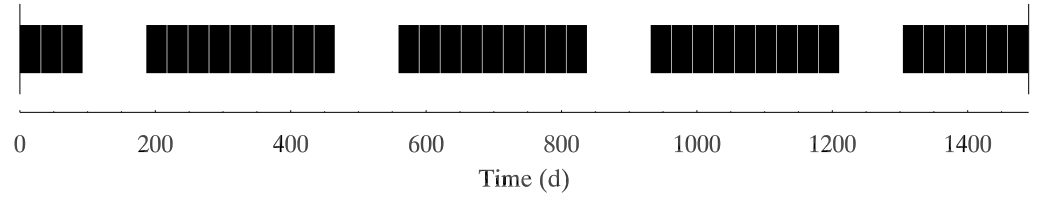

Figure 1.24: Simulated time coverage of a target falling on Module 3, over four satellite orbits. Black blocks indicate observations. Large white gaps show the relative duration of 'downtime' while the star lies on Module 3, for three consecutive Kepler months out of every twelve. Small white lines show the relative duration of monthly downlinks, and can be used to separate LC data into 'months' like the SC data.

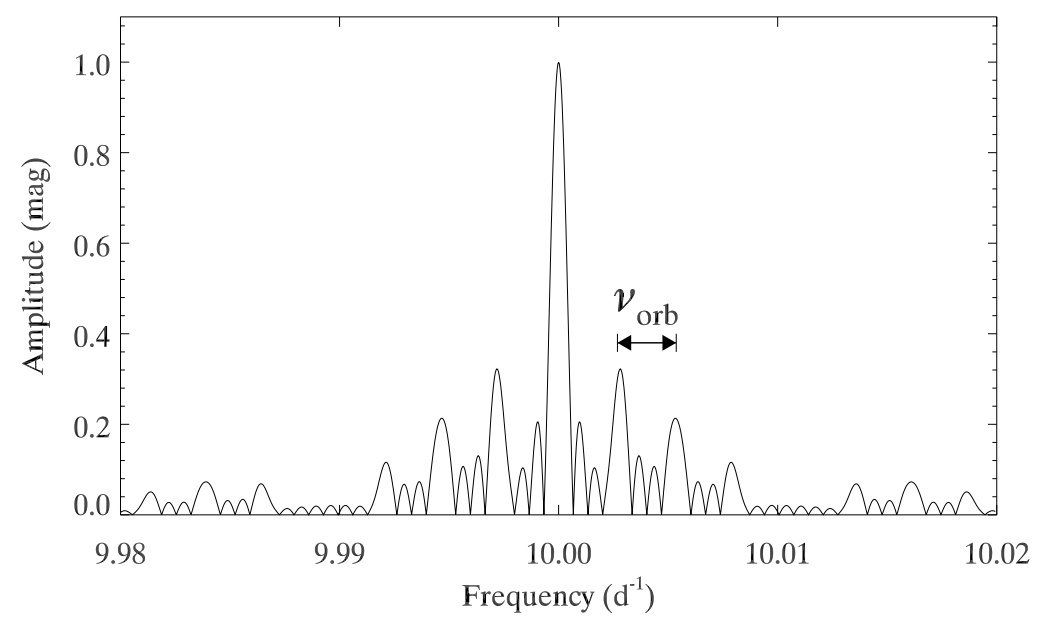

Figure 1.25: Cycle count ambiguities for a single-mode star that falls on Module 3. To within the frequency precision, sidelobes are separated from the main peak and neighbouring sidelobes by Kepler's orbital frequency, $\nu_{\text {orb}}$. 


\section{CHAPTER 1}

\subsubsection{Overcoming aliasing in ground-based data}

Even the worst-case scenario for Kepler, whose actual duty cycle is around 92 per cent (reduced to around 69 per cent for stars falling on Module 3), is better than what is commonly achieved from the ground, though some ground-based campaigns do reach >90 per cent duty cycles for short time spans, except for the Sun (Mosser \& Aristidi, 2007). Let us set aside the difference in attainable precision, for the moment, and focus only on the continuity of observations. Single-site photometry from the ground suffers greatly from gaps associated with Earth's rotation and with bad weather. One cannot conduct such observations during the day, but the target may still only be visible for part of the night. Let us construct two examples in which a target is observable for $8 \mathrm{~h}$ per night (with optimal weather conditions) and for the same time interval we used before: 0.1 Kepler orbits $(=37.25 \mathrm{~d})$. Our first example is for a pulsation frequency at $3 \mathrm{~d}^{-1}$, and the second at $9.1357 \mathrm{~d}^{-1}$, chosen to be some arbitrary number that is not a simple fraction or integer multiple of $1 \mathrm{~d}^{-1}$. The examples are plotted in Fig. 1.26.

Even though the real frequencies are different, the cycle count aliases in the Fourier transform are separated from the true peaks by $1 \mathrm{~d}^{-1}$ (sidereal day). This is expected, given that our sampling window has a periodicity of $1 \mathrm{~d}$ - the day/night cycle on Earth. While the true frequency is still discernible as the highest-amplitude peak in each panel, it is plain to see that with multiple pulsation frequencies in the same star, the Fourier transform becomes a mess. If the true frequency is fitted and removed from the data (pre-whitened), all of these cycle count aliases disappear in the noise-free case. (Real ground-based observations, where noise is high and observations run for a few hours per night, on a few consecutive nights, and are not made again until some months later, have significantly larger cycle count ambiguities that are occasionally insurmountable - see e.g. Kurtz 1981; Martinez et al. 1995.) If the wrong frequency is fitted, power still remains in the other peaks, as shown in 


\section{CHAPTER 1}

Fig. 1.27, but the real frequency no longer has the highest amplitude of the remaining peaks. Additionally, at low-amplitude where the effects of noise may push an alias to an amplitude higher than that of the true frequency, the true frequency might not be identifiable.

In reality, some strategies exist to reduce the influence of the cycle count aliases. One such strategy would be to observe the star from different sites in the same

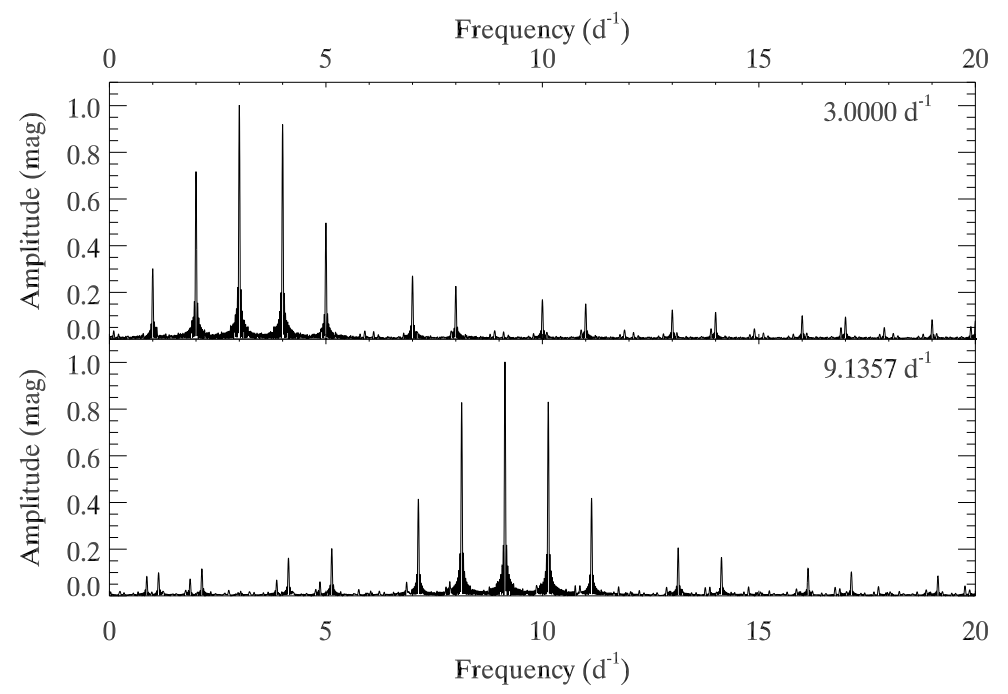

Figure 1.26: Cycle count ambiguities in the Fourier domain for two simulated, noise-free, ground-based data runs, comprising $8 \mathrm{~h}$ per night over $37.25 \mathrm{~d}$. In the upper panel, the pulsation frequency was chosen to be $3 \mathrm{~d}^{-1}$, and in the lower panel $9.1357 \mathrm{~d}^{-1}$ to avoid having a fraction or multiple of the 1-d sampling window cycle. In both cases, cycle count aliases are seen separated from the true peaks (the highest amplitude peak in each panel) by $1 \mathrm{~d}^{-1}$.

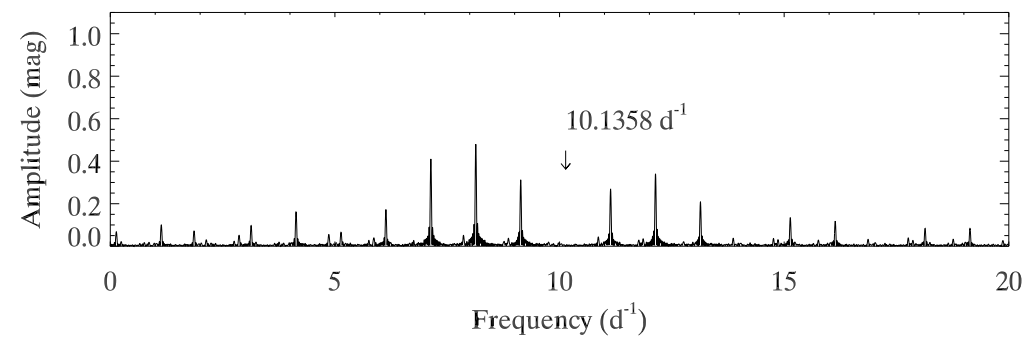

Figure 1.27: A copy of the lower panel of Fig. 1.26, here with the wrong frequency $\left(10.1358 \mathrm{~d}^{-1}\right)$ pre-whitened. That frequency was determined using a non-linear leastsquares algorithm for a frequency at approximately the real frequency plus $1 \mathrm{~d}^{-1}$. The real frequency of $9.1357 \mathrm{~d}^{-1}$ no longer has the highest amplitude. 


\section{CHAPTER 1}

24-h period, as has been done with Whole Earth Telescope (WET) campaigns ${ }^{10}$. Schwarzenberg-Czerny (2012) suggested another strategy: planning observations such that, if only a short amount of observing can be done for a given star on consecutive nights, the amplitudes of the cycle-count ambiguities are much reduced, without increasing total observing time, if those observations are scattered about different times of night on those consecutive nights. The SuperWASP survey does this (though not for this reason) by taking irregularly timed observations with the aim of surveying the whole sky down to $\sim 15$ th mag once per night, at different times each night. Shortening the cadence of the observations, on the other hand, does not improve the cycle count aliasing problems.

It is also possible to reduce cycle count aliases with some data processing techniques. In order to address this, we should discuss why the alias patterns separated by $1 \mathrm{~d}^{-1}$ occur in the first place. The Fourier transform is a convolution of the window function with the continuous function describing the stellar pulsation (the full formalism will be described in $\S 1.5)$. The cleanest Fourier transform - the one most closely resembling the continuous function describing the stellar pulsation is thus the one with a spectral window whose distribution of peaks features a sinc function (only) at the true frequency. When the data are gapped, like single-site ground-based data, there are sidelobes at $\pm 1 \mathrm{~d}^{-1}$ in the spectral window like those in the top panel of Fig. 1.28. These convolve with the continuous function to produce spectral window aliases (i.e. cycle count ambiguities) separated by $1 \mathrm{~d}^{-1}$ from the real pulsation frequency. The amplitude of these spectral window aliases is determined by the amplitude ratio of the sidelobes in the spectral window, to its central peak. Filling in the gaps in observations, by including data from another site, reduces the amplitude of these sidelobes as greater time coverage reduces our cycle count ambiguity (Fig. 1.28, middle panel). The sidelobes are further affected by applying weights to the data.

\footnotetext{
${ }^{10}$ http://www.physics.udel.edu/ darc/wet/
} 


\section{CHAPTER 1}
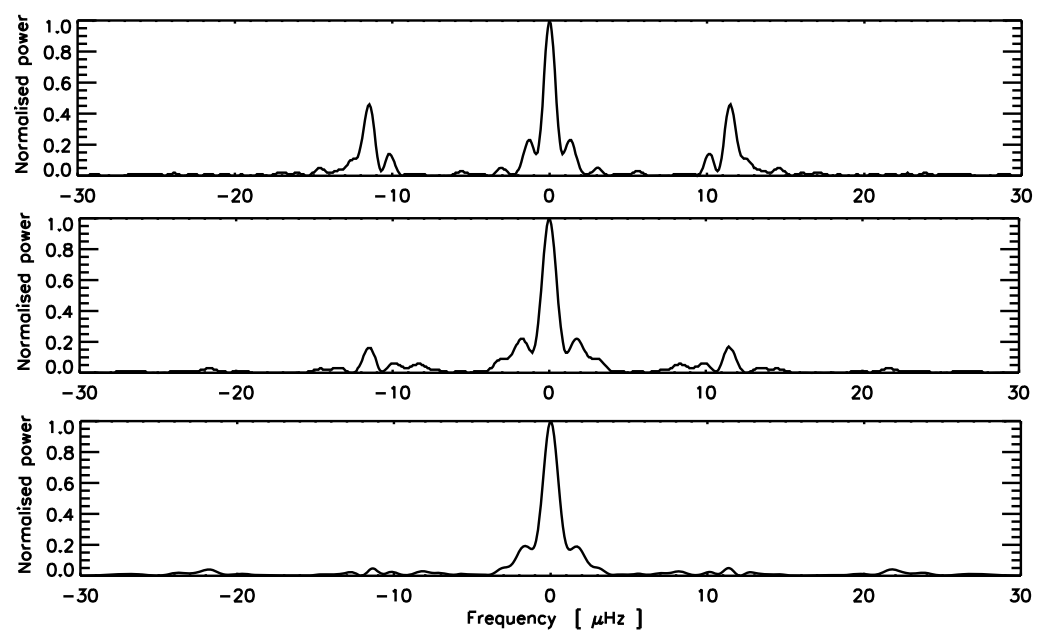

Figure 1.28: Comparison of the spectral windows of the time series of the [singlesite] CORALIE data (top panel) and the one of the combined [mutli-site] time series of the CORALIE, UVES and UCLES data with standard weights (middle panel) and sidelobe-optimised weights (bottom panel). The daily aliases are already much reduced in the case of the combined time series with standard weights, since UCLES nights fill several gaps in the CORALIE and UVES time series. Figure and caption from de Meulenaer et al. (2010, their figure 2). Permission obtained from A\&A.

When observing from different sites, the observations are weighted according to which afford the higher precision. The resulting spectral window, which takes weights into account, then has strong sidelobes, i.e. applying weights actually increases the amplitude of spectral window aliases. Bedding et al. (2004) addressed this problem by iterating over some 'adjustment factors' to minimise the amplitudes of the sidelobes in the spectral window. Observations that fill gaps, i.e. that are in the centre of longer runs, are weighted more heavily, and these weight adjustments are calculated on a nightly basis. This process was adopted by de Meulenaer et al. (2010) to produce the bottom panel of Fig. 1.28, where the sidelobe amplitudes have been reduced. The result of this optimisation is a small decrease in frequency resolution, and a modest increase in noise at high frequency. Bedding et al. rightly claimed that the improvement in the spectral window produces a much clearer Fourier spectrum, and that this compensates for the reduction in signal-to-noise ratio. 


\section{CHAPTER 1}

\subsubsection{An aside: choosing a step-size}

When calculating the Fourier transform, one must choose the size of the step in frequency at which values of the amplitude will be calculated. The software package PERIOD04 allows the user to choose the step size, with a default 'high-precision' setting of $1 / 20 T$, where $T$ is the observation time span of the data. The frequency resolution itself is $\sim 1 / T$. For long data sets, such as the $1000+\mathrm{d}$, continuous data sets that are available with Kepler, such a small step size is not normally required. Particularly if the data are SC, those having thirty-times more data points, one can encounter computation-time issues, and the user might wish to consider increasing the step size. Mostly, taking a step size of $10^{-4} \mathrm{~d}^{-1}$ is more than sufficient, especially when a non-linear least-squares fitting algorithm is later used. Choosing a step size becomes important when investigating peaks that are not well-separated, such as the Nyquist aliases we will investigate in the next section.

\subsection{The Nyquist frequency and super-Nyquist as- teroseismology with Kepler}

Most of the information in this section comes from Murphy, Shibahashi E Kurtz (2013), and so, beginning on the next page, that paper is provided here, as published. A short discussion follows afterwards. I coordinated and wrote the majority of the paper, with some editing from co-authors, and I ran simulations of Kepler's orbit around the Sun that were jointly coded and interpreted by D. Kurtz and me. These simulations, along with the study of real-data observations that I provided, aided the development of the theory. Observations and theory were pursued simultaneously, each supporting the other. Section 2 of the paper was predominantly written by H. Shibahashi. 


\title{
Super-Nyquist asteroseismology with the Kepler Space Telescope
}

\author{
Simon J. Murphy, ${ }^{1 \star}$ Hiromoto Shibahashi ${ }^{2}$ and Donald W. Kurtz ${ }^{1}$ \\ ${ }^{1}$ Jeremiah Horrocks Institute, University of Central Lancashire, Preston PR1 2HE, UK \\ ${ }^{2}$ Department of Astronomy, The University of Tokyo, Tokyo 113-0033, Japan
}

Accepted 2013 January 15. Received 2013 January 15; in original form 2012 November 5

\begin{abstract}
Barycentric corrections made to the timing of Kepler observations, necessitated by variations in light arrival time at the satellite, break the regular time-sampling of the data - the time stamps are periodically modulated. A consequence is that Nyquist aliases are split into multiplets that can be identified by their shape. Real pulsation frequencies are distinguishable from these aliases and their frequencies are completely recoverable, even in the super-Nyquist regime, i.e. when the sampling interval is longer than half the pulsation period. We provide an analytical derivation of the phenomenon, alongside demonstrations with simulated and real Kepler data for $\delta$ Sct, roAp and sdBV stars. For Kepler data sets spanning more than one Kepler orbital period (372.5 d), there are no Nyquist ambiguities on the determination of pulsation frequencies, which are the fundamental data of asteroseismology.
\end{abstract}

Key words: asteroseismology-methods: data analysis - stars: oscillations - stars: variables: $\delta$ Scuti-stars: variables: general.

\section{INTRODUCTION}

It is well known that all light emitted or received is Doppler shifted by the motion of the emitting or receiving body. In astronomy the Doppler shift from the emitting body is used to deduce radial velocity; the Doppler shift of the receiving body, the Earth, is removed from the observations by correcting the times of observation to the Solar System barycentre. All of this is standard practice. The Doppler shift from emitting bodies has traditionally been used to deduce orbital motion for binary stars and for exoplanets with spectroscopic radial velocities, and with $O-C$ analyses in the cases of binary pulsars and orbiting pulsating stars (Hulse \& Taylor 1975; see also Sterken 2005b). Recently, Shibahashi \& Kurtz (2012) have shown how this orbital Doppler shift can be seen directly in the Fourier transform of light variations in binary pulsating stars and used to derive the mass function without the need of spectroscopic observations. Now, we show here, both analytically and in practice, that the Doppler shift at the receiving body - in this case the Kepler Space Telescope - allows the unique identification of periodic frequencies in the emitting body without any Nyquist ambiguity.

The Nyquist frequency of equally spaced data represents an upper limit on a range of frequencies over which a Fourier transform is unique. It is well defined as $f_{\mathrm{Ny}}=1 /(2 \Delta t)$, where $\Delta t$ is the sampling interval between two consecutive points; this is also known as the 'cadence' of the data. In perfectly equally spaced data there is an infinity of frequencies that fit the data equally well. External physical constraints are mandatory to select the frequency range that is appropriate for pulsating stars. Commonly, the cadence is

^E-mail: smurphy6@uclan.ac.uk selected so that the known plausible frequency range for the type of star lies in the range $\left[0, f_{\mathrm{Ny}}\right]$, and the infinity of ambiguous higher frequency Nyquist aliases are ignored as unphysical.

However, it is not possible to adjust the observing cadence for Kepler mission data to accommodate the study of pulsating stars in different frequency regimes. The Kepler mission's prime goal of detecting Earth-like planets in the habitable zone led to a design where Kepler data are available in two cadences: long-cadence (LC) at $29.43 \mathrm{~min}$, and short-cadence (SC) at $58.85 \mathrm{~s}$, with corresponding Nyquist frequencies of 24.469 and $734.07 \mathrm{~d}^{-1}$, respectively. The number of SC observing slots is limited to 512 by telemetry constraints, and most of those observing slots are needed for higher time resolution studies of exoplanet transits. With this limitation most classical pulsators observed by Kepler have used LC, even though many such pulsators - such as $\delta$ Sct, roAp, sdBV, pulsating white dwarf and $\beta$ Cep stars - have pulsation modes with frequencies that can exceed the LC Nyquist frequency. Murphy (2012) showed some of the difficulties encountered with Nyquist aliases when studying $\delta$ Sct stars with Kepler LC data; Dawson \& Fabrycky (2010) did the same for planet searches with radial velocity data.

Now, we show in this paper how the correction of the times of observations of Kepler data to the Solar System barycentre generates a time-dependent Nyquist frequency, and that this completely removes all Nyquist alias ambiguities in the amplitude spectrum. For data sets longer than one Kepler orbit, true pulsation frequencies can always be distinguished from all Nyquist aliases. The observing cadence for Kepler data is not a barrier to asteroseismic modelling of pulsation frequencies because of Nyquist ambiguity - there is none. In practice, of course, higher frequencies have reduced amplitude with LC data (see Murphy 2012), and that affects the signal-to-noise 
ratio, which decreases with increasing frequency. Thus, as stated by Eyer \& Bartholdi (1999), in the irregular-sampling case, the frequency limit becomes dominated by exposure time (and intrinsic amplitude), not sampling rate, where with Kepler the effective exposure time is 91 per cent of the sampling interval for both cadences.

The Kepler spacecraft is in a 372.5 d, heliocentric Earth-trailing orbit. Four times per orbit the satellite must perform a roll to keep its solar panels facing the Sun, so the data are divided into quarters (denoted $\mathrm{Q} n$ ) and a brief gap in observations occurs. In addition to the data downlink that takes place at each quarterly roll, two more downlinks occur at approximately 30-d intervals in the middle of each quarter, effectively dividing the data into three 'months' per quarter (thus denoted Qn.m for SC data). ${ }^{1}$ These events represent the most frequent gaps in Kepler data which are otherwise nearly continuous at their micromagnitude precision. Other gaps due to occasional safe-mode events have occurred, and we refer the reader to the Data Characteristics Handbook ${ }^{2}$ for more information. However, the important property of the observations with respect to the gaps is that observations are always taken at regular intervals according to the spacecraft's clock, which can be expressed as some integer product with $\Delta t$ from some (fixed) arbitrary start time, i.e. $t_{n}=t_{0}+n \Delta t$. In this regard, the Kepler data may be described as equally spaced.

Timing onboard the spacecraft is in Julian Date, but the spacecraft is in orbit around the Solar System's barycentre and as such the arrival times of photons from the Kepler field are shifted seasonally by up to $\pm 200 \mathrm{~s}$ from those of the Solar System barycentre. Although data are sampled regularly onboard, the time stamps are subsequently modified when converted into Barycentric Julian Date (BJD) and hence become irregularly sampled: no longer can the observation times be represented in the form $t_{n}=t_{0}+n \Delta t$ for integer values of $n$. The effect on super-Nyquist frequencies has been observed by Baran et al. (2012), and described as a 'smearing' of the frequencies. In this paper we show how the satellite motion results in multiplets being generated out of the Nyquist aliases, and how this can be used to distinguish these aliases from real pulsation frequencies.

\section{ANALYTICAL DERIVATION}

\subsection{Discrete Fourier transform}

\subsubsection{General description}

Let us consider first a general case of discrete data $\left\{x\left(t_{n}\right)\right\}$, which can be regarded as the following function of any value of $t$ :

$x_{N}(t) \equiv \sum_{n=0}^{N} x(t) \delta\left(t-t_{n}\right)$,

where $x(t)$ on the right-hand side is a continuous function, $\delta\left(t-t_{n}\right)$ is Dirac's delta function and the number of measurements is $N+$ 1. It is well known that the Fourier transform of $x_{N}(t)$ is expressed as a convolution of the Fourier transform of $x(t)$ and the sampling window spectrum $W_{N}(\omega)$ :

$$
\begin{aligned}
\frac{1}{N+1} F_{N}(\omega) & \equiv \frac{1}{N+1} \int_{t_{0}}^{t_{N}} x_{N}(t) \exp (\mathrm{i} \omega t) \mathrm{d} t \\
& =\left(F * W_{N}\right)(\omega),
\end{aligned}
$$

${ }^{1} \mathrm{LC}$ data are not separated into different files around these months like the SC data are, but the gaps are still present.

${ }^{2}$ Available at http://keplergo.arc.nasa.gov/Documentation.shtml where

$F(\omega) \equiv \int_{-\infty}^{\infty} x(t) \exp (\mathrm{i} \omega t) \mathrm{d} t$

is the Fourier transform of the continuous function $x(t)$ and

$W_{N}(\omega) \equiv \frac{1}{N+1} \sum_{n=0}^{N} \exp \left(\mathrm{i} \omega t_{n}\right)$.

Here, we have used the following expression of the delta function:

$\delta(t)=\int_{-\infty}^{\infty} \exp (-\mathrm{i} \omega t) \mathrm{d} \omega$.

That is, the Fourier transform of discretely sampled data is equal to the convolution of the Fourier transform of the original continuous function and the window function. If we assume $x(t)=\cos \left(\omega_{0} t+\right.$ $\phi)$, i.e. pulsation with a single mode of angular frequency $\omega_{0}$, regarding this as a model of a pulsating star, then

$F(\omega)=\frac{1}{2}\left\{\delta\left(\omega+\omega_{0}\right)+\delta\left(\omega-\omega_{0}\right)\right\}$.

Thus the Fourier transform of the sample series $F_{N}(\omega)$ consists of a superposition of the shape of the window spectrum, shifted by $\pm \omega_{0}$.

\subsubsection{The case of a uniform cadence}

If the sampling is taken with a uniform cadence with a time interval $\Delta t$, then $t_{n}=t_{0}+n \Delta t$, and

$\left|W_{N}(\omega)\right|=\frac{1}{N+1}\left|\frac{\sin \{(N+1) \omega \Delta t / 2\}}{\sin (\omega \Delta t / 2)}\right|$.

The window spectrum $\left|W_{N}(\omega)\right|$ has a series of sharp, high peaks at $\omega=n \omega_{\mathrm{S}}$, where

$\omega_{\mathrm{S}} \equiv \frac{2 \pi}{\Delta t}$

and $n$ denotes integers (see Fig. 1). Then $F_{N}(\omega)$ has sharp peaks at $\omega=n \omega_{\mathrm{S}} \pm \omega_{0}$. These apparent multiple peaks are the Nyquist aliases, and they look identical except for their frequencies.

The peak in the range of $\left[0, \omega_{\mathrm{S}} / 2\right]$ corresponds to the true angular frequency as long as $2 \omega_{0}<\omega_{\mathrm{S}}$ (here, we take $\omega_{0}>0$ ). Hence,

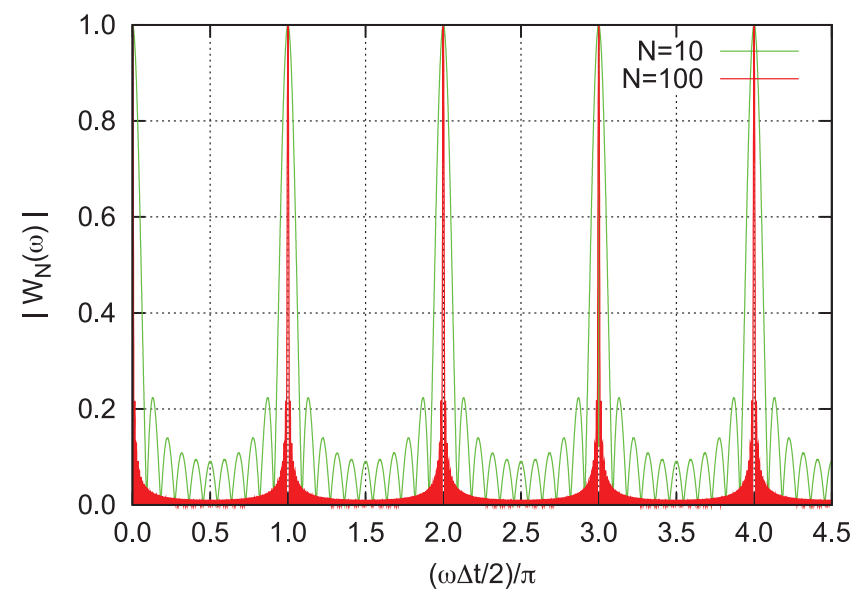

Figure 1. The sampling window spectrum $W_{N}(\omega)$ in the case of sampling with a constant time interval. The abscissa is $\omega \Delta t /(2 \pi)$, where $\Delta t$ is the sampling time interval. The red curve shows the case of $N=100$ data points, while the green curve shows the case of $N=10$. The window function shows conspicuous peaks at $\omega=n \omega_{\mathrm{S}}$, where $\omega_{\mathrm{S}}=2 \pi / \Delta t$ is the sampling angular frequency and $n$ denotes integers. 
for a given angular frequency $\omega_{0}$, if we select the sampling rate $\Delta t<\left(2 \pi / \omega_{0}\right) / 2$, we can identify uniquely the true frequency from the Fourier transform of the sampled data. This condition of $\Delta t<$ $\left(2 \pi / \omega_{0}\right) / 2$ is a sufficient condition for unique identification of the true frequency, not a necessary condition.

Now let us consider a case that the sampling rate is given in advance. In this case, if the true angular frequency is lower than $\omega_{\mathrm{S}} / 2$, the lowest frequency peak of the Fourier transform of the sampled data gives the true angular frequency. Note that this is again a sufficient condition, not a necessary condition. The Nyquist frequency is this frequency limit, under which we can identify uniquely the true angular frequency:

$$
f_{\mathrm{Ny}} \equiv \frac{1}{2} \frac{\omega_{\mathrm{S}}}{2 \pi}=\frac{1}{2 \Delta t}
$$

The reason why the true frequency is not uniquely identified without external constraints in the case of sampling with a uniform cadence is the fact that all the Nyquist aliases look identical and are indistinguishable. Inversely, if we could distinguish the individual Nyquist aliases, we would be able to identify uniquely the true frequency even if $\omega_{0}>\omega_{\mathrm{S}} / 2$. We will see in the case of Kepler data this is indeed possible.

\subsubsection{Sampling with a periodically modulated time interval}

In the case of Kepler, observations are taken at regular intervals according to the clock on-board the spacecraft. Data sampling is made at

$t_{n}=t_{0}+n \Delta t$,

where $n=0, \ldots, N$, and $\Delta t$ is a constant. The LC data have $\Delta t=$ $29.4 \mathrm{~min}$. However, since Kepler is orbiting around the Solar System barycentre, the resulting annual variation in the distance between the stars and the spacecraft leads to modulation in the phase of observed stellar pulsation, and the time stamps are converted to barycentre arrival times. As a consequence, the time stamp interval of the Kepler data is periodically modulated.

To make the modelling simple, we ignore in this section the difference between the barycentre and the heliocentre. Also, we approximate Kepler's orbit as a circle with a radius of $a=1 \mathrm{au}$. Then, the arrival time of the light from the star to the spacecraft delays from that to the heliocentre by

$\delta t(t)=\frac{a}{c} \cos \beta \cos \{\lambda \odot(t)-\lambda\}$,

which is often called the Rømer delay, in honour of Ole Rømer, who discovered observationally the fact that the speed of light is finite by comparing the observed timing of the eclipses of Jupiter's satellite Io with the estimate made at his time (Sterken 2005a). Here, $\lambda$ and $\beta$ are the ecliptic longitude and latitude of the star, $\lambda \odot(t)$ is the geocentric ecliptic longitude of the Sun and $c$ denotes the light speed (see Fig. 2). With the present assumption,

$\lambda \odot(t)=\Omega\left(t-t_{0}\right)$,

where $\Omega \equiv 2 \pi / 365 \mathrm{rad} \mathrm{d}^{-1}$ and $t_{0}$ is the vernal equinox passage time of the Sun. It is instructive to write down here, for later use, the light time with the equatorial coordinates,

$$
\begin{aligned}
\delta t(t)= & \frac{a}{c} \cos \lambda \odot \cos \alpha \cos \delta \\
& +\sin \lambda \odot(\cos \varepsilon \sin \alpha \cos \delta+\sin \varepsilon \sin \delta),
\end{aligned}
$$

where $\alpha$ and $\delta$ are the right ascension and the declination of the star and $\varepsilon$ is the obliquity of the ecliptic.

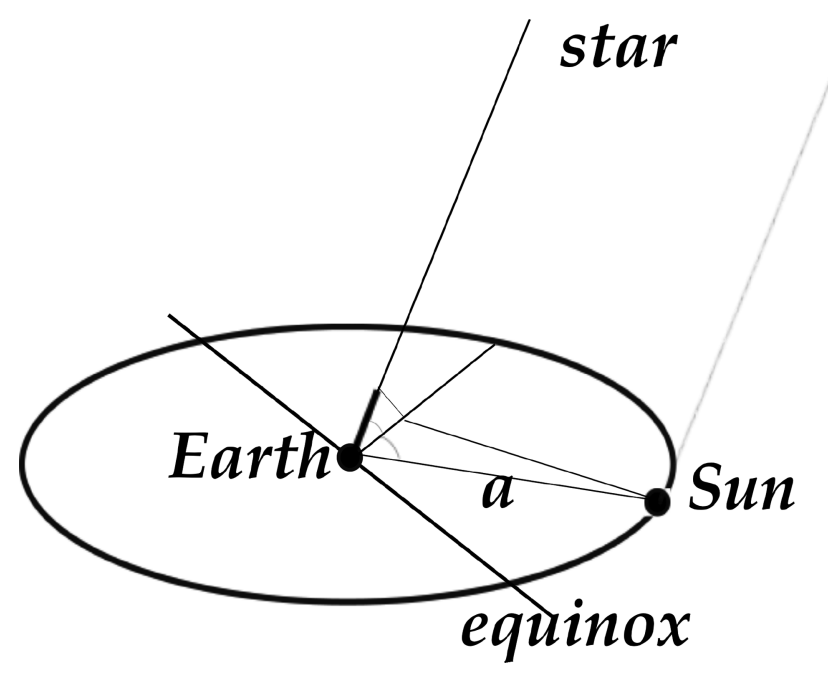

Figure 2. A schematic picture showing geometric relations among the Earth, the Sun and the star. The Sun is moving around the Earth on the ecliptic plane. The geocentric ecliptic longitude is measured from the vernal equinox. The star's position is $(\lambda, \beta)$, and the geocentric ecliptic longitude of the Sun is $\lambda \odot(t)$. The difference in the path length is shown with a thick segment. The path difference is obviously given as $a \cos (\lambda-\lambda \odot) \cos \beta$.

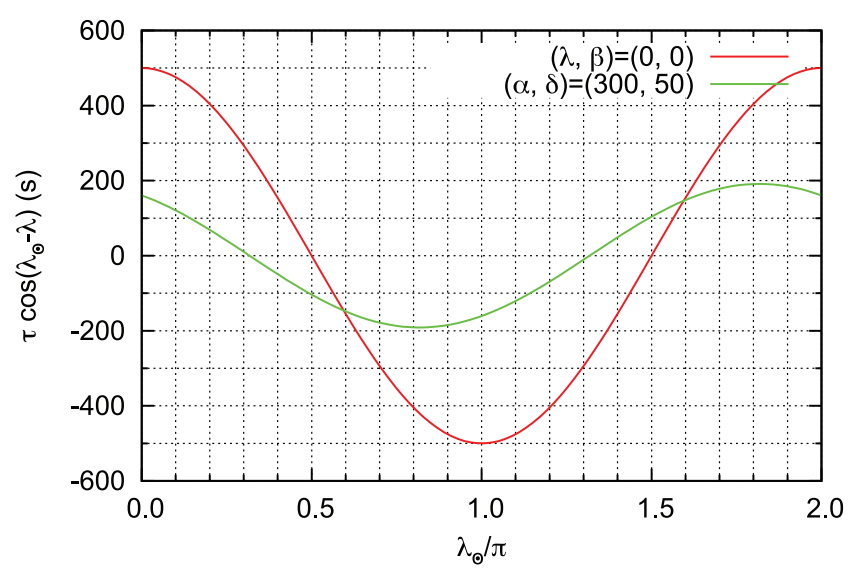

Figure 3. The time correction for the case of $\lambda=0^{\circ}, \beta=0^{\circ}$ (red) and for the Kepler field-of-view $\left(\alpha=300^{\circ}, \delta=50^{\circ}\right)$ (green) as a function of the Sun's ecliptic longitude $\lambda \odot$.

By taking account of this light time effect, the Kepler data are stored as a function of the arrival time at the heliocentre: ${ }^{3}$

$t_{\odot, n} \equiv t_{n}+\tau \cos \left(\Omega t_{n}-\lambda\right)$,

where

$\tau \equiv \frac{a}{c} \cos \beta$

gives the amplitude of modulation of the time stamps of the Kepler data. The second term on the right-hand side of equation (14) is the heliocentric time correction. Fig. 3 shows this time correction (the Rømer delay) for the field of $\lambda=0^{\circ}, \beta=0^{\circ}$ (red) and for the

\footnotetext{
${ }^{3}$ Strictly speaking, the data are stored as a function of the Barycentric Dynamical Time (TDB) referenced to the Solar System barycentre. The difference between the time referenced to the heliocentre and that to Solar System barycentre is $\lesssim 4 \mathrm{~s}$. The main cause of this difference is the acceleration of the Sun due primarily to Jupiter and Saturn.
} 
Kepler field-of-view (green). The case of $\beta=0^{\circ}$ gives the maximum correction; $\tau=500 \mathrm{~s}$. In the case of the Kepler field, $\tau \simeq 190 \mathrm{~s}$.

\subsection{The window spectrum of periodically modulated sampling}

Our aim is to carry out Fourier analysis of the Kepler data of pulsating stars. Since the Fourier transform of the sample series $F_{N}(\omega)$ is a superposition of the window spectrum, the problem is essentially what the window spectrum $W_{N}(\omega)=(N+1)^{-1} \sum_{n=0}^{N} \exp \left(\mathrm{i} \omega t \odot_{, n}\right)$ looks like in the case of periodically modulated sampling. The problem is then how to treat the terms $\exp (\mathrm{i} \omega \tau \cos \Omega t)$ and $\exp (i \omega \tau \sin \Omega t)$.

\subsubsection{Bessel coefficients}

These terms can be expressed with a series expansion in terms of Bessel functions of the first kind with integer order. With the help of Jacobi-Anger expansions

$\cos ( \pm \xi \cos \varphi)=J_{0}(\xi)+2 \sum_{n=1}^{\infty}(-1)^{n} J_{2 n}(\xi) \cos 2 n \varphi$

and

$\sin ( \pm \xi \cos \varphi)= \pm 2 \sum_{n=0}^{\infty}(-1)^{n} J_{2 n+1}(\xi) \cos (2 n+1) \varphi$,

we easily obtain the following relations:

$$
\begin{aligned}
\cos (\vartheta \pm \xi \cos \varphi)= & \sum_{n=-\infty}^{\infty}(-1)^{n}\left\{J_{2 n}(\xi) \cos (\vartheta+2 n \varphi)\right. \\
& \left.\mp J_{2 n+1}(\xi) \sin \{\vartheta+(2 n+1) \varphi\}\right\}
\end{aligned}
$$

and

$$
\begin{aligned}
\sin (\vartheta \pm \xi \cos \varphi)= & \sum_{n=-\infty}^{\infty}(-1)^{m}\left\{J_{2 n}(\xi) \sin (\vartheta+2 n \varphi)\right. \\
& \left.\mp J_{2 n+1}(\xi) \cos \{\vartheta+(2 n+1) \varphi\}\right\} .
\end{aligned}
$$

Here $J_{n}(\xi)$ denotes the Bessel function of the first kind of integer order $n$.

\subsubsection{Analytic expression of the window spectrum}

By applying these relations, after somewhat lengthy but straightforward manipulations, we eventually get

$$
\begin{aligned}
(N+1)^{2}\left|W_{N}(\omega)\right|^{2} & =\sum_{k=-\infty}^{\infty}\left|J_{k}(\omega \tau) \frac{\sin \{(N+1)(\omega+k \Omega) \Delta t / 2\}}{\sin \{(\omega+k \Omega) \Delta t / 2\}}\right|^{2} \\
& +\left[\sum_{k=-\infty}^{\infty} \sum_{k^{\prime}=-\infty}^{\infty} J_{2 k}(\omega \tau) J_{2 k^{\prime}}(\omega \tau)\right. \\
& \times \cos \left\{2\left(k-k^{\prime}\right) \Omega \frac{N \Delta t}{2}-2\left(k-k^{\prime}\right) \lambda\right\} \\
& +\sum_{k=-\infty}^{\infty} \sum_{k^{\prime}=-\infty}^{\infty} J_{2 k+1}(\omega \tau) J_{2 k^{\prime}+1}(\omega \tau)
\end{aligned}
$$

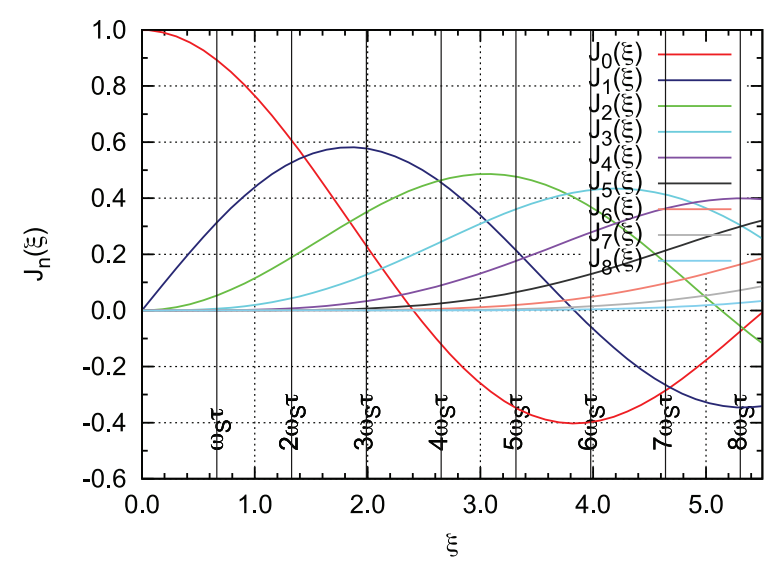

Figure 4. The Bessel coefficients $J_{n}(\xi)$ with $n=0, \ldots, 8$ for $\xi=[0: 5.5]$. The vertical lines show $\xi=n \omega_{\mathrm{S}} \tau(n=0, \ldots, 8)$, at which the window spectrum has sharp multiplet peaks (see text).

$$
\begin{aligned}
& \times \cos \left\{2\left(k-k^{\prime}\right) \Omega \frac{N \Delta t}{2}-2\left(k-k^{\prime}\right) \lambda\right\} \\
& +\sum_{k=-\infty}^{\infty} \sum_{k^{\prime}=-\infty}^{\infty} J_{2 k}(\omega \tau) J_{2 k^{\prime}+1}(\omega \tau) \\
& \times \sin \left[\left\{2 \omega+\left[2\left(k+k^{\prime}\right)+1\right] \Omega\right\} \frac{N \Delta t}{2}\right. \\
& \left.\left.-\left\{2\left(k+k^{\prime}\right)+1\right\} \lambda\right]\right] \\
& \times\left|\frac{\sin \{(N+1)(\omega+k \Omega) \Delta t / 2\}}{\sin \{(\omega+k \Omega) \Delta t / 2\}}\right|^{2},
\end{aligned}
$$

where $\sum^{\prime}$ means summation for $k^{\prime}$ except for when $k^{\prime}=k$.

The Bessel coefficients of different orders are orthogonal to each other (see Fig. 4). Hence, among the terms on the right-hand side, the first term is dominant. Then,

$$
\begin{aligned}
&\left|W_{N}(\omega)\right| \simeq \frac{1}{N+1} \\
& \quad \times \sum_{k=-\infty}^{\infty}\left|J_{k}(\omega \tau) \frac{\sin \{(N+1)(\omega+k \Omega) \Delta t / 2\}}{\sin \{(\omega+k \Omega) \Delta t / 2\}}\right| .
\end{aligned}
$$

This means that the window spectrum $\left|W_{N}(\omega)\right|$ consists of a singlet sharp peak at $\omega=0$ and multiplets of sharp peaks at $\omega=n \omega_{\mathrm{S}}+$ $k \Omega$, where $n$ and $k$ are integers and $\omega_{\mathrm{S}} \equiv 2 \pi / \Delta t$. The amplitude of each peak is $\left|J_{k}\left(n \omega_{\mathrm{S}} \tau\right)\right|$, since $\Omega \ll \omega_{\mathrm{S}}$ (see Table 1 ).

It should be stressed here that $\left|W_{N}(\omega)\right|$ is highly dependent on frequency $\omega$ through $\xi \equiv \omega \tau$, the argument of the Bessel function. For $\xi \ll 1$, the dominant term is only $J_{0}(\xi) \sim 1-\xi^{2} / 4$ and all the higher order Bessel functions are negligibly small. However, with the increase of $\xi$, the first-order Bessel function $J_{1}(\xi)$ comes larger as $J_{1}(\xi) \sim \xi / 2$, and eventually it becomes larger than $J_{0}(\xi)$ around $\xi \simeq 1.5$. But the higher order terms $J_{k}(\xi)$ for $k \gtrsim 4$ are still negligibly small. This means that $\left|W_{N}(\omega)\right|$ looks like a triplet with an equal spacing of $\Omega$ if $\omega \lesssim(2 \tau)^{-1}$. With the further increase of $\xi$, the second-order Bessel function $J_{2}(\xi)$ also becomes non-negligible. As a consequence, $\left|W_{N}(\omega)\right|$ comes to look like a quintuplet. This tendency continues further: the high-order Bessel functions become non-negligible with the increase of $\omega$. Therefore, the individual Nyquist aliases are distinguishable from each other. 
Table 1. The window spectrum.

\begin{tabular}{|c|c|c|c|}
\hline$n$ & $k$ & $\omega$ & Amplitude \\
\hline 0 & 0 & 0 & 1 \\
\hline \multirow[t]{4}{*}{1} & 0 & $\omega_{\mathrm{S}}$ & $J_{0}\left(\omega_{S} \tau\right)$ \\
\hline & \pm 1 & $\omega_{\mathrm{S}} \pm \Omega$ & $J_{1}\left(\omega_{S} \tau\right)$ \\
\hline & \pm 2 & $\omega_{\mathrm{S}} \pm 2 \Omega$ & $J_{2}\left(\omega_{\mathrm{S}} \tau\right)$ \\
\hline & $\cdots$ & $\cdots$ & $\cdots$ \\
\hline \multirow[t]{5}{*}{2} & 0 & $2 \omega_{\mathrm{S}}$ & $J_{0}\left(2 \omega_{\mathrm{S}} \tau\right)$ \\
\hline & \pm 1 & $2 \omega_{\mathrm{S}} \pm \Omega$ & $J_{1}\left(2 \omega_{\mathrm{S}} \tau\right)$ \\
\hline & \pm 2 & $2 \omega_{\mathrm{S}} \pm 2 \Omega$ & $J_{2}\left(2 \omega_{\mathrm{S}} \tau\right)$ \\
\hline & \pm 3 & $2 \omega_{\mathrm{S}} \pm 3 \Omega$ & $J_{3}\left(2 \omega_{\mathrm{S}} \tau\right)$ \\
\hline & $\cdots$ & $\cdots$ & $\cdots$ \\
\hline \multirow[t]{6}{*}{3} & 0 & $3 \omega_{\mathrm{S}}$ & $J_{0}\left(3 \omega_{\mathrm{S}} \tau\right)$ \\
\hline & \pm 1 & $3 \omega_{\mathrm{S}} \pm \Omega$ & $J_{1}\left(3 \omega_{\mathrm{S}} \tau\right)$ \\
\hline & \pm 2 & $3 \omega_{\mathrm{S}} \pm 2 \Omega$ & $J_{2}\left(3 \omega_{\mathrm{S}} \tau\right)$ \\
\hline & \pm 3 & $3 \omega_{\mathrm{S}} \pm 3 \Omega$ & $J_{3}\left(3 \omega_{\mathrm{S}} \tau\right)$ \\
\hline & \pm 4 & $3 \omega_{\mathrm{S}} \pm 4 \Omega$ & $J_{4}\left(3 \omega_{\mathrm{S}} \tau\right)$ \\
\hline & $\cdots$ & $\cdots$ & $\cdots$ \\
\hline$\cdots$ & $\cdots$ & $\cdots$ & \\
\hline
\end{tabular}

This characteristic is different from the case of sampling with a uniform cadence.

\subsubsection{Nyquist frequency is no longer the detection limit}

We have shown in Section 2.1.1 that the Fourier transform of the discretely sampled data $x_{N}(t)$ is given as the convolution of the Fourier transform of the original continuous data $x(t)$ with that of the window spectrum $W_{N}(\omega)$. Hence the Fourier transform of discrete data with a periodically modulated sampling interval essentially consists of multiplets around the Nyquist aliases of $W_{N}(\omega)$ at $n \omega_{\mathrm{S}} \pm$ $\omega_{0}$, where $n$ denotes integers. It should be noted here that the shape of the multiplets is independent of the frequency of the mode $\omega_{0}$, as long as the amplitude and the frequency of the mode are stable. It should also be stressed that only the peak at $\omega=\omega_{0}$ with $n=$ 0 is a single peak, while the other Nyquist aliases are multiplets; $n \omega_{\mathrm{S}} \pm \omega_{0} \pm k \Omega$, where $n \neq 0$ and $k$ denotes integers (see Table 2). Hence, by finding the unique singlet, we can distinguish the true pulsation frequency, $\omega_{0}$, from its Nyquist aliases. Note also that this feature is true not only for the case of $\omega<\omega_{\mathrm{S}} / 2$ but also for the case of $\omega>\omega_{\mathrm{S}} / 2$. That is, a pulsation frequency higher than the Nyquist frequency, which is still defined as $1 /(2 \pi \Delta t)$, is uniquely determined when the sampling rate is periodically modulated, irrespective of the number of multiples of the Nyquist frequencies crossed. ${ }^{4}$ The Nyquist frequency is no longer the upper limit of frequency determination in such a case. This has been mathematically proved here.

\subsection{Window spectrum for the Kepler LC data}

In the case of the Kepler field, $\tau \simeq 190 \mathrm{~s} \simeq 2.20 \times 10^{-3} \mathrm{~d}$. For the range of $\omega /(2 \pi)=[0,200] \mathrm{d}^{-1}\left(\omega=\left[0,1.26 \times 10^{3}\right] \mathrm{rad} \mathrm{d}^{-1}\right)$,

\footnotetext{
${ }^{4}$ For truly irregularly (randomly) sampled data, the lowest frequency above which real frequencies are truly indistinguishable from aliases is equal to the reciprocal of the accuracy to which time is measured (Koen 2010). For Kepler this is around $10^{7} \mathrm{~d}^{-1}$, so we can distinguish aliases from real frequencies for all frequencies within the star that are physically meaningful.
}

Table 2. Fourier transform of the data sampled with a periodically modulated interval.

\begin{tabular}{|c|c|c|}
\hline$n$ & Angular frequency & Amplitude \\
\hline \multirow[t]{4}{*}{-1} & $-\omega_{\mathrm{S}}+\omega_{0}$ & $J_{0}\left(\omega_{\mathrm{S}} \tau\right)$ \\
\hline & $-\omega_{\mathrm{S}}+\omega_{0} \pm \Omega$ & $J_{1}\left(\omega_{\mathrm{S}} \tau\right)$ \\
\hline & $-\omega_{\mathrm{S}}+\omega_{0} \pm 2 \Omega$ & $J_{2}\left(\omega_{\mathrm{S}} \tau\right)$ \\
\hline & $\cdots$ & $\cdots$ \\
\hline 0 & $\omega_{0}$ & 1 \\
\hline \multirow[t]{4}{*}{1} & $\omega_{\mathrm{S}}-\omega_{0}$ & $J_{0}\left(\omega_{\mathrm{S}} \tau\right)$ \\
\hline & $\omega_{\mathrm{S}}-\omega_{0} \pm \Omega$ & $J_{1}\left(\omega_{\mathrm{S}} \tau\right)$ \\
\hline & $\omega_{\mathrm{S}}-\omega_{0} \pm 2 \Omega$ & $J_{2}\left(\omega_{\mathrm{S}} \tau\right)$ \\
\hline & $\cdots$ & $\cdots$ \\
\hline \multirow[t]{4}{*}{1} & $\omega_{\mathrm{S}}+\omega_{0}$ & $J_{0}\left(\omega_{\mathrm{S}} \tau\right)$ \\
\hline & $\omega_{\mathrm{S}}+\omega_{0} \pm \Omega$ & $J_{1}\left(\omega_{\mathrm{S}} \tau\right)$ \\
\hline & $\omega_{\mathrm{S}}+\omega_{0} \pm 2 \Omega$ & $J_{2}\left(\omega_{\mathrm{S}} \tau\right)$ \\
\hline & $\cdots$ & $\cdots$ \\
\hline \multirow[t]{5}{*}{2} & $2 \omega_{\mathrm{S}}-\omega_{0}$ & $J_{0}\left(2 \omega_{S} \tau\right)$ \\
\hline & $2 \omega_{\mathrm{S}}-\omega_{0} \pm \Omega$ & $J_{1}\left(2 \omega_{\mathrm{S}} \tau\right)$ \\
\hline & $2 \omega_{\mathrm{S}}-\omega_{0} \pm 2 \Omega$ & $J_{2}\left(2 \omega_{\mathrm{S}} \tau\right)$ \\
\hline & $2 \omega_{\mathrm{S}}-\omega_{0} \pm 3 \Omega$ & $J_{3}\left(2 \omega_{\mathrm{S}} \tau\right)$ \\
\hline & $\cdots$ & $\cdots$ \\
\hline \multirow[t]{5}{*}{2} & $2 \omega_{\mathrm{S}}+\omega_{0}$ & $J_{0}\left(2 \omega_{\mathrm{S}} \tau\right)$ \\
\hline & $2 \omega_{\mathrm{S}}+\omega_{0} \pm \Omega$ & $J_{1}\left(2 \omega_{\mathrm{S}} \tau\right)$ \\
\hline & $2 \omega_{\mathrm{S}}+\omega_{0} \pm 2 \Omega$ & $J_{2}\left(2 \omega_{S} \tau\right)$ \\
\hline & $2 \omega_{\mathrm{S}}+\omega_{0} \pm 3 \Omega$ & $J_{3}\left(2 \omega_{S} \tau\right)$ \\
\hline & $\cdots$ & $\cdots$ \\
\hline$\ldots$ & $\ldots$ & $\ldots$ \\
\hline
\end{tabular}

Table 3. Parameters for the Kepler LC sampling.

\begin{tabular}{cc}
\hline Parameters & Values \\
\hline$\tau$ & $2.20 \times 10^{-3} \mathrm{~d}$ \\
$\Delta t$ & $2.10 \times 10^{-2} \mathrm{~d}$ \\
$\omega_{\mathrm{S}}$ & $3.02 \times 10^{2} \mathrm{rad} \mathrm{d}^{-1}$ \\
$\Omega$ & $1.72 \times 10^{-2} \mathrm{rad} \mathrm{d}^{-1}$ \\
\hline
\end{tabular}

$\omega \tau=[0,2.77]$ rad. Fig. 4 shows the Bessel coefficients $J_{n}(\omega \tau)$ with $n=0, \ldots, 8$ for $\omega \tau=[0,5.5]$. From this figure, it is obvious that $J_{n}(\omega \tau)$ with $n \geq 8$ are negligibly small in this range.

Let us consider the case of LC of Kepler. If we take $\Delta t=30 \mathrm{~min}=$ $2.1 \times 10^{-2} \mathrm{~d}$, then $\omega_{\mathrm{S}} \equiv 2 \pi / \Delta t=3.016 \times 10^{2} \mathrm{rad} \mathrm{d}^{-1}$. Then $\omega_{\mathrm{S}} \tau=0.663 \mathrm{rad}$. As for the modulation, $\Omega=2 \pi / 365 \mathrm{rad} \mathrm{d}^{-1}=$ $1.72 \times 10^{-2} \mathrm{rad} \mathrm{d}^{-1}$, then $\Omega / \omega_{\mathrm{S}}=5.69 \times 10^{-5}$. Parameters for the Kepler LC sampling are given in Table 3.

Fig. 5 shows the dependence of $\mid J_{k}(\omega \tau) \sin \{(N+1)(\omega+$ $k \Omega) \Delta t / 2\} / \sin \{(\omega+k \Omega) \Delta t / 2\} \mid$ on $\omega / \omega_{\mathrm{S}}$ for $k=0, \ldots, 8$. Here, we assumed $N=10^{5}$, i.e. the observational time-span was assumed to be $\sim 5.75 \mathrm{yr}$. In such a case, the high peaks are so sharp that their self-cross terms dominate over the other cross terms. As seen in Fig. 5, the high peaks appear as multiplets around $\omega=n \omega_{\mathrm{S}}$, where $\omega_{\mathrm{S}}=2 \pi / \Delta t$ and $n$ denotes integers. The multiplets have the equal splitting of the orbital frequency, $\Omega\left(\Omega / \omega_{\mathrm{S}}=5.69 \times 10^{-5}\right.$ in Fig. 5).

Strictly speaking, each multiplet has infinite peaks. However, the tiny amplitude peaks are practically undetectable, and each multiplet looks like a finite number of components; the first Nyquist alias looks like a triplet, the second looks like a quintuplet and so on. The degree of the apparent multiplicity becomes higher with the 

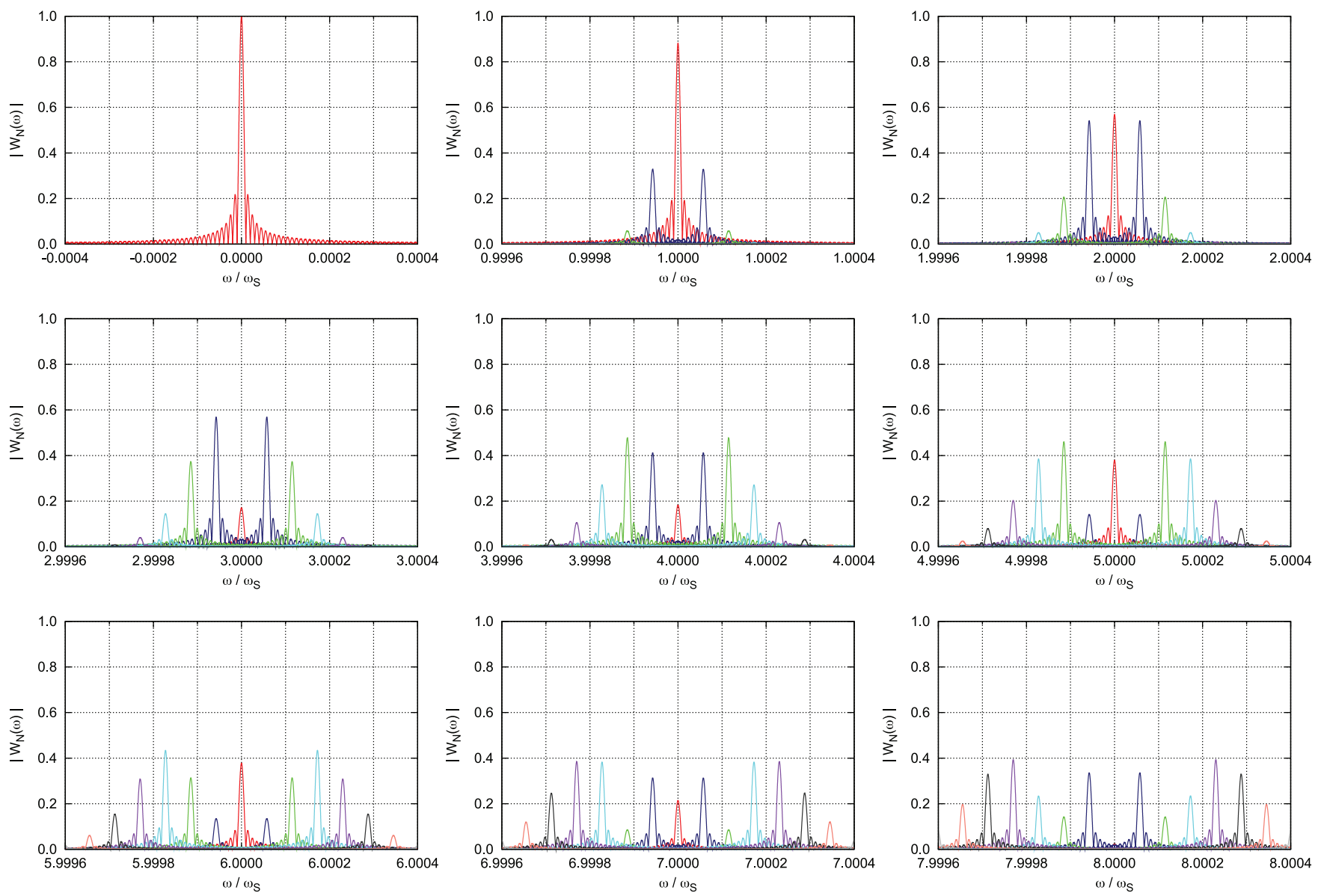

Figure 5. The dependence of $(N+1)^{-1}\left|J_{k}(\omega \tau) \sin \{(N+1)(\omega+k \Omega) \Delta t / 2\} / \sin \{(\omega+k \Omega) \Delta t / 2\}\right|$ on $\omega / \omega_{\mathrm{S}}$, for $k=0$, . ., 8 , where $\omega_{\mathrm{S}} \equiv 2 \pi / \Delta t$. The colour codes, corresponding to the order of the Bessel coefficients $k$, are in common with Fig. 4 . The parameters are $\Delta t=30 \mathrm{~min} ; \omega_{\mathrm{S}}=3.016 \times 10^{2}$ rad $\mathrm{d}^{-1}$. $\Omega=2 \pi / 365 \mathrm{rad} \mathrm{d}^{-1} ; \Omega / \omega_{\mathrm{S}}=5.69 \times 10^{-5} . \tau=190 \mathrm{~s}=2.20 \times 10^{-3} \mathrm{~d} ; \omega_{\mathrm{S}} \tau=0.663 \mathrm{rad} ; N=10^{5}$.

increase of $n$, because the Bessel coefficients with $n \neq 0$ become important with the increase of $\omega$.

The amplitudes of the peaks in the individual multiplet are determined by $\omega_{\mathrm{S}} \tau$. Since $\tau \simeq 190 \mathrm{~s} \simeq 2.20 \times 10^{-3} \mathrm{~d}, \omega_{\mathrm{S}} \tau=0.663$, $2 \omega_{\mathrm{S}} \tau=1.326,3 \omega_{\mathrm{S}} \tau=1.989,4 \omega_{\mathrm{S}} \tau=2.652,5 \omega_{\mathrm{S}} \tau=3.315$, $6 \omega_{\mathrm{S}} \tau=3.978,7 \omega_{\mathrm{S}} \tau=4.641$ and $8 \omega_{\mathrm{S}} \tau=5.304$. The Bessel coefficients at these values are graphically seen in Fig. 4.

\subsection{Multiplets of the Kepler LC data}

It should be stressed here again that only the peak at $\omega=\omega_{0}$ with $n=0$ is a single peak. The aliases with frequencies $\pm \omega_{\mathrm{S}} \pm \omega_{0}$ look like a triplet, while those with $2 \omega_{\mathrm{S}} \pm \omega_{0}$ are a quintuplet. The aliases associated with $3 \omega_{\mathrm{S}}, 4 \omega_{\mathrm{S}}, 5 \omega_{\mathrm{S}}$ and $6 \omega_{\mathrm{S}}$ look like a septuplet, a nonuplet, an undecuplet and a tredecuplet, respectively.

\section{EXAMPLES WITH SIMULATED DATA}

\subsection{Importance of a large observational time-span}

With the real Kepler data we are limited in our observational timespan by the length of the mission thus far. Stars that have been observed continuously since the mission began will have been covered for three complete orbits of the satellite, at the time of writing. However, with simulated data we are not so restricted. In Fig. 6 we show the importance of a large observational time-span on resolving the multiplets into which Nyquist aliases are split. Although these simulated data are without noise, it is clear that when one orbit (four mission quarters) of data is available, aliases are no longer single peaks, but are split into sets of partially resolved peaks and can thus be distinguished from real peaks (which are neither split nor similarly distorted). With two or more orbits, an obvious multiplet emerges, but the full width at half-maximum of each peak is still large compared to the separation of the multiplet peaks, hence the window pattern of each peak can interfere with its neighbours. Increasing the number of orbits further sharpens the peaks and resolves the pattern for comparison with the analytical patterns we derived, shown in Fig. 5.

\subsection{Effect of varying coordinates}

It is the barycentric time corrections that cause the splitting of Nyquist aliases. For a satellite that orbits around the Solar System barycentre in the ecliptic plane, these time corrections are determined by the ecliptic latitude of the target star, because this determines the difference in light arrival time at the satellite and the barycentre. We show how the multiplet shape changes with varying ecliptic latitude in Fig. 7. In the more familiar celestial coordinates, both right ascension and declination affect the ecliptic latitude, so we expect the arrival times and thus the multiplet shape to change when either coordinate is varied. Since neither of these coordinates 


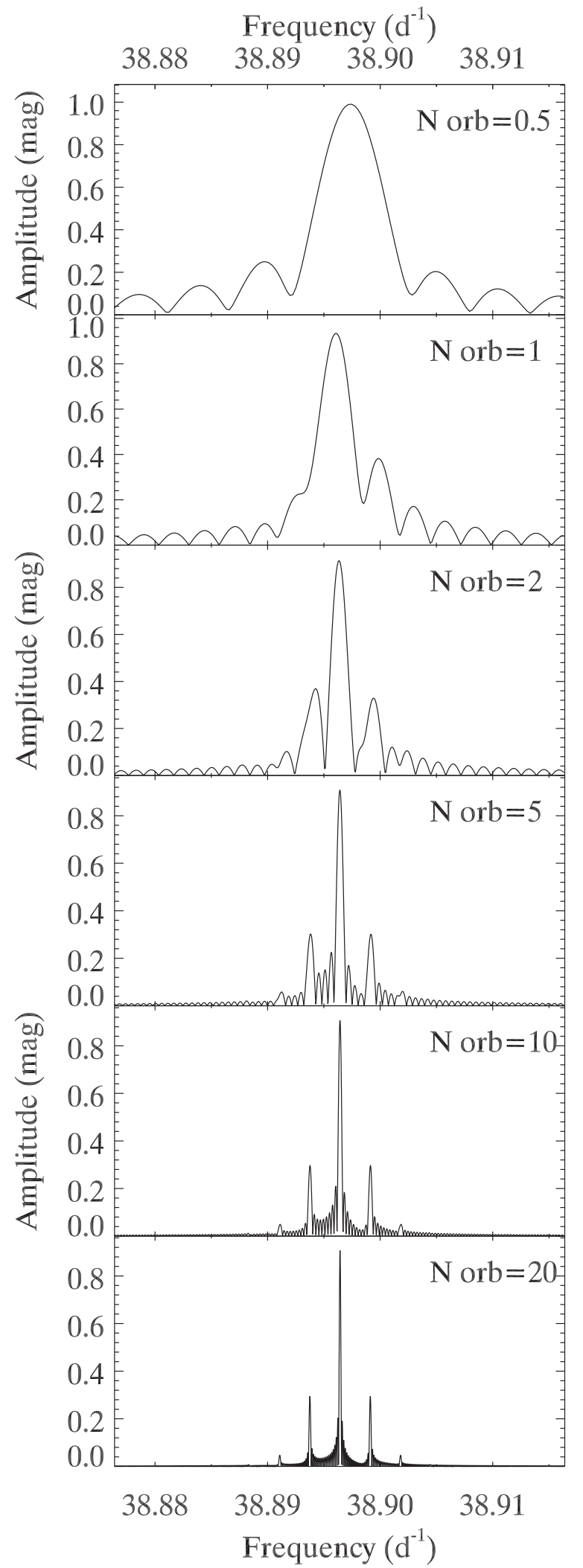

Figure 6. Simulated data showing the frequency resolution achieved with different numbers of orbits of the satellite. For short observational timespans, the window pattern of each member of the multiplet distorts the shape of its neighbours. Calculated for Kepler field coordinates, at the first Nyquist alias of a $10 \mathrm{~d}^{-1}$ pulsation frequency.

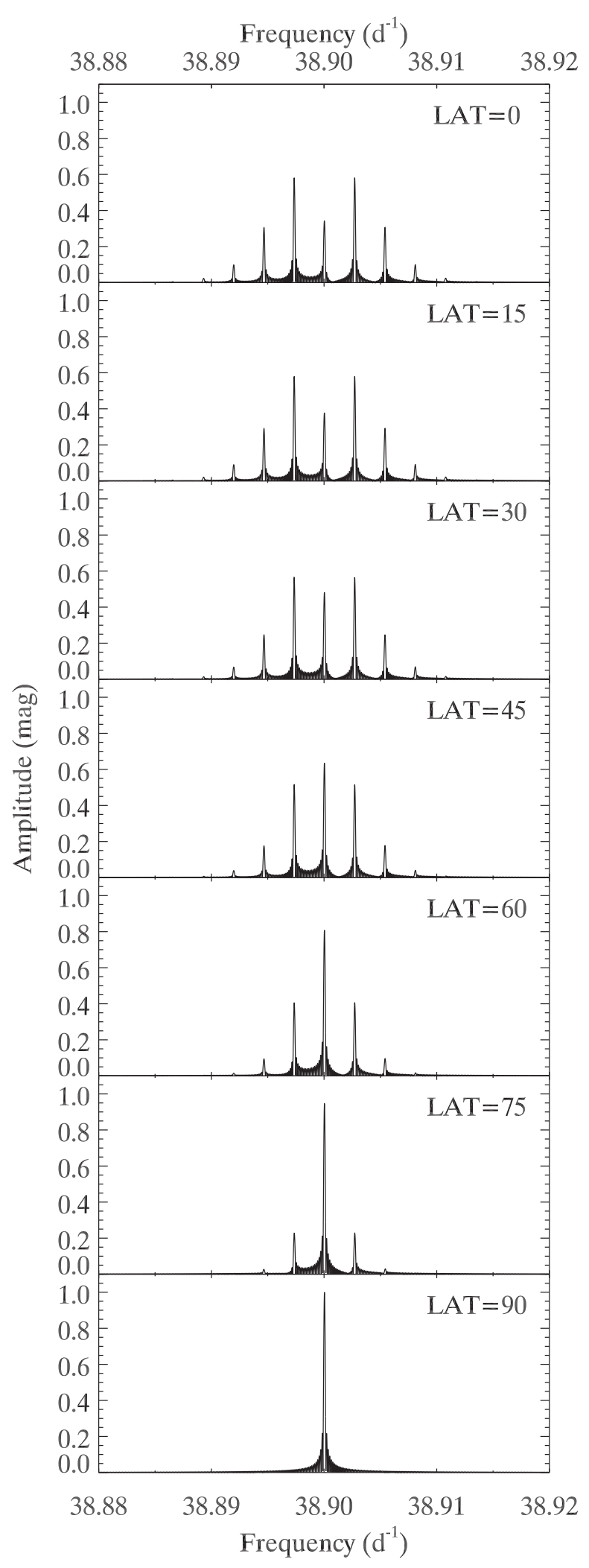

Figure 7. Variation in multiplet shape for changing ecliptic latitude. Note that, because the ecliptic is inclined to the equator, varying either right ascension or declination will change the ecliptic latitude. At an ecliptic latitude of $90^{\circ}$, a single peak is seen because there is no longer any difference in light arrival time. Calculated for 20 orbits, at the first Nyquist alias of a $10 \mathrm{~d}^{-1}$ pulsation frequency. 
changes greatly over the Kepler field, the multiplet shape is almost identical, too.

\section{SUPER-NYQUIST ASTEROSEISMOLOGY}

\subsection{Demonstration with real data}

We now compare our theory with real Kepler data by examining the case of a high-amplitude $\delta$ Sct (HADS) star. These stars are the main-sequence counterparts to the classical Cepheids, having large amplitudes and normally few pulsation modes - often the fundamental radial mode alone or with the first radial overtone. Sometimes harmonics of the frequencies of these modes are seen, as with the case we present here: that of KIC 6861400 . We used the LC data from quarters 1 through 9 (Q1-9), which have been processed with the PDC-MAP pipeline (Smith et al. 2012; Stumpe et al. 2012). The star features three independent mode frequencies and many harmonics of the two mode frequencies with the highest amplitudes. We applied a pre-whitening procedure, in which the frequencies are fitted with least-squares routines and subtracted from the data in the time domain, to all harmonics and kept only the highest amplitude mode of the three, leaving a single, highamplitude peak to make our example. No data points were deleted.

As expected, there is one alias in each frequency range $\left[n f_{\mathrm{Ny}},(n+\right.$ 1) $f_{\mathrm{Ny}}$ ] (upper panel, Fig. 8), for integer $n$. Even values of $n$, counting from $n=0$ at $0 \mathrm{~d}^{-1}$, are multiples of the sampling frequency. In
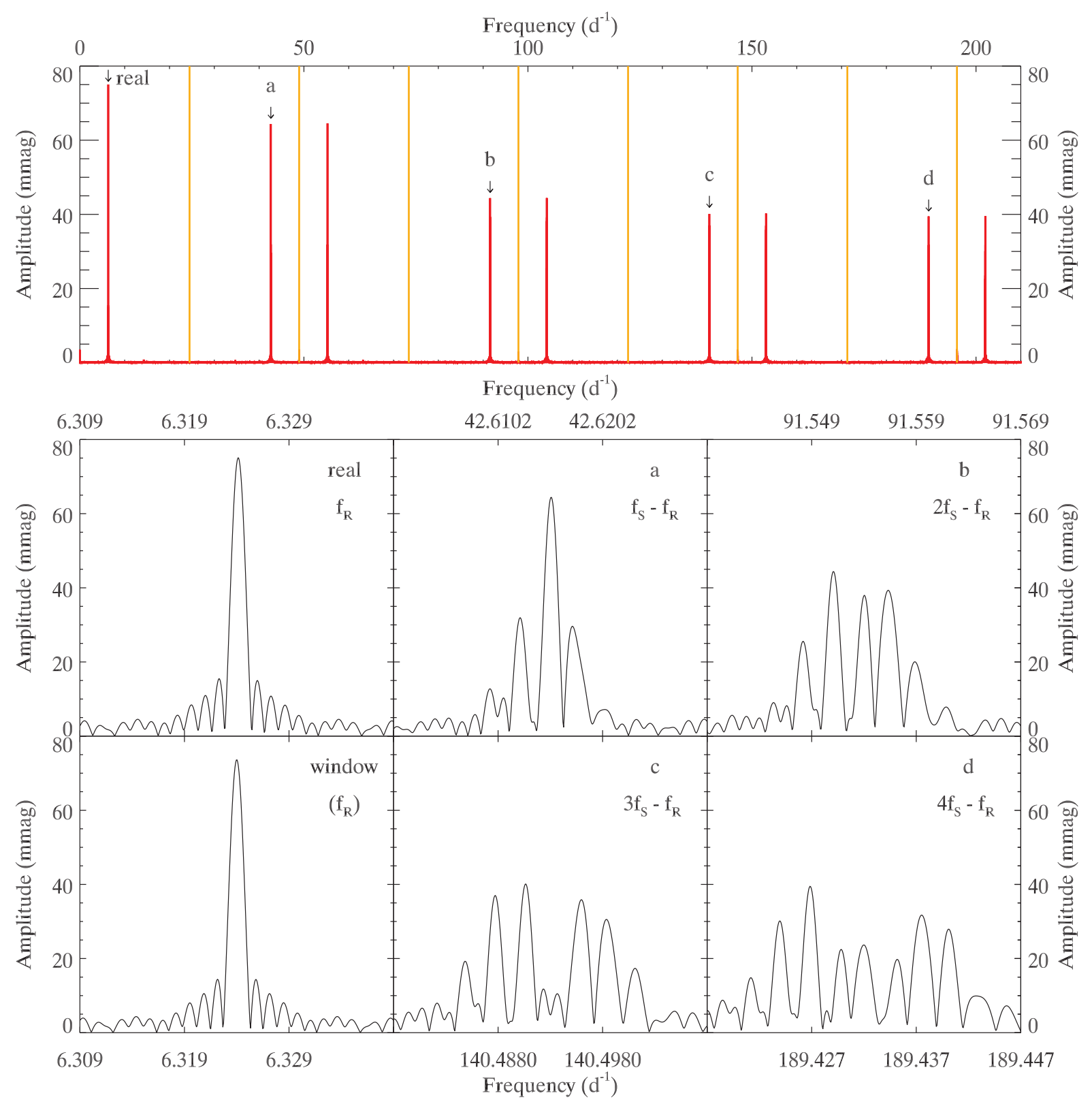

Figure 8. The upper panel shows the frequency distribution of Nyquist aliases. In the lower panels, which are all to the same scale in frequency and amplitude, the left-hand column shows the real peak, as labelled in the upper panel, and its window function. Panels (a)-(d) show aliases (also labelled in the upper panel), and their relationship to the sampling frequency and the real frequency. The pattern of the number of peaks, $p$, as a function of the coefficient of the Nyquist frequency straddled, $n$, is apparent: $p=2 n+1$. As such, there are three peaks at $f=f_{\mathrm{S}}+f_{\mathrm{R}}$, three at $f_{\mathrm{S}}-f_{\mathrm{R}}$, five each at $2 f_{\mathrm{S}} \pm f_{\mathrm{R}}$ and so on, as shown in Fig. 5. The example shown is KIC 6861400, using Q1-9 PDC-MAP data. 


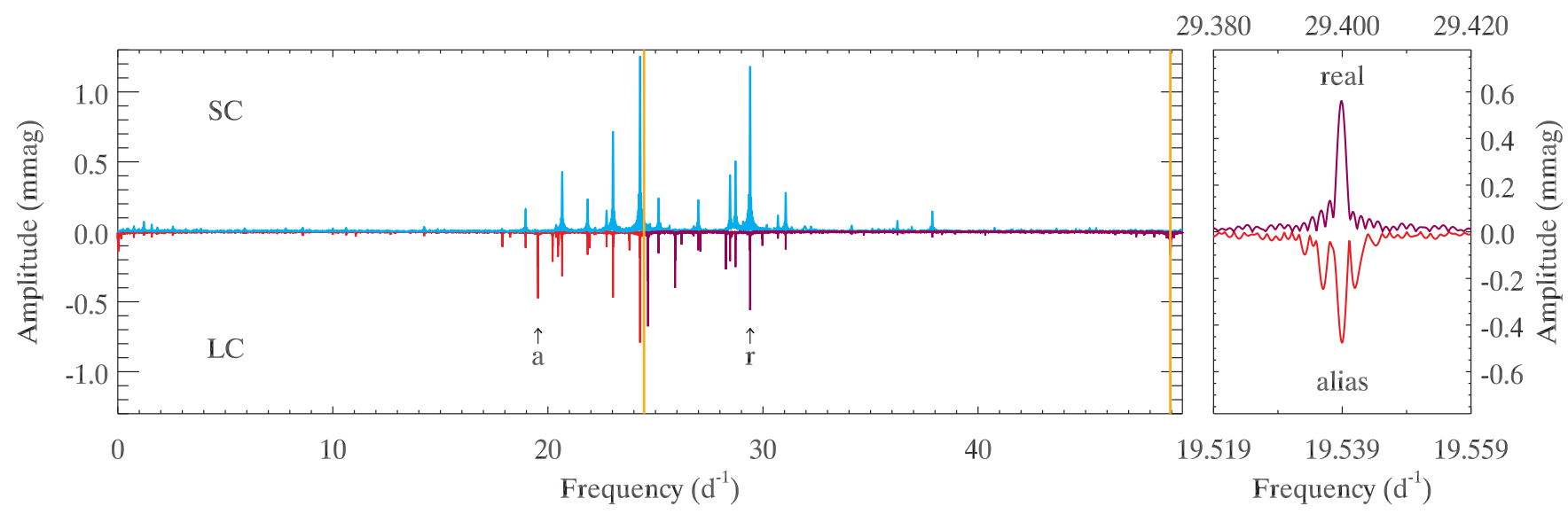

Figure 9. In the left-hand panel, the SC (top) and LC (bottom) data are displayed. LC Nyquist frequency and sampling frequency are represented with vertical orange lines. The LC data lying above their Nyquist frequency are coloured magenta; those below are red. There are Nyquist aliases in the LC data where real peaks have been aliased across the Nyquist frequency. By comparison with the SC data, we can see these aliases have no real partner in the SC data. An example of this is the alias peak labelled ' $a$ ', corresponding to the real peak labelled ' $r$ ' - there is no peak in the SC data at the same frequency as peak ' $a$ '. We zoom in on ' $a$ ' and ' $r$ ' from the LC data in the right-hand panel. The real peak takes on the typical sinc-function shape as expected in the Fourier transform. The effect of periodically modulated sampling, however, is that the aliased peak is split into a multiplet, provided the time-span of the data is at least one complete Kepler orbit. The example given is the $\delta$ Sct star KIC 8590553. SC data cover Q4.3 only, but LC data are from Q0 to 9.

Fig. 8, two peaks straddle each of these, and are identical in the Fourier transform. For this reason, only the peak to the left of each multiple of the sampling frequency is presented in the lower panels for a closer look. The multiplet structure is clearly present in all but the real peak, which is represented by a unique singlet peak. The spacing in the multiplets is approximately equal to the orbital frequency of the satellite, but due to the small number of orbits covered (2.25), the frequency resolution is insufficient to separate fully the window patterns of the members of the multiplets. The window pattern of each peak of the multiplet thus interferes with neighbouring multiplet members, and distorts the shape somewhat, as we showed in Fig. 6.

As the frequency increases when we study consecutive aliases of higher $n$, the argument to the Bessel functions is also higher and the higher order (higher $k$ ) terms become more significant. As such, the number of peaks seen increases. The amplitudes of the more distant members of the multiplet increase - we begin to see the expected quintuplets, septuplets and so on (cf. Section 2.4), as these peaks' amplitudes climb above the noise level. The amplitude ratios of the peaks with respect to each other also change. In panel (a), the central peak is dominant, but it is absent in panel (c), where only the window pattern of the first-order sidelobes can be seen in its place. The amplitudes of the Bessel orders that determine these peaks' amplitudes were shown as a function of frequency in Fig. 5.

The frequency dependence of the shape of the multiplet is on the sampling frequency straddled, only. The multiplet generated at the first Nyquist alias of a pulsation frequency at $20 \mathrm{~d}^{-1}$ looks exactly the same that of a $10-\mathrm{d}^{-1}$ frequency, and so on. Only the frequency of the alias changes, not the multiplet shape.

\subsection{Distinguishing real peaks from Nyquist aliases}

The difficulty in observing $\delta$ Sct stars with Kepler is that the typical pulsation frequency range of $\delta$ Sct stars spans both sides of the LC Nyquist frequency. Those pulsation frequencies above the LC Nyquist frequency are aliased to lower frequencies in the Fourier transform, meaning that they overlap with the real peaks. Distinguishing these aliases from the real peaks is impossible with data that are truly equally spaced in time (cf. Section 2.1.2), even if gaps exist in the data (Koen 2010). It has been argued that SC data are required to study the $\delta$ Sct stars so that this problem of overlapping real and aliased frequencies is removed, because the SC Nyquist frequency is so much higher than the LC one. With a periodically modulated sampling, however, one can use the shape of a peak to determine whether it is real or aliased. Fig. 9 shows how a real peak exists as a single peak in the Fourier transform, but a Nyquist alias will be a multiplet (resolved or not) split by the satellite's orbital frequency.

Use of this technique to distinguish Nyquist aliases opens up asteroseismic possibilities for many Kepler targets that have been observed in LC only. With data sets that have time-spans greater than one Kepler orbital period there is no Nyquist ambiguity in selecting the true pulsation frequencies for all types of pulsating stars. While there are still benefits of SC over LC data (Murphy 2012), the SC data are not needed to resolve Nyquist aliases.

\subsection{Beyond the Nyquist frequency}

The rapidly oscillating Ap stars are a population of chemically peculiar A-type stars that pulsate with frequencies much higher than $\delta$ Sct stars - frequencies between about 70 and $250 \mathrm{~d}^{-1}$. As such, when viewed with LC data, these pulsations are many multiples of the Nyquist frequency away from falling into the range $\left[0, f_{\mathrm{Ny}}\right]$. It is still possible, however, to identify the true frequency $\left(f_{\mathrm{R}}=\omega_{0} / 2 \pi\right)$ of the pulsations with LC data, even though the sampling period is much longer than the pulsation period, if the Fourier transform is calculated over a frequency range that includes $f_{\mathrm{R}}$.

We present an example in Fig. 10 using the star KIC 10195926, whose roAp pulsations have periods among the longest known and whose frequency spectrum features an oblique dipole mode at $\sim 84 \mathrm{~d}^{-1}$ that is rotationally split into a septuplet (Kurtz et al. 2011). We have run a high-pass filter on the data to pre-whiten the low frequency content. Thus the Q1-11 LC data presented contain only the high-frequency pulsation. With this figure we present a few final examples of the application of the technique to LC data.

First, it is clear that despite being substantially higher in frequency than the Nyquist frequency, the real peak is recoverable and frequency analysis can be conducted without SC data. Secondly, 


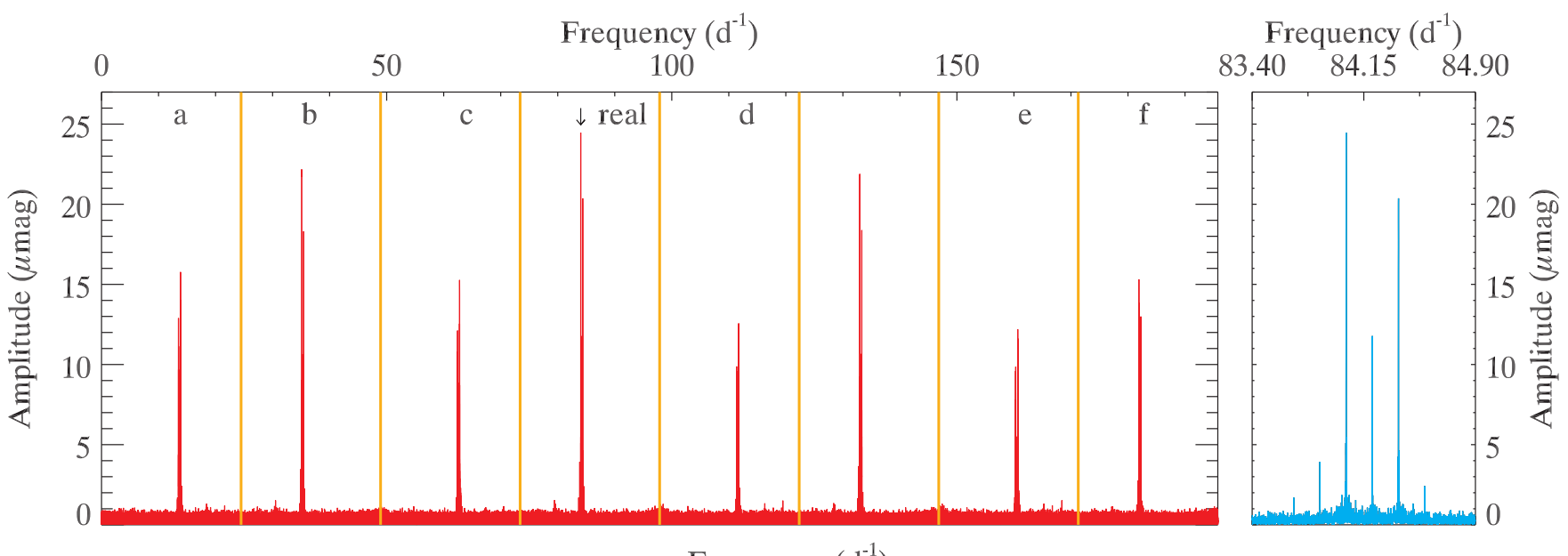

Frequency $\left(d^{-1}\right)$

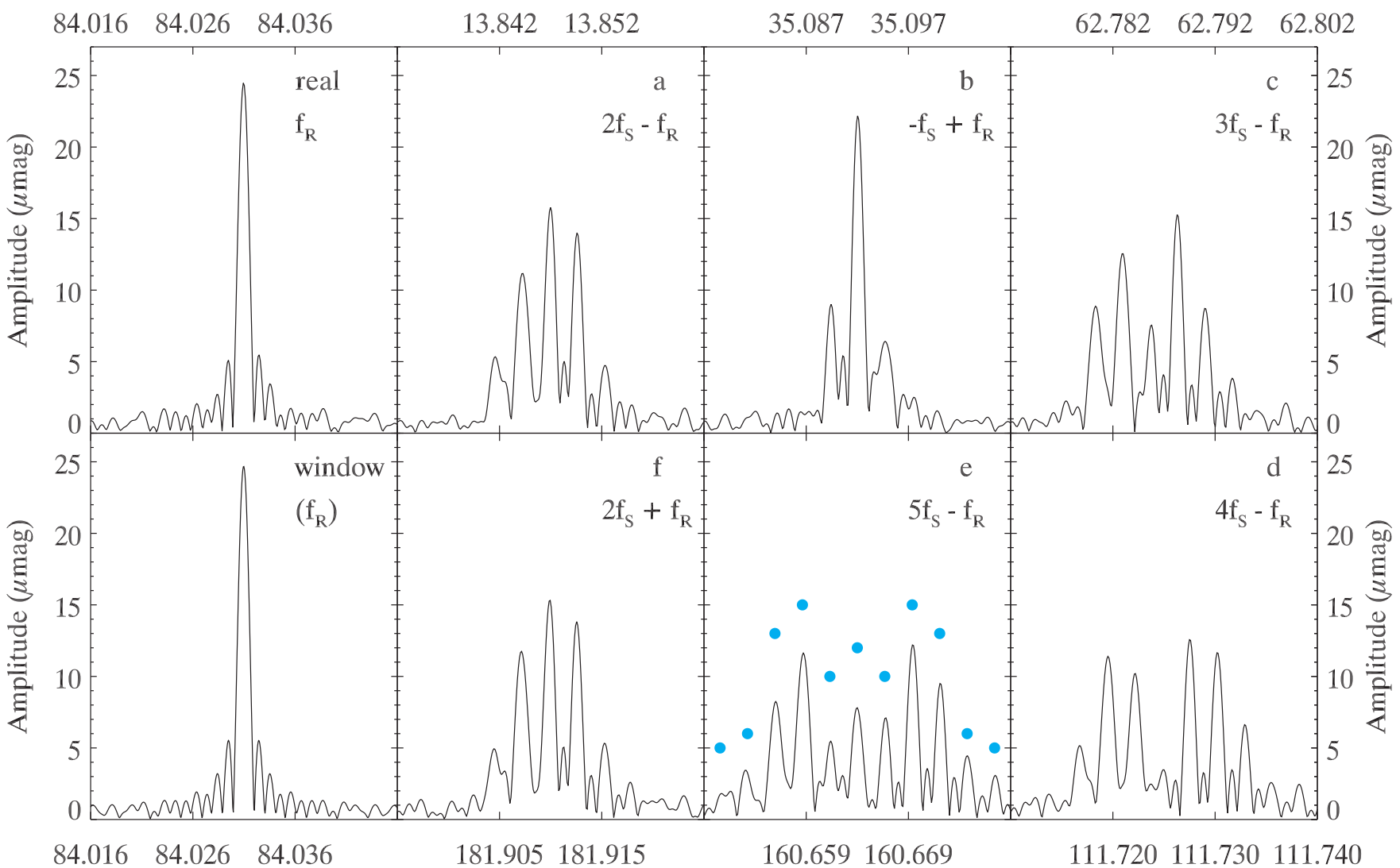

Figure 10. In this example of the roAp star KIC 10195926, a real multiplet of peaks appears around $84 \mathrm{~d}^{-1}$, and is shown zoomed in the top right-hand panel. The many aliases thereof are visible along with the real multiplet in the top left. The Nyquist frequency and its integer multiples are indicated as vertical orange lines. In the lower panels, we demonstrate the effect of periodically modulated sampling on the shape of the highest amplitude peak in each dipole-mode multiplet, all to the same scale. The real peak (far-left, top) has the typical sinc-function shape, even in these LC data and well beyond the Nyquist frequency. It can be compared to the window function in the far-left, bottom panel. The remaining lower panels, (a)-(f), correspond to labels in the upper panel, and are in frequency order, clockwise. The data are Q1-11 LC data. See the text for further explanation.

peaks with the same relationship to the sampling frequency, i.e. the pairs matching $n f_{\mathrm{S}} \pm f_{\mathrm{R}}$, are split into multiplets of the same shape, e.g. the multiplets corresponding to $2 f_{\mathrm{S}}-f_{\mathrm{R}}$ and $2 f_{\mathrm{S}}+f_{\mathrm{R}}$ have the same shape, as is seen by comparing the lower panels (a) and (f). Thirdly, even though the peaks have low amplitudes (being only about an order of magnitude higher in amplitude than the noise), it is a testament to the quality of Kepler data and confirmation of the validity of our theory that we can see so many orders of each multiplet. Specifically, panel (e) of Fig. 10 corresponds to the fifth Nyquist alias. We thus expect $5 n+1=11$ equally spaced peaks to be visible. We plot blue circles at intervals of $(1 / 372.5) \mathrm{d}^{-1}$, i.e. the orbital frequency, from the central component of the multiplet. Each multiplet member can be seen to lie at its predicted position, beneath a blue circle. There are 11 blue circles, and all but the leftmost 
one corresponds to a clear multiplet member. This leftmost member is of low amplitude, barely above the noise level and is therefore not resolved from another noise or window-pattern peak. Its counterpart, under the rightmost blue circle, is identifiable. If we had a longer observational time-span the peaks would sharpen and crosstalk between their window patterns would decrease, likely leading to the final unresolved member becoming more distinguished.

\subsection{Distinction from other modulation}

Periodic amplitude and frequency modulation of a stellar pulsation signal both generate multiplets in the frequency domain. The critical factor that distinguishes the Nyquist aliases we describe here from frequency modulation multiplets in binary stars as described by Shibahashi \& Kurtz (2012), or amplitude modulation multiplets that are seen in, for example, roAp stars and Blazhko RR Lyrae stars, is that the Nyquist alias multiplets are split by exactly the Kepler orbital frequency (1/372.5 d), within the frequency resolution. It is exceedingly unlikely that other targets will have the same modulation frequencies, hence show the same splitting. In the unlikely event that such a coincidental frequency were found in some star, then careful examination of the sidelobe amplitudes and phases would clearly distinguish the cases. Section 2 provides all the necessary information, should amplitude and phase need to be examined in this rare case.

\section{APPLICATION TO THE KEPLER SC DATA}

In the case of Kepler SC data, the sampling time interval is much shorter (at $58.9 \mathrm{~s}$ ) than in LC mode. Hence in SC we have $\omega_{\mathrm{S}}=$ $9.217 \times 10^{3} \mathrm{rad} \mathrm{d}^{-1}$, and then $\omega_{\mathrm{S}} \tau=20.3 \mathrm{rad}$ and $\Omega / \omega_{\mathrm{S}}=1.866 \times$ $10^{-6}$. The SC Nyquist frequency is $734.07 \mathrm{~d}^{-1}(=8496.18 \mu \mathrm{Hz})$.

Since the argument of the Bessel coefficients, $\omega_{\mathrm{S}} \tau$, is 30 times larger in the case of Kepler SC than that of LC, the multiplet becomes much higher order even in the case of the lowest Nyquist alias, and also the amplitude of each peak shrinks as the power is distributed into more peaks. In the case of the lowest Nyquist alias, at $\omega=\omega_{\mathrm{S}}$, the multiplicity is as high as 50 , and the amplitude is reduced to about 20 per cent of that of the true peak since the amplitude of the Bessel coefficients $J_{k}(\xi)$ asymptotically decreases as $\sim[2 /(\pi \xi)]^{1 / 2}$. This means that, in the case of Kepler $\mathrm{SC}$, each of the Nyquist aliases in the power spectrum looks like a 'forest' of peaks, while the true peak is a singlet five times higher in amplitude than the 'forest'. Hence the singlet true peak looks obviously and conspicuously different from the aliases, irrespective of whether the true frequency is higher or lower than the Nyquist frequency. The true peak should be more easily distinguished than in the LC case.

Fig. 11 shows the window spectrum for the lowest Nyquist alias at $\omega=\omega_{\mathrm{S}}$ in the case of $N=10^{5}$. Most of the peaks therein are as short as 20 per cent of the true peak, whose amplitude is normalized as unity, or much shorter. The multiplet would look like an unresolved, broad-band plateau if the resolution were lower.

As a real example, we show in Fig. 12 the alias peak in the SC data of our previous roAp star example. The upper panel demonstrates the appearance of the oblique dipole mode in the frequency range beyond the Nyquist frequency. One can see that the Nyquist aliases are at least six in number, where the seventh is at the noise level. Each peak looks like a thick pillar. That these are aliases is obvious because there is a forest where there would be a single peak if we were looking at the real pulsation frequency. The dashed red lines in the upper panel show the region that is plotted in the lower panel.

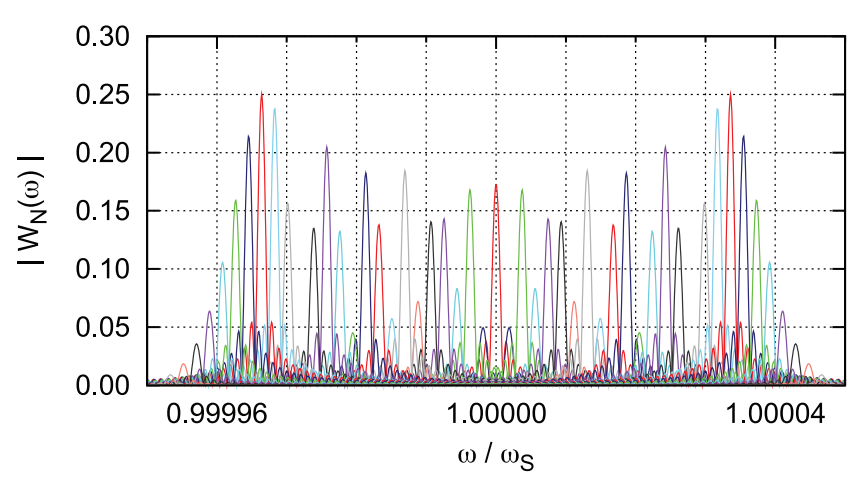

Figure 11. The window spectrum for the lowest Nyquist alias at $\omega=\omega_{\mathrm{S}}$ of the Kepler SC data.
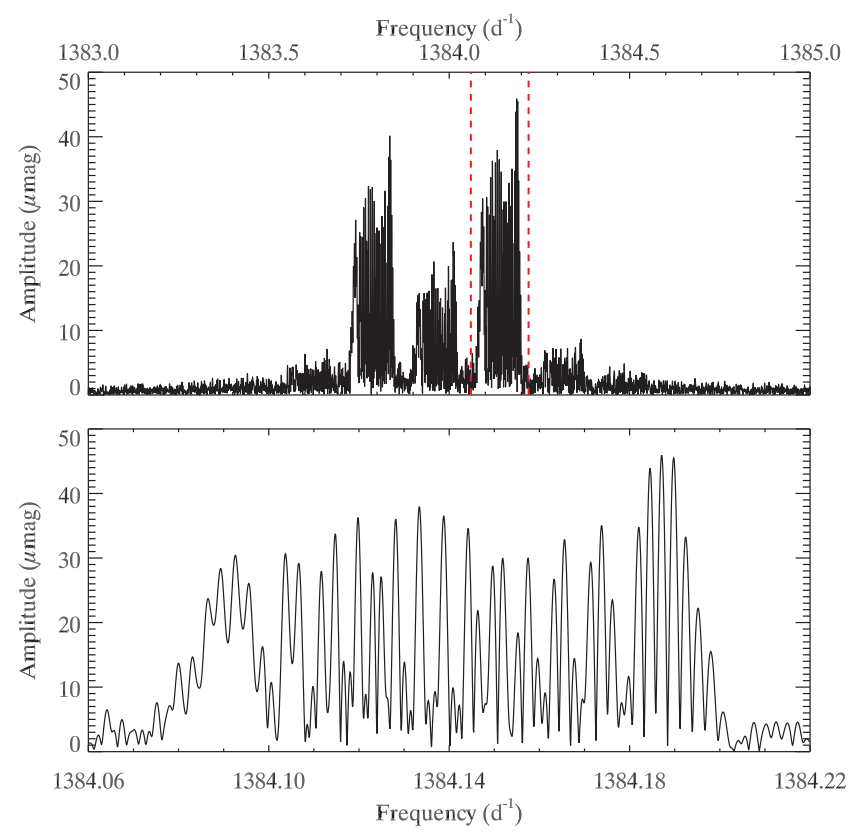

Figure 12. The Nyquist alias of Kepler SC data of the roAp star KIC 10195926. The frequency of this alias is $f_{S}-f_{\mathrm{R}}$. The upper panel shows what the mode looks like as a whole. The region delimited by the dashed red lines is shown in the lower panel, zooming in on the 'forest'.

As seen in this zoomed-in plot, the shape of the 'forest' matches reasonably well with the expected window spectrum shown in Fig. 11, in particular, for the right half (on the left some peaks are unresolved in the real data) the relative amplitudes of each peak fit well with the expectation, as does the amplitude ratio of the alias to the true peak: the multiplet has amplitudes that range from around $30 \mu \mathrm{mag}$ for the unresolved part to $45 \mu \mathrm{mag}$ for the righthand side, while the amplitude of the true peak in the Q6-12 SC data is $168 \mu \mathrm{mag}$. The anticipated amplitude reduction to around 20 per cent is confirmed.

We provide one more example: that of aliasing of a pulsation frequency above the SC Nyquist frequency. We use the subdwarf B star KIC 10139564 that was examined by Baran et al. (2012). In this star, non-linear combinations of mode frequencies lead to combination frequencies above the SC Nyquist frequency. It should be stressed that these frequencies are still real frequencies that describe the light variations of the star. Baran et al. correctly identified aliases of a few of these in their fig. 20. The aliases identified were of the form $f_{\mathrm{R}}-f_{\mathrm{S}}$, and their Fourier calculations were based on 

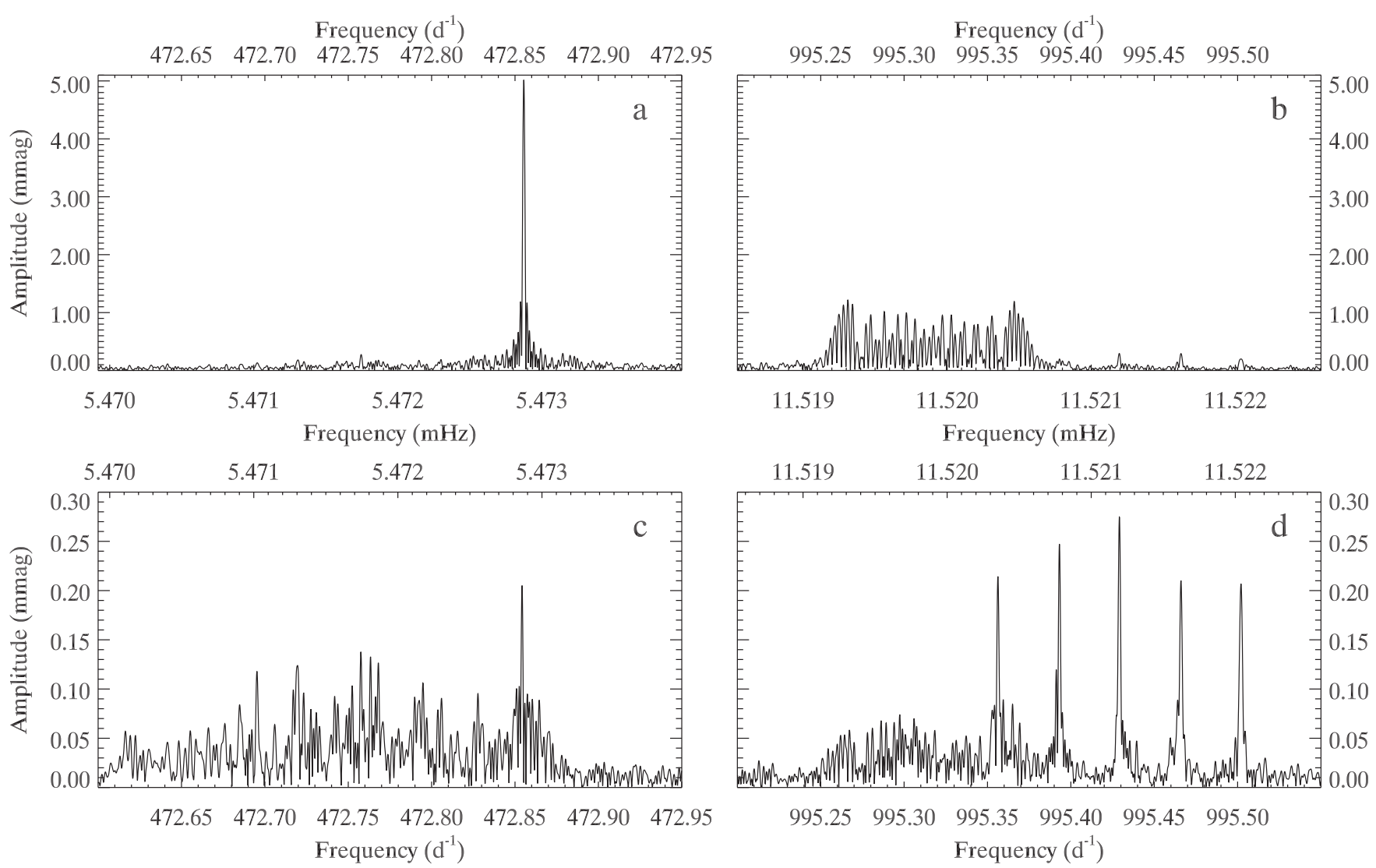

Figure 13. Horizontally adjacent panels have the same amplitude scale; vertically adjacent panels cover the same frequency region. All panels are to the same scale in frequency. The tall peak of panel (a) is the ' $f_{18}$ ' of Baran et al. (2012), and is pre-whitened in the lower panels, only. The Fourier transform is calculated on Kepler Q5-12 SC data, processed with pipeline version 8.0/8.1. Further explanation is provided in text.

626000 data points covering $462.5 \mathrm{~d}$ of SC data. Here, we extend the analysis to $10^{6}$ data points covering $739 \mathrm{~d}$.

In addition to the combination frequencies at $995.43 \mathrm{~d}^{-1}$ reported by Baran et al. (2012), lies a Nyquist alias centred at $995.31 \mathrm{~d}^{-1}$. This frequency to which this Nyquist alias belongs was pre-whitened by those authors (identified there as $f_{18}=472.855 \mathrm{~d}^{-1}$ ) and so its Nyquist alias does not appear in their fig. 20. We present the situation in Fig. 13.

Panel (a) shows ' $f_{18}$ ' of Baran et al. (2012). In lower panels only, $f_{18}$ has been pre-whitened. Its Nyquist alias, $f_{\mathrm{S}}-f_{18}$, is the broad forest in panel (b), of substantially lower amplitude than the real peak, and in accordance with our theoretical expectations regarding both location in frequency and reduced amplitude. Also visible on the right of panel (b) are some low-amplitude peaks that are combination frequencies in this $\mathrm{sdB}$ star. If $f_{18}$ is pre-whitened, its alias multiplet disappears from panel (b); what remains is visible in panel (d) and includes those combination frequencies. Finally, those combination frequencies have Nyquist aliases of their own, but since those (real) combination frequencies are so low in intrinsic amplitude, their Nyquist aliases just contribute to the noise left in panel (c) - Baran et al. noticed and commented upon this increased noise, describing it as 'low-amplitude complex signal'.

What we are seeing is two sets of frequencies $\left(f_{18}\right.$ and the combination frequencies) that lie almost equidistant from and on opposite sides of the SC Nyquist frequency, such that the aliases of one set fall right next to the real frequency of the other set, and vice versa. Panel (b) in particular highlights how easily these Nyquist aliases can be distinguished from real peaks, even (and indeed especially) in this very high frequency regime.

\section{CONCLUSIONS}

The barycentric time corrections applied to Kepler data break the regularity of the time interval between consecutive observations Kepler data are not equally spaced in time. For data spanning at least one orbital period, periodically modulated sampling causes multiplets to be generated out of Nyquist aliases, whereas real peaks remain as singlets, irrespective of whether they lie above or below the Nyquist frequency. Multiplicity of the Nyquist aliases, along with relative amplitudes between multiplet members, depends on the number of multiples of the Nyquist frequency crossed, the amplitude of the sampling modulation and the observational time-span of the data.

We have theoretically derived the expected shapes of the alias multiplets and shown that the observed patterns are in agreement with the theory. We investigated the shape as a function of the number of multiples of the Nyquist frequency crossed, and obtained the following results. In real Kepler LC data, where noise is small but none the less present, the Nyquist aliases are split into multiplets whose members number $2 n+1$, where $n$ is the number of multiples of the Nyquist frequency crossed. In the SC case, multiplicity is substantially higher and amplitudes are further reduced. Multiplet members are equally separated in frequency by the orbital frequency 
of the satellite in both cadences. The shape of the multiplet is independent of the actual pulsation frequency.

The resulting amplitude reduction upon aliasing precludes this method from being applicable to frequencies of very low inherent amplitudes, such as those of solar-like oscillators - the amplitude reduction leaves no significant signal. The major consequence of our findings is that LC data may be used to study $\delta$ Sct stars and roAp stars with real pulsation frequencies above the LC Nyquist frequency. This is also true using $\mathrm{SC}$ data for much higher frequency pulsators such as sdBV stars and pulsating white dwarf stars that have frequencies above the SC Nyquist frequency. The requirement is that continuous data sets are needed that have time-spans greater than the orbital period of Kepler. This is the case for many hundreds of stars observed by Kepler in LC that previously were thought to have insurmountable ambiguities in their frequencies.

\section{ACKNOWLEDGEMENTS}

SJM acknowledges the financial support of the STFC. This work was carried out with partial support from a Royal Society UK-Japan International Joint program and also from a JSPS Japan-UK Joint
Research project. We thank P. Degroote and S. Bloemen for their useful comments.

\section{REFERENCES}

Baran A. S. et al., 2012, MNRAS, 424, 2686

Dawson R. I., Fabrycky D. C., 2010, ApJ, 722, 937

Eyer L., Bartholdi P., 1999, A\&AS, 135, 1

Hulse R. A., Taylor J. H., 1975, ApJ, 195, L51

Koen C., 2010, MNRAS, 401, 586

Kurtz D. W. et al., 2011, MNRAS, 414, 2550

Murphy S. J., 2012, MNRAS, 422, 665

Shibahashi H., Kurtz D. W., 2012, MNRAS, 422, 738

Smith J. C. et al., 2012, PASP, 124, 1000

Sterken C., 2005a, in Sterken C., ed., ASP Conf. Ser. Vol. 335, The LightTime Effect in Astrophysics: Causes and Cures of the O-C Diagram. Astron. Soc. Pac., San Francisco, p. 181

Sterken C., 2005b, in Sterken C., ed., ASP Conf. Ser. Vol. 335, The LightTime Effect in Astrophysics: Causes and Cures of the O-C Diagram. Astron. Soc. Pac., San Francisco, p. 215

Stumpe M. C. et al., 2012, PASP, 124, 985

This paper has been typeset from a $\mathrm{T}_{\mathrm{E}} \mathrm{X} / \mathrm{LT} \mathrm{E} \mathrm{X}$ file prepared by the author. 


\section{CHAPTER 1}

\subsubsection{The rescue of Module 3}

One of the conditions of super-Nyquist asteroseismology is a continuous data set. So far, we have dismissed gaps in the Kepler data as being insignificant in its application, but within the context of the lack of influence that the gaps have on the timing of observations. Now we address the other influence that larger gaps have.

We highlighted in $§ 1.4 .3$ that cycle count ambiguities affect otherwise continu-

ous light curves for the $\frac{4}{21}$ stars that fall on Module 3, and that those cycle count ambiguities are each separated by the orbital frequency (cf. Fig. 1.25). We have seen in this present section that Nyquist aliases are also split into multiplets, and are also separated by the orbital frequency. We will now demonstrate that even stars on Module 3 are suitable subjects for super-Nyquist asteroseismology. The question we need to address is: how do we disentangle the two equally separated and superimposed contributions to the Fourier transform?

Let us first demonstrate that at high-frequency, where Nyquist multiplets have much different amplitude ratios from the cycle count ambiguities, the two are indeed distinguishable. Fig. 1.29 does this. The Fourier transform of simulated data for a star on Module 3 is a superposition of the spectral window at the Nyquist alias, with the Nyquist multiplet produced by periodically modulated sampling.

In the high-frequency case, where the shape of the spectral window is much different from the Nyquist alias multiplet, prewhitening the central frequency of the multiplet will, of course, leave highly significant residuals. However, the most important alias we need to distinguish is the $f_{\mathrm{S}}-f_{\mathrm{R}}$ Nyquist alias multiplet. Here, the spectral window looks similar in morphology to the alias multiplet, so direct visual inspection does not make the alias obvious (compare the upper and middle panels of Fig. 1.30). However, one can prewhiten the central frequency and again see that substantial residuals remain, as illustrated in the lower panel of Fig. 1.30. 


\section{CHAPTER 1}

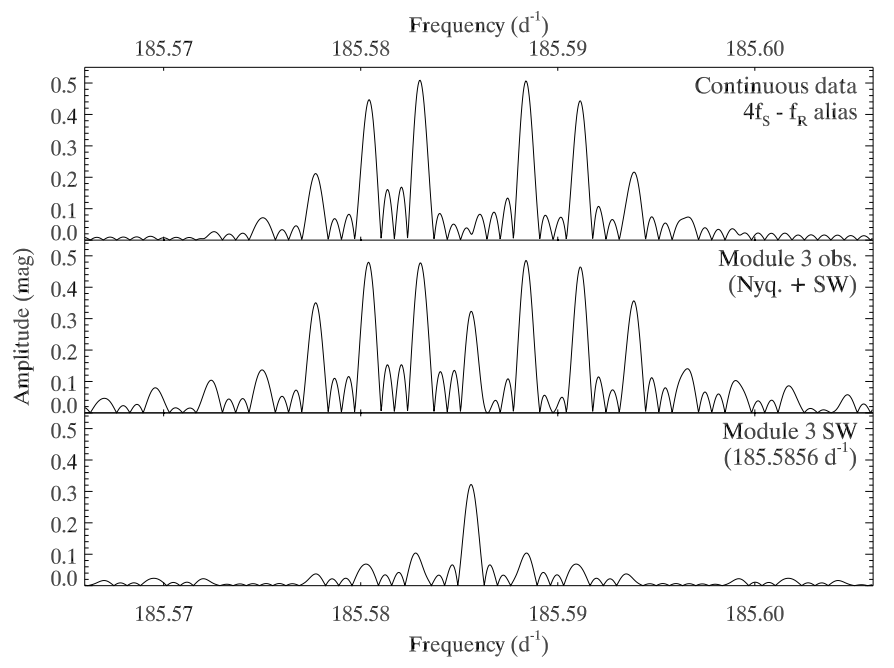

Figure 1.29: Upper panel: the expected Nyquist alias multiplet at $4 f_{\mathrm{S}}-f_{\mathrm{R}}$, for $f_{\mathrm{R}}=10 \mathrm{~d}^{-1}$, based on four orbits of simulated, LC, continuous data (for a star not affected by the Module 3 failure). Middle panel: the same Fourier calculation as in the upper panel, but for a star on Module 3. This is a superposition of the Nyquist multiplet and the spectral window (SW). Lower panel: the SW only, for the star on Module 3, folded in frequency at $4 f_{\mathrm{S}}-f_{\mathrm{R}}=185.5856 \mathrm{~d}^{-1}$ and scaled in amplitude to be the same height as the central peak in the middle panel.

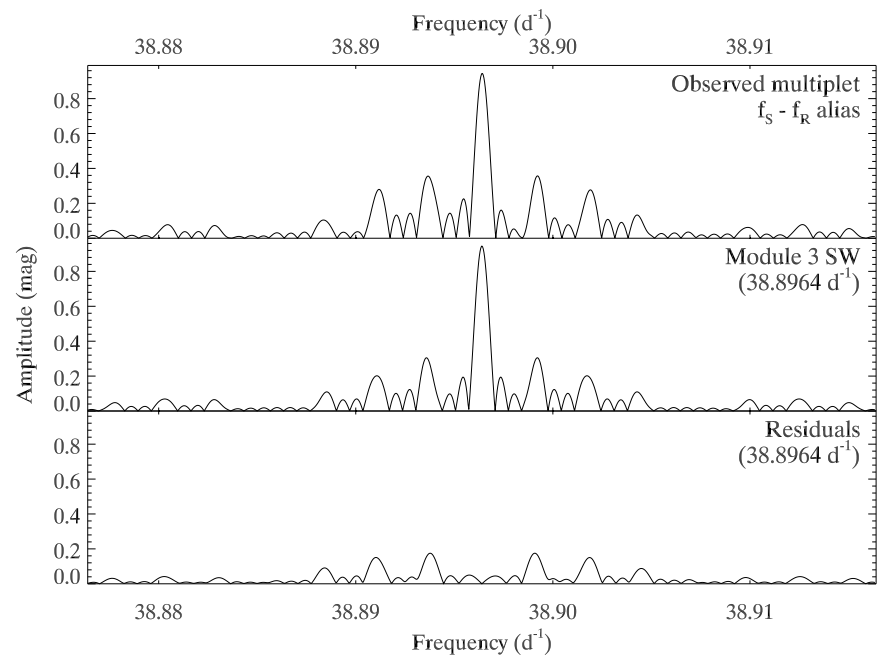

Figure 1.30: Upper panel: the observed Nyquist alias multiplet at $f_{\mathrm{S}}-f_{\mathrm{R}}$, for $f_{\mathrm{R}}=10 \mathrm{~d}^{-1}$, based on four orbits of simulated, LC data for a star affected by the failure of Module 3. Middle panel: spectral window at the frequency of the central component. Lower panel: the central component has been prewhitened using nonlinear least-squares fitting, and the residuals are plotted. 


\section{CHAPTER 1}

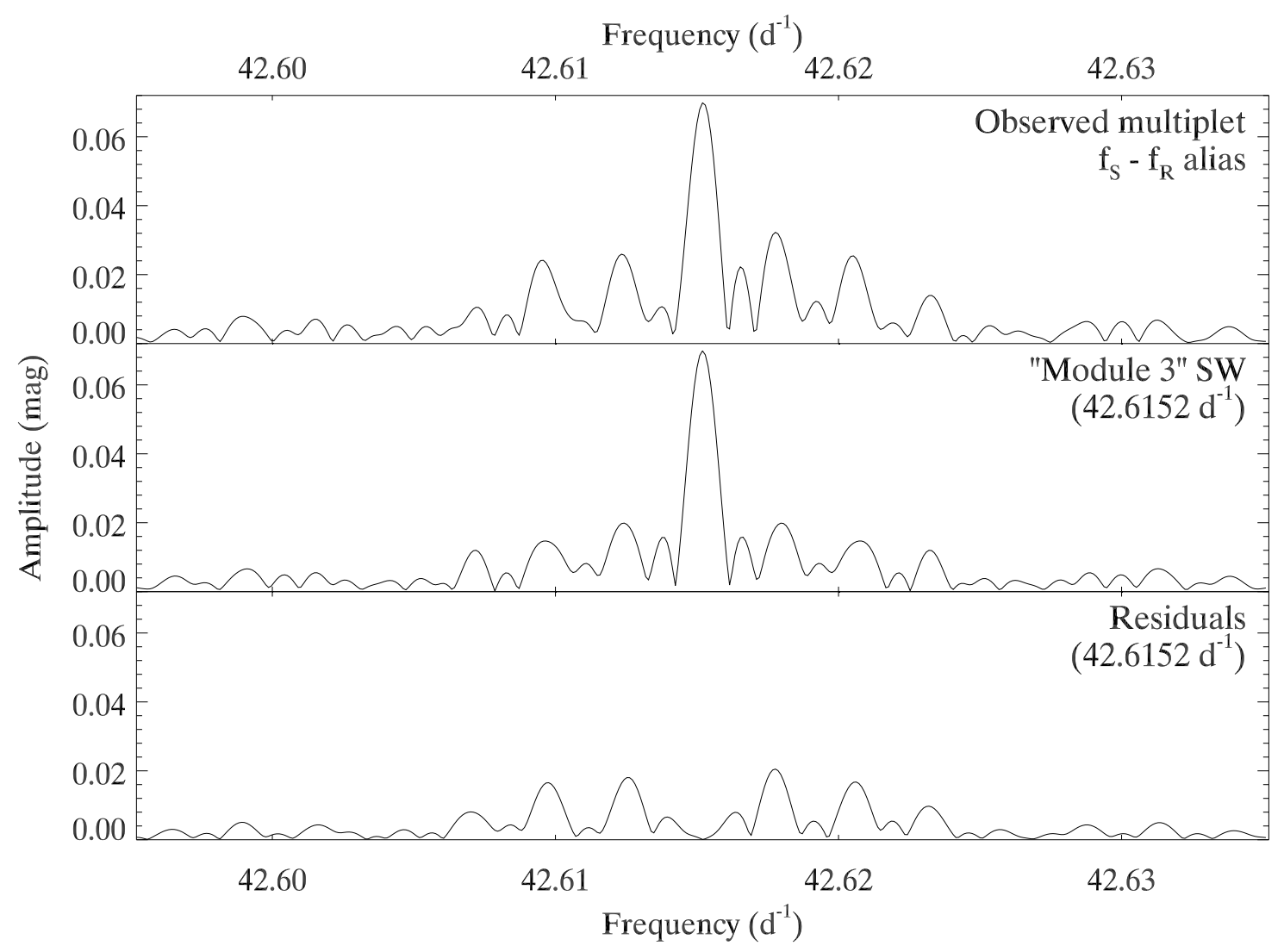

Figure 1.31: Upper panel: the observed Nyquist alias multiplet at $f_{\mathrm{S}}-f_{\mathrm{R}}$, for $f_{\mathrm{R}}=6.324 \mathrm{~d}^{-1}$, based LC data for a HADS star whose data distribution mimics the failure of Module 3 (see text). Middle panel: spectral window at the frequency of the central component. Lower panel: the central component has been prewhitened using non-linear least-squares fitting, and the residuals are plotted.

For a real example, we revisit the HADS star from the super-Nyquist asteroseismology paper. We use the Q1-12 data, but omit the Q2, Q6 and Q10 data from our analysis to simulate the star being on Module $3 .{ }^{11}$ This is a simplified example because the pulsation amplitude is high and other frequencies were pre-whitened, ${ }^{12}$ but a Nyquist alias is still distinguishable from the real peak when data outages similar to Module 3 gaps are present - the residuals after prewhitening the central component are statistically significant over the noise (Fig. 1.31).

\footnotetext{
${ }^{11}$ The fact that Module 3 was still functioning in Q2 is irrelevant in this example.

${ }^{12}$ To paraphrase Gray \& Desikachary (1973), the singly periodic case is the simplest because the periodogram is exactly the window transform translated to that single frequency.
} 


\section{CHAPTER 1}

\subsection{Chapter summary}

In this chapter we have seen how the Kepler data are revolutionary in asteroseismic analyses, without really commenting on the sheer number of targets being observed. Specific case studies will be presented in other chapters.

We saw how the SC data are superior to their LC counterparts, offering not only greater time resolution, but also higher precision on pulsation frequencies, amplitudes and phases.

The performance of the PDC pipelines, new and old (MAP and LS), was discussed and shown to be substantially better than the SAP data on which only basic calibration is performed. Issues with injected noise and low-frequency artefacts were presented and it appears that the PDC-MAP pipeline still has a long way to go. It is unlikely that a perfect pipeline will be developed, that treats instrumental artefacts without at all modifying stellar signal, but it remains true that the PDC-LS pipeline treated global (affecting all light curves) trends, such as the return to operating focus and temperature after a quarterly roll, much more successfully.

We investigated the noise of the Kepler PDC-MAP LC data for various quarters, Kp magnitudes and effective temperatures. The dependence of the logarithm of the median of the noise, $\log \left(\sigma_{\mathrm{M}}\right)$ was found to scale with Kp mag as $\log \left(\sigma_{\mathrm{M}}\right) \propto+0.18 K p$. Granulation and activity were found to increase with decreasing temperature between $7500 \mathrm{~K}$ and $6000 \mathrm{~K}$, but to be temperature independent above $7500 \mathrm{~K}$. The quality of each Kepler quarter was investigated by finding the mean noise level for each quarter. It was found that the pipeline acts to smooth the variation in quality between quarters, such that the major difference between noise in each quarter is the time span of (number of points in) that quarter. Determination of the grass level is affected by pulsation, so a population of 'constant' stars was isolated - 44.1 per cent of stars in the $\delta$ Sct instability strip had no Fourier peak above $50 \mu$ mag and are 'constant' by this definition. 


\section{CHAPTER 1}

We examined the presence of gaps in the data and determined that they do not limit asteroseismology with Kepler data. The largest gaps encountered that are common to all stars correspond to safe mode events, which, up to Q13, have been no larger than one-fifth of an observing quarter in length. The concept of adjusting weights in favour of consecutive observations to overcome aliasing difficulties in ground-based data was discussed.

Until recently the Nyquist frequency has been a limiting factor in the study of many classes of pulsating stars with Kepler observations. We have seen that the barycentric corrections to time-stamps of Kepler data, necessitated to remove the Doppler shift of arriving photons at the receiving satellite, leads to periodically modulated sampling. When multiple Kepler orbits of data are available, this causes Nyquist aliases to be split into multiplets that are distinguishable from the real pulsation frequencies whose peaks remain as singlets. The result is true even if the pulsation frequency is well beyond the Nyquist frequency. The LC data were shown to have reduced amplitudes compared with the SC data because of the lower sampling rate (leading to under-sampling), and for this reason the solar-like oscillators are precluded from application of this method. But for many hundreds of stars with LC data that were previously thought to have Nyquist ambiguities ( $\delta$ Sct and roAp stars), or could be misinterpreted from such ambiguities (a $\delta$ Sct star pulsating at high frequency could be misidentified as a $\gamma$ Dor star), this result opens up an avenue for further study. The same is true of the SC data for the pulsating white dwarfs and sdBV stars.

\section{A Published Content}

In this appendix, the papers that were adapted in $\S 1.2$ are provided in their published format, noting that the papers are superseded by that section. 


\title{
An examination of some characteristics of Kepler short- and long-cadence data
}

\author{
Simon J. Murphy ${ }^{\star}$ \\ Jeremiah Horrocks Institute, University of Central Lancashire, Preston PR1 2HE
}

Accepted 2012 January 26. Received 2012 January 25; in original form 2011 August 31

\begin{abstract}
A close comparison of Kepler short- and long-cadence data released prior to 2011 November 1 has shown some subtle differences that make the short-cadence data superior to their longcadence counterparts. The inevitable results of a faster sampling rate are present: the shortcadence data provide greater time resolution for short-lived events like flares, and have a much higher Nyquist frequency than the long-cadence data; however, they also contain fewer high-amplitude peaks at low frequency and allow a more precise determination of pulsation frequencies, amplitudes and phases. The latter observation indicates that Kepler data are not normally distributed. Moreover, a close inspection of the Pre-search Data Conditioned long-cadence data shows residuals that have increased noise on time-scales important to asteroseismology, but unimportant to planet searches.
\end{abstract}

Key words: asteroseismology - methods: data analysis - methods: observational techniques: photometric - stars: activity - stars: oscillations.

\section{INTRODUCTION}

The Kepler Space Telescope is in a 372.5-d heliocentric Earthtrailing orbit, collecting white-light photometric data for a sample of $\sim 160000$ stars covering a field of view of $115 \mathrm{deg}^{2}$. The core goal of the mission is detection of Earth-like planets orbiting Sunlike stars within the habitable zone. The details of the mission goals and design are described by Koch et al. (2010) and Borucki et al. (2010).

Secondary to the main goal of the mission is asteroseismology, which can provide valuable information on the host stars that is important in planet characterization. With transit depths, only the ratio of the planet and host star radii is available, but asteroseismology allows detailed analysis of the star's interior if the star pulsates, and for solar-like oscillators can yield the star's radius to better than 3 per cent (Stello et al. 2009) - even approaching 1 percent in some cases (Gilliland et al. 2010a). Through asteroseismology, Kepler also promises significant advances in stellar astrophysics with the dedication of $\sim 1$ per cent of observations to asteroseismic study (Gilliland et al. 2010a).

Kepler data are available in two cadences, long cadence (LC) and short cadence (SC). Each cadence is composed of multiple 6.02-s exposures with associated 0.52 -s readout times (Gilliland et al. 2010b). The LC data integrate over 270 exposures to give 29.4-min observations, whereas the SC data contain nine exposures giving one data point every $58.9 \mathrm{~s}$. Both cadences are stored on-board

^E-mail: smurphy6@uclan.ac.uk and downlinked to Earth roughly every $32 \mathrm{~d}$, introducing gaps up to $\sim 24 \mathrm{~h}$ in length while the photometer is not collecting data. Kepler completes one quarter of its orbit after three downlinks, and must then perform a quarterly roll to keep its solar panels pointing towards the Sun and its radiator pointing to deep space. Kepler data are therefore organized into quarters and thirds around those rolls and downlinks. LC data quarters are denoted by Q $n$, and SC quarters by Qn.m to notify which third (or 'month') of that quarter the data correspond to.

Pre-Q9, Kepler data were available in two forms: (i) 'raw' flux, of which Simple Aperture Photometry (SAP) flux is the preferred nomenclature, and for which basic calibration is performed distinguishing it from the truly raw flux, and (ii) Pre-search Data Conditioned (PDC) 'corrected' flux. The PDC data were created as a step towards facilitating planetary transit searches and should be used only with caution in astrophysical analyses because some stellar variability can be modified in the light curves by the PDC pipeline, pertaining to data releases 11 and earlier. This is discussed in Section 5. Post-Q9, PDC has been superseded by another pipeline, PDC MAP. New quarters of data will contain PDC MAP rather than the old PDC fluxes, and older quarters of data are due to be reprocessed with this pipeline and made public by 2012 July.

There are many advantages of SC data over LC data, but hardware limitations restrict SC slot allocation to 512 slots at any given time. Here we discuss the following advantages of SC data: increased sampling rate, higher Nyquist frequency, fewer low-frequency artefacts, and reduced errors on frequency, amplitude and phase determinations in the Fourier spectrum. We also discuss the difference in distribution of data points between SC and LC data and look at 
the differences in the PDC and SAP-flux data. Initial characteristics of the LC and SC data can be found in Jenkins et al. (2010) and Gilliland et al. (2010b), respectively. For a detailed, recent review of Kepler noise properties, see Gilliland et al. (2011) and references therein.

\section{SAMPLING RATE}

There are 30 times more SC points than LC points in a quarter, arising from the longer integration time used for LC data. Two well-known effects this has on time series analysis are the time resolution available and the associated Nyquist frequency.

\subsection{Time resolution}

The primary mission goal of planet detection requires the increased sampling rate to time transits more precisely; signs of gravitational perturbation seen as changes in transit duration may lead to subsequent detections of other planets orbiting the same star. Holman \& Murray (2005) calculate that under the gravitational influence of other Solar system bodies, Earth's apparent transit time for an observer viewing along the orbital plane would appear to decrease by around $650 \mathrm{~s}$ for two in 10 of its orbits, depending on the relative position of each planet and the observer with respect to orbital phase. The effect is even greater for planets orbiting farther from their star (up to $\sim 6000 \mathrm{~s}$ for Mars) and for planets orbiting less massive stars. The sensitivity required for such detections is easily met by Kepler, where transit durations would vary by 11 and 101 SC points for the Earth and Mars cases, respectively, even where errors from photon statistics on such a transit duration reach $\sim 500 \mathrm{~s}$, equivalent to eight SC points (Holman \& Murray 2005). Indeed, the first previously unknown planet to be detected using this technique, Kepler-19c, does not appear to transit its star (Ballard et al. 2011), but leaves a clear sinusoidal deviation of the transit times once the transits of Kepler-19b are subtracted out.

The higher sampling rate is useful for astrophysical studies too, particularly for short-lived events like flares. The star KIC 12406908 is in Kepler Asteroseismic Science Consortium ${ }^{1}$ Working Group 7 (Cepheid variables), but is probably misclassified. It is one of the $\sim 15$ per cent of the Kepler Input Catalogue stars that have no fundamental parameters listed, that is no $T_{\text {eff }}$ or $\log g$ values are available. Fig. 1 shows a 7.2-h sample of the light curve of the largest flare in Q3.1. The LC data have been plotted underneath the SC data for comparison, and a shift in magnitude has been created for demonstrative purposes only. The longer integration time in LC has the effect of averaging the SC points, and undersamples the 0.056-mag flare. The shape of the flare, including its erratic nature as its luminosity output rises and falls numerous times across the event, is lost in the LC data. Only a rough approximation of its magnitude and duration would be determinable without the SC data. Events with such short time-scales can clearly only be studied in SC.

\subsection{Nyquist frequency}

The most important benefit of SC data to asteroseismology is the higher Nyquist frequency, and the exquisite quality of the Kepler data provides a nice opportunity to demonstrate this. Many asteroseismic targets pulsate at frequencies higher than the Nyquist frequency of the LC data $\left(24.469 \mathrm{~d}^{-1} ; 283.21 \mu \mathrm{Hz}\right)$, and cannot

\footnotetext{
${ }^{1}$ http://astro.phys.au.dk/KASC/
}

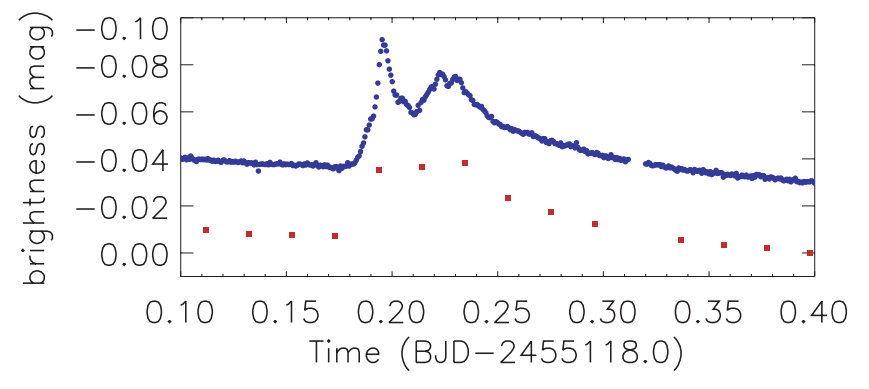

Figure 1. A large-amplitude flare on KIC 12406908. The LC data (red squares) are plotted beneath the SC data (blue circles) for comparison. The change in brightness is precise, but not accurate - the dimmest LC point was chosen as the zero-point for the graph, and all SC points are offset for clarity. The SC data used are Q3.1 PDC flux (see Section 5 for more details on PDC flux), and the LC data are simulated by averaging bins of 30 consecutive SC points.

be studied reliably due to aliasing problems. Solar-like oscillations and roAp star pulsations only occur at frequencies much higher than this. Straddling both sides of the LC Nyquist frequency are the $\delta$ Sct stars. These stars pulsate in low-order $\mathrm{p}$ modes (pressure modes) and typically have frequencies in the range 4-50 $\mathrm{d}^{-1}(46-579 \mu \mathrm{Hz}$; Breger 2000), although the highest published frequency for a $\delta$ Sct star is currently $79.5 \mathrm{~d}^{-1}(920 \mu \mathrm{Hz}$; Amado et al. 2004).

The Nyquist frequency is equal to half the rate at which a signal is being sampled. Since LC has a point every $29.4 \mathrm{~min}$, there are 48.9 points per day, and the Nyquist frequency is therefore $24.5 \mathrm{~d}^{-1}$ $(284 \mu \mathrm{Hz})$. Hence, if in the Fourier spectrum a signal is detected with a frequency higher than the Nyquist frequency, it is not fully sampled, and an alias will be detected at $2 f_{\text {Nyquist }}-f_{\text {signal. }}$. It is not always obvious that these detected frequencies are 'reflections' of frequencies higher than $f_{\text {Nyquist }}$, and can sometimes be interpreted as real pulsation frequencies.

One is naturally cautious when any frequencies are detected in LC near the Nyquist frequency, as the star could have pulsation frequencies above the Nyquist frequency even if the detected frequencies are real and not reflections. However, for much lower frequencies the possibility of a reflection seems more remote.

KIC 10977859 is a $\delta$ Sct star in which the SC data show only highorder p modes, and a lack of pulsations at frequencies below the LC Nyquist frequency (Fig. 2, upper panel). When only the LC data are considered (if SC data were not available, for instance), the spectrum looks entirely different. Nyquist frequency limitations mean that in LC data the true frequencies of high-order $\mathrm{p}$ modes would not be discernible, but a huge number of peaks are visible below the LC Nyquist frequency instead (Fig. 2, lower panel), strongly implying the star pulsates in low-order p modes and maybe g modes (gravity modes), too. What is even more misleading in this case, and makes the situation problematic, is the absence of peaks in the periodogram of the LC data between 16 and $24.4 \mathrm{~d}^{-1}(185-282 \mu \mathrm{Hz})$, fooling the observer into believing these are low-order p modes with only a small likelihood of signals at higher frequencies - there is no warning of what lies beyond the LC Nyquist frequency.

One must exercise extreme caution when analysing LC data if there are no SC data to test for aliasing problems associated with the LC Nyquist frequency.

\section{LOW-FREQUENCY PEAKS}

Another benefit of SC data over LC is the reduced number of highamplitude peaks at low frequency. Such peaks can arise naturally 

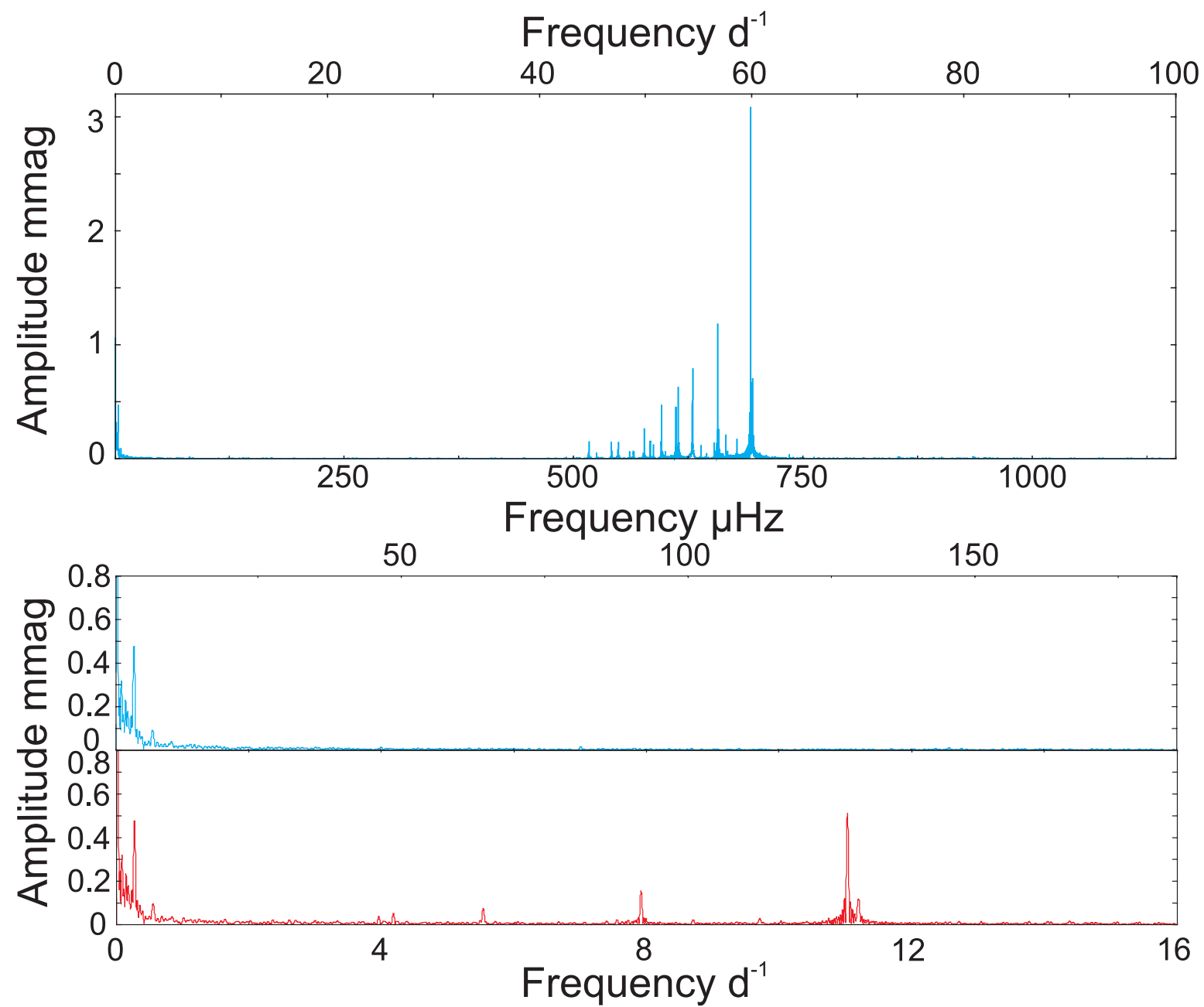

Figure 2. Upper panel: the Fourier spectrum of the $\delta$ Sct star KIC 10977859 for Q1, showing only high-order, high-frequency p modes, and a lack of pulsations at lower frequencies. Lower panels: a magnification of the spectrum between 0 and $16 \mathrm{~d}^{-1}$ for the same star, in SC (blue, middle) and LC (red, bottom). The amplitude of the real peaks in the top panel is up to five times greater than corresponding peaks in the bottom panel, indicating a significant amplitude reduction in reflected peaks. No points were removed from these SAP-flux data, which were analysed using the statistical package PERIOD04 (Lenz \& Breger 2004).

from long time-scale processes such as differential velocity aberration, with stars moving across the CCD by up to 1.5 pixels, which results in a different flux fraction being captured by the $\mathrm{CCD}$, and the amount of background contaminating light changing. García et al. (2011) cite CCD degradation as a cause of long time-scale drifts too, but CCD degradation is likely to arise from high-energy cosmic ray impacts and will often, as a result, be more of a step-function than a long-term trend. SAP-flux data do contain strong instrumental trends that dominate at low frequency, rendering the difference in prevalence of low-frequency peaks between the two cadences insignificant in SAP flux. However, the LC PDC-flux data, from which instrumental trends have mostly been removed, still contain some relatively high-amplitude peaks at low frequency.

In order to demonstrate this phenomenon, a nearly constant star was selected to minimize the number of peaks seen as a result of pulsations. The nature of any frequency peaks in the Fourier transform depends greatly on the length of the data set. For one of the stars analysed, KIC 9390100, the LC Q2 data span 88.9 d, but the SC Q2.2 data only span $30.0 \mathrm{~d}$ - the star was not observed in SC during Q2.1 and Q2.3. It was therefore necessary to truncate the length of the LC data to that of the SC Q2.2 data. Regardless of cadence, each quarter is divided into thirds by Kepler's downlinking process, thereby the cadences corresponding to Q2.1 and Q2.3 in the LC data were easily removed. No outliers were removed from either data set. A third data set was made for comparison by binning sets of 30 consecutive SC points and replacing them with a single point; the fluxes of the SC points were added and their times averaged. This 'merging' process creates points that are entirely concurrent with the LC data (for every LC point there is a merged point at exactly the same time), and the merged and Kepler LC data sets shown in Fig. 3 contain exactly the same number of points. To achieve this, two more points had to be removed from the LC data set, at BJD $=2455042.3243$ and 2455051.4377 , because undefined SC flux values in these bins did not allow accurate merged data points to be created, but Kepler LC points existed at those times. The $29 \times 2$ $\mathrm{SC}$ data points corresponding to each of these LC points were also removed for a fair comparison in this PDC data example.

At lower frequencies (below $2 \mathrm{~d}^{-1}$, and especially below $0.4 \mathrm{~d}^{-1}$ ), the SC and LC spectra are significantly different (Fig. 3). There are a few coherent peaks common to both cadences, but the LC data 


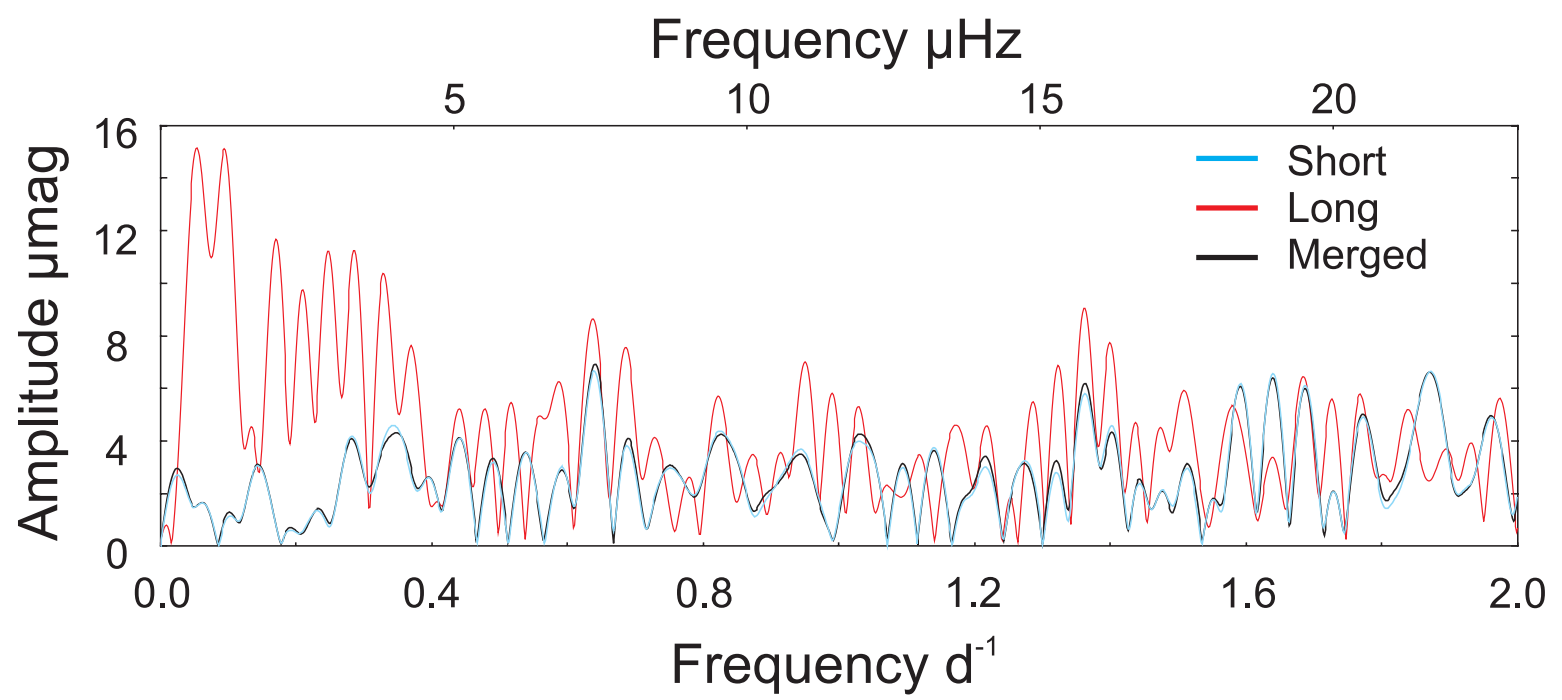

Figure 3. The PDC SC data (blue) are plotted on top of the PDC LC data (red) of Q2.2 for the near-constant star KIC 9390100 for frequencies 0-2 d ${ }^{-1}$. Plotted in black are the LC data created from merging the PDC SC data. The blue and black lines almost completely overlap, but the displacement of the red line indicates the extent of artificial low-frequency peaks introduced by the PDC data pipeline.

have many more high-amplitude ${ }^{2}$ peaks at those frequencies under $2 \mathrm{~d}^{-1}$. This difference between SC and LC Kepler data is often encountered, and requires treating before frequency analysis.

At higher frequencies (above $2 \mathrm{~d}^{-1}$ ), the two amplitude spectra are almost the same, and can be seen converging in the figure. The merged data, which one expects to be similar to the LC data because they are created by integrating fluxes over the same cadence numbers, mimic the SC data very well over the entire frequency range. Slight differences can be accounted for by considering that there are occasionally SC data points next to data gaps that do not get incorporated into merged data, because the merged data must be concurrent with the LC data for a direct comparison.

The Kepler LC data should not behave exactly like the merged data, because LC and SC data go through a different calibration process (involving such methods as dark/bias subtraction and flat-field removal), but the scale of the difference suggests that discrepancies do remain after the PDC-correction procedure. These discrepancies are not seen to the same extent in the SAP-flux data; the difference in peak heights in the PDC example in Fig. 3 is $12 \mu$ mag ( 400 per cent) compared with $6 \mu \mathrm{mag}$ in SAP flux ( 2 per cent), noting that instrumental trends dominate in SAP flux. That some discrepancies remain in the PDC data is not surprising, as they were designed to facilitate planet finding, not asteroseismology. While the residual peaks contribute significantly to the noise on time-scales important to asteroseismology, planetary transit searches are not greatly affected. Gilliland et al. (2011) compared noise on 6.5-h time-scales, chosen to be representative of planet transit durations; this corresponds to a frequency of $3.7 \mathrm{~d}^{-1}$, but all three lines in Fig. 3 are already converging at $2 \mathrm{~d}^{-1}$. In fact, a planet orbiting a Sun-like star and having a transit time of $1 \mathrm{~d}-$ a time-scale where the discrepancies are an important source of excess noise - would have a semimajor axis of $3.4 \mathrm{au}$ and a period of $6.3 \mathrm{yr}$. The detection and confirmation of such a planet is much beyond the design capabilities of Kepler unless the mission competes successfully for an extension.

\footnotetext{
${ }^{2}$ Relatively speaking, amplitudes of $10^{-5}$ mag are normally considered tiny in the analyses of $\delta$ Sct stars!
}

In addition to contributions to the total noise level, the lowfrequency artefacts can cause other problems, an example of which is in automatic frequency extraction procedures that select the highest amplitude peak or peaks in the Fourier spectrum to classify a star. If the spectrum is dominated by non-astrophysical low-frequency peaks, then false classification may occur and lead to incorrect statistics on both dominant frequencies and amplitudes.

A solution to the problem is on the way. PDCMAP sees $\sim 10-20$ per cent improvement in signal-to-noise ratio on the 6.5-h time-scales reported in Gilliland et al. (2011) (Jon Jenkins, private communication), and supports their conclusions that the major contributor to the observed excess noise still originates in the stars themselves.

\section{PEAK WIDTHS, AMPLITUDES AND ERRORS}

The width of a peak in the Fourier spectrum of a data set of length $T$ can be approximated by $1 / T$ (the Rayleigh criterion). For data sets of equal length, the greater number of points in SC data has no effect on the width of the peak, but the amplitude of peaks is different. As mentioned in Section 2, the longer integration time of the LC data causes an averaging effect that is more notable for shorter period events or pulsations. The same effect reduces the amplitude of peaks in the periodogram of LC data, as shown in Fig. 4. The percentage difference in amplitude between SC and LC increases with higher frequencies.

This effect can be explained mathematically. For a Fourier peak with true amplitude $A_{0}$, it can be shown (see Supporting Information with the online version of the paper) that the observed amplitude, $A$, is described by the equation

$A=\frac{\sin \pi / n}{\pi / n} A_{0}$,

for $n$ points per cycle. Using KIC 3437940 (from Fig. 4) as a numerical example, the ratio of the observed amplitude in $\mathrm{SC}$ to that of $\mathrm{LC}, A_{\mathrm{SC}} / A_{\mathrm{LC}}$, is 1.08 for the peak at $10.5 \mathrm{~d}^{-1}$, but $A_{\mathrm{SC}} / A_{\mathrm{LC}}$ increases to 1.34 for a hypothetical peak at $20 \mathrm{~d}^{-1}$.

KIC 10977859 (Fig. 2) demonstrates the same effect for highfrequency pulsations that are reflected in the Nyquist frequency. 


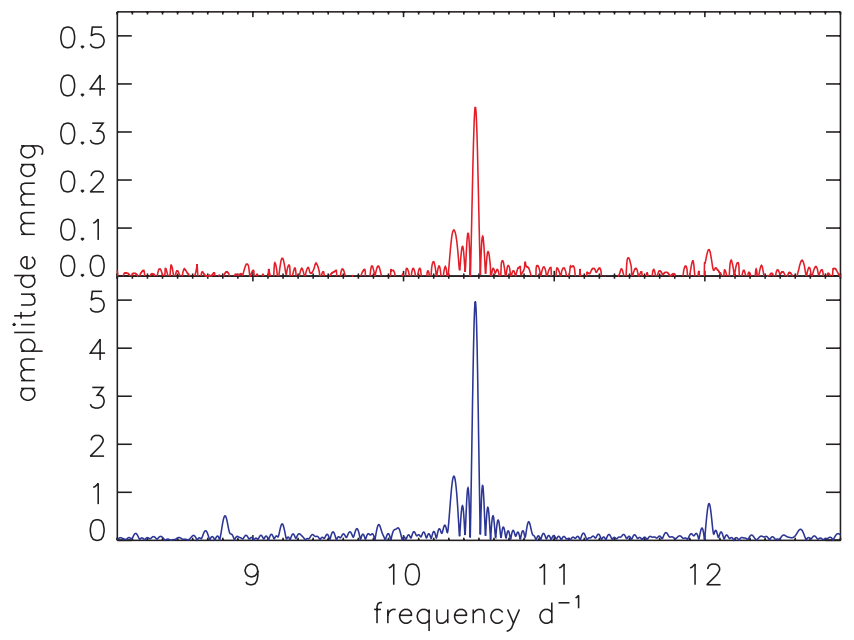

Figure 4. The Fourier transform of the LC Q3.2 (truncated) data for KIC 3437940 is plotted at the bottom in blue, and the difference between the amplitudes of the SC and LC data for the same month is plotted above in red, showing that the SC data have slightly higher amplitude peaks due to the shorter integration time and the averaging effect inherent within LC data. Note the change in scale on the vertical axis by a factor of 10 between the two plots.

The highest peak in the bottom panel is located in frequency just where one expects from the reflection of the highest peak in the top panel (to well within one full width at half-maximum), and has an amplitude reduction in agreement with equation (1) to within the least-squares errors. One can therefore do asteroseismology on $p$ modes above the LC Nyquist frequency using LC data, providing at least one month of SC data is available to overcome the aliasing problem.

Having a greater number of points allows a more precise determination of pulsation frequencies, amplitudes and phases. To give a quantitative example, one month of LC data was compared to SC data for the star KIC 3437940 and points were only removed to truncate the LC Q3 data set to exactly the same time-span as the SC Q3.2 data set - as was done in Section 3 for KIC 9390100. The errors on frequency, amplitude and phase for the different cadences are summarized in Table 1, and are of the order of 5 times greater for the LC data. The result applies to both SAP and PDC flux, indicating the precision difference is not a result of the greater variance at low frequency presented in Fig. 3. This is an important distinction pointing to greater quality of the SC data, and implies the data are not normally distributed. Degroote et al. (2009) found a similar result for CoRoT noise properties.

The scatter of points in SC data is greater than that of LC data. There are 30 times more points in SC data, so the scatter of points

Table 1. The formal least-square errors on frequency, amplitude and phase for Q3.2 SC and LC data of the star KIC 3437940, with the LC errors being greater in each case by a factor of $\sim 5$. The least-square errors were calculated with PERIOD04.

\begin{tabular}{lccc}
\hline Cadence & $\begin{array}{c}\text { Frequency error } \\
\left(\times 10^{-5} \mathrm{~d}^{-1}\right)\end{array}$ & $\begin{array}{c}\text { Amplitude error } \\
(\mu \mathrm{mag})\end{array}$ & $\begin{array}{c}\text { Phase error } \\
\left(\times 10^{-3} \mathrm{rad}\right)\end{array}$ \\
\hline SC PDC & 4.9 & 14 & 0.4 \\
LC PDC & 25.8 & 70 & 2.2 \\
SC SAP & 4.6 & 13 & 0.4 \\
LC SAP & 25.8 & 70 & 2.2 \\
\hline
\end{tabular}

is expected to be $\sqrt{30}$ times greater if the noise is assumed to be white. ${ }^{3}$ This is particularly notable in those stars that are approximately constant. In those that pulsate, one has to be careful when discarding those points that appear to be outliers - there should be more outliers in SC because of the greater number of points, but sampling the brightness variations more often produces higher amplitude peaks in both the light curve and the Fourier transform. If one 'sigma clips' the data too closely from the beginning, the extrema of those peaks in the light curve might be lost. Clipping at $3 \sigma$ is too tight - if the data were normally distributed, one discards one in 200 points that naturally belong to the distribution in this manner; these points may lie further from the mean or fit, but are not necessarily erroneous outliers. A discussion of the validity of sigma clipping and other outlier removal procedures can be found in Hogg, Bovy \& Lang (2010).

\section{PDC VERSUS SAP FLUX}

Data files downloaded through either the Kepler Asteroseismic Science Operations Centre ${ }^{4}$ or the National Aeronautics and Space Administration multimission archive ${ }^{5}$ contain times of observations, SAP flux, PDC flux (or PDC MAP, depending on data release) and the errors on those fluxes. The SAP-flux data show instrumental trends, but the PDC flux light curves correct some of these. In both cases, bad cadences are flagged in the .fits data file format. García et al. (2011) discuss the process of correcting Kepler light curves in more detail and specifically its application to asteroseismic analysis, even creating their own, separate, pipeline. A more thorough discussion of the PDC pipeline with more general applications can be found in the Kepler Data Characteristics Handbook ${ }^{6}$ (Christiansen $\&$ van Cleve 2011). Here, we focus on a few common issues, not including the information concerning processing of LC data that was presented in Section 3.

\subsection{Instrumental effects}

The most significant effects on Kepler light curves are the following: differential velocity aberration (discussed in Section 3); loss of fine pointing, which leads to small gaps in the light curves in both SAP and PDC flux; reaction wheel zero-crossings, during which the spacecraft shakes for a day or so (not corrected in PDC data); cosmic ray events, which cause a step-function change in the flux level, which decays exponentially back to $90-100$ per cent of the original level (corrected in PDC data); monthly Earth downlinks, identifiable by a gap in the data of up to $\sim 24 \mathrm{~h}$ followed by an exponential increase/decrease in flux level as the telescope returns to science operating focus and temperature (corrected in PDC data); and attitude tweaks, which have thus far occurred only twice in the entire mission, during science operations in Q2, and are no longer expected to be a problem (not fully corrected in PDC data). Further details on these effects can be found in the Kepler Data Characteristics Handbook, which also keeps track of lists of known spurious peaks belonging to the SC and LC data.

\footnotetext{
${ }^{3}$ The noise is not white, but this serves as a useful approximation.

${ }^{4}$ http://kasoc.phys.au.dk/

${ }^{5}$ http://archive.stsci.edu/kepler/

${ }^{6}$ http://archive.stsci.edu/kepler/manuals/Data_Characteristics_Handbook_ 20110201.pdf
} 


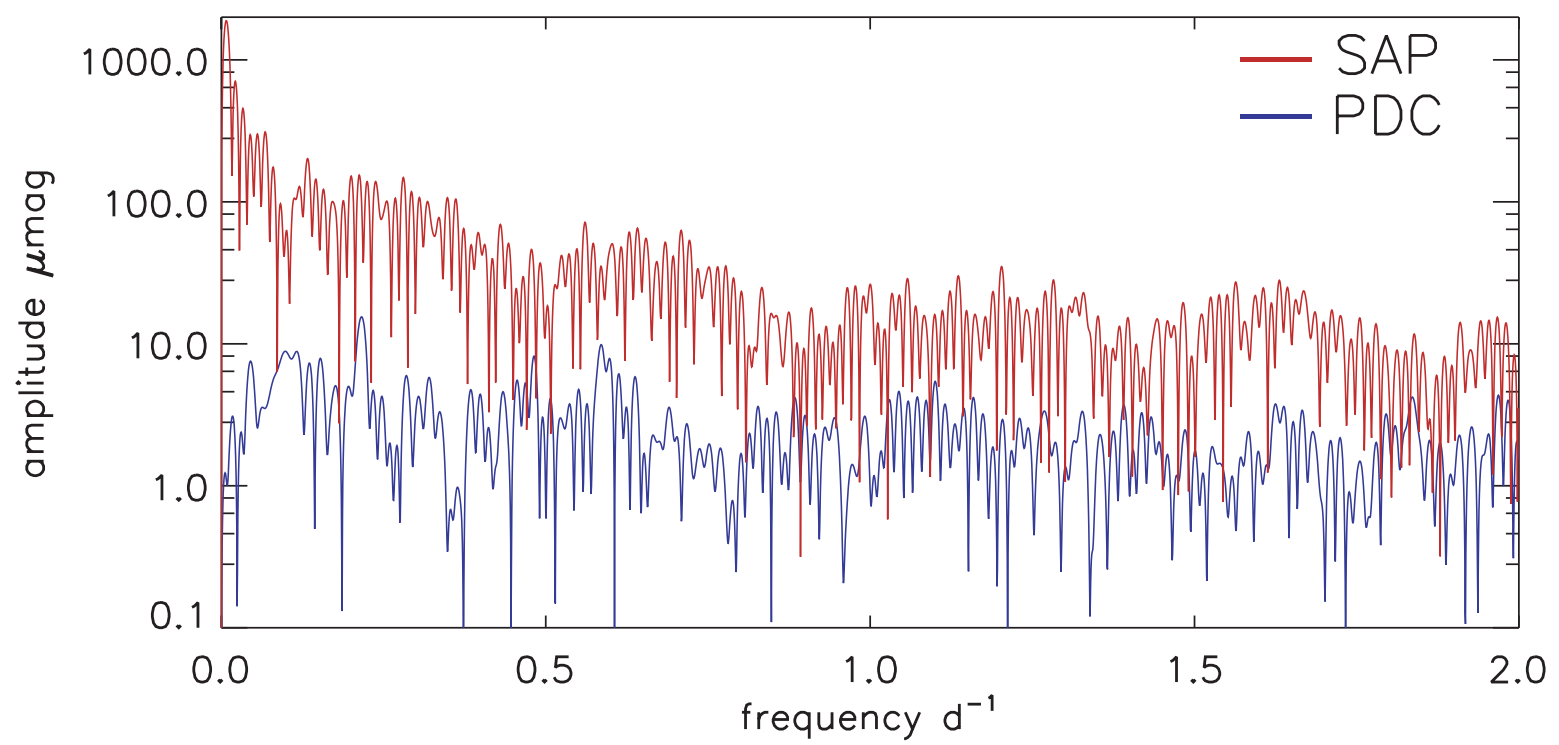

Figure 5. The PDC-flux data (blue) have been plotted over the SAP-flux data (red) for the Kepler object KIC 7450391 during Q2. No points were removed from either data set. The low-frequency peaks in PDC LC data mentioned in Section 3 are present but insignificant by comparison to the difference between PDC and SAP data.

\subsection{Optimal aperture corrections}

In SAP- and PDC-flux data alike, the amount of flux in the defined aperture changes from quarter to quarter, and discontinuities in flux are seen as a result. The PDC data contain corrections for both: (a) the amount of light contributed from the intended target and not contaminating stars: the median flux over a month or quarter is multiplied by ( 1 - contamination) and subtracted from each cadence; and (b) the fraction of flux not captured by the 'optimal aperture', which is defined to maximize the signal-to-noise ratio rather than to capture all light from the star. The SAP-flux data contain no such corrections.

\subsection{Effect on data analysis}

Whilst analysing the effect of cadence on the prevalence of lowfrequency peaks in the periodogram, the PDC-flux data were compared with the SAP-flux data. Fig. 5 illustrates the pervasiveness of low-frequency peaks in the SAP data compared with the much flatter PDC flux, for the near-constant star KIC 7450391, using the full Q2 LC data set and not removing outliers. This puts some perspective on the problematic LC PDC-flux data, in that the SAP-flux data are dominated much more substantially by artificial peaks than are the PDC-flux data.

\section{CONCLUSION}

The SC data are almost always better than the LC data. The SC slot availability is the major limitation one faces when using and obtaining SC data. We have seen the necessity of the increased sampling rate of SC data for resolving short time-scale events such as flares, and for precise transit timing.

The Nyquist frequency of LC data can be problematic and certainly limiting in asteroseismic analysis. Pulsations detected near the Nyquist frequency can be indicative of higher frequency pulsations that require $\mathrm{SC}$ data, and also that peaks in the periodogram may be reflections of peaks from above the Nyquist frequency. However, the lack of pulsations near the Nyquist frequency cannot rule out the presence of higher order $\mathrm{p}$ modes, and SC data may still be required. When $\mathrm{SC}$ data are not available to check for frequencies above the LC Nyquist frequency, one must be aware that observed signals could be reflections from beyond the LC Nyquist frequency.

There is an amplitude difference between SC and LC data associated with the longer integration time of LC data. The result is that peaks in the periodogram have slightly higher amplitudes in $\mathrm{SC}$ data, and the percentage difference between the two cadences grows with increasing frequency.

Effects that are not directly concerned with the different sampling rates have also been observed. LC PDC-flux data often contain spurious peaks of non-astrophysical origin at very low frequency that are not always present with similar amplitudes in SC PDC data. This can affect studies of long-period brightness variations arising from such things as spots on slow rotators. Automatic frequency extraction can suffer from these artificial peaks.

The greater number of points in SC data does not produce narrower peaks in the periodogram and cannot improve resolution of two closely spaced frequencies. Frequencies can be determined with greater precision though, as can their corresponding amplitudes and phases. For this to be true, the data cannot be normally distributed.

The usefulness of PDC-flux data was also discussed. Noise in the periodogram can be vastly reduced by analysing the PDC-flux data instead of SAP-flux data. The PDC-flux data have fewer drifts, jumps and outliers, generating cleaner light curves and Fourier spectra, but may also modify astrophysical signals. It is therefore recommended to cross-examine results obtained with PDC-flux data with those from SAP-flux data, as advised in the Prefatory Admonition of the Data Characteristics Handbook, to check that genuine pulsation frequencies have not been missed as a result of accidental removal in the data processing pipeline. The PDC-flux data do not necessarily remove all jumps and outliers, so it is still recommended to manually check light curves for such artefacts prior to analysis. Moreover, it is recommended that investigators analyse the subset of data points that have a quality flag of zero, meaning the cadences are 'good'. Of the observations made during Kepler's 92 per cent duty cycle, $\sim 95$ per cent of data points in 'well-behaved' quarters might be described as 'good' for a typical 13th magnitude star. Data 
generated in this method will supersede the present PDC-flux data for asteroseismic analysis.

Finally, the PDC pipeline leaves artificial peaks in the LC data. There are relatively high-amplitude peaks at low frequency $\left(<2 \mathrm{~d}^{-1}\right)$ in the Fourier transform of the LC PDC-flux data. Such time-scales are important to asteroseismology, but unimportant for the transits of potentially habitable planets. Nevertheless, improvement is expected in data processed in Quarter 9 Release 12 and subsequent releases, through the PDC MAP pipeline.

\section{ACKNOWLEDGMENTS}

I would like to thank Don Kurtz for advice and discussions, and acknowledge the financial support of the STFC via the PhD studentship programme.

\section{REFERENCES}

Amado P. J., Moya A., Suárez J. C., Martín-Ruiz S., Garrido R., Rodríguez E., Catala C., Goupil M. J., 2004, MNRAS, 352, L11

Ballard S. et al., 2011, ApJ, 743, 200

Borucki W. J. et al., 2010, Sci, 327, 977

Breger M., 2000, in Breger M., Montgomery M., eds, ASP Conf. Ser. Vol. 210, Delta Scuti and Related Stars. Astron. Soc. Pac., San Francisco, p. 3

Christiansen J., van Cleve J. E., 2011, Kepler Data Characteristics Handbook Degroote P. et al., 2009, A\&A, 506, 111
García R. A. et al., 2011, MNRAS, 414, L6

Gilliland R. L. et al., 2010a, PASP, 122, 131

Gilliland R. L. et al., 2010b, ApJ, 713, L160

Gilliland R. L. et al., 2011, ApJS, 197, 6

Hogg D. W., Bovy J., Lang D., 2010, preprint (arXiv e-prints)

Holman M. J., Murray N. W., 2005, Sci, 307, 1288

Jenkins J. M. et al., 2010, ApJ, 713, L120

Koch D. G. et al., 2010, ApJ, 713, L79

Lenz P., Breger M., 2004, in Zverko J., Ziznovsky J., Adelman S. J., Weiss W. W., eds, Proc. IAU Symp. 224, The A-Star Puzzle. Cambridge Univ. Press, Cambridge, p. 786

Stello D. et al., 2009, ApJ, 700, 1589

\section{SUPPORTING INFORMATION}

Additional Supporting Information may be found in the online version of this article:

Appendix. Amplitude reduction derivation.

Please note: Wiley-Blackwell are not responsible for the content or functionality of any supporting materials supplied by the authors. Any queries (other than missing material) should be directed to the corresponding author for the article.

This paper has been typeset from a $\mathrm{T}_{\mathrm{E}} \mathrm{X} / \mathrm{LT}_{\mathrm{E}} \mathrm{X}$ file prepared by the author. 


\title{
Kepler Fourier concepts: The performance of the Kepler data pipeline
}

\author{
S.J. Murphy \\ Jeremiah Horrocks Institute, University of Central Lancashire, Preston PR1 2HE, UK
}

Received 2012 Oct 18, accepted 2012 Oct 22

Published online 2012 Dec 3

Key words methods: data analysis - stars: oscillations

Given the extreme precision attainable with the Kepler Space Telescope, the mitigation of instrumental artefacts is very important. In an earlier paper (Murphy 2012), the characteristics of Kepler data were discussed in light of their effect on asteroseismology. We continue this discussion now that data processed with the new PDC-MAP pipeline are publicly available; users should use the latest data reductions available at the Mikulski Archive for Space Telescopes (MAST), not just for PDC, but also for improvements in the attached meta-data. We discuss the injection of noise in the frequency range $0-24 \mathrm{~d}^{-1}$ (up to $\sim 277 \mu \mathrm{Hz}$ ) by the PDC-LS pipeline into $\sim 15 \%$ of light-curves.

(C) 2012 WILEY-VCH Verlag GmbH \& Co. KGaA, Weinheim

\section{Introduction}

The exquisite precision and long time-base of Kepler data make for excellent demonstrations of some fundamental concepts particular to Fourier transforms. Many of these were presented in Murphy (2012, hereafter Paper I), which addressed characteristics of data of different sampling rates, namely of the Kepler short- and long-cadence data, with 58.9-s and 29.4-min exposures, respectively.

Paper I demonstrated some basic Fourier concepts as applied to Kepler data, such as the importance of having shortcadence (SC) data for investigating $\delta$ Sct stars because of the aliasing generated by the low long-cadence (LC) Nyquist frequency, and also how the study of flares, for instance, can benefit from the increased time-resolution (see, e.g. Balona 2012). Furthermore, Paper I showed how SC data allow for a more precise determination of pulsation frequencies, amplitudes and phases, but do not offer greater frequency resolution, and how the observed pulsation amplitudes in LC data suffer an amplitude-reduction effect due to under-sampling. Also presented therein was the performance of the Presearch Data Conditioning (PDC) pipeline, whose job it is to remove instrumental systematic signatures whilst preserving the astrophysics, compared to the Simple Aperture Photometry (SAP) data, which undergo only basic calibration. It was shown that the PDC data of Data Release 11 and earlier showed much lower noise than their SAP counterparts, but that spurious low-frequency peaks were evident in the LC PDC data in non-pulsating stars.

This article offers a continuation from Paper I in which we further address a newly-discovered and characterised form of noise injection in the older version of the PDC pipeline, which used least-squares algorithms to process the data, and has thus been renamed PDC-LS to alleviate confu-

\footnotetext{
^ Corresponding author: smurphy6@uclan.ac.uk
}

sion - this characterisation is presented in Sect. 2. An early performance review of the newer, Maximum A Posteriori PDC pipeline (PDC-MAP) is presented in Sect. 3. We discuss the benefits of continuous Kepler coverage in Sect. 4. The data presented in this paper pertain to Data Release 11 (or earlier) for PDC-LS, and to Data Release 14 for PDCMAP.

\section{Noise injection by PDC-LS}

At the time of writing, all Kepler quarters have now been reprocessed with the newer PDC-MAP pipeline and are available on MAST, but PDC-MAP does not yet treat SC data, meaning that $\mathrm{SC}$ data are only available in the least-squares format. For further reading on the pipelines, the papers of Stumpe et al. (2012) and Smith et al. (2012) are recommended, along with the Kepler Data Characteristics Handbook $^{1}$. In this section we provide examples of how the PDCLS pipeline injects noise into the data, how to detect it, and the on-going efforts to handle the problem.

We demonstrate the way in which PDC noise injection can distort light curves in Fig. 1, and how this affects the Fourier transform in Fig. 2. One of the characteristic features of the injected noise is the drop-off in power around $24 \mathrm{~d}^{-1}$. Specifically, in a logarithmic plot of the power spectrum an extremely rapid reduction in power is seen (Fig. 3), and this was used to identify stars affected by the noise injection. Indeed, this power-reduction was observed in $15 \%$ of the stars analysed - those with SC data available and Kepler Input Catalogue (KIC) temperatures between 5500 and $9500 \mathrm{~K}$. The power-reduction is hard to detect in LC due to the low Nyquist frequency, but we stress that the LC data do show the injected noise.

\footnotetext{
1 Available at http://keplergo.arc.nasa.gov/Documentation.shtml.
} 

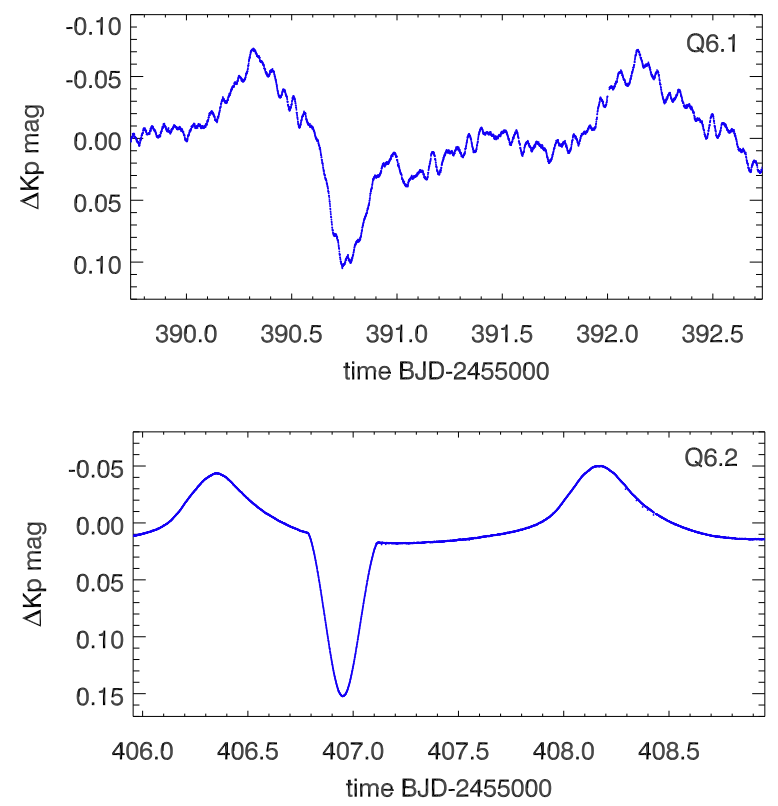

Fig. 1 (online colour at: www.an-journal.org) Three-day segments of the light curves for the detached eclipsing binary system KIC 11285625 in SC PDC flux. In Q6.1 the pipeline is injecting noise, distorting the light curve, whereas the Q6.2 light curve looks comparatively very clean. The corresponding Fourier transforms are shown in Fig. 2. It is well-known that PDC-LS does not treat binaries well, but the noise injection is not limited to eclipsing binary systems.

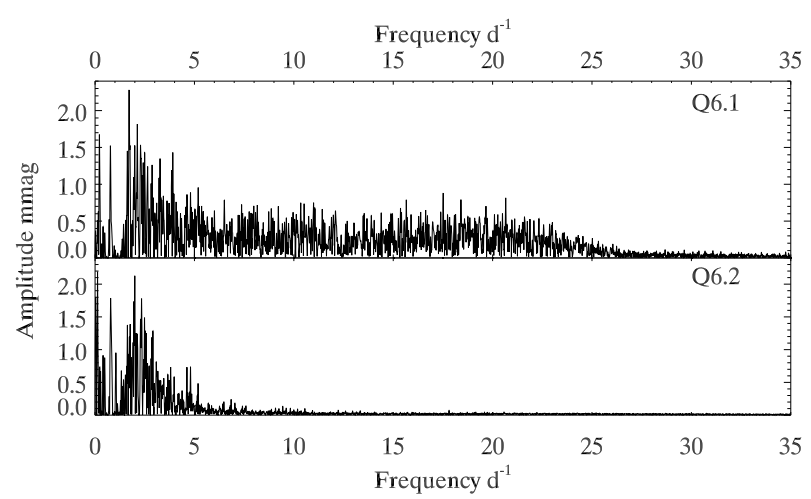

Fig. 2 The prewhitened Fourier transforms for Q6.1 and Q6.2 corresponding to the light curves in Fig. 1, and typical for stars in which the noise injection is seen. Pulsations and many harmonics of the orbit have been prewhitened; the appearance of the Fourier transform below $\sim 5 \mathrm{~d}^{-1}$ is strongly affected by those harmonics that remain. Upper panel: in the affected Q6.1 data, there is an elevated "grass level" - the amplitude of the Fourier peaks that somewhat resemble mown grass - up to a frequency of $\sim 24 \mathrm{~d}^{-1}$ after which there is a sharp drop-off in power. Lower panel: the Q6.2 data do not suffer the noise injection. The sharp power-reduction is not noticeable in LC data (not shown) because of the low Nyquist frequency of $24.4 \mathrm{~d}^{-1}$.

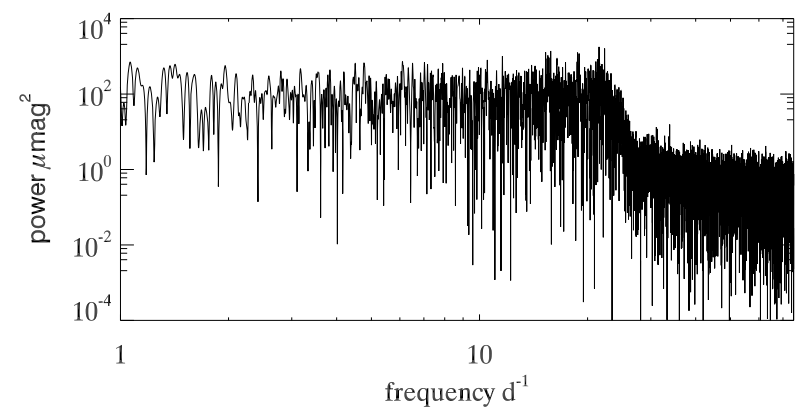

Fig. 3 The injected noise is easily identified by a rapid power reduction at $24 \mathrm{~d}^{-1}$ in the Fourier transform, plotted here in log-log space. The example given is KIC 3429637 Q7 SC data, after prewhitening, that is, fitting and removing the statistically significant (signal-to-noise $\geq 4$ ) sine curves from this $\delta$ Sct star.

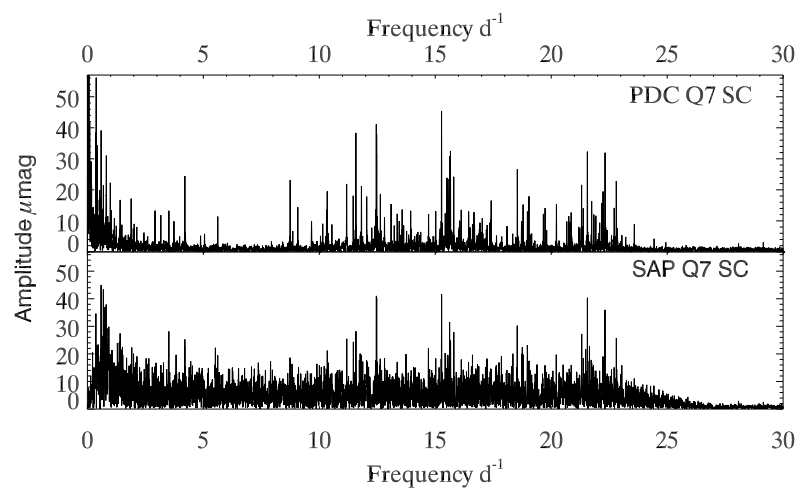

Fig. 4 Linear Fourier transforms for KIC 3429637, showing the extent of the injected noise: the grass level is $\sim 15 \mu$ mag in the PDC data, compared to just $2 \mu$ mag in the SAP data. The decrease in power at $\sim 24 \mathrm{~d}^{-1}$ is clear. The effect is present in Q7 in both SC and LC PDC data for this star, but not present at all in Q8.

Figure 4 shows how for the star KIC 3429637 the PDCLS pipeline injects noise into the SC Q7 data. The noise is restricted solely to PDC data and affects Q7 but not Q8 for this star. The grass level (defined in Fig. 2 caption) is higher in PDC Q7 LC than in SAP Q7 LC (after pre-whitening), so the effect is present in LC too, even if the low Nyquist frequency hides the power drop.

It is not known why some stars are affected while others are not, nor why, for the same star, only some quarters are affected and that these vary for different affected stars. No correlation in fraction of stars affected was seen with $T_{\text {eff }}$, nor was any correlation seen with sky/CCD position (Fig. 5).

Sometimes the pipeline fails to fit a light curve and passes the light curve through without modification. In the lower panel of Fig. 2, it can be seen that although noise is not injected, low-frequency peaks, which are often caused by trends in the data, are not removed either. In this case, the light curve has perhaps emerged untouched by the pipeline.

The Kepler Science Office is working extremely hard to continually improve the pipeline, and every effort is made to visually inspect the light-curves at each data release. 


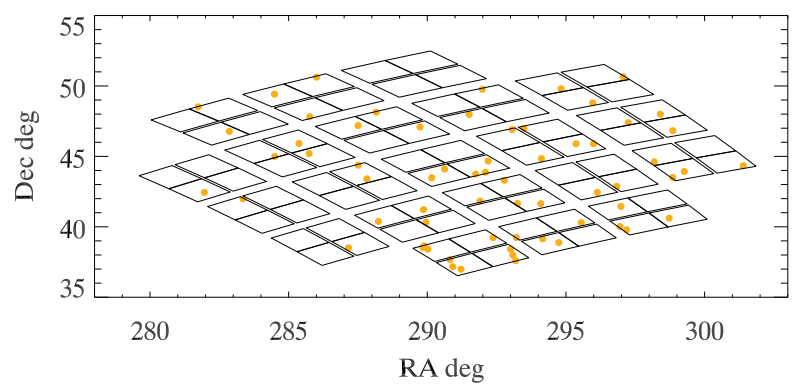

Fig. 5 (online colour at: www.an-journal.org) The spatial distribution of the affected stars corresponds to the spatial density of all stars across Kepler's CCDs.

\section{Performance of PDC-MAP}

At the time of writing, PDC-MAP has only very recently become available for public data. In comparing PDC-MAP with the older PDC-LS and the SAP data (Fig. 6), it is clear that PDC-MAP has strong trends (over many cadences) following the horizontal discontinuities in the data that arise from the scheduled data downlinking events. In the older pipeline these trends were fixed quite well. Such teething problems might be expected with new software, somewhat similarly to how the Copernican model of the solar system initially struggled to out-perform the Ptolemaic model because of the substantially larger amount of time invested in improving the latter. PDC-MAP identifies systematic behaviour common to many stars to create a fit to subtract from the data, but there appears to be a great variation between the response of different pixels to the thermal effects that cause the trends, and this effect is convolved with differential velocity aberration, making the light-curves particularly hard to treat.

Paper I investigated the difference between SAP and PDC-LS data in Fourier space, noting that the noise in the latter was reduced by factors of 10-100 over the former. Here, we extend that comparison to also include the PDCMAP data (Fig. 7). It appears that although PDC-MAP is slightly noisier at very low frequency due to the remaining thermal and focusing effects, at higher frequency (above $\sim 2 \mathrm{~d}^{-1}$ ) it out-performs PDC-LS, and has the added reassurance that it preserves stellar variability more satisfactorily than PDC-LS. From Quarter 13 onwards, a cubic fit will also be calculated and subtracted from the data in a pipeline version called 'multi-scale' MAP. It is therein assumed that long-term trends on the scale of around one month are systematic and are thus removed.

\section{The benefits of continuous Kepler coverage of $\delta$ Sct stars}

Kepler SC slots are oversubscribed, such that even though some SC data are desirable (though not required - to be demonstrated in a future publication) to resolve the LC

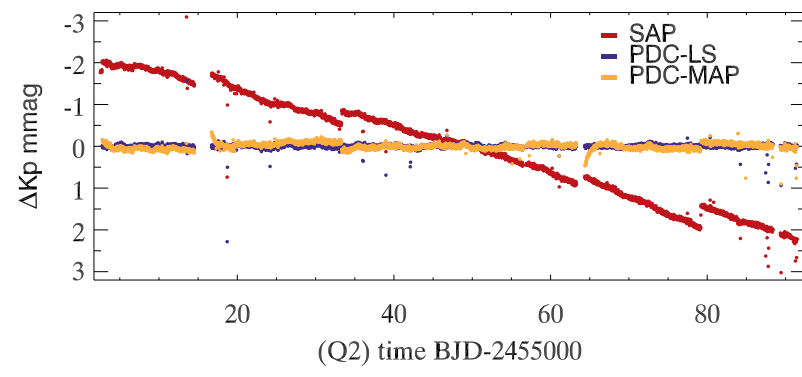

Fig. 6 (online colour at: www.an-journal.org) Although PDCMAP removes many of the long-term trends satisfactorily, it has initially struggled to mitigate thermal effects. For this reason, small exponential thermal recoveries are seen at the data downlink events in PDC-MAP, just as they do in the SAP data. The example shown is KIC 7450391 - a non-pulsating late A-type star, for Kepler Quarter 2, noting that Q2 is the most extreme example and that in other Quarters PDC-MAP does much better than this.

Nyquist aliasing issues, it is mostly only feasible to continuously study a star in LC. Here we shall address the benefits of doing so.

The $\delta$ Sct stars are not particularly stable pulsators. Their amplitudes and frequencies are subject to change, and the literature contains numerous examples such as $4 \mathrm{CVn}$ (Breger 2000), whose pulsation amplitudes have been observed to change unpredictably for decades. With continuous, space-based observations of such high precision, the potential for observing such changes is great. Murphy et al. (2012a) have used eight Quarters of Kepler data to investigate the pulsational amplitude growth of the $\rho$ Pup star KIC 3429637. Such observations may prove indispensable in understanding mode interactions and the details of the driving mechanism and damping in operation in $\delta$ Sct stars.

In addition, Shibahashi \& Kurtz (2012) have described a method of deriving the mass function of a binary pair using the frequency-modulation (FM) technique on Fourier data, i.e. without the need of spectroscopy. Specifically, if one has continuous LC data covering at least a whole orbit, and one of those stars pulsates (preferably at high amplitude and frequency), then by using the sidelobes present in the Fourier transform one can calculate the parameters of that orbit. So far, only three "FM stars" are known: the prototype in Shibahashi \& Kurtz (2012, KIC 4150611), a highly non-linear $\delta$ Sct star with many combination frequencies (KIC 11754974; Murphy et al. 2012b), and a third star currently under study by Kurtz \& Shibahashi. Telting et al. (2012) have found a pulsating sdB in a binary for which the sidelobes were only of borderline significance and thus direct application of the FM technique was ineffective. Under the right conditions, however, it is even possible to detect Jupiter-mass planets orbiting $\delta$ Sct stars with this technique.

Finally, continuous coverage in eclipsing binary systems, or even transiting planets is essential to precisely determine the orbital period and even allow detections of additional bodies through Transit Timing Variations (Ballard et al. 2011). For this reason, the news that the Kepler Space 


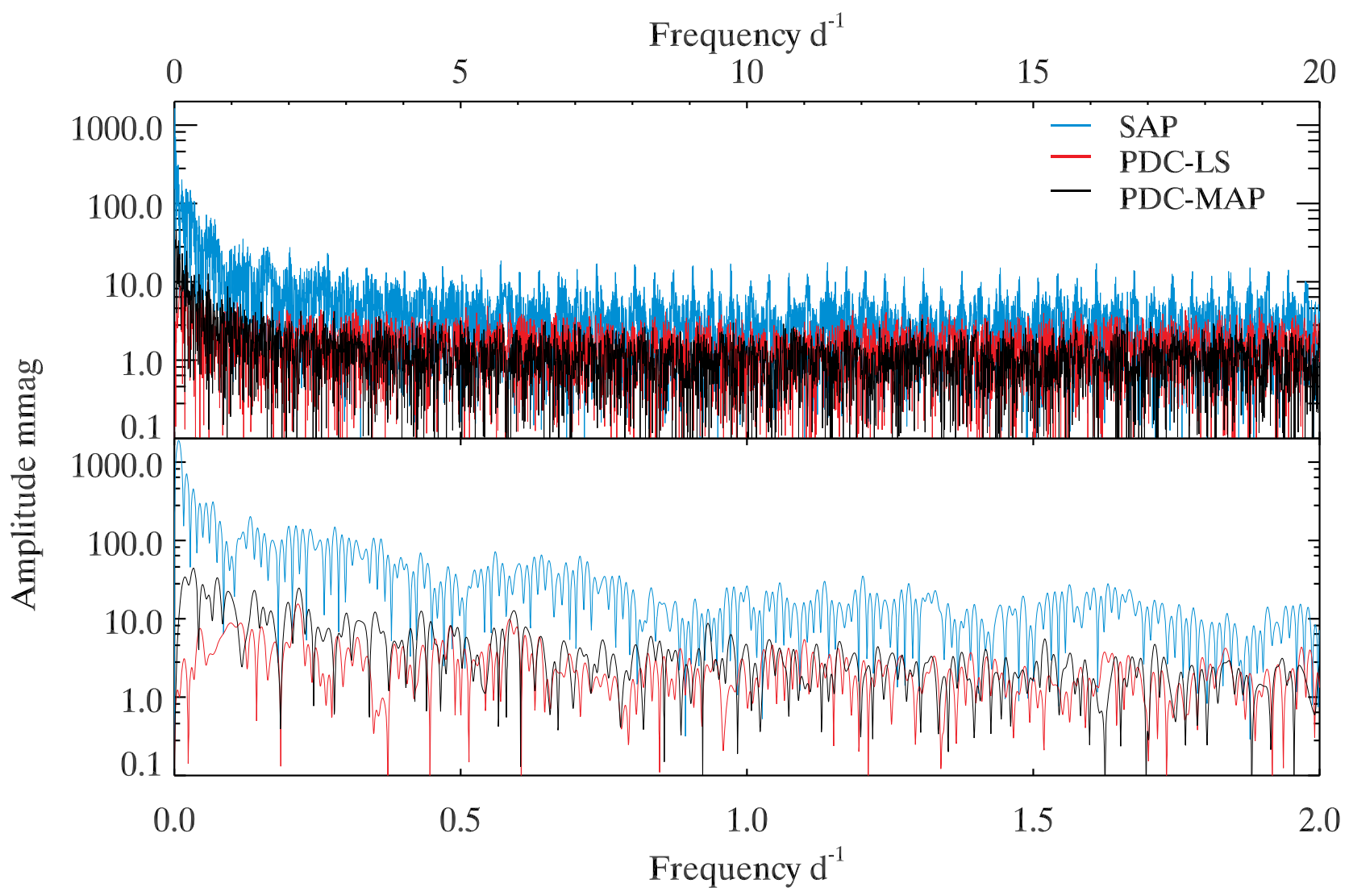

Fig. 7 (online colour at: www.an-journal.org) Overlaid Fourier plot for SAP, PDC-LS and PDC-MAP for KIC 7450391, Quarter 2, corresponding to the light curves in Fig. 6. Upper panel: below $\sim 2 \mathrm{~d}^{-1}$ PDC-MAP is slightly noisier than PDC-LS, but has lower noise above that frequency. Lower panel: zoom-in on the region below $2 \mathrm{~d}^{-1}$. SAP is substantially noisier, and the convergence in performance of PDC-MAP and PDC-LS can be seen as low as $0.5 \mathrm{~d}^{-1}$. PDC-MAP can thus be said to be at least as good as PDC-LS on time-scales important to astrophysics.

Mission is receiving funding for an extension is particularly exciting.

Acknowledgements. SJM acknowledges the financial support of the STFC and of the ESF, and would like to thank the Kepler Science Office for their continued efforts in improving the pipeline. In particular, he would like to thank Fergal Mullally for the bilateral discussions on the pipeline.

\section{References}

Ballard, S., et al.: 2011, ApJ 743, 200

Balona, L.A.: 2012, MNRAS 422, 1092
Breger, M.: 2000, in: L. Szabados, D. Kurtz (eds.), The impact of large-scale surveys on pulsating star research, ASPC 203, IAU Colloq. 176, p. 421

Murphy, S.J.: 2012, MNRAS 422, 665

Murphy, S.J., Grigahcene, A., Niemczura, E., Kurtz, D.W., Uytterhoeven, K.: 2012a, astro-ph/1209.1493

Murphy, S.J., et al.: 2012b, in prep.

Shibahashi, H., Kurtz, D.W.: 2012, MNRAS 422, 738

Smith, J.C., Stumpe, M.C., Van Cleve, J.E., et al.: 2012, PASP 124, 1000

Stumpe, M.C., Smith, J.C., Van Cleve, J.E., et al.: 2012, PASP 124, 985

Telting, J.H., Østensen, R.H., Baran, A.S., et al.: 2012, A\&A 544, A1 


\section{Chapter 2}

\section{An observational review of rotation in A stars}

\section{Abstract}

Rotation is a key physical process operating in the A stars. Angular rotational velocities reach their maximum at about A5, with some stars rotating at a large fraction of their break-up velocity. Slow rotators are also seen at spectral type A, and these tend to be chemically peculiar. The literature on rotation in the A stars is reviewed, including the mechanisms by which a star's rotation can be braked. For Ap stars, braking is understood, but too few progenitors are observed. For the Am stars, the tidal braking mechanisms proposed in the literature induce mixing that is incompatible with observed abundance anomalies. Unexpectedly, the literature review revealed evidence that A stars accelerate for the first third of their main-sequence life, confirming the results of Zorec \& Royer (2012). The possibility of determining rotational velocities from the Fourier transforms of Kepler light curves is also discussed. 


\section{CHAPTER 2}

\subsection{Introductory remarks}

The stars of spectral type A do not necessarily remain so throughout their life on the main sequence. The principal direction of evolution across the HR diagram for stars at this spectral type is towards cooler temperatures, i.e. to the right (see Fig. 2.1). Thus stars born at late B will evolve to become early-A stars and stars at late A with luminosity class $\mathrm{V}$ will become early-F giants. For this reason, and others, a review of the rotational velocities of A stars naturally extends into neighbouring regions of the HR diagram. For reference, the mass of a typical star at A0 is $\sim 2.5 \mathrm{M}_{\odot}$. To aid the reader, Appendix 2.A provides a calibration of spectral type, temperature, photometric indices and colours that has been assembled from the literature.

A-type stars show a range of spectral peculiarities, most common of which are the Am and Ap phenomena. As such, stars without 'abnormal' spectra are referred to as 'normal' stars, even though at some spectral types, normal stars do not constitute the majority. Throughout this review, a normal star simply means not classified as abnormal (mostly Am or Ap) in some way.

The A stars possess a wide range of equatorial rotational velocities, from a few $\mathrm{ms}^{-1}$ ( $\gamma$ Equ rotates around once per century; Leroy et al. 1994) right up to $400 \mathrm{~km} \mathrm{~s}^{-1}$ for the most massive stars (Zorec \& Royer, 2012). Since many stellar properties are correlated with rotation, stars are commonly described as either 'fast' or 'slow' rotators about a dividing line of $120 \mathrm{~km} \mathrm{~s}^{-1}$. This line marks the observational limit up to which chemically peculiar A stars are found, as we shall see in this review. 


\section{CHAPTER 2}

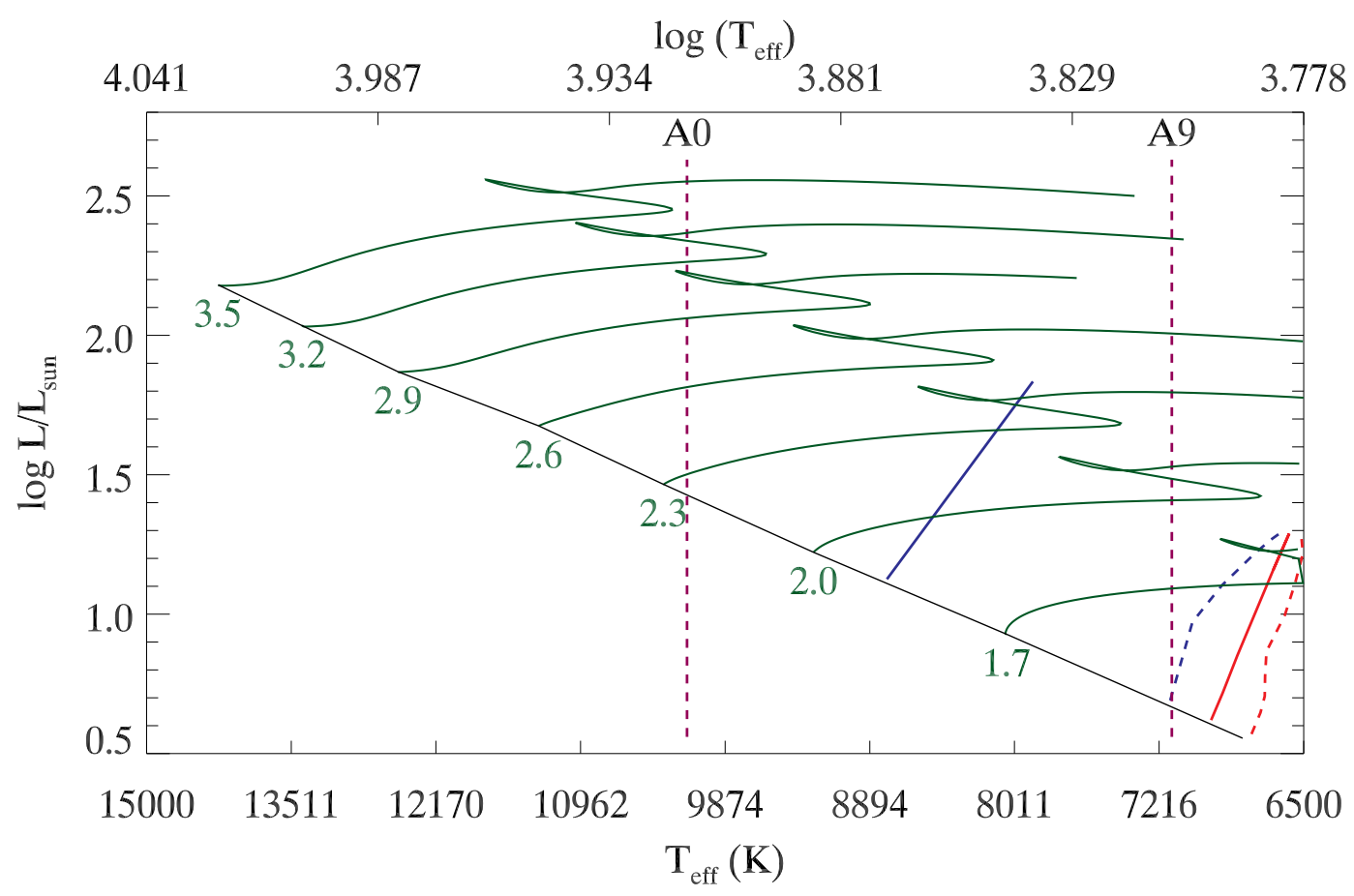

Figure 2.1: HR diagram covering the late-B to early-F region (spectral types A0 and A9 are delimited). A star at A0 has a surface temperature of $\sim 10150 \mathrm{~K}$, and at A9 $7150 \mathrm{~K}$. Evolutionary tracks are plotted in green, with their corresponding masses (in $\mathrm{M}_{\odot}$ ) written just below the zero-age main sequence (black). The principal direction of evolution for the B stars is to the right, such that they become A stars. For reference, the $\delta$ Sct instability strip's red and blue edges are plotted as solid lines, and the $\gamma$ Dor strip as dashed lines. Tracks were calculated using time-dependent convection (TDC) models, and contributed by A. Grigahcène (priv. comm.). 


\section{CHAPTER 2}

\section{$2.2 \quad$ Velocity distributions}

\subsubsection{Is the distribution bimodal?}

It has long been observed that there is an excess of slow rotators among the A stars. Conti (1965) concluded that at least two separate velocity distributions are represented among the $\mathrm{A}$ stars, and that the slow rotators among the late-A stars were the metallic-lined Am stars. Similarly, Wolff (1967) showed that the correlation between chemical peculiarity and rotation in late-B and early-A stars was consistent only with Ap stars being slow rotators, i.e. not fast rotators seen pole-on.

Perhaps the best illustrations of the bimodal nature of the rotational distributions of A stars come from Abt \& Morrell (1995) and Zorec \& Royer (2012). In the latter, Maxwellians are used to describe the distributions of rotational velocities. From observations in the former it is then clear that at least two Maxwellians are required to describe the distribution empirically (Fig. 2.2), with the slowly rotating population being chemically peculiar $(\mathrm{CP})$, that is, all Am or Ap stars. It is noteworthy, however, that Preston (1970) argued that if the Ap stars with rotational velocities of years belong to the same slow-rotator distribution, then they are over-represented in observations by an order of magnitude.

Zorec \& Royer (2012), on the other hand, clearly showed a slowly rotating population of stars for masses above $\sim 2.5 \mathrm{M}_{\odot}$, after removing known $\mathrm{CP}$ and close binary stars from their sample (Fig. 2.3). Thus they have found a subsample of slow rotators among the normal stars, despite claims that the bimodal distribution for late-B stars can be explained by a set of slowly rotating Ap stars and a set of rapidly rotating, normal B stars (Abt, Levato \& Grosso, 2002). Either way, one can see that the situation is more complex than a superposition of one or two Maxwellian distributions. 


\section{CHAPTER 2}

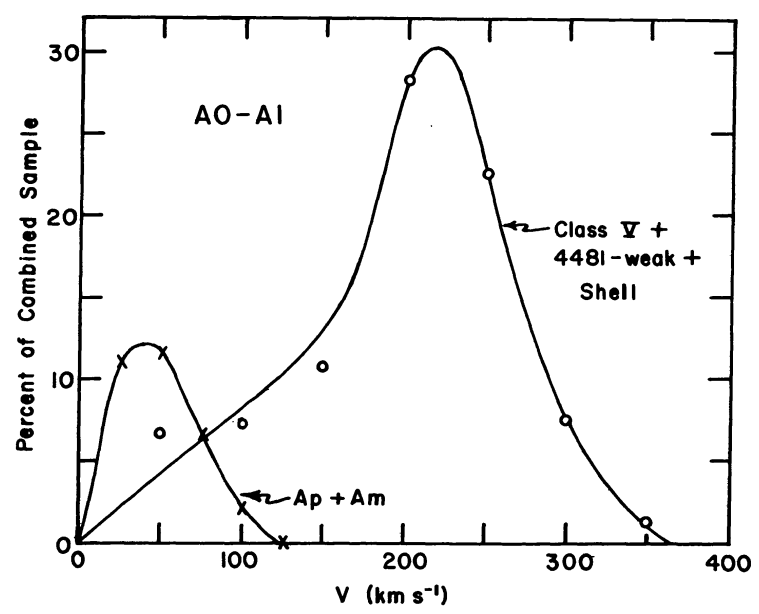

Figure 2.2: Distributions of equatorial rotational velocities, $V$, for two samples of A0-A1 stars. The right distribution is for 188 normal class V stars, 58 stars with weak $\lambda 4481$ lines and five shell stars; the distribution on the left is for $46 \mathrm{Ap}+\mathrm{Am}$ stars whose peculiar abundances are thought to be due to diffusion. The areas under the curves are proportional to their relative frequencies in the Bright Star Catalogue. Figure and caption from Abt \& Morrell (1995, figure 4). Permission obtained from author; reproduced by permission of the AAS.

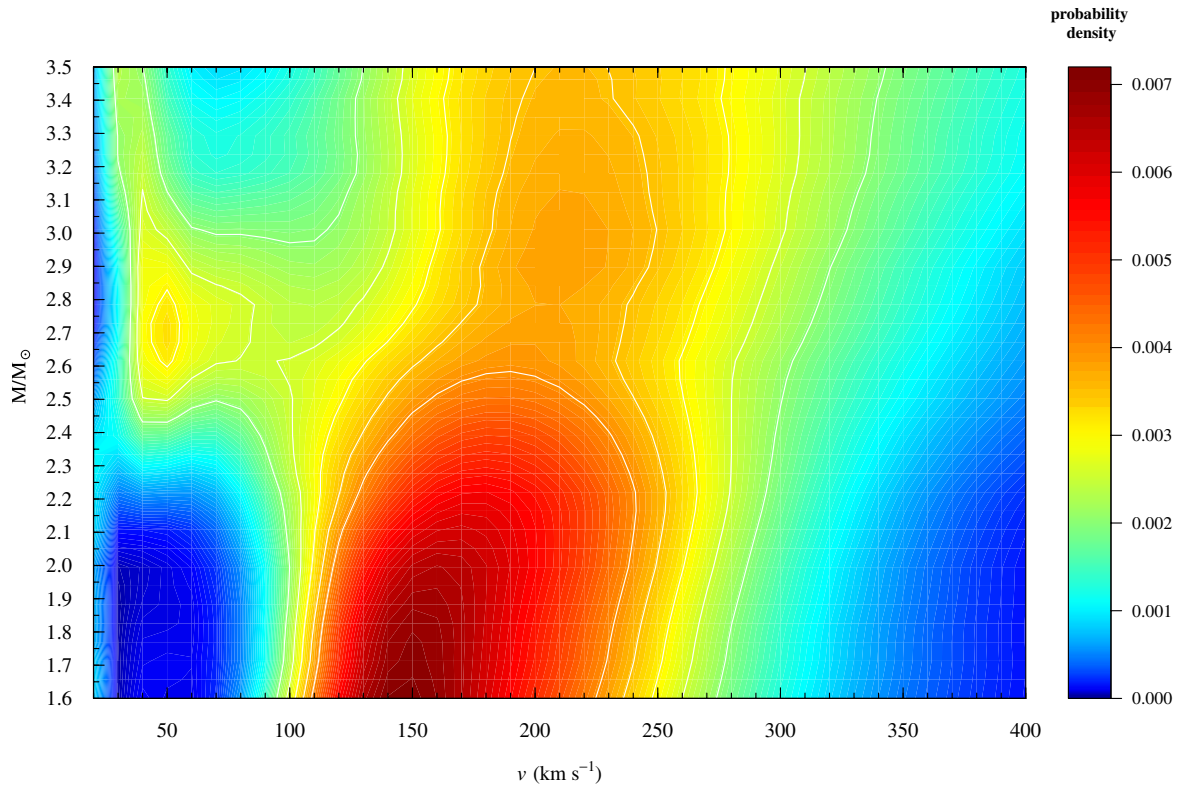

Figure 2.3: Distribution of true rotational velocities, $v$, for normal stars as a function of the stellar mass, $M / \mathrm{M}_{\odot}$. The colour scale represents the density in the onedimensional normalised distributions. The bluer the region, the smaller the number of stars, and vice-versa for the red-coloured scale. White solid lines follow iso-density contours. Figure and caption from Zorec \& Royer (2012, their figure 7). Permission obtained from A\&A. 


\section{CHAPTER 2}

\subsubsection{Variations between observers}

The two most active 'groups' in studying the rotational velocities of A stars in the past two decades have been the Royer group (Royer et al. 2002a,b; Royer, Zorec \& Gómez 2007; Zorec \& Royer 2012), and Abt and his collaborators (Abt \& Morrell 1995; Abt 2004, 2009). Here we shall explain the methods and discuss the results of the groups.

Both groups have sufficiently large samples to correct statistically for inclination. Abt's sample is based on the 1700 northern stars for which half-widths of the Mg II 4481 and Fe I 4476 lines were determined by Abt \& Morrell (1995), using the Slettebak system - a catalogue of $v \sin i$ vs. FWHM of many lines for late-O to late-F stars - which has seen wide use, but whose $v \sin i$ values are regarded to be slightly underestimated (Royer 2009, and references therein). The more recent papers from the Royer group used a combination of Abt, Levato \& Grosso's (2002) data, with binaries and CP stars removed, and data from Royer et al. (2002a,b), in which rotational velocities were derived for 777 southern stars from Fourier transforms of several line profiles in the $4200-4500 \AA$ range. The magnesium doublet was not chosen by the latter authors because at low rotational velocities the intrinsic separation of the doublet becomes visible and the line width is no longer sensitive to rotational broadening. The error on the Fourier method is judged to be 6 per cent, and the main difference in rotational velocities between the Abt \& Morrell and Royer et al. samples occurs for $v \sin i$ below $25 \mathrm{~km} \mathrm{~s}^{-1}$. A systematic shift of $5.3 \mathrm{~km} \mathrm{~s}^{-1}$ as well as a 15 per cent difference in $v \sin i$ is observed between the two samples, which implies, according to Royer et al., that Abt \& Morrell's velocities are slightly too low. Abt \& Boonyarak (2004) maintain that Abt \& Morrell's velocities are valid. Nevertheless, there now exists a 'corrected' version of Abt \& Morrell's catalogue, taking into account the underestimation of the Slettebak system, in Royer et al. (2002b). Finally, 


\section{CHAPTER 2}

Royer, Zorec \& Gómez (2007) note that differences in mean, true equatorial velocities from their method compared to the literature can reach $\Delta v=50 \mathrm{~km} \mathrm{~s}^{-1}$. Their thorough comparisons and tests imply the literature values are underestimated.

Even once the differences in $v \sin i$ from different observing methods are taken into account, discrepancies in results still remain. Abt, Levato \& Grosso (2002) found that only 0.3 per cent of B stars have rotational velocities in excess of twothirds of break-up velocity, whereas Royer, Zorec \& Gómez (2007) showed a distinctly different case for the B9 stars: approximately 30 per cent appear to be above the $\Omega / \Omega_{c}>2 / 3$ threshold (Fig. 2.4), where $\Omega_{c}$ is the critical angular velocity corresponding to break-up. An explanation for this difference is that B9 stars are substantially closer to the maximum of $\Omega / \Omega_{c}$ at A5 than are the group of Abt et al.'s B stars as a whole. That maximum at A5 is particularly clear in the middleright panel of Fig. 2.4, where all stars are fast rotators. Closer inspection of some of the numbers in Royer, Zorec \& Gómez (2007) might reveal another cause of the discrepancies. They only removed $16 \mathrm{CP}$ and 33 close binary stars from their total of 190 stars in the A7-A9 range. If one assumes all of those close binaries are Am stars (see $\S 2.4$ ), then they find only a quarter of stars in this range to be Am. Yet it is widely observed that at about A8, Am stars comprise about 50 per cent of the A-star population (e.g. Smith, 1973). One must also consider that Royer, Zorec \& Gómez are considering luminosity classes V and IV only. The proportion of Am stars increases with age (North, 1993; North et al., 1997), but only up to the terminal-age main sequence (TAMS), so Royer, Zorec \& Gómez are probably not missing many of those. Perhaps their disregard for the more evolved objects, covering $\rho$ Pup stars and metallic A-F giants, makes up some of the deficit and they do not have a strong selection bias.

Alternatively, it is possible that Royer, Zorec \& Gómez (2007) have isolated in their 'normal slow rotators' subgroup those Ap stars that are yet to develop some 


\section{CHAPTER 2}

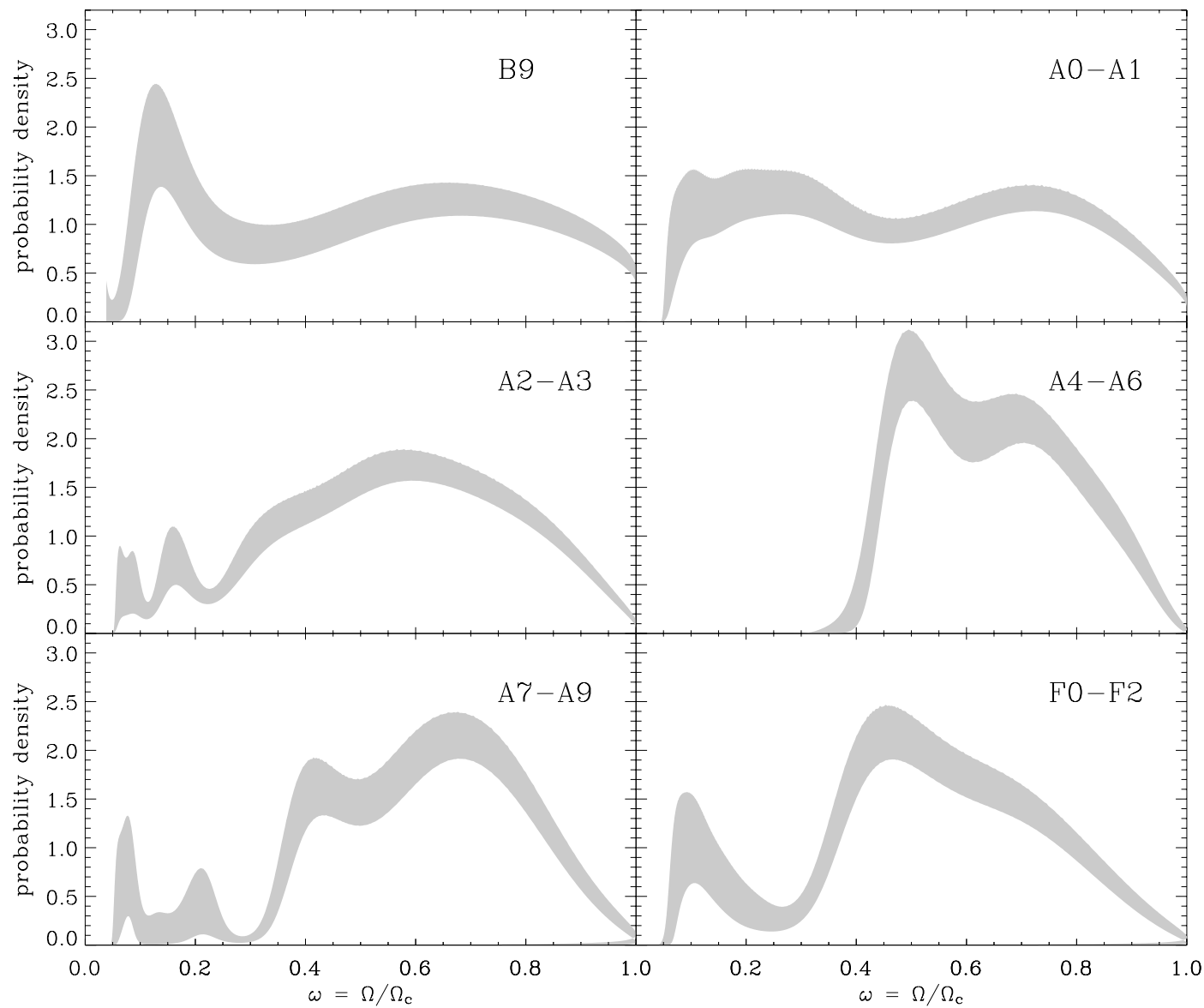

Figure 2.4: Distributions of angular velocities as a fraction of the critical velocity for breakup, $\Omega / \Omega_{c}$, derived from $v$ distributions in Royer, Zorec \& Gómez (2007). $\omega=1$ thus corresponds to break-up. The area under a band over a particular angular velocity interval corresponds to the fraction of stars in that interval. Only the variability band of each probability density function is displayed - the width of the band can be thought of as the size of the error bars, and the computed values lie approximately at the centre of each band. Figure from Royer, Zorec \& Gómez (2007, their figure 10). Permission obtained from A\&A. 


\section{CHAPTER 2}

chemical peculiarity. Specifically, it takes the cluster Ap SrCrEu stars half of their main-sequence life to reach the same frequency as is observed in field stars (Abt, 2009). The ages of Royer, Zorec \& Gómez's 'normal slow rotators' subgroup were not determined beyond the fact that they are main-sequence objects, so at least some of them could be Ap progenitors.

To settle the debate, we can refer to comparisons of rotational velocities in two other surveys: Uesugi \& Fukuda (1982) and Erspamer \& North (2003). The latter two sources are in good agreement with one another. In comparison, the results of Abt \& Morrell (1995) are lower, particularly for $v \sin i>100 \mathrm{~km} \mathrm{~s}^{-1}$, whereas the results of Royer et al. (2002a) are in better agreement. In terms of systematic errors (neglecting sample coverage), all sources are satisfactorily mutually consistent.

\subsubsection{Evolution}

Since we have just commented on the increase in CP prevalence with age, and slow rotation is correlated with the Am and Ap phenomena, let us review the effect of evolution on A star rotational velocities.

Wolff \& Simon (1997) compared the mean rotational velocities of field stars with those of young stars in Orion, the $\alpha$ Per cluster, the Pleiades and the Hyades. They showed that there is no appreciable decline in rotational velocity on the main sequence among the 1.9 to $2.4-\mathrm{M}_{\odot}$ stars, but that for stars between 1.3 and $1.6 \mathrm{M}_{\odot}$ the decrease in $v \sin i$ ranges from 30-50 per cent (Fig. 2.5). If one assumes equal braking rates, the result is explained by the difference in nuclear time-scales between the two mass ranges.

If the coupling between the surface and the interior is sufficiently strong, Wolff \& Simon (1997) claim a star will rotate as a solid body. However, how strong is the coupling when stars rotate at a substantial fraction of the break-up velocity? Wolff \& Simon used strong coupling as an explanation for the constant velocities of 


\section{CHAPTER 2}

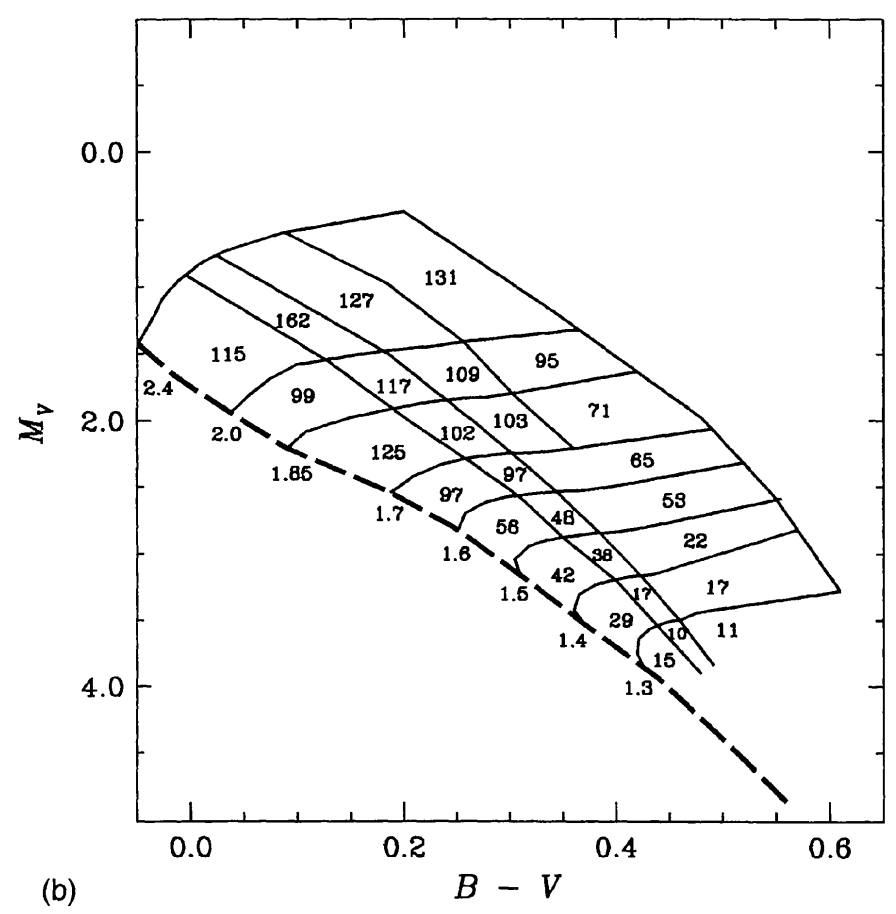

Figure 2.5: The average rotational velocities of stars as a function of mass and age. The heavy dashed line represents the zero-age main sequence (ZAMS). The nearly horizontal lines represent evolutionary tracks with the masses indicated just to the left of the ZAMS. The lines nearly parallel to the ZAMS are used to separate the stars into different groups according to the extent of their evolution away from the ZAMS. Numbers enclosed are the average $v \sin i$ for the enclosed populations. Figure and caption from Wolff \& Simon (1997, figure 3b). Permission obtained from author.

stars above $1.6 \mathrm{M}_{\odot}$ during their main-sequence lifetime, but Royer, Zorec \& Gómez (2007) have shown that the mean value of the fraction of critical angular velocity, $\Omega / \Omega_{c}$, is around $2 / 3$ for A4-A9 stars, and about $1 / 2$ for F0-F2 (Fig. 2.4). It is hard to imagine that these stars rotate as solid bodies, particularly near the end of their main-sequence lives. Indeed, Zorec \& Royer (2012) show that stars of $2.0 \mathrm{M}_{\odot}$ and higher rotate with more rotational energy at the TAMS than a critical rigid rotator can bear, and must therefore be rotating differentially. Even the Sun rotates differentially and it is a slow rotator, so the rigid rotator hypothesis seems untenable.

How do the observational results of Wolff \& Simon (1997), reproduced in Fig. 2.5, here, compare to the more recent ones of Zorec \& Royer (2012), who also investigated 


\section{CHAPTER 2}

the evolution of rotational velocities in A stars? The latter authors used uvby $\beta$ photometry and Hipparcos parallaxes to determine $T_{\text {eff }}$ and $\log L / \mathrm{L}_{\odot}$, and then used stellar evolution models to calculate masses and ages ${ }^{1}$. They found that normal stars in the $2.0-2.4 \mathrm{M}_{\odot}$ range appear to rotate more quickly with time spent on the main sequence $\left(t_{\mathrm{MS}}\right)$. Specifically, they found a monotonic increase in mean age across the $50-110 \mathrm{~km} \mathrm{~s}^{-1}$ velocity interval, suggesting the faster rotators of the interval are older, and implying some sort of spin-up from angular momentum redistribution among the slow rotators (Fig. 2.6). The trend diminishes with increasing mass. However, the CP stars belonging to the slow rotator population in any mass range from 2.0 to $3.5 \mathrm{M}_{\odot}$ showed only slight changes in rotational velocity with age, and showed moderate decelerations for those $\mathrm{CP}$ stars belonging to the fast rotator population (albeit with larger errors).

Zorec \& Royer calculated models for a variety of stellar structures, using different approaches to rotation. Specifically, they looked at the cases of rigid and differential rotation for different ZAMS rotation rates. Differential rotation can be either simple, where the specific angular momentum, $J / M$, is lower than the critical value that a rigid rotator can possess, or neat, where that critical value is exceeded. Their rigid rotator models are two-dimensional and were constructed for $M=1.5$ and $3.0 \mathrm{M}_{\odot}$, conserving total angular momentum throughout the main-sequence phase. Both masses showed only a marginal increase in $v / v_{\text {ZAMS }}$ over the first two-thirds of $t_{\mathrm{MS}}$, but rapid increases of over 0.4 in $v / v_{\text {ZAMS }}$ in the final third (Fig. 2.7). Conversely, stars in the simple differential rotation regime were forecast to show monotonic decreases of 40 per cent $\left(1.5-\mathrm{M}_{\odot}\right.$ case $)$ or greater $\left(3-\mathrm{M}_{\odot}\right.$ case $)$ over the entire $t_{\mathrm{MS}}$.

In the differentially rotating case, the specific angular momentum becomes increasingly centrally condensed, meaning the surface rotational velocity decreases

\footnotetext{
${ }^{1}$ their quoted errors on $T_{\text {eff }}, L / \mathrm{L}_{\odot}, M / \mathrm{M}_{\odot}$, and age were on the order of $2,15,3$, and 10 per cent, respectively. Their models are detailed in the two appendices of that paper.
} 


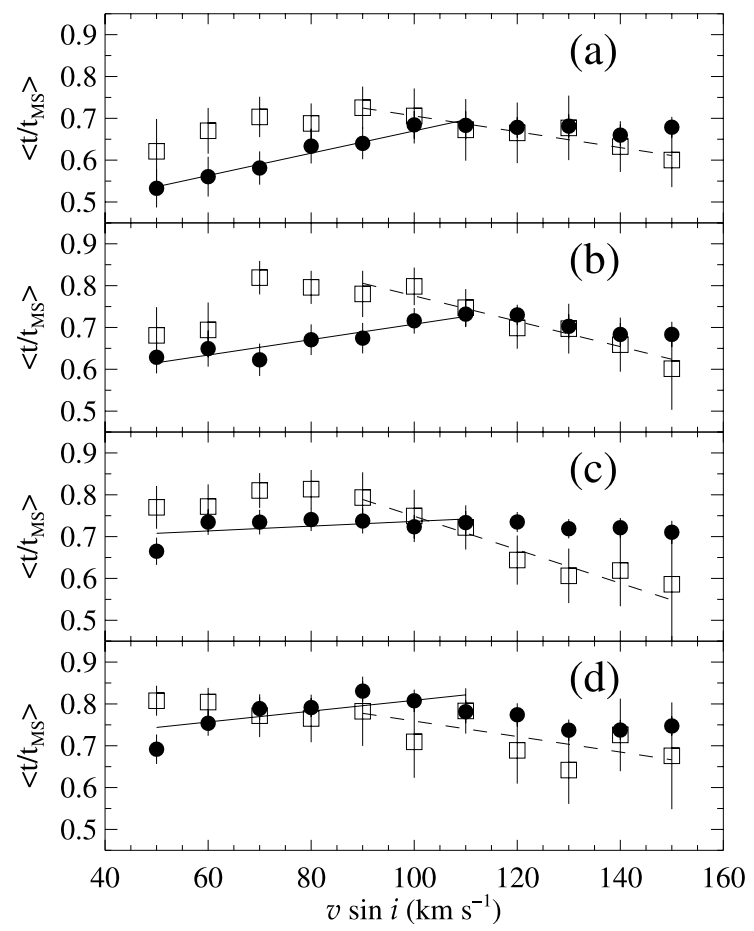

Figure 2.6: Mean age $<t / t_{\mathrm{MS}}>$ per bin of $v \sin i$ for different mass intervals: a) $2<M / M_{\odot}<2.4$; b) $2.2<M / M_{\odot}<2.6$; c) $2.35<M / M_{\odot}<2.85$; d) $2.55<$ $M / M_{\odot}<3.05$. Filled circles stand for normal stars, whereas open squares represent CP stars. The bins in $v \sin i$ are $40 \mathrm{~km} \mathrm{~s}^{-1}$ wide. Solid lines are the results of linear fits on normal stars with $v \sin i \leq 110 \mathrm{~km} \mathrm{~s}^{-1}$ and dashed lines the results of the fits for CP stars with $v \sin i \geq 90 \mathrm{~km} \mathrm{~s}^{-1}$. Figure and caption from Zorec \& Royer (2012, their figure 8). Permission obtained from A\&A.

strongly as a function of $t_{\mathrm{MS}}$, almost irrespective of mass. The results for differential rotation are fairly independent of the value of $J$, but by definition rotational velocities become critical in a rigid rotator when $J=J_{\text {crit }}^{\text {TAMS }}$.

Zorec \& Royer then compared their observational results, for which values of $J / M$ were calculated, to their models. They confirmed that their 'low rotational energy regime' assumption, in which differential rotators have less total angular momentum than the critical value for a rigid rotator, is valid. They highlighted that spin-up of surface angular velocities is only possible with differential rotation, where the core is rotating more quickly than the envelope and angular momentum is transported outwards. This is entirely plausible if a star is braked more strongly at the surface during the pre-main sequence phase (due to, say, disc interaction), 


\section{CHAPTER 2}

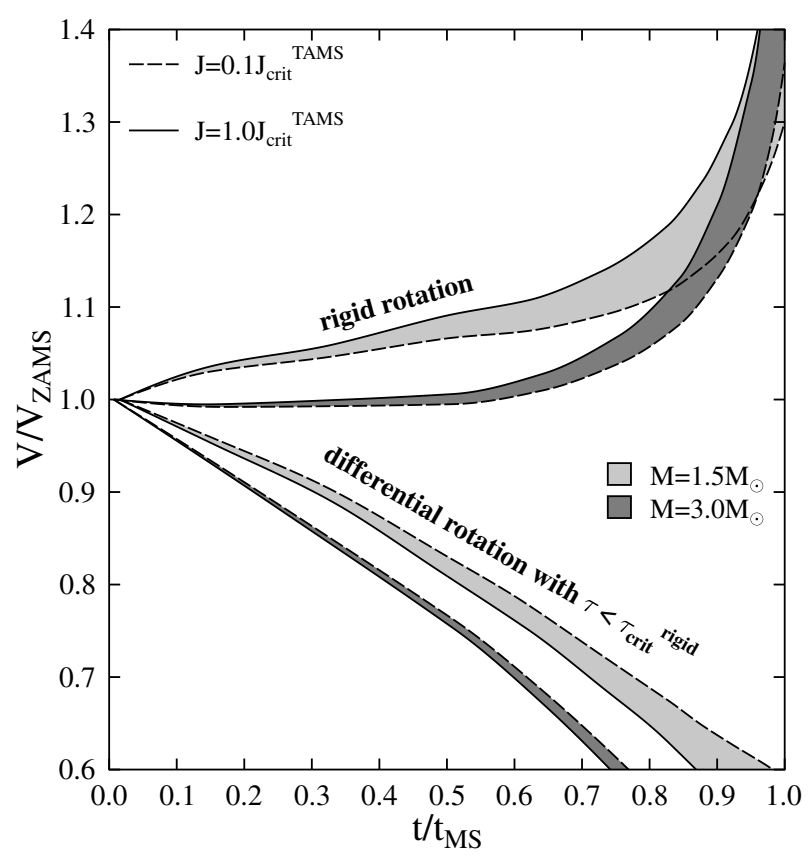

Figure 2.7: $v / v_{\text {ZAMS }}$ ratio of equatorial rotational velocities as a function of the $t / t_{\mathrm{MS}}$ ratio in the main sequence for stars with masses $M=1.5 \& 3 \mathrm{M}_{\odot} \cdot v_{\mathrm{ZAMS}}$ is the equatorial rotational velocity on the ZAMS, $t$ is the stellar age and $t_{\mathrm{MS}}$ is the time that a star of given mass $M$ can remain on the main sequence. Two extreme cases are represented: stars evolving as rigid rotators (instantaneous complete angular momentum redistribution) and as differential rotators without any angular momentum redistribution. Dashed lines represent the limits for $J / M \rightarrow 0$, while the solid lines are for stars that on the ZAMS have a specific angular momentum that in the TAMS becomes a critical one. $\tau$ is the ratio of kinetic to gravitational potential energy in the star. Figure and caption from Zorec \& Royer (2012, their figure 10). Permission obtained from A\&A.

than at the core. Their observations of rotational velocities increasing in the first third of the main-sequence lifetime (Fig. 2.8) can be explained by a rigid, rapidly rotating core transferring angular momentum to the surface. The turning point then represents the phase where the core and surface are rotating at the same rate. The more massive stars then decelerate efficiently (possibly as neat differential rotators for cases of high angular momentum) in a way consistent with angular momentum being conserved in shells with no redistribution. The lower $\left(2.0-\mathrm{M}_{\odot}\right)$ mass stars do not decelerate efficiently and end up with TAMS velocities greater than they were on the ZAMS. 


\section{CHAPTER 2}

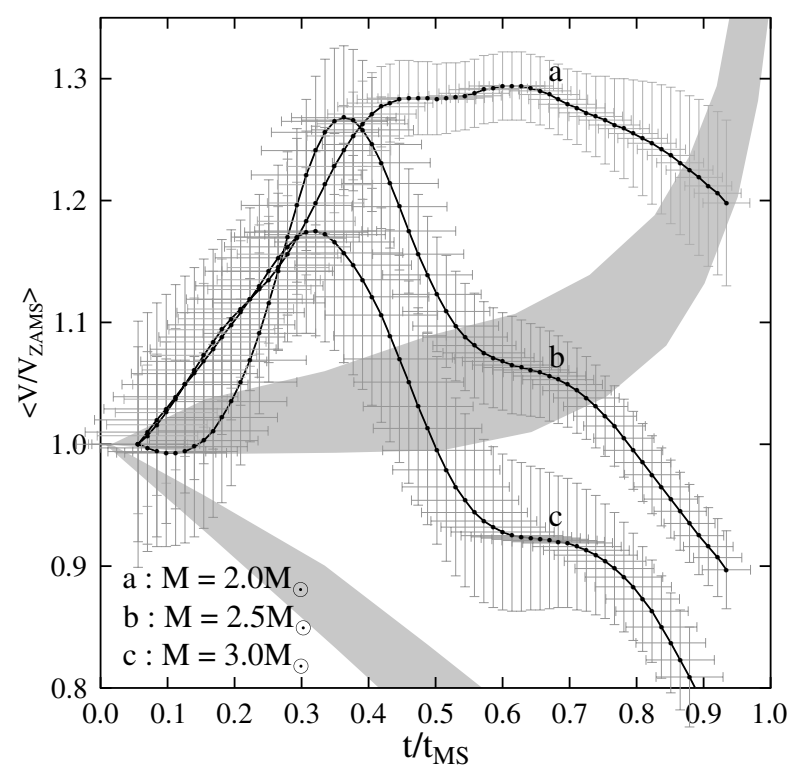

Figure 2.8: Evolution of the equatorial velocity ratio $\left\langle v / v_{\text {ZAMS }}\right\rangle$ in the mainsequence lifetime span, where $v_{\text {ZAMS }}$ is the true equatorial velocity of stars on the ZAMS. The shaded regions correspond to the theoretical evolution of the $v / v_{\text {ZAMS }}$ ratios shown in Fig. 2.7 (this document): rigid rotators (middle), simple differential rotators (lower left corner). Figure and caption from Zorec \& Royer (2012, their figure 15). Permission obtained from A\&A.

If we investigate the mass range of $\sim 1.8$ to $2.0 \mathrm{M}_{\odot}$ more closely, we have four information sources that are in agreement. From the observations of Zorec \& Royer (2012), we expect an initial spin-up starting at the ZAMS, to a peak in the central third of the main-sequence lifetime, followed by a weaker deceleration in the latter third of the main-sequence band (cf. Fig. 2.8). Inspection of the numbers of Wolff \& Simon (1997, cf. Fig. 2.5, here) for this mass range agrees with that prediction, but with a stronger final deceleration than we would expect from Zorec \& Royer (2012). A third source of information comes from Erspamer \& North (2003), who analysed $v \sin i$ and $\log g$ for a sample of $\mathrm{A}$ and $\mathrm{F}$ stars using the ELODIE and CORALIE echelle spectrographs and Hipparcos data. Their uncertainty of 0.2 dex in $\log g$ is not favourable (though not intrinsically poor, either), and there would be much benefit in having more data, but still the data show an agreement with $v \sin i$ initially increasing and then decreasing in the latter half of the main-sequence 


\section{CHAPTER 2}

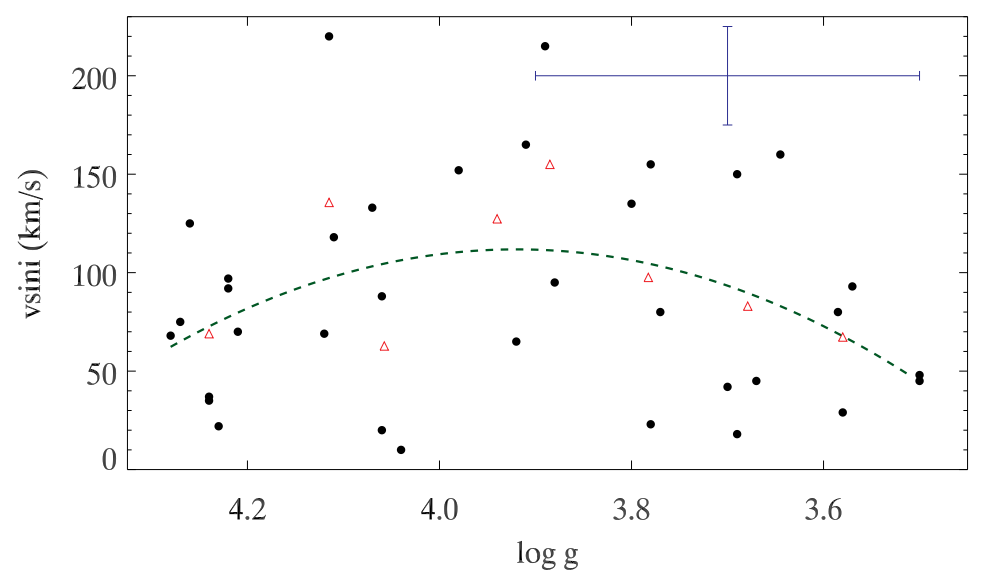

Figure 2.9: A replot of figure 14 of Erspamer \& North (2003), showing the variation of $v \sin i$ with $\log g$ over the main-sequence band, which extends from about $\log g=4.3$ to 3.5 for the 1.8 to $2.0-\mathrm{M}_{\odot}$ range. Original data shown as filled black circles. Added in the upper right is a typical error bar representing a conservative 0.2 dex in $\log g$ and $25 \mathrm{~km} \mathrm{~s}^{-1}$ in $v \sin i$ (estimated - Erspamer \& North do not quote exact errors). The dashed line is a second-degree polynomial fit to the data, the significance of which is described in the text. Open red triangles represent the binned data.

band. We illustrate that agreement in Fig. 2.9, which is a replot of their figure 14, noting the main-sequence band stretches from about 4.3 to 3.5 in $\log g$. Here, a second-degree polynomial has been fitted to the data to aid the eye. Although the correlation is not tight $\left(R^{2}=0.03\right.$ for a second-order polynomial, vs. 0.003 for a straight line), one must consider the large star-to-star variation in $v \sin i$ and the difficulty in obtaining precise $\log g$, which will affect any observational attempt to confirm the result. For this reason, the original data were binned into 0.1-dex bins in $\log g$ and added to Fig. 2.9. This shows a clearer correlation to the fit. Finally, the fourth source is Slettebak (1955), who in his figure 6 showed mean rotational velocities against spectral type and luminosity class, much like Wolff \& Simon (1997) did later, but Slettebak had a coarser grid. Erring strongly on the side of caution, Slettebak did not note the acceleration as astrophysical. 


\section{CHAPTER 2}

\subsubsection{Inferring velocity distributions from Fourier transforms of Kepler data}

The rotational velocity distribution of the A stars is well studied, and, as we saw in $\S 2.2 .1$, and will continue to discuss later in this chapter, determines the emergence of chemical peculiarities in these stars. Its importance has drawn many studies, both spectroscopic (e.g. Abt \& Morrell 1995) and photometric (e.g. Balona 2011). In the latter, Balona used Kepler data for several hundred A0-A8 field stars, finding about 8 per cent of them had light curves resembling those of cool stars with spots. A further 20 per cent had dominant low frequencies (example provided in Fig. 2.10) that were also attributed to spots or "some other corotating structure". By assuming the dominant low frequency is due to rotation, the distribution of low frequencies (Fig. 2.11) can be matched with A-star rotational distributions from the literature.

To this end, I prepared an observing proposal to obtain spectra for a sample of A stars that did not pulsate, in order to remove the influence of combination
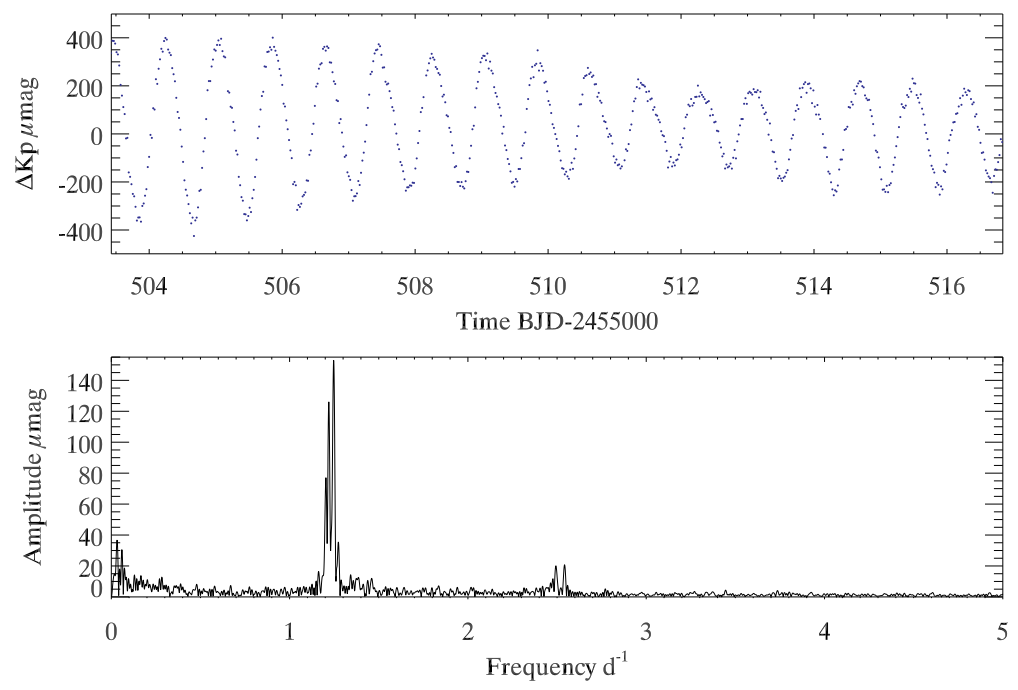

Figure 2.10: Lower panel: example of the low-frequency peaks seen in the Fourier transform, with a segment of the corresponding light curve (upper panel). Example given is KIC 11443271, LC, Q7 PDC-MAP data. 


\section{CHAPTER 2}

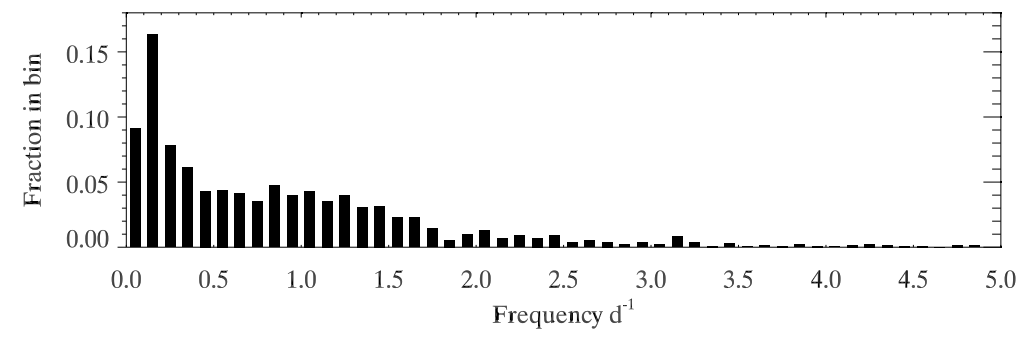

Figure 2.11: Histogram of the frequencies of the Fourier peaks, for stars satisfying $7000 \leq \mathrm{KIC} T_{\text {eff }} \leq 10000 \mathrm{~K}$. Break-up velocity for a $2-\mathrm{R}_{\odot}$ star occurs at $\sim 4 \mathrm{~d}^{-1}$.

frequencies (combination frequencies are discussed in detail in Chapter 4, but for now can be considered a property or consequence of pulsation). This has the added benefit of obtaining spectra for stars that are likely, through their lack of pulsation, to be chemically peculiar. This particular idea is developed in the next chapter. Although only a fraction of the target list was observed due to bad weather, the obtained spectra were supplemented with ground based observations of suitable resolution and wavelength coverage that were originally taken for other purposes. If the spectroscopic and Fourier-inferred rotational velocity distributions agree, the low frequency variations could offer a constraint on the inclination angle for individual stars, or give an indication of the equatorial rotational velocity without the need for time-consuming ground-based follow-up. In particular, if the Fourier transform peak corresponds to a rotation frequency, then a suitable radius estimate should, of course, yield a value of $v_{\text {rot }}$ that is greater than the measured $v \sin i$ for every single star that can be measured, except, perhaps, for some unlikely combinations of latitudinal differential rotation and inclination.

Before the analysis could be completed, Balona, a co-investigator in the project, proceeded with the Fourier analysis independently, publishing the results in a singleauthor paper (Balona, 2013). While the spectroscopic analysis remains as future work, the present discussion focusses on Balona's study. The frequency distribution he found is a match to that shown here in Fig. 2.11, and Balona showed this to 


\section{CHAPTER 2}

match the rotational velocity distribution of A stars in the literature, specifically that of the Glebocki, Gnacinski \& Stawikowski (2000) catalogue.

In Balona's paper, the physical mechanism most strongly proposed for the production of the observed light variation is spots, and specifically spots that are akin to those seen in cooler stars. Surface magnetic fields can be generated at the base of the convective envelopes of cool stars, and these fields lead to the production of spots of cooler temperature that cause a reduction in observed intensity when they are on the observed hemisphere. The scientific interest in Balona's paper is that such spots are not expected in A stars, whose surface convection is inefficient at late-A and nearly absent for stars earlier than this. Without the surface convection, how can spotted-star signatures be generated in the light curve? There are actually many ways to achieve this, although the presence of spots intrinsic to a single A star, that result from activity (i.e. they are not Ap spots), is yet to be demonstrated as one of them.

In his figure 3, Balona (2013) presents some examples of periodograms of hot A stars, with the implication that the frequencies below $\sim 2.5 \mathrm{~d}^{-1}$ that are therein illustrated arise from spots on the rotating surface. KIC temperatures are well known to be underestimated for stars hotter than around F0, so selecting a $T_{\text {eff }}$ range up to $10000 \mathrm{~K}$ results in some of the 'A stars' presented being B stars, further eliciting an explanation of a physical mechanism capable of generating spots in such hot stars. It turns out that for the 'spotted' stars that were checked for this discussion, other mechanisms are viable: ellipsoidal variables are capable of producing Fourier transforms like these, though detailed fitting is still required. Some of Balona's spotted stars were clearly slowly pulsating B (SPB) stars, whose instability strip lies at late-B. A background giant could easily contaminate the light curve and generate a similar signal, too, because the amplitudes of the peaks are often small fractions of a mmag, but again detailed testing is required. Modelling light curves of late-B stars 


\section{CHAPTER 2}

with spots has been attempted, but with limited success (Degroote et al., 2011), and a physical mechanism for the production of spots that fit the observations is still lacking.

Spots in cool stars migrate to different latitudes and change size, and there are usually multiple spots on any given hemisphere at any given time (except, perhaps, at the minimum of the activity cycle). This leads to phased light curves that change with time, unlike those of Ap stars (discussed in $\S 2.3$ ), whose spots are not free to migrate, and of ellipsoidal variables. The Fourier transform, in turn, shows a broader peak or set of unresolved peaks in the former case, but sharp peaks in the latter cases. Harmonics may also be present if there is some variability happening multiple times per cycle, such as antipodal Ap spots that are both observable at different rotation phase, or an elongated hemisphere of an ellipsoidal variable that is visible twice per orbit. In these ways, one might distinguish the spots that Balona proposes from those of Ap stars or from ellipsoidal variables, but he does not attempt such a distinction; it is shown that the amplitudes of the low frequencies are significantly variable in some cases, though. Even if a phased light curve shows temporal evolution, it must be demonstrated that the spots are on the A star, and

not a contaminant. Although KIC contamination is not reliable at small $(\lesssim 0.01)$ values, at large values one cannot ignore contamination. Balona made no rejection of contaminated objects, yet a check of some of his example stars shows objects with contamination exceeding a few tenths.

In conclusion, it may be possible to infer rotational velocities from Fourier transforms, but that this is generated by spots akin to those in cool stars as Balona (2013) suggests is yet to be demonstrated, and a substantial fraction of his 'spotted' stars can be shown to be binaries, pulsators or contaminated objects. 


\section{CHAPTER 2}

\subsection{The magnetic Ap stars}

The frequency of Ap stars among field stars peaks at 13 per cent at about B9 (Wolff, 1983), where the distribution of the cooler Ap SrCrEu stars overlaps with that of the hotter Ap Si stars. Spectral classification tends to lead to later types being assigned than would be inferred from the photometric classification $(B-V)$. Since many of these objects are actually B stars, the class is sometimes called "the ApBp stars", though cool Ap stars are even found at early-F.

The field strengths in magnetic Ap stars typically range from 0.3 to $30 \mathrm{kG}$ (Braithwaite et al., 2010), and are responsible for most of the observed peculiarities in these objects, either indirectly, by reducing the rotation speeds of these stars to values low enough that meridional circulation no longer efficiently mixes the chemical composition, as we shall see in $\S 2.3 .2$, or directly by concentrating certain elements into anomalous abundance patches on the surface.

\subsubsection{The oblique rotator model}

The Rigid or Oblique Rotator Model (originally proposed and dismissed by Babcock in 1949, developed in a time-series photometric, spectroscopic and polarimetric case study by Stibbs (1950), yet more often attributed to Deutsch 1956) describes a rigid rotator - one with no large-scale differential flows - whose observed magnetic field strength changes as the star rotates, providing the rotational axis is not along the line of sight. The magnetic field is inclined to (or oblique to) the rotational axis, and plays a dominant role in stabilising against convective motions near the surface. This reduces mixing of material, and allows the segregation of elements through atomic diffusion (i.e. gravitational settling and radiative levitation). The magnetic field also concentrates rare-earth elements into anomalous abundance patches or 'spots' on the surface, whose photometric intensity and spectral line profiles differ from the surrounding photosphere. As the star rotates and these antipodal spots, 


\section{CHAPTER 2}

located near or on the magnetic poles, move with respect to the line of sight, periodic changes are observed in the atmospheric properties, as illustrated in the aforementioned case study by Stibbs (1950). These variables are known as $\alpha^{2} \mathrm{CVn}$ stars. The apparently dipolar magnetic fields in these stars are strong, the strongest being Babcock's star (HD 215441; $67 \mathrm{kG}$ ), whose geometry has been reviewed by Landstreet et al. (1989). Although the fields appear dipolar, Braithwaite (2008) showed that roughly axisymmetric, stable magnetic fields consisting of a poloidal and a toroidal component can take on a dipolar appearance, implying the fields can be strongly non-dipolar.

Moss (1990) performed simulations of the evolution of the angle between magnetic and rotational axes, $\chi$, and found the angle to increase over $t_{\mathrm{MS}}$. North (1985) observed the distribution of $\chi$ to be apparently random on the ZAMS, but increasingly bimodal with time, depending on whether toroidal or poloidal magnetic fields are present. In both cases, it appears that the time-scale for $\chi$ drift is only slightly shorter than $t_{\mathrm{MS}}$, because the initial random distribution of $\chi$ is recoverable yet an increasing bimodality is observable. Wade (1997), too, found intermediate values of $\chi$ for stars as old as $10^{9} \mathrm{yr}$, but Landstreet \& Mathys (2000) found that the slowly rotating Ap stars (periods longer than a month) generally had magnetic and rotational axes aligned to within about $20^{\circ}$, whereas $\chi$ was much larger in the shortperiod Ap stars. Hubrig, North \& Schöller (2007) investigated obliquity angles for stars either side of the $3.0-\mathrm{M}_{\odot}$ boundary. They agreed that the slower rotators tend to have fields aligned or nearly aligned with the rotational axis, regardless of mass, but that otherwise the evolution of obliquity is different for high- and low-mass Ap stars. Landstreet \& Mathys (2000) discussed many potential causes for the alignment of fields with the rotational axis in slow rotators, with some causes arising from rotational braking through magnetic processes (accretion, stellar wind) occurring in the pre-main sequence phase. This is the subject of the next section. 


\section{CHAPTER 2}

\subsubsection{Pre-main sequence evolution}

The seminal paper of Stȩpień (2000) covered the pre-main sequence evolution of Ap stars in some detail. The following discussion is derived mostly from Stȩpień (2000), with some smaller contributions from other works.

Stȩpień sought to explain the observational result that Ap stars are slow rotators in light of the fact that they appear not to be braked on the main sequence (Hartoog, 1977; North, 1984, 1985). He determined that of all the possible reasons for the slow rotation he presented, only one was testable and satisfies the observations: that they emerge from the intense contraction phase of stellar formation with angular momenta similar to those of normal stars, but lose most of it in the pre-main sequence phase.

In the stellar interior, fossil magnetic fields are destroyed in convective zones (and replaced with dynamo fields) but can persist in stars with fully radiative envelopes on time-scales much longer than the $t_{\mathrm{MS}}$ of an intermediate-mass star. In stars more massive than about $1.5 \mathrm{M}_{\odot}$, the fully convective contraction phase is probably too short $\left(<10^{5} \mathrm{yr}\right)$ to destroy the fossil field. Ap magnetic fields had already been determined to be fossil fields from observational considerations of the uniform distribution of Ap stars across the main sequence (North, 1993; North et al., 1997; Wade, 1997). In addition, Leroy et al. (1994) supposed that a fossil field theory fits better than a dynamo one for the Ap stars because stars with similar field strengths are observed with periods ranging from $4 \mathrm{~d}$ to $77+\mathrm{yr}$.

Three major factors are important in the braking of Ap stars in the pre-main sequence phase: torque exerted by the interaction with the disc, accretion from the disc onto the star, and a magnetised wind. Stȩpień found that only for weak magnetic fields does accretion dominate period changes in early phases of the pre-main sequence evolution. Later in the pre-main sequence evolution, disc locking becomes very important and is the limiting factor of any spin-down, allowing rotational periods to become several times longer than those of normal stars, but not longer than 


\section{CHAPTER 2}

that. Indeed, it is disc locking that prevents most stars reaching rotational periods of years.

The role of the disc is complicated, in that it initially acts to spin up the star, but later acts to maintain a constant rotational velocity, and thus acts counter to spin-down mechanisms imposed by both the wind and the magnetic field. When stars possess both magnetic fields and accretion discs, they will accrete matter along magnetic field lines, and thus enhance their surface content of refractory elements.

Stępień examined the effect of switching off the disc early. Since the disc's early effect is to spin up the star, early switch-off in massive stars would produce magnetic stars with abnormally high rotational velocities because the pre-main sequence phase is fairly short, and there is insufficient time for other factors (wind, magnetic field) to spin the star down again. Conversely, lower-mass stars that spend longer in the pre-main sequence phase will have very long rotational periods when the wind and magnetic field operate without opposition from the disc.

The property to which angular momentum loss is most sensitive is the strength of the magnetic field, because it affects each spin-down mechanism. The field interacting with the disc itself produces a torque on the star, and any magnetised wind will also carry away stellar angular momentum. Stȩpień found that a threefold increase in magnetic field strength produces a 300-fold increase in ZAMS rotational period for a 1.5 to $2.0-\mathrm{M}_{\odot}$ star. Indeed, early disc switch-off has a substantially greater effect in these lower mass stars only if the field strength is high. It is these extreme circumstances that might produce Ap stars with periods on the order of a century. Such stars are more common than any of the rotational distributions presented in $\S 2.2$ predict, though this is likely contributed to by an observational selection effect (Mathys, 2004) due to the systematic search for stars with magnetically resolved lines (such as the search conducted by Mathys et al. in 1997) preferentially detecting very slow rotators. 


\section{CHAPTER 2}

Although the pre-main sequence braking works efficiently for magnetic Ap stars, it doesn't explain the slow velocities of metallic-lined (Am) stars or the non-magnetic Ap stars. The former are explained via tidal deceleration in binaries, which will be addressed in $\S 2.4 .2 \& 2.4 .3$, but the latter remain unexplained.

\subsubsection{Main sequence evolution}

There is disagreement in the literature over the main-sequence evolution of Ap stars, mostly in terms of their distribution across the HR diagram. We mentioned in $\S 2.3 .2$ that Ap stars had been observed with a uniform distribution across the main sequence (North, 1993; North et al., 1997; Wade, 1997). Similarly, North (1998) concluded that there is no indication of any loss of angular momentum on the main sequence for Ap Si stars, and Hubrig, North \& Mathys (2000) found no correlation between Ap rotational periods and fraction of main-sequence lifetime completed, confirming Stȩpień's work showing the angular momentum evolution to happen in the pre-main sequence phase. Hubrig, North \& Medici (2000) looked for progenitors of Ap stars on the main sequence. They found no statistically significant difference between the $v \sin i$ of young and old A stars in the $1.7-2.5 \mathrm{M}_{\odot}$ range, but did find a marginally significant population of slow rotators among the young A stars. All of these finds argue against the evolution of Ap rotational velocities on the main sequence.

Hubrig, North \& Mathys (2000), on the other hand, found that magnetic Ap stars with masses below $3 \mathrm{M}_{\odot}$ were concentrated towards the centre of the main-sequence band - they have completed 30 per cent of their main-sequence life before magnetic fields become detectable. Although Hubrig, North \& Mathys found no evidence for magnetic braking on the main sequence, the observational results of this study appear questionable in light of other studies. For instance, if the Ap progenitor already rotates slowly before presenting an observable magnetic field, why is it not 


\section{CHAPTER 2}

an Am star? Abt (2009) comprehensively addresses this question and concludes these normal slow rotators are the Ap SrCrEu stars yet to develop peculiarity. If a strong magnetic field is required for the pre-main sequence braking, then why does it disappear for the first 30 per cent of $t_{\mathrm{MS}}$ ? Hubrig, North \& Medici (2000) suggested the magnetic field could be complicated, either disorganised or buried below the surface. Furthermore, it could be that the type of extreme abundances that attract observational surveys do not develop in the first 30 per cent of $t_{\mathrm{MS}}$, but this is contrary to long-standing theoretical work that the stability generated by magnetic fields facilitates the establishment of chemical peculiarities within $10^{6} \mathrm{yr}$ (Michaud et al., 1976); some claim even in just days for Sr in the high atmosphere (Braithwaite et al., 2010). Ultimately, the search for Ap progenitors in general suffers from insufficient data: Hubrig, North \& Mathys determined that an extension of their sample to $\sim 2200$ stars would be required to generate a $3 \sigma$ detection.

Kochukhov \& Bagnulo (2006) re-opened the debate with public data for $150 \mathrm{CP}$ stars with accurate Hipparcos parallaxes. They found the highest number density of $\mathrm{CP}$ stars was in the $2.5-3.0 \mathrm{M}_{\odot}$ bin, and that at these masses the $\mathrm{CP}$ stars occupy the full width of the main-sequence band. At masses lower than this the $\mathrm{CP}$ stars are absent in the final tenth of $t_{\mathrm{MS}}$, but there is no agreement with the findings of Hubrig, North \& Mathys (2000) that no Ap stars are found younger than $t / t_{\mathrm{MS}}=0.3$. Later analyses found that Ap stars with $M \leq 3.0 \mathrm{M}_{\odot}$ were indeed clustered around the middle of the main-sequence band, but that Ap stars more massive than $3 \mathrm{M}_{\odot}$ occupy the full width of the main-sequence (Hubrig et al., 2006; Hubrig, North \& Schöller, 2007).

\subsection{Binaries}

The binary frequency of A stars lies at around 35 per cent (Abt, 2009), and is not abnormally high. Table 2.1 shows the observed binary frequency for main-sequence 


\section{CHAPTER 2}

Table 2.1: Summary of observed spectroscopic binary frequencies for main-sequence stars. Table and caption from Abt (2009, table 1). Permission obtained from author, and reproduced by permission of the AAS.

\begin{tabular}{lrccc}
\hline Type & Sample & $\begin{array}{c}\text { Binaries } \\
\text { Found }\end{array}$ & $\begin{array}{c}\text { Freq. } \\
(\%)\end{array}$ & Source \\
\hline O & 227 & 54 & $24 \pm 3$ & Mason et al. 1998 \\
B2-B5 & 48 & 19 & $40 \pm 9$ & Abt \& Levy 1978 \\
A0-A9 & 119 & 41 & $35 \pm 5$ & Abt 2009 \\
F7-G8 & 175 & 38 & $22 \pm 3$ & Abt \& Willmarth 2006 \\
M & 64 & 8 & $13 \pm 4$ & Marcy \& Benitz 1989 \\
\hline
\end{tabular}

stars. In this section we will discuss the effect of binarity on the rotation rates of the A stars.

\subsubsection{Observational results}

Abt \& Boonyarak (2004) collected orbital data for 400 stars of types B (162 stars) and A (238 stars). They found that "the amount of angular momentum lost, presumably by tidal effects, decreases with increasing binary separation and increases with stellar ages or types." Their study is significant in that they look not just at close binaries, but those whose orbital periods lie between 4 and $500 \mathrm{~d}$ such that they cover periods where synchronisation is not expected. In fact, the authors were surprised to observe tidal effects up to these long periods, given that for A stars, theoretical limits for circularisation and synchronisation were 1.58 and $0.95 \mathrm{~d}$, respectively (Zahn, 1984).

The amount of angular momentum lost as a result of being in partially synchronised $^{2}$ binaries of types A6-F0 was found to range from 90 per cent at $P_{\text {orb }}=5.5 \mathrm{~d}$, to 40 per cent at $P_{\text {orb }}=100 \mathrm{~d}$. The authors added that no significant differences in losses were found for single-lined (SB1) or double-lined (SB2) binary systems,

\footnotetext{
${ }^{2}$ The meaning of 'partially synchronised' is not explicit in that paper. It sometimes means stars that are synchronised at periastron, when orbits are highly eccentric, but in this case perhaps means stars that show some tidal braking but are not fully synchronised.
} 


\section{CHAPTER 2}

so these effects are not sensitive to the secondary masses. However, the results are purely observational and do not require that the braking occurs on the main sequence; they are open to interpretation and perhaps the spin-down occurs during formation.

At periods exceeding $500 \mathrm{~d}$, the rotational velocities are a statistical match with single stars, i.e. no braking is observed, but A0-A5 binaries with periods of $4 \mathrm{~d}$ to $500 \mathrm{~d}$ rotate on average at $31 \pm 2$ per cent of single star velocities (the numbers for B-type and A6-F0 stars are $67 \pm 5$ and $41 \pm 15$ per cent, respectively).

Kogure (1981) looked at the period distribution of binary systems with A0-A9 V-III primaries (from various catalogues), for the purpose of deriving a periodrotational velocity relationship, and found the maximum of the period distribution to lie at $P_{\text {orb }} \approx 5 \mathrm{~d}$. Kouwenhoven et al. (2005) observed $199 \mathrm{~A}$ and late-B members of the nearby Scorpius OB2 association in the near-infrared, though their method appears to have been sensitive to long periods only, covering ranges of several decades to several thousands of years, thus having the opposite period sensitivity to spectroscopic surveys. No period distribution could therefore be inferred. However, among their results they found that the mass ratio, $q$, of secondary to primary covers the whole range from 0.1 to 1 for primaries between 1.5 and $2 \mathrm{M}_{\odot}$, and from 0.1 to 0.75 for 2 to $3-\mathrm{M}_{\odot}$ stars. The normalised mass-ratio distribution, $f(q)$, is described empirically as $f(q)=q^{-0.33}$, and is important in braking calculations (cf. $\S 2.4 .2$ ). They also found a lower limit to the multiplicity (not duplicity) of A stars of 0.4, in agreement to that of Abt (2009) once one accounts for the duplicity/multiplicity difference.

To obtain larger-number and complete statistics on the short-period binary distribution, Mazeh, Tamuz \& North (2006) constructed a 938-star subsample of eclipsing binaries with main sequence B-type primaries from OGLE photometric data of the LMC in an automated way. They find a "somewhat surprising" flat distribution 


\section{CHAPTER 2}

in $\log P$ between 2 and $10 \mathrm{~d}$ (Fig. 2.12), after trimming their sample to improve homogeneity and correcting for detection probability. As such, although their results are based on the eclipsing binaries, the corrections applied in the lower panel of Fig. 2.12 extend its applicability to all LMC binaries, not just eclipsing ones. One cannot just extend the distribution to A stars, but at least for the early-A stars, it is hard to see that the distribution would change drastically.

\subsubsection{Short-range tidal braking}

Working on the idea that the dominant non-adiabatic damping mechanism operating in stars with convective envelopes is turbulent friction, Zahn (1977) derived an expression for the time-scale of synchronisation, $t_{\mathrm{syn}}$, of low-mass (i.e. convective envelope) main-sequence binaries: $t_{\text {syn }} \sim q^{-2}(d / R)^{6}$, where $d$ is the orbital separation and $R$ the radius of the star to be synchronised (Langer, 2009). The stars with radiative envelopes, however, are damped radiatively by tidally induced gravity-mode pulsations on time-scales of $t_{\text {syn }} \sim q^{-2}(1+q)^{-5 / 6}(d / R)^{17 / 2}$ (Zahn, 1975). We are concerned here with the latter time-scale.

The time-scale increases rapidly with decreasing $q$, the process being twice as efficient for $q=1$ as $q=0.75$, but the most important parameter is the binary separation, $d / R$, here expressed in stellar radii, for which the exponent is high. The mechanism decreases extremely quickly in efficiency for greater separation, and is only effective at ranges closer than $\sim d / R=6$, corresponding to a physical separation of $<0.07 \mathrm{AU}\left(P_{\text {orb }}<2.5 \mathrm{~d}\right)$ for a $2.5-\mathrm{R}_{\odot}$ primary.

\subsubsection{Medium-range tidal braking}

The short-range mechanism accredited to Zahn is not the full story in tidal braking. The theoretical limits calculated by Abt \& Boonyarak (2004) based on Zahn (1984), for circularisation and synchronisation periods of 1.58 and $0.95 \mathrm{~d}$, respectively, do not 
CHAPTER 2
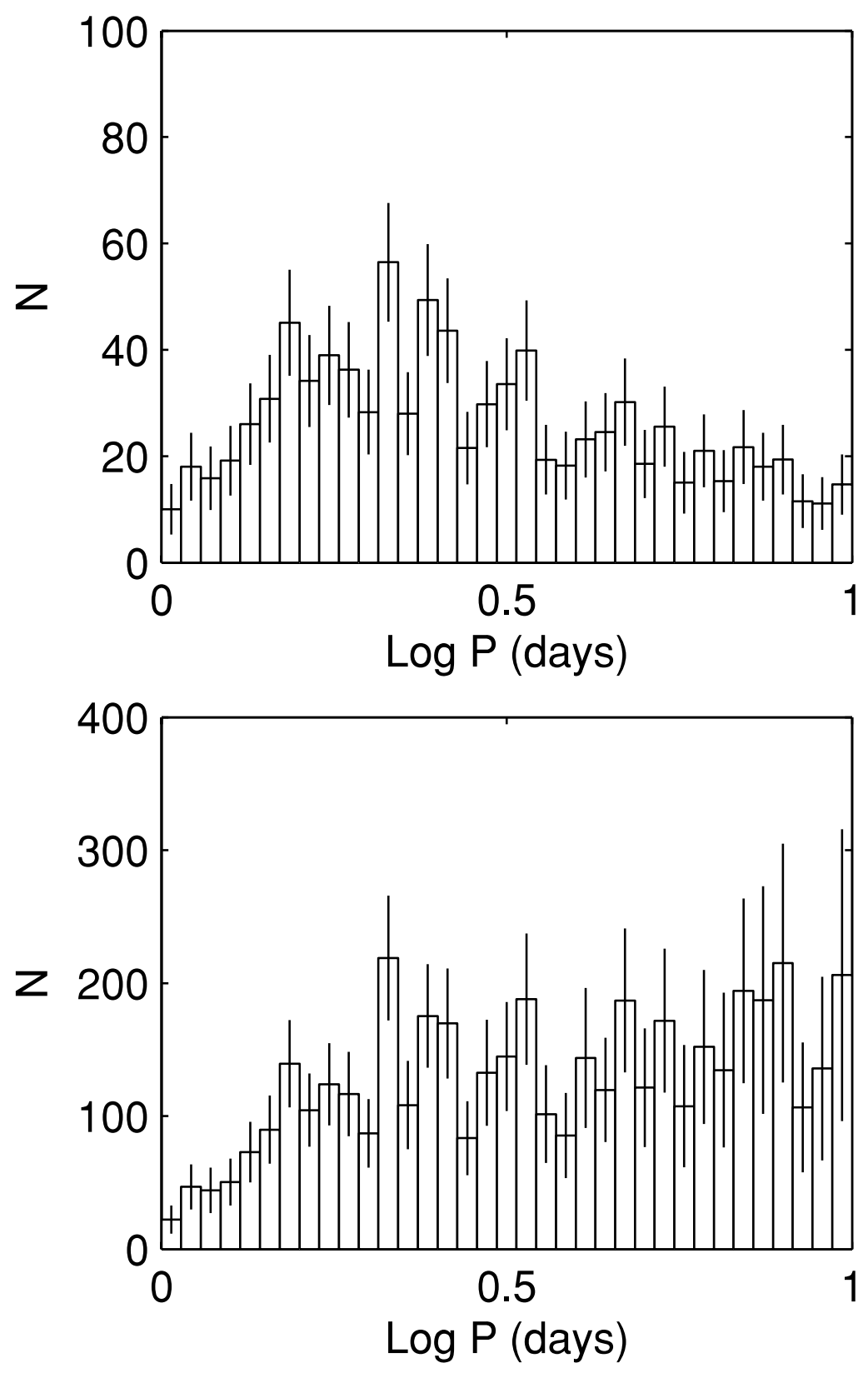

Figure 2.12: Top panel: raw period distribution of the bias-free sample. Bottom panel: period distribution corrected for the detection probability. Figure and caption from Mazeh, Tamuz \& North (2006, figure 1). Permission obtained from Astrophys. Space Sci. (Springer). 


\section{CHAPTER 2}

perfectly match the observations. Abt, Levato \& Grosso (2002) observed values of 4.9 and $2.5 \mathrm{~d}$ instead, and found rotational velocities reduced, but not synchronised, for up to twice these periods. In $\S 2.4 .1$ we pointed out the severe braking observed by Abt \& Boonyarak (2004) for periods up to 500 d. Some other mechanism would appear to be braking these stars effectively at much larger distances than Zahn's.

Tassoul \& Tassoul (1992b) presented such a mechanism. So-called 'Ekman pumping' is a mass-transfer process (hence transporting angular momentum) between the surface and the inviscid interior. It allows effective synchronisation within a time much shorter than $t_{\mathrm{MS}}$ by redistributing angular momentum within the star. Largescale meridional flows are set up from equator to pole at the surface, but from pole to equator in the interior. The equatorial material of high angular momentum is thus regularly replaced with lower-angular momentum material from the interior, and the star is spun-down exponentially in the rotating frame. Rieutord (1992) made calculations refuting the Ekman-pumping mechanism, but the Tassouls showed Rieutord's calculations to have omitted tidally driven currents, inappropriately assumed solid-body rotation, and to have failed to incorporate tidal lag.

Tassoul \& Tassoul (1992a) explained their mechanism in a way that is more easily accessible to the general reader: the presence of a binary companion elongates the primary along the line connecting the two. A particle rotating around an axis perpendicular to this line thus travels in an ellipse, rather than a circle, and accelerates and decelerates along its path. Large-scale meridional flow thus comes into existence in a non-synchronous binary component. The resulting replacement of high angular velocity fluid with low angular velocity fluid is more efficient than viscous friction, spinning the star down efficiently. The synchronisation time can be calculated as follows:

$$
t_{\mathrm{syn}}(\mathrm{yr})=\frac{14.4 \times 10^{-N / 4}}{q(1+q)^{3 / 8}}\left(\frac{\mathrm{L}_{\odot}}{L}\right)^{1 / 4}\left(\frac{\mathrm{M}_{\odot}}{M}\right)^{1 / 8}\left(\frac{R}{\mathrm{R}_{\odot}}\right)^{9 / 8}\left(\frac{d}{R}\right)^{33 / 8}
$$




\section{CHAPTER 2}

where the constant factor $10^{N}$ is the ratio of the macroscopic and microscopic viscosities (typically $N=0$ for radiative envelopes and $N=10$ for convective ones).

Tassoul \& Tassoul (1992a) compared their theory with Zahn's. While Zahn's mechanism has dependencies of $(d / R)^{6}$ for a convective envelope and $(d / R)^{8.5}$ for a

radiative one, the Ekman-pumping mechanism has a dependency of $(d / R)^{4.125}$. The latter is therefore effective at longer ranges than the other two, being effective up to $(d / R) \sim 20$ rather than $(d / R) \sim 6$. It can remain operative up to $\sim 100$-d periods for early-type stars without causing full synchronisation beyond $P_{\text {orb }}=15-25 \mathrm{~d}$. It is thus compatible with the observations described in $\S 2.4 .1$.

Some qualifications are applicable to the Tassouls' mechanism. Their equations require the stellar radius to be constant over the braking time-scale. For longer periods, this time-scale is longer and the validity breaks down because some radius evolution occurs. An expanding radius affects conservation of specific angular momentum and acts against synchronisation. Furthermore, moderately small eccentricities were assumed, so that just like Zahn's (1977) mechanism, the assumptions are invalid for moderate or high eccentricities. (One must realise, of course, that for high eccentricity pseudo-synchronous rotation is all that is possible.)

Witte \& Savonije (2001) developed another medium-range mechanism that works specifically on eccentric binaries. It works when an orbital harmonic becomes locked on a resonance, is sustained in that resonance state for a prolonged time, and affects the spin and orbit significantly in that time. The harmonic remains locked in resonance, exciting gravity-mode oscillations, until either the power redistribution in the orbital harmonics becomes too large or some other frequency comes into resonance and pushes the other one out (cf. heartbeat stars, Thompson et al. 2012). Witte \& Savonije find that for a simulated $10-\mathrm{d}$ orbit of two $10-\mathrm{M}_{\odot}$ stars, the accumulated time during which at least one star is locked in resonance spans 64 per cent of the total evolution time, while 81 per cent of the total eccentricity decrease is accounted 


\section{CHAPTER 2}

for during the locking periods: the total decrease in this case being from $e=0.6$ to 0.2 in just a few Myr.

With a period of $20 \mathrm{~d}$, i.e. doubled from the previous simulation, the effectiveness of locking at reducing the eccentricity is quartered, but still important. Although in the first $4 \mathrm{Myr} P_{\text {orb }}$ drops by a quarter and eccentricity by a fifth (from 0.60), the rotation rates do not decrease over this time and $4 \mathrm{Myr}$ is roughly one eighth of the main-sequence lifetime for a $10-\mathrm{M}_{\odot}$ star.

However, we are concerned with stars of lower mass than this. How might the results change for a $2.0-\mathrm{M}_{\odot}$ star? Witte \& Savonije do not comment on this, but intuitively it seems that the braking mechanism would become less effective with a lower-mass primary. We must also consider, though, that the main-sequence lifetime of a $2.0-\mathrm{M}_{\odot}$ star is far longer than that of a $10-\mathrm{M}_{\odot}$ star, so the mechanism can act for much longer. Hence if time is measured in fractional main-sequence lifetime, then there is little reason to suspect the mechanism would not work for lower-mass stars. No comment was provided on the effect of decreasing $q$, either. It would certainly serve to decrease the efficiency, but it is not known by how much.

The orbital harmonic locking mechanism is much more efficient for higher eccentricities, and, although it doesn't brake rotational velocities greatly, it has a large effect on the orbital period. As such that may bring the stars close enough and begin to circularise the orbits so that Zahn's short-range mechanism or the Ekmanpumping mechanisms can reduce the spin to observed levels. We will take a closer (simple) look at angular momentum transfer in binary orbits in Appendix 2.B of this chapter, but a full-scale theoretical investigation is beyond the scope of this work.

\subsection{Metallic-lined A stars (Am stars)}

The classical Am stars are those whose CaII K line types appear too early for their hydrogen line types, and metallic-line types appear too late, such that the 


\section{CHAPTER 2}

spectral types inferred from the Ca II K and metal lines differ by five or more spectral subclasses. They are observed to be slow rotators with $v \sin i<120 \mathrm{~km} \mathrm{~s}^{-1}$, and although some Am stars are observed with higher rotational velocities, there is a strong observed correlation between slow rotation and abundance anomalies, and the standard Am-star model requires it. This section pertains specifically to the rotational velocity properties of Am stars, and their presence in binary systems. More details on the line strength anomalies and the peculiar abundance model are given in Chapter 3.

\subsubsection{Presence in binaries}

The slow rotation of the Am stars is largely brought about by their presence in short-period (1d $\leq P \leq 10 \mathrm{~d}$ ) binary systems (Abt, 1967). The exact fraction of Am stars in binaries is not certain. Historically, the number was believed to be high - Abt (1961) measured 22 of 25 (= 88 per cent) stars in his survey to be binaries, and Vauclair (1976) put the number at about 80 per cent - but more recent surveys with less observational bias put the percentage between about 60 (North et al., 1998) and 54 (Debernardi, 2000). In another study (Debernardi et al., 2000), the binarity of 19 Am stars in the Praesepe and Hyades clusters was established to have a lower limit of 63 per cent, and the authors could not definitively rule out that the percentage of single Am stars might be zero. This binary frequency is to be compared to the much lower value of 35 per cent of A stars as a whole (Abt, 2009, cf. Table 2.1 here). It is the tidal interaction in close binaries that brakes the stars' rotational velocities, with different mechanisms being dominant at different stellar separations $(\S 2.4 .2$ \& $\S 2.4 .3)$.

Since Am stars require slow rotation to facilitate gravitational settling and radiative diffusion (e.g. Baglin et al., 1973), those Am stars that are not in binaries and thus not tidally braked require an explanation for their slow rotation - how do single 


\section{CHAPTER 2}

Am stars come to possess such small rotational velocities? The majority of A stars have rotational velocities higher than the observed upper limit of Am star velocities, but could the single Am stars simply lie at the lower end of a normal distribution of rotational velocities, low enough to fall below the observed Am star rotational velocity limit and thus require no braking? Arguments presented in $\S 2.2 .1$ suggest the distribution is multimodal, and that the population of Am and Ap stars belong to their own Maxwellian, not the same as the normal stars. In $\S 2.3 .2$ it was found that pre-main sequence braking is extremely efficient when a star possesses a magnetic field, but that some slow rotators emerge from the pre-main sequence phase without strong magnetic fields, and that the fields are perhaps buried. Maybe these stars can become single Am stars?

The 'single Am' problem remains an unsolved one. One of the greatest difficulties lies in eliminating the presence of a companion for a large sample of 'single' Am stars $^{3}$. Abt (1979) presented a different hypothesis based on a study of Am stars in clusters. While he did find Am stars in the youngest cluster, implying the Am star spectral peculiarities develop in less than $10^{6}$ y (after the suggested age correction of Abt 2009 is applied), he found that in the youngest clusters the Am stars have $v \sin i$ far greater than the observed limit of field stars of $\sim 120 \mathrm{~km} \mathrm{~s}^{-1}$. Though this has never been confirmed, it would mean the Am stars do not have to be slowly rotating, but perhaps that in most cases the mechanism causing the spectral peculiarities also causes the slow rotation. The hypothesis is interesting, and Abt derived an empirical rotational braking relation: $\left\langle v \sin i>\propto T^{-0.28}\right.$, where $T$ is the cluster age in years, but Am star theory requires slow rotation and the postpeculiarity braking hypothesis finds little mention in the literature. The consensus is that that study was based on too few data points (e.g. Stȩpień, 2000).

\footnotetext{
${ }^{3}$ Unlike our Solar System, where Jupiter contains most of the angular momentum, A stars rotate so rapidly that a planet of Jupiter's mass and orbital parameters would contain on the order of 1 per cent of the angular momentum of the star, thus distribution of angular momentum into planetary systems is not a viable mechanism for achieving a slowly rotating single A star.
} 


\section{CHAPTER 2}

\subsubsection{Implications on mixing}

The various mechanisms for tidal braking in $\S 2.4$ each impose large amounts of mixing of material in the stars concerned. The Witte \& Savonije (2001) method, involving orbital harmonics in resonance with g modes, sees the low-order orbital harmonics grow to high amplitudes during resonance crossings, and "these crossings could possibly induce large-scale mixing." Similarly, the meridional circulation set up during Ekman-pumping (Tassoul \& Tassoul, 1992a,b) will also thoroughly mix material. Zahn's 'turbulent friction' mechanism (Zahn, 1975, 1977), involves coupling between convective motions and tidal oscillations (at least, it does in reality it was not realistically treated in the initial derivation). Thus all three tidal braking mechanisms act to mix the stellar material, contrary to observations that Am stars are vertically stratified in elemental abundances, and are largely found in close binaries. Perhaps it is the case that the mixing halts once the stars attain synchronisation, but this would be testable in that no Am star should be observed outside of synchronisation - we have just seen that this is not the case, and Murphy et al. (2012) have recently presented a case-study of an intriguing single Am star observed by Kepler, KIC 3429637. This will be presented in Chapter 5. Further discussion of those mixing processes not induced by binarity will be discussed in Chapter 3 .

\subsection{The Kraft break}

The A stars rotate much more quickly than the late-F and G stars. Even many of the slowly rotating A stars are not slow by comparison with the average G-dwarf; the normal A stars rotate faster than those by an order of magnitude or more. The two regimes have their 'transition' at around spectral type $\mathrm{F} 5 \mathrm{~V}$, that is, normal stars earlier than about F5 rotate quickly and those later than F5 rotate slowly. This boundary is known as the Kraft break, after Robert Kraft who first explained 


\section{CHAPTER 2}

the phenomenon (Kraft, 1967).

From observations of over 200 field stars Kraft showed that a break in rotational velocities of over an order of magnitude occurs over a very small mass range. The mean $v \sin i$ of $1.3-\mathrm{M}_{\odot}$ stars lies at around $20 \mathrm{~km} \mathrm{~s}^{-1}$, compared to $\sim 75 \mathrm{~km} \mathrm{~s}^{-1}$ for a 1.6- $\mathrm{M}_{\odot}$ star, as shown in Fig. 2.13.

To be specific, this relationship of $v \sin i$ with mass was found for stars without chromospheric CaII K line emission, which is an important indicator of age and activity in the late-F and G stars - Ca II K line emission is seen in the younger stars with more active convection zones. The average rotational velocity was seen to be higher in stars with CaII K line emission than without. Kraft's results thus show that young stars are braked by magnetic winds coupled to the surface hydrogen convection zone, such that once they reach an age where the stellar activity has lessened, they have been strongly braked. Stars of later spectral types will have spent more time on their Hyashi tracks in a fully convective state, and thus reach the ZAMS having been braked substantially more. The braking rate depends on

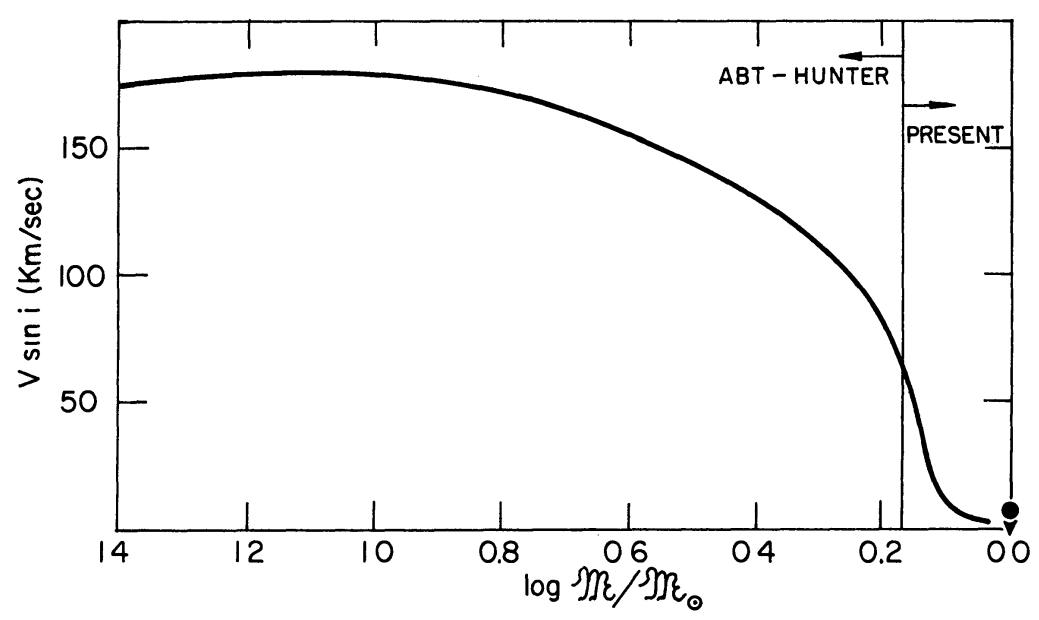

Figure 2.13: $<v \sin i>$ as a function of $\log M / \mathrm{M}_{\odot}$ for field stars. The masses were determined from the work of Abt \& Hunter (1962) and Kraft's $v \sin i$ values were adopted. The greatest rate of change of $v \sin i$ with mass occurs for $\log M / \mathrm{M}_{\odot}=0.13$ $\left(M=1.35 \mathrm{M}_{\odot}\right)$ corresponding to spectral type $\sim$ F5 V. Figure from Kraft (1967, figure 2). Permission obtained from author, and reproduced by permission of the AAS. 


\section{CHAPTER 2}

both the convection zone overturn time-scale and the depth of that convection zone (see Sills, Pinsonneault \& Terndrup 2000 for a well-referenced introductory review). Early-F stars do not have a deep Hydrogen convection zone on the main sequence, and so are not braked by magnetically coupled winds after reaching the ZAMS.

Irwin \& Bouvier (2009) emphasize the importance of the circumstellar disc on the pre-main sequence in regulating the rotational period. We saw this argument earlier from Stȩpień (2000) for the Ap stars. The duration of the pre-main sequence phase for which the disc is present depends on the stellar mass, in that the less massive stars spend longer in this phase and are influenced by the presence of the disc for longer. The disc acts to maintain the stellar rotational period at a constant value; in the absence of the disc, the star would spin up as it contracts, meaning that the disc acts to slow rotation, whether it be through disc locking or accretion-driven stellar winds. This argument of pre-main sequence braking being strongly mass dependent furthers the conclusions of the model of braking through a magnetic wind during the main-sequence phase, and explains satisfactorily why the Kraft break is observed: the mechanisms that are more efficient on the pre-main sequence have less time to act in the more massive (earlier) F stars, so those stars leave this phase having been braked less, and they continue to be braked less on the main sequence because they have only shallow surface convection zones with which magnetic winds can couple. The complete picture of rotation vs. spectral type is shown in Fig. 2.14.

\subsection{Chapter Summary}

We have seen that the distribution of rotational velocities in A stars ranges widely from essentially zero right up to $400 \mathrm{~km} \mathrm{~s}^{-1}$. The distribution cannot be represented empirically by a single Maxwellian; indeed, even two Maxwellians are insufficient because there exists separate populations of (i) normal, fast rotators; (ii) normal, slow rotators; and (iii) chemically peculiar (CP) slow rotators. CP fast rotators are 


\section{CHAPTER 2}

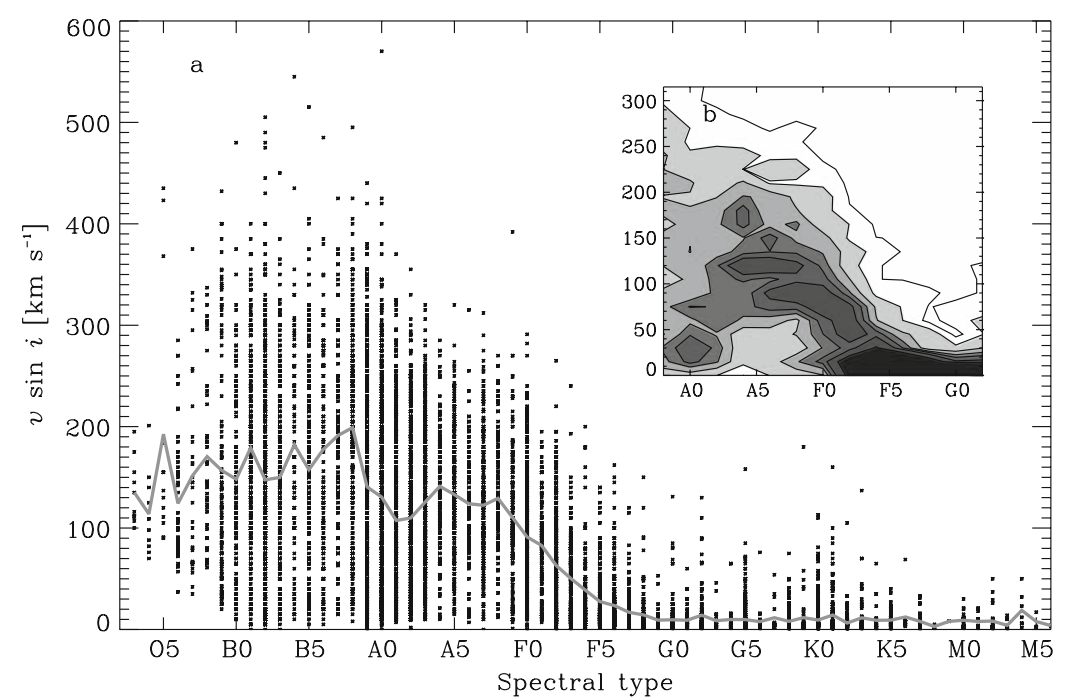

Figure 2.14: (a) Distribution of $v \sin i$ as a function of spectral type. The individual values (crosses) represent individual stars. The variation of the average $v \sin i$ per spectral type (solid thick line) is also over-plotted. (b) The sub-panel in the upperright corner represents the color-coded scale density of points in the same diagram $v \sin i$-spectral type; both axes have the same scale as in (a). Darker tones stand for higher densities. The Kraft break is evident at $\sim F 5$ as a high density of slow rotators and lack of fast rotators. The over-density of slow rotators at around A0 is the population of Ap stars. Figure and part of caption from Royer (2009, figure 1). Reproduced with kind permission of Springer Science and Business Media.

generally not observed.

There is a discrepancy in $v \sin i$ determinations by different observers, with both systematic shifts and fractional differences. In particular, the results of Royer, Zorec \& Gómez (2007) are high compared to the literature values, especially those of Abt \& Morrell (1995). The former authors' results are the most recent, and they had the opportunity to compare them to those of other groups. A comparison of two other sets of rotational velocities having stars in common with Abt \& Morrell indicated the values of the latter are underestimated.

The evolution of rotational velocities among A stars is dissimilar to that observed for cooler stars. Only the late-A stars see substantial braking whilst approaching the TAMS. Intermediate A stars appear to brake little in the latter half of the time spent on the main sequence $\left(t_{\mathrm{MS}}\right)$, but more massive stars are braked quite efficiently 


\section{CHAPTER 2}

after $t / t_{\mathrm{MS}}=1 / 3$.

All stars between 2 and $3 \mathrm{M}_{\odot}$ are observed to accelerate while $t / t_{\mathrm{MS}}<1 / 3$. This is explained as angular momentum distribution outward from a core that rotates faster on the ZAMS than the surface does. A reinvestigation of older studies, namely those of Slettebak (1955), Wolff \& Simon (1997), and Erspamer \& North (2003), has been performed here and shows agreement with the findings of Zorec \& Royer (2012).

It may be possible to infer rotation frequencies from the Fourier transform of the light curve. Work to investigate this link, whose origin is unclear, is ongoing. Balona (2013) interprets the evidence as the presence of spots on the surface of 40 per cent of A stars and some B stars, but an analysis of several of his candidates for spots showed that binarity, pulsation or contamination were equally satisfactory matches to the observations, and require no new physics.

We examined the cause of slow rotation in Ap stars. Magnetic field strength is the main factor determining the extent of braking in the pre-main sequence phase, with the disc lifetime also playing an important role. The observed Ap star periods can be explained through pre-main sequence braking and, for the extremely slow rotators, with an additional observational selection bias. No unambiguous evidence for main-sequence braking of Ap stars has been presented, though the development time-scale for some spectral peculiarities can give the appearance of Ap stars lying only in the central third (measured from ZAMS to TAMS) of the main-sequence band. A 3- $\sigma$ identification of Ap progenitors requires a larger sample than has currently been obtained.

We discussed the distribution of binary periods, which was recently observed to be approximately flat in $\log P_{\text {orb }}$ for orbital periods between 2 and $10 \mathrm{~d}$. Mass ratios appear to be able to take on a wide range at mid-A, with an empirical dependency $f(q)=q^{-0.33}$. At periods exceeding $500 \mathrm{~d}$, rotational velocities resemble those of 


\section{CHAPTER 2}

single stars. Between 4 and $500 \mathrm{~d}$, increasingly-long orbital periods see decreasing amounts of braking. Below $4 \mathrm{~d}$, orbits are observed to be fully synchronised.

Three mechanisms were presented for tidal braking: (I) The 'orbital harmonic resonances' mechanism works by locking orbital harmonics in resonances, during which they greatly reduce the eccentricity and period of the orbit. Resonances can account for over 50 per cent of the total evolution time. This mechanism does not slow the rotation but can reduce the orbit to a range where the other two mechanisms are efficient. (II) The 'Ekman-pumping' mechanism operates with largescale motions acting to replace high angular momentum material at the surface with lower angular momentum material from the interior. This is more effective than simple viscous friction, and efficiently brakes the stars within ranges of up to $\frac{d}{R} \sim 20$, i.e. separations up to 20 times the primary star's radius. (III) Zahn's 'turbulent friction' mechanism is very efficient but requires separations below $\frac{d}{R}=6$.

Each of the mechanisms proposed for tidal braking would appear to mix the material too much to be compatible with Am star observations. Nevertheless, we reviewed the frequency of Am stars that are in binaries, the most recent estimates being on the order $55-65$ per cent. It is very difficult to definitively rule out a companion from systems that appear to be single, so the binary fraction could be substantially higher than this.

The A and early-F stars rotate much more quickly than late-F and $G$ stars. The Kraft break, occurring roughly at spectral type F5 V (corresponding to 1.3$\mathrm{M}_{\odot}$ stars) delimits the regimes of fast rotation in hotter stars, and slow rotation in cooler stars. The causes are differences in the duration of the pre-main sequence phase, during which deceleration is efficient, and the presence of deep hydrogen convection zones in the cooler stars, which facilitate braking on the main sequence though magnetic winds coupled to the convection zones. 


\section{CHAPTER 2}

\section{A Spectral type calibrations}

In astronomy there are many different photometric systems in use, each with different filters. Systems come into and fall out of use over time; the nature of highprecision, ground-based, $V$-band photometry in the latter half of the previous century saw the narrow-band Strömgren filters become widely used. Now, many surveys in the infrared and ultraviolet favour broader filters, and all-sky surveys are adopting the Sloan system. Sloan may be the future, but much archived data exists in older formats that have seen more extensive calibration in the literature. It is these systems for which literature calibrations are assembled in Table 2.2 in this appendix for reference. Calibrations of the main sequence under the Sloan system would be highly valuable but are yet to be performed, and so are not provided here.

The photometric parameters in Table 2.2 are useful in many ways. The Balmer discontinuity parameter, $c_{1}=(u-v)-(v-b)$, is an indicator of luminosity; $m_{1}=$ $(v-b)-(b-y)$ is the line-blanketing parameter, an indicator of metallicity; $\beta$ is a narrow-band index measuring the $\mathrm{H} \beta$ line strength, mostly free from line blanketing and interstellar extinction, an indicator of temperature for stars between A3 and F2 (Joshi et al., 2006), and mildly sensitive to gravity due to pressure broadening of the line. Smalley \& Dworetsky (1995) calculated synthetic $\beta$ values for a grid of $T_{\text {eff }}$ and $\log g$ in their table 7 . Another temperature indicator is $b-y$, which is "reasonably free of effects of [line] blanketing" (Crawford, 1975), but is affected by reddening. The $B-V$ index is calibrated so that $\mathrm{A} 0$ stars have $B-V \equiv 0$, but the values in Table 2.2 remain true to source.

Two indices from these values are commonly used to diagnose chemical peculiarity: $\delta m_{1}=m_{1}($ standard $)-m_{1}$ (observed $)$, for a given $\beta$; and $\delta c_{1}$, rather confusingly defined in the opposite sense from $\delta m_{1}$ (the poor observer!), as $\delta c_{1}=$ $c_{1}$ (observed) $-c_{1}$ (standard), again for a given $\beta$. The standard relations can be found in Crawford $(1975,1978,1979)$ for the F, B and A stars, respectively. A more 


\section{CHAPTER 2}

Table 2.2: Calibrations from the literature of spectral type, absolute visual magnitude $M_{V}$, colour $(B-V)$, and effective temperature for the stars around spectral type A at luminosity class V. Data taken from Gray \& Corbally (2009) for $M_{V}$ calibration against spectral type; from Fitzgerald (1970), who provides $U B V$ data for many spectral types; and Bessell (1979), who provides calibrations of $T_{\text {eff }}$ against $B-V$. The latter were fitted with a cubic polynomial, and $T_{\text {eff }}$ was calculated for each $B-V$ quoted in the table, before being rounded to the nearest $50 \mathrm{~K}$. Values of $\beta$ and the de-reddened indices $(b-y)_{0}, m_{0}$ and $c_{0}$ are taken from Crawford's (1975; $1978 ; 1979)$ 'standard relations' that specifically correspond to the ZAMS, and their definitions are explained in this appendix; a few values had to be interpolated.

\begin{tabular}{lccccccc}
\hline $\begin{array}{l}\text { Spectral } \\
\text { Type }\end{array}$ & $\begin{array}{c}M_{V} \\
\text { mag }\end{array}$ & $\begin{array}{c}B-V \\
\text { mag }\end{array}$ & $\begin{array}{c}\text { Teff } \\
\mathrm{K}\end{array}$ & $\begin{array}{c}\beta \\
\mathrm{mag}\end{array}$ & $\begin{array}{c}(b-y)_{0} \\
\mathrm{mag}\end{array}$ & $\begin{array}{c}m_{0} \\
\mathrm{mag}\end{array}$ & $\begin{array}{c}c_{0} \\
\mathrm{mag}\end{array}$ \\
\hline B8 & 0.0 & -0.11 & 12000 & 2.748 & & 0.118 & 0.66 \\
B8.5 & 0.4 & -0.09 & 11600 & 2.772 & & 0.122 & 0.75 \\
B9 & 0.7 & -0.07 & 11200 & 2.795 & & 0.126 & 0.83 \\
B9.5 & 1.0 & -0.04 & 10650 & 2.827 & & 0.134 & 0.97 \\
A0 & 1.4 & -0.01 & 10150 & 2.861 & & 0.154 & 1.01 \\
A1 & 1.6 & 0.02 & 9750 & 2.873 & & 0.162 & 1.05 \\
A2 & 1.9 & 0.05 & 9300 & 2.885 & 0.029 & 0.169 & 1.08 \\
A3 & 2.0 & 0.08 & 8950 & 2.871 & 0.055 & 0.172 & 1.08 \\
A4 & & 0.12 & 8500 & 2.864 & 0.071 & 0.184 & 1.05 \\
A5 & 2.1 & 0.15 & 8200 & 2.841 & 0.090 & 0.195 & 0.96 \\
A6 & 2.2 & 0.17 & 8000 & 2.833 & 0.099 & 0.198 & 0.93 \\
A7 & 2.3 & 0.20 & 7800 & 2.824 & 0.107 & 0.201 & 0.90 \\
A8 & 2.4 & 0.27 & 7300 & 2.789 & 0.132 & 0.194 & 0.87 \\
A9 & 2.5 & 0.30 & 7150 & 2.779 & 0.145 & 0.193 & 0.83 \\
F0 & 2.6 & 0.32 & 7000 & 2.768 & 0.158 & 0.191 & 0.79 \\
F1 & 2.8 & 0.34 & 6950 & 2.736 & 0.204 & & \\
F2 & 3.0 & 0.35 & 6900 & 2.704 & 0.250 & & \\
F3 & 3.1 & 0.41 & 6650 & 2.690 & 0.263 & & \\
F4 & 3.3 & 0.42 & 6600 & 2.674 & 0.277 & & \\
F5 & 3.4 & 0.45 & 6500 & 2.665 & 0.290 & & \\
\hline
\end{tabular}




\section{CHAPTER 2}

negative $\delta m_{1}$ index indicates a stronger metallicity, and is typical in Am stars. A very small $(\lesssim 0.1)$ or negative $\delta c_{1}$ index is also seen in most Am stars, but not exclusively - both peculiar stars and binaries have small or negative $\delta c_{1}$ indices. Am stars have a smaller $\delta c_{1}$ index because of line blanketing, which describes the effect of metal lines on the spectrum and, due to the increase in density of spectral lines at bluer wavelengths, removes more flux at shorter wavelengths than longer ones. Since Am stars have stronger metal lines, line blanketing is greater, and the spectrum is reddened, causing $\delta c_{1}$ to decrease. Hence the trend noticed by Crawford (1979) that stars with larger negative $\delta m_{1}$ have smaller than average $\delta c_{1}$.

Occasionally the indices $(b-y)_{0}, c_{0}$ and $m_{0}$ appear in the literature, and are used in Table 2.2. They indicate $(b-y), c_{1}$ and $m_{1}$ values that have been corrected for reddening (Crawford, 1975). $(B-V)_{0}$ is also occasionally seen, and a 'colour excess', defined as $E(B-V)=(B-V)_{0}-(B-V)$ is commonly used to describe interstellar reddening. It is noteworthy that reddening for Kepler targets is negligible (Molenda-Żakowicz et al., 2009).

Literature values of $M_{V}$ vs. spectral type are not particularly consistent. A large difference is found by Gray \& Corbally (2009) and Adelman (2004) for the early-A stars, for instance. Calibrations are frequently borrowed from various sources in a hard-to-trace way. For this reason I have calculated a new calibration for luminosity class V objects, using the Tycho-2 Spectral Type Catalog (Wright et al., 2003) and Hipparcos parallaxes. Existing calibrations of $M_{V}$ with Hipparcos data against spectral type include that of Jaschek \& Gomez (1998), unknown to this author at the time of the investigation with Tycho-2 data, where the luminosity class was the primary subject of investigation rather than the temperature subclass of the spectral type. Jaschek \& Gomez used only MK standards, which when filtering out stars without parallaxes or with large errors, left them with only 96 stars in the range B0-F5. We shall see here that the Tycho-2 data have afforded numbers superior by 


\section{CHAPTER 2}

two orders of magnitude. ${ }^{4}$

The Tycho-2 Spectral Type Catalog contains over 350000 stars with spectral types. The positional matching of these stars is described as 'stringent', with 97.5 per cent of the stars being matched to within $1^{\prime \prime}$ of star positions from other cross-referenced catalogues. The catalogue also provides $B$ and $V$ magnitudes. The $V$ magnitudes were used here with Hipparcos parallaxes to determine absolute magnitudes, and to calibrate these against spectral type. A calibration in $B-V$ has not yet been performed but is a possible direction for future work.

About half of the stars in the Tycho-2 catalogue do not have both spectral types and luminosity classes. Many more are not of spectral types B8-F5, and so were not of interest to the current study. Chemically peculiar stars were excluded, and any objects with two spectral types given (whether arising from uncertainty, binarity or some other source) were also rejected in the interest of creating a calibration for normal, single, luminosity-class $\mathrm{V}$ objects. These selection criteria reduced the initial 350000 stars to about 35000 . A further requirement was that stars used must be given the object type ' $*$ ' in the Simbad database, further eliminating known binaries or multiple systems, peculiar stars other than the common Am or Ap types, and importantly, known variable stars. Finally, not all stars in the Tycho2 catalogue have Hipparcos parallaxes available; those stars without parallaxes, or whose parallaxes were below 3 mas were rejected, since the errors on individual parallax measurements are on the order 1 mas. The final number of usable stars was 8722.

Although Simbad offers other sources for spectral type and $V$, the values used were strictly the Tycho-2 values. This has two main benefits: (1) the spectral type sources are few in number (a result of the aforementioned selection criteria was that only three sources within the catalog were used: the catalogues of Jaschek, Conde \&

\footnotetext{
${ }^{4}$ The main conclusion of Jaschek \& Gomez (1998), incidentally, was that the MK standards ought to be revised to eliminate the stars deviating furthest from a direct relation between luminosity class and $M_{V}$.
} 


\section{CHAPTER 2}

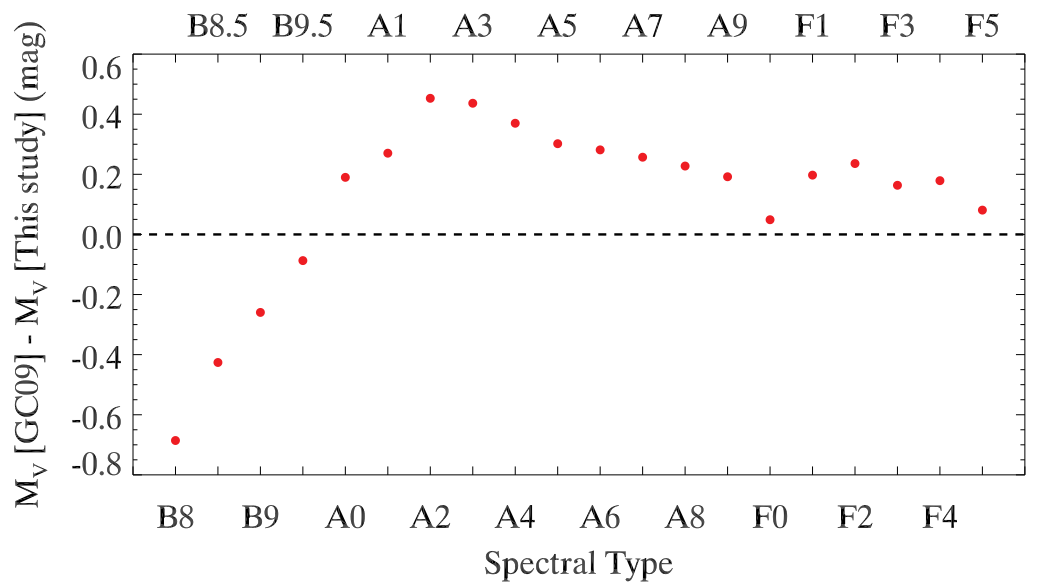

Figure 2.15: Difference in $M_{V}$ between Gray \& Corbally (2009), "GC09", and this study, for each spectral type analysed.

de Sierra (1964) and Kennedy (1983), and all five volumes of The Michigan Catalog (Houk \& Cowley, 1975; Houk, 1978, 1982; Houk \& Smith-Moore, 1988; Houk \& Swift, 1999)), thus spectral types are internally consistent. (2) The Tycho-2 $V$ values are given to a higher precision than most of the Simbad values, and have just one source.

It is noteworthy that the number of stars for each spectral type is neither uniform nor does it follow the initial mass function. It is typical for the spectral types to match MK anchor points ${ }^{5}$, rather than lie between them. For this reason, there are very few stars at F1 (24) compared to 915 and 881 at F0 and F2, respectively.

The results of the calibration are given in Table 2.3, alongside the literature calibrations of Gray \& Corbally (2009) and Adelman (2004). Fig. 2.15 offers an easy comparison between the Gray \& Corbally (2009) literature values and the values from this study.

The effect on the results of deficiencies in the method can be explained qualitatively, but a quantification of the differences compared to literature values is not

\footnotetext{
${ }^{5} \mathrm{MK}$ standards are divided into anchor points, primary, and secondary standards. Anchor points are those that have not changed since the initial definition of the system. See Appendix A of Gray \& Corbally (2009).
} 


\section{CHAPTER 2}

Table 2.3: Calibration of spectral type vs. absolute magnitude $\left(M_{V}\right)$. Columns two and three are literature values from Gray \& Corbally (2009) and Adelman (2004), respectively. Column four contains the mean value of $M_{V}$ from this study of Tycho data, across the number of stars in that spectral-type bin as indicated in the rightmost column. The fifth (penultimate) column contains the results of a cubic fit to those mean values, resulting in a more steady change of $M_{V}$ with spectral type. The cubic-fit values are the ones plotted in Fig. 2.15.

\begin{tabular}{lccccc}
\hline $\begin{array}{l}\text { Spectral } \\
\text { Type }\end{array}$ & $\begin{array}{c}M_{V}(\text { Gray }) \\
\text { mag }\end{array}$ & $\begin{array}{c}M_{V}(\text { Adelman }) \\
\text { mag }\end{array}$ & $\begin{array}{c}M_{V}>\text { Tycho } \\
\text { mag }\end{array}$ & $\begin{array}{c}M_{V} \text { Cubic } \\
\text { mag }\end{array}$ & \# of stars \\
\hline B8 & 0.0 & & 0.61 & 0.69 & 159 \\
B8.5 & 0.4 & & 0.72 & 0.83 & 77 \\
B9 & 0.7 & & 1.05 & 0.96 & 401 \\
B9.5 & 1.0 & & 1.20 & 1.09 & 193 \\
A0 & 1.4 & 0.7 & 1.29 & 1.21 & 903 \\
A1 & 1.6 & & 1.45 & 1.33 & 553 \\
A2 & 1.9 & 1.3 & 1.40 & 1.45 & 304 \\
A3 & 2.0 & & 1.49 & 1.56 & 307 \\
A4 & & & 1.72 & 1.68 & 127 \\
A5 & 2.1 & 2.0 & 1.67 & 1.80 & 153 \\
A6 & 2.2 & 2.1 & 1.71 & 1.92 & 68 \\
A7 & 2.3 & 2.3 & 1.92 & 2.04 & 111 \\
A8 & 2.4 & 2.4 & 2.00 & 2.17 & 93 \\
A9 & 2.5 & 2.5 & 2.44 & 2.31 & 331 \\
F0 & 2.5 & 2.6 & 2.54 & 2.45 & 915 \\
F1 & 2.8 & & 2.55 & 2.60 & 24 \\
F2 & 3.0 & & 2.84 & 2.76 & 881 \\
F3 & 3.1 & & 2.96 & 2.94 & 1378 \\
F4 & 3.3 & & 2.79 & 3.12 & 46 \\
F5 & 3.4 & & 3.19 & 3.32 & 1678 \\
\hline
\end{tabular}




\section{CHAPTER 2}

possible because the literature values most likely suffer the same deficiencies. One such deficiency is the effect of stellar evolution on the findings. By selecting stars of luminosity class $\mathrm{V}$ only, the calibration uses stars across the whole main-sequence band, which is about one magnitude wide. Considerable dispersion therefore exists in the data.

The number of sources for spectral types is small, but those catalogues may contain stars classified by various classifiers. The extent to which they were checked by the authors is not certain. Even the best classifiers might disagree, use different sensitivity criteria, or make errors. While the lowest luminosity A0 star is going to be of class $\mathrm{V}$, there is no guarantee that no class IV-V stars made it into the class $\mathrm{V}$ population, or even that the classifier actually distinguished between classes $\mathrm{V}$ and IV-V. Small luminosity class errors are easily made, particularly with noisy or lowresolution spectra (Gray, priv. comm.). Since medium to high-resolution spectra are usually degraded by convolving with a Gaussian for the purpose of spectral typing, the uncertainty on spectral subclass is not improved beyond 1-2 subclasses, as measured by agreement of different, standard diagnostics (Catanzaro et al., 2010).

Using Hipparcos parallaxes offers precise data for a huge number of stars. The idea to limit the analysis to stars with parallaxes above 3 mas reduces the percentage error on the parallaxes used, but the brightness goes as $1 / r^{2}$. A more conservative approach would be to use a higher parallax limit, even if it affords fewer stars for the analysis.

The stars used in this analysis were chosen to be normal and single. That the stars are single is clearly important for establishing the correct brightness, as is the contribution from background giants or optical pairs, but that is much harder to quantify. That the stars are normal is also important. The peculiar A stars rotate slowly, so by removing them the population that remains is rotating more quickly than the average A star. Faster rotating stars are gravity darkened around 


\section{CHAPTER 2}

the equator (but gravity brightened at the poles - the flux has to go somewhere). Although this would appear to cancel out for randomly orientated rotational axes, one must consider that statistically there is a much larger solid angle (in latitude and longitude) from which a star appears to be 'equator on' than 'pole on'. Hence through gravity darkening, one expects the selection of normal stars (rapid rotators) to cause a systematic shift in brightness at spectral types where peculiar stars are most common: around A0 for the Ap stars and around A8 for the Am stars. It is not clear how literature calibrations have treated the chemically peculiar stars and binaries.

A future study to calibrate the main sequence using similar data would utilise the precise $B-V$ values, which are continuous, unlike the discrete spectral types, and a new calibration would be drawn for the bottom of the main-sequence band (i.e. the zero age main sequence) as was done in the seventies by Crawford (1975, 1978, 1979).

\section{B Angular momentum transfer}

In this appendix we shall discuss the effect of transferring angular momentum between orbit and rotation, and show that large changes in rotational velocity can occur with relatively small corresponding changes in orbital separation.

\section{B.1 Orbital angular momentum}

For two stars of masses $m_{1}$ and $m_{2}$ treated as point masses in a circular orbit, the orbital angular momentum of the system is

$$
L=L_{1}+L_{2}=m_{1} v_{1} r_{1}+m_{2} v_{2} r_{2}
$$




\section{CHAPTER 2}

where $v$ and $r$ denote the orbital velocities and orbital radii. Let us adopt the simplest scenario, where the two stars are identical. Eq. 1 simplifies to

$$
L=2 m v_{\text {orb }} r
$$

We must remember that each star orbits the barycentre of the system, which lies at the midpoint of the line connecting the two stars. Let us eliminate the orbital velocity

$$
L=2 m \frac{2 \pi r}{P} r=4 \pi m r^{2} P^{-1} \quad\left(=2 m \omega r^{2}\right)
$$

and by considering that the orbit is Keplerian, eliminate the period

$$
L_{\mathrm{orb}}=4 \pi m r^{2}\left(\frac{4 \pi^{2} r^{3}}{2 G m}\right)^{-1 / 2}=\left(8 G m^{3} r\right)^{1 / 2}
$$

\section{B.2 Rotational angular momentum}

For the rotational angular momentum, let us assume the star has a uniform density. We shall focus on a single star when analysing the effect of orbital braking. The angular momentum of that star is:

$$
L_{\mathrm{rot}}=I \omega=\frac{2}{5} m R^{2} \omega=\frac{2}{5} m R v_{\mathrm{rot}}
$$

where $R$ is the stellar radius.

\section{B.3 Transfer of momentum}

We have assumed that both stars are identical, and now we shall assume that $m=$ $2 \mathrm{M}_{\odot}$ and $R=2 \mathrm{R}_{\odot}$. We can derive an expression for the transfer of momentum 


\section{CHAPTER 2}

between rotation and orbit, in either direction, by considering equations 2.2 and 2.3.

$$
\begin{aligned}
\Delta L_{\mathrm{orb}} & =\Delta L_{\mathrm{rot}} \\
\left(8 G m^{3}\right)^{1 / 2} \Delta r^{1 / 2} & =\frac{2}{5} m R \Delta v_{\mathrm{rot}}
\end{aligned}
$$

and substituting our assumed stellar parameters

$$
80 \Delta r^{1 / 2} \approx \Delta v_{\text {rot }}
$$

This implies that for a reduction in rotational velocity of $10 \mathrm{~km} \mathrm{~s}^{-1}$, the orbital radius would have to increase by $\sim 10^{4} \mathrm{~m}$. For a hypothetical $10 \mathrm{~d}$ orbit, whose radius is $\sim 10^{10} \mathrm{~m}$, the fractional increase in orbital radius is only $10^{-6}$, while the rotational velocity will have reduced by $10 \%$ if the star were initially rotating at $100 \mathrm{~km} \mathrm{~s}^{-1}$. That is, substantial braking can occur without large changes in orbital radius.

What then happens when the orbit shrinks, as main-sequence binary orbits are often observed to do? The dominant orbital shrinkage mechanisms are not the reverse of the dominant rotational angular momentum changes. Instead, the orbital decay is driven by cases of mass loss where the secondary collects the mass lost from the primary. Roche-lobe overflow, for instance, causes large changes in the orbit over short time-scales, and not all mass lost is necessarily accreted onto the secondary. Even the angular momentum loss from a stellar wind, where total mass lost is small, will be effective for reducing the orbital period, and on yet longer time-scales gravitational-wave radiation plays a (smaller) role (Hilditch, 2001). 


\section{CHAPTER 2}

\section{Acknowledgements}

SJM would like to thank Antonio García Hernández and especially Don Kurtz for their useful comments, and Richard Gray for the discussion on spectral type calibrations. Appendix A to this chapter made extensive use of the SIMBAD database and the VizieR catalogue access tool (Ochsenbein, Bauer \& Marcout, 2000), operated at CDS, Strasbourg, France. 


\section{Chapter 3}

\section{A selective review of spectral peculiarities in the A stars}

\section{Abstract}

The literature on Am stars is extensively reviewed, paying particular attention to abundance anomalies and the state of Am-star models in light of pulsations found in many of these objects. A new study into the non- $\delta$ Sct A stars using Kepler data, and the spectroscopic classification of two thirds of them as chemically peculiar stars is also performed with complementary abundance analyses. Although there are chemically normal $\gamma$ Dor stars in the $\delta$ Sct instability strip without $\delta$ Sct pulsation, the least variable stars (without $\gamma$ Dor pulsation either) are chemically peculiar, and some strongly so. The $\rho$ Pup stars of spectral type A/F are reviewed. For this group there is a consensus that they are the evolved Am stars. This chapter also reviews a class of B stars - it offers one of the few reviews of the 'sn' stars, whose spectra display both sharp and nebulous lines, and proposes a link between these and the Ap stars. Additionally, the two main theories for the origin of abundance anomalies 


\section{CHAPTER 3}

in $\lambda$ Boo stars are discussed and it is suggested that both of them imply the $\lambda$ Boo stars contain a high fraction of pulsators - a suggestion that is backed up by observations in the literature.

\subsection{Introduction}

The region of the HR diagram occupied by the A stars features a large array of spectral peculiarities, with peculiar stars making up 50 per cent of the stellar population at some temperature subclasses. These spectral peculiarities are observed as line strength anomalies. To quote from Wolff's (1983) well known monograph on the A stars: "The fundamental question, of course, is whether the line strength anomalies reflect true abundance anomalies or whether they can be explained in terms of unusual excitation and ionisation conditions in an atmosphere of normal composition." Even 30 years ago, Wolff weighed the evidence and found it to favour the first of those two possibilities, though we now know in addition to that, the atmospheres of peculiar stars are stratified and must be modelled as such. It is the aim of this chapter to review the progress made in the field of spectral peculiarities in the A stars since then, and we shall see that although our fundamental understanding has not changed much, we are really honing the details.

Wolff dedicated many pages in her monograph to the Ap stars. These are the most peculiar of the A stars, some of which exhibit abundance anomalies of factors of $10^{5}$ for some elements, particularly rare earth metals of the lanthanide series. The two most common types of Ap stars are the relatively hot Ap Si stars, featuring silicon overabundances in stars with $10000 \leq T_{\text {eff }} \leq 16000 \mathrm{~K}$, and the cooler Ap SrCrEu stars (8-12000 K), some of which pulsate as the so-called rapidly oscillating $A p$ or roAp stars (Kurtz, 1982). With an exceptionally low $T_{\text {eff }}$ of $6400 \mathrm{~K}$ (Shulyak et al., 2010), Przybylski's star (HD 101065) is the coolest Ap star known and is the roAp prototype (Kurtz, 1978a). The fraction of Ap stars reaches a peak of 0.13 at 


\section{CHAPTER 3}

B9, where those two populations overlap. Although Ap stars are covered extensively in the literature, full reviews are hard to come by. For the interested reader, Wolff's monograph is still highly recommended; the Ap stars will not be reviewed in this chapter.

A-type stars with normal abundances, i.e. those that are not chemically peculiar, tend to be $\delta$ Sct stars. The $\delta$ Sct stars have pulsations driven by the opacity mechanism operating on the second ionisation zone of helium. A review of $\delta$ Sct and related stars will be presented in the next chapter; in this chapter we shall see how peculiar abundances affect stellar pulsation, particularly in the Am stars. The Am stars are metallic-lined A stars that are most commonly found around A8, where at $(b-y)=0.14$ their fraction reaches its maximum value of $\sim 0.5$ (Smith 1973; cf. appendix to previous chapter).

The well known Am and Ap stars are not the only peculiar stars found at and around spectral type A. At early-F, one finds the $\rho$ Pup stars - these are evolved Am stars (e.g. Gray \& Garrison, 1989a). Throughout the A-star regime, one can find the metal-weak $\lambda$ Boo stars making up $\sim 2$ per cent of the population. At even earlier spectral types, a couple of dozen of the lesser-known 'sn' stars have been recorded. These three classifications of peculiarity are also reviewed in this chapter.

Thus the layout of this review is as follows: we begin with a discussion of what constitutes a 'normal' star in $\S 3.2$, and how our choice of the Sun as the star against which to normalise abundances may affect our results. $\S 3.3$ contains an extensive review of the Am stars, including the observed abundance anomalies, the current state of the models and the challenges posed by observations of pulsating Am stars. In that section, the hypothesis that the non- $\delta$ Sct stars are necessarily chemically peculiar is investigated. A short summary of the $\rho$ Pup stars, discussing their nature as the evolved Am stars, is presented in $\S 3.4$, and alternative theories for their nature are discussed. A concise review of the $\lambda$ Boo literature is given in $\S 3.5$, and a similar 


\section{CHAPTER 3}

review of the somewhat smaller literature on the 'sn' stars is offered in $\S 3.6$. Novel inferences from the review of chemical peculiarities are provided with a summary in $\S 3.7$.

\subsection{What is a normal A star?}

A normal A star is simply one that does not have any spectral peculiarity. However, their study is not so simple. Investigations into peculiar stars are often hampered by the lack of studies on normal stars (e.g. Hill \& Landstreet 1993; Varenne \& Monier 1999; Fossati et al. 2007; Gebran, Monier \& Richard 2008; Gebran et al. 2010). There is, of course, a very good reason. One is naturally more drawn to the peculiar, but it is the rapid rotational velocities of normal stars that makes them difficult to characterise. Spectral lines that would be used for abundance analyses in the slow rotators become blends as they are Doppler broadened in the moderate and fast rotators, and even a line-free continuum becomes hard to find.

The A stars display significant star-to-star variations in abundances (Hill \& Landstreet, 1993; Gebran et al., 2010; Fossati et al., 2008b), more so than for F-stars (Gebran, Monier \& Richard, 2008), that further hinders the work of the spectroscopist. In this section, we seek to outline what constitutes a 'normal' A star, what the spectrum of such an object looks like and how it changes across spectral type A.

A-type stars are demarcated from B stars where He lines are no longer visible, i.e. at A0, where the CaII K line also becomes a notable feature in the spectrum. The hydrogen Balmer lines are also particularly strong in the A stars, reaching a broad maximum at around A2 under the MK system.

As one progresses from an A1 to an F0 star, a few easily spotted changes in the spectrum occur, as we can see in Fig. 3.1: the hydrogen lines become weaker, in both depth and width; the Ca II K line rapidly increases in strength, overtaking the 


\section{CHAPTER 3}

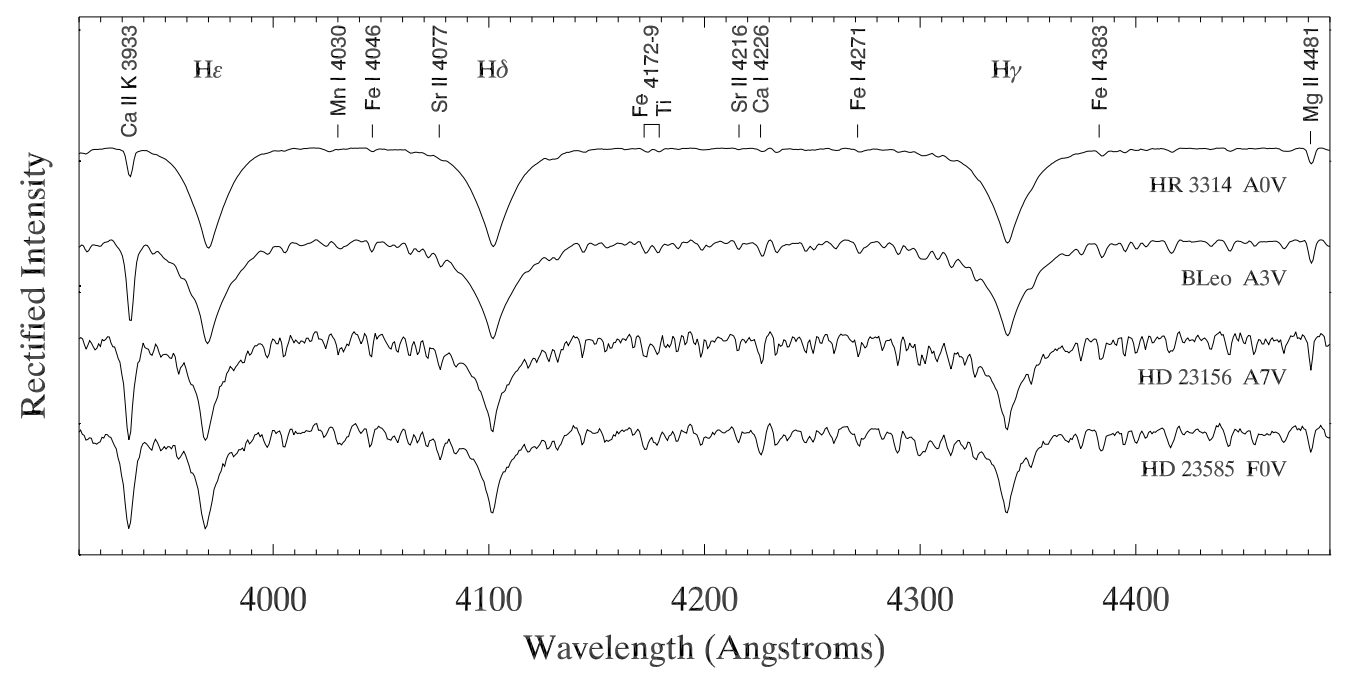

Figure 3.1: A spectral comparison for A stars of different temperature subclasses. Each star is a spectral standard, and was obtained from the online repository of 1.8-A standard star spectra taken at the Dark Sky Observatory: http://stellar. phys.appstate.edu/Standards/std1_8.html.

hydrogen lines in depth at late A; and the strength of neutral metal lines gradually increases. The latter is particularly evident in the Fe I lines. The singly ionised Mg II 4481 profile, however, is fairly insensitive to temperature. The apparent change in depth of this line is due to rotational velocity (see Fig. 3.2), making this line particularly useful in determining $v \sin i$. Other labelled lines of Fig. 3.1 will be discussed later.

All of the stars in Fig. 3.1 are of luminosity class V. A-type stars in the Kepler field are mostly classes V-III. From class V to class III, the hydrogen lines become narrower and the lines of ionised metals become stronger. One normally uses the hydrogen lines and the luminosity-sensitive, blended lines of Fe II and Ti II at $\lambda 4172-9$ in assigning a luminosity class. The strength of this blend can be compared to the Fe 4271 line, which is less sensitive to luminosity. At early-A, the hydrogen lines are particularly sensitive to luminosity, allowing distinction of upper and lower main-sequence stars (i.e. Va and $\mathrm{Vb}$ ) but at late-A the hydrogen lines have lost that 


\section{CHAPTER 3}

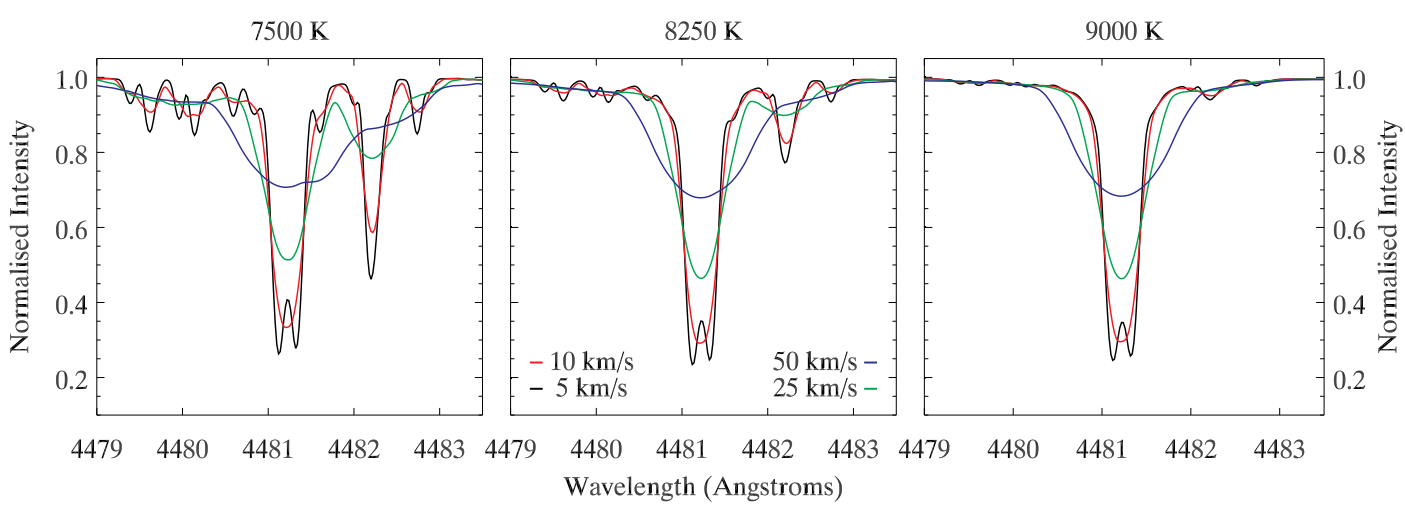

Figure 3.2: A comparison of the Mg II 4481 line in synthetic spectra of stars of different $T_{\text {eff }}$ (shown above each panel), where $\log g$ was fixed at 4.0. Lines of different colour show the usefulness of this line in determining $v \sin i$. Synthetic spectra were calculated and broadened using the SPECTRUM package (Gray, 1999), using atmosphere models computed by Castelli \& Kurucz (2004).

sensitivity. From luminosity class V to IV to III, the depth of the Fe/Ti II blend changes from being weaker than, to comparable to, to stronger than the Fe I 4271 line. The reader is referred to Gray \& Corbally (2009) for more information.

Before we begin discussing the abundance anomalies of chemically peculiar stars, we must look at the abundance pattern seen for the normal A stars against which we shall compare. Russell (1995) reported that for $\delta$ Sct stars, which have a tendency to be normal rather than chemically peculiar that we shall discuss later, carbon is underabundant by up to 1 dex with respect to the solar value. Gebran et al. (2010) investigated chemical abundances for 15 elements of $16 \mathrm{~A}$ and $28 \mathrm{~F}$ dwarfs in the Hyades cluster. Their data (available online ${ }^{1}$ ) for the normal A stars are presented in Fig. 3.3, from which it is clear that the normal A stars do not have solar abundances. Specifically, they are enriched in heavier elements. As Murphy et al. (2012) explained ${ }^{2}$, this can have multiple causes. One is that the study of Gebran et al. (2010) focussed on the Hyades cluster, and those cluster stars could

\footnotetext{
${ }^{1}$ http://vizier.cfa.harvard.edu/viz-bin/VizieR?-source=J/A+A/523/A71

${ }^{2}$ The discussion in this paragraph and the next uses text taken directly from Murphy et al. (2012). That material was written by this author, who also owns the copyright.
} 


\section{CHAPTER 3}

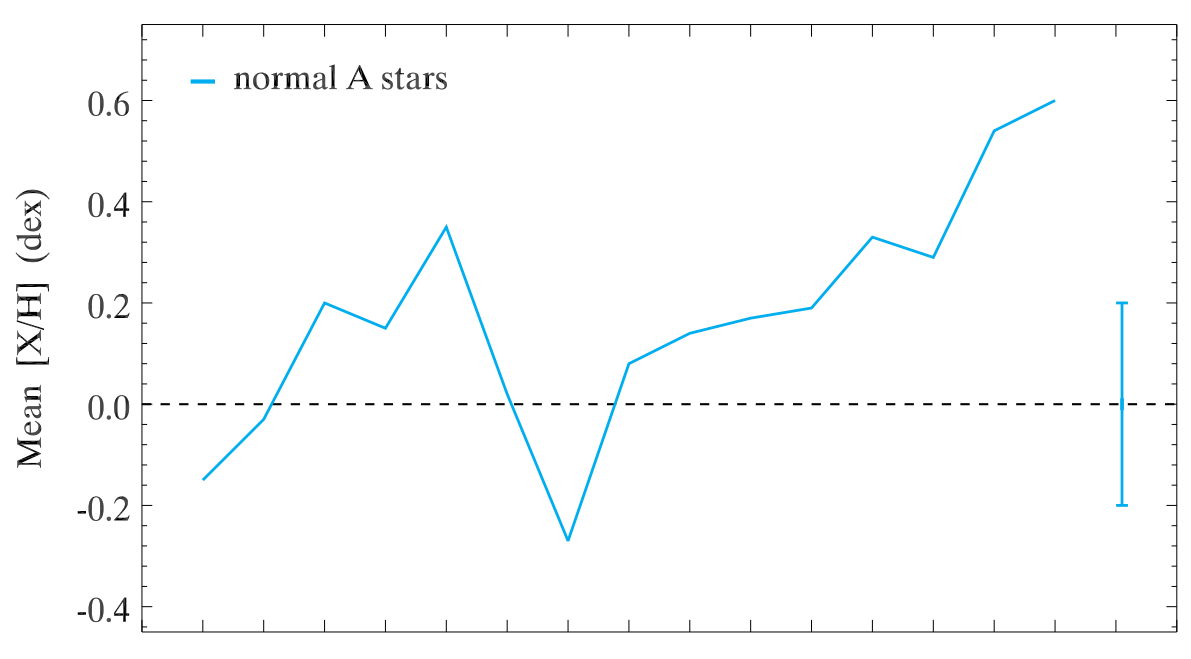

C $\mathrm{O}$ Na Mg Si Ca Sc Ti Cr Mn Fe Ni $\mathrm{Sr}$ Y $\mathrm{Zr}$

Figure 3.3: The mean elemental abundance of various elements for the normal A stars in the Hyades cluster, as determined from the data of Gebran et al. (2010). Heavier elements are significantly overabundant. The error bar represents a typical abundance uncertainty per star, but the uncertainty for the mean of the population is substantially smaller.

be systematically enriched in heavier elements. The abundances of nearly all heavier elements are known to be correlated with iron (Hill \& Landstreet, 1993). The $\left[\frac{F e}{H}\right]$ of the Hyades cluster is not well agreed upon. Boesgaard \& Friel (1990) determined a super-solar metallicity $\left\langle\left[\frac{F e}{H}\right]\right\rangle=0.127 \pm 0.022$ dex, yet Varenne \& Monier (1999) found a much lower value of $-0.05 \pm 0.03$ dex when investigating 29 F-dwarfs. Gebran et al. themselves find $\frac{F e}{H}=0.19$ dex above solar. A recent independent calculation (Carrera \& Pancino, 2011) based on three K-giants yields $\left\langle\left[\frac{F e}{H}\right]\right\rangle=+0.11 \pm 0.01$ dex and is close to the mean of previous studies collated by those authors. Thus Murphy et al. (2012) concluded that a slightly super-solar Fe abundance and the correlation of elemental abundances with Fe are contributors to the overabundances of metals in the normal Hyades A stars. However, they also identified another cause. 


\section{CHAPTER 3}

\subsubsection{The peculiar Sun}

Elemental abundances are most commonly quoted with respect to the Sun. Indeed, the standard notation $\left[\frac{F e}{H}\right]$ means $\log \left(\frac{F e}{H}\right)_{\star}-\log \left(\frac{F e}{H}\right)_{\odot}$. However, using the Sun as a standard star has the weakness of assuming the Sun is typical. Meléndez et al. (2009) found the Sun to be depleted in refractory elements by $\sim 20$ per cent relative to volatile elements compared to solar twins. Specifically, the lighter, volatile elements (e.g. C, $\mathrm{N}$ and $\mathrm{O}$ ) are enriched by $\sim 0.05$ dex and the heavier, refractory elements depleted by $\sim 0.03$ dex. Their findings were confirmed in another study (Ramírez, Meléndez \& Asplund, 2009), and the hypothesis that planetary formation is the cause is independently theoretically supported (Chambers, 2010) in that the solar depletions are correlated with an element's condensation temperature in the way one would expect if planet formation were the cause. However, that view has been challenged (González Hernández et al., 2010, 2012), because similar behaviour was observed for samples of solar twins with and without planets. Regardless of the origin, the peculiar solar abundance therefore also contributes to the apparent overabundance of heavy elements in normal A stars, and we may assume that a specific physical effect in A-star photospheres is not at play. Hence we must keep the peculiar Sun in mind when making abundance analyses of A stars; we will develop upon this idea in the next section.

It is worth a small detour to highlight the implications of a peculiar solar abundance pattern. Hill (1995) looked at compositional differences among the A stars, and later Fossati et al. (2008b) performed abundance analyses for seven $\delta$ Sct stars. Both studies found small but clear overabundances of most of the elements that are overabundant in Fig. 3.3. Similarly, Hill \& Landstreet (1993) found increasing overabundance with atomic number for Fe-peak elements and beyond in their investigation of narrow-lined A stars. The conclusions of all of these studies can be explained by the concept of the peculiar Sun. 


\section{CHAPTER 3}

\subsubsection{The effect of rotation on spectral type assignment}

Many of the spectral peculiarities found in A stars are determined by stellar rotational velocity. We shall look at the correlation between rotation and abundance anomalies in the next section. Before we do, let us look quickly at how rotation might affect spectral type assignment for normal stars. Central to this idea is the theory of gravity darkening. A rotating star is oblate, with a larger equatorial radius than its polar radius. Thus the poles have a higher effective gravity and correspondingly higher temperature. Since $L \propto T^{4}$, the poles are gravity brightened and the equator is gravity darkened. We established in the previous chapter that normal stars are generally the more rapid rotators, so we expect them to display this phenomenon. If one takes a normal, non-rotating star on the ZAMS, and subsequently spins it up, it will move off the (non-rotating) ZAMS line. If seen pole on, the star will appear brighter but of the same temperature. Conversely, if seen from the equator the star will move at constant luminosity to a cooler spectral type (Gray \& Corbally, 2009, specifically figure 5.4). Gray \& Garrison (1987, 1989a,b) estimated an A2 star could become an F0 star if one could observe such a spin up. Royer, Zorec \& Gómez (2007) put the difference between equator-on and pole-on observations at about $800 \mathrm{~K}$, or four spectral subclasses.

\subsection{Am stars}

The first recognition of Am stars was by Titus \& Morgan (1940), who identified "under-luminous" A stars with very strong metal lines in their spectra ${ }^{3}$. The anomalous weakness of the CaII K line was also emphasized. Since for A stars the Ca II $\mathrm{K}$ line grows in strength towards later spectral types, a weak $\mathrm{K}$ line is described as being 'too early' for the hydrogen line type. Similarly, the metal lines grow towards

\footnotetext{
${ }^{3}$ The anomalous luminosity of Am stars is discussed throughout this review, but cf. in particular the discussion in $\S 3.3 .6$.
} 


\section{CHAPTER 3}

later types, such that anomalously strong metal lines are 'too late' for the hydrogen line type. In order to have an objective identification criterion, classical Am stars are defined as having 5 or more spectral subclasses between the CaII K line type and the metal line type. Thus Am stars are usually given three spectral types, e.g. Am kA8hA9mF3 V, corresponding to the $\mathrm{K}$ line, hydrogen lines and metallic lines, respectively. The Am phenomenon also occurs in the $\mathrm{F}$ stars, and these are referred to as Fm stars in a similar way. While 'Fm' specifically refers to an F star, 'Am' is often used as a collective for the combined Am + Fm population.

The marginal Am stars (or Am: stars, pronounced "Am colon") show fewer than five spectral subclasses between the CaII K and metallic lines. Such stars are therefore less extreme examples of the classical Am stars, with milder abundance anomalies. Kurtz (1978b) showed the majority of the Am: stars lie at the blue edge of the $\delta$ Sct instability strip (his figure 2), which makes them difficult to distinguish from the 'hot Am stars' whose primary characteristics are Am anomalies in stars hotter than A4. Am anomalies are known in stars as early as A2 with $T_{\text {eff }}=10200 \mathrm{~K}$ $\left(\right.$ Smith, 1974) ${ }^{4}$. Marginal Am stars are also found at the cool edge of the instability strip, however, with spectra similar to the historical $\delta$ Del stars. We refer the reader to Gray \& Garrison (1989a) for a discussion of why the $\delta$ Del classification has been dropped and why the evolved Am stars are now known as $\rho$ Pup stars. ${ }^{5}$

In Chapter 2 we saw that the Am stars are slow rotators, as was first noted in a small sample by Slettebak (1954), and confirmed with more stars (Slettebak, 1955). Under the paradigm of slow rotation, mixing mechanisms are inefficient, allowing helium to gravitationally settle (Baglin et al., 1973), and radiation pressure to cause

\footnotetext{
${ }^{4} 10200 \mathrm{~K}$ is hot for an A2 star, being more like B9.5

${ }^{5}$ When describing marginal Am stars, this author prefers not to use the term 'proto-Am', which is preferred elsewhere (e.g. Gray \& Corbally, 2009). This is because of the potential confusion with the way 'proto' is used in relation to its greek root protos, meaning 'first', as in 'proto-star' for a forming star. Although it is true that a classical Am star must first pass through a phase of marginal abundance anomalies, and 'proto' has connotations of something not yet properly or fully formed, 'proto-Am' would appear to rule out a marginal Am star being in a state where abundance anomalies are disappearing.
} 


\section{CHAPTER 3}

overabundances of Fe-peak and heavier elements. Specifically, ions with absorption lines near the peak wavelength of the spectral energy distribution are radiatively levitated towards the surface, and those that are easily ionised into an argon-like configuration sink. For a given atmospheric turbulence, abundance anomalies in heavy elements are greater for more massive stars, since $\log g$ is lower and $g_{\text {rad }}$ is higher (Richer, Michaud \& Turcotte, 2000). The abundance pattern is the same, but with larger zero offsets.

\subsubsection{Abundance anomalies}

The abundance anomalies reported for Am stars are well illustrated with the aforementioned data of Gebran et al. (2010), plotted again in Fig. 3.4 for the Am stars against the abundances of the normal A stars for comparison. Those authors found $\mathrm{C}$ and $\mathrm{O}$ to be deficient and Fe-peak elements and beyond to be enriched. In accordance with long-standing characterisations (Conti, 1965; Smith, 1970), they found Sc to be particularly underabundant and Sr to be particularly overabundant. Also in agreement with Smith (1970), they found Ca to be normal in a large number of Am stars. This might seem strange, given the use of the Ca II K line in the classification of Am stars, and warrants a small diversion.

The CaII $\mathrm{K}$ line is an example of a resonance line, which is one that arises from the ground state and obeys both of the following quantum-mechanical selection rules: (i) $\Delta S=0$, which states that the total electronic spin should not change during a radiative transition; and (ii) $\Delta L= \pm 1$, meaning that the angular momentum quantum number should change by \pm 1 . Transitions violating the latter rule are forbidden lines, while those breaking the former are intercombination lines. Resonance lines do not only occur for neutral species - as we know from the Ca II $\mathrm{H}$ and $\mathrm{K}$ lines - nor are they always strong. It is important that the resonance line also arises from the dominant ionisation state in the line forming region. Resonance 


\section{CHAPTER 3}

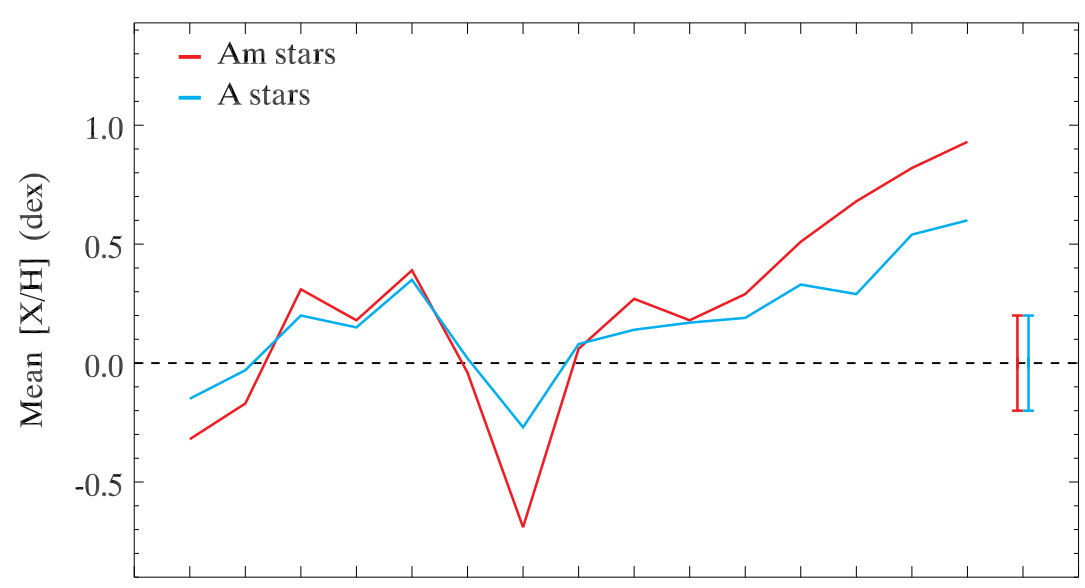

C O Na Mg Si Ca Sc Ti Cr Mn Fe Ni $\mathrm{Sr}$ Y $\mathrm{Zr}$

Figure 3.4: The mean elemental abundance of various elements for the normal A and Am stars in the Hyades cluster, as determined from the data of Gebran et al. (2010). Although heavier elements $(Z \geq 26 \equiv \mathrm{Fe})$ are overabundant in A stars with respect to the Sun (cf. $§ 3.2 .1$ ), they are made even more anomalous in Am stars though radiative levitation. Sc is dramatically underabundant, but Ca is only 0.1 dex below normal. Volatile elements $\mathrm{C}$ and $\mathrm{O}$ gravitationally settle, leaving sub-solar abundances at the photosphere. Error bars represent uncertainties on individual elements for individual stars, but lines drawn are for the ensemble.

lines are not necessarily in the optical, either. Many common ions have resonant lines in the UV. This is true of the Lyman series for hydrogen, where Ly $\alpha$ lies at $1216 \AA$. Since the Ca II K line is a resonant line and is the dominant ionisation state in A-star photospheres it is highly sensitive to the $\mathrm{Ca}$ abundance, which is why only slight depletions in Ca lead to large changes in line strength.

Returning to the discussion of abundance anomalies, it is sometimes convenient to quantitatively assess the strength of abundance anomalies, rather than as simply 'strong' or 'mild'. This has been attempted with line strength anomalies by defining $\alpha=$ Sc II 4246.8 / Sr II 4215.5 (Conti, 1965). That Sc is found deficient in most Am stars and Sr enhanced makes $\alpha$ a doubly sensitive parameter, leading to $\alpha \ll 1$ for the Am stars yet $\alpha \sim 1$ for normal stars. Smith (1970) suggested an alternative ratio, of Sc II 4320.7 / Y II 4309.6, because this ratio is insensitive to differences in $T_{\text {eff }}$ and $\log g$. In practice, however, these line ratios are difficult to apply, because spectra 


\section{CHAPTER 3}

of different signal-to-noise may show different anomaly strengths for the same star, and for stars with only mild anomalies the noise can overwhelm the result (such is the difficulty of using just one or two pairs of lines). Stateva, Iliev \& Budaj (2012) suggest using the more robust quantifier $[\mathrm{Ca} / \mathrm{Fe}](=[\mathrm{Ca} / \mathrm{H}]-[\mathrm{Fe} / \mathrm{H}])$ as a peculiarity indicator, because this ratio is also doubly sensitive to the Am phenomenon and they point out that $\mathrm{Ca}$ and Fe respond in the same way to changing temperature in that the lines of both elements get weaker with increasing temperature. By using multiple lines and spectral synthesis methods, the value of $[\mathrm{Ca} / \mathrm{Fe}]$, and thus the degree of peculiarity, can be much more reliably determined than with line ratios. However, one must consider the minutia of this idea: The response of line strength and abundance to temperature are two rather different things, as level populations change at different rates for different elements - the strength of a line might change because the ionisation balance is shifting, not because there is a different amount of iron in the line forming region for a star of different temperature. The difference in ionization and excitation potentials also matters - lines chosen for the $[\mathrm{Ca} / \mathrm{Fe}] \mathrm{de}-$ termination should have as small a difference in those potentials as possible (Smith, 1970). As it happens, Fossati et al. (2008b) found there is little evidence for abundance (not line strength) changes with respect to $T_{\text {eff }}$ for any element except $\mathrm{Y}$, and Richer, Michaud \& Turcotte (2000) found overabundant elements to show no $T_{\text {eff }}$ dependence. So provided appropriate lines are chosen, the use of a $[\mathrm{Ca} / \mathrm{Fe}]$ discriminant should be fine. However, another factor remains: many Am stars are actually approximately normal in terms of Ca abundance. This might not be problematic, in that some alpha elements $(\mathrm{Mg}, \mathrm{Si}, \mathrm{Ca})$ have been shown to correlate in abundance with Fe in normal A stars (Gebran et al., 2010), so that quantification of peculiarities should not be hampered by stars of differing global metallicity. In conclusion, there is certainly some use in quantifying peculiarities and the potential to do it exists, but one must think extremely carefully about how to go about it. 


\section{CHAPTER 3}

\section{Dependence on $v \sin i$}

Much work has gone into assessing the correlation between abundances and $v \sin i$, yet there is no consensus. Takeda et al. (2008) found that peculiarities decrease with increasing $v \sin i$, until about $100 \mathrm{~km} \mathrm{~s}^{-1}$ where they disappear. Fossati et al. (2008b) agreed, with the exception of Sc which they found to be anti-correlated with $v \sin i$ (Fossati et al., 2008a). Conversely, Gebran et al. (2010) found no particular evidence for correlation or anti-correlation between $\mathrm{C}, \mathrm{O}, \mathrm{Mg}$, Sc, Fe or $\mathrm{Y}$ with $v \sin i$. In reality, it may be that the star-to-star abundance variations are too high to reveal any statistically significant underlying correlation, or that rotation is not the only controlling factor; perhaps some other mechanism or parameter (such as age) has an effect on abundances.

\section{Dependence on Age}

In the models of Richer, Michaud \& Turcotte (2000), abundances appear to depend only on $Z$ at young $(\sim 5 \mathrm{Myr})$ ages. At $70 \mathrm{Myr}$ there is an offset between $Z=0.02$ and $Z=0.03$ models, but abundance patterns are similar. By $670 \mathrm{Myr}$ there is little difference at all in heavy elements, and only slight differences for the underabundant elements. Thus after a brief transition phase, it appears that Am abundances depend little on main-sequence age or $Z$. A similar conclusion was reached by Smith (1972), who discovered many Am stars in the young Orion 1c association, of age 2-6 Myr (Bally, 2008). Old Am stars have also been observed, such as those in the Praesepe cluster, of age $\sim 600 \mathrm{Myr}$ (Kraus \& Hillenbrand, 2007), so the findings of Richer, Michaud \& Turcotte can be tested with observations.

\section{Influence of binarity}

We saw in Chapter 2 that tidal deceleration in a binary system is the favoured mechanism for braking an A star's rotational velocity sufficiently for it to become 


\section{CHAPTER 3}

an Am star. We discussed that the mechanism is not completely satisfactory, and evidence of Am stars in clusters as young as a few Myr, i.e. in clusters too young for a tidal braking mechanism to have had sufficient impact, argues against binarity being the sole braking mechanism, implying not all Am stars are necessarily binaries. Other evidence exists for single Am stars. The pulsations of Am stars could reveal their binary nature: a pulsator influenced by orbital motion in a binary pair should exhibit signs of frequency modulation in its Fourier transform (Shibahashi \& Kurtz, 2012). However, Murphy et al. (2012) found no evidence of frequency modulation in long Kepler data sets for the pulsations of the marginal Am star KIC 3429637, which is further evidence of the existence of single Am stars.

For those Am stars known to be binaries, does the abundance pattern change as a function of binary parameters such as the orbital period or eccentricity? Using their [Ca/Fe] indicator, Stateva, Iliev \& Budaj (2012) found that it does. Specifically, Am peculiarities were seen to increase with increasing orbital eccentricity, but essentially no trend was seen with orbital period. The former result confirms the earlier findings of some of the same authors (Iliev et al., 1998), but on close inspection the correlation is not robust against small number statistics, and certainly not in light of the large star-to-star variations in abundance that were reported earlier in this section.

\subsubsection{Modelling Am stars}

Until recently, models of Am stars had not qualitatively reproduced observed abundance anomalies, and consistently predicted abundances that were too peculiar. Great progress was made with the introduction of models with turbulence in what are dubbed the New Montreal Models (Richer, Michaud \& Turcotte, 2000; Turcotte et al., 2000), whereas the previous models that focussed more on meridional circulation are described here as "pre-2000 models". 


\section{CHAPTER 3}

Several mechanisms determine the chemical separation in the Am stars. Diffusion (microscopic, thermal, and differential radiative acceleration) and gravitational settling act to separate elements whereas the mixing processes (mostly meridional circulation and turbulence) act against that separation. In the pre-2000 models, Michaud et al. (1983) determined that meridional circulation was the primary mechanism competing against helium settling, but those authors required mass loss to make their model work. However, the requirement for mass loss was not the only problem. The extent to which circulation operated restricted the allowable contribution of turbulence to an insignificant level (in order to agree with observations), yet Zahn (1992) argued that in non-magnetically braked stars, which includes the Am stars, the star rapidly reaches a circulation-free state. Furthermore, Charbonneau (1993) determined that meridional circulation does not have a significant effect on the diffusion of metals once the He II ionisation zone has disappeared. It was clear that models incorporating turbulence as an alternative mixing process needed to be developed.

The introduction of turbulence into Am star models by Richer, Michaud \& Turcotte (2000) brought the models qualitatively in-line with observations. Although the peculiarities remained overestimated by a factor of 3 , the abundance pattern matched the observations. They found that once the enhancement of Fe-peak elements reaches a factor of 4, the Fe-peak opacity bump is sufficient that an Fe-peak element convection zone develops at around $200000 \mathrm{~K}$, which is linked to the surface (H I and HeI) convection zone by overshoot. A schematic description of the near-surface region of an Am star under the pre-2000 and New Montreal Models is presented in Fig. 3.5.

Théado et al. (2009) called for smaller enhancements of iron and other overabundant elements than in the Montreal models, because the latter models do not 


\section{CHAPTER 3}

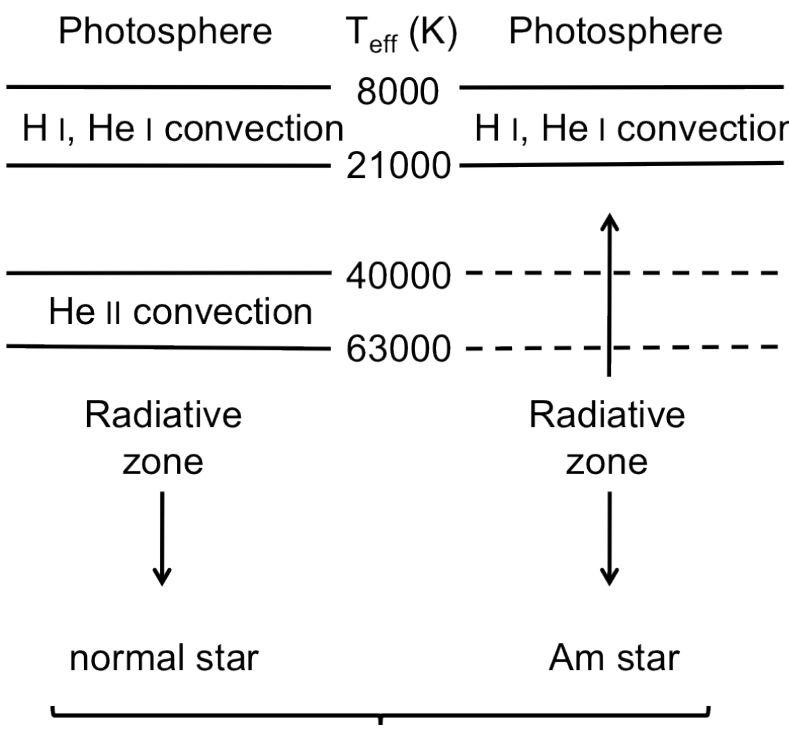

Pre-2000 models

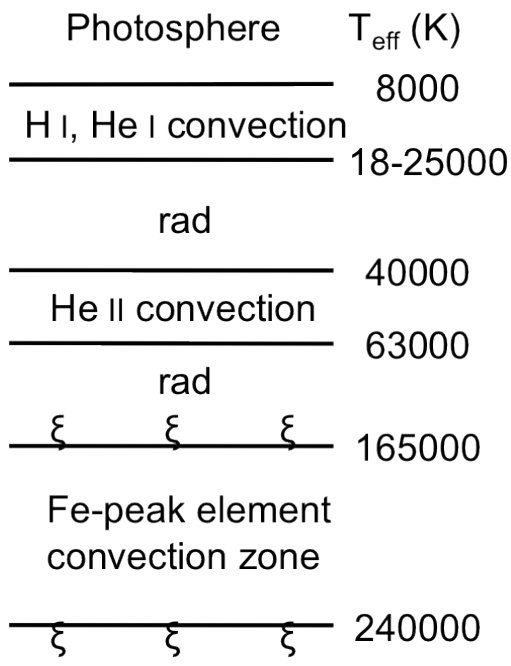

New Montreal Model

Figure 3.5: Left: the near-surface region of a normal A star, showing a surface convection zone of $\mathrm{HI}$ and HeI, and a subsurface convection zone of He II with a radiative zone beneath (based on Smith 1971, his figure 11). Centre: in pre-2000 models, the gravitational settling of He leads to the radiative zone extending right up to the surface convection zone (based on Gray \& Corbally 2009, their figure 5.13). Right: the New Montreal Models (Richer, Michaud \& Turcotte, 2000; Turcotte et al., 2000) feature an Fe-peak element convection zone that appears once the He II convection zone has subsided and radiative levitation has enhanced Fe-peak element abundances sufficiently. This Fe-peak element convection zone is linked to the surface convection zone through overshoot, and qualitatively describes observed abundance anomalies in Am stars. Temperatures are inferred from Richer, Michaud \& Turcotte (2000, their figure 9$)$, correspond to a star of $2 \mathrm{M}_{\odot}$, and depend on age. 


\section{CHAPTER 3}

properly treat the inverse $\mu$ gradient created by radiative acceleration of heavy elements. The effect of levitating iron-peak elements above less dense material invokes thermohaline convection, an additional source of mixing (Vauclair, 2008). Models with thermohaline convection see less dramatic increases in iron abundance (factors of $\sim 10$ rather than 150), and correspondingly smaller increases in opacity. As such, the iron abundance is always close to the critical value required for convection (Théado et al., 2009). Once the critical value is exceeded, convection mixes the material again until the iron enhancement is low enough to stop the convective driving. In $1.5-\mathrm{M}_{\odot}$ models, Théado et al. found the Fe opacity bump alternates between radiative and convective states for the duration of the main sequence. However, after $400 \mathrm{Myr}$ the 1.7 - and $1.9-\mathrm{M}_{\odot}$ models have continuously convective Fe opacity bumps up to the TAMS. It is noteworthy that the reduction in iron enhancement acts to bring the models more in line with observations.

The Montreal models demand many CPU hours, making them impractical for use as asteroseismic models that must be computed in large numbers to reach a fine-tuned match to observations. Théado et al. (2012) described Toulouse-Geneva Evolution Code (TGEC) models, which trade accuracy for savings in computation time, and demonstrated a good match to the Montreal models.

In summary, the theory of Am star near-surface regions, inferred from models that best match the observations, can be described thusly: the slow-rotation paradigm rapidly leads to a scenario in which meridional circulation is inefficient in mixing the star, becoming altogether insignificant once enough helium has drained to suppress the convection of the He II partial ionisation zone. The dominant mixing mechanism is then turbulence, which by itself does not prevent the radiative levitation of heavy elements. The accumulation of these elements leads to an opacity bump, which, depending on the mass and age of the star, introduces an (intermittent) Fe-peak element convection zone near $200000 \mathrm{~K}$. This zone only appears after 


\section{CHAPTER 3}

the disappearance of the He II convection zone, and limits the rate of further draining of helium, while enhancing the abundances of Fe-peak elements in the line-forming region through convective overshoot. Even though helium is drained (reaching a minimum abundance of 0.114 at $750 \mathrm{Myr}$ in a $1.9-\mathrm{M}_{\odot}$ Montreal model that is stable at that time), pulsations are not excluded just because the He II convection zone has disappeared. Far from it - we will examine the evidence for pulsating Am stars shortly. With evolution, the superficial helium abundance increases in the Am stars again. Meanwhile, the He II ionisation zone shifts deeper into the star (in mass fraction) towards regions of higher helium abundance. Thus models predict that stability against pulsation in Am stars subsides naturally with evolution, i.e., without the need for dredge-up of material or mass loss.

\subsubsection{Pulsating Am stars}

For a long time, observations indicated that Am stars do not pulsate. Breger (1970) originally pointed this out, when none of the 11 Am stars belonging to the Praesepe and Hyades clusters that he observed were pulsating. In a larger sample of $19 \mathrm{Am}$ stars inside and 7 outside the $\delta$ Sct instability strip, still no pulsations were detected. However, in a 93-star sample of non-peculiar stars, 30 per cent were $\delta$ Sct stars (at the $0.01 \mathrm{mag}$ limit). The observational exclusion of pulsation in classical Am stars remained, despite several counterexamples that were all dismissed by Kurtz et al. (1976), until 1989 when the classical Am star HD 1097 was shown to be a $\delta$ Sct star (Kurtz, 1989).

Marginal Am stars and the evolved Am ( $\rho$ Pup) stars, on the other hand, are frequently observed to pulsate as is predicted in the models. Kurtz (1978b) came to this conclusion with the presentation of some pulsating Am: stars, and led a particularly detailed case study of HD 40765 - a $\rho$ Pup star that is also a high-amplitude 


\section{CHAPTER 3}

$\delta$ Sct (HADS) star (Kurtz et al., 1995). There it was found that spectral peculiarities still remain in the presence of a peak-to-peak surface radial velocity range of $14 \mathrm{~km} \mathrm{~s}^{-1}$. This might appear to conflict with the stability required for diffusion to operate. Diffusion velocities are generally much less than $\mathrm{cm} \mathrm{s}^{-1}$, thus it would appear that pulsation in chemically peculiar stars can be so laminar that turbulence on the order of $\mathrm{cm} \mathrm{s}^{-1}$ is not generated. This was considered problematic in Amstar models until Turcotte et al. (2000) identified that it is velocity gradients that are important in turbulence generation, not the surface radial velocities themselves. This hypothesis indicates that pulsating Am stars should possess low-overtone and low-degree modes only. High-overtone and high-degree modes have many surface or near-surface nodes, which implies velocity gradients in the envelope that could generate turbulence and mix away the abundance anomalies.

A large scale survey of pulsations in Am stars was recently carried out with SuperWASP (Smalley et al., 2011), and has created much discussion in the field. Those authors found 197 of 1620 Am stars (12 per cent) to pulsate as $\delta$ Sct stars or $\delta$ Sct $+\gamma$ Dor hybrids at amplitudes above 1 mmag. Using Kepler data, Catanzaro \& Balona (2012) investigated the number of stars pulsating in various temperature bands within the $\delta$ Sct instability strip. They used a slightly higher amplitude limit of $1.5 \mathrm{mmag}$ and compared the number of Am stars that pulsate as a fraction of the total number of Am stars, as inferred from Smalley et al. (2011), to the number of $\delta$ Sct stars as a fraction of all stars in the $\delta$ Sct instability strip. They determined the fractions to be approximately equal. Thus Catanzaro \& Balona paint a picture of "the helium problem in the A-type stars" - the pulsations indicate there is no appreciable settling of helium.

To what extent is this the case? By calculating Fourier transforms of the same data over the frequency range $5-24 \mathrm{~d}^{-1}$ and sorting by the highest amplitude peak in this region, I have recomputed the number of stars pulsating above the $1.5 \mathrm{mmag}$ 


\section{CHAPTER 3}

limit imposed by Catanzaro \& Balona (2012). As discussed in Chapter 1, the method is robust against common instrumental artefacts operating at low frequency in the Kepler data, and focusses on the frequency region of maximum power for most $\delta$ Sct stars. Some $\delta$ Sct stars have their maximum power above the Kepler LC Nyquist limit, but aliases of these peaks will occur in the investigated frequency region provided the pulsations are not in the range $43-53 \mathrm{~d}^{-1}$. Kepler SC data indicate very few $\delta$ Sct stars have their maximum power in this frequency region, so the method is sound, even though a frequency-dependent amplitude reduction is seen in the LC data when the Nyquist frequency is exceeded (cf. Chapter 1, §1.2.2 / Fig. 1.5). My calculations are in satisfactory agreement with Catanzaro \& Balona: in the temperature range with the highest fraction of $\delta$ Sct stars, $7500-8000 \mathrm{~K}$, they found $\delta$ Sct stars make up 21 per cent of the population compared to my 24 per cent (at the $1.5 \mathrm{mmag}$ level). However, the SuperWASP amplitude detection limit quoted by Smalley et al. (2011) is $1 \mathrm{mmag}$, not $1.5 \mathrm{mmag}$, which makes a sizeable difference to the numbers, increasing the fraction of pulsators to almost 1 in 3 in my calculations.

The difference between the number of pulsators at the 1.5- and 1.0-mmag level does not invalidate or solve the "helium problem". Is there another explanation? We have learned that pulsation is not only commonplace, but is theoretically expected in the marginal and evolved Am stars. Does the sample of Am stars investigated with SuperWASP data include these? The catalogue Smalley et al. (2011) used is the Renson \& Manfroid (2009) catalogue of Ap, HgMn and Am stars. In this catalogue, compiled from many literature sources, $\delta$ Del stars are recorded. Since Gray \& Garrison (1989a) ruled the class obsolete due to lack of homogeneity, what do we make of these entries? First, let it be said that there are few of them. Some of them are probably evolved Am stars, but many evolved Am stars are probably not indicated in the catalogue, because Am: stars are not indicated. The certainty with which classifications are provided by Renson \& Manfroid (2009) is denoted with a 


\section{CHAPTER 3}

flag: an asterisk in the catalogue indicates a certain classification, and a question mark denotes an uncertain one. Smalley et al. (2011) do not specify whether they include the questionable identifications, which one expects comprise many of the marginal Am stars in which pulsations are expected, and which make up about one third of all of the Am stars in the Renson \& Manfroid catalogue. As it happens, 72 of 227 stars in the SuperWASP sample were of the uncertain identification (Smalley, priv. comm.), though this does not mean that they are not Am stars. Low signalto-noise might also lead a classifier to indicate an uncertain classification. However, it seems likely that on the average, the 72 uncertain Am stars make up some of the Am: stars that we should see in the sample, but not necessarily all of them. Whether this really affects the claimed number of 'pulsating Am stars' is unclear. Smalley et al. do acknowledge, however, that 24 per cent of their pulsating Am stars are actually Fm $\delta$ Del stars. These should not be counted towards the statistics because they are expected to pulsate. In light of this discussion, the number of classical Am stars seen to be pulsating with SuperWASP data has dwindled and the helium problem is not as severe as it may seem. Having said that, the existence of any pulsating classical Am stars defines the problem.

Another study of pulsation in non-magnetic chemically peculiar stars has recently been undertaken. Paunzen et al. (2013) analysed photometric data from the STEREO spacecraft for 558 non-magnetic chemically peculiar stars, 44 of which were recorded as variables in the Smalley et al. (2011) sample. Of those 44 'variables', Paunzen et al. (2013) could detect variability in only one star, while 8 were clearly constant and the remainder could not be reliably classified. Although this could be interpreted as an overinterpretation of variability in the SuperWASP sample, 38 per cent of the classifiable Paunzen et al. (2013) sample were pulsators. Thus one expects more than 1 in 9 of the classifiable 44 'variable' stars to pulsate, but no real weight can be attached to these small-number statistics. Furthermore, that no 


\section{CHAPTER 3}

reliable classification as either constant or pulsating could be made for 339 of 558 stars analysed calls for caution in interpreting the Paunzen et al. (2013) result - could it be that, instead of being 'without a reliable detection', these light curves could just have a higher amplitude detection limit? If so, the fraction of constant stars among those 'unclassifiable' light curves would be higher. Paunzen et al. state that the unclassifiable stars are likely non-variable, but, unfortunately, do not comment on the amplitude detection limit in their data. I analysed a pseudo-random sample of 67 of the 'unclassifiable' stars ${ }^{6}$, which showed that most of the Fourier transforms have grass levels of 1-2 mmag, so 3-4 mmag pulsations would be detectable. Four stars appeared to be $\delta$ Sct stars, with three more as candidates (of borderline significance), but instrumental artefacts prevent certain classifications. The other 60 (90 per cent) are non-pulsators at this amplitude detection limit. Thus, through my analysis and discussion with one of the authors (K.T. Wraight), the fraction of constant non-magnetic CP stars (which does include HgMn stars) is 85-90 per cent at about the 4 mmag level in STEREO data. This is in contrast to the SuperWASP result, when one considers the different amplitude (detection) limits.

A summary of the above findings is presented in Table 3.1.

Table 3.1: Comparison of the number of $\delta$ Sct pulsators found with different instruments at various amplitude limits among populations of A stars.

\begin{tabular}{lllcc}
\hline Source & Instrument & Type of Stars & $\begin{array}{c}\delta \text { Sct pulsators } \\
\%\end{array}$ & $\begin{array}{c}\text { Amp. limit } \\
\text { mmag }\end{array}$ \\
\hline Smalley+2011 & SWASP & "Am" & 12 & $\sim 1$ \\
Catanzaro+2012 & Kepler & $7500-8000 \mathrm{~K}$ & 21 & 1.5 \\
This work & Kepler & $7500-8000 \mathrm{~K}$ & 24 & 1.5 \\
This work & Kepler & $7500-8000 \mathrm{~K}$ & 31 & 1.0 \\
This work & STEREO & non-magnetic CP & $10-15$ & $\sim 4$ \\
\hline
\end{tabular}

\footnotetext{
${ }^{6}$ thanks to K.T. Wraight for providing the STEREO data
} 


\section{CHAPTER 3}

\subsubsection{Non- $\delta$ Sct stars as Am star candidates}

One can invert the usual research question. Instead of asking "Do the Am stars pulsate?" one can ask "Are the non- $\delta$ Sct stars in the $\delta$ Sct instability strip chemically peculiar?" In order to answer this question, a sample of Kepler stars without p-mode pulsations was observed with the HERMES high-resolution instrument on the Mercator telescope. These observations were supplemented with other non- $\delta$ Sct stars observed with the same instrument for other projects, but that had KIC temperatures in or near the $\delta$ Sct instability strip. The investigation and observations are ongoing, and preliminary results are reported here.

Inspection for pulsation was carried out with Kepler LC Q5 data. Using LC data to look for $\delta$ Sct pulsation does not limit the analysis in any sense related to the low Nyquist frequency of the LC data, since pulsation frequencies higher than the Nyquist frequency have detectable aliases. To be classed as 'non- $\delta$ Sct', a star must not have statistically significant frequencies between 5 and $24 \mathrm{~d}^{-1}$, but variability with frequencies below $5 \mathrm{~d}^{-1}$ was deemed acceptable. Ultimately, there is no theoretical exclusion between the Am phenomenon and the $\gamma$ Dor phenomenon, so it is irrelevant whether the star pulsates as a $\gamma$ Dor star as long as it does not pulsate as a $\delta$ Sct star. We remind the reader that by accepting pulsations below $5 \mathrm{~d}^{-1}$, we are also accepting pulsations between 43 and $53 \mathrm{~d}^{-1}$, but we know of no single $\delta$ Sct star pulsating at exclusively these frequencies in the Kepler data, and thus the probability of accidental inclusion of such a star in our small sample is negligible.

Spectral classification provides an excellent 'first look' at the nature of a star before detailed (and more time-consuming) atmospheric parameters and abundances are derived. As part of this project, I have classified 80 stars on the MK system, a subsample of which was spot-checked by an expert classifier (R.O. Gray). The agreement in our classifications is good, but I would benefit from a larger set of 


\section{CHAPTER 3}

MK standards dispersed at shorter intervals. Myself, Gray and collaborators will be observing these in 2013-2014. Classifications provided here have been iterated with Gray, and provide the required information on peculiarity. The wider set of 80 classifications of the HERMES sample will be published along with detailed atmospheric parameters and abundances in a later paper (Niemczura et al., in prep.).

We must ascertain whether each non- $\delta$ Sct star lies in (or on the boundary of) the $\delta$ Sct instability strip. If the star lies much beyond either edge of the strip, then we reach the trivial solution that the star does not pulsate because it is not in the pulsational instability strip. Location within the strip can be determined with the MK type: a luminosity class $\mathrm{V}$ object between A5 and F1 lies within conservative boundaries of the instability strip. With detailed atmospheric parameters, membership can be determined as a function of $T_{\text {eff }}$ and $\log g$. The distribution of non- $\delta$ Sct stars with HERMES data on a $T_{\text {eff }}-\log g$ diagram is shown in Fig. 3.6, where one can see the trivial solution applies to many of the stars.

The derived MK spectral types for the non- $\delta$ Sct stars tend to have temperature sub-classes much earlier than the corresponding KIC temperatures suggest they should have, especially for hotter stars. This systematic offset has been reported in the literature (Pinsonneault et al., 2012). Until spectroscopic temperatures are derived for all candidates, we have a subset of stars in the middle of the instability strip, according to KIC temperatures and supported by MK type, that we expect will still lie within the strip when more accurate temperatures are derived from spectroscopy. Ten such stars are analysed here, and are the stars represented by cyan squares in Fig. 3.6. Their Fourier transforms and spectral types are presented in Fig. 3.7.

The sample of ten stars has six chemically peculiar stars, and four normal stars. The least variable stars to appear to be chemically peculiar, where 'variable' refers to both $\gamma$ Dor and $\delta$ Sct pulsation, but excludes very low frequency variation at the 


\section{CHAPTER 3}

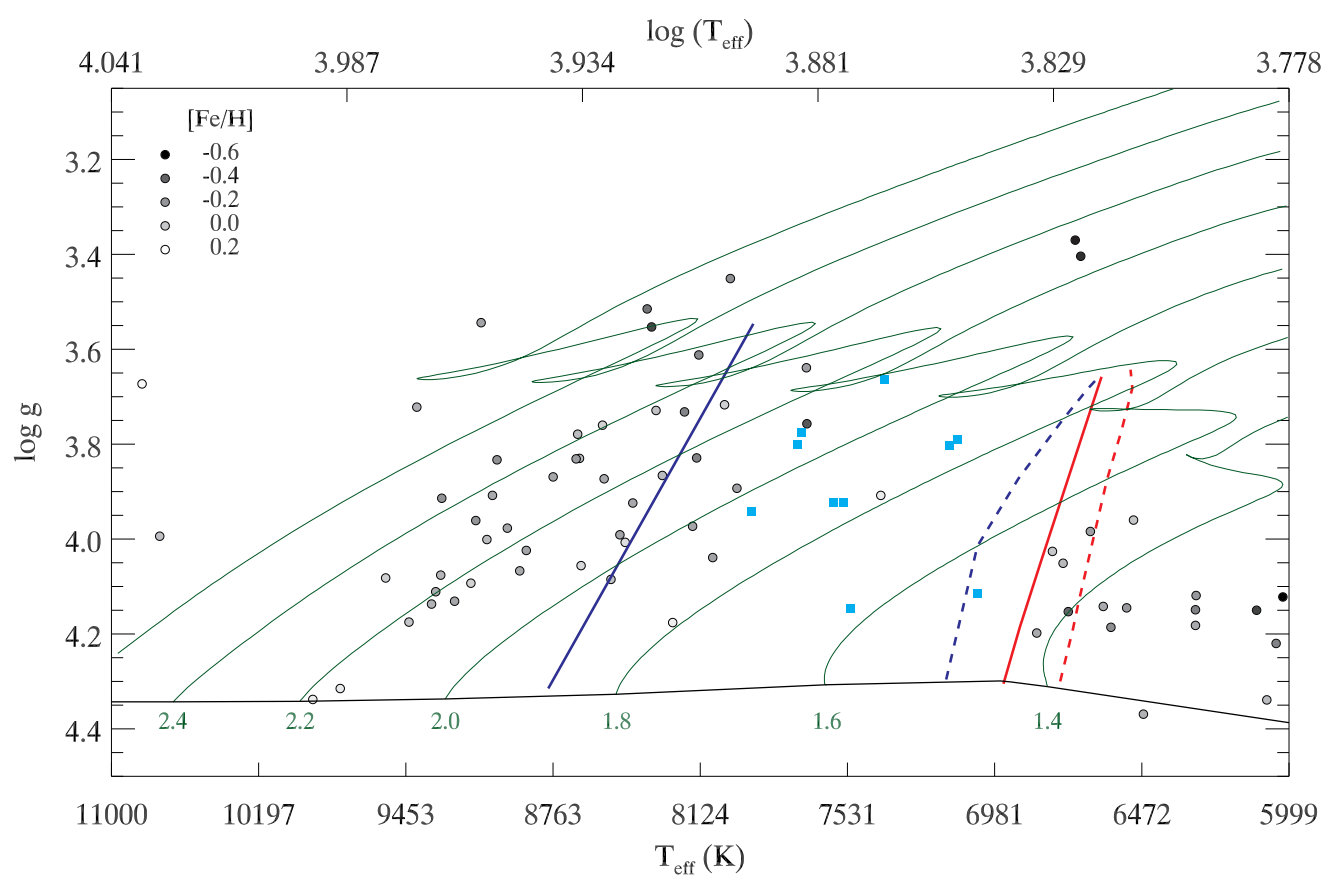

Figure 3.6: $T_{\text {eff }}-\log g$ diagram using KIC parameters for non- $\delta$ Sct stars (circles and squares) observed by HERMES. The $T_{\text {eff }}$ scale is logarithmic. Evolutionary tracks (green) starting at the ZAMS (black) are drawn, with their masses (in $\mathrm{M}_{\odot}$ ) indicated below the ZAMS. The solid blue and red lines mark the edges of the $\delta$ Sct instability strip, and the $\gamma$ Dor instability strip is delimited with dashed lines. Instability strips are calculated with TDC models. Cyan squares identify stars analysed in closer detail in the present analysis; the fill of the grayscale circles corresponds to the (unreliable) KIC metallicity of the remainder of the sample. Most of the non- $\delta$ Sct stars in our sample lie outside the $\delta$ Sct instability strip. 


\section{CHAPTER 3}

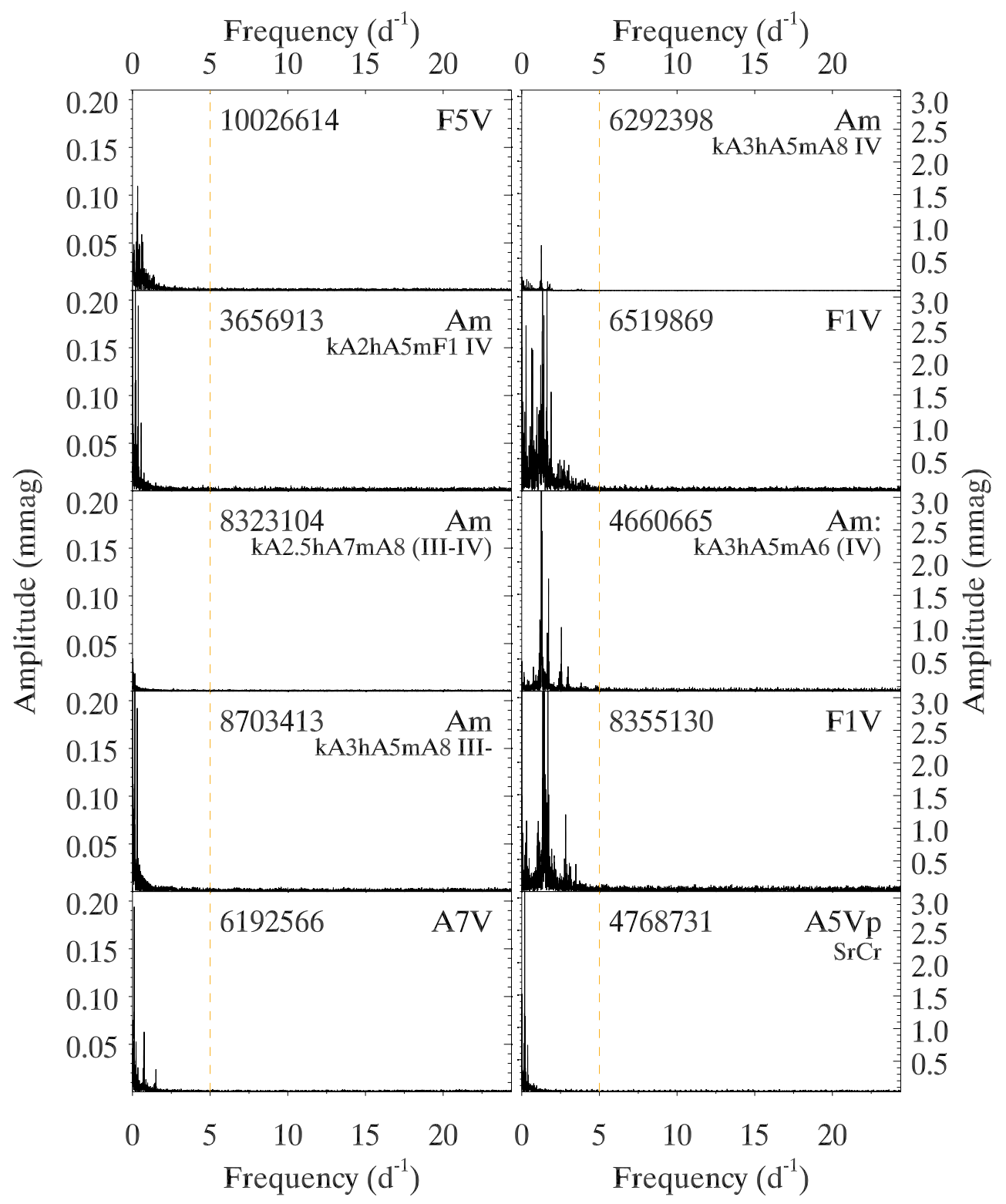

Figure 3.7: Fourier transforms of ten non- $\delta$ Sct stars in the $\delta$ Sct instability strip. Dashed orange lines indicate the $5 \mathrm{~d}^{-1}$ cut-off. Notice the change in y-axis scale between left and right panels. KIC numbers are labelled in the upper-left of each panel, and spectral types in the upper right. Am stars are classified in the usual way, with brackets around the luminosity type if the star exhibits the anomalous luminosity effect (see $\S 3.4$ ). Some non- $\delta$ Sct stars are chemically normal. 


\section{CHAPTER 3}

far left of some Fourier transforms that is probably due to binarity. However, the $\gamma$ Dor stars with high amplitudes ( $~ 3$ mmag, middle-right in Fig. 3.7) do not have strong peculiarities. There is no known exclusion between $\gamma$ Dor pulsation and Am peculiarity, as the convective flux blocking mechanism that drives $\gamma$ Dor pulsations is not directly affected by gravitational settling of helium taking place in Am stars, so it is intriguing that the high amplitude $\gamma$ Dor stars are chemically normal. Perhaps there is a link between the pulsation mechanism or convection efficiency required for $\gamma$ Dor modes with mmag amplitudes, and the mixing of the line-forming region caused by such a convective zone, that hinders the segregation of elements. Little can be said from these small number statistics; investigation of the larger sample is required.

The three $\gamma$ Dor stars aside, there are two remaining non-peculiar stars from the ten-star sample: KIC 10026614 and KIC 6192566. The former has a spectral type of F5V, and is too cool to exhibit $\delta$ Sct pulsation. KIC 6192566, on the other hand, is an interesting case. At A7V, this star lies well inside the $\delta$ Sct instability strip. Its Johnson $(B-V)$ value of 0.21 , calculated from Tycho data, agrees with the spectral type of A7 for a luminosity class V object. So why does this star not pulsate, given that it is a normal star? It turns out that this is an SB2 system (components yet to be determined), which complicates the spectroscopic analysis. The SB2 nature is expected to lead to Am peculiarity, but also hinders the demonstration of that peculiarity through an abundance analysis. Whether normal, non-pulsators can exist in the $\delta$ Sct instability strip is still uncertain. This is an open question that will be developed with the larger sample.

The sample also includes stars with strong peculiarities, three of which are illustrated alongside MK standards in Fig. 3.8. The temperature subclass of the hydrogen line type of these three stars is A5, but the Am stars have Ca II K line types that are a few temperature subclasses earlier than A5, and metal line types that 


\section{CHAPTER 3}

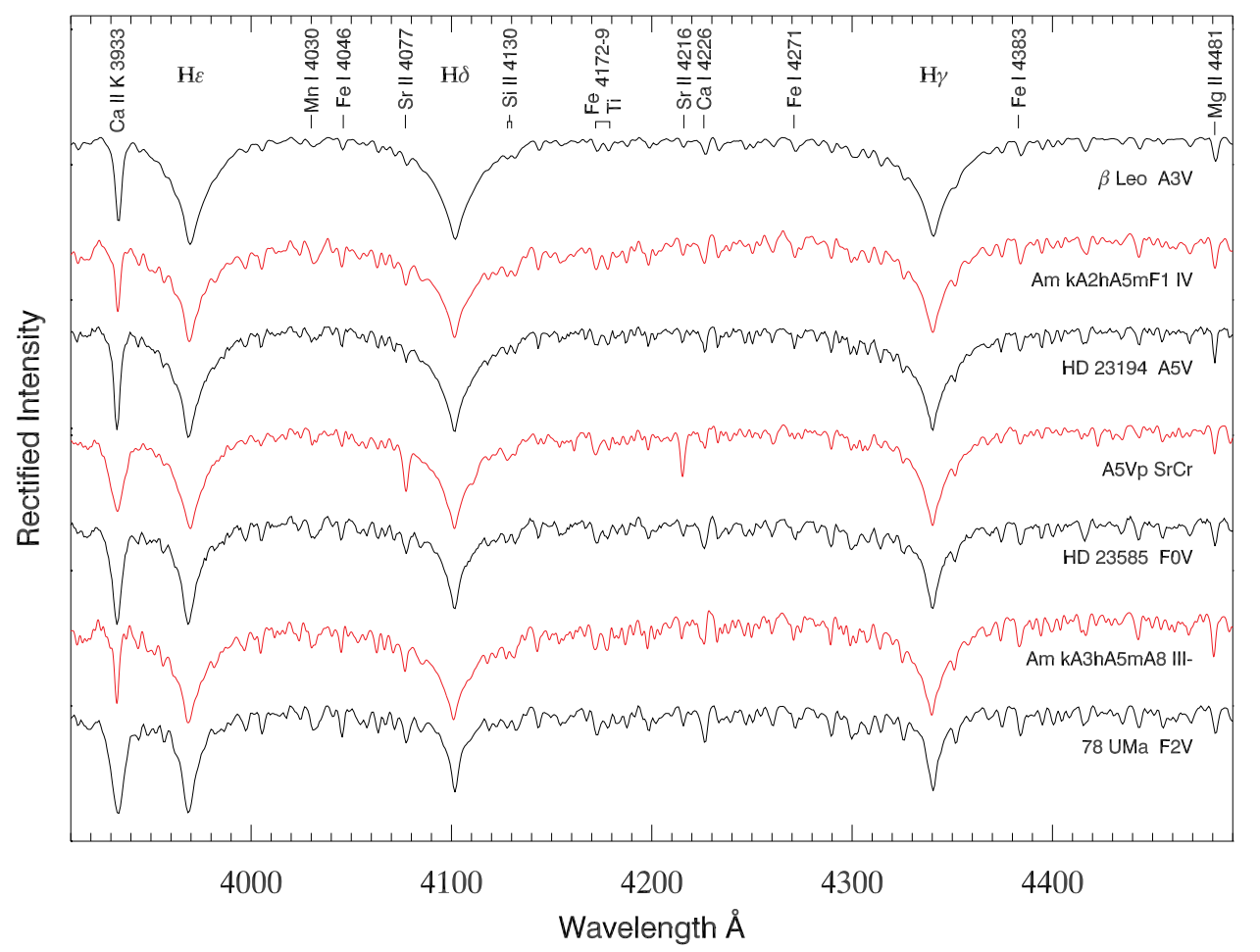

Figure 3.8: Spectral comparison for three peculiar stars from the sample (red; top to bottom) KIC 3656913, KIC 4768731 and KIC 8703413, against MK standards (black). Notice the exceptionally strong Sr lines in the peculiar star KIC 4768731, and the weakness in the CaII K line yet strong iron lines in the Am stars.

are multiple temperature subclasses later; strontium and iron lines are particularly enhanced (cf. $\S 3.3 .1)$. The star KIC 4768731 is an Ap SrCr star. The Sr II $\lambda \lambda 4077$ \& 4216 lines are remarkably strong, while the other metal lines match the hydrogen line type. The Ca II K line is abnormally broad but shallow. The Si II 4130 doublet is abnormally strong, but otherwise Si lines (e.g. $\lambda \lambda 4002 \& 4028$ ) are normal. The star also has roAp pulsations among the longest known, and I will be co-author on a paper describing this object.

The abundances of some of these stars have been examined, and for four stars are presented in Fig. 3.9. At A5, the Am star KIC 6292398 is on the hot border of the $\delta$ Sct instability strip. The other three stars, including the two $\gamma$ Dor stars at F1 $\mathrm{V}$, lie within the instability strip. The $\gamma$ Dor stars have solar abundances, within the 


\section{CHAPTER 3}

errors, but the Am stars are more peculiar than the mean Am star sample of Gebran et al. (2010). Thus the least variable stars seen with Kepler have the potential to be among the most peculiar Am stars. The four stars also happen to be slow rotators. This is no surprise for the Am stars, but that two slowly rotating, non- $\delta$ Sct F1 dwarfs are not chemically peculiar is unexpected. Although we are yet to answer the question "are the non-pulsating stars in the $\delta$ Sct instability strip necessarily chemically peculiar?", a slightly rephrased question, "are the non- $\delta$ Sct stars in the $\delta$ Sct instability strip chemically peculiar?", does have an answer from this study: not all of them.

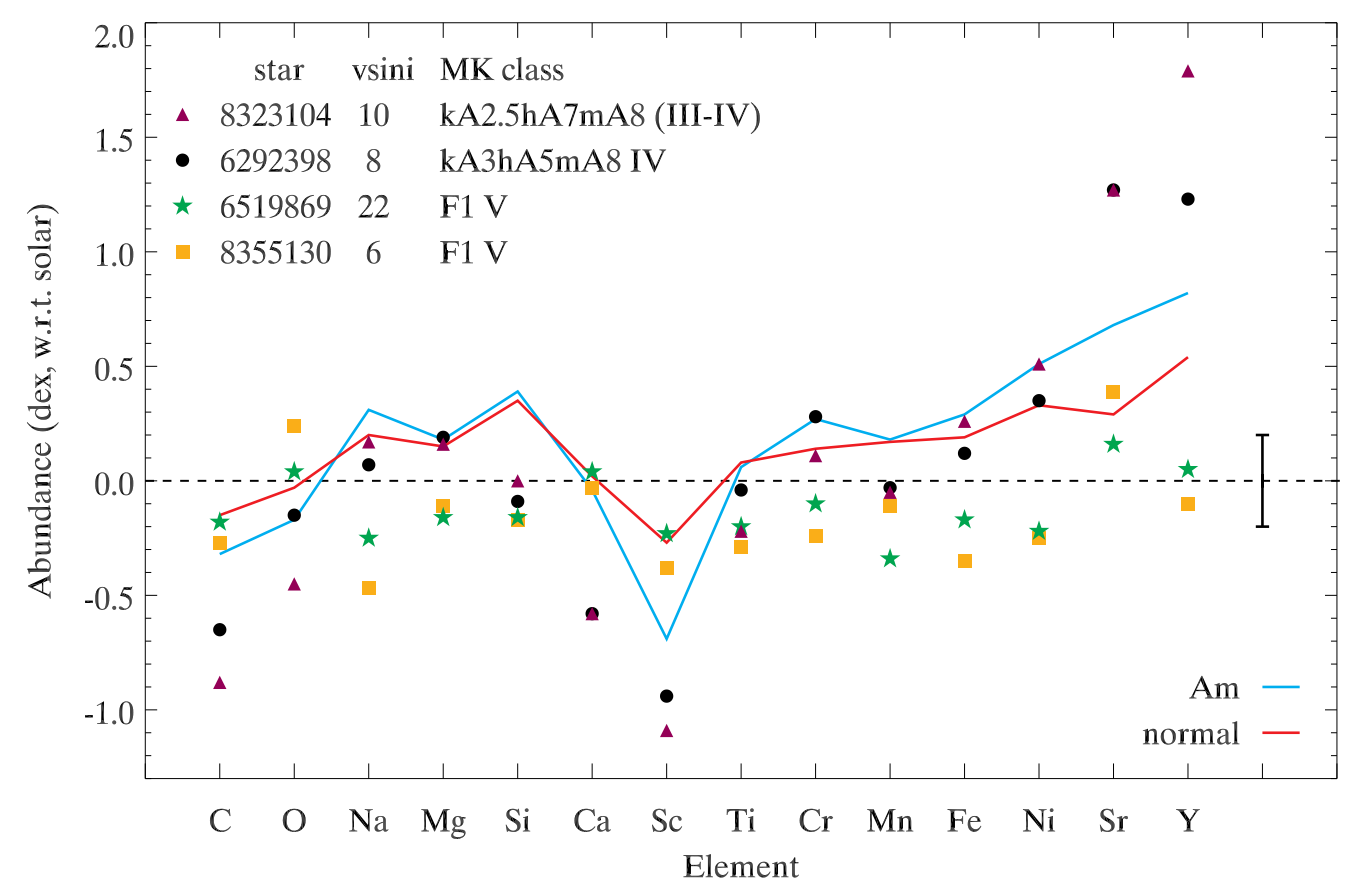

Figure 3.9: Abundances with respect to the Sun for four of the ten stars examined. For comparison, red and blue lines show mean abundances for the Hyades Am and normal A dwarfs (from Gebran et al. 2010), respectively. The error bar (right) shows the typical uncertainty for a single measurement. Notice each star is a slow rotator. 


\section{CHAPTER 3}

\subsubsection{Microturbulence}

Microturbulence refers to turbulence on a scale smaller than the mean free path of a photon, $\bar{l}$, which is related to the opacity, $\kappa$, and local density, $\rho$, as

$$
\bar{l}=\frac{1}{\kappa \rho} .
$$

Microturbulence has been seen as an important parameter in atmospheric characterisations, being at least as important as gravity in the luminosity classification of giants (Gray, Graham \& Hoyt, 2001). Its effect, therein described, is the broadening of spectral lines and desaturation of line cores by Doppler shifting photons from the line core to the wings. In early-type stars, the advent of non-LTE analyses alleviated the need for an adjustable, free, microturbulence parameter, leading Gray, Graham \& Hoyt (2001) to describe microturbulence as somewhat of a 'fudge-factor'. Additionally, Asplund et al. (2000) created 3D, radiative-hydrodynamical convective simulations of solar granulation that matched observed line profiles without the need of micro- and macroturbulence, indicating that in late-type stars the role of microturbulence in altering line profiles is fulfilled by convection. There is disagreement on the significance of this, with proponents of microturbulence arguing that velocity fields exist (Landstreet et al., 2009), whether modelled as granules in 3D models or micro- and macroturbulence in 1D models. The following discussion of microturbulence in Am stars is therefore tentatively provided, as one of a parameter whose influence is not only poorly understood, but may also prove to be redundant.

In Am stars the microturbulence velocity $(\xi)$ required to match the observed spectra is peculiar, generally higher than in the normal stars (Smalley et al., 2011), and is a 'symptom' of atmospheric velocity fields (Landstreet et al., 2009). As Fig. 3.10 illustrates, the required $\xi$ value rises from $1 \mathrm{~km} \mathrm{~s}^{-1}$ at $6000 \mathrm{~K}$ to $4 \pm 2 \mathrm{~km} \mathrm{~s}^{-1}$ at $8000 \mathrm{~K}$, decreasing to $1-2 \mathrm{~km} \mathrm{~s}^{-1}$ at $10000 \mathrm{~K}$ (see also Takeda et al., 2008). At 


\section{CHAPTER 3}

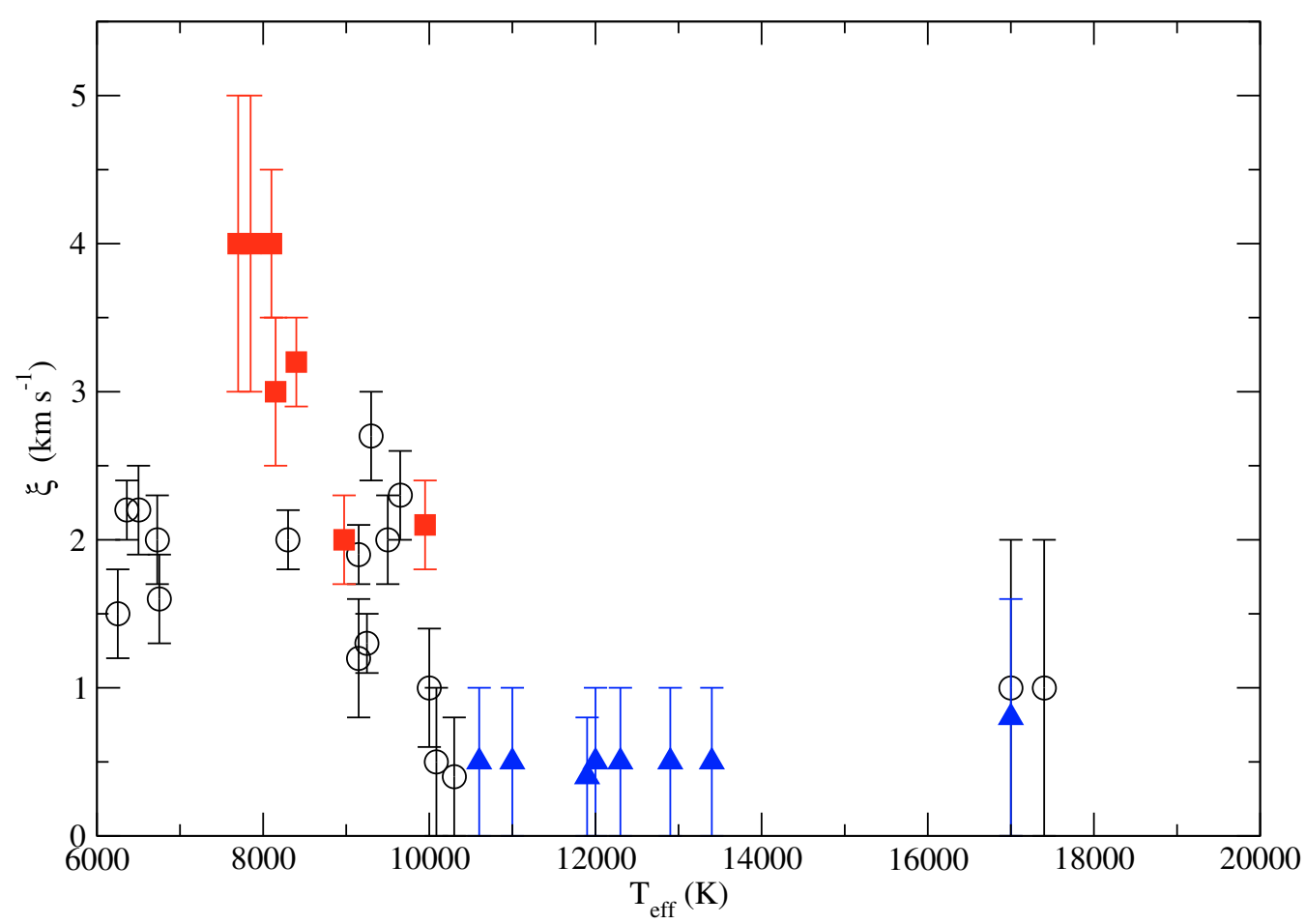

Figure 3.10: Microturbulence parameter $\xi$ as a function of stellar $T_{\text {eff }}$ for main sequence stars in the sample. Open black symbols are normal stars, filled red squares are Am stars, and filled blue triangles are HgMn stars. Figure and caption from Landstreet et al. (2009, their figure 2). Permission obtained from A\&A.

first glance, $\xi$ appears to be higher in the Am stars, but the lack of overlap between the normal and Am samples at $8000 \mathrm{~K}$ in their figure leaves the question open. The traditional interpretation is that $\xi$ is greater in stars with inefficient convection; $\mathrm{B}$ stars have purely radiative photospheres and $\mathrm{F}$ stars have efficient convection (Smalley, 2004). Coupry \& Burkhart (1992) claimed that the peak in $\xi$ at A5 is not related to the 'importance' of the convection zone, but noted that it coincides with both the classical instability strip and with maximum angular rotational velocities. They, too, acknowledged that Am stars require higher values of $\xi$, though this author points out the apparent contradiction with the $\xi$ peak corresponding to high angular rotational velocities and it being higher in the slowly rotating Am stars. Coupry 


\section{CHAPTER 3}

\& Burkhart suggested $\xi$ might be higher in Am stars as a result of the way that Am stars were modelled with scaled solar abundances rather than self-consistently determined ones. Indeed, Lane (1981) found that when calculated with spectral synthesis methods, $\xi$ for Am stars was much more in line with that of normal stars than when computed with classical curve-of-growth analyses. It is no surprise, then, that recent studies are finding progressively smaller discrepancies in $\xi$ between Am and normal stars - Gebran \& Monier (2007) determined that while some Am stars had higher $\xi$ than normal stars, in others $\xi$ was 'normal'... if indeed it has a physical meaning at all.

\subsubsection{Other observed properties of Am stars}

\section{Anomalous absolute magnitudes}

Budaj (1996) claimed there is some evidence that Am stars have larger radii and/or ages than those characterising normal A stars on the main sequence. That claim is based upon the conclusions of Gomez et al. (1981) that Am stars lie about 1 magnitude above the main sequence, depending on the main sequence used; the actual value varies from about 1.3 visual magnitudes (above the ZAMS) to 0.8 visual magnitudes (above the 'mean main sequence') as defined by Allen (1973). If radius is the sole cause of the difference in brightness, then Am stars are about 1.5 times larger than normal stars of the same temperature. Why would that be? One might propose larger radii on the basis of higher opacity. The accumulation of metals might raise the opacity, which acts to inflate a star's radius. However, this alone cannot account for a 50 per cent increase in radius. An alternative explanation is that they are more evolved than the normal stars against which they were compared, but we have seen that Am stars can take on a variety of ages, as young as a few Myr. Gravitational energy released from helium draining is also insufficient to cause the unusually high brightness. Thus the most convincing explanation appears to be 


\section{CHAPTER 3}

observer bias. Similar investigations into the same phenomenon yielded much less extreme results, e.g. Kurtz (1978b) obtained a value of $\Delta M_{V}=0.49 \pm 0.01 \mathrm{mag}$, i.e. the Am stars lie half a magnitude above the ZAMS, which makes sense given the main sequence is about a magnitude across. Given the Am stars rotate slowly, we might expect gravity darkening to play a smaller role in these stars than normal A stars - this could also explain the anomalous brightness. Incidentally, Catanzaro \& Balona (2012) issued a good reminder that using the $c_{1}$ index along with the Crawford calibrations for $M_{V}$ (Crawford, 1975) to estimate a luminosity leads to an anomalously low luminosity for the Am stars.

\section{Lack of magnetic fields}

The presence of magnetic fields strongly influences the abundances of some elements via the Lorentz force, as evidenced by the extreme peculiarities of the magnetic Ap stars. Stars cooler than early-F have only weak global magnetic fields, but in tangled flux tubes fields can be strong. Stars hotter than early-F that do have magnetic fields show strong, global and dipolar fields (Kurtz, 2000). However, that is not to say that all A stars do have magnetic fields, by any means. The Am stars do not: Shorlin et al. (2002) found no evidence for magnetic fields in 25 Am stars, nor in any of 22 of the normal B, A and F stars of their sample. Fossati et al. (2007) looked for magnetic fields in eight Praesepe Am stars, but discovered none. It would thus appear clear that the Am stars do not form a continuum of spectral peculiarity with the hotter and magnetic Ap stars.

\section{$3.4 \quad \rho$ Pup stars}

The $\rho$ Pup stars were distinguished as a class when Gray \& Garrison (1989a) isolated a class of unusually late and apparently evolved Am stars. They named these stars after the brightest prototype, $\rho$ Puppis. The need for the new classification arose 


\section{CHAPTER 3}

from the inhomogeneity of the $\delta$ Del stars, whose spectra were often indistinguishable from those of normal or marginal Am stars.

Stars of the $\rho$ Pup class show similar spectroscopic abundances to Am stars, but with later spectral types (mid-F) and cooler temperatures. The hydrogen line type of the vast majority of Am stars is no later than F2, but some members of the $\rho$ Pup class were best matched by an F5 hydrogen line type (Gray \& Garrison, 1989a). The stars were also inferred to be evolved, either through the size of the $c_{1}$ index or by consideration of the luminosity class in spite of the 'anomalous luminosity effect', which describes the discrepancy in luminosity class obtained for Am (and $\rho$ Pup) stars when considering different regions of the spectrum - Am stars appear to be of brighter luminosity classes in the violet spectral region than in the blue (Abt \& Morgan, 1976). ${ }^{7}$ However, the 'evolving Am star' hypothesis for the $\rho$ Pup stars is not the only one.

An alternative hypothesis for the $\rho$ Pup stars was that they were related to the Ba-dwarf stars (McGahee, 2010), whose peculiarities arise from the accretion of material from a post-AGB star. The link between Ba stars and late Am stars was discussed by North, Berthet \& Lanz (1994) through spectroscopic investigation of the Sr II 4077-strong stars, and the separation of those stars into various classes. One of those classes is the Ba dwarfs, in which only s-process elements, namely Ba and Sr, are overabundant. The $\rho$ Pup stars (and by extension the Am stars that have similar abundances), on the other hand, show an excess of the r-process element, $\mathrm{Eu}$, too, which essentially rules out a mass transfer hypothesis similar to that seen in the Ba dwarfs (McGahee, 2010). This provides independent verification of the invalidity of the mass transfer hypothesis origin for Am and $\rho$ Pup star abundance anomalies. The young age at which some Am stars are observed is another, though no particularly young $\rho$ Pup stars are reported.

\footnotetext{
${ }^{7}$ The anomalous luminosity effect was first described as the 'spurious luminosity effect' by Barry (1970), who stated the phenomenon was well-known, even at that time.
} 


\section{CHAPTER 3}

McGahee (2010) discovered the distribution of the $\rho$ Pup stars as a function of temperature is bimodal. The classification of a $\rho$ Pup star is dependent on late hydrogen line types for a star with Am characteristics, but Fig. 3.11 illustrates that very few $\rho$ Pup stars are identified at F3 or F4, with most being around F1 or at F5. The classifications were performed by two different observers (Houk and Mermilliod - stars were selected from: volumes 4 (Houk \& Smith-Moore, 1988) and 5 (Houk \& Swift, 1999) of the Michigan Catalog; and the photometric catalog of Mermilliod, Mermilliod \& Hauck (1997)). Observer bias does not explain the distribution, as the trend fits both observers' data well. We saw in the appendix to the previous chapter that stars are often given spectral types that exactly match MK standards, thus F5 is extremely well-populated and F4 very underpopulated. However, F3 is also one of the more commonly given spectral types, so the bimodality of the distribution cannot be explained in this way, unless for some unlikely reason Mermilliod and Houk did not use any F3 or F4 standards. One final consideration along the same lines is that there was only a loose criterion for what constituted a $\rho$ Pup star in the classifications. If the F2 temperature class were a strict boundary for what is a $\rho$ Pup star and what is an Am star, we would see no $\rho$ Pup stars earlier than F2 and the observed distribution would be much different. Although it is a concern, it still does not explain the lack of $\rho$ Pup stars at F3 or F4.

McGahee (2010) had her own interpretations of the bimodality. She said it suggested that the $\rho$ Pup and Am/Fm stars may be two disjunct groups, thus adding motivation for exploring the Ba dwarf, mass transfer hypothesis for the origin of the $\rho$ Pup stars. She also claimed the bimodality could result from the evolutionary process itself, if the stars are distributed around the post-MS contraction phase, but the jury is still out pending further investigations.

The pulsations in $\rho$ Pup stars would also agree with their status as evolved Am stars. The suggestion is they developed deeper convection zones as they expanded 


\section{CHAPTER 3}

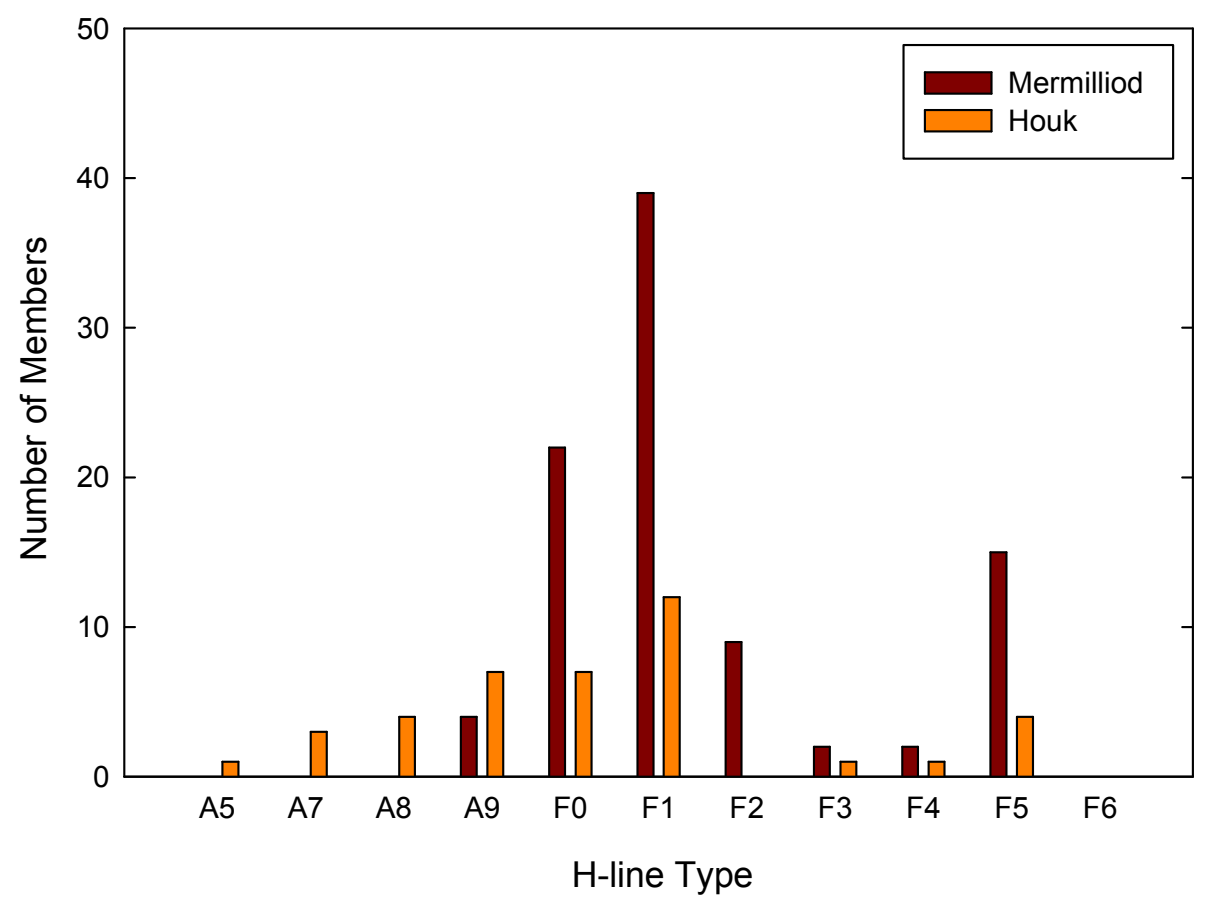

Figure 3.11: Distribution of $\rho$ Pup stars as a function of the hydrogen line type. The distribution is clearly bimodal in stars from both catalogues, which are volumes 4 (Houk \& Smith-Moore, 1988) and 5 (Houk \& Swift, 1999) of the Michigan Catalog; and the photometric catalog of Mermilliod, Mermilliod \& Hauck (1997). Slightly concerning is the number of $\rho$ Pup stars appearing at relatively early spectral types (see text). Figure from McGahee (2010, her figure 4.7). Reproduction permission obtained from author. 


\section{CHAPTER 3}

and cooled whilst leaving the main sequence (Kurtz, 1976), but that their abundance anomalies have not yet been erased. This was the stage attributed to the object KIC 3429637 (Murphy et al. 2012; provided as a case study in Chapter 5), whose consistent pulsational amplitude growth was believed to be tracing real-time evolution in a $\rho$ Pup star.

\section{$3.5 \lambda$ Boo stars}

The $\lambda$ Boo stars are a spectroscopically distinct class of stars with surface underabundances of metals in general, but normal abundances of $\mathrm{C}, \mathrm{N}$ and $\mathrm{O}$. Their metal-poor spectra are characterised by broad but shallow hydrogen line wings and very weak Mg II 4481 lines, even in light of the deficiencies of other metals (Gray, 1988). The group extends from late-B to early-F dwarfs, with low space velocities that suggest they are Population I objects (e.g. Hauck \& Slettebak, 1983). Bohlender \& Landstreet (1990) found no evidence for magnetic fields in $\lambda$ Boo stars at the 90-95 per cent confidence level.

Interest in the $\lambda$ Boo stars increased dramatically in the late 1990s. Around this time, Paunzen \& Gray (1997) began a spectroscopic survey to search for additional members of the then-small $\lambda$ Boo group, using Gray's (1988) extensively refined definition of the class. A total of 732 stars were classified in the process (Paunzen et al., 2001), resulting in the discovery of 26 new members and confirmation of 18 candidates from the literature (Paunzen, 2001), almost tripling the number of $\lambda$ Boo stars known at that time. Thus Paunzen inferred the percentage of $\lambda$ Boo stars among galactic field A stars to be 1.5 per cent out to 75 pc (Paunzen, 2001). Later, their incidence was put at 2.0-2.5 per cent in a more robust analysis by Gray \& Corbally (2002). Meanwhile, the abundances of the $\lambda$ Boo stars were being investigated in detail. Heiter (2002) determined the abundances of $12 \lambda$ Boo stars, which are presented here in Fig. 3.12. About sixty $\lambda$ Boo stars are known to date. 


\section{CHAPTER 3}

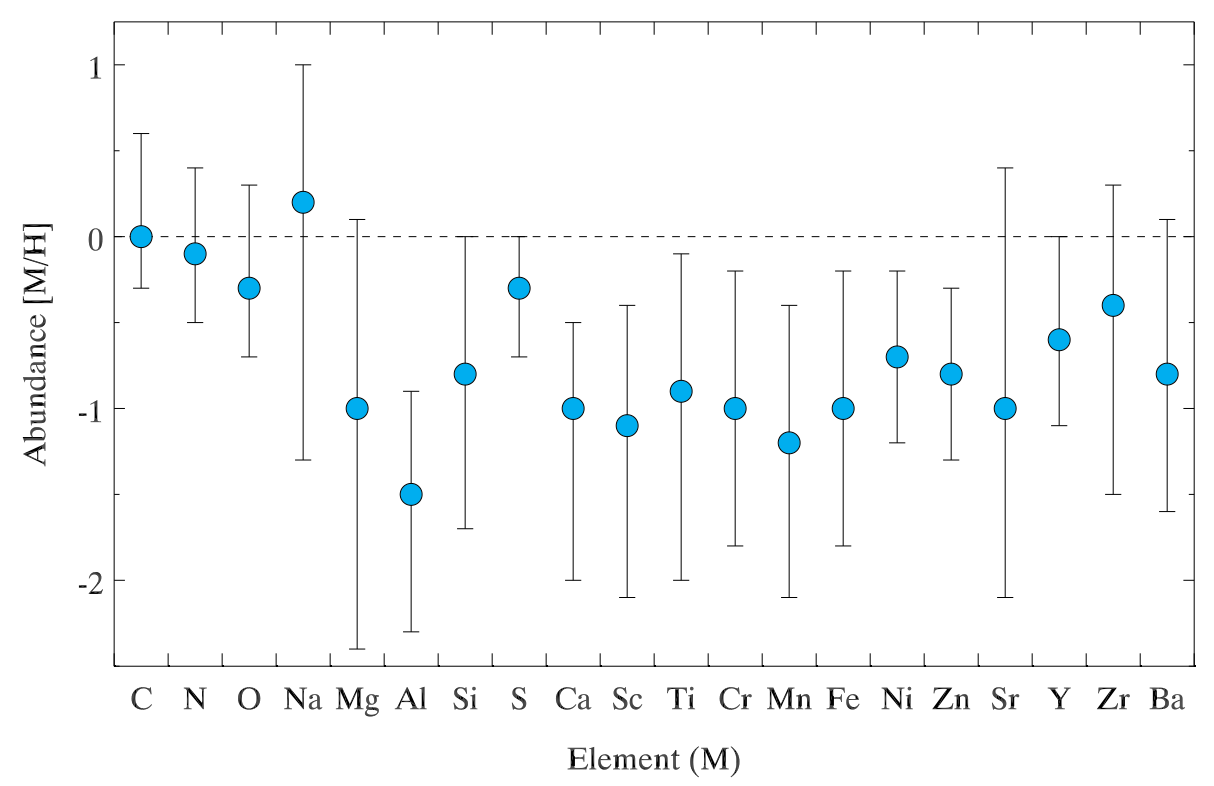

Figure 3.12: Mean abundances for the $\lambda$ Boo stars, from the twelve-star sample of Heiter (2002). Bars represent the maximum and minimum values among the sample to indicate the range of abundances observed; they are not error bars. The trend of metal deficiency but normal $\mathrm{C}, \mathrm{N}, \mathrm{O}, \mathrm{Na}$ and $\mathrm{S}$ is clear, and the ranges in abundance for each element also show that the star-to-star scatter is large for these stars.

Knowing detailed abundance characteristics of the group has allowed membership confirmation for individual stars, and importantly, for stars in binary systems. For a long time, the evolutionary status of $\lambda$ Boo stars was controversial (Gomez et al., 1998, and references therein). The Paunzen survey had already found some $\lambda$ Boo stars in the young OB1 association (Paunzen \& Gray, 1997), and Paunzen (1997) argued that $\lambda$ Boo stars fit pre-main-sequence evolutionary tracks well. Using abundance analyses, Iliev et al. (2002) characterised two binary systems, for which both stars in each system had $\lambda$ Boo abundances. They were able to derive ages, pointing to a $~ 1-G y r$ (i.e. not pre-main sequence) pair and a much younger 0.01-0.1 Gyr pair. This had important bearings on the long-disputed origin of the abundance anomalies.

Two main hypotheses exist for the origin of the $\lambda$ Boo phenomenon: diffusion combined with either (a) mass loss; or (b) accretion. The mass loss hypothesis was 


\section{CHAPTER 3}

put forward by Michaud \& Charland (1986), who showed that a mass loss rate of $10^{-13} \mathrm{M}_{\odot} \mathrm{yr}^{-1}$ in the models leads to underabundances of most metals. However, their models indicated it takes a few hundred Myr of mass loss to produce abundance anomalies that quantitatively match the observations, which reduces the viability of this mechanism in light of the discovery of very young $\lambda$ Boo stars. ${ }^{8}$ It is also unclear why mass loss should operate only in a small number of stars, so as to reproduce the observed fraction of $\lambda$ Boo stars.

The Michaud \& Charland (1986) models did not incorporate the mixing processes of meridional circulation or turbulence. This is problematic, since unlike Am stars, the mean $v \sin i$ of $\lambda$ Boo stars is not small and might lead to significant meridional circulation (see e.g. Charbonneau 1992, p42). Indeed, following a discussion of circulation in AmFm stars compared with in $\lambda$ Boo stars, Charbonneau (1993) called for the mass loss hypothesis, at least in the form developed by Michaud \& Charland (1986), to be abandoned. None the less, it is intriguing to note that Michaud \& Charland found that mass loss rates as high as those required for $\lambda$ Boo peculiarity also prevent the gravitational settling of helium (see also Charbonneau 1993). This has important consequences for pulsation - the He II convection and partial ionisation zone is well-supplied with helium with which to drive $\delta$ Sct pulsations. We will return to this shortly, after discussing the origin of the abundance anomalies.

Accretion was first suggested as an explanation for the $\lambda$ Boo phenomenon when Venn \& Lambert (1990) noticed the abundance pattern matches that of the interstellar medium (ISM) where metals condense out into dust grains. The suggestion was examined in closer detail by Kamp \& Paunzen (2002), who found that dust is efficiently Rutherford scattered away from the star, while gas is efficiently accreted. The distance scales involved in those processes naturally explain why $\lambda$ Boo stars are restricted to a fairly narrow portion of the HR-diagram - stellar winds prevent

\footnotetext{
${ }^{8}$ Young $\lambda$ Boo stars also disprove the hypothesis of Andrievsky (1997), that the $\lambda$ Boo stars arise from contact binaries of the W UMa type.
} 


\section{CHAPTER 3}

accretion in hotter stars, while convection will act to thoroughly mix the photospheres of the cooler stars, whose radiation fields are also too weak to prevent dust accretion. Conversely, the mass loss hypothesis does not explain the spectral range of the class. The accretion hypothesis allows abundance anomalies to develop very rapidly in the continued presence of accretable material, and meridional circulation is insufficient to immediately mix away the abundance anomalies that are developed through accretion (Charbonneau, 1993), requiring some $10^{7}$ yr to remove the abundance anomalies in a late-A star rotating at $200 \mathrm{~km} \mathrm{~s}^{-1}$ (Turcotte \& Charbonneau, 1993; Kamp \& Paunzen, 2002). Slower rotators actually erase the accretion signature more quickly, because chemical separation accumulates metals to be mixed back into the surface convection zone (Turcotte \& Charbonneau, 1993). Since the mainsequence lifetime of a mid-A star is some $2 \mathrm{Gyr}$, a quick time-scale analysis indicates an upper limit of $\frac{10^{7}}{2 \times 10^{9}}=0.005$ (i.e. 1 in 200) stars should be $\lambda$ Boo stars, whereas the observed fraction is closer to 0.02. Clearly, the accretion episode is longer lived than the time required post-accretion to erase the abundance anomalies.

King (1994) provided independent support for the accretion hypothesis, based on a search of the IRAS (Neugebauer et al., 1984) database for $\lambda$ Boo stars showing infrared excess. Martínez-Galarza et al. (2009) modelled the observed infrared excess for six $\lambda$ Boo stars, finding support for the accretion hypothesis, but not being able to distinguish between ISM accretion or the presence of a circumstellar debris disk. The accretion model is far from perfect, though. Heiter, Weiss \& Paunzen (2002) found a mismatch between ISM and $\lambda$ Boo abundances that would argue against the accretion hypothesis, but called their results 'inconclusive', pending further observational data and theoretical developments.

It is worth mentioning that Gray \& Corbally (2002) found a significant null result for $\lambda$ Boo stars existing in intermediate-age clusters. The consequences of which are as follows: for the accretion mechanism, presence in a cluster must somehow 


\section{CHAPTER 3}

inhibit accretion, probably through the rapid dispersion of diffuse interstellar clouds through photo-evaporation and molecular outflows - the dissipation time for gas in clusters is at most $10^{7} \mathrm{yr}$ (Palla \& Stahler, 2000). For the mass loss mechanism, which should be internal to the star and not influenced by environment, the null result implies this mechanism is not responsible for producing $\lambda$ Boo stars. It was proposed (Paunzen, 2001), but later refuted for reasons just mentioned (Gray \& Corbally, 2002), that both mass loss and accretion support $\lambda$ Boo peculiarity, but at different main-sequence stages. Contrarily, it has also been suggested over the years that neither mechanism may be correct, so we should consider an alternative solution to the problem.

A list of members of the $\lambda$ Boo class was collected by Gerbaldi, Faraggiana \& Lai (2003), who found the class to be heterogeneous. Their list contained many 'remarks' on the spectra of the various members, arguing the case for misclassification of about half of the stars. They also acknowledged a lack of agreement between lists of members compiled by different authors. One particularly frequent remark was that the spectrum was 'composite', i.e. of a binary or multiple system. That $\lambda$ Boo spectra might not represent peculiar stars at all, but rather a composite spectrum that appears peculiar when interpreted as a single star, has been favoured by Faraggiana \& Bonifacio $(1999,2005)$. $\lambda$ Boo abundances were reproduced in their models because metal lines exhibit large variations in strength in the A stars, but $\mathrm{C}, \mathrm{N}$ and O lines do not - an early A star (whose metal lines are weak) dominates the flux and washes out the metal lines of the late-A companion, whereas if the composite is interpreted as a single mid-A star then the metal lines would seem weak while $\mathrm{C}, \mathrm{N}$ and $\mathrm{O}$ remain normal, leading to a $\lambda$ Boo classification. The discovery of at least 22 composite spectra in a sample of $132 \lambda$ Boo candidates led Faraggiana et al. (2004) to state that "some, if not all, the $\lambda$ Boo candidates are not chemically peculiar at all". Gerbaldi \& Faraggiana (2004) noted that disentangling the binaries 


\section{CHAPTER 3}

is hard because there are few, weak metal lines, and these are 'washed out' due to high $v \sin i$ values (the rotational velocity distribution of these stars is indistinguishable from normal A stars according to Paunzen 2001), implying there may be many undetected binaries among the $\lambda$ Boo candidates. However, support for the composite spectrum hypothesis would appear to be dwindling, given that Stütz \& Paunzen (2006) found that for 90 per cent of the group members, the composite spectrum hypothesis cannot explain the observations, and Griffin, Gray \& Corbally (2012) reached the verdict that "The composite-spectrum binary hypothesis does not explain the $\lambda$ Bootis stars" in their eponymous paper.

We shall conclude this review of the $\lambda$ Boo class with a promised description of their pulsation properties. We mentioned that the $\delta$ Sct driving region should be well stocked with helium if mass loss is operative. Furthermore, as far as the more favoured accretion hypothesis is concerned, continual accretion of gas keeps the star's surface regions, which include the He II partial ionisation zone, well stocked with helium. This holds even if the star rotates slowly enough that rotationally induced mixing cannot prevent gravitational settling of helium. I predict the consequence is an increase in pulsational driving, implying that these stars should: (a) be more likely to pulsate than normal (i.e. non-peculiar) stars of the same temperatures; and/or (b) show richer pulsation spectra than normal $\delta$ Sct stars.

To analyse hypothesis (a), literature observations were reinvestigated. The incidence of pulsators among the class has been observed to be high: figure 1 of Weiss et al. (2000) shows at least 66 per cent of $\lambda$ Boo stars in the $\delta$ Sct instability strip pulsate, compared to just 56 per cent for normal stars (based on numbers from Chapter 1), and with a much more restrictive amplitude detection limit. Therefore, at the $50 \mu$ mag limit that was applied in Chapter 1, the fraction of $\lambda$ Boo stars that pulsate is most likely even higher. Hypothesis (b) will require spectral types for metal-poor 


\section{CHAPTER 3}

Kepler $\delta$ Sct stars, to determine which of these are $\lambda$ Boo stars, if any, and then compare the mode density of their Fourier transforms with non- $\lambda$ Boo stars. A telescope proposal has been submitted to evaluate this hypothesis.

Pulsations are important to the study of $\lambda$ Boo stars, as they may help constrain evolutionary status. They have already helped confirm that the interior abundances of the star must be solar, and that the peculiarity is a surface phenomenon (Weiss et al., 2000), unlike for the SX Phe stars (cf. Murphy et al. 2013). Weiss \& Paunzen (2000) described the pulsational properties of $\lambda$ Boo stars to be similar to the $\delta$ Sct stars, with both radial and non-radial modes being excited. Spectroscopic study of $\lambda$ Boo stars is not only warranted to study pulsations - if $\lambda$ Boo stars really are formed by the accretion mechanism, the presence of a (recent) circumstellar disk might well point to these objects being planet hosts, particularly since their abundances are conspicuously similar to those of the "peculiar Sun" mentioned in $\S 3.2 .1$. A case in point would be the dusty $\lambda$ Boo star HR 8799 that plays host to multiple planets.

\section{6 'sn' stars}

The 'sn' stars are so-named because they have both sharp (s) and nebulous (n) spectral lines (Abt, 1979). They occupy a narrow absolute magnitude range, between -2.8 and $+1.3 \mathrm{mag}$, thus 'sn' spectra are only seen in B stars (Abt, 1979). Specifically, they are found at B2-B9, and luminosity classes V to III (Mermilliod, 1983), thus the age interval they occupy is wide: $2 \times 10^{6}$ to $2.7 \times 10^{8} \mathrm{yr}$. Mermilliod (1983) found two-thirds of the 'sn' stars to lie near the ZAMS, and one third to lie nearer the TAMS.

Only He I lines are found nebulous in the 'sn' stars, whilst lines of Ti II, Ca II, Si II, C II and Fe II are sharp (Mermilliod, 1983). Each of the three 'sn' stars studied by Shore, Brown \& Sonneborn (1987) shows C IV variations - in stars that are too 


\section{CHAPTER 3}

cool for photospheric C IV - indicating this line must be produced in a stellar wind. Given that the helium lines place these stars at B8, Shore, Brown \& Sonneborn commented that the 'sn' stars appear to be a subgroup of the He-weak stars, noting that their hydrogen lines appear strange (they have wings that are too weak and diffuse for their core strength). Analysis of the C II 4267 line pointed to them being the slowest rotators among the He-weak stars - a conclusion that was also reached by Abt (1979), based on the requirement of slow rotation to produce the sharp lines. Mermilliod (1983) found that the group do indeed have low rotational velocities: 12 of the 16 stars he studied had $v \sin i<50 \mathrm{~km} \mathrm{~s}^{-1}$. A list of 'sn' stars with their rotational velocities is collected in an Appendix (3.A) to this chapter.

Mermilliod (1983) excluded binarity and pole-on hypotheses for the slow rotation, but also deemed Abt's hypothesis of these being shell stars to be unlikely because shell stars are normally fast rotators (their rotational velocities can be inferred from the Mg II 4481 profile, which samples the underlying star and not the shell). Another of Mermilliod's reasons for rejecting the shell hypothesis was a lack of evidence of magnetic fields, but Shore, Brown \& Sonneborn found magnetic fields to be driving the winds observed in all three of their stars. In any case, sharp lines are also seen in shell stars (see Gray \& Corbally 2009 for a description of spectral characteristics of shell stars), in spite of their slow rotation, so it is not time to reject the shell hypothesis yet.

One description of the 'sn' stars on which Abt and Mermilliod (1983) agree, not that there was any bitter debate, is that the 'sn' stars might be related to the ApBp phenomenon. Mermilliod (1983) thought the 'sn' stars could be on the boundary of becoming peculiar, and that initial conditions would determine the age distribution of these objects. It has caught the attention of this author that the mean cluster fraction of 'sn' stars is 13 per cent (Abt, 1979), which happens to be the very same frequency as the peak of the Ap-star distribution (e.g. Wolff 1983). Abt (2009) 


\section{CHAPTER 3}

determined the normal, slow-rotators of early-A spectral types can be explained as Ap SrCrEu stars that are yet to fully develop their peculiarities - he concluded it takes $186 \mathrm{Myr}$ for the frequency of Ap stars in clusters to reach that of field stars. Since the principal direction of evolution of B stars on the HR diagram is towards cooler temperatures and into the A class, it may be that 'sn' stars are proto-Ap stars. The upper limit of the ages of 'sn' stars given by Mermilliod (1983), $2.7 \times 10^{8} \mathrm{yr}$, supports this idea. A cross-examination of high-resolution spectra of 'sn' and Ap stars of similar ages would be worth pursuing, and inspection of Mermilliod's sample for magnetic fields could be worthwhile in investigating the link with the Ap stars. ${ }^{9}$ It would be interesting to see if the known 'sn' stars all exhibit C IV variations, like the Shore, Brown \& Sonneborn (1987) sample, by examining any IUE spectra of these stars.

\subsection{Chapter summary}

The peculiar abundances of the Sun and the hypothesis that the depletion of refractories could be explained by the solar system's planetary formation history have intriguing implications for the $\lambda$ Boo stars, whose surface metal content is also depleted. If the accretion hypothesis for the $\lambda$ Boo stars stands, then the separation of dust and gas in the circumstellar environment could lead to these stars having a higher incidence of planets than normal A stars. Furthermore, if mass loss plays any role in creating the observed abundance anomalies in these stars, then helium is prevented from gravitationally settling. This has important implications for the incidence of pulsators among the $\lambda$ Boo stars, which we have shown to be high. Thus we have two reasons for which a spectroscopic search in the Kepler field for $\lambda$ Boo stars would be extremely useful.

\footnotetext{
${ }^{9}$ Since the Ap SrCrEu stars can pulsate (these are the roAp stars), I had wondered whether the 'sn' stars might pulsate too, but their temperatures are too hot to put them in (or even near) the roAp instability strip.
} 


\section{CHAPTER 3}

Abundance analyses of Am stars and other peculiar stars are also affected by normalisation against those of the Sun. It is suggested that such analyses should be calculated with respect to normal A stars instead, as was once standard practice, with consideration given to the scaling of abundances with iron. As such, a selfconsistent study into the abundances of a large number of normal A stars would be valuable to progress in the field. The challenge is finding stars with $v_{\text {eq }}$ high enough to inhibit development of peculiarities, but low enough that rotational broadening of lines does not prevent a detailed abundance analysis.

The degree to which Am stars are peculiar is strongly correlated with $v \sin i$, but after an initial transition phase, only weakly dependent on age on the main sequence. The literature paints a clear picture that as the Am stars reach the end of their main-sequence life, they become $\rho$ Pup stars (formerly $\delta$ Del stars) and their abundances anomalies become milder. Their stability against pulsation also decreases, and recently many Am stars were found to pulsate in SuperWASP data (Smalley et al., 2011), some of which were actually $\delta$ Del stars rather than classical Am stars. Indeed, the degree of peculiarity associated with 'Am' stars in the SuperWASP sample is unclear, and many might be marginal rather than classical Am stars. Both Am: and $\delta$ Del stars are expected to pulsate, so the apparent conflict between stable, helium-depleted models of classical Am stars, and observed pulsations in non-classical Am stars - the so-called 'helium problem in Am stars' (Catanzaro \& Balona, 2012) - is not as big a problem as they suggest.

Recent developments to Am star models are the calculations of radiative accelerations of individual elements and their near-surface abundances in the presence of convection. The most recent addition to models is thermohaline convection, an often-neglected mixing mechanism. Pulsation in marginal Am stars is not expected to generate additional turbulence if the velocity gradients are small. I have thus highlighted the implication that pulsations in Am stars should be of low overtone 


\section{CHAPTER 3}

and low degree, as higher overtone and higher degree modes have many nodes on or near the surface, producing steeper velocity gradients in the envelope.

A sample of non- $\delta$ Sct stars was inspected for peculiarity. Of ten stars investigated, the trivial solution of not lying within the $\delta$ Sct instability strip applied to one, and six of the others were chemically peculiar. Interestingly, stars with $\gamma$ Dor pulsation but not $\delta$ Sct pulsation had milder or no abundance anomalies. One of the ten targets was slowly rotating and not pulsating, lying right in the middle of the $\delta$ Sct instability strip. Unless low inclination explains the slow rotation, the absence of chemically peculiarity is a challenge to Am-star theory; otherwise, the lack of pulsations is a challenge to pulsation theory. The star happens to be an SB2 system, which complicates the pending abundance analysis.

The 'sn' stars are a group of mid- and late-B type stars with both sharp and diffuse lines that rotate slowly compared to normal stars at that spectral type. They are young objects, slightly younger than the other group of slowly rotating stars at late-B: the Ap stars. The development time-scale for Ap SrCrEu stars approximately matches the upper age limit of $2.7 \times 10^{8} \mathrm{yr}$ put on the 'sn' stars (Mermilliod, 1983), though the time-scale is much shorter for Ap Si stars. Since stars that are late-B on the ZAMS become early-A stars in the middle of their main-sequence life, we arrived at the interesting conclusion that 'sn' stars might be the proto-Ap stars, yet to develop their peculiarities. A search of 'sn' stars for magnetic fields like those in Ap stars is to be arranged. 


\section{A List of 'sn' stars}

Table 3.2: A list of the 'sn' stars, assembled predominantly from Mermilliod (1983), which in turn was largely assembled from Abt (1979). Two stars (HD 5737 \& HD 79158) from Shore, Brown \& Sonneborn (1987) were added, and supplemented with measurements from SIMBAD. The ':' character indicates an uncertain classification. Stars below the mid-table break are in the Orion Association.

\begin{tabular}{rlrr}
\hline HD & Spectral Type & $\begin{array}{r}\mathrm{V} \\
\mathrm{mag}\end{array}$ & $\begin{array}{r}v \sin i \\
\mathrm{~km} \mathrm{~s}^{-1}\end{array}$ \\
\hline 5737 & B7IIIp (?) & 4.30 & 20 \\
37000 & B5V & 7.49 & - \\
37058 & B2V & 7.32 & 5 \\
37129 & B2.5V & 7.14 & 50 \\
161480 & B6Vp (CII) & 7.68 & 25 \\
20961 & B9.5V & 7.64 & 25 \\
21071 & B7V & 6.09 & $<40$ \\
21699 & B5Vp (He wk) & 5.48 & 40 \\
22136 & B8IV: & 6.89 & 25 \\
23408 & B7III & 3.86 & 35 \\
23873 & B9.5IV & 6.59 & 85 \\
23950 & B9IVp (Hg,Mn) & 6.07 & $75:$ \\
79158 & B9 & 5.29 & 50 \\
162515 & B9V & 6.56 & 95 \\
162586 & B8V & 6.13 & $<40$ \\
162679 & B9V & 7.16 & $<40$ \\
162804 & B9V & 7.02 & $<40$ \\
162678 & B9V & 6.38 & $<40$ \\
205116 & B9.5V & 6.83 & 90 \\
\hline 35502 & B5IVp (He wk) & 7.32 & 285 \\
35792 & B3V & 9.77 & 60 \\
36351 & B2.5V & 5.46 & 45 \\
36392 & B3V & 7.56 & 40 \\
36429 & B4IVp (He wk) & 7.56 & 80 \\
36430 & B2.5V & 6.20 & 25 \\
36485 & B2V & 6.83 & 85 \\
36954 & B4V & 8.80 & 190 \\
37151 & B8Vp (Si) & 7.38 & - \\
37232 & B2.5V & 6.11 & 120 \\
37321 & B5V & 7.09 & 100 \\
37889 & B2.5V & 7.63 & - \\
\hline & & & \\
\hline
\end{tabular}




\section{CHAPTER 3}

Table 3.3: A list of the 'sn' star candidates, from Mermilliod (1983), identified as such because they are normal slow rotators.

\begin{tabular}{rlrr}
\hline HD & Sp.T. & $\begin{array}{r}\mathrm{V} \\
\mathrm{mag}\end{array}$ & $\begin{array}{r}v \sin i \\
\mathrm{~km} \mathrm{~s}^{-1}\end{array}$ \\
\hline 74146 & B5V & 5.20 & 50 \\
74195 & B3IV & 3.64 & 20 \\
74560 & B3IV & 4.84 & $<45$ \\
45546 & B2V & 5.05 & 45 \\
295102 & B9V & 8.68 & $<40$ \\
45627 & A0V & 8.88 & 50 \\
60969 & B5IV & 7.01 & $\leq 40$ \\
60999 & B9V & 8.75 & $\leq 40$ \\
NGC2422-79 & B5V & 7.76 & $\leq 40$ \\
NGC2516- 2 & A0IV-V & 8.80 & 40 \\
NGC2516- 5 & B8.5IV & 8.54 & 50 \\
\hline
\end{tabular}




\title{
Chapter 4
}

\section{A pulsation review of delta Scuti and related stars}

\author{
Abstract \\ The $\delta$ Sct stars are by far the most common type of pulsating stars at \\ spectral type A. In this, the most extensive review of $\delta$ Sct stars in the \\ Kepler era, the following topics are discussed: the $\delta$ Sct instability strip; \\ pulsation amplitudes and frequency ranges of the class; mode selection \\ and identification; and the influence of rotation, evolution, and metallic- \\ ity on pulsation. The $\gamma$ Dor variables and the overlap of their instability \\ strip with the $\delta$ Sct instability strip are outlined, before a short descrip- \\ tion of especially interesting case-studies is presented, pointing to further \\ reading.
}

\subsection{Introduction}

Asteroseismology is the most informative method with which we can observe the stellar interior. Other than solar neutrinos, it is the only method. Oscillation frequencies present in stars' light curves are the fundamental data of asteroseismology, 


\section{CHAPTER 4}

and permit us to fine-tune stellar models. Although ground-based support is still required, particularly in constraining stellar atmospheric parameters and for direct mode identification, the existence of nearly continuous, micromagnitude-precision, space-based photometry is revolutionising our capacity to study stars.

The A stars exhibit a range of pulsation phenomena. This is where many of the classical pulsators - those driven by the 'heat-engine' mechanism - are found. Under this mechanism, first proposed by Eddington (1917), a layer heats up on compression, increasing the region's opacity and driving radius expansion. The expanded region later cools, causing an opacity drop and re-compression. The dependence on opacity has lead to the mechanism being known as the opacity-, or simply $\kappa$ mechanism. The RR Lyrae variables, rapidly oscillating Ap (roAp) stars and $\delta$ Scuti stars are all examples of A stars driven by the $\kappa$-mechanism. Classical Cepheid variables are also driven by the $\kappa$-mechanism, but at later spectral types. The $\gamma$ Doradus variables make up a large number of variables at early-F spectral types, but are driven by a convective-flux modulation mechanism (Guzik, Bradley \& Templeton, 2000). Some stars show pulsations driven by more than one mechanism and are known as hybrids (Handler, 2012).

At the rate at which progress occurs with Kepler, to cover all types of pulsating stars in one review would be impractical. Here we will focus mostly on the $\delta$ Sct stars, which are the most populous class of pulsating variables of spectral type A. The instability strip of the $\gamma$ Dor stars overlaps with the cool edge of the $\delta$ Sct instability strip, and many hybrids of these classes are found. Strictly speaking, most of the $\gamma$ Dor and some of the $\delta$ Sct stars are of spectral type F, but since the stellar properties are continuous over the spectral-type boundary and until the Kraft break (see Chapter 2), we do not make the distinction.

We will refer to pulsation modes using quantum numbers: $n$ is the overtone or radial order of the mode, indicating the number of radial nodes; $\ell$ is the degree, 


\section{CHAPTER 4}

indicating the number of surface nodes; and $m$ is the azimuthal order, with $|m|$ indicating the number of surface nodes that are also lines of longitude. We shall see later that the sign of $m$ indicates whether the mode is prograde or retrograde, and we use the convention that prograde modes have negative $m$ values and higher frequencies in the observer's frame. Pressure modes ( $\mathrm{p}$ modes) are denoted with positive values of $n$, and gravity modes (g modes) with negative values. One sometimes writes $p_{1}, p_{2}, \ldots$, to denote the $n$-value of the p modes. The g modes are always non-radial, that is, they have $\ell \geq 1$. Spherically symmetric stars have degenerate pulsation frequencies, in that all modes with the same $(n, \ell)$ have the same frequency, regardless of $m$. Rotation and magnetic fields can break the symmetry and lift this degeneracy, however.

The $\delta$ Sct stars are the main-sequence counterparts to the classical Cepheid variables, located at the junction between the classical Cepheid instability strip with the main sequence. Indeed, the high amplitude delta Scuti (HADS) stars, arbitrarily defined as $\delta$ Sct stars with peak-to-peak light variations exceeding 0.3 mag, follow the same period-luminosity relationship as first overtone classical Cepheids with no discontinuity (Soszynski et al., 2008).

In this review we will discuss observational selection effects affecting studies of $\delta$ Sct stars $(\S 4.2)$; the extent of the $\delta$ Sct instability strip ( $\S 4.3)$; mode selection and identification (§4.4), including mode visibility, frequency distributions, mode density, the effect of rotation and the existence of combination frequencies. In $\S 4.5$ we look at related variables such as the HADS, SX Phe and $\gamma$ Dor stars. We finish with a mention of a few important case studies of $\delta$ Sct stars in $\S 4.6$.

\subsection{Observational selection effects}

The $\delta$ Sct stars have been observed from the ground for decades. A combination of better instrumentation and improving observing techniques yielded increasingly 


\section{CHAPTER 4}

richer frequency spectra, largely because the noise level in the observations dropped. Before space-based observations, it was thought that all stars in the $\delta$ Sct instability strip might pulsate below the 1-mmag detection limit at the time (e.g. Breger 2000b), and certainly that the frequency spectra would be far richer at lower amplitude. The Kepler mission has dropped the detection limit down to the 1-10 $\mu$ mag level, and has certainly proven the latter prediction to be true. However, multiple selection effects were biassing our knowledge of $\delta$ Sct stars.

The first $\delta$ Sct stars to be found were those with high amplitudes, as these required less precise photometry. High amplitudes have long been associated with slow rotation: Dickens (1968) suggested that rotation must limit the pulsation amplitude in a manner dependent on the ratio of centrifugal force to gravity. Moreover, fast rotation causes turbulence, which favours non-radial modes over radial ones (Breger, 2000b). Since radial modes can achieve higher amplitudes than non-radial ones (Dziembowski, 1977a), fast rotation may therefore inhibit high-amplitude pulsation.

There was another selection effect in place, in that kinematic studies favour stars having sharp-lined spectra. The majority of $\delta$ Sct stars that were discovered through such kinematic studies were therefore slow rotators (Sterken \& Jaschek, 1996). Indeed, the average $v \sin i$ of the $\delta$ Sct stars was established at $35 \mathrm{~km} \mathrm{~s}^{-1}$ in the early 1960s (McNamara, 1961), but is indicated to be $\sim 160 \mathrm{~km} \mathrm{~s}^{-1}$ now (cf. Chapter 2).

Even now, when dedicated searches for $\delta$ Sct stars take place, some selection effects remain. One's ability to detect variability from the ground depends on the brightness of the object, and brighter A stars are closer - certainly within the thin disk. Although the thin disk contains stars of a variety of ages and metallicities, it is fair to say that the higher metallicity of the thin disk will bias the metallicities of $\delta$ Sct stars discovered nearby. Certainly, claims that $\delta$ Sct stars are generally metal rich must consider them relative to non-pulsating A stars, rather than the 


\section{CHAPTER 4}

Sun, because of two important factors: the decreased lifetime of A stars compared to the Sun indicates these stars may be a generation younger and consequently composed of more metal-rich material, more so even than the Sun, which appears to be slightly metal rich for its age (Holmberg, Nordström \& Andersen, 2009); and the Sun itself is depleted in refractory elements by $\sim 20$ per cent relative to volatile elements compared to solar twins (Meléndez et al., 2009), which has consequences for abundance analyses of $\delta$ Sct stars, as was discussed in Chapter 3 . Of course, the metallicity is important to stellar models of $\delta$ Sct stars, as we shall see in the next section.

One must be extremely careful when deriving the properties of a group not to be hampered by a selection bias. Such biases have created problems in the past, and one must remember that standards such as the Sun can be unusual in their own right.

\subsection{Instability strip}

The observational $\delta$ Sct instability strip extends between spectral types A2V to F0V and A3III to F5III (Kurtz, 2000), and covers not only the full height of the mainsequence band, but also pre-main sequence regions. Most $\delta$ Sct stars that are near the ZAMS have $7380<T_{\text {eff }}<8600 \mathrm{~K}, \log g \approx 4.30(\mathrm{cgs})$ and masses in the range $1.2-$ $2 \mathrm{M}_{\odot}$; whereas stars at the top of the $\delta$ Sct instability strip have $6360<T_{\text {eff }}<7530 \mathrm{~K}$ and $\log g=3.25$ (Rodríguez \& Breger 2001, Handler \& Shobbrook 2002). Pre-main sequence stars can also show $\delta$ Sct pulsations when they cross this instability strip (e.g. Zwintz 2008), pulsating in mostly non-radial modes (Handler, 2009). The Kepler field contains no such stars, though. Balona \& Dziembowski (2011) found a gap between the ZAMS and the $\delta$ Sct stars observed by Kepler that increases with temperature and is indicative of the age of the field.

The blue edge of the strip is delimited mostly by the excitation of modes with 


\section{CHAPTER 4}

$n=6$ or 7 in the hotter stars (Dupret et al., 2005). The hotter the star, the closer the HeII driving zone moves toward the surface, until at some point there is no longer a large enough zone to drive pulsations. Kepler observations have found $\delta$ Sct pulsation in stars hotter than the published borders, but uncertainties in $\mathrm{KIC}$ temperatures prohibit a firm conclusion from being drawn, and a detailed study to redefine an observational blue edge is required. The red edge is even more complicated to parametrize. It is delimited by g modes of progressively higher radial order and degree. Time Dependent Convection (TDC; Dupret et al. 2004) is required to analyse the red edge - models with Frozen Convection (FC; see, e.g., Li 2000) do not actually indicate a red edge of the $\delta$ Sct instability strip, in that stabilisation is never achieved when one goes to cooler $T_{\text {eff }}$. The TDC models indicate that cooler models that have larger convective envelopes act to stabilise modes and create a red edge. Thus the instability strip shown in Fig. 4.1 is taken from Dupret et al. (2005)

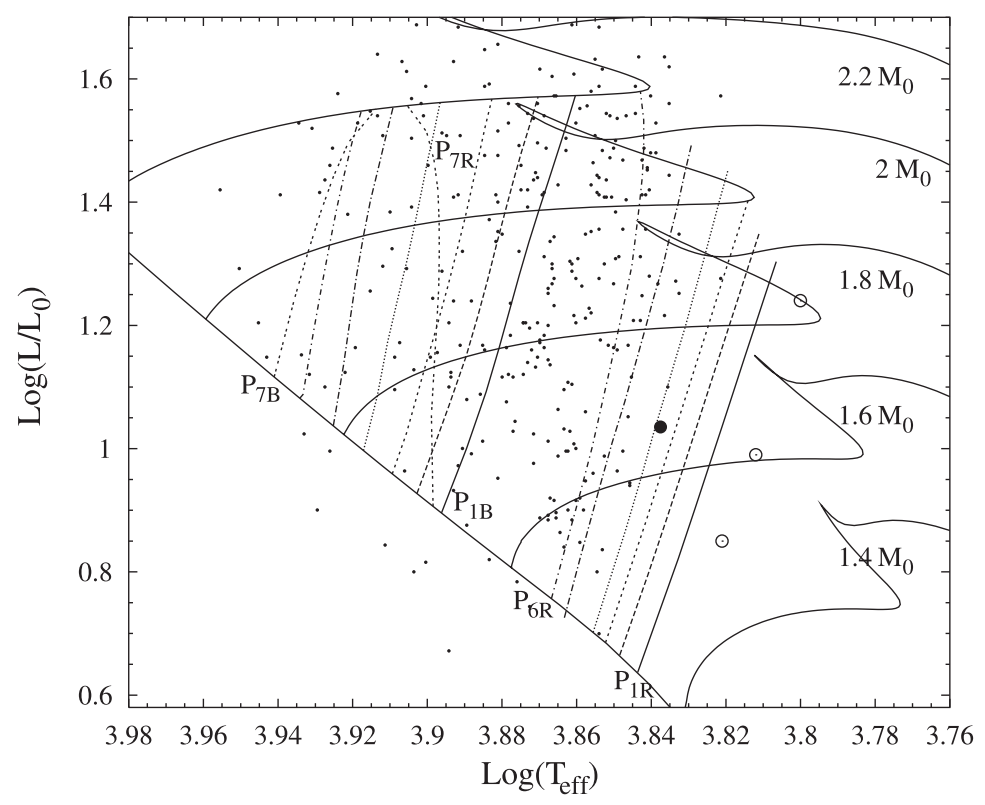

Figure 4.1: Blue and red edges of the $\delta$ Sct theoretical instability strip. Lines represent Time Dependent Convection (TDC) results for radial modes from $p_{1}$ to $p_{7}$ for models with $\alpha=1.8$. The small points correspond to observations. For comparison, we also give the red edges for the fundamental radial mode obtained by Xiong et al.

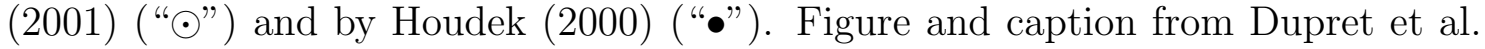
(2005, their figure 2). Permission obtained from A\&A. 


\section{CHAPTER 4}

who calculated TDC models for different values of the mixing-length parameter $\alpha$. It is important to recognise, however, that Kepler observations have shown the instability strip to be wider than TDC predicts (Uytterhoeven et al., 2011). In the chemically peculiar stars, which are drained of helium, the reduction in driving efficiency sees the red and blue edges move closer together.

The most important parameter influencing the evolutionary tracks and instability strip edges in these models is the mixing length parameter, $\alpha_{\mathrm{MLT}}$, from the BöhmVitense (1958) Mixing Length Theory. This parameter describes the number of pressure scale heights travelled by a convective parcel of gas in the stellar interior. In the models, the convective envelope is thinner for lower $\alpha_{\mathrm{MLT}}$ at a given $T_{\text {eff }}$ (Dupret et al., 2005). Thus the theoretical red edges are displaced to lower $T_{\text {eff }}$ when $\alpha_{\text {MLT }}$ decreases because convection plays less of a roll to stabilise against pulsation for a given temperature. The value giving the best fit to ground-based observations of $\delta$ Sct stars is $\alpha_{\mathrm{MLT}}=1.8$ (Dupret et al., 2005). They state that using the correct value of $\alpha_{\mathrm{MLT}}$ in the modelling is much more important than the use of TDC over FC models, although TDC becomes increasingly important as pulsation frequencies increase and temperatures decrease. Much lower values of $\alpha_{\mathrm{MLT}}$ have been preferred by some authors not using TDC models $\left(\alpha_{\mathrm{MLT}} \approx 0\right.$, Daszyńska-Daszkiewicz et al. 2005; $\alpha_{\mathrm{MLT}}=0.5$, Casas et al. 2006, 2009).

The next most important parameter is metallicity $(Z)$. With lower $Z$, evolutionary tracks shift to higher effective temperature and higher surface gravity (Fig. 4.2). Although higher surface gravities are ordinarily associated with denser bodies, i.e. those with smaller radii and correspondingly lower luminosities, the luminosity is much more sensitive to temperature than to radius ( $T^{4}$ vs. $R^{2}$ ), so metal-poor stars of the same mass and age are more luminous, too. The higher luminosity causes core hydrogen to be exhausted more quickly, and the time spent on the main sequence is slightly reduced. 


\section{CHAPTER 4}

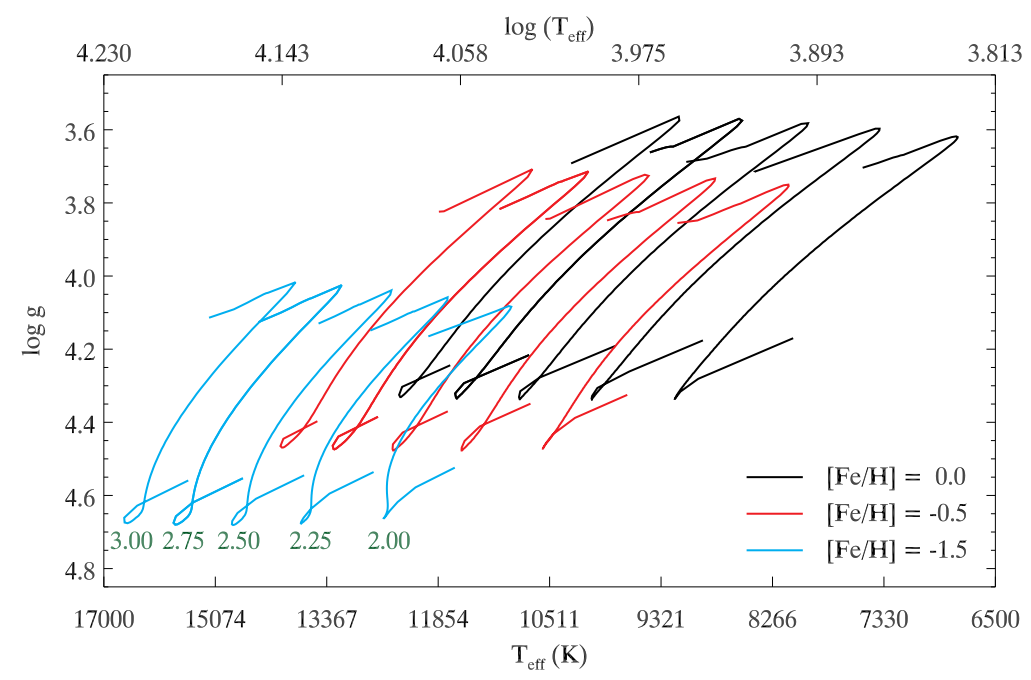

Figure 4.2: The effect of metallicity on evolutionary tracks for stars of $2.0-3.0 \mathrm{M}_{\odot}$. Note that although $\log g$ increases for lower- $Z$ stars, so does $T_{\text {eff }}$ such that for their age, the metal-poor stars are more luminous than solar-metallicity stars. Based on tracks provided by J.C. Suarez (priv. comm.).

McNamara (2000) showed that stars of lower metallicities have shorter pulsation periods (his figure 1). Stability against pulsation occurs at cooler temperatures for lower $Z$ stars (Grigahcène et al., 2006), but since the driving zone for $\delta$ Sct stars is the He II partial ionisation zone, metallicity plays only a small role in shifting the instability strip edges. Instead, it is the evolutionary tracks that are shifted, meaning stars of a given mass cross the red or blue edge at different ages, and will pulsate at different stages of their main-sequence lives.

Another important parameter affecting the evolutionary tracks is core overshoot. Core convective overshoot lengthens the time spent on the main-sequence, and one goal of $\delta$ Sct star models is to constrain this parameter. The effect of a large overshooting parameter is that the tracks shift to higher $T_{\text {eff }}$, and, because core material is replenished, the main sequence widens (Grigahcène et al., 2006).

The $\delta$ Sct instability strip overlaps with the instability strip of the rapidly oscillating Ap (roAp) stars, and most notably with that of the $\gamma$ Dor stars. While this leads to hybrid pulsators in the $\delta$ Sct- $-\gamma$ Dor case (we will cover hybrid pulsation 


\section{CHAPTER 4}

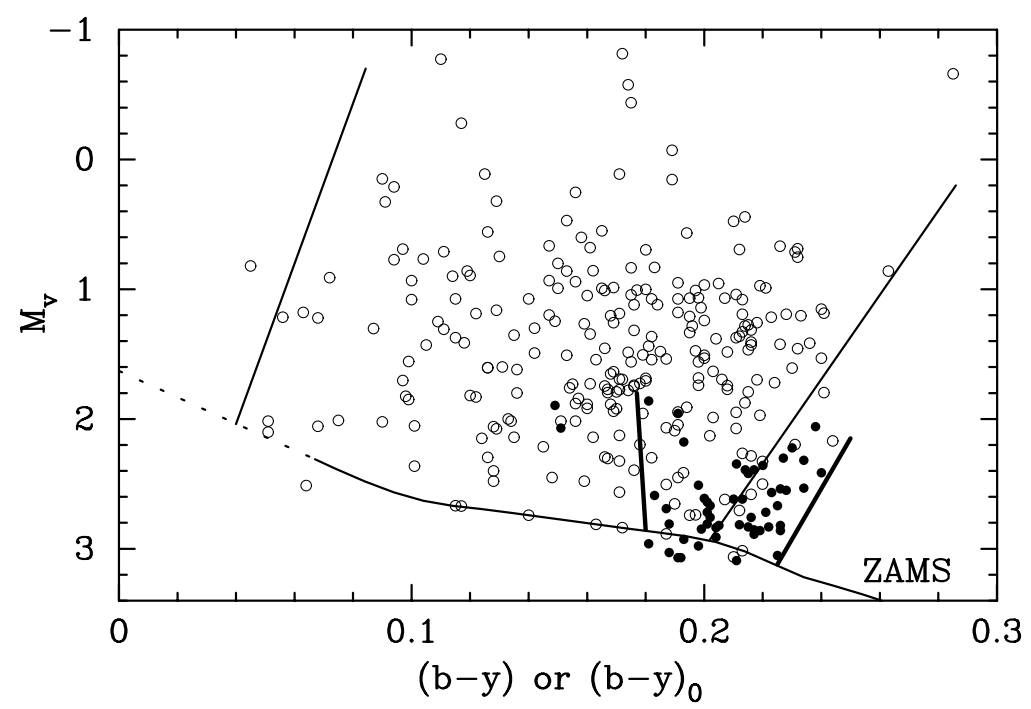

Figure 4.3: The location of $\delta$ Sct and $\gamma$ Dor stars in a colour-magnitude diagram. The open circles represent all $\delta$ Sct stars for which Strömgren colours and Hipparcos parallaxes are available, whereas the filled circles show all $\gamma$ Dor stars with the same information. The Zero-Age Main Sequence (ZAMS) is indicated, taken from the standard relations by Crawford $(1975,1979)$, and is extrapolated towards low $(b-y)$ (dotted part of the ZAMS). The observational borders of the two pulsational instability strips (Rodríguez \& Breger 2001; Handler \& Shobbrook 2002) with the thicker lines corresponding to the $\gamma$ Dor strip, are also shown. Figure and caption from Handler (2005, his figure 1). Permission obtained from Springer.

in $\S 4.5 .3)$, non-adiabatic analyses indicate $\delta$ Sct pulsation is suppressed by magnetic fields exceeding $1 \mathrm{kG}$ in roAp stars (Saio, 2005). Fig. 4.3 shows the overlap of the $\delta$ Sct and $\gamma$ Dor instability strips, populated with some pulsators known from ground-based data, in a colour-magnitude diagram. There are also many stars in the $\delta$ Sct instability strip that do not pulsate at all. Even in the centre of the strip (7000-8000 K), Balona \& Dziembowski (2011) determined only 30 to 50 per cent of stars to be pulsating (at some unclear amplitude limit, presumably $100 \mathrm{ppm}$ ), and Catanzaro \& Balona (2012) claimed even fewer pulsate. In Chapter 1, we found that 44 per cent of stars in the $T_{\text {eff }}$ range $7000-8500 \mathrm{~K}$ have no peak above $50 \mu$ mag. 


\subsection{Mode selection and identification}

\subsubsection{Observability of modes}

The number of surface nodes of a pulsation mode is equal to its degree, $\ell$. The regions equidistant from two nodes are called antinodes, and antinodes on either side of the same node will have opposite amplitudes (or, the same amplitude with opposite phase, if one prefers). Thus if both of those antinodes are in view their opposing effects on the observed luminosity partially cancel out. Since higher degree modes feature more nodes on the surface, and therefore more antinodes, the partial photometric cancellation is greater - a smaller fraction of uncancelled antinodes can be distributed over the boundary of the observable and unobservable hemispheres. For this reason, the modes that are directly observable in $\delta$ Sct stars are restricted to low $\ell$ (Dziembowski, 1977a). Effects such as limb and gravity darkening, which further modify the flux observed from particular regions, can influence the extent of the partial cancellation, but only a little. The result is that the amplitudes of even $\ell$ modes are greatly reduced for $\ell \geq 4$, and all odd $\ell$ modes would have zero amplitude were it not for limb darkening (Aerts, Christensen-Dalsgaard \& Kurtz, 2010, their figure 1.5), with the exception of the $\ell=1$ modes, whose amplitudes can actually exceed those of radial modes, depending on the location of the node with respect to the observer. Nevertheless, Balona \& Dziembowski (1999) described the disk-averaging effect as "not very strong" with other terms becoming important for $\ell \gtrsim 6$, and showed that in Strömgren $u$ and $y$ filters, modes with $\ell>6$ still have amplitudes of tens of $\mu$ mag. Daszyńska-Daszkiewicz, Dziembowski \& Pamyatnykh (2006) show that for $\ell \lesssim 16$ (even) and $\lesssim 11$ (odd), non-linear limb-darkening laws lead to a disk-averaging effect that reduces amplitudes by a factor $<1000$, such that modes with intrinsic amplitudes of several mmag with these $\ell$ values would be observable with Kepler data. Furthermore, at increasingly high rotation rates 


\section{CHAPTER 4}

the node spacing of modes with moderate $\ell-|m|$ can become uneven, reducing the efficiency of the disk-averaging effect (Lignières \& Georgeot, 2009). That is not to say high-degree modes should be observed with high amplitude, but rather that they can be visible at some (low) amplitude.

Daszyńska-Daszkiewicz et al. (2002) presented different evidence for the importance of moderate $\ell$ modes to be considered in asteroseismic analyses. For moderate rotation velocities, typical of $\delta$ Sct stars, modes with $\ell>2$ acquire substantial $\ell \leq 2$ components and are thus easily detected. Specifically, mode interaction in the presence of moderate rotation allows modes whose spherical degrees differ by two or four to interact, such that the higher degree mode acquires a large component of the lower-degree mode. Fig. 4.4, reproduced from Reese et al. (2013), illustrates this excellently. ${ }^{1}$ Although it is not clear to what amplitudes $\ell=9$ modes are excited in their model, they appear to reach amplitudes similar to those of $\ell=1$ modes, which in turn can be the highest possible for $\delta$ Sct stars.

Models indicate that very high degree modes should be excited; Dziembowski (1977b) found the maximum of instability occurs for $\ell \sim 500$ for $\delta$ Sct stars. Although modes of such high degree cannot be detected, modes with degrees as high as 20 were spectroscopically confirmed in $\tau$ Peg (Kennelly et al., 1998). Even still, there remains an issue that $\delta$ Sct stars appear not to pulsate in modes of all degrees that are predicted to be unstable (e.g. Goupil et al. 2005). We already stated that some stars in the instability strip appear not to pulsate at all, and so it is clear that pulsation-damping mechanisms are not fully understood.

The two leading mechanisms for amplitude limitation (which for non-pulsating stars implies an amplitude limit below the detection limit), are mode coupling and

\footnotetext{
${ }^{1}$ Footnote to Fig. 4.4: Reese et al. (2013) provided a distinction between the critical and Keplerian break-up rotation rates: "the former uses true gravity (excluding the centrifugal force) at the equator, $g_{\text {eq }}$, based on the actual distribution of matter, to calculate the break-up rotation rate, whereas the latter uses its Keplerian approximation $\frac{G M}{R_{\mathrm{eq}}^{2}}$, which amounts to assuming spherical symmetry for the distribution of matter." They go on to mention that Roxburgh (2004) pointed out the Keplerian approximation slightly underestimates the true gravity, such that $\Omega_{\mathrm{C}}>\Omega_{\mathrm{K}}$.
} 


\section{CHAPTER 4}
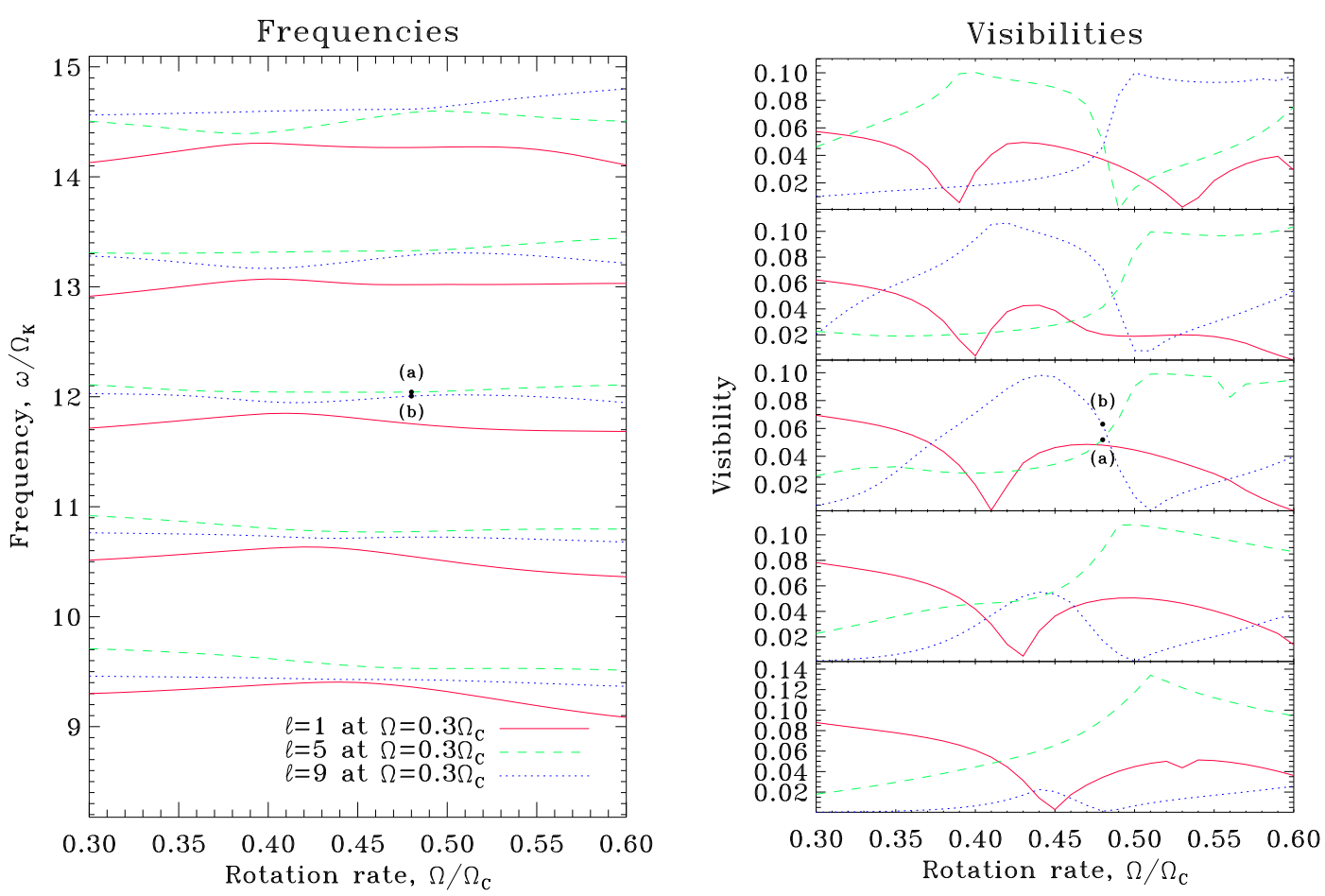

Figure 4.4: A set of interactions between $\ell=1,5$ and 9 modes with $m=1$, for 30 per cent of the critical rotation frequency, $\Omega_{\mathrm{C}}=\left(g_{\mathrm{eq}} / R_{\mathrm{eq}}\right)^{1 / 2}$. The left panel shows the frequencies as a fraction of the Keplerian break-up rotation rate, $\Omega_{\mathrm{K}}=\left(G M / R_{\mathrm{eq}}^{3}\right)^{1 / 2}$ (see footnote 1 ). The right panel shows the visibility (in terms of a normalised contribution to fractional change in luminosity) in the CoRoT photometric band for $i=30^{\circ}$. Points (a) and (b) mark two modes undergoing an avoided crossing. Figure from Reese et al. (2013, their figure 11), permission obtained from A\&A.

saturation of the driving mechanism. In the former scenario, energy is transferred between modes in a parametric resonance (Dziembowski \& Krolikowska, 1985), for which amplitudes are further limited by rotation if $v_{\text {eq }}>20 \mathrm{~km} \mathrm{~s}^{-1}$ (Dziembowski, Krolikowska \& Kosovichev, 1988), which encompasses the majority of $\delta$ Sct stars. Buchler, Goupil \& Hansen (1997) further investigated mode coupling, and showed resonances can even occur between $\mathrm{p}$ and g modes. The g modes exist in the deep interior and do not attain large amplitudes near the surface, thus it is easily possible for p-mode amplitudes to be limited by modes that are not even detectable at the surface. 


\section{CHAPTER 4}

Saturation of the driving mechanism does not appear to be an adequate explanation on its own, in that it is hard to imagine that this mechanism is limiting for the non-pulsating stars. Another suggestion is that for some reason these stars pulsate only in modes of degrees too high to be observable. This suggestion, like the mode-coupling and saturation hypotheses, still does not explain why some stars are observed to pulsate and some do not. It only changes the question.

\subsubsection{Mode selection and frequency distribution}

Our discussion has been limited to the visibility of non-radial modes, mostly, but the radial pulsators have amplitudes 10-100 times greater than non-radial pulsators (Breger, 2000b). That is not to say that radial modes always have high amplitudes, but the highest-amplitude modes in many stars can be identified as the fundamental radial mode, or first and second overtones; it is the hotter $\delta$ Sct stars that tend to pulsate in higher (up to $n \sim 7$ ) radial overtones (Pamyatnykh, 2000).

In a statistical study into the $\delta$ Sct stars observed by Kepler, Balona \& Dziembowski (2011) compared the observed frequency of maximum amplitude against that from the models (Fig. 4.5). The models indicate the trend for hotter stars to pulsate at higher frequencies, but the observations show hot stars can have their frequency of maximum amplitude at lower frequency, too. Observed frequencies of maximum power have a much larger range than predicted in the models, further highlighting that competition between pulsation excitation and damping mechanisms is not fully understood.

Balona \& Dziembowski (2011) also investigated the frequency range over which the stars pulsate, with curious results (Fig. 4.6). Firstly, the models indicate that for any given temperature, the frequency range of pulsation can differ by up to $30 \mathrm{~d}^{-1}$, though no explanation is provided. For instance, it is not stated whether the helium abundance, age or some other parameter determines the range over which 


\section{CHAPTER 4}

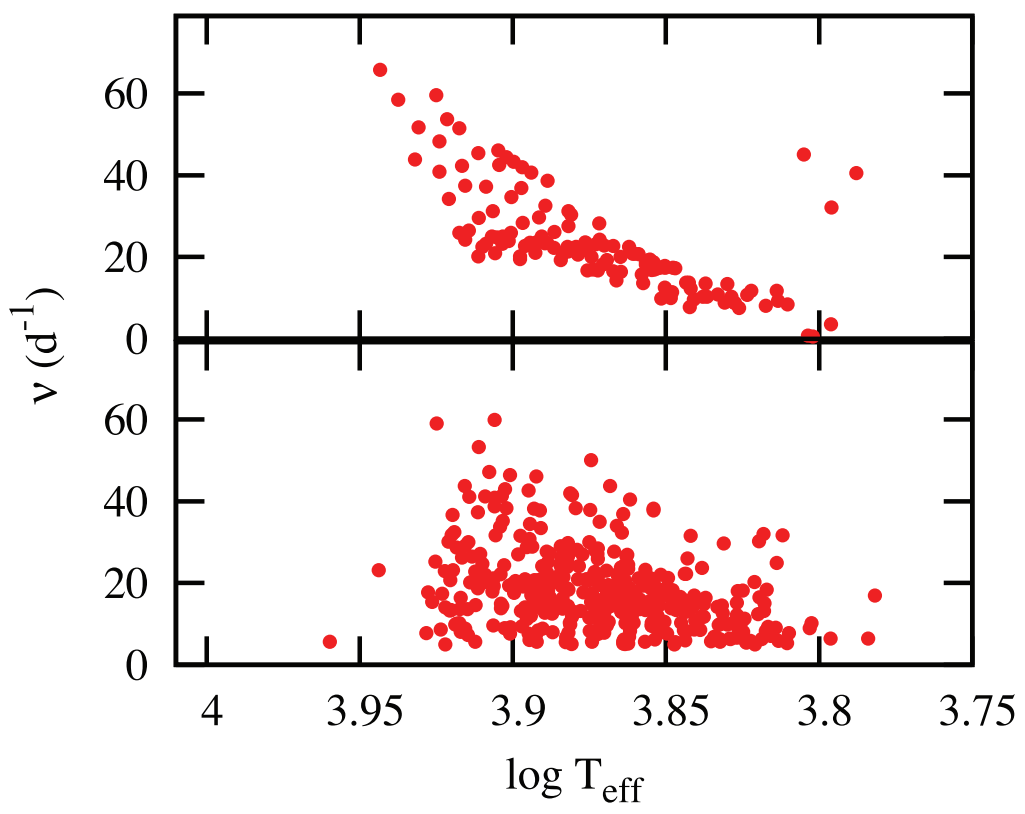

Figure 4.5: Top panel: the frequency with highest growth rate for $0 \leq \ell \leq 2$ as a function of effective temperature from the models. Bottom panel: the frequency of maximum amplitude, $\nu$, as a function of effective temperature for Kepler $\delta$ Sct stars. Figure and caption from Balona \& Dziembowski (2011, figure 2), permission obtained from MNRAS.

pulsation is seen. Secondly, the observations do not match the models at all and no convincing explanation is provided for that, either. It appears that pulsation mode frequencies from the models have been compared with observed frequencies of any kind, and it is important to recognise that the two are not the same. For instance, harmonics (of form $n f_{i}$ ) or more generally combination frequencies $\left(a f_{i} \pm b f_{j}\right)$ are included in the observed frequencies, but these are not mode frequencies, and so are not counted in the models. This is important because the presence of even the simplest combinations, those with $(a, b)=(1,1)$, has a profound effect on the frequency range measured, and renders the comparison meaningless. Furthermore, much attention has been given to low frequencies in A-F stars, pulsating or not, and these 'rotational light variations' are distinctly claimed not to be pulsation modes (Balona, 2011). It is unclear whether these non-mode frequencies were included in the frequency range determinations, too. 
CHAPTER 4

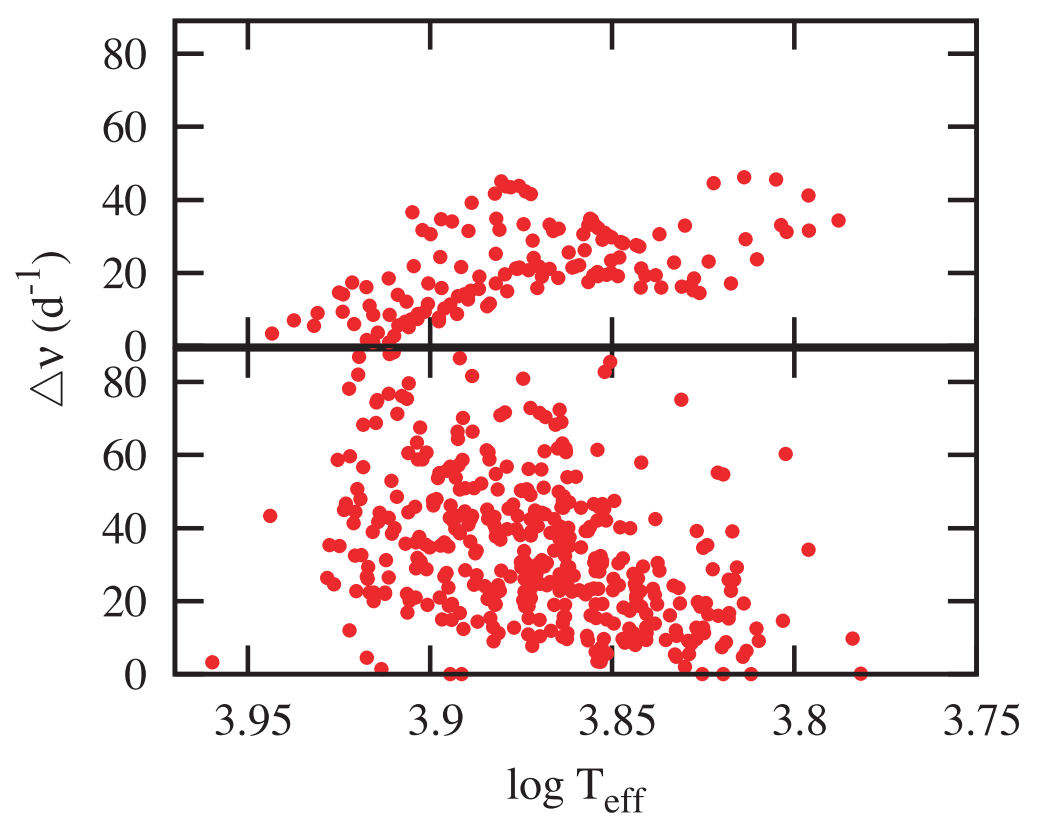

Figure 4.6: Top panel: the frequency range of unstable modes for $0 \leq \ell \leq 2$ as a function of effective temperature from the models. Bottom panel: the frequency range, $\Delta \nu$, as a function of effective temperature for Kepler $\delta$ Sct stars. Figure and caption from Balona \& Dziembowski (2011, figure 3), permission obtained from MNRAS. 


\section{CHAPTER 4}

Radial modes in $\delta$ Sct stars show a preferred, non-equal spacing. The spacing approaches an asymptote in frequency for high $n(\sim 20)$ only, as in the solar-like oscillators. Using frequencies extracted from the light curve of the $\delta$ Sct star FG Vir, Breger, Lenz \& Pamyatnykh (2009) calculated frequency differences between each and every frequency. They found that the non-radial modes also have a preferred frequency spacing, matching that of the radial ones - the non-radial modes cluster around the radial modes. Similar evidence for 'characteristic spacing' between modes of consecutive radial order was presented earlier for both XX Pyx (Handler et al., 1997) and FG Vir (Breger et al., 1999b), and with space-based data is seeing a resurgence in the literature (e.g. García Hernández et al. 2009). We return to this at the end of $\S 4.4 .5$.

\subsubsection{Mode identification}

Modelling $\delta$ Sct stars is difficult because of the 'almost overwhelming' parameter space to be explored (Guzik, Templeton \& Bradley, 1998), which includes the helium $(Y)$ abundance, heavy element abundance $(Z)$, element mixture (both through atomic diffusion and initial abundances), and internal rotation profile. Add to that the atmospheric parameters $T_{\text {eff }}$ and $\log g, v \sin i$ (i.e., inclination is unknown and convolved with the rotation velocity), the extent of core overshoot, and atmospheric stratification in the case of chemically peculiar stars. Then one has the task of matching the observed frequencies with theoretical ones from the models. Such factors create the need for as many observational constraints as possible, including direct mode identification.

Mode identification is extremely limited with white-light photometry alone. Multicolour photometry, radial velocity curves and high-resolution spectroscopy are other tools deployed in asteroseismology to identify modes.

Multicolour photometry seeks to identify phase differences and amplitude ratios 


\section{CHAPTER 4}

between pulsation frequencies in different colour filters. Analytic expressions for this technique were formed by Dziembowski (1977a), Balona \& Stobie (1979), and Stobie \& Balona (1979). Use of the technique for mode discrimination was presented by Stamford \& Watson (1981). Watson (1988) demonstrated the applicability to many classes of pulsators, and later Garrido, Garcia-Lobo \& Rodriguez (1990) and Balona \& Evers (1999) refined the technique and applied it to $\delta$ Sct stars. Mode identification from multicolour photometry is well discussed in Chapter 6 of Aerts, Christensen-Dalsgaard \& Kurtz (2010). The results obtained with this method are very sensitive to convection treatment, which in itself has been used to probe stellar convection (Daszyńska-Daszkiewicz, Dziembowski \& Pamyatnykh, 2003; DaszynskaDaszkiewicz et al., 2004). The phase lag between maximum brightness and minimum radial velocity also helps to identify modes - this is why spectroscopy is often sought with simultaneous photometry, a task that was made easier with the abundance of Kepler data. Surface radial velocity variations induced by pulsations act to periodically Doppler-shift spectroscopic line profiles, leading to the use of spectroscopy to identify modes of high degree that was mentioned earlier. These so-called LPV (Line-Profile Variation) analyses were established by Osaki (1971) with the $\beta$ Cep stars, but much work in the development of the technique was done by Aerts, so the reader is once again referred to the thorough text of Aerts, Christensen-Dalsgaard \& Kurtz (2010). Since this thesis is primary concerned with Kepler data, we will leave the discussion of multicolour and spectroscopic mode identification at this point, and discuss next the mode identification that can be made with white-light photometry.

Theoretical mode frequencies for the $\delta$ Sct stars are well studied. Therefore, when two frequencies of high amplitude are detected in a $\delta$ Sct star, one can use the frequency ratio (or its reciprocal, the period ratio) to try to identify the modes. Petersen \& Christensen-Dalsgaard (1996) calibrated the theoretically expected frequency ratio $(=0.77)$ for the fundamental radial mode $\left(\mathrm{F}\right.$; period $\left.\Pi_{0}\right)$ and first radial 


\section{CHAPTER 4}

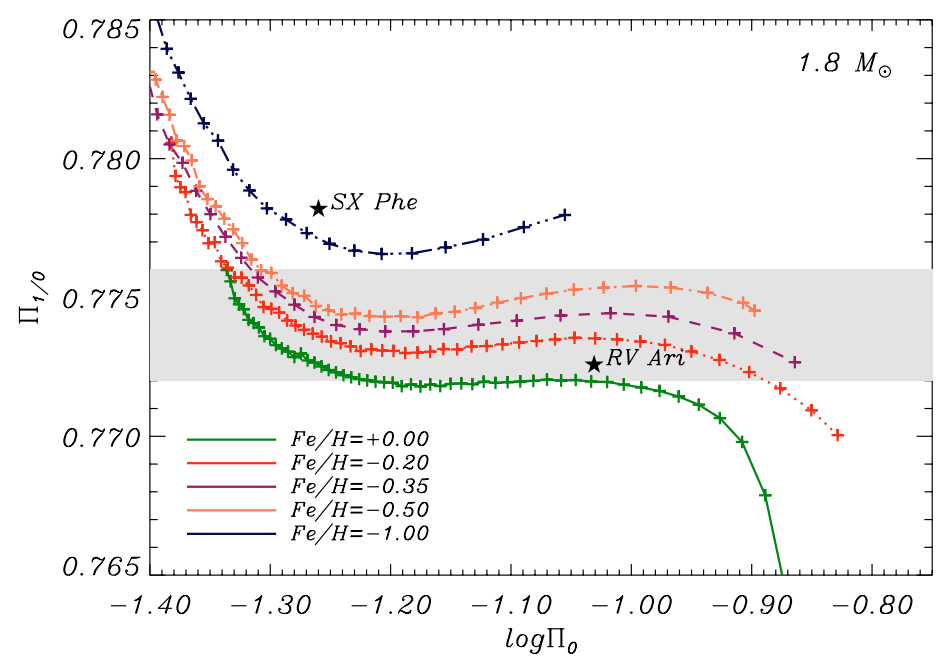

Figure 4.7: Typical Petersen diagram $\left(\Pi_{0}\right.$ in $\left.\mathrm{d}\right)$ containing different tracks of $1.8-\mathrm{M}_{\odot}$ evolutionary models computed with different initial metal content $[\mathrm{Fe} / \mathrm{H}]$, from solar composition (bottom, solid line) to -1.00 (top, triple dot-dashed line). Crosses represent non-rotating models. The shaded area corresponds to typical values found for Pop. I $\delta$ Sct stars. The two filled-star symbols represent the observed $\Pi_{1 / 0}$ of the double-mode HADS stars RV Ari and SX Phe, as an illustration of (Pop. I) and (Pop. II) stars, respectively. Figure and caption from Suárez, Garrido \& Goupil (2006, their figure 2), permission obtained from A\&A.

overtone $\left(10\right.$; period $\left.\Pi_{1}\right)$ with stars of different metallicity. They plot their results on a period-ratio diagram, which is now more commonly known as a Petersen diagram after their work ${ }^{2}$. A similar diagram is shown in Fig. 4.7 from Suárez, Garrido \& Goupil (2006). The diagram shows how metallicity modifies the period ratios, in that metal-poor stars have higher frequency ratios, and notice that for frequencies common in $\delta$ Sct stars, ratios under about 0.772 are not found. If the stellar metallicity is well constrained, one can identify the $\mathrm{F}$ and $1 \mathrm{O}$ modes in this manner (if they are excited), or even the $1 \mathrm{O}$ and second radial overtone $(2 \mathrm{O})$ modes when the frequency ratio is 0.80 .

This mode identification method often works for the HADS stars because high

\footnotetext{
${ }^{2}$ The diagrams were first discussed by Petersen (1978), but it was Art N. Cox who brought the term 'Petersen diagram' into general use through his conference talks and eventually in his journal articles. Published use of the term dates back to the note on HD 161796 in 1982 (Fernie, 1982). Footnote from Murphy et al. (2013).
} 


\section{CHAPTER 4}

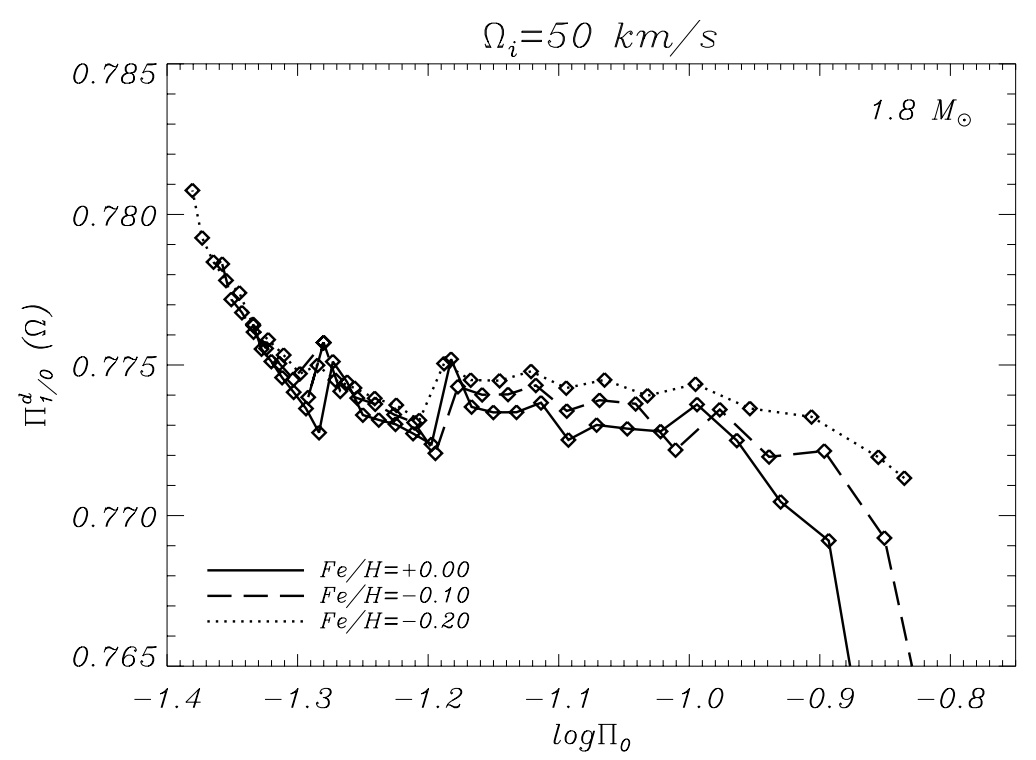

Figure 4.8: The influence of mode interaction on the Petersen diagram. Contrast against the comparatively smooth non-rotating models in Fig. 4.7. Figure from Suárez, Garrido \& Moya (2007, their figure 5). Permission obtained from A\&A.

amplitude modes are present, such modes are often radial (and hence give a positive I.D. as the F/1O or 1O/2O modes), and rotation is slow. Suárez, Garrido \& Goupil (2006) also showed how moderate rotation ( of $\Omega=50 \mathrm{~km} \mathrm{~s}^{-1}$ ) causes the frequency ratio to change by more than 0.001 , causing tracks of different metallicities to overlap (though the effect is much less pronounced at $\Omega=25 \mathrm{~km} \mathrm{~s}^{-1}$ ). When the modeinteraction effects of close frequencies are included, which are stronger at higher rotation rates, the situation worsens. For most values of $\log \Pi_{0}$, mode interaction has little effect on $\Pi_{1}$, and almost no effect on $\Pi_{0}$, but for $\log \Pi_{0} \approx-1.2$ (corresponding to a frequency of $15.8 \mathrm{~d}^{-1}$ ) the effect is very strong (Fig. 4.8), causing a difference in $\Pi_{1 / 0}$ of almost 0.002 at $50 \mathrm{~km} \mathrm{~s}^{-1}$ (Suárez, Garrido \& Moya, 2007). The conclusion is, although the frequency ratio can be useful, there is a certain degeneracy and uncertainty introduced by rotation and mode interaction. One would need good photometry for the mode frequencies, and spectroscopy for an indication of $Z$ and $v \sin i$, before the Petersen diagram is of diagnostic use. 


\section{CHAPTER 4}

\subsubsection{Mode density}

Owing partly to the effects of mode interaction, mode density is one of the current problems in $\delta$ Sct research. As the amplitude detection limit for these stars is being not only continually lowered, but with space missions has taken a drop of a factor of over a hundred, the number of detected frequencies has increased rapidly. So rapidly, in fact, that the reports of a few hundred modes being excited in the same star, for two $\delta$ Sct stars observed by CoRoT (HD 50844 by Poretti et al. 2009; and HD 174936 by García Hernández et al. 2009), incited discussions in the literature that tried to explain or even discredit those findings. One such attempt is that of Moya \& Rodríguez-López (2010), who determined from energy considerations that it is indeed possible for a star to excite so many modes to the observed amplitudes simultaneously. Kallinger \& Matthews (2010), on the other hand, sought to explain the mode density as granulation. Surface convection is still expected in the cool $\delta$ Sct stars, albeit in a very thin layer - hence the need for TDC models as outlined in $§ 4.3$ - though Kallinger \& Matthews do note the number of granulation cells on the stellar surface is more important than the depth of the convection zone itself. Nevertheless, the granulation hypothesis received mixed support: Balona \& Dziembowski (2011) state that evidence for granulation in the A stars is weak, citing Balona (2011), even though the latter reference actually concluded that there is a gradual increase in overall stellar noise level in the A stars with decreasing effective temperature. This was interpreted as the increasing importance of granulation in cooler A stars. The case was verified in Chapter 1, where the noise as a function of temperature shows evidence for granulation for stars cooler than $7500 \mathrm{~K}$ (Fig. 1.17 there).

Let us re-examine the case for granulation for HD 50844. Poretti et al. (2010) argued that the noise excess observed in HD 50844 cannot be due to granulation because of the star's spectral type. Since they had two A2 stars, one exhibiting 


\section{CHAPTER 4}

noise excess (HD 50844), the other not (HD 171586), they concluded granulation cannot be responsible because it would operate equally in both stars. They may have a point, in that is it unheard of to observe granulation at a spectral type as early as A2. However, inspection of the $(b-y), \beta$ and $c_{1}$ indices from Hauck \& Mermilliod (1998) for HD 50844 indicates the star is almost certainly of late-A type, in agreement with Poretti et al.'s derived $T_{\text {eff }}$, and consistent with the observed $\delta$ Sct pulsation (the theoretical $\delta$ Sct instability strip does not extend to spectral types as early as A2; cf. Fig. 2.1 in Chapter 2 and, e.g. Uytterhoeven et al. 2011). HD 50844 is thus, contrary to their argument, a valid candidate in which to observe granulation. For the record, $(b-y)$ and $\beta$ values put the non-pulsating star HD 171586 exactly at $\mathrm{A} 2$, where one expects neither $\delta$ Sct pulsation nor granulation, rendering the statement in their discussion that "noise excess is not observed in constant stars" moot. An investigation into whether granulation is more prevalent in $\delta$ Sct stars vs. non-pulsating stars inside the $\delta$ Sct instability strip would be worthy of pursuit. Furthermore, the noise excess seen by Poretti et al. (2010) is similar to the noise injected by the Kepler PDC-LS pipeline, as illustrated in Chapter 1 (§ 1.2.4), leading this author to suggest that an instrumental origin for the extreme mode density has not been definitively ruled out. That a CoRoT target observed at the same time as HD 50844 does not show the same noise excess is not sufficient evidence to suggest the noise excess is an extremely dense set of pulsational modes.

We know granulation is a possible explanation; is mode density really a viable explanation, too? Balona \& Dziembowski (2011) carried out an independent investigation into the mode density of $\delta$ Sct stars, and found that the extreme mode density reported by Poretti et al. (2009) and García Hernández et al. (2009) in CoRoT data is only reproduced in very small frequency regions in the Kepler $\delta$ Sct stars. It seems likely that an instrumental contribution to the CoRoT power spectra was occurring.

What does mode density have to do with mode interaction? It is easy to see that 


\section{CHAPTER 4}

the more modes present, the more closely-spaced in frequency they will be, thus the more likely it is they will interact. Evolution contributes directly to the increasing mode density - more modes become unstable in $\delta$ Sct stars as they evolve. Handler (2005) presented a model example where the number of unstable frequencies predicted increased three-fold between the ZAMS and TAMS. Furthermore, interactions proceed with evolution: as $\delta$ Sct stars evolve, the convective cores shrink and the convective envelopes expand, causing a frequency increase of g modes but a frequency decrease of p modes (Handler, 2009). Rather than crossing over smoothly in frequency, these modes interact to produce 'mixed modes' - modes that have a p-mode character in the envelope and a g-mode character in the interior. When the frequencies become close enough, one mode is bumped to a different frequency and the other takes its initial place (Aizenman, Smeyers \& Weigert, 1977). These 'avoided crossings' were demonstrated in Fig. 4.4.

\section{Rotational splitting}

As we mentioned in $\S 4.4 .3$, the interaction strength is dependent on rotation, but this is not the only contribution from rotation. Since modes with the same $(n, \ell)$ are only degenerate in frequency in the spherically symmetric case, the departure from spherical symmetry induced by rotation causes the modes of different azimuthal order to split (Ledoux, 1951). That is, $2 \ell+1$ modes will be seen instead of just one peak, though not all will have the same amplitude: prograde modes, under the definition as those with the highest frequencies in the observer's reference frame and the most-negative $m$ values, are theoretically expected to be more unstable (Hansen, Cox \& Carroll, 1978), both for white dwarfs and main-sequence stars. For the main sequence stars there are unconfirmed observational reports (Smith, 1982) that this is the case. For the white dwarfs, or specifically the DOV star PG 1159-035 (Winget et al., 1991), the results are inconclusive, with the average power in $\ell=1$ 


\section{CHAPTER 4}

modes showing $m= \pm 1$ values having identical amplitudes, but the $l=2$ modes having much more power in retrograde modes. One must remember that inclination plays a role - the exact morphology of rotationally split multiplets depends on both excitation and orientation of the rotation axis (also called the inclination angle, $i$ ). This entanglement offers extra information, either on $i$ or mode excitation, if either one of those can be inferred by other means.

Although rotational splitting adds to the mode density, and thus the greater potential for mode interaction, it offers another key benefit. If the rotationally split multiplet of modes with the same $(n, l)$ values has predictable splitting, related to the rotation frequency of the star, then one can use this splitting to infer the rotation velocity, and in turn the inclination of the star if $v \sin i$ is observed. In the reference frame of the observer, the formula

$$
\omega=\omega_{0}+\left(1-C_{L}\right) m \Omega+D_{L} \frac{m^{2} \Omega^{2}}{\omega_{0}}
$$

adapted from Dziembowski \& Goode $(1992)^{3}$, is commonly used to describe the effects rotation up to second order. Here, the observed frequency, $\omega$, is related to the true pulsational frequency, $\omega_{0}$, and the angular rotation frequency, $\Omega$, through two Ledoux coefficients $\left(C_{L}\right.$ and $\left.D_{L}\right)$, which depend on mode character and were calculated by Saio (1981). At first order, one can see that consecutive $m$ values are spaced in frequency by an equal amount. However, at second order the rotationally split frequencies should not have exact spacings. Dziembowski \& Goode (1992) developed the calculations of the expected departure from equal spacings in $\delta$ Sct stars, and Murphy et al. (2013) used these to confirm mode identification for KIC 11754974.

Nevertheless, non-equal spacing is not commonly observed. This could be a

\footnotetext{
${ }^{3}$ modified so that here, in the observer's frame, the most negative m-values represent the prograde modes with the highest frequencies.
} 


\section{CHAPTER 4}

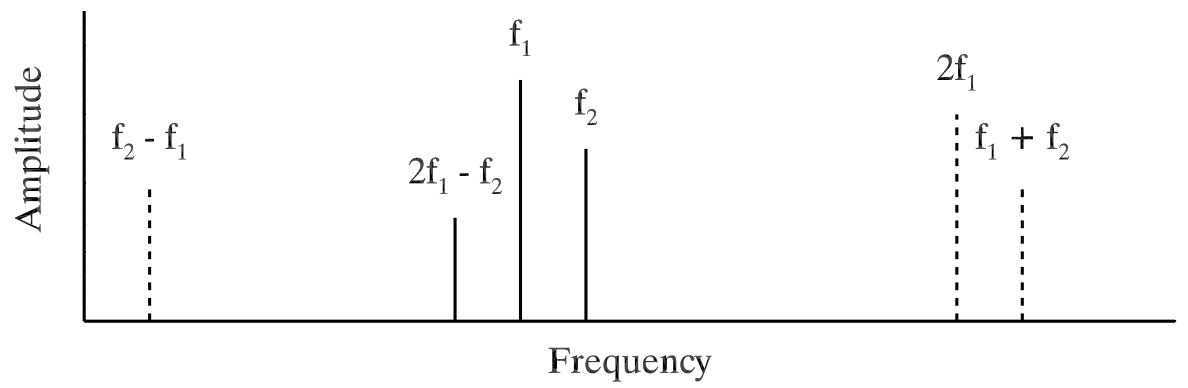

Figure 4.9: Combination modes can generate an equally spaced triplet. Based on figure 1 of Breger \& Kolenberg (2006).

selection effect: an observer is less likely to notice or label a set of peaks (particularly in a triplet) as being a rotationally split multiplet if the splittings are not equal. Hence the majority of multiplets identified will be equally spaced. Additionally, case studies of $\delta$ Sct stars tend to focus on slower rotators where the second order effects are not dominant, and departure from equal spacing will be small. Again, this results in most multiplets being recorded as equally spaced. But the situation is further complicated by combination frequencies. An exactly equally split multiplet can be generated if two close frequencies, $f_{1}$ and $f_{2}$ (where $f_{2}$ lies at a higher frequency than $f_{1}$ ), are excited to moderate amplitudes in a $\delta$ Sct star (Breger \& Kolenberg, 2006). They may have a combination, $2 f_{1}-f_{2}$, at an observable amplitude that appears in the Fourier transform to be a part of a triplet (Fig. 4.9). In addition, nonlinear theory not only permits, but indicates an increased likelihood of equally spaced modes due to resonant coupling (Buchler, Goupil \& Hansen, 1997). This, and the uncertain intrinsic amplitudes of modes of different $m$ (for the same $n, \ell$ ), makes modelling of $\delta$ Sct stars rather difficult.

The rotational splitting will vary from mode to mode when the rotation rate depends on latitude or depth. Hence for low rotational velocities, rotation can be treated with a perturbative approach to account for the spherical asymmetry, and the inclination is calculable in the models from rotationally split multiplets. For fast rotators, however, the situation is far more complicated. Here, rotationally split 


\section{CHAPTER 4}

multiplets are more widely spaced in frequency, becoming overlapped. Modes take on entirely different natures resulting in a higher mode density, and the treatment is far more complex. We refer the reader to the work of Reese and collaborators (e.g. Reese et al. 2013) for further reading on rapidly rotating pulsators.

\subsubsection{The amplitude detection limit}

Let us return to our discussion of mode density and the amplitude detection limit. It might appear at first that the reduction of the amplitude detection limit has created problems in that we now have too much information. It is the sensitivity to such information, and the nearly uninterrupted photometry, for which the arrival of space-based observations were hailed long in advance. But concern was also expressed that the oscillation spectra would be dominated by high degree modes of unknown azimuthal order, rendering mode identification an impossible task (Daszyńska-Daszkiewicz, Dziembowski \& Pamyatnykh, 2006).

Have we reached the limit of what is detectable, i.e. modes with amplitudes equal to the photospheric noise? Before the advent of space telescopes, we suspected that there would be modes with amplitudes below the ground-based detection limit because if one binned the stars by pulsation amplitude, $A$, the lowest amplitude bin (corresponding to, say, $A<0.005 \mathrm{mag}$ ) would contain many more stars than the next bin up. Two explanations resulted: there are many constant (non-pulsating) stars in the $\delta$ Sct instability strip, or there are many pulsators whose amplitudes were not yet detectable. It turns out that both were true.

In Chapter 1, the number of non-pulsating stars at the $50 \mu$ mag level, whose KIC $T_{\text {eff }}$ puts them inside the $\delta$ Sct instability strip, was determined to be 44 per cent. However, KIC temperatures can be unreliable, so a ground-based spectroscopic follow-up on the non-pulsating stars is now in preparation to determine whether these stars truly lie inside the $\delta$ Sct instability strip. Preliminary results of the 


\section{CHAPTER 4}

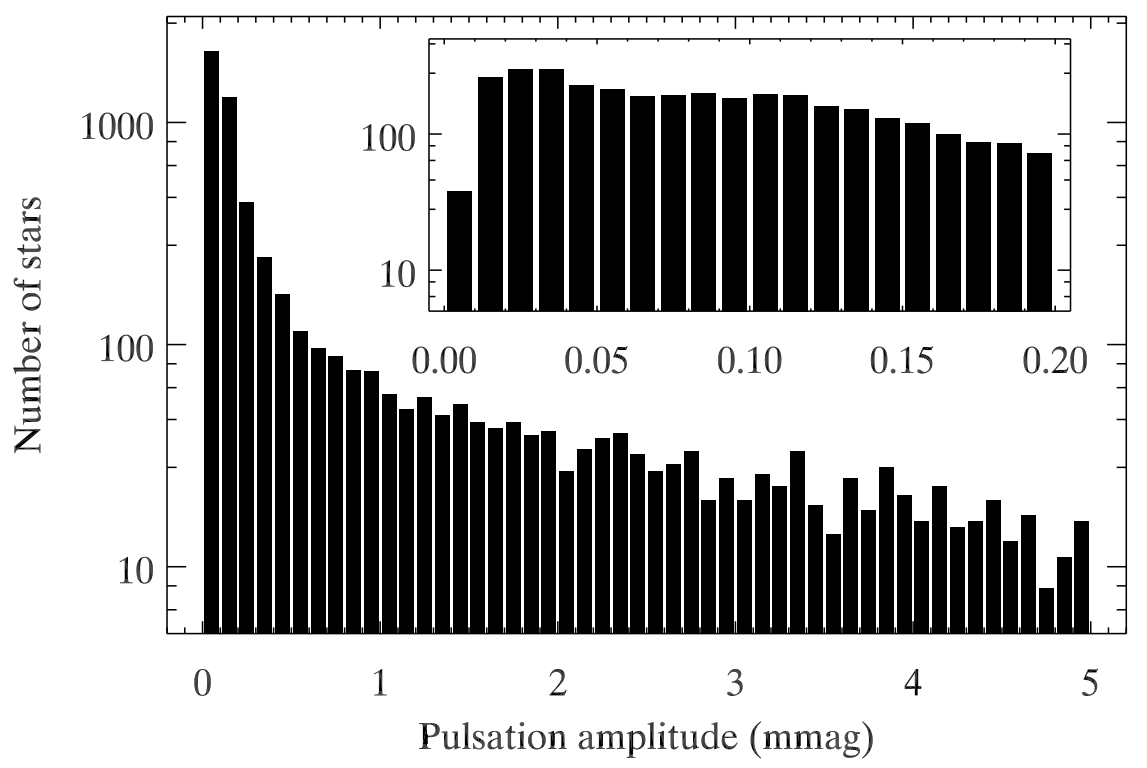

Figure 4.10: Highest amplitude in each Fourier transform of PDC MAP Q3 data, for stars with KIC temperatures between 6500 and $8500 \mathrm{~K}$, in 0.1 mmag bins. The inset covers amplitudes below $0.2 \mathrm{mmag}$ and the bin size is reduced to $0.01 \mathrm{mmag}$. Kepler's sensitivity limit is clearly reached in the leftmost two bins of the inset.

inspection of these stars for peculiarity were presented in Chapter 3 .

Many stars do pulsate, but below the ground-based limit. The amplitudes of pulsators around and below the ground-based limit are shown in Fig. 4.10. Data were taken for Q3 only to avoid counting the same star twice, with Q3 chosen for covering a large number of stars in the $T_{\text {eff }}$ range $(6500$ to $8500 \mathrm{~K})$, which itself was chosen as representative of the $\delta$ Sct instability strip. Q3 also has a mean noise level that was algorithmically determined using non-pulsating stars to be representative of the midpoint for the presently available Kepler quarters (Chapter 1). From Fig. 4.10 it is clear that the number of pulsators per bin keeps increasing towards lower amplitudes, but grows rapidly towards low amplitudes until reaching Kepler's sensitivity limit. Some of these non-pulsating A stars, without appreciable granulation or stellar winds, are probably the least variable objects on the HR diagram.

Have we made things more difficult by obtaining more sensitive data? A mode 


\section{CHAPTER 4}

density that is too high may make mode identification more challenging, but those modes are still present if the detection limit is at a higher amplitude. Mode interaction still occurs and affects the frequencies observed, so there is nothing negative about having more sensitive data. Indeed, observing more frequencies allows calculations of mode spacings. García Hernández et al. (2009; 2013 in prep.) searched for periodicities in the frequencies by using Fourier transforms of frequency positions and by using Echelle diagrams, respectively, whereas Breger et al. (1999b) calculated histograms of frequency differences. The frequency spacing, if interpreted as being similar to the large separation in solar-like oscillations, i.e. as being the difference in frequency between modes of the same degree of consecutive radial order, can be used as a discriminant between stellar models and, in the case of the $\delta$ Sct star HD 174966 observed with CoRoT, García Hernández et al. (2013, in prep.) were able to infer the mean stellar density, $\bar{\rho}$, with a 6 per cent uncertainty.

\subsubsection{Combination frequencies and phases}

We have seen the amplitude distribution of $\delta$ Sct pulsators in Fig. 4.10, though that was limited to only the highest-amplitude peak for each star. Some examples of multiperiodic $\delta$ Sct stars seen with Kepler are shown in Fig 4.11. Mono-periodic $\delta$ Sct stars are seldom seen with Kepler. Beating is common in $\delta$ Sct light curves, resulting from multiple peaks of similar amplitudes in the same frequency regime. In the higher amplitude pulsators, where amplitudes are tens of mmag or more, harmonics and combination frequencies are common.

Mode interactions modify the shape of the light curve. This generates combination frequencies. Those light curves exhibiting a steeper rise and shallower fall in brightness require higher order harmonics to describe them (Balona, 2012a). The example Balona used was data from the asteroseismic study of a $\delta$ Sct star observed with CoRoT and analysed by Poretti et al. (2011). The example is recreated here. 


\section{CHAPTER 4}
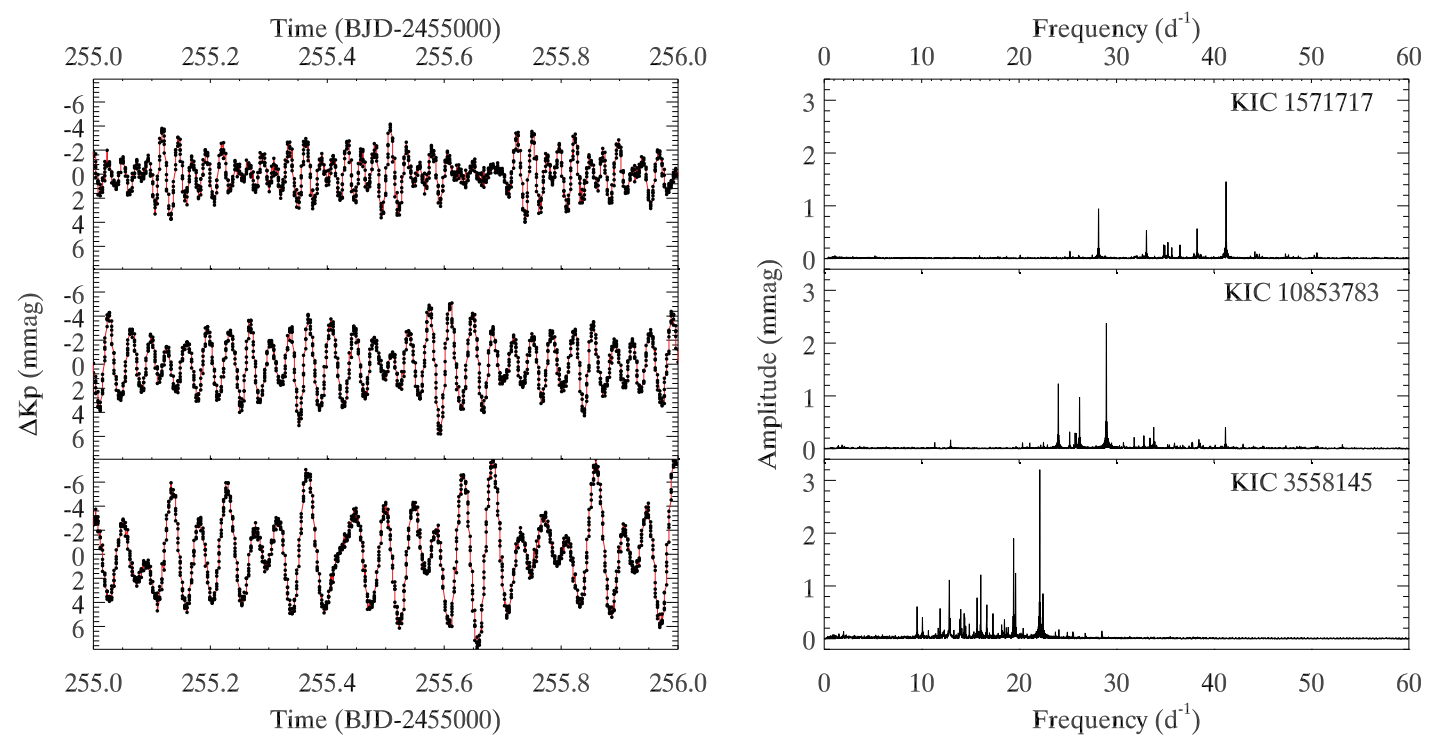

Figure 4.11: Three typical $\delta$ Sct pulsators. One-day segments of their light curves from Q4.3 are shown in the left panels, with the red lines provided to guide the eye, and corresponding Fourier transforms are shown in the right panels for the whole of Q4.3. That the stars show a pattern of the higher frequency pulsators having lower amplitudes is coincidental.

Table 4.1 contains the frequencies, amplitudes and phases of the highest amplitude peak in that data set along with its harmonics. The light variation explained by these is plotted in Fig. 4.12, and compared to a case of random phases.

Clearly the phases of the harmonics are very important, but it is not yet clear exactly what extra information they can provide. Balona (2012a) investigated the phase-frequency relationship further by looking at the phase of a combination frequency relative to its parents. Building on the mathematical description of Buchler, Goupil \& Hansen (1997), combination terms with frequency $f=n_{\mathrm{i}} f_{\mathrm{i}}+n_{\mathrm{j}} f_{\mathrm{j}}$ should have a relative phase, $\phi_{\mathrm{r}}=\phi_{\mathrm{c}}-\left(n_{\mathrm{i}} \phi_{\mathrm{i}}+n_{\mathrm{j}} \phi_{\mathrm{j}}\right)$, where $\phi_{\mathrm{c}}$ is the phase calculated by least-squares fitting. Balona (2012a) showed that for two interacting modes, the relative phases of the harmonics of a given mode decrease linearly with increasing harmonic order (i.e. frequency). Following arbitrary zero-point shifts of $\pm 2 m \pi$, 


\section{CHAPTER 4}

Table 4.1: Data for the highest amplitude peak and its harmonics for

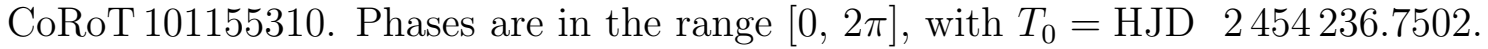
Table partially reproduced from Poretti et al. (2011, their table 1). Permission obtained from A\&A.

\begin{tabular}{rrcc}
\hline ID & $\begin{array}{c}\text { Frequency } \\
\mathrm{d}^{-1}\end{array}$ & $\begin{array}{c}\text { Amplitude } \\
\text { mag }\end{array}$ & $\begin{array}{c}\text { Phase } \\
\text { rad }\end{array}$ \\
\hline$f_{1}$ & 7.949172 & 0.137632 & 5.3775 \\
$2 f_{1}$ & 15.898343 & 0.041859 & 2.3933 \\
$3 f_{1}$ & 23.847515 & 0.014404 & 5.6197 \\
$4 f_{1}$ & 31.796687 & 0.004433 & 2.4116 \\
$5 f_{1}$ & 39.745859 & 0.002146 & 5.4146 \\
$6 f_{1}$ & 47.695030 & 0.001150 & 2.2493 \\
$7 f_{1}$ & 55.644203 & 0.000552 & 5.3327 \\
$8 f_{1}$ & 63.593373 & 0.000317 & 2.3551 \\
$9 f_{1}$ & 71.542544 & 0.000130 & 5.3127 \\
$10 f_{1}$ & 79.491717 & 0.000100 & 2.6673 \\
\hline
\end{tabular}

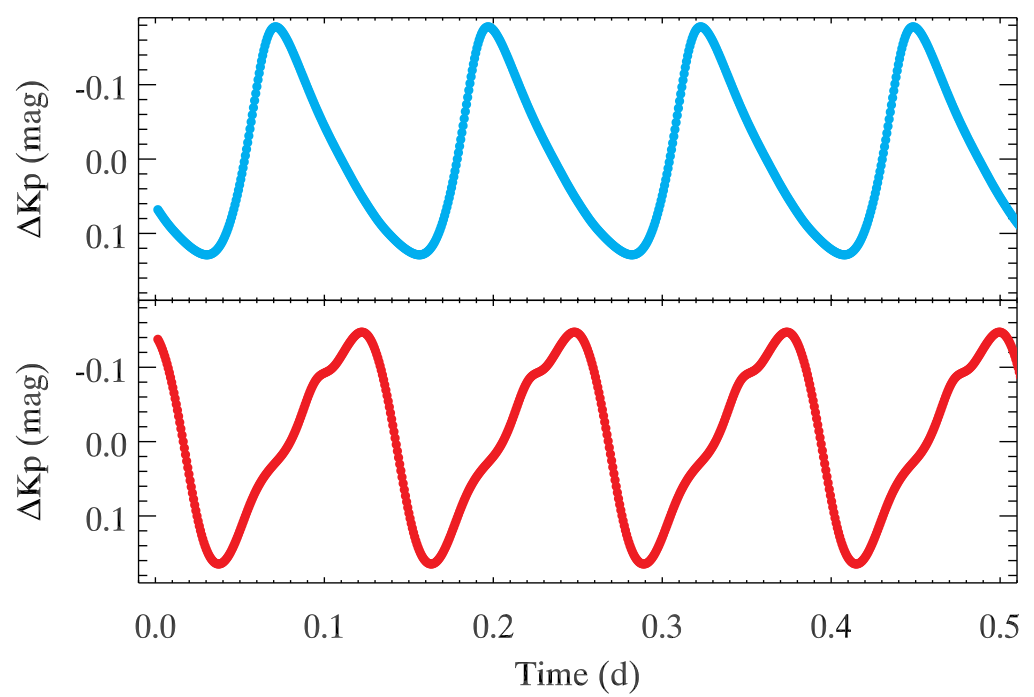

Figure 4.12: The upper panel shows the asymmetry seen in high-amplitude pulsators, where the harmonics are required to explain the steeper rise and shallower fall in brightness. Harmonics are well known in physics, e.g. in musical instruments. The phases of the harmonics are clearly important, since in the lower panel the same frequencies and amplitudes (those of Table 4.1) are plotted but with random phases; the same shape is not observed when phases are randomised. 


\section{CHAPTER 4}

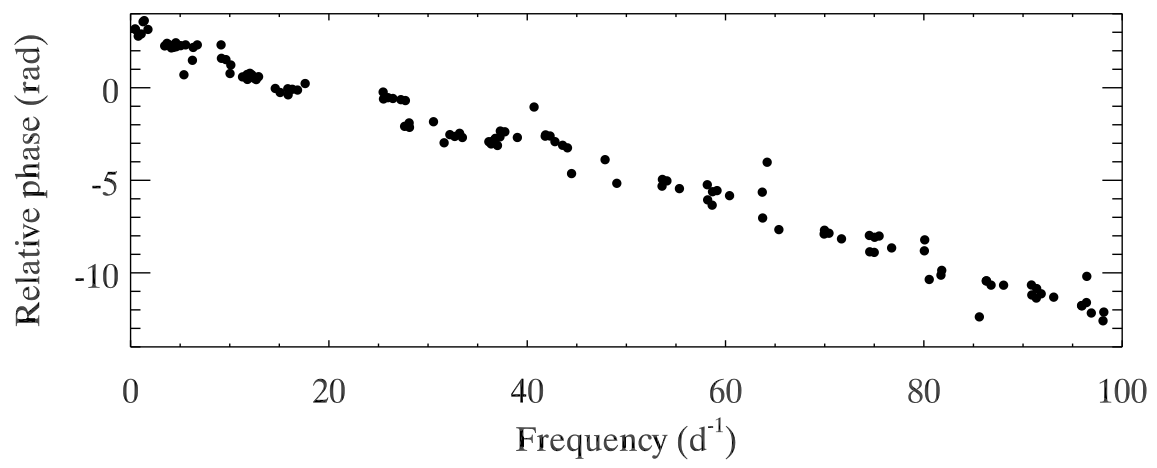

Figure 4.13: Relative phases are negatively correlated with frequency for the combination frequencies. Those points located far from the others might be interpreted as independent frequencies instead of combinations. Error bars for phases are shown, but are generally much smaller than the plot symbols. Phases and errors are the formal least-squares values and were calculated with respect to the mid-point of the

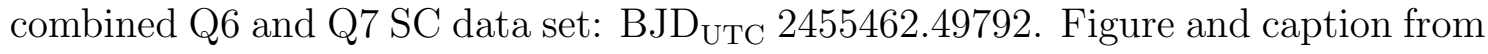
Murphy et al. (2013, their figure 10).

where $m$ is some small integer, the relative phases fall on a straight line, as confirmed by Murphy et al. (2013) (Fig. 4.13, this review). However, Murphy et al. also called for caution against over-interpreting the data because the phase uncertainties with the long and ultra-precise Kepler data sets are very small. The effect of such small error bars is to reduce the significance of the observational evidence substantially - the point with the median deviation in Fig. 4.13 lies over $27 \sigma$ away from the calculated linear fit. However, a linear fit cannot be produced for random phases.

\subsection{Related Variables}

\subsubsection{HADS stars}

The high-amplitude delta Scuti (HADS) stars are a subgroup of the $\delta$ Sct stars whose peak-to-peak light variation exceeds $0.3 \mathrm{mag}$. That designation is arbitrary and was formed long before space-based observations, which have confirmed that the vast majority of $\delta$ Sct stars are low amplitude $(\sim 1 \mathrm{mmag})$ non-radial pulsators. The 


\section{CHAPTER 4}

absence of high amplitude pulsators in the Kepler field has led to other properties, such as the dominance of radial modes and slow rotation, to be used as HADS-star criteria. The latter two properties are not independent. We mentioned in $\S 4.2$ that fast rotation induces turbulence which favours selection of non-radial modes, which have lower observed amplitudes.

HADS are concentrated in the centre of the $\delta$ Sct instability strip in a well-defined region (Petersen \& Christensen-Dalsgaard, 1996; McNamara, 2000), but close to the TAMS. They follow the same period-luminosity relationship as the brighter Cepheid variables with no discontinuity (Soszynski et al., 2008). The HADS probably evolve from the main sequence $\delta$ Sct stars, and therefore show no abundance anomalies in spite of their slow rotation, which is a natural consequence of evolution. Their pulsational instability grows as they evolve (Kurtz, 1976).

HADS have been identified as Pop. I and as Pop. II stars (see Poretti et al. 2005; McNamara \& Feltz 1978, respectively). They make up 0.24 per cent of the stars in the $\delta$ Sct instability strip (based on ground-based observations; Lee et al., 2008), and having the highest amplitudes, they make good tests of evolutionary theory of frequency and period modulation when decades of observations are available. Pulsation periods are predicted to increase during evolution (Andreasen, Hejlesen \& Petersen, 1983), as are the number of mixed modes (Dziembowski \& Krolikowska, 1990). The work of Andreasen, Hejlesen \& Petersen, who investigated theoretical evolution sequences, and that of Rodríguez et al. (1995), who looked at period changes and evolution in the $\delta$ Sct instability strip, have been important in the development of the theory of pulsational evolution; for an excellent review of those and other works, see Breger (2000b). 


\section{CHAPTER 4}

\subsubsection{SX Phe stars}

The SX Phe variables are the metal-poor Pop. II relatives of the $\delta$ Sct stars. They pulsate with substantially shorter periods than the Pop I variables - Nemec et al. (1995) studied five extremely metal poor $([\mathrm{Fe} / \mathrm{H}]=-2.41 \mathrm{dex})$ SX Phe stars in NGC 5053 and four were pulsating in their fundamental radial mode with frequencies in the range 25-30 $\mathrm{d}^{-1}$. Early (ground-based) observations of SX Phe stars indicated they were single-mode radial pulsators, and those members of the class obey a period-luminosity relation that can be used to determine cluster distances, as Pych et al. (2001) did for the cluster M55. However, those same authors also found SX Phe stars that were multiperiodic, non-radial pulsators.

SX Phe stars can generally be classified into one of two groups: those resembling halo stars, and those resembling old disk stars in terms of kinematics and metallicities (Nemec \& Mateo, 1990). Their masses are low compared to $\delta$ Sct stars.

Balona \& Nemec (2012) searched for SX Phe stars in the Kepler field, discriminating by high spatial velocities and distance from the galactic plane. Low metallicities could not be used as a discriminant because low-metallicity $\delta$ Sct stars exist in the LMC (Garg et al., 2010), but without being SX Phe (i.e., Pop. II) stars. Balona \& Nemec then investigated the pulsation amplitudes of the SX Phe stars and found the literature claims that SX Phe stars were also HADS stars are incorrect - the high amplitudes were a selection bias. From light curves alone, the SX Phe stars are indistinguishable from $\delta$ Sct stars.

Explanations for the origin of the SX Phe stars are inconclusive. Their space velocities point to Pop. II but they have anomalously large masses and young ages for such objects. Suggestions include stellar mergers, or strong internal mixing within the atmosphere of a single star. As such, these 'blue stragglers', so-named because their colours are bluer than the main-sequence turnoff for their cluster, may

offer an opportunity to study the internal structure of stars with strong mixing 


\section{CHAPTER 4}

histories. The difficulty of doing such, however, will mean that these investigations may have to wait until further progress is made with 'normal' $\delta$ Sct stars.

\subsubsection{Gamma Doradus variables}

\section{A short history}

The $\gamma$ Dor variables are a relatively new group, only being recognised as a separate class of variable in the mid-1990s (Krisciunas, 1993; Balona et al., 1994) and defined later that decade (Kaye et al., 1999). They were officially named as $\gamma$ Dor variables during the meeting on Stellar Pulsation held in Cape Town, in 1995 (Rodríguez et al., 2006). The prototype of the group was observed to be similar in effective temperature and luminosity to the $\delta$ Sct stars (Balona et al., 1994), but its pulsation periods were too long to fit neatly into that group (Cousins, 1992). The multicolour photometry and spectra obtained by Cousins allowed him to place the star on the main sequence, and propose that it belonged to Pop. I. Balona et al. (1994) commented that the only mechanism capable of producing the two closely spaced main frequencies in $\gamma$ Doradus, at 1.32098 and $1.36354 \mathrm{~d}^{-1}$, is non-radial pulsation. They also highlighted that the long periods and significant light variations imply low order g modes.

The nature of $\gamma$ Doradus was initially uncertain. Balona et al. (1994) had found that the light curve could be modelled as a single-period pulsator with a doublewave light curve, which allowed it to be classified as a W UMa binary with no need for a new class of variables. But they declared the single-period theory was unlikely because in simulations it failed to accurately reproduce the observed periodogram. They also found no clear radial velocity variation to $\pm 2 \mathrm{~km} \mathrm{~s}^{-1}$, essentially ruling out a companion. Furthermore, a model with two spots and differential rotation was rejected, but a scenario featuring two antipodal spots with unequal areas and $90^{\circ}$ inclination was found to be agreeable. 


\section{CHAPTER 4}

Cousins (1994) found strong evidence for large changes in temperature that were in phase with light curve variation. He used this as further weight towards the spotted star hypothesis, speculating that a magnetic field could be the anchor of such spots. The spotted-star hypothesis soon fell out of favour for need of highly stable and very large spots (Balona, Krisciunas \& Cousins, 1994), and the single period pulsator hypothesis was also therein dismissed upon detection of a new third period. The accepted cause of the pulsations became the high order, low-degree g modes that now typify the class (Kaye et al., 1999).

To bring the history to the space-based photometry era, some 70 confirmed and 88 candidate $\gamma$ Dor stars are now known from ground-based studies (Uytterhoeven et al., 2011). Hareter et al. (2010) detected $34 \gamma$ Dor stars with CoRoT. With Kepler, Balona et al. (2011) found at least $245 \gamma$ Dor stars, and up to a thousand candidates whose variability is explained equally well by rotating spotted stars as by pulsation. Some light curves of $\gamma$ Dor stars observed with Kepler are presented in Fig. 4.14. From the range of frequency instability alone, the class could be confused with oscillating red giants (compare Fig. 4.14 with Fig. 4.15), and the light curves of both rotating red giants and main sequence stars with spots could easily be confused with $\gamma$ Dor light curves. However, red giant Fourier transforms are easily distinguished because they show a distinct granulation signature (high noise at low frequency, decreasing strongly by $\sim 5 \mathrm{~d}^{-1}$ ), and many clustered peaks of lower signalto-noise. These 'clustered peaks' belong to a Lorentzian profile, whose width carries information on the lifetime of the mode.

\section{Driving mechanism}

The driving mechanism of the pulsations is what is known as the convective fluxblocking mechanism (Guzik, Bradley \& Templeton, 2000), by which radiative flux is modulated by the convection zone. The driving is most efficient in the middle of the 


\section{CHAPTER 4}
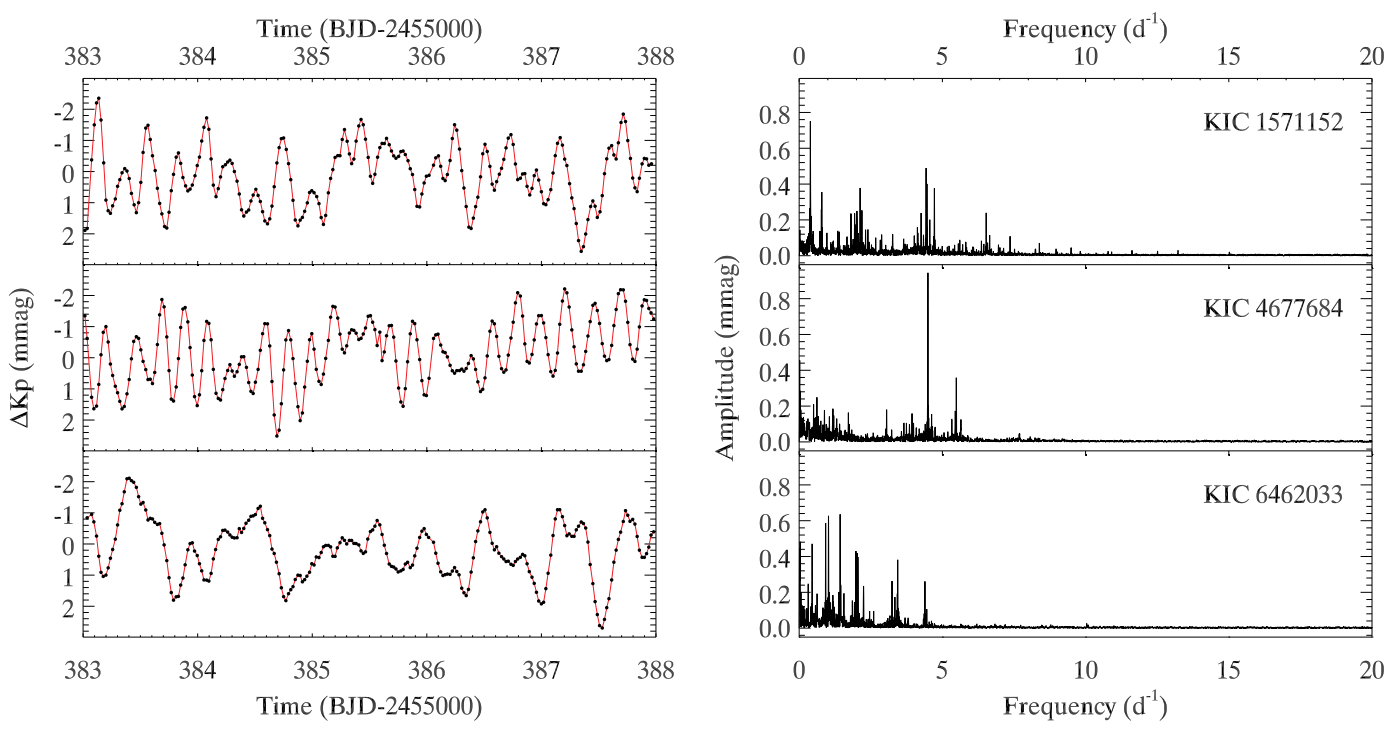

Figure 4.14: 5-d light curve segments from Q6 for the $\gamma$ Dor stars KIC 1571152, 4677684 and 6462033, as classified by Uytterhoeven et al. (2011). The red lines are provided to guide the eye. KIC 1571152 was also studied by Tkachenko et al. (2012) who confirmed its location within the $\gamma$ Dor instability strip with spectroscopy. The stars are clearly multiperiodic from their Fourier transforms of the entire Q6 PDCMAP data.
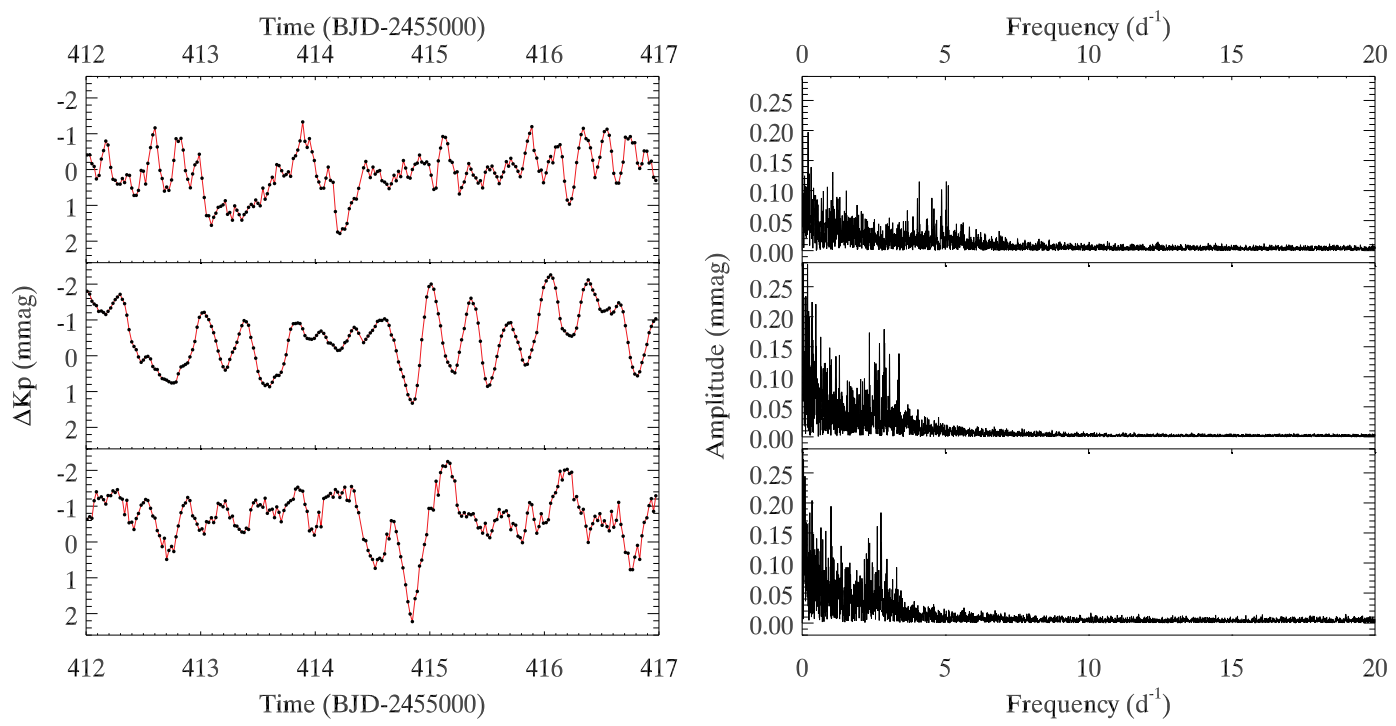

Figure 4.15: 5-d segments of light curves of some red giants observed with Kepler. The red lines are provided to guide the eye. Although they can be confused with $\gamma$ Dor stars, granulation, broad power profiles and signal-to-noise ratios can be used to separate the two, if atmospheric parameters are not available. Fourier transforms are calculated on the entire Q6 PDC-MAP data for each star. 


\section{CHAPTER 4}

strip $($ at $(b-y)=0.2$, or $6900 \mathrm{~K})$, where the temperature of the base of the convection zone leads to a thermal relaxation time that is equal to the pulsation period (Dupret et al., 2005). The $\gamma$ Dor instability strip has its blue edge at temperatures where the convective envelope becomes thin, its thermal relaxation time becomes shorter than the pulsation period, and the driving becomes inefficient; at the red edge, radiative damping of g modes surpasses the driving (Balona et al., 2011).

Balona et al. (2011) divided the light curves of stars of the class into two main groups: SYM for the symmetric light curves, and ASYM, for those stars with large variations in maximum brightness, and only small variations in minimum brightness. It is thought that the asymmetry of the light curve arises from saturation of the driving mechanism. ASYM light curves are also seen for HADS and RRab stars, and it is intuitive that under the 'saturation hypothesis' the high-amplitude pulsators should have asymmetric light curves. Murphy et al. (2013) demonstrated the asymmetry of an SX Phe light curve with HADS-like properties in their figure 3.

Only $5 \gamma$ Dor stars investigated by Hareter et al. (2010) showed recurrent period spacings (that is, the same period spacing seen in many places throughout the Fourier spectrum), which they claimed indicates that asymptotic spacing of g modes is destroyed by rotation and chemical composition gradients in the star.

\section{Frequencies, temperatures, and $\gamma$ Dor $-\delta$ Sct hybrids}

The $\gamma$ Dor stars are found between spectral types A7 and F5, and at luminosity classes IV-V and V (Dupret et al., 2005). The centroid of the $\gamma$ Dor stars on the HR diagram lies nearly on the red edge of the $\delta$ Sct instability strip (cf. Fig. 4.3), therefore about half of all $\gamma$ Dor stars fall within the $\delta$ Sct instability strip. Theoretically, one would expect these stars to show both p-mode and g-mode pulsations in what are called 'hybrid' stars, but very few hybrids have been observed from the ground.

The $\delta$ Sct stars have pulsation frequencies between about 5 and $75 \mathrm{~d}^{-1}$, which 


\section{CHAPTER 4}

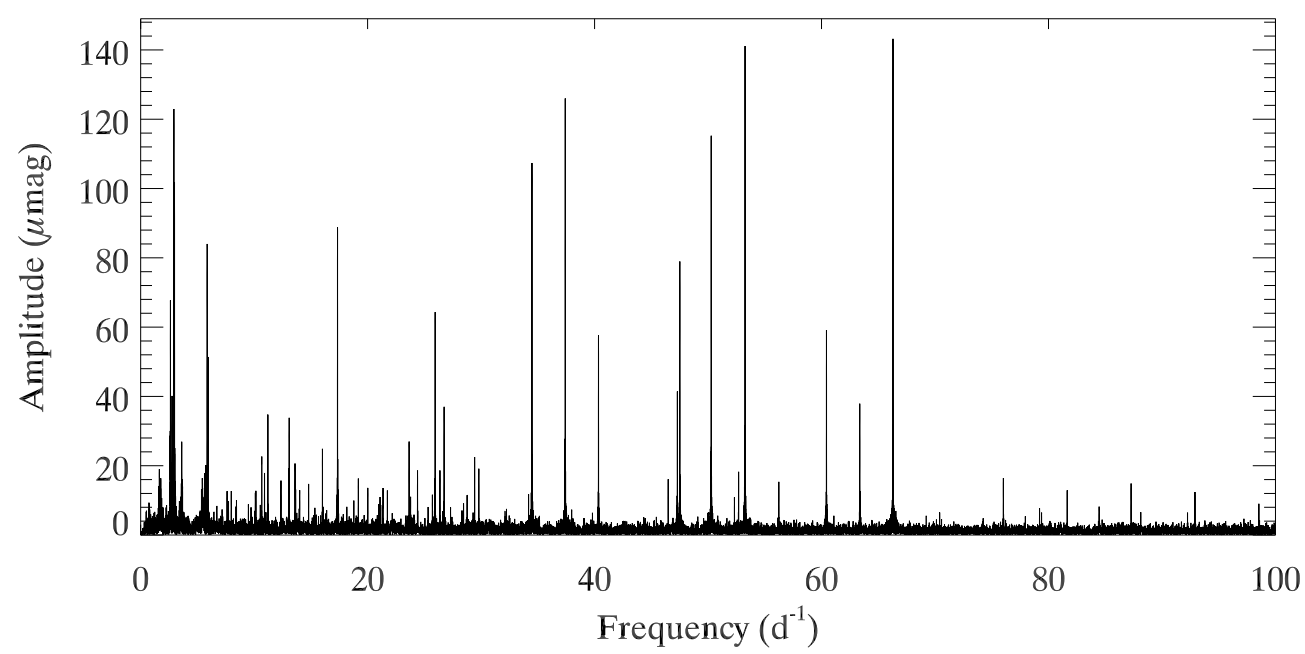

Figure 4.16: KIC 8054146 (Q6, SC, PDC-LS data shown) - a $\gamma$ Dor- $\delta$ Sct hybrid with no frequency gap around $5 \mathrm{~d}^{-1}$. The obvious triplets can be explained by combination frequencies and resonance excitation (cf. $\S 4.4 .4$ ). This star has also been the subject of an independent case study (Breger et al., 2012) for the relationships between its high and low frequencies.

compares with 0.3 and $4 \mathrm{~d}^{-1}$ for the $\gamma$ Dor stars (Handler, 2005) ${ }^{4}$. It is easy to conceive of a continuum of pulsation frequencies, where stars whose frequencies straddle the $5 \mathrm{~d}^{-1}$ border would be hybrids. Stars with frequencies spanning through the whole range are seen with Kepler, an example of which is provided in Fig. 4.16. The models do not predict a continuum, however. There is a frequency gap in the models between the two pulsation regimes, in which modes are predicted to be stable (Dupret et al., 2005).

In their variability survey of Kepler A-F stars, Uytterhoeven et al. (2011) applied the following criteria to the hybrid classification:

1. The stars show frequencies in both the $\delta$ Sct $\left(>5 \mathrm{~d}^{-1}\right)$ and $\gamma$ Dor $\left(<5 \mathrm{~d}^{-1}\right)$ regime;

2. The amplitudes in the two regimes are comparable or do not differ by a factor of more than 5 to 7 (on a case-by-case judgement); and

\footnotetext{
${ }^{4}$ The $\gamma$ Dor variables also overlap in frequency with the post-giant, low-mass $\left(<1 \mathrm{M}_{\odot}\right)$, RR Lyrae variables (Breger, 2000b).
} 


\section{CHAPTER 4}

3. At least two independent frequencies are detected in each regime with amplitudes above $100 \mathrm{ppm}$.

Grigahcène et al. (2010) went further and called stars $\delta$ Sct or $\gamma$ Dor in the pure case, but $\delta$ Sct $/ \gamma$ Dor hybrids if the higher frequencies were dominant (compared to the low frequencies present) and $\gamma$ Dor $/ \delta$ Sct hybrids if the lower frequencies were dominant. It is not clear whether this form of classification offered anything helpful or meaningful, or just added additional terminology.

The Uytterhoeven et al. description has a very important qualifier - the peaks must be independent. One can often find low frequency peaks in the Fourier transform of $\delta$ Sct stars that are combination frequencies, and that do not mean the star is a hybrid. In all, those authors found 63 per cent of the A-F stars could be characterised as either $\delta$ Sct, $\gamma$ Dor or hybrid stars $(27,13$, and 23 per cent of their sample, respectively). Their positions on a $T_{\text {eff }}-\log g$ diagram are shown in Fig. 4.17, along with the $\gamma$ Dor and $\delta$ Sct instability strips from Dupret et al. (2005) that were created using TDC models, tuned to match ground-based data. It appears that with more sensitive data, the observational instability strips are wider than the models predicted, or rather, than ground-based observations indicated. However, the KIC parameters on which the $T_{\text {eff }}$ and $\log g$ of Fig. 4.17 are based have large errors $\left( \pm 200 \mathrm{~K}\right.$ in $T_{\text {eff }}, \pm 0.5$ dex in both $\log g$ and [Fe/H]; Molenda-Żakowicz et al. 2010) as well as systematic offsets of about $200 \mathrm{~K}$ for A-F stars (Pinsonneault et al., 2012).

The hybrids are very much a new area of research. It is not known to what extent one form of pulsation influences the other. For instance, Hareter et al. (2010) found that 'only in a few cases' do the hybrids pulsate with frequencies above $30 \mathrm{~d}^{-1}$. Handler \& Shobbrook (2002) concluded that $\gamma$ Dor stars are less likely to be $\delta$ Sct stars than non- $\gamma$ Dor stars in the same area of the lower instability strip, but in hindsight this may just be affirmation that few hybrids are detectable from groundbased data. Hareter et al. (2011) presented evidence against the $\delta$ Sct- $\gamma$ Dor hybrids 


\section{CHAPTER 4}

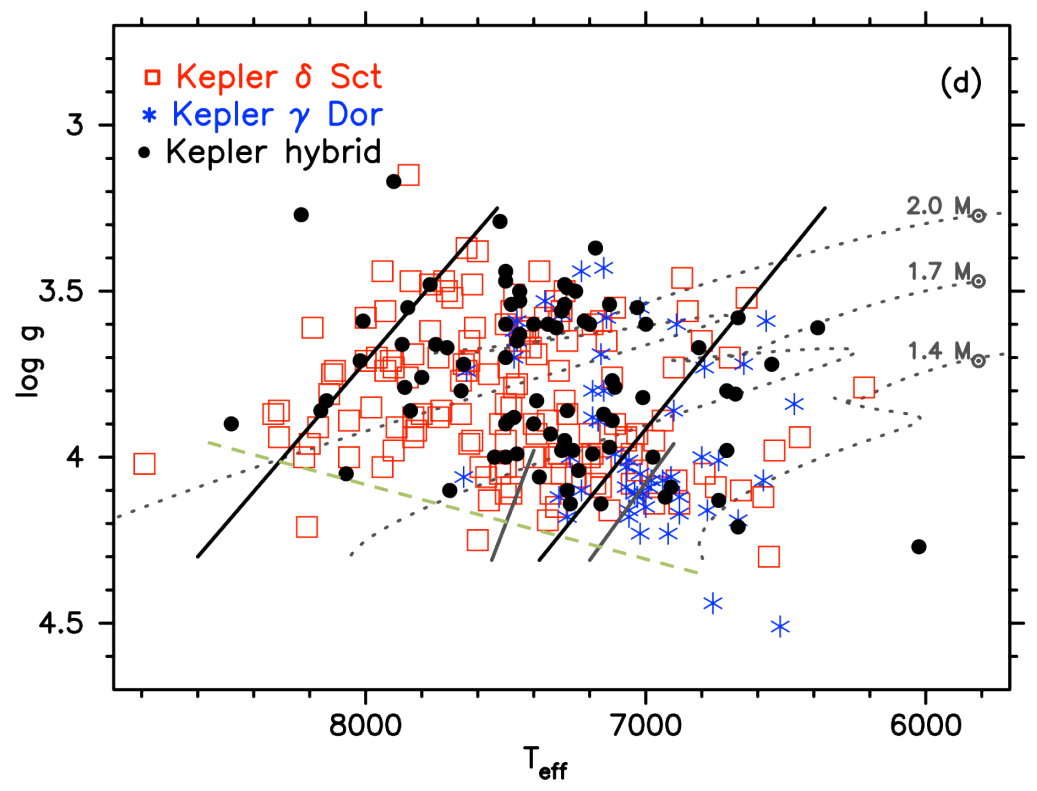

Figure 4.17: The distribution of $\delta$ Sct, $\gamma$ Dor and $\delta$ Sct- $\gamma$ Dor hybrids with amplitudes above $1 \mathrm{mmag}$, as seen with Kepler data. Short-dashed black lines are evolutionary tracks, starting on the ZAMS. A long-dashed green line is drawn to indicate the age of the Kepler field, as was discussed in $\S 4.3$. Figure adapted from Uytterhoeven et al. (2011, their figure 10). Permission obtained from A\&A.

being chemically peculiar, but $\gamma$ Dor and $\delta$ Sct pulsations have also been observed in (candidate) Ap stars (Balona et al., 2010).

Finally, we recall that the Kepler field was chosen not to lie wholly in the galactic plane. Although evolved $\delta$ Sct stars are found, the $\gamma$ Dor instability strip does not extend as far from the ZAMS. It is therefore likely that the incidence of $\gamma$ Dor pulsators is higher among A-F stars than Kepler data suggest. Indeed, in $\S 4.3$ we noted that Balona \& Dziembowski (2011) found a gap between the ZAMS and the $\delta$ Sct stars with the highest $\log g$ values, which increases with higher temperature, even though the $\delta$ Sct instability strip is known to extend down to the ZAMS and below; pre-MS $\delta$ Sct stars are known. 


\section{CHAPTER 4}

\subsection{Selected case studies}

Pulsations offer much more than a constraint on stellar models - they allow us to select between them. One improves the physics of the stellar models by modelling the most complete, most accurate and most extensive observations of pulsations, along with supplementary constraints on global stellar properties. Thus detailed case studies are essential to the progress sought in $\delta$ Sct star research.

Some case studies represent fleeting insights into single targets, such as those targets observed by CoRoT that will not be observed again for whatever remains of the mission's life time. Kepler, too, will not run forever: at the present time it is in safe-mode to mitigate the failure of another reaction wheel. It will leave a lasting legacy of multiple years of nearly continuous observations, greatly outclassing ground-based photometry in all aspects, except perhaps timebase for a few special targets studied by dedicated observers. The process of obtaining as much data as possible for single targets, not limited to only photometric data, is known as the 'direct approach' (Breger, 2000b) and has been administered by the Whole Earth Telescope and the Delta Scuti Network.

It is not feasible to talk in detail about all of the targets that have garnered such close attention, not only due to page limitations but because the field is being revolutionised. What was once unsurpassed coverage of many hundreds of hours worth of data (e.g. $926 \mathrm{~h}$ for the star FG Vir in 2003/2004; Breger et al. 2005) is to be exceeded by more than an order of magnitude with Kepler - some targets have now been observed for $\sim 30000$ hours and counting, at a 90-95 per cent duty cycle. It is then appropriate to limit this discussion to only the most extensive groundbased studies, in addition to some existing Kepler case studies. A brief summary is thus provided below for the $\delta$ Sct stars $4 \mathrm{CVn}, 44$ Tau and FG Vir, along with four Kepler targets whose analysis I have led during the PhD programme. 


\section{CHAPTER 4}

\subsection{1 $4 \mathrm{CVn}$}

The cool, evolved $\delta$ Sct star 4 CVn remains the best studied to date. During 1996 and 1997, the Delta Scuti Network obtained 529 h of data for this star (Breger et al., 1999a; Breger, 2000b), resulting in the detection of 34 frequencies. From 2005 to 2009, the star was observed for five months of the year (Breger, 2009). No less than 72 frequencies were detected from the frequency analysis of these data, of which 60 had amplitudes below 1 mmag - from ground-based data(!). Breger and his colleagues looked for period and amplitude modulation on a variety of timescales, combining these data with other sets going as far back as 1966 (Breger, 2000a). The amplitudes of almost all modes were shown there to be 'extremely variable', with a few modes being suppressed almost completely and reappearing some time later. The disappearance of one mode coincided with the appearance of another, illustrating the concept of resonant mode coupling.

In his most recent paper on the star, Breger (2009) found evidence of period variability on a time-scale of decades, perhaps pointing to the presence of a stellar cycle, though a full cycle was not observed. Interestingly, frequencies belonging to modes of $m=-1$ changed at similar rates but in with the opposite sign as those belonging modes of $m=+1$. The rate of change of period, $\frac{d P}{d t}$, was thus found to depend on the $m$ value, and was two orders of magnitude greater than that expected from stellar evolution. Time-series spectroscopy was able to identify five modes (Castanheira et al., 2008), and more recent spectroscopy has revealed the star to be in a binary system (Schmid et al., 2012).

\subsubsection{FG Vir}

Even more pulsation frequencies were detected for the star FG Vir. The tally stood at 79 in the year 2005 (Breger et al., 2005), following many extensive photometric campaigns, yielding $494 \mathrm{~h}$ of data in 1995/1996 (Viskum et al., 1998; Breger et al., 


\section{CHAPTER 4}

1999b), $398 \mathrm{~h}$ in 2002 (Breger et al., 2004), and $926 \mathrm{~h}$ in 2003/2004 (Breger et al., 2005). The extensive time-base offered sensitivity to closely-spaced frequencies, which turned out to be important given the distribution of the pulsation frequencies. Although frequencies were found to cover a wide range $\left(6-44 \mathrm{~d}^{-1}\right)$, they were not distributed at random. We mentioned earlier how Breger, Lenz \& Pamyatnykh (2009) used data from FG Vir to discover that non-radial modes tend to cluster around radial ones. Breger \& Pamyatnykh (2006) also investigated the rather high number of close frequency pairs using this favourable frequency resolution, concluding their origin is astrophysical, not accidental. With this star, Breger and coauthors solved the long-standing problem that pulsation models predicted far more modes to be excited than were observed. The solution was a combination of the requirement for excellent frequency resolution alongside mmag (or better) precision.

For a main-sequence $\delta$ Sct star, FG Vir appears to be a slow rotator with $v \sin i=$ $21.6 \pm 0.3 \mathrm{~km} \mathrm{~s}^{-1}$ (Zima et al. 2006, in agreement with the earlier results of Mantegazza, Poretti \& Bossi 1994; Mittermayer \& Weiss 2003), but the inclination is quite low (19 $\pm 5^{\circ}$; Zima et al. 2006; Mantegazza \& Poretti 2002) so that $v_{\text {eq }}=$ $66 \pm 16 \mathrm{~km} \mathrm{~s}^{-1}$. The moderate rotation explains the high amplitude of the pulsation. The highest-amplitude mode has an amplitude of over 30 mmag in the $v$ filter, and a few combination frequencies are observed. Photometric and spectroscopic mode identification was carried out by Daszyńska-Daszkiewicz et al. (2005) and Zima et al. (2006); based on their findings and comparisons to convection models, the former authors were able to infer that convective transport is inefficient in this star. Even in spectroscopic analyses, only modes of low $\ell$ were found. Later, Lenz et al. (2008a) examined intrinsic mode amplitudes for this star and 44 Tau, finding the intrinsic radial displacement of $\ell=2$ modes to be comparable to the fundamental modes. With such dedicated study, FG Vir thus became a testbed for various theories surrounding $\delta$ Sct pulsation. 


\section{CHAPTER 4}

\subsubsection{Tau}

As $\delta$ Sct stars go, 44 Tau is an exceptionally slow rotator, with $v \sin i=2 \pm 1 \mathrm{~km} \mathrm{~s}^{-1}$ (Zima et al., 2007). Antoci et al. (2007) found a pole-on view (with $i<10^{\circ}$ ) to have a low probability of only 1.5 per cent. The star's slow rotation led Breger (2000c) to describe 44 Tau as "one of the most promising candidates for $[\delta$ Sct] asteroseismology". It is classified as an F2 IV star, which matches the spectroscopic $T_{\text {eff }}(7000 \mathrm{~K})$ and $\log g(3.6 \mathrm{dex})$ from Zima et al. (2007) well. For a long time, the evolutionary stage of 44 Tau was not known, and the $\log g$ value did not offer a unique solution. Main-sequence models and models in the post-main sequence expansion phase failed to match all of the observations simultaneously (Lenz et al., 2008b). A summary of the deficiencies of past modelling attempts is provided by Lenz et al. (2010), in the same article in which they announce a satisfactory match with a model in the post-main sequence contraction phase.

The most recent and extensive frequency analysis, on the back of seven years of photometric study, is that of Breger \& Lenz (2008). Those authors found 15 independent pulsation modes, including a g mode, in addition to 34 combination frequencies. They also studied amplitude variability and found the radial modes to be stable, but the $\ell=1$ modes to undergo strong amplitude variability, with each $\ell=1$ mode behaving similarly to the others. They found that precession of the rotation axis could not explain the variability (through changing observational inclination angle) because axisymmetric $(m=0)$ modes behaved in the same way as the non-axisymmetric modes. Beating between close frequencies could not be ruled out with the existing data set, and mode-coupling / resonance effects were found to be an unlikely cause. The future of observations of stars like 44 Tau in the era of space-based photometry is unclear, despite a declaration in the most recent paper on this star (Lenz et al., 2010) that their results were "very promising" and that more data were in demand. 


\section{CHAPTER 4}

\subsubsection{KIC 9700322}

KIC 9700322 is a relatively high-amplitude $\delta$ Sct star compared with other Kepler $\delta$ Sct stars, with the main two frequencies having amplitudes of around 30 mmag. As such, the star shows many combination terms and some modulation by what is interpreted to be the rotation frequency. Published studies have been written by Breger et al. (2011) and Guzik \& Breger (2011), and we refer the reader to those works for the full analysis. This author began a continuation of those works but insufficient extra information has been gathered thus far to warrant publication.

\subsubsection{KIC 3429637}

KIC 3429637 (HD 178875) is a $\delta$ Sct star whose light curve demonstrated continuous amplitude growth. Instrumental causes of the amplitude growth were eliminated; the origin was instead determined to be intrinsic to the star, i.e. pulsational. Furthermore, spectroscopic observations revealed the star to be mildly chemically peculiar. TDC models were used on an individual $\delta$ Sct star for the first time in this study, which concluded that the observations were consistent with the star being an evolved Am star ( $\rho$ Pup star).

The full study (Murphy et al., 2012) is presented as a contribution to this thesis and is appended as-published in $\S 5.3$. I led and coordinated the project, selecting the star for detailed study. The resulting frequency analysis and spectral classification were my work. E. Niemczura obtained the high resolution spectrum, under time awarded to K. Uytterhoeven, and conducted an abundance analysis, writing Sections 3.1 and 3.2 of the paper. I presented the spectroscopic data and wrote the rest of the paper, with the exception of Section 6, which was led by A. Grigahcène. D. Kurtz in particular assisted with editing of the paper and discussion of its content. 


\section{CHAPTER 4}

\subsubsection{KIC 11754974}

KIC 11754974 is another relatively high-amplitude $\delta$ Sct star that shares many characteristics with the HADS stars. It is metal-poor, and has high spatial velocities, in common with SX Phe variables. The star shows a remarkable number of combination frequencies, but a lack of high quality spectroscopic data has hindered progress on this particular star. Murphy et al. (2013) used TDC and rotating models, amongst other means, to determine the star is probably a fundamental radial mode pulsator, but multicolour photometry is required before mode identification can be completed. This cool star is the primary of a non-eclipsing 343-d binary system, which was determined from orbital effects on the pulsation frequencies alone.

The full analysis is also appended as-published in $\S 5.4$. I initially found the star using my Kepler catalogues, described in the next chapter, and although I led the study, large contributions were made from co-authors. The time- and computationally-intensive frequency analysis was my work; I coordinated heavily with A. Pigulski on the O-C work that he produced for Section 2.1 and I performed the FM analysis of Section 2.2. Many different efforts went into determining the atmospheric parameters of the star, which I collated into Section 3. Section 4 on the pulsation characterisation was entirely my own work, with supervision from D. Kurtz. Section 5 was a joint effort of J.C. Suárez, G. Handler, A. Grigahcène and myself. The manuscript was my work with editing from many authors, but from D. Kurtz and A. Pigulski in particular.

\subsubsection{KIC 10977859}

KIC 10977859 has some very high pulsation frequencies for a $\delta$ Sct star. For this reason, Murphy (2012a) used the star as an example of the difficulties one can encounter with Nyquist aliasing with the Kepler LC data. The high frequencies make the star intriguing, indicating it is near the blue edge of the $\delta$ Sct instability 


\section{CHAPTER 4}

strip. Amplitude variability is also evident. Although the study is in its infancy, spectroscopic and photometric data are all in hand, and although at $8100 \pm 100 \mathrm{~K}$ the star is not especially hot for a $\delta$ Sct star, its $\operatorname{low} \log g$ value of $3.7 \pm 0.2(\operatorname{cgs})$ puts it at the upper left edge of the $\delta$ Sct instability strip. Analysis of this target, to include a full abundance analysis, is ongoing.

\subsection{Chapter Summary}

The $\delta$ Sct instability strip extends from early-A to mid-F spectral types, extending above, below and across the main sequence. The blue edge is found where hotter temperatures place the He II ionisation zone increasingly close to the surface until it can no longer drive pulsations. Here, modes with $n=6$ or 7 are found. At the red edge, convection dampens pulsation.

Modes with the highest amplitudes tend to be radial, and these are found in the slow rotators where the damping influence of turbulence on radial modes is small. Non-radial modes tend to be of low degree $(\ell=1$ or 2$)$, because disk-averaging effects partially cancels modes of higher degree. Having said that, many mechanisms operate that counter this partial cancellation, including mode interaction that allows high- $\ell$ modes to take on a low- $\ell$ character. Even so, these modes will not be observed with high amplitudes.

Modelling $\delta$ Sct stars is very difficult. There are many free parameters, which, even with good observational constraints, produce a very diverse set of models. Poorly understood damping and excitation mechanisms for different modes also contribute to the complexity. Models benefit greatly from mode identification, which requires multicolour photometry and high-resolution spectroscopy. Time-series spectroscopy is of great use but is impractical for even the bright Kepler targets. With white light (Kepler) photometry one can use period ratios to identify radial modes.

With such incredibly precise data, space missions like Kepler and CoRoT are 


\section{CHAPTER 4}

revealing light curves containing many frequencies. Some stars show suspiciously high mode densities that might be better explained as a combination of granulation with pulsation, than by pulsation alone. Alternatively, instrumental injection of spurious signal could be a contributor. Even in the non-suspicious cases, modelling so many frequencies in the Kepler data is difficult. The problem is caused partly by rotational splitting, which is very important for the $\delta$ Sct stars, whose rotational velocity distribution has its mode at $165 \mathrm{~km} \mathrm{~s}^{-1}$. Rotational splitting removes the frequency degeneracy of the spherically symmetric case and splits modes of the same $(n, \ell)$ values into multiplets of different $m$ values. At first order these multiplets should be equally spaced, but (calculable) departure from equal spacing is expected at second order. That few non-equally spaced multiplets are reported in the $\delta$ Sct literature is probably observer bias.

Having reduced the noise level so dramatically, we asked the question: has Kepler reached the limit of what is detectable from $\delta$ Sct stars? That is, does stellar photospheric noise set a background noise level below which the precision of one's data becomes irrelevant? The fact that granulation can be seen to play a role in noise levels in the cool $\delta$ Sct stars would imply this is the case, but for the hotter $\delta$ Sct stars in particular, we concluded that Kepler's sensitivity limit is reached (i.e., the noise level is set by the satellite's precision) because the amplitude distribution for $\delta$ Sct stars flattens out at very low amplitude. However, that the distribution rapidly rises before flattening out implies there are many constant stars, too.

Combination frequencies and harmonics are very important in asteroseismic analyses, in that they must not be interpreted as independent modes, but their usefulness is unclear. Certainly, they are more often present in the high-amplitude $\delta$ Sct (HADS) stars, defined as $\delta$ Sct stars with peak-to-peak light variations exceeding $0.3 \mathrm{mag}$. These stars are evolved, though not all $\delta$ Sct stars become HADS; other factors like rotation velocity are also important. SX Phe stars, on the other hand, 


\section{CHAPTER 4}

are not necessarily HADS stars; on the contrary, literature determinations that this was the case have been dispelled as observational selection effects. SX Phe stars are the Pop. II $\delta$ Sct stars that have large space velocities and low metallicities. These 'blue stragglers' probably originate as stellar mergers.

The $\gamma$ Dor variables pulsate at frequencies lower than those of the $\delta$ Sct stars, but the instability strips of the two classes partly overlap. Here one sees hybrid stars, pulsating in both frequency regimes. $\gamma$ Dor stars have pulsations driven by a flux blocking mechanism, whereas the $\kappa$-mechanism drives $\delta$ Sct pulsation. The hybrids are of great scientific interest, because the $\delta$ Sct pulsations provide information (mostly) on the surface layers, and the $\gamma$ Dor pulsations probe deep into the star.

Finally, the importance of case studies was addressed. A few ground-based case studies were noted, and four particular Kepler targets attracted special mention; for two of them, publications are appended to the thesis in Chapter 5. If real progress is made in the field of $\delta$ Sct stars, Kepler will certainly have a great role to play, and it is through intense case studies with ground-based follow-up and detailed modelling that advances will be made. However, now that observations have taken a sudden leap in precision, we are waiting for theory to catch up. Substantial progress will not be made with $\delta$ Sct stars until we can produce models that take both rotation and TDC into account with non-adiabatic oscillation calculations. Ultimately, for a rotating star, $T_{\text {eff }}$ and $\log g$ are a function of latitude, making this treatment particularly necessary - when we see a star we see a whole hemisphere, so we see a range of $T_{\text {eff }}$ and $\log g$ for the same star. Furthermore, chemical separation is a process to be incorporated into the models, but is a tier lower in priority for models of chemically normal $\delta$ Sct stars. 


\title{
Chapter 5
}

\section{Kepler catalogues and published case studies}

\begin{abstract}
This chapter features two published case studies of individual $\delta$ Sct stars led by this author. Detailed author contributions were given in $\S 4.6$. The case studies are presented as published, and are stand-alone journal articles, but are additionally contextualised by the review of the literature on pulsating stars that was presented in the previous chapter. Also described and presented are catalogues of Kepler data that I have used to select stars for case studies.
\end{abstract}

\subsection{Description of Kepler catalogues}

The large number of A stars observed by Kepler necessitates an efficient method of sifting through the data. For this purpose, I created Kepler catalogues. They broadly consist of a light curve and Fourier transform, along with various metadata: the KIC number, mission quarter, observing cadence, $T_{\text {eff }}$ and other stellar parameters. Each page represents one observing quarter (LC) or month (SC) for a given 


\section{CHAPTER 5}

star.

The semi-automated routine designed to create the catalogues allows for any stars to be grouped into a catalogue. Their first application was in cataloging classes of pulsators for each KASC working group. This was later extended to Heartbeat stars and to public Kepler data when they became available. Coupled with the algorithm described in Chapter 1 for selecting non-pulsating stars, catalogues were created for those, too. A four-page extract from a catalogue of stars showing amplitude variability is provided overleaf.

For stars with SC data, two Fourier transforms are plotted: one in the range 0$24 \mathrm{~d}^{-1}$ (i.e. up to half the LC sampling frequency), and one in the range $0-100 \mathrm{~d}^{-1}$. For LC data, only the first of those two is plotted. Moderate amplitude or frequency variability is easy to spot because the frequency scale is the same for every observing quarter, although different limits on the frequency range can be entered to look for roAp stars, for example. One can flip pages back and forward for a blink comparison of neighbouring observing quarters. The amplitude scale is relative, at 110 per cent of the highest peak. The frequency and amplitude of the highest peak are also printed to the pdf catalogue.

The extract chosen here fulfils multiple purposes. The star, KIC 10977859, was described in $\S 4$ 4.6.7. Notice that on the annotated first page (Q8, LC, PDC-MAP data) the Fourier transform indicates variability at frequencies around $5-10 \mathrm{~d}^{-1}$. The following page (SC data) shows no such variability, indicating the peaks in the LC Fourier transform were Nyquist aliases. This example was made in Chapter 1 (Fig. 1.2). The amplitude variability of the frequency at $54 \mathrm{~d}^{-1}$ is obvious when one compares adjacent pages (months) of SC data. The frequency range, which at 45$60 \mathrm{~d}^{-1}$ is high for a $\delta$ Sct star, is also easily noticed. When multiple stars exist in the same catalogue, selection of stars pulsating at high frequencies is straightforward.

The published case studies immediately follow the catalogue extract. 


$$
\text { cadence (Ilc = long; slc = short) }
$$

\section{kic10977859 Q8 IIc MAP}

KIC number Quarter pipeline

KIC metadata
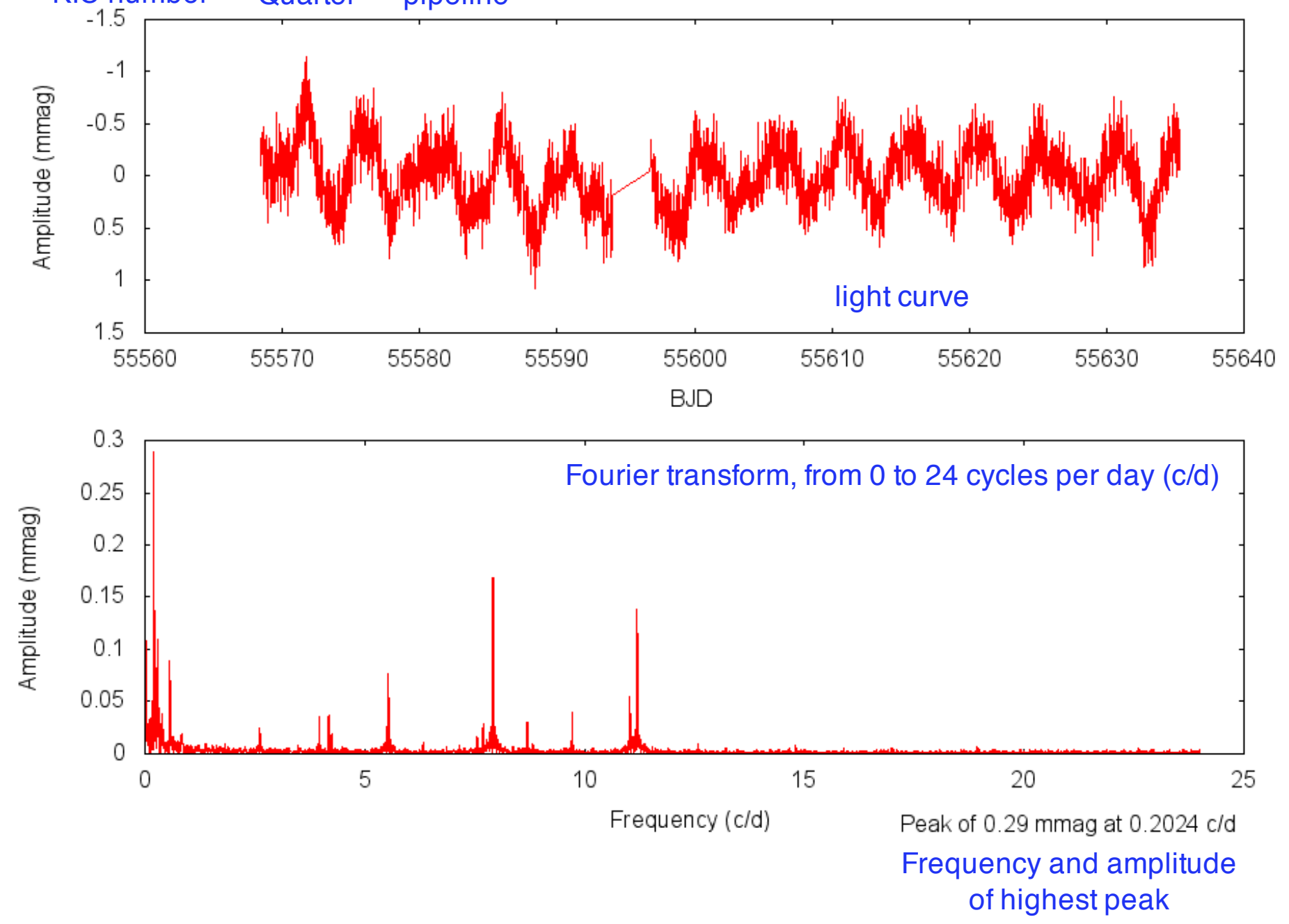

Fourier transform, from 0 to 100 cycles per day (c/d) [For SC data only] 
$\begin{array}{llll}\text { kic10977859 Q8.1 slc MAP } & \text { Kp mag }=8.808 & \log g=3.935 & \text { Teff }=8052 \\ \text { contam. }=0.004 & {[\mathrm{Fe} / \mathrm{H}]=0.153} & \text { radius }=2.232\end{array}$
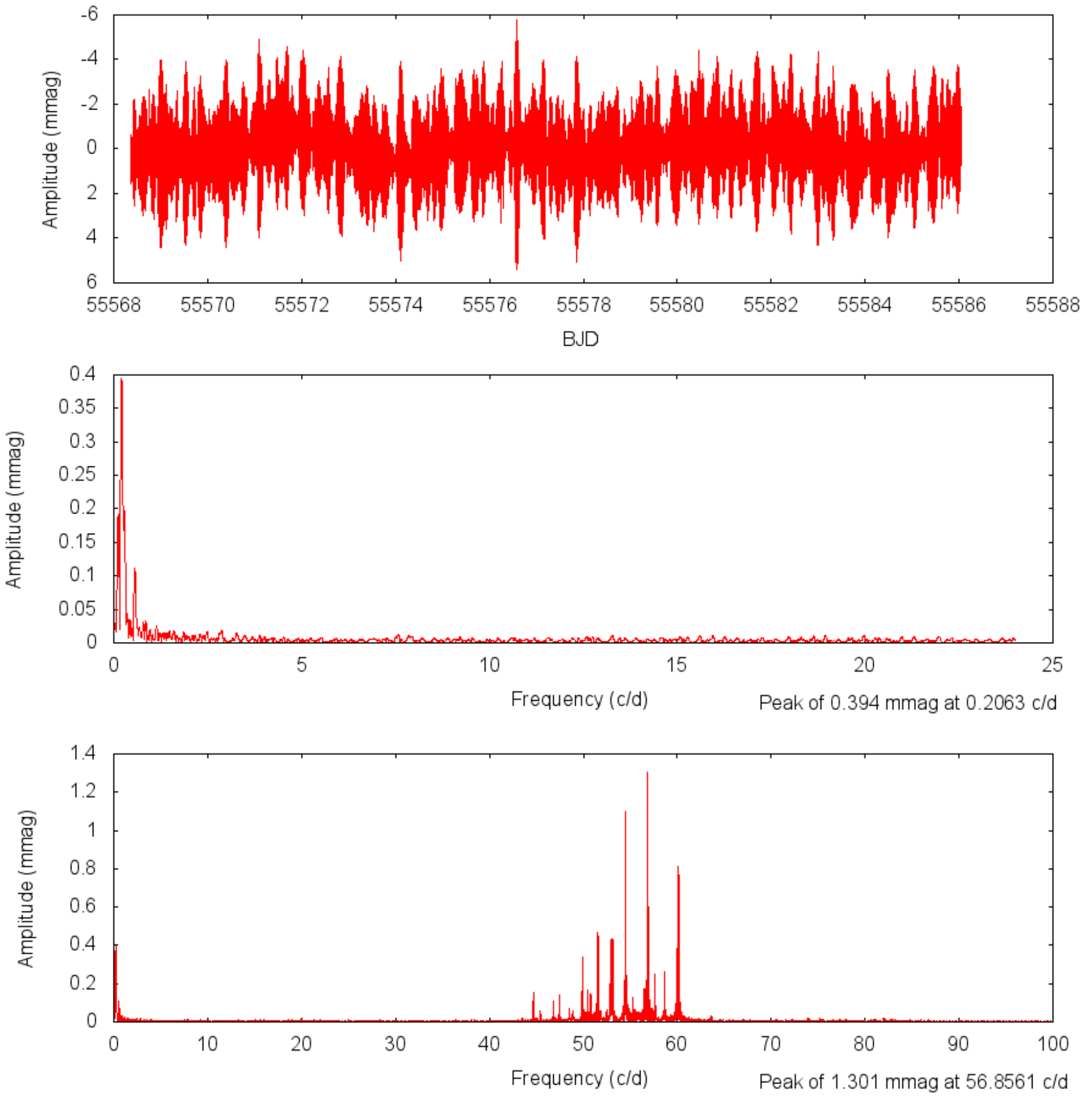

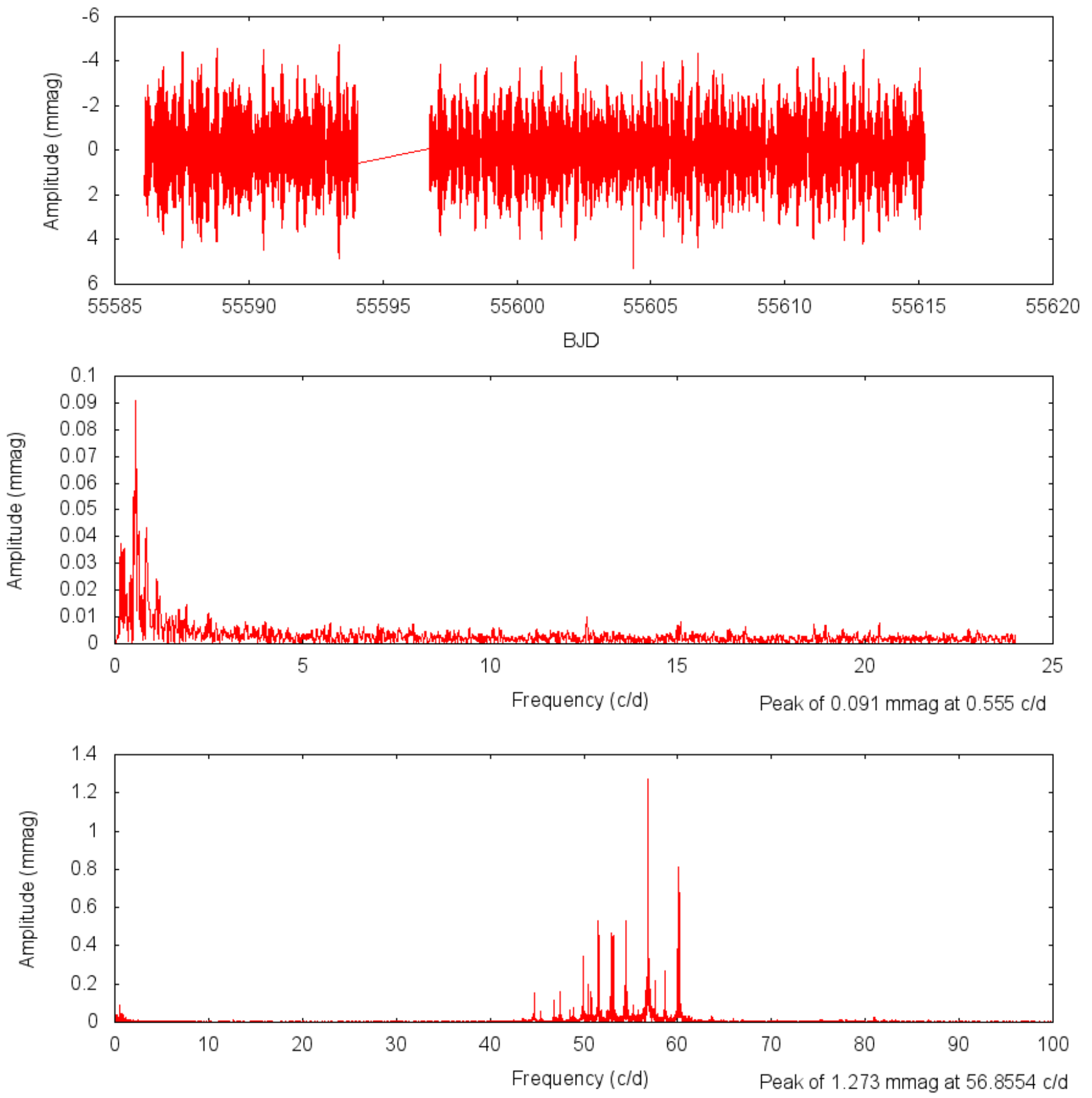

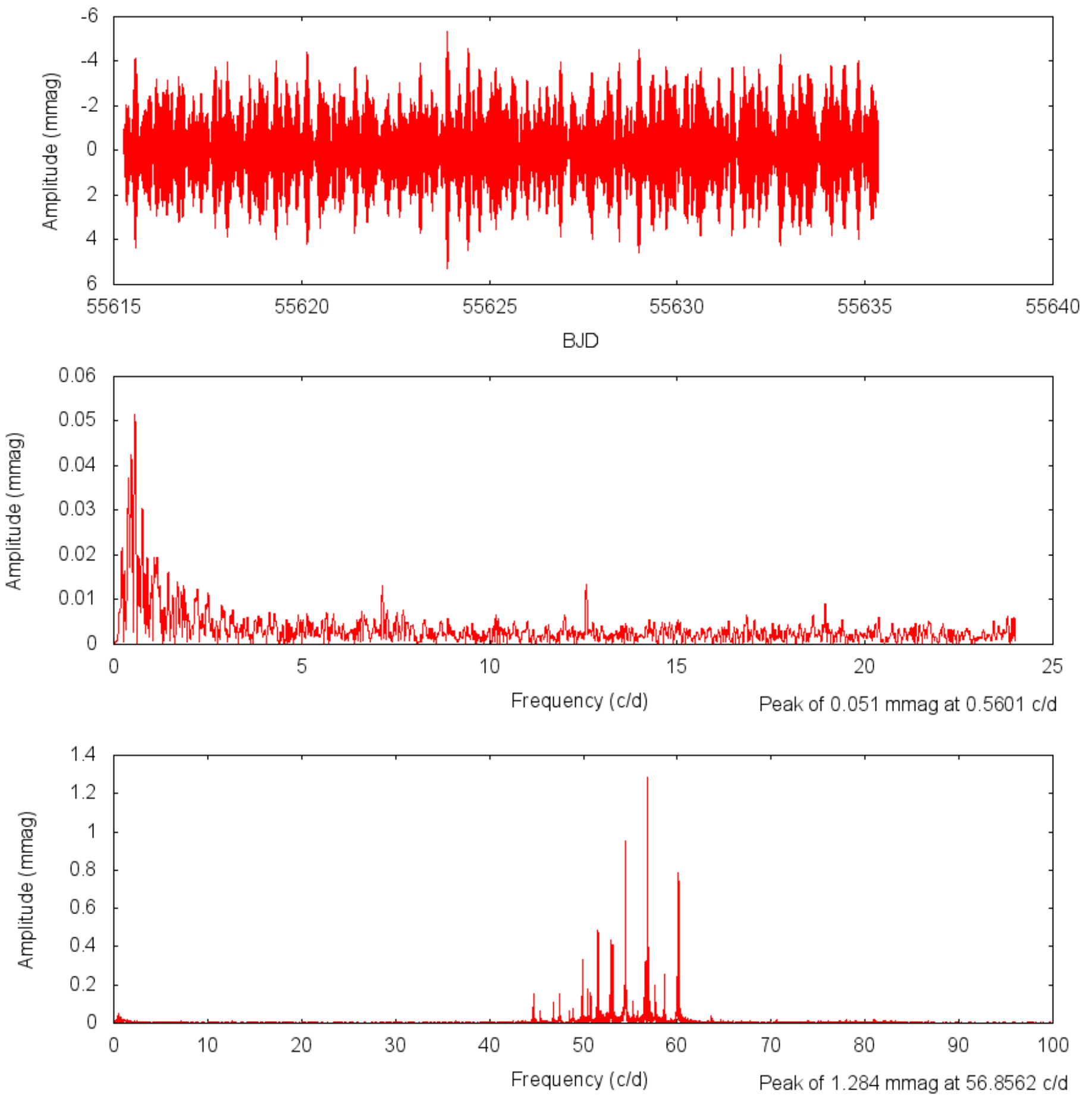


\title{
Pulsational amplitude growth of the star KIC 3429637 (HD 178875) in the context of Am and $\rho$ Pup stars
}

\author{
Simon J. Murphy, ${ }^{1 \star}$ A. Grigahcène,,${ }^{2,3}$ E. Niemczura, ${ }^{4}$ D. W. Kurtz ${ }^{1}$ \\ and K. Uytterhoeven ${ }^{5,6}$ \\ ${ }^{1}$ Jeremiah Horrocks Institute, University of Central Lancashire, Preston PR1 2HE \\ ${ }^{2}$ Centro de Astrofísica, Faculdade de Ciências, Universidade do Porto, Rua das Estrelas, 4150-762 Porto, Portugal \\ ${ }^{3}$ Kavli Institute for Theoretical Physics and Department of Physics Kohn Hall, University of California, Santa Barbara, CA 93106, USA \\ ${ }^{4}$ Astronomical Institute, Wrocław University, Kopernika 11, 51-622 Wrocław, Poland \\ ${ }^{5}$ Instituto de Astrofísica de Canarias (IAC), Calle Via Lactea $s / n, 38205$ La Laguna, Tenerife, Spain \\ ${ }^{6}$ Dept. Astrofísica, Universidad de La Laguna (ULL), Tenerife, E-38206 La Laguna, Spain
}

Accepted 2012 September 5. Received 2012 September 4; in original form 2012 August 1

\begin{abstract}
KIC 3429637 (HD 178875) is a $\delta$ Sct star whose light curve shows continuous pulsational amplitude growth in Kepler mission photometry. Analysis of the three largest amplitude peaks in the Fourier transform indicates different growth rates for all three. We have ruled out instrumental causes, and determine the amplitude growth to be intrinsic to the star. We calculate time-dependent convection models and compare them with the observations. We confirm earlier characterizations that KIC 3429637 is a marginal Am star through the analysis of new spectroscopic data. With the data presently available, a plausible cause of the amplitude growth is increasing pulsational driving as evolutionary changes shift the He II driving zone deeper in this $\rho$ Puppis star. If this model is correct, then we are watching real-time stellar evolutionary changes.
\end{abstract}

Key words: stars: chemically peculiar - stars: individual: HD 178875 - stars: oscillations stars: variables: $\delta$ Scuti.

\section{INTRODUCTION}

The $\delta$ Sct stars are low-overtone pressure mode pulsators on or near the main sequence between spectral types A2V to F0V and A3III to F5III (Kurtz 2000), whose pulsation frequencies range from 3 to $80 \mathrm{~d}^{-1}$ (i.e. $18 \mathrm{~min}$ to $8 \mathrm{~h}$ in period). Their pulsations are driven by the opacity $(\kappa)$ mechanism operating on the He II partial ionization zone. These intermediate-mass $\left(1.5-2.5 \mathrm{M}_{\odot}\right)$ stars are mostly Population I (Pop I) stars, but some Pop II $\delta$ Sct stars do exist and are known as SX Phe variables (see e.g. Templeton, Basu \& Demarque 2002).

The $\delta$ Sct instability strip is located at the junction between the classical Cepheid instability strip and the main sequence. It is not populated solely with $\delta$ Sct variables with normal spectra, however. The $\delta$ Sct stars have as their neighbours the following: the photometrically less variable, chemically peculiar, classical metallic-line (Am) stars; the marginal Am (Am:, pronounced 'Am colon') stars with slightly milder abundance anomalies; the early or hot Am stars; the $\rho$ Pup stars (evolved Am stars); the Ap stars (where the 'p' means peculiar); the $\gamma$ Dor stars; the $\lambda$ Boo stars; and the 'normal'

^E-mail: smurphy6@uclan.ac.uk
A-stars. This paper concerns KIC 3429637, a $\rho$ Pup star showing $\delta$ Sct pulsations.

\subsection{The Am stars}

The classical Am stars are those whose Ca II K-line types appear too early for their hydrogen line types, and metallic-line types appear too late, such that the spectral types inferred from the Ca II $\mathrm{K}$ line and metal line differ by five or more spectral subclasses. Such stars are therefore commonly given three spectral types, e.g. Am kA8hA9mF3, corresponding to the $\mathrm{K}$ line, hydrogen lines and metallic lines, respectively. The Am group makes up a significant fraction of late A-stars - up to 50 per cent at A8 (Smith 1973; Smalley et al. 2011), and features abundance anomalies typically of factors of \pm 10 (Abt 2009). Historically, the classical Am stars were thought not to pulsate, but with the micromagnitude photometric precision of the space-based Kepler mission six of the 10 known Am stars in the Kepler field of view are observed to pulsate (Balona et al. 2011a), and Smalley et al. (2011) found 200 of 1600 metallic-line A and F dwarfs to be pulsating from SuperWASP data.

The Am stars rotate sufficiently slowly that turbulent motions do not prevent gravitational settling of helium (Baglin et al. 1973). When enough helium is drained from the $\mathrm{He}$ II partial ionization 
zone, the star no longer pulsates. Meanwhile, ions with absorption lines near the peak wavelength of the photon energy distribution are radiatively levitated towards the surface, and others, notably $\mathrm{He}$, $\mathrm{C}, \mathrm{Ca}$ and $\mathrm{Sc}$, gravitationally settle, thus providing the abundance anomalies (Kurtz 1989).

The marginal Am stars show fewer than five spectral subclasses between the $\mathrm{Ca}$ II $\mathrm{K}$ and metallic lines. Such stars are therefore less extreme examples of the classical Am stars, with milder abundance anomalies. Kurtz (1978) showed that the majority of the Am: stars lie at the blue edge of the $\delta$ Sct instability strip (his fig. 2), which makes them difficult to distinguish from the 'hot Am stars' whose primary characteristics are Am anomalies in stars hotter than A4. Marginal Am stars are also found at the cool edge of the instability strip, however, with spectra similar to the historical $\delta$ Del stars. We refer the reader to Gray \& Garrison (1989) for a discussion of why the $\delta$ Del classification has been dropped and why the evolved Am stars are now known as $\rho$ Pup stars.

Kurtz (1978) presented members of the $\rho$ Pup class, which also pulsate. A particularly good case study of a $\rho$ Pup star that is also a high-amplitude $\delta$ Sct star is that of HD 40765 (Kurtz et al. 1995). There it was found that spectral peculiarities still remain in the presence of a peak-to-peak surface radial velocity range of $14 \mathrm{~km} \mathrm{~s}^{-1}$.

\subsection{Models of pulsating Am stars}

The introduction of time-dependent convection in the modelling of $\delta$ Sct star pulsations was a major step towards a quantitative comparison between theory and observations. Dupret et al. $(2004,2005)$ have successfully explained the red edge of the $\delta$ Sct instability strip by using these models. This approach was successfully applied to three $\delta$ Scuti stars by Dupret et al. (2005). The $\delta$ Sct stars observed by Kepler offer a higher level test for the theory. In this work we apply time-dependent convection models to a $\delta$ Sct star observed by Kepler for the first time.

Before we move on to the specific case of KIC 3429637, let us discuss the issue of pulsation coincident with metallicism, i.e. of the $\rho$ Pup stars. In the Am stars it was thought that there should be insufficient helium left in the He II partial ionization zone for $\delta$ Sct pulsations to be driven, but this is becoming increasingly doubted (e.g. Catanzaro \& Balona 2012). Kurtz (1989) discussed the theoretical exclusion of metallicism and pulsation in detail before announcing the first case of an extreme classical Am star showing $\delta$ Sct pulsations. It appears that pulsation in chemically peculiar stars with pulsation velocities of several hundred $\mathrm{m} \mathrm{s}^{-1}$ can be so laminar that turbulence of the order of $\mathrm{cm} \mathrm{s}^{-1}$ is not generated. If turbulence were generated, the diffusion process would become ineffective and the stars would become homogenized. Turcotte et al. (2000) addressed the issue, noting that it is velocity gradients that generate turbulence, and not the speed of displacement itself - fast but uniform displacement will not become turbulent. Their models, the New Montreal Models, which take diffusion of elements up to $\mathrm{Ni}$ into account, find stable stars with $\mathrm{He}$ abundances as low as 0.114 at 750 Myr. Specifically, He is still substantially present in the He II driving region in these models, and the iron-peak-element opacity bump is increased relative to standard models and contributes to excitation of longer period modes. As the stars evolve, they begin to pulsate naturally [i.e. without invoking dredge-up or hypothetical mass-loss (Turcotte et al. 2000)] because of the way that radius expansion causes the He II ionization zone to shift inward in mass fraction where more residual He is present (Cox, Hodson \& King 1979). The evolution leads to an increase in period of observed pulsation modes, and Turcotte et al. concluded that generally the effect of diffusion is to stabilize against p-mode pulsations but excite $\mathrm{g}$ modes. No extensive observational study covering a large sample of stars has been carried out to investigate pulsation frequency ranges in light of abundance anomalies.

In spite of all the strengths of diffusion theory (see e.g. Kurtz 2000), the diffusion models still fail to reproduce the recent observations of the commonality of pulsating Am stars (Smalley et al. 2011) satisfactorily, though we note that the disparity is not as great as it seems because 24 per cent of their 'pulsating Am' sample were actually 'Fm $\delta$ Del' stars, and that pulsations are expected to occur naturally in these evolved stars according to modern diffusion models. We refer the reader to the work of Balona et al. (2011b) for further discussion and observations on the edges of the instability strip for pulsating Am stars.

\subsection{The Kepler space mission}

The Kepler space mission features an array of 42 CCDs covering a $115 \mathrm{deg}^{2}$ area of the sky between the constellations of Cygnus and Lyra. It captures white-light photometric data from $\sim 150000$ stars, with the objective of detecting transiting planets that orbit within the habitable zone (Koch et al. 2010). The attained precision is around the micromagnitude level, and, with a duty cycle $>92$ per cent, the mission offers vast advances in astrophysics through the study of stellar pulsations. With photometry alone, transiting studies only yield the ratio of the planet and host-star radii, but asteroseismology can determine stellar radii to within $\sim 1$ per cent in some cases (Gilliland et al. 2010). Keeping that in mind, about 1 per cent of observations are allocated to asteroseismology.

Kepler data are available in two cadences: long cadence (LC) with effective 29.4-min integrations and short cadence (SC) with 58.9$\mathrm{s}$ integrations. The near-continuous observations are interrupted every $32 \mathrm{~d}$ (one 'month') for data downlinking, and every three months corresponds to one quarter of Kepler's 372.5-d heliocentric, Earth-trailing orbit. LC data are therefore available in quarters (denoted Qn), and SC data are further subdivided into months (denoted Qn.m). Each quarter the satellite performs a roll to keep its solar panels pointed towards the Sun and its radiator pointed towards deep space. Stars subsequently fall on to a different detector module when the symmetrical focal plane rotates. The unfortunate failure of module 3 early in the mission means that a small fraction $(\sim 4 / 21)$ of the field of view is only observable for three quarters of the year.

\section{PHOTOMETRIC OBSERVATIONS}

Kepler data are available in abundance for KIC 3429637, which is the ninth brightest in the $\delta$ Sct working group of the Kepler Asteroseismic Science Consortium (KASC). LC public data are available from Q1 through Q8, and SC public data are available for each month of Q7 and Q8. The LC data, with just over 30000 data points covering $670.33 \mathrm{~d}$, have a duty cycle of 91.4 per cent. The light curve for the Pre-search Data Conditioned (PDC) LC data is displayed in Fig. 1, from which it is immediately clear that the amplitude of the light variation is growing with time. We used only the least-squares version of the PDC pipeline (i.e. PDC-LS).

There are two obvious potential sources of the apparent amplitude growth: (1) the amplitude growth is intrinsic to the star, i.e. astrophysical; and (2) the amplitude growth is in some way instrumental. PDC data are not created to facilitate asteroseismology, but rather to prepare the data for planet searches. As such, the data are not to be used for asteroseismology without caution that the pipeline 

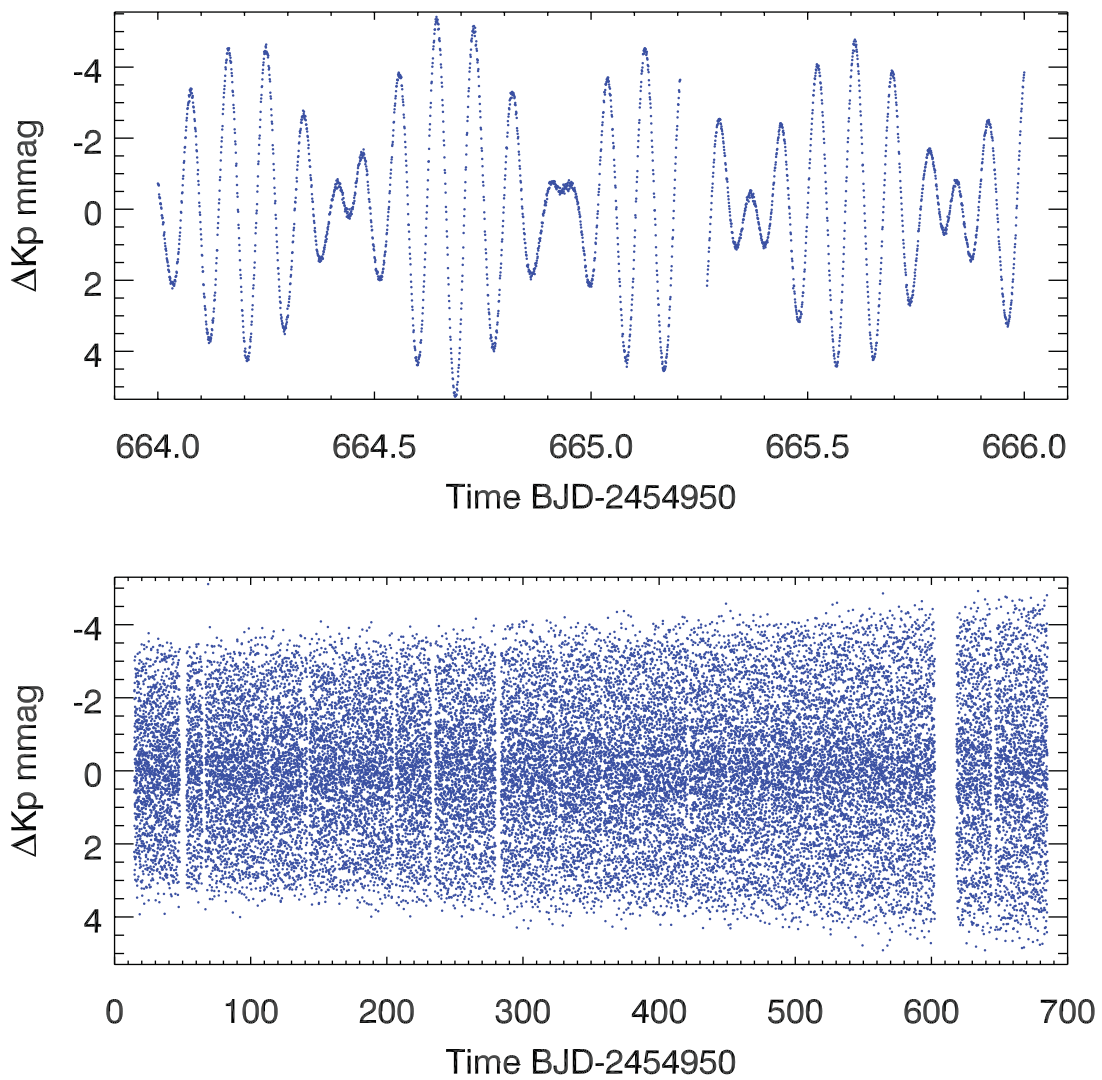

Figure 1. Upper panel: the $\delta$ Sct pulsations of KIC 3429637 in SC PDC from Q8. Kp denotes Kepler magnitude, and BJD is Barycentric Julian Date. Lower panel: the unedited light curve of the LC PDC data from Q1 to Q8. The amplitude of light variation is clearly growing with time, as can be seen from the unresolved envelope of the pulsations.

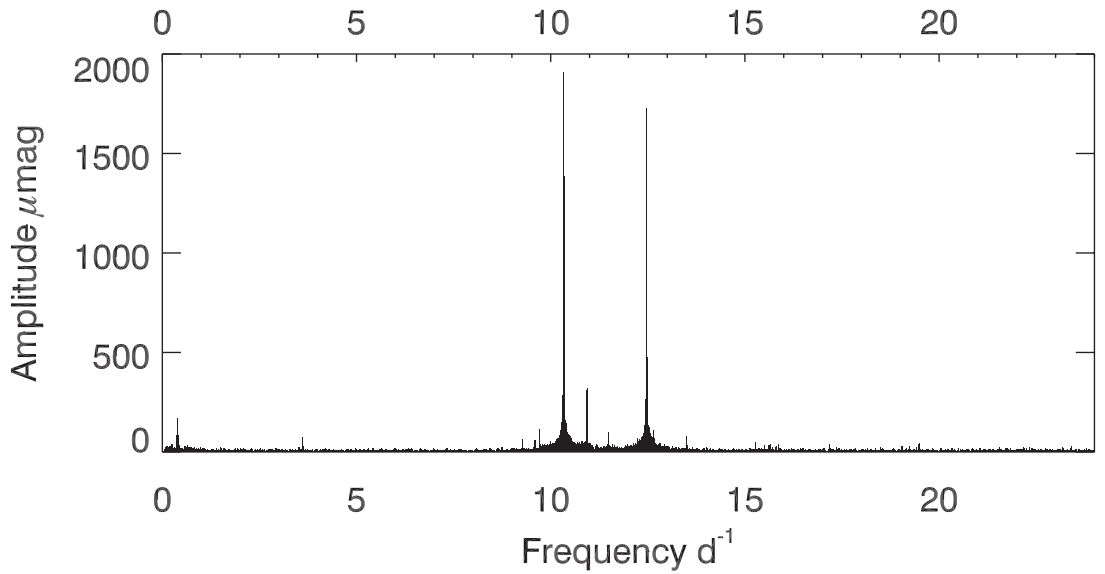

Figure 2. The Fourier transform of the unedited Q5 LC time string. The three highest amplitude peaks, in descending amplitude order, are $f_{1}, f_{2}$ and $f_{3}$.

might modify stellar variability. To test whether the pipeline caused the amplitude growth we compared the PDC data to the simple aperture photometry (SAP) data, for which only basic calibration has been performed and which is deemed suitable for asteroseismology, provided that instrumental trends are corrected for. Since instrumental trends generally affect low frequencies only, these are not an issue in the periodogram at the frequencies of pulsation shown in the Fourier transform of the data in Fig. 2. The amplitudes of the pulsations in the PDC data were compared to the uncorrected SAP data, by means of a non-linear least-squares fit of the frequencies to each quarter of data using the software package PERIOD04
(Lenz \& Breger 2004). The amplitude growth was the same in both data sets.

The star has two dominant frequencies $\left(f_{1}=10.337 \mathrm{~d}^{-1}\right.$ and $f_{2}=$ $12.472 \mathrm{~d}^{-1}$ ), with amplitudes growing between 1.5 and $2.5 \mathrm{mmag}$, and a third mode that lies between them $\left(f_{3}=10.936 \mathrm{~d}^{-1}\right)$ with an amplitude of $\sim 0.3 \mathrm{mmag}$ - observable without any pre-whitening. These frequencies are typical for $\delta$ Sct stars. As an additional check that the amplitude growth is astrophysical and not instrumental, the amplitude of that third peak was tracked across multiple quarters for comparison. It was found that although the two main peaks continued to grow in amplitude across all quarters, $f_{3}$ showed a 


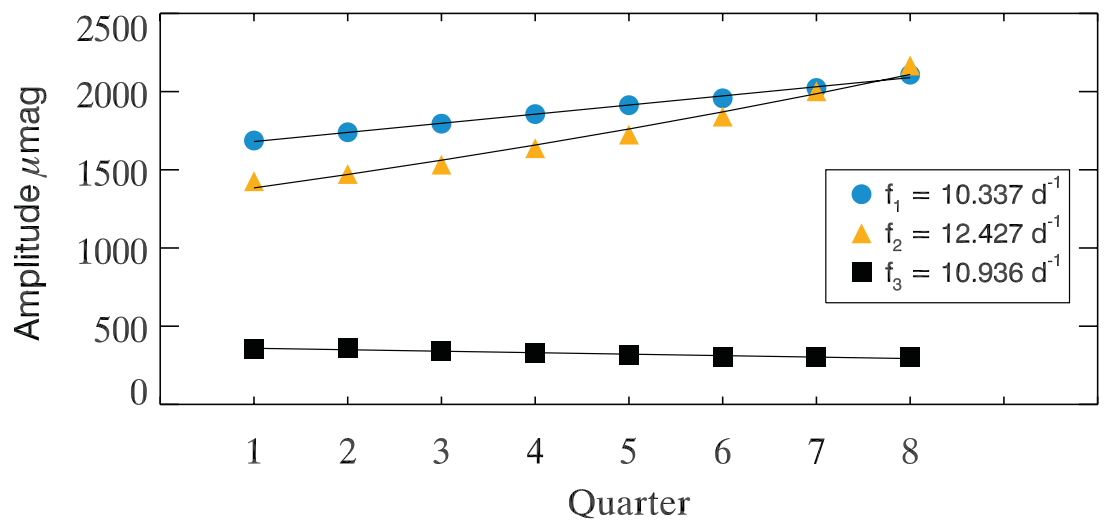

Figure 3. The growth of amplitude of $f_{1}$ and $f_{2}$, and decrease in amplitude of $f_{3}$ in LC PDC-LS flux as a function of time. Linear fits are shown for $f_{1}$ and $f_{3}$, and an exponential fit is shown for $f_{2}$. Errors on amplitude are of the order of a few micromagnitudes, and are much smaller than the plot symbols.

continual decrease in amplitude, as seen in Fig. 3. This proves that the growth in amplitude of the light variation is astrophysical. In addition to these three main frequencies, some $\sim 40$ statistically significant peaks with lower amplitudes exist in the data. We tracked the amplitudes of only the next four highest amplitude modes, and have hence tracked all modes for which modelling was performed (see Section 6). The amplitudes of these four modes, not shown in Fig. 3, show changes of no more than $30 \mu$ mag across the eight quarters and the changes appear to be noise dominated, so the amplitudes of peaks with lower statistical significance were not tracked.

The optimum aperture for a star is defined such that the signalto-noise ratio of the light captured from that star is maximized. This does not, therefore, capture all light from that star, because pixels further from the centre of the star contain less of the star's light and therefore relatively higher noise levels. It is possible, as a direct consequence of the choice of optimum aperture, that slightly more light from this star is being captured in each quarter and slightly less of a neighbouring star that is contributing towards the total light, which would create the effect of amplitude growth of all pulsation frequencies in the star's Fourier spectrum. Although not all modes grow in amplitude, a check is necessary. The Kepler Input Catalogue (KIC; Latham et al. 2005; Brown et al. 2011) lists the contamination for this star to be 0.037 , meaning that about 3.7 per cent of the light in the aperture of this star comes from one or more neighbours rather than the target star itself. To rule out the hypothesis that the amplitude growth is due to changing flux fractions of any neighbour, we took the target pixel light curves of Q1 to Q5 and defined a mask for each one that covered every pixel available for the star for that quarter. We found that just as for the PDC LS pipeline data, with our custom mask the amplitudes of the dominant modes grew each quarter and the amplitude of the third mode that lies between them continued to decrease.

In the Catalogue of the Components of Double and Multiple Stars (CCDM; Dommanget \& Nys 1994), KIC 3429637 is listed as a double star with a separation of 1.58 arcsec. The primary has $V=7.8$ and the secondary has $V=12.7$, thus the secondary has a spectral type around early K. Having a Hipparcos parallax of $3.75 \pm 0.58$ mas, the stars are well separated with an orbital period $>1000 \mathrm{yr}$, so tidal effects on the primary's rotation period are unimportant. Without radial velocities, we must treat KIC 3429637 as if it were a single star. Furthermore, there is no evidence at present to rule out chance alignment.

We wish to examine the possibility that the amplitude change is caused by the secondary. According to the CCDM, the dimmer star contributes just 1 per cent of the light coming from the pair. For this dimmer star to cause an amplitude change of $\sim 30$ per cent is impossible: one would have to remove it 30 times to exact such a change.

\section{SPECTROSCOPIC OBSERVATIONS}

Various methods exist for inspection of a star for chemical peculiarity, and definitions of peculiarity in the literature are complex. In his Am star review, Conti (1970) summarized the peculiarities as 'deficient $\mathrm{Ca}(\mathrm{Sc})$ or overabundant heavier elements, or both'. Indeed, Conti (1965) used the line depth ratio of Sc II $\lambda 4246.8 / \mathrm{Sr}$ II $\lambda 4215.5$ as an indicator of peculiarity and Smith (1970) went further and used another criterion Sc II $\lambda 4320.7 / \mathrm{Y}_{\text {II }} \lambda 4309.6$ because of the potential for blending of the Sc II line with the Fe I line (at $\lambda 4247.3$ ) in fast rotators; Smith (1970) noted that a substantial fraction of Am stars may be nearly normal in Sc and $\mathrm{Ca}$. Modern abundance surveys are consistent with long-established characterizations: Gebran et al. (2010) found that 'All Am stars in the Hyades are deficient in $\mathrm{C}$ and $\mathrm{O}$ and overabundant in elements heavier than Fe but not all are deficient in calcium and/or scandium.'

Another method for detection of peculiarity is the Strömgren $\Delta m_{1}$ index. A-stars with negative $\Delta m_{1}$ indices are commonly Am stars, with more negative values indicating a more chemically peculiar star. Using uvby parameters for KIC 3429637 from Olsen (1983) and Hauck \& Mermilliod (1998) $\left(b-y=0.189, m_{1}=0.215\right.$, $\left.c_{1}=0.849\right)$, noting that both sources agree to that precision, the $\Delta m_{1}$ index for this star was calculated using table 1 of Crawford (1979) for calibration as $\Delta m_{1}=-0.028$, which indicates an Am/Am: star. Our evaluation is consistent with that of Abt (1984), who classified the star as $\mathrm{Am}(\mathrm{F} 2 / \mathrm{A} 9 / \mathrm{F} 3)$ - a marginal Am star given that the metallic-line type and $\mathrm{Ca}$ II $\mathrm{K}$ line type differ by fewer than five spectral subclasses. We note that although the Crawford $\Delta m_{1}$ calibration is for main-sequence stars, it may also be applied to evolved stars.

Atmospheric parameters for KIC 3429637 were calculated by Catanzaro et al. (2011) as part of their spectroscopic survey of potential Kepler asteroseismic targets in the $\delta$ Sct and $\gamma$ Dor instability strips. They estimated an equivalent spectral type of F0 III (from fundamental parameters), $v \sin i=50 \pm 5 \mathrm{~km} \mathrm{~s}^{-1}$, and used a variety of methods to determine $T_{\text {eff }}$ and $\log g$. The weighted mean of their values for $T_{\text {eff }}$ and $\log g$ are $7300 \pm 200 \mathrm{~K}$ and $3.16 \pm 0.25$ (CGS), respectively. Catanzaro et al. also computed masses for this star: a canonical value of $3.6_{-0.6}^{+0.7} \mathrm{M}_{\odot}$ and a non-canonical value (that is, with convective overshooting: $\lambda_{\mathrm{OV}}=0.2 H_{p}$ ) of $3.2_{-0.5}^{+0.6} \mathrm{M}_{\odot}$. They 
Table 1. The elemental abundances for KIC 3429637 as compared to the solar values of Asplund et al. (2009). Columns 4 and 5 display the abundances of this star and the Sun, respectively, with their standard deviations. For those elements in column 4 whose abundances were determined from only one or two lines, an accurate uncertainty estimate cannot be made so we provide the mean uncertainty of our other abundances (0.12) instead. Column 6 is the difference between columns 4 and 5. Column 7 contains $[\mathrm{M} / \mathrm{H}]$ for the normal A-stars from Gebran et al. (2010) for the elements they analysed. In their paper, these abundances were relative to the solar values of Grevesse \& Sauval (1998), but here we correct them to the latest and most precise abundances: Asplund et al. (2009). Column 8 contains the difference between the abundances of this star and a normal A-star. The final column is like column 8 , but with a metallicity correction applied - it represents the difference between KIC 3429637 and a normal star of the same Fe/H (see in-text explanation, Section 3.3).

\begin{tabular}{cccccrrrr}
\hline Element & Z & No. of lines & Abundance & Solar value & {$[\mathrm{M} / \mathrm{H}]$} & A-stars & $\Delta M$ & $\Delta M_{\text {corr }}$ \\
\hline $\mathrm{C}$ & 6 & 6 & $8.03 \pm 0.16$ & $8.43 \pm 0.05$ & $-0.40 \pm 0.17$ & -0.06 & -0.25 & -0.49 \\
$\mathrm{Na}$ & 11 & 1 & $6.07 \pm 0.12$ & $6.24 \pm 0.04$ & $-0.17 \pm 0.13$ & 0.29 & -0.37 & -0.04 \\
$\mathrm{Mg}$ & 12 & 6 & $7.53 \pm 0.15$ & $7.60 \pm 0.04$ & $-0.07 \pm 0.16$ & 0.13 & -0.22 & 0.07 \\
$\mathrm{Si}$ & 14 & 4 & $7.32 \pm 0.17$ & $7.51 \pm 0.03$ & $-0.19 \pm 0.17$ & 0.39 & -0.54 & -0.08 \\
$\mathrm{~S}$ & 16 & 1 & $7.67 \pm 0.12$ & $7.12 \pm 0.03$ & $0.55 \pm 0.12$ & - & - & - \\
$\mathrm{Ca}$ & 20 & 11 & $6.34 \pm 0.09$ & $6.34 \pm 0.04$ & $0.00 \pm 0.10$ & 0.04 & -0.02 & -0.03 \\
$\mathrm{Sc}$ & 21 & 11 & $2.97 \pm 0.10$ & $3.15 \pm 0.04$ & $-0.18 \pm 0.11$ & -0.25 & 0.09 & -0.38 \\
$\mathrm{Ti}$ & 22 & 29 & $4.82 \pm 0.13$ & $4.95 \pm 0.05$ & $-0.13 \pm 0.14$ & 0.15 & -0.21 & -0.11 \\
$\mathrm{~V}$ & 23 & 2 & $4.43 \pm 0.12$ & $3.93 \pm 0.08$ & $0.50 \pm 0.14$ & - & - & - \\
$\mathrm{Cr}$ & 24 & 25 & $5.50 \pm 0.20$ & $5.64 \pm 0.04$ & $-0.14 \pm 0.20$ & 0.17 & -0.28 & -0.05 \\
$\mathrm{Mn}$ & 25 & 6 & $5.24 \pm 0.14$ & $5.43 \pm 0.05$ & $-0.19 \pm 0.15$ & 0.13 & -0.36 & -0.16 \\
$\mathrm{Fe}$ & 26 & 80 & $7.35 \pm 0.11$ & $7.50 \pm 0.04$ & $-0.15 \pm 0.12$ & 0.19 & -0.34 & 0.00 \\
$\mathrm{Ni}$ & 28 & 17 & $6.43 \pm 0.12$ & $6.22 \pm 0.04$ & $0.21 \pm 0.13$ & 0.36 & -0.12 & 0.39 \\
$\mathrm{Cu}$ & 29 & 2 & $3.92 \pm 0.12$ & $4.19 \pm 0.04$ & $-0.27 \pm 0.13$ & - & - & - \\
$\mathrm{Zn}$ & 30 & 2 & $4.37 \pm 0.12$ & $4.56 \pm 0.05$ & $-0.19 \pm 0.13$ & - & - & - \\
$\mathrm{Sr}$ & 38 & 2 & $3.97 \pm 0.12$ & $2.87 \pm 0.07$ & $1.10 \pm 0.14$ & 0.39 & 0.81 & 1.41 \\
$\mathrm{Y}$ & 39 & 3 & $2.94 \pm 0.01$ & $2.21 \pm 0.05$ & $0.73 \pm 0.05$ & 0.57 & 0.19 & 1.01 \\
$\mathrm{Zr}$ & 40 & 3 & $2.98 \pm 0.03$ & $2.58 \pm 0.04$ & $0.40 \pm 0.05$ & 0.62 & -0.20 & 0.10 \\
$\mathrm{Ba}$ & 56 & 3 & $3.08 \pm 0.13$ & $2.18 \pm 0.09$ & $0.90 \pm 0.16$ & - & - & - \\
$\mathrm{La}$ & 57 & 1 & $0.92 \pm 0.12$ & $1.10 \pm 0.04$ & $-0.18 \pm 0.13$ & - & - & - \\
\hline & & & & & & & - & - \\
& & & & & & & - & - \\
\end{tabular}

estimated the luminosity to be $20 \pm 6 \mathrm{~L} \odot$. Additional parameters from the KIC, roughly derived from broad-band photometry, are $R=4.1 \mathrm{R} \odot$ and $[\mathrm{Fe} / \mathrm{H}]=0.12$. It is immediately apparent from the luminosity class and $\log g$ parameters that the star is a giant, and thus an evolved Am, or $\rho$ Pup, star. A comparison of the $m_{1}$ value of this star with those studied by Kurtz (1976, his fig. 3) strongly implies that this star is of the $\rho$ Pup type. We return to this in Section 4.

\subsection{New spectroscopic observations}

A medium-resolution optical spectrum of the investigated object was obtained using the cross-dispersed, Fibre-fed Échelle Spectrograph (FIES) on the 2.5-m Nordic Optical Telescope, at Roque de los Muchachos, La Palma during the run of 2010 August 3-5 (proposal: 61-NOT7/10A). FIES offers an optical spectrum from 3700 to $7300 \AA$, with a spectral resolution of 46000 in a single exposure. The signal-to-noise ratio of the spectrum is about 100 at $5500 \AA$.

The spectrum was reduced using standard procedures of FIEStool, which consists of bias subtraction, extraction of scattered light produced by the optical system, division by a normalized flatfield and wavelength calibration. After reduction, the spectrum was normalized to the continuum by using SPLAT, the spectral analysis tool from the Starlink project (Draper et al. 2005).

\subsection{Atmospheric parameters' determination}

To perform an abundance analysis, one needs to determine an appropriate atmospheric model of the star, which requires the knowledge of its effective temperature $T_{\text {eff }}$, surface gravity $\log g$ and metallicity. The necessary atmospheric models were computed with the line-blanketed local thermodynamic equilibrium (LTE) ATLAS9 code (Kurucz 1993a), which treats line opacity with opacity distribution functions. The synthetic spectra were computed with the SYNTHE code (Kurucz 1993b) over the wavelength ranges 4000-5850 and 6100-6800 A, excluding those segments contaminated with telluric lines. Both codes, ATLAS 9 and SYNTHE, were ported under GNU Linux by Sbordone (2005) and are available online. ${ }^{1}$ The stellar line identification and the abundance analysis were performed on the basis of the line list from Castelli \& Hubrig (2004). ${ }^{2}$

We derived $T_{\text {eff }}$ for KIC 3429637 using the sensitivity of hydrogen line wings to temperature, following the method proposed by van't Veer-Menneret \& Megessier (1996). The effective temperature was estimated by computing the ATLAS 9 model atmosphere which gives the best match between the observed $\mathrm{H} \delta, \mathrm{H} \gamma, \mathrm{H} \beta$ and $\mathrm{H} \alpha$ line profiles and those computed with sYNTHE. The same value of $T_{\text {eff }}$ was obtained from the analysis of iron lines. In this method effective temperature, surface gravity and microturbulence $(\xi)$ are determined by the comparison of the abundances obtained from neutral and ionized iron lines. The analysis is based on Fe lines because they are the most numerous in the stellar spectrum. In general, we require that the abundances measured from $\mathrm{Fe}_{\mathrm{I}}$ and $\mathrm{Fe}$ II lines yield the same result. The absorption lines of $\mathrm{Fe}$ I depend mainly on $T_{\text {eff }}, \xi$ and metallicity, and are practically independent of $\log g$, whereas the Fe II lines are mostly sensitive to $\log g$. First, we adjust $\xi$ until we see no correlation between iron abundances and line intensity for the Fe I lines. Secondly, $T_{\text {eff }}$ is changed until we see

\footnotetext{
${ }^{1}$ wwwuser.oat.ts.astro.it/atmos/

2 http://wwwuser.oat.ts.astro.it/castelli/grids.html
} 
Table 2. Fundamental atmospheric parameters determined from the FIES spectrum.

\begin{tabular}{cccc}
\hline $\begin{array}{c}T_{\text {eff }} \\
(\mathrm{K})\end{array}$ & $\begin{array}{c}\log g \\
(\mathrm{CGS})\end{array}$ & $\begin{array}{c}\xi \\
\left(\mathrm{km} \mathrm{s}^{-1}\right)\end{array}$ & $\begin{array}{c}v \sin i \\
\left(\mathrm{~km} \mathrm{~s}^{-1}\right)\end{array}$ \\
\hline $7300 \pm 100$ & $3.0 \pm 0.1$ & $4.0 \pm 0.5$ & $51 \pm 1$ \\
\hline
\end{tabular}

no trend in the abundance versus excitation potential of the atomic level causing the Fe I lines. $\xi$ and $T_{\text {eff }}$ are not independent of each other. Then $\log g$ is obtained by fitting the Fe II and $\mathrm{Fe}_{\mathrm{I}}$ lines and by requiring the same abundances from both neutral and ionized lines.

The abundances of chemical elements were determined by the spectrum synthesis method. Our analysis follows the methodology presented in Niemczura, Morel \& Aerts (2009) and relies on an efficient spectral synthesis based on a least-squares optimization algorithm. This method allows for the simultaneous determination of various parameters involved with stellar spectra and consists of the minimization of the deviation between the theoretical flux distribution and the observed normalized one. The synthetic spectrum depends on the stellar parameters, such as $T_{\text {eff }}, \log g, \xi, v \sin i$ and the relative abundances of the elements. Some of these parameters have similar influence on stellar spectra and have to be known before the determination of the abundances of chemical elements. $T_{\text {eff }}, \log g$ and $\xi$ are the input parameters. All other aforementioned parameters can be determined simultaneously because they produce detectable and different spectral signatures. The $v \sin i$ values are determined by comparing the shapes of observed metal line profiles with the computed profiles, as shown by Gray (2005).

For chemical abundance analysis we used short segments of the spectrum to isolate individual spectral features, and in a few cases, some blended features. In the case of blended lines, more than one chemical element can influence the line profile. Only the most important elements for characterizing Am star peculiarities were considered. Every selected part of the spectrum was analysed by the spectrum synthesis method described above. We iteratively adjusted radial velocity, $v \sin i$, and chemical abundances until the determined parameters remained the same within 2 per cent for three consecutive iterations, thus determining the closest match between the calculated and observed spectrum. Finally, we determined the average values of radial velocity, $v \sin i$, and abundances of 20 elements with error estimates (Table 1). Table 2 summarizes our determined atmospheric parameters, and we plot our spectrum against comparisons in Fig. 4.

\subsection{Discussion of spectroscopic results}

The line ratios of Conti (1965) and Smith (1970) were inconclusive on the Am: nature of the star, necessitating the full abundance analysis (Table 1 ). We note normal $\mathrm{Ca}$ and nearly normal $\mathrm{Fe}$, but slightly deficient $\mathrm{Sc}$, strongly deficient $\mathrm{C}$, and highly overabundant $\mathrm{Sr}, \mathrm{Y}$ and $\mathrm{Ba}$, which would collectively confirm that the star is chemically peculiar. However, observations of Gebran et al. (2010) suggest that even normal A-stars are overabundant in Sr, $\mathrm{Y}$ and $\mathrm{Zr}$ with respect to solar values. This effect of overabundant heavy elements can have multiple origins as discussed in the following.

(1) The study of Gebran et al. focused on the Hyades cluster, and those cluster stars could be systematically enriched in heavier elements. The abundances of nearly all heavier elements are known to be correlated with iron (Hill \& Landstreet 1993). The [Fe/H] of the Hyades cluster is not well agreed upon. Boesgaard \& Friel (1990) determined a supersolar metallicity $\langle[\mathrm{Fe} / \mathrm{H}]\rangle=0.127 \pm 0.022 \mathrm{dex}$; however, Varenne \& Monier (1999) found a much lower value of $-0.05 \pm 0.03$ dex when investigating $29 \mathrm{~F}$ dwarfs. Gebran et al. themselves find $\mathrm{Fe} / \mathrm{H}=0.19$ dex above solar. A recent independent calculation (Carrera \& Pancino 2011) based on three K giants yields $\langle[\mathrm{Fe} / \mathrm{H}]\rangle=+0.11 \pm 0.01 \mathrm{dex}$ and is close to the mean of previous studies collated by those authors. We may therefore safely conclude at least a slightly supersolar Fe abundance and that correlation with $\mathrm{Fe}$ is a contributor.

(2) Using the Sun as a standard star has the weakness of assuming that the Sun is typical. Meléndez et al. (2009) found the Sun to be depleted in refractory elements by $\sim 20$ per cent relative to volatile elements compared to solar twins. Specifically, the lighter, volatile elements (e.g. C, $\mathrm{N}$ and $\mathrm{O}$ ) are enriched by $\sim 0.05 \mathrm{dex}$ and the heavier, refractory elements depleted by $\sim 0.03 \mathrm{dex}$. Their findings were confirmed in another study (Ramírez, Meléndez \& Asplund 2009), and the hypothesis that planetary formation is the cause is independently theoretically supported (Chambers 2010). The peculiar solar abundance therefore also contributes to the

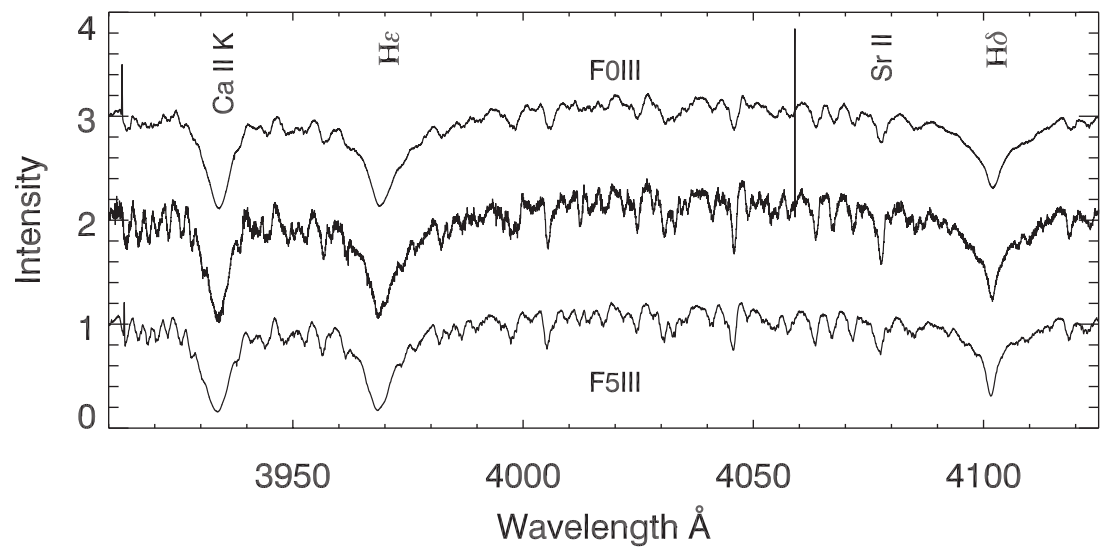

Figure 4. Spectrum of KIC 3429637 (centre) with two comparisons: the F0 III star HD 7312, and the F5 III star HD 65925, though neither is an MK-standard star. Catanzaro et al. (2011) listed KIC 3429637 as F0 III m. The hydrogen line type is a good match to F0/A9. Despite being of the Am type, note that the Ca II $\mathrm{K}$ line is no weaker than the F0 comparison (cf. Table 1, in which calcium is completely normal). The metal lines are much stronger, though, and are even stronger than the F5 comparison. In particular, the Sr II $\lambda 4077$ line is much deeper, owing to the Am nature. The comparison spectra were obtained at 'http://www.eso.org/sci/observing/tools/uvespop/field_stars_uptonow.html', normalized at $3910.0 \AA$ and vertically separated by 1 unit. 
apparent overabundance of heavy elements in normal A-stars, and we may assume that a specific physical effect in A-star photospheres is not at play.

(3) One must also consider that the abundances of heavier elements are derived from fewer lines, and are therefore at greater risk of having erroneous values caused by blends.

Nevertheless, for those elements that were studied by Gebran et al. we also show the difference between KIC 3429637 and normal A-star abundances, relative to the Sun in Table 1. Mn, Ni, Sr and $\mathrm{Ba}$ are strongly correlated with $\mathrm{Fe}$ (Gebran, Monier \& Richard 2008), and $\mathrm{Y}$ and $\mathrm{Zr}$ increase rapidly with $\mathrm{Fe} / \mathrm{H}$ (Gebran et al. 2010), thus the chemical peculiarities of KIC 3429637 would be even more pronounced were it not for its low iron abundance. Therefore in the final column of Table 1 we tentatively provide the abundance differences as if KIC 3429637 had the same $\mathrm{Fe} / \mathrm{H}$ as the mean value of the 'normal' A-stars to which we are comparing, namely $[\mathrm{Fe} / \mathrm{H}]$ $=0.19$. The correction is calculated from the data tables provided in the online material of Gebran et al. (2010). Since A-stars are known to show large star-to-star variations in abundances compared to F-stars (Gebran, Monier \& Richard 2008), the correlation between $[\mathrm{M} / \mathrm{H}]$ and $[\mathrm{Fe} / \mathrm{H}]$ is not always tight, hence the 'tentative' provision. Importantly, the elements for which the correlation is tighter are those that are the most peculiar. Fossati et al. (2008b) found that there is no temperature effect on abundance patterns, thus we need not worry about any temperature difference between KIC 3429637 and the mean of the A-stars against which we are comparing.

We did not take non-LTE effects into account in our calculations, yet these effects can be important. Given the importance of calcium in Am star spectra, we considered non-LTE corrections for this element, but they are small (Mashonkina, Korn \& Przybilla 2007). For iron, non-LTE corrections are significant (0.2-0.3 dex) for Fe I, but not Fe II, and are positive (Rentzsch-Holm 1996). The corrections would bring our $\mathrm{Fe}$ abundance closer to the solar value. Conversely, corrections for carbon are negative (Rentzsch-Holm 1996) and would further exaggerate the carbon deficiency seen in this star and expected in Am stars.

All three of our $T_{\text {eff }}, \log g$ and $v \sin i$ determinations agree extremely well with the weighted means of those presented in Catanzaro et al. (2011), and $\xi$ is slightly high for a star of this temperature (Takeda et al. 2008). We note, however, that in Am stars $\xi$ is expected to be slightly higher than in normal stars (Coupry \& Burkhart 1992; Gebran \& Monier 2007). The former authors pointed out that the $\xi$ distribution reaches a maximum around A5, where the angular rotational velocity distribution also reaches a maximum. This could implicate rotational instabilities in generating microturbulence, but if that were true Am stars would not be observed to have higher $\xi$ values. Pace et al. (2006) defined the relation $\xi=-4.7 \log \left(T_{\text {eff }}\right)+$ $20.9 \mathrm{~km} \mathrm{~s}^{-1}$ for horizontal branch stars, in which radiative levitation is also important - this would give an expected $\xi$ of $2.7 \mathrm{~km} \mathrm{~s}^{-1}$, but the relation is not appropriate for the whole main sequence, and might even be used as a diagnostic to distinguish horizontal branch stars from main-sequence stars.

The low $\log g$ value indicates that this star is evolved. Interestingly, the value of $v \sin i$ is high for an evolved Am (i.e. $\rho$ Pup) starAm main-sequence stars are already slow rotators, with the Am/Ap star unimodal rotation velocity distribution peaking at $\sim 60 \mathrm{~km} \mathrm{~s}^{-1}$ after statistical correction for inclination (Abt \& Morrell 1995). During evolution, stars transfer angular momentum inward through radius expansion, slowing the surface rotation further. Hence by accepting the fact that the star is evolved, the high $v \sin i$ argues against the type of close binary system common among Am stars. ${ }^{3}$ Instead, the hypothesis of a single A-star with a subaverage rotation velocity is favoured for this case, in which this star was rotating slowly enough to develop some peculiarities.

It is well established that a large fraction of Am stars are found in binaries and that tidal braking slows the stellar rotation, allowing diffusion to occur. With the exclusion of Sc (Fossati et al. 2008a), peculiarities are more extreme in more slowly rotating stars (Takeda et al. 2008). The observed moderate $v \sin i$ value is indeed compatible with the fact that KIC 3429637 is not an extreme Am star, as the moderate abundance anomalies imply. Notwithstanding, $\rho$ Pup stars do not have extreme abundances, regardless of $v \sin i$. However, Stateva, Iliev \& Budaj (2012) found that peculiarity is also slightly correlated with orbital eccentricity: more eccentric orbits produce more anomalous abundances, but we do not expect this to have any significance for wide binaries. Attempts to use the frequency-modulation technique of Shibahashi \& Kurtz (2012) to infer orbital parameters were unsuccessful - orbital sidelobes were not present. This indicates that either the star is in a binary system with an orbital period longer than the data set, i.e. $P_{\text {orb }}>700 \mathrm{~d}$, or the star is not binary at all.

\section{ASTROPHYSICAL CAUSE OF AMPLITUDE GROWTH}

The different amplitude growth rates of each mode, visible in Fig. 3, can be used to determine a time at which the mode amplitudes were too low to be detectable from the ground, assuming that the growth rates are well behaved. A linear growth rate gave the best fit to $f_{1}$, but an exponential growth rate was more appropriate to $f_{2}$, which is growing much more rapidly than the lower frequency mode. Taking the limit of ground-based detection as $1 \mathrm{mmag}$ in the amplitude spectrum, $f_{1}$ would only have become detectable $\sim 2.6$ yr ago, and $f_{2}$ around $11 \mathrm{yr}$ ago. We might conclude that these modes would have been undetectable in 1984 at the time when Abt spectroscopically observed the star to be metallic lined, although no photometric investigation into variability was carried out. However, Breger (2000) observed the evolved $\delta$ Sct star $4 \mathrm{CVn}$ over three decades and found mode amplitudes to be unpredictable and highly variable. One highamplitude mode in that star disappeared altogether and re-emerged with a random phase. With so few studies of amplitude growth rates, and the unpredictable behaviour seen in $4 \mathrm{Cvn}$, we cannot predict how KIC3429637 will behave or how it behaved in the past. This star is most certainly worthy of continued observation to monitor the amplitude changes.

In any case, the current rates of growth indicate that the star has (a) not been pulsating for very long, and has just crossed some instability threshold; (b) undergone large amplitude variations over long periods of time, possibly in some cyclic nature; or (c) none of the above. We do not have enough data to pursue hypothesis (b), in that we have not observed a full cycle of variation in the light curve, but since the amplitude growth is not the same for each mode, nor does it appear to be sinusoidal, the cyclical amplitude variation hypothesis is unsupported by the present evidence. If we assume this star to be like $4 \mathrm{CVn}$, then continued observations are

${ }^{3}$ Tidal braking is most significant when the orbital period decreases below $10 \mathrm{~d}$ - the tidal braking mechanism proposed by Zahn (1975) denotes that braking time-scales depend on the stellar separation to the power 8.5. This is confirmed observationally by the fact that SB2 systems are circular for periods below $\sim 8 \mathrm{~d}$ (Debernardi 2000). 


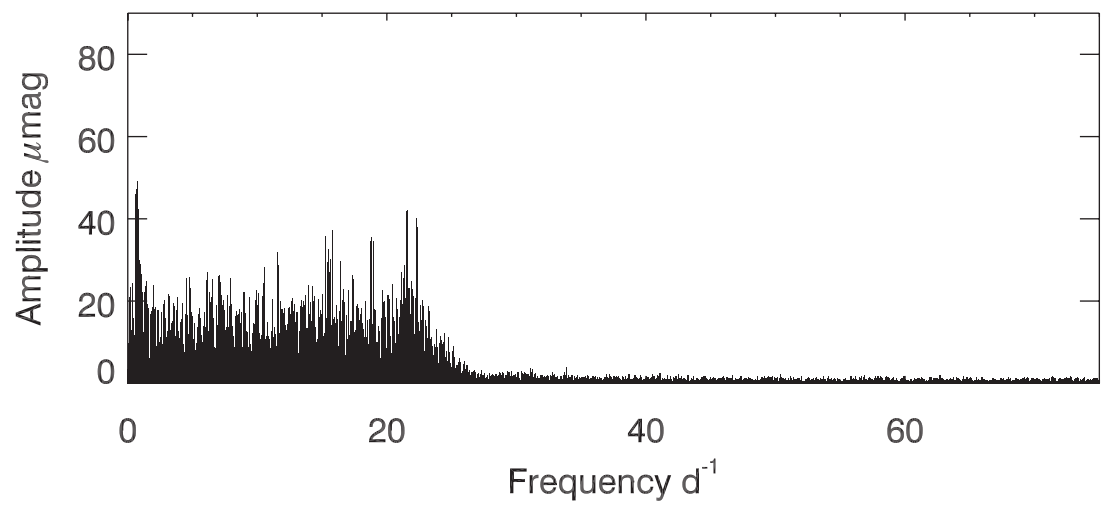

Figure 5. There is a rapid decrease in noise level at around $24 \mathrm{~d}^{-1}$. Excess noise is injected by the PDC-LS pipeline at frequencies below this limit. There are no peaks remaining below that frequency with signal-to-noise ratio $>4.0$, and no peaks have been removed at all above $24 \mathrm{~d}^{-1}$, hence the effect has not been caused by preferential pre-whitening. Note that here we have pre-whitened many more frequencies than were modelled in order to demonstrate the effect more clearly.

required to analyse the changing amplitudes. In the meantime, let us examine hypothesis (a) from a theoretical point of view.

Even if the pulsations are not especially laminar, the destruction of the chemical abundance anomalies may take time, such as in the case of $o$ Leo A, which is an evolved Am star whose abundance anomalies have not yet been erased (Michaud, Richer \& Richard 2005). KIC 3429637 could well have been a classical Am star on the main sequence, which is now at or approaching a stage of rapid evolution in which its chemical peculiarities are being erased and its pulsation amplitudes are growing. Temporal evolution of abundance anomalies is another understudied area of Am star research; KIC 3429637 would make a perfect case study. Existing studies (e.g. Abt 1979; Gebran et al. 2010) have mostly focused on multiple coeval stars in one cluster and contrasting clusters of different ages. In these cases the scatter in abundances of given elements is large enough that no real difference of any element with age is prominent. Focusing on single, regularly observed targets such as KIC 3429637 is thus potentially astrophysically rewarding.

\section{NOISE IN THE RESIDUALS}

Spectroscopic data show KIC 3429637 to be cool for a $\delta$ Sct pulsator, and progressively cooler A-type stars have increasingly deep surface convection zones (see Kallinger \& Matthews 2010 for a discussion). We used the Q7.1 SC data to look for such a signature of granulation in the form of a rapid change in noise with frequency. We present the results in Fig. 5. After extensive investigation, the rapid decrease in noise seen between 20 and $30 \mathrm{~d}^{-1}$ was determined not to arise from granulation, but rather the noise is injected by the Kepler PDC-LS ${ }^{4}$ pipeline for this particular Kepler quarter. It seems that approximately 15 per cent of stars in the $\delta$ Sct instability strip are afflicted with the same problem, which affects both LC and SC data, but not necessarily all quarters for a given star. A more detailed description will be given in a future publication (Murphy, in preparation).

The Q8 data for this star contain no evidence for 'granulation signature', implicating noise injection by the pipeline over an astrophysical origin. The drop in noise at $\sim 24 \mathrm{~d}^{-1}$ in SC data is

\footnotetext{
4 'LS' describes the least-squares algorithm used by the pipeline. We refer the reader to the papers of Stumpe et al. (2012), Smith et al. (2012) and the Kepler Data Characteristics Handbook for more information on the pipeline, and to Murphy (2012) for its effect on asteroseismic analyses.
}

characteristic for this noise injection. The Nyquist frequency of LC data prevents the drop being seen in that cadence, but the noise is still present. The new PDC maximum a posteriori (PDC-MAP) data will fix this for the LC data when the old data are reprocessed, but for now there is no implementation of MAP for SC.

\section{STRUCTURE AND OSCILLATION MODELS}

In our theoretical computations we used the Code Liégeois d'Évolution stellaire CLÉS (Scuflaire et al. 2008), where the input physics is as follows: the equation of state is the superior ' $\mathrm{CEFF}$ ' (see e.g. Christensen-Dalsgaard \& Daeppen 1992); the opacity tables are OPAL opacities from Iglesias \& Rogers (1996) and Alexander \& Ferguson (1994) for high and low temperatures, respectively. The relative mixture of chemical composition from Grevesse \& Noels (1993) was used, while the convection was treated using mixing-length theory (MLT; Böhm-Vitense 1958). Our models include neither rotation nor diffusion. For our non-adiabatic, nonradial oscillation calculations we used the MAD code (Dupret 2001), where Gabriel's treatment of time-dependent convection (TDC) has been implemented (Gabriel 1996).

We consequently produced a grid, where $\ell=0-3$ eigenfrequency spectra are computed for each of the models. For the selection of the best model we used the maximum likelihood method as described by Grigahcène et al. (2012).

\subsection{Results and discussion}

Using Kepler data, Uytterhoeven et al. (2011) showed how A-F pulsators can have such complex frequency spectra that they are challenging targets for any asteroseismic study. We consequently need as many constraints as possible for any theoretical modelling. Although the number of detected frequencies is reduced in KIC 3429637 compared to what is usually detected in this type of star, its modelling is no less complicated.

We performed careful and detailed modelling, varying all the parameters (mass, metallicity, mixing-length parameter $\alpha_{\mathrm{MLT}}$, etc.) to obtain the combination giving the maximum value of the likelihood function. We explored different possibilities, taking into account the detected frequencies and their associated amplitudes, and the position of the star in the Hertzsprung-Russell (HR) diagram. The main requirements on our models were as follows: (1) fitting the maximum number of the observed frequencies, (2) modes should 
Table 3. Properties of the best model.

\begin{tabular}{cccccccccc}
\hline $\begin{array}{c}M \\
\left(\mathrm{M}_{\odot}\right)\end{array}$ & $\begin{array}{c}T_{\text {eff }} \\
(\mathrm{K})\end{array}$ & $\log \left(L / \mathrm{L}_{\odot}\right)$ & $\log g$ & $\begin{array}{c}R \\
\left(\mathrm{R}_{\odot}\right)\end{array}$ & $\begin{array}{c}\text { Age } \\
(\mathrm{yr})\end{array}$ & $X$ & $Z$ & $\alpha_{\mathrm{MLT}}$ & $\alpha_{\mathrm{Ov}}$ \\
\hline 2.178 & 7452 & 1.570 & 3.648 & 3.666 & $8.982 \mathrm{E}+08$ & 0.7360 & 0.0149 & 2.0 & 0.2 \\
\hline
\end{tabular}

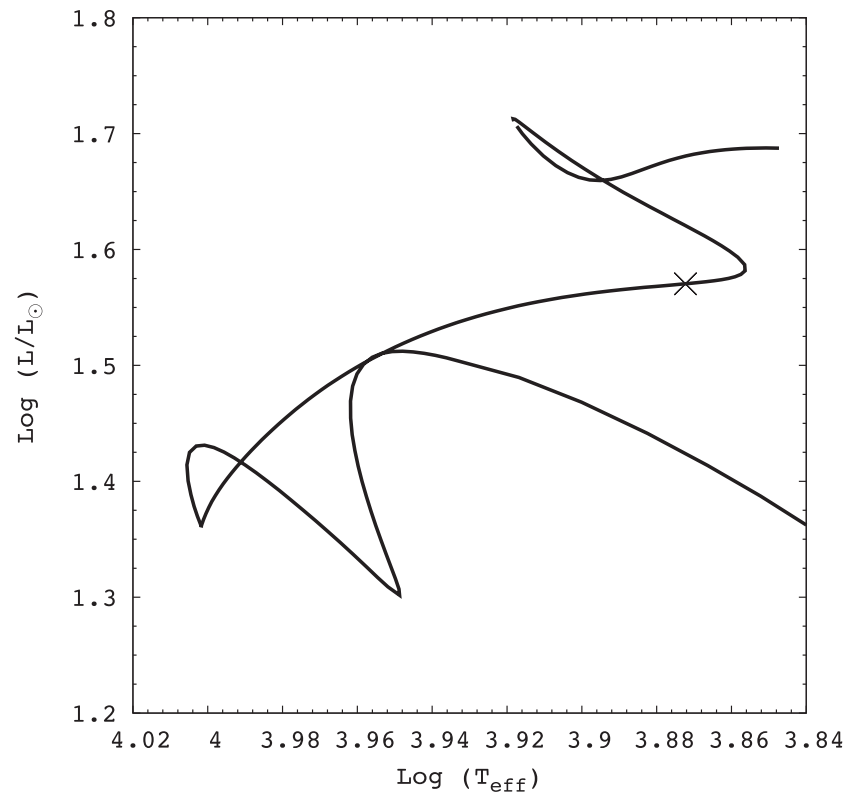

Figure 6. Position of the best model on its evolutionary track in the HR diagram, showing the evolutionary stage of the star. As this is a model, there are no error bars.

be unstable and (3) the global parameters should be inside the observational photometric error box. In Table 3 we show the main properties of the best obtained model. All the parameters are within the spectroscopic uncertainties. We show the star's position on the HR diagram and its evolutionary stage in Fig. 6.

Some important results come out from our comparison between theoretical time-dependent convection models and observations. First, we managed to obtain a model where seven of the eight highest amplitude frequencies are excited. The lowest frequency mode $\left(f_{8}=0.39448 \mathrm{~d}^{-1}\right)$ is predicted to be stable (i.e. not excited). This mode might not be caused by oscillation - at these low frequencies, instrumental origins are common (Murphy 2012, his fig. 3). The origin does not appear to be rotational or equal to the difference in frequency between any two other modes in Table 4. The alternative to instrumental origin is that it is indeed a pulsation frequency, in which case the conclusion is that some physics is missing from the model. We did not model all $40+$ statistically significant frequencies due to the overwhelming complexity involved in doing so.

Secondly, we found that the value of the mixing-length parameter giving the maximum value for the likelihood function is $\alpha_{\mathrm{MLT}}=$ 2, i.e. different from the solar value in Grigahcène et al. (2012). It is known that $\alpha_{\mathrm{MLT}}$ should vary from star to star and also with temperature as the internal structure changes from a solar-like one to those of A- and F-stars. As this star is the first $\delta$ Sct star to be treated with TDC models, coverage of $\alpha_{\mathrm{MLT}}$ for $T_{\text {eff }}>6000 \mathrm{~K}$ is currently unavailable, but for cooler stars we refer the reader to the works of Grigahcène et al. (2012) and Trampedach \& Stein (2011) for deeper discussion.
Table 4. Identification of oscillating modes. Negative $n$ values denote mixed modes with g-mode-like character. An additional observed frequency, $f_{8}=0.39448 \mathrm{~d}^{-1}$, was modelled but was not found to be excited. Formal least-squares frequency precision is $3 \times 10^{-6} \mathrm{~d}^{-1}$ for $f_{1}$ and $f_{2}$, and $2 \times 10^{-5} \mathrm{~d}^{-1}$ for $f_{3}$. Since our models do not include rotation to lift the degeneracy, all modes presented are $m=0$

\begin{tabular}{lccrcr}
\hline ID & $\begin{array}{c}f_{\text {Obs }} \\
\left(\mathrm{d}^{-1}\right)\end{array}$ & $\ell$ & $n$ & $\begin{array}{c}f_{\text {Theo }} \\
\left(\mathrm{d}^{-1}\right)\end{array}$ & $\begin{array}{r}f_{\text {Obs }}-f_{\text {Theo }} \\
\left(\mathrm{d}^{-1}\right)\end{array}$ \\
\hline 1 & 10.33759 & 0.0 & 3.0 & 10.36443 & -0.02684 \\
& & 1.0 & 0.0 & 10.45143 & -0.11384 \\
& & 3.0 & -3.0 & 10.22146 & 0.11613 \\
& & 2.0 & -1.0 & 10.50785 & -0.17027 \\
2 & 12.47161 & 0.0 & 4.0 & 12.4716063 & $2.0 \mathrm{E}-07$ \\
& & 3.0 & -1.0 & 12.45554 & 0.01607 \\
3 & 10.93641 & 3.0 & -2.0 & 10.93305 & 0.00336 \\
& & 1.0 & 1.0 & 10.94367 & -0.00725 \\
4 & 9.71214 & 3.0 & -4.0 & 9.71185 & 0.00029 \\
& & 2.0 & -2.0 & 9.71928 & -0.00714 \\
5 & 12.65066 & 1.0 & 2.0 & 12.82501 & -0.17435 \\
& & 0.0 & 4.0 & 12.47161 & 0.17905 \\
& & 3.0 & -1.0 & 12.45554 & 0.19512 \\
& & 2.0 & 1.0 & 12.89238 & -0.24172 \\
6 & 13.50229 & 3.0 & 0.0 & 13.67304 & -0.17074 \\
7 & 11.48287 & 2.0 & 0.0 & 11.65746 & -0.17459 \\
\hline
\end{tabular}

A third remark regards the fitting of the frequency values. In Table 4 we list the theoretical frequencies together with the observed frequencies and mode identifications. Four of the observed frequencies are very well fitted by the theoretical modes - the difference is less than $0.03 \mathrm{~d}^{-1}(0.35 \mu \mathrm{Hz})$. For the other three the difference is less than $0.2 \mathrm{~d}^{-1}$. However, an ambiguity arises for some modes in that there are multiple mode identification possibilities of similar likelihood. This indicates that ground-based multicolour photometric or spectroscopic observation campaigns are necessary to complete the mode identification.

Finally, while the asteroseismic effective temperature agrees with the spectroscopic one to within less than $2 \sigma$, the $\log g$ value from the models (3.65) does not agree particularly well with that from spectroscopy $(3.0 \pm 0.1)$. Similar model behaviour was seen in Grigahcène et al. (2012).

\subsection{Conclusions from modelling}

We have compared the observed frequencies of KIC 3429637 to state-of-the-art $\delta$ Sct models. The results of this comparison are the following. (1) All the observed modes (those listed in Table 4) are predicted to be excited in our model except the lowest frequency. (2) Four out of seven frequencies are fitted to within 0.26 per cent. (3) The varying amplitudes between quarters might be explained by mode interaction - the modelled frequencies are all rather close and are perhaps subject to resonances as described in e.g. Dziembowski \& Krolikowska (1985). We identify many potential mixed modes, and it is noteworthy that g modes that are not even visible in the 
Fourier transform have the potential to affect p-mode frequencies (Buchler, Goupil \& Hansen 1997).

\section{CONCLUSIONS}

The high-precision Kepler data indicate two dominant modes of growing amplitude, with different growth rates, and a third mode of intermediate amplitude whose amplitude decreases. The mode amplitude growth appears to be intrinsic to the star.

Our spectroscopic data confirm literature classifications that this star is of the marginal Am type. The heavy elements are overabundant with respect to solar values, and the light elements are underabundant - as one expects for Am stars. Notably, calcium is normal.

Mode amplitudes in $\delta$ Sct stars can change by large amounts on short time-scales, with changing growth rates (cf. $4 \mathrm{CVn}$ ). The amplitude growth in this star appears much less chaotic than for $4 \mathrm{CVn}$, and we have proposed evolution as the dominant cause, in that pulsation amplitudes are expected to increase naturally in evolving Am stars as the He II convection zone deepens and picks up residual helium. We cannot definitively rule out that mode interaction is the cause, where energy is being transferred from one or more modes into those whose amplitudes are growing, except to say that we see no modes decreasing in amplitude by comparable amounts to those by which the dominant modes are growing. As such, this explanation seems viable only if one or more unseen modes - perhaps $g$ modes or high-degree p modes - are transferring the energy.

For the first time, time-dependent convection has been used in models of a $\delta$ Sct star observed with Kepler. Despite the narrow observational constraints, the modelling was difficult. We determine a $2.18 \mathrm{M}_{\odot}, 7450-\mathrm{K}$ model with $Z=0.0149$ to be the best solution (cf. Table 3), with the star being 94 per cent of the way through its main-sequence lifetime (see Fig. 6), and we match four of seven mode frequencies to within 0.26 per cent (Table 4). Multicolour photometry and/or time series spectroscopy would facilitate mode identification, and this target is most worthy of continual follow-up in Kepler's LC mode, not least because it offers a chance to witness stellar evolution on human time-scales.

\section{ACKNOWLEDGMENTS}

This research has made use of the SIMBAD data base, operated at CDS, Strasbourg, France. Calculations have been partially carried out in Wroclaw Centre for Networking and Supercomputing (http://www.wcss.wroc.pl), grant No. 214. Based on data from the Kepler Space Telescope, for which we are extremely grateful, and on observations made with the Nordic Optical Telescope, operated on the island of La Palma jointly by Denmark, Finland, Iceland, Norway and Sweden, in the Spanish Observatorio del Roque de los Muchachos of the Instituto de Astrofísica de Canarias (IAC).

SJM would like to acknowledge the financial support of the STFC. AG acknowledges the KITP staff of UCSB for their warm hospitality during the research programme 'Asteroseismology in the Space Age'. This research was supported in part by the National Science Foundation of the United States under Grant No. NSF PHY05-51164. EN acknowledges support from Polish MNiSW5 grant N N203 405139. KU acknowledges financial support by the Spanish National Plan of R\&D for 2010, project AYA2010-17803. We thank the Spanish Night-Time Allocation Committee (CAT) for awarding time to the proposal 61-NOT7/10A.

\section{REFERENCES}

Abt H. A., 1979, ApJ, 230, 485

Abt H. A., 1984, ApJ, 285, 247

Abt H. A., 2009, AJ, 138, 28

Abt H. A., Morrell N. I., 1995, ApJS, 99, 135

Alexander D. R., Ferguson J. W., 1994, ApJ, 437, 879

Asplund M., Grevesse N., Sauval A. J., Scott P., 2009, ARA\&A, 47, 481

Baglin A., Breger M., Chevalier C., Hauck B., Le Contel J. M., Sareyan J.

P., Valtier J. C., 1973, A\&A, 23, 221

Balona L. A. et al., 2011a, MNRAS, 413, 2403

Balona L. A. et al., 2011b, MNRAS, 414, 792

Boesgaard A. M., Friel E. D., 1990, ApJ, 351, 467

Böhm-Vitense E., 1958, Zeit. Astrophys., 46, 108

Breger M., 2000, in Szabados L., Kurtz D., eds, ASP Conf. Ser. Vol. 203, IAU Colloq. 176: The Impact of Large-Scale Surveys on Pulsating Star Research. Astron. Soc. Pac., San Francisco, p. 421

Brown T. M., Latham D. W., Everett M. E., Esquerdo G. A., 2011, AJ, 142, 112

Buchler J. R., Goupil M., Hansen C. J., 1997, A\&A, 321, 159

Carrera R., Pancino E., 2011, A\&A, 535, A30

Castelli F., Hubrig S., 2004, A\&A, 425, 263

Catanzaro G., Balona L. A., 2012, MNRAS, 421, 1222

Catanzaro G. et al., 2011, MNRAS, 411, 1167

Chambers J. E., 2010, ApJ, 724, 92

Christensen-Dalsgaard J., Daeppen W., 1992, A\&AR, 4, 267

Conti P. S., 1965, ApJ, 142, 1594

Conti P. S., 1970, PASP, 82, 781

Coupry M. F., Burkhart C., 1992, A\&AS, 95, 41

Cox A. N., Hodson S. W., King D. S., 1979, ApJ, 231, 798

Crawford D. L., 1979, AJ, 84, 1858

Debernardi Y., 2000, in Reipurth B., Zinnecker H., eds, IAU Symp. 200, The Formation of Binary Stars. Astron. Soc. Pac., San Francisco, p. 161 Dommanget J., Nys O., 1994, Communications de l'Observatoire Royal de Belgique, 115, 1

Draper P. W., Allan A., Berry D. S., Currie M. J., Giaretta D., Rankin S., Gray N., Taylor M. B., 2005, in Shopbell P., Britton M., Ebert R., eds, ASP Conf. Ser. Vol. 347, Astronomical Data Analysis Software and Systems XIV. Astron. Soc. Pac., San Francisco, p. 22

Dupret M. A., 2001, A\&A, 366, 166

Dupret M.-A., Grigahcène A., Garrido R., De Ridder J., Scuflaire R., Gabriel M., 2005, MNRAS, 361, 476

Dupret M.-A., Grigahcène A., Garrido R., Gabriel M., Scuflaire R., 2004, A\&A, 414, L17

Dziembowski W., Krolikowska M., 1985, Acta Astron., 35, 5

Fossati L., Bagnulo S., Landstreet J., Wade G., Kochukhov O., Monier R., Weiss W., Gebran M., 2008a, A\&A, 483, 891

Fossati L., Kolenberg K., Reegen P., Weiss W., 2008b, A\&A, 485, 257

Gabriel M., 1996, Bull. Astron. Soc. India, 24, 233

Gebran M., Monier R., 2007, in Kupka F., Roxburgh I., Chan K., eds, IAU Symp. 239, Convection in Astrophysics. Cambridge Univ. Press, Cambridge, p. 160

Gebran M., Monier R., Richard O., 2008, A\&A, 479, 189

Gebran M., Vick M., Monier R., Fossati L., 2010, A\&A, 523, A71

Gilliland R. L. et al., 2010, PASP, 122, 131

Gray D. F., 2005, The Observation and Analysis of Stellar Photospheres. Cambridge Univ. Press, Cambridge

Gray R. O., Garrison R. F., 1989, ApJS, 69, 301

Grevesse N., Noels A., 1993, in Prantzos N., Vangioni-Flam E., Casse M., eds, Origin and Evolution of the Elements. Cambridge Univ. Press, Cambridge, p. 15

Grevesse N., Sauval A. J., 1998, Space Sci. Rev., 85, 161

Grigahcène A., Dupret M.-A., Sousa S. G., Monteiro M. J. P. F. G., Garrido R., Scuflaire R., Gabriel M., 2012, MNRAS, 422, L43

Hauck B., Mermilliod M., 1998, A\&AS, 129, 431

Hill G. M., Landstreet J. D., 1993, A\&A, 276, 142

Iglesias C. A., Rogers F. J., 1996, ApJ, 464, 943

Kallinger T., Matthews J. M., 2010, ApJ, 711, L35 
Koch D. G. et al., 2010, ApJ, 713, L79

Kurtz D. W., 1976, ApJS, 32, 651

Kurtz D. W., 1978, ApJ, 221, 869

Kurtz D. W., 1989, MNRAS, 238, 1077

Kurtz D. W., 2000, in Breger M., Montgomery M., eds, ASP Conf. Ser. Vol. 210, Delta Scuti and Related Stars. Astron. Soc. Pac., San Francisco, p. 287

Kurtz D. W., Garrison R. F., Koen C., Hofmann G. F., Viranna N. B., 1995, MNRAS, 276, 199

Kurucz R., 1993a, Kurucz CD-ROM No. 13, ATLAS9 Stellar Atmosphere Programs and $2 \mathrm{~km} \mathrm{~s}^{-1}$ Grid. Smithsonian Astrophysical Observatory, Cambridge, MA

Kurucz R., 1993b, SYNTHE Spectrum Synthesis Programs and Line Data. Kurucz CD-ROM No. 18. Smithsonian Astrophysical Observatory, Cambridge, MA

Latham D. W., Brown T. M., Monet D. G., Everett M., Esquerdo G. A., Hergenrother C. W., 2005, BAAS, 37, 110.13

Lenz P., Breger M., 2004, in Zverko J., Ziznovsky J., Adelman S. J., Weiss W. W., eds, IAU Symp. 224, The A-Star Puzzle. Cambridge Univ. Press, Cambridge, p. 786

Mashonkina L., Korn A. J., Przybilla N., 2007, A\&A, 461, 261

Meléndez J., Asplund M., Gustafsson B., Yong D., 2009, ApJ, 704, L66

Michaud G., Richer J., Richard O., 2005, ApJ, 623, 442

Murphy S. J., 2012, MNRAS, 422, 665

Niemczura E., Morel T., Aerts C., 2009, A\&A, 506, 213

Olsen E. H., 1983, A\&AS, 54, 55
Pace G., Recio-Blanco A., Piotto G., Momany Y., 2006, A\&A, 452, 493

Ramírez I., Meléndez J., Asplund M., 2009, A\&A, 508, L17

Rentzsch-Holm I., 1996, A\&A, 312, 966

Sbordone L., 2005, Mem. Soc. Astron. Ital. Suppl., 8, 61

Scuflaire R., Théado S., Montalbán J., Miglio A., Bourge P.-O., Godart M., Thoul A., Noels A., 2008, Ap\&SS, 316, 83

Shibahashi H., Kurtz D. W., 2012, MNRAS, 422, 738

Smalley B. et al., 2011, A\&A, 535, A3

Smith J. C. et al., 2012, PASP, 124, 1000

Smith M. A., 1970, ApJ, 161, 1181

Smith M. A., 1973, ApJS, 25, 277

Stateva I., Iliev I. K., Budaj J., 2012, MNRAS, 420, 1207

Stumpe M. C. et al., 2012, PASP, 124, 985

Takeda Y., Han I., Kang D. I., Lee B.-C., Kim K.-M., 2008, J. Korean Astron. Soc., 41,83

Templeton M., Basu S., Demarque P., 2002, ApJ, 576, 963

Trampedach R., Stein R. F., 2011, ApJ, 731, 78

Turcotte S., Richer J., Michaud G., Christensen-Dalsgaard J., 2000, A\&A, 360,603

Uytterhoeven K. et al., 2011, A\&A, 534, A125

van’t Veer-Menneret C., Megessier C., 1996, A\&A, 309, 879

Varenne O., Monier R., 1999, A\&A, 351, 247

Zahn J.-P., 1975, A\&A, 41, 329

This paper has been typeset from a $\mathrm{T}_{\mathrm{E}} \mathrm{X} / \mathrm{LT}_{\mathrm{E}} \mathrm{X}$ file prepared by the author. 


\title{
Asteroseismology of KIC 11754974: a high-amplitude SX Phe pulsator in a 343-d binary system
}

\author{
S. J. Murphy, ${ }^{1,2 \star ~ A . ~ P i g u l s k i ~}{ }^{3}$ D. W. Kurtz, ${ }^{1}$ J. C. Suárez, ${ }^{4}$ G. Handler, ${ }^{5}$ \\ L. A. Balona, ${ }^{6}$ B. Smalley, ${ }^{7}$ K. Uytterhoeven, ${ }^{8,9}$ R. Szabó, ${ }^{10}$ A. O. Thygesen,,${ }^{11,12}$ \\ V. Elkin, ${ }^{1}$ M. Breger, ${ }^{13,14}$ A. Grigahcène, ${ }^{4}$ J. A. Guzik, ${ }^{15}$ J. M. Nemec ${ }^{16}$ \\ and J. Southworth ${ }^{7}$ \\ ${ }^{1}$ Jeremiah Horrocks Institute, University of Central Lancashire, Preston PRI 2HE, UK \\ ${ }^{2}$ Centro de Astrofísica, Faculdade de Ciências, Universidade do Porto, Rua das Estrelas, P-4150-762 Porto, Portugal \\ ${ }^{3}$ Instytut Astronomiczny, Uniwersytet Wroctawski, Kopernika 11, PL-51-622 Wroctaw, Poland \\ ${ }^{4}$ Instituto de Astrofísica de Andalucía, Camino Bajo de Huétor 50, Granada E-18008, Spain \\ ${ }^{5}$ Nicolaus Copernicus Astronomical Center, Bartycka 18, PL-00-716 Warsaw, Poland \\ ${ }^{6}$ South African Astronomical Observatory, PO Box 9, Observatory 7935, Cape Town, South Africa \\ ${ }^{7}$ Astrophysics Group, Keele University, Staffordshire ST5 5BG, UK \\ ${ }^{8}$ Instituto de Astrofísica de Canarias, E-38200 La Laguna, Tenerife, Spain \\ ${ }^{9}$ Department Astrofísica, Universidad de La Laguna, E-38206 La Laguna, Tenerife, Spain \\ ${ }^{10}$ Konkoly Obs., Research Centre for Astronomy and Earth Sciences, HAS, H-1121 Budapest, Konkoly Thege Miklós út 15-17, Hungary \\ ${ }^{11}$ Zentrum Für Astronomie der Universität Heidelberg, Landessternwarte, Königstuhl 12, D-69117 Heidelberg, Germany \\ ${ }^{12}$ Nordic Optical Telescope, Apartado 474, E-38700 Santa Cruz de La Palma, Santa Cruz de Tenerife, Spain \\ ${ }^{13}$ Institut für Astronomie, Türkenschanzstraße 17, A-1180 Wien, Austria \\ ${ }^{14}$ Department of Astronomy, University of Texas, Austin, TX 78712, USA \\ ${ }^{15}$ Los Alamos National Laboratory, XTD-2 MS T-086, Los Alamos, NM 87545-2345, USA \\ ${ }^{16}$ Department of Physics and Astronomy, Camosun College, Victoria, British Columbia V8P 5J2, Canada
}

Accepted 2013 April 4. Received 2013 April 3; in original form 2013 March 19

\begin{abstract}
The candidate SX Phe star KIC 11754974 shows a remarkably high number of combination frequencies in the Fourier amplitude spectrum: 123 of the 166 frequencies in our multifrequency fit are linear combinations of independent modes. Predictable patterns in frequency spacings are seen in the Fourier transform of the light curve. We present an analysis of $180 \mathrm{~d}$ of short-cadence Kepler photometry and of new spectroscopic data for this evolved, late A-type star. We infer from the 1150-d, long-cadence light curve, and in two different ways, that our target is the primary of a 343-d, non-eclipsing binary system. According to both methods, the mass function is similar, $f(M)=0.0207 \pm 0.0003 \mathrm{M}_{\odot}$. The observed pulsations are modelled extensively, using separate, state-of-the-art, time-dependent convection (TDC) and rotating models. The models match the observed temperature and low metallicity, finding a mass of $1.50-1.56 \mathrm{M}_{\odot}$. The models suggest that the whole star is metal poor, and that the low metallicity is not just a surface abundance peculiarity. This is the best frequency analysis of an SX Phe star, and the only Kepler $\delta$ Sct star to be modelled with both TDC and rotating models.
\end{abstract}

Key words: asteroseismology -stars: individual: KIC 11754974-Stars: oscillations - stars: Population II - stars: variables: $\delta$ Scuti.

\section{INTRODUCTION}

The goal of the Kepler mission is to find Earth-like planets in the habitable zone (Koch et al. 2010). The methodology inherent in this task is the simultaneous, space-based observation of $\sim 150000$ stars in a $115 \mathrm{deg}^{2}$ field of view for transiting events.

^E-mail: smurphy6@uclan.ac.uk
Kepler observations are made in two cadences: most observations take place in long-cadence (LC) format, where photometric readouts are co-added for $29.4 \mathrm{~min}$. Short-cadence (SC) observations have effective integration times of $58.8 \mathrm{~s}$. These cadences make the data excellent for asteroseismic investigations. Kepler data are organized into quarters, denoted $\mathrm{Q} n$, and SC data are further subdivided into three one-month segments, denoted Qn.m.

The success of the Kepler mission lies in its unprecedented $\mu$ mag photometric precision. Even for a star as faint as KIC 11754974 
$(\mathrm{Kp}=12.7 \mathrm{mag})$, the subject of this work, the average noise amplitude in the Fourier transform is $\sim 5 \mu$ mag at low frequency, decreasing gradually to $\sim 2 \mu \mathrm{mag}$ for frequencies above about $70 \mathrm{~d}^{-1}$.

Kepler photometry not only offers higher precision, but also provides nearly continuous observations. Progress in the analysis of $\delta$ Sct stars is no longer hampered by the insurmountable time gaps in the data associated with ground-based observations. Most stars now have almost continuous observations in LC mode from the commissioning run of Q0 right through to the most recent quarter at the time of writing (Q13), spanning over $1150 \mathrm{~d}$; the same is true for some targets in SC mode. This long time-span is particularly important for resolving peaks that are very closely spaced in frequency, and for studies of frequency or amplitude modulation, among other reasons (see e.g. Murphy 2012b). Completely alleviating Nyquist ambiguities with Kepler data can now also be added to that list (Murphy, Shibahashi \& Kurtz 2013).

The $\delta$ Sct stars are located at the junction of the classical Cepheid instability strip and the main sequence (MS), with pulsation periods between $18 \mathrm{~min}$ and $8 \mathrm{~h}$. They are driven by the opacity mechanism in the He II partial ionization zone. SX Phe stars are the Population II counterparts of the $\delta$ Sct stars, characterized by low metallicity and high tangential velocities (Nemec \& Mateo 1990; Balona \& Nemec 2012). Within the last decade, observations of SX Phe stars have revealed that they are not necessarily intrinsically high-amplitude pulsators (Olech et al. 2005; Balona \& Nemec 2012), but show amplitude distributions similar to the Population I $\delta$ Sct stars, viz., having peak pulsation amplitudes of a few mmag (see Uytterhoeven et al. 2011 for a statistical study). Traditionally, $\delta$ Sct stars with amplitudes above 0.3 mag were termed high-amplitude Delta Scuti (HADS) stars, and were found to pulsate mostly in one or two radial modes. Their high amplitudes are attributed to their evolved stages (e.g. Petersen \& Christensen-Dalsgaard 1996, who describe HADS stars as being in the immediate post-MS stage). KIC 11754974 has a maximum peak-to-peak light variation of only 0.24 mag, based on tangential velocities was identified as an SX Phe star by Balona \& Nemec (2012) and was the highest amplitude Kepler SX Phe star they identified. In this work, we find KIC 11754974 to have properties in common with both HADS and SX Phe stars.

\section{BINARITY OF KIC 11754974}

\subsection{Doppler-shifted frequencies}

Our target falls on Kepler's failed Module 3, meaning it is only observed for three quarters of each year. For our frequency analysis, we utilized the higher sampling of the SC data, the longest uninterrupted run of which is Q6 and Q7, covering about $180 \mathrm{~d}$. In the course of analysing the combined Q6+Q7 data set, it appeared that in Fourier spectra a significant residual signal occurs near the strongest terms after their subtraction. This indicated frequency and/or amplitude changes which prompted us to carry out the analysis separately for shorter data sets. Both LC and SC data were used in this analysis; they were split into $30-50 \mathrm{~d}$ subsets. For each subset, the frequency set containing the 72 strongest terms (20 independent and 52 harmonics and combinations) was fitted by means of non-linear least squares. The resulting frequencies of the strongest modes in different subsets were then compared. The result is shown in Fig. 1, where we plot the fractional frequency change of the four frequencies with the highest amplitudes in a given time subset, relative to their amplitudes in the arbitrarily chosen reference subset, here the SC Q6 data. As can be seen, the frequencies

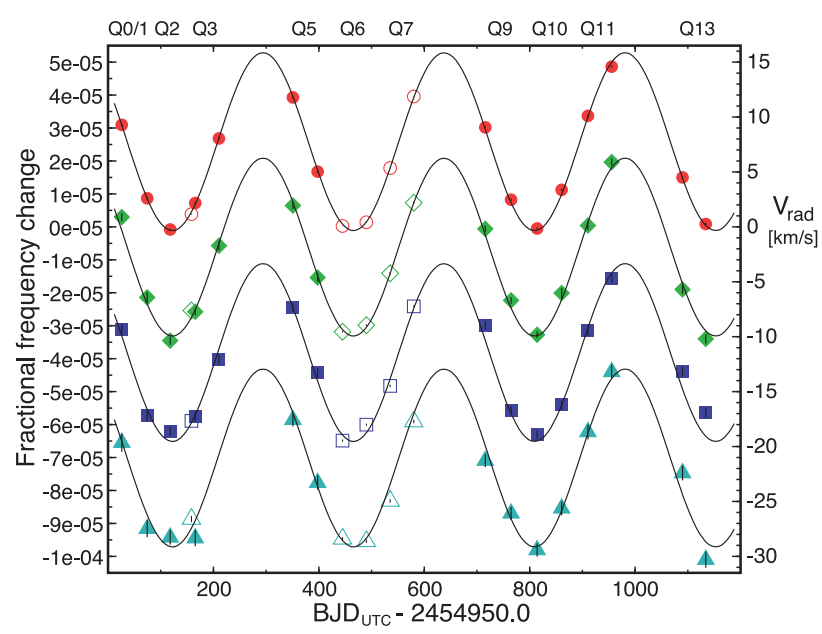

Figure 1. Temporal changes in the frequency of the four strongest modes excited in KIC $11754974, f_{1}, f_{2}, f_{3}$ and $f_{4}$, top to bottom. The values derived from LC and SC data are plotted with filled and open symbols, respectively. The sinusoid with a period of $343 \mathrm{~d}$ fitted to the $f_{1}$ data is also shown. For clarity, an offset has been added to the $f_{2}, f_{3}$ and $f_{4}$ data; the sinusoid has also been shifted accordingly. The right-hand side ordinate shows radial velocities calculated from the Doppler effect.

of all four of the strongest modes behave in the same manner and can be described by a sinusoid with a period of about $343 \mathrm{~d}$.

The only plausible explanation of the changes seen in Fig. 1 is the motion of KIC 11754974 in a binary system. The frequency changes can be interpreted in terms of the Doppler effect and transformed to radial velocities via the equation

$V_{\mathrm{rad}}=c \frac{f_{i}-f_{\mathrm{ref}}}{f_{\mathrm{ref}}}$,

where $c$ represents the speed of light. These radial velocities can be used to derive parameters of the spectroscopic orbit of the pulsating primary. It can be seen from Fig. 1 that the peak-to-peak radial velocity of KIC 11754974 amounts to about $16 \mathrm{~km} \mathrm{~s}^{-1}$.

In order to derive parameters of the spectroscopic orbit, however, we proceeded in another way. Instead of using radial velocities calculated from frequencies, we decided to use the $\mathrm{O}-\mathrm{C}$ diagram for the times of maximum light. The advantage of using the $\mathrm{O}-\mathrm{C}$ diagram is that in this diagram the changes of period accumulate. For large orbital-to-pulsation period ratios, spectroscopic elements can be derived from the $\mathrm{O}-\mathrm{C}$ diagram more accurately than when using radial velocities calculated from the Doppler effect.

Since the amplitude of the strongest mode is several times larger than the amplitudes of the next three modes, we decided to use only the times of maximum light of the strongest mode. First, all terms with amplitudes higher than $40 \mathrm{ppm}$ except for $f_{1}$ and its harmonics were removed from the data. The residuals were divided into 5-20 d non-overlapping subsets. For each subset, a sinusoid with frequency $f_{1}$ and its harmonics were fitted in order to obtain amplitude and phase information. The time of maximum light was calculated from the phase of $f_{1}$ with the epoch adopted to be the nearest to the average time of observation in a given subset. The resulting observed (O) times of maximum light were compared to the calculated (C) times of maximum light according to the following ephemeris:

$\mathrm{C}(E)=2454950.021179+0.0611817444 \times E$,

where $E$ is the number of cycles elapsed from the initial epoch, in BJD using Coordinated Universal Time (UTC; Eastman, Siverd \& 


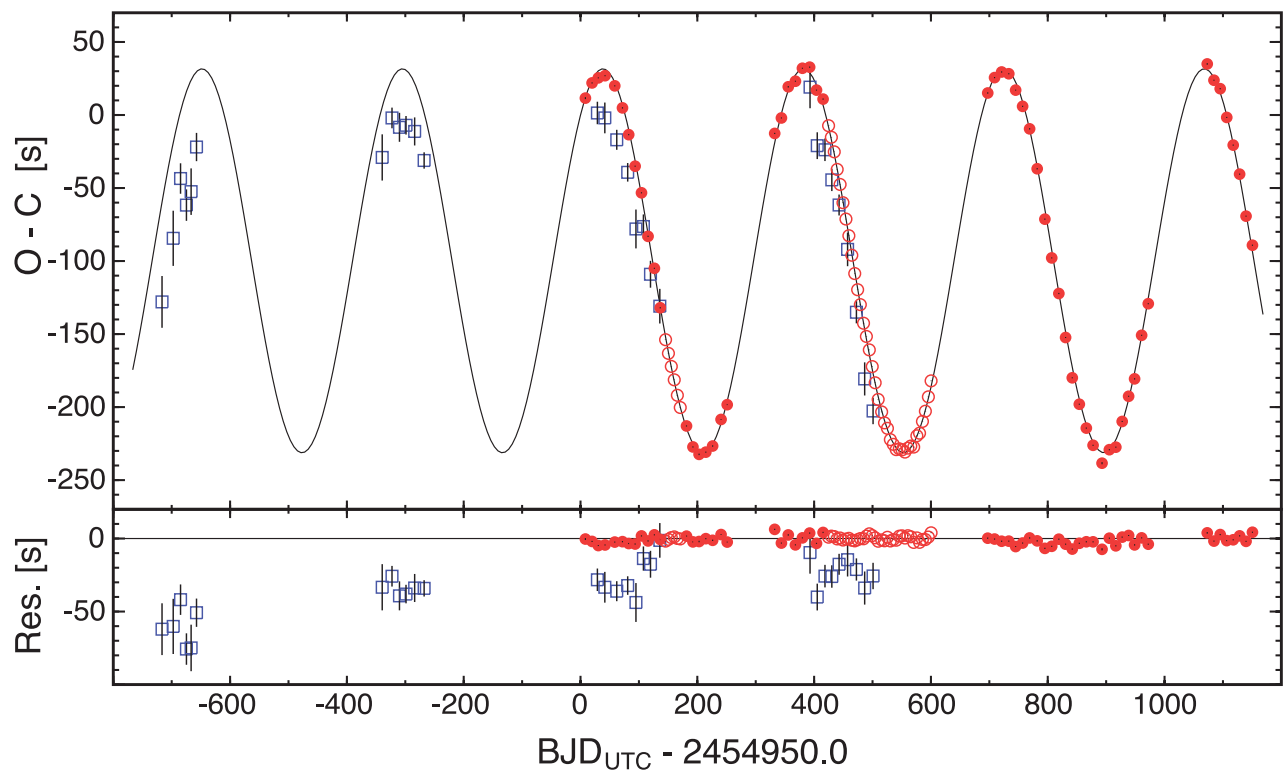

Figure 2. The $\mathrm{O}-\mathrm{C}$ diagram for the times of maximum light of the strongest mode of KIC 11754974 (upper panel). The filled and open circles denote Kepler LC and SC data, respectively. The open squares represent the 2007-2010 WASP data. The lower panel shows the residuals from the eccentric orbit fit, shown as a continuous line in the upper panel. The parameters of the fit are given in Table 1.

Table 1. Parameters of the spectroscopic orbit derived from the $\mathrm{O}-\mathrm{C}$ diagram. $T_{0}$ is given in BJD UTC.

\begin{tabular}{cc}
\hline Parameter & Value \\
\hline$T_{0}$ & $2454999 \pm 37$ \\
$e$ & $0.013_{-0.006}^{+0.011}$ \\
$\omega\left(^{\circ}\right)$ & $102 \pm 38$ \\
$K_{1}\left(\mathrm{~km} \mathrm{~s}^{-1}\right)$ & $8.35 \pm 0.04$ \\
$P_{\text {orb }}(\mathrm{d})$ & $343.27 \pm 0.34$ \\
$P_{0}(\mathrm{~d})$ & $0.0611817444 \pm 0.0000000011$ \\
$a_{1} \sin i(\mathrm{au})$ & $0.2634 \pm 0.0013$ \\
$f(M)\left(\mathrm{M}_{\odot}\right)$ & $0.02069 \pm 0.00031$ \\
\hline
\end{tabular}

Gaudi 2010). As such, our Kepler times do not suffer the timing error discovered in 2012 November. ${ }^{1}$

The Wide Angle Search for Planets (WASP) is a multisite, multicamera imaging system obtaining photometric data with an accuracy better than 1 per cent for objects with $7 \leq V \leq 11.5$ (Pollacco et al. 2006). Lower quality data are obtained for stars with $11.5 \leq$ $V \leq 15$. Although the Kepler data have much higher precision and duty cycle, the WASP observations have time-spans extending back to years before Kepler came online. We utilized this longer timebase of the WASP data to confirm our $\mathrm{O}-\mathrm{C}$ results, the values of which are plotted in Fig. 2.

Using the more precise Kepler times of maximum light (for both LC and SC data), we fitted the $\mathrm{O}-\mathrm{C}$ parameters assuming an eccentric orbit. The parameters of the fit are given in Table 1. The meaning of the parameters is standard: $T_{0}$ denotes the epoch of periastron passage; $e$, eccentricity; $\omega$, longitude of periastron; $K_{1}$, half-range of the primary's radial velocity variation; $P_{\text {orb }}$, the orbital period; $P_{0}$, the pulsation period; $a_{1}$, the semimajor axis of primary's absolute orbit; and $f(M)$, the mass function. The residuals from the

\footnotetext{
${ }^{1}$ http://archive.stsci.edu/kepler/timing_error.html
}

fit are equal to $3.28 \mathrm{~s}$ for the LC and $1.47 \mathrm{~s}$ for the SC data. At least part of the residual scatter comes from the small-amplitude variation due to modes that were not subtracted from the data.

The lower panel of Fig. 2 indicates that the WASP data have large residuals (in time) when compared with the Kepler data. We tested whether the shift is intrinsic by comparing two stars in which such shifts were deemed to be unlikely - we chose two relatively bright, short-period W UMa stars, namely KIC 8554005 and KIC 9392683 (1SWASP J191805.45+444115.4 and 1SWASP J190413.72+455657.6, respectively). We investigated the strongest (second) harmonic in the Fourier transform of each star, and define a parameter DT $=T_{\max }($ WASP $)-T_{\max }$ (Kepler), where $T_{\max }$ represents the time of maximum light closest to the mean epoch of a given data set. For KIC 8554005, we determine DT $=-5.8 \pm 5.2 \mathrm{~s}$ and for $\mathrm{KIC} 9392683 \mathrm{DT}=-13.7 \pm 6.0 \mathrm{~s}$, and note that DT is therefore zero to within $1-2 \sigma$. For KIC 11754974 , we found DT $=-34.2 \pm 2.8 \mathrm{~s}$ from all data or $-27.2 \pm 2.1 \mathrm{~s}$ if the first season is excluded. We therefore conclude that the shift for KIC 11754974 is intrinsic and probably occurs because the WASP and Kepler bands are different; the central wavelengths are $\sim 550 \mathrm{~nm}$ for WASP and $\sim 660 \mathrm{~nm}$ for Kepler. We note that WASP and Kepler use heliocentric and barycentric Julian date, respectively, and that contributes up to a couple of seconds to the offset.

One can see that the orbit has a small eccentricity. The secondary is likely to be a low-mass MS star. Assuming that the primary's mass lies in the range $1.5-2.5 \mathrm{M}_{\odot}$, we get $M_{2}=0.42-0.58 \mathrm{M}_{\odot}$ for inclination $i=90^{\circ}$ and $M_{2}=0.65-0.87 \mathrm{M}_{\odot}$ for $i=45^{\circ}$. This indicates a K- or early M-type secondary companion. Its contribution to the total flux is therefore not larger than a few per cent unless the inclination is very low. This reduces the possibility of detection of variability of the secondary if present at all.

Having calculated the orbital elements, we have corrected the BJD times for SC Q6 and Q7 data for the light-time effect in the binary system. Because in different quarters different detectors and apertures are used, it is expected that fluxes from different quarters are not directly comparable and need to be scaled. Using the 
amplitudes of the strongest modes, we found that the amplitudes derived from Q7 data are larger by a factor of 1.0087 than those obtained from Q6 data. Therefore, the Q7 data were divided by this factor prior to combining. Subsequently, this high-duty cycle combined Q6+Q7 SC data set was subject of time series analysis (Section 4), where we have used only these adjacent SC quarters to avoid aliasing difficulties.

\subsection{Frequency-modulated stars}

The effect of the binary system on light arrival times is that of frequency modulation for the pulsation modes. Shibahashi \& Kurtz (2012) showed how a frequency multiplet is induced around each pulsation mode as a result of the binarity. The frequency spacing can be used to infer $P_{\text {orb }}$, and the amplitudes and phases of the frequency multiplet give $T_{0}, e$ and $f(M)$, provided the data set is longer than $P_{\text {orb }} f(M)$ is calculable using the ratio of the amplitudes of the first orbital sidelobes to those of the central pulsation frequency (Shibahashi \& Kurtz 2012). We used this method to check our binary parameters, and we will show that the results we obtain using this method are entirely consistent with those derived from the $\mathrm{O}-\mathrm{C}$ diagram. We applied this methodology to the Kepler LC Q0-13 data set, spanning $1153 \mathrm{~d}$ (3.36 orbits).

We started with the highest amplitude peak in the Q0-13 data set, which lies at $16.344745 \mathrm{~d}^{-1}$ with an amplitude of $51.79 \mathrm{mmag}$. We extracted this peak and its orbital sidelobes and applied non-linear least-squares fitting routines to improve the frequencies, amplitudes and phases. From the mean spacing of the multiplet, we derive $P_{\text {orb }}=343.66 \pm 0.48 \mathrm{~d}$, which is consistent with that derived via the $\mathrm{O}-\mathrm{C}$ diagram. Given the slightly lower uncertainty on $P_{\text {orb }}$ from the latter method, we used the $(\mathrm{O}-\mathrm{C})$-calculated value of $P_{\text {orb }}$ to force-fit the sidelobes to be exactly equally spaced. We chose the zero-point in time, such that the sidelobes have the same phase with respect to each other, but are $\pi / 2$ out of phase with the central frequency, thus demonstrating the triplet is orbital in nature. Table 2 displays the results for this frequency and three others, each of which shows a frequency triplet split by the orbital frequency.
The mass function is calculable as

$f\left(m_{1}, m_{2}, \sin i\right)=\left(\frac{A_{+1}+A_{-1}}{A_{0}}\right)^{3} \frac{P_{\mathrm{osc}}^{3}}{P_{\mathrm{orb}}^{2}} \frac{c^{3}}{2 \pi G}$,

where the amplitudes of the central, higher and lower frequency components of the triplet are denoted $A_{0}, A_{+1}$ and $A_{-1}$, respectively; $P_{\text {osc }}$ is the oscillation period; and all quantities are in SI units. Via this method we find $f(M)=0.02069 \pm 0.00036 \mathrm{M}_{\odot}$, which is exactly the value calculated via the $\mathrm{O}-\mathrm{C}$ method. Equation (23) of Shibahashi \& Kurtz (2012) allows one to calculate $a_{1} \sin i$, too, as

$a_{1} \sin i=\frac{P_{\mathrm{osc}}}{2 \pi} \alpha c$.

Application of this formula yields $a_{1} \sin i=0.2629 \pm 0.0051 \mathrm{au}$, in good agreement with the $\mathrm{O}-\mathrm{C}$ value.

The fact that such information is discernible from the Fourier transform highlights the importance of having continuous long-term observations available for $\delta$ Sct stars, whose high-amplitude, highfrequency pulsations produce sidelobes with high signal-to-noise $(\mathrm{S} / \mathrm{N})$.

We also looked for eclipses in the SC data at the times when the stars are aligned to the line of sight. The possibility of finding such a shallow eclipse in the residuals of the light curve of a binary with this long a period, even when our multifrequency fit was subtracted, was very small. Although we did not find an eclipse, for such a wide system this provides only a slight constraint on the orbital inclination (an upper limit that is near $90^{\circ}$ ).

\section{SPECTROSCOPIC OBSERVATIONS}

We obtained three spectra of KIC 11754974 with the FIES spectrograph at the $2.5-\mathrm{m}$ Nordic Optical telescope (NOT). The star is too faint for high-resolution observations with these facilities so to obtain spectra of a higher $\mathrm{S} / \mathrm{N}$ we observed with medium resolution $(R=25000)$. Even still, observing conditions rendered the $\mathrm{S} / \mathrm{N}$ ratio of the obtained spectra low - the $\mathrm{S} / \mathrm{N}$ ratio of the averaged spectrum, estimated from the noise level of reduced 1D spectra, is

Table 2. A least-squares fit of the frequency triplets for the four highest amplitude modes. The sidelobes are fixed at frequencies of $v_{\text {osc }} \pm v_{\text {orb }}$. The zero-point in time, $t_{0}=\mathrm{BJD}_{\mathrm{UTC}} 24555331.39590$, was chosen so that the sidelobes have equal phases and the difference in phase between the sidelobes and central peak is $\pi / 2$. Column 4 thus shows that the difference in phases of the sidelobes with each other is zero within the errors, and Column 5 demonstrates that their phase difference from the central peak is $\pi / 2$ within the errors. Column 6 contains the amplitude ratios of the sidelobes to the central frequency. Since these values are small compared to unity, they are approximately equal to $\alpha$, the amplitude of the phase modulation. Column 7 shows the expected theoretical outcome that the ratio of $\alpha$ to the oscillation frequency is the same for all triplets. We note that for the first triplet, the amplitudes of the sidelobes are only equal at the $4 \sigma$ level, and that the errors provided are overestimated because there are still many frequencies left in the data.

\begin{tabular}{|c|c|c|c|c|c|c|}
\hline $\begin{array}{l}\text { Frequency } \\
\qquad\left(d^{-1}\right)\end{array}$ & $\begin{array}{l}\text { Amplitude } \\
\text { (mmag) }\end{array}$ & $\begin{array}{l}\text { Phase } \\
\text { (rad) }\end{array}$ & $\begin{array}{c}\phi_{+1}-\phi_{-1} \\
\quad(\mathrm{rad})\end{array}$ & $\begin{array}{c}\left\langle\phi_{+1}-\phi_{-1}\right\rangle-\phi_{0} \\
(\mathrm{rad})\end{array}$ & $\alpha=\left(A_{+1}+A_{-1}\right) / A_{0}$ & $\begin{array}{c}\alpha / \nu_{\text {osc }} \\
\left(\times 10^{-3} \mathrm{~d}\right)\end{array}$ \\
\hline 16.341823 & $3.964 \pm 0.048$ & $-1.6554 \pm 0.0120$ & & & & \\
\hline 16.344745 & $51.792 \pm 0.049$ & $-0.0545 \pm 0.0009$ & $-0.011 \pm 0.017$ & $-1.606 \pm 0.008$ & $0.157 \pm 0.003$ & $9.58 \pm 0.16$ \\
\hline 16.347667 & $4.148 \pm 0.048$ & $-1.6663 \pm 0.0115$ & & & & \\
\hline 21.396067 & $1.116 \pm 0.048$ & $0.9328 \pm 0.0427$ & & & & \\
\hline 21.398989 & $11.046 \pm 0.049$ & $2.5642 \pm 0.0044$ & $0.012 \pm 0.060$ & $-1.626 \pm 0.030$ & $0.204 \pm 0.012$ & $9.54 \pm 0.58$ \\
\hline 21.401911 & $1.140 \pm 0.048$ & $0.9443 \pm 0.0418$ & & & & \\
\hline 20.904493 & $0.766 \pm 0.048$ & $0.0139 \pm 0.0623$ & & & & \\
\hline 20.907414 & $8.320 \pm 0.049$ & $1.6598 \pm 0.0059$ & $0.045 \pm 0.086$ & $-1.623 \pm 0.043$ & $0.189 \pm 0.016$ & $9.02 \pm 0.78$ \\
\hline 20.910336 & $0.803 \pm 0.048$ & $0.0589 \pm 0.0595$ & & & & \\
\hline 20.940632 & $0.584 \pm 0.048$ & $2.1123 \pm 0.0818$ & & & & \\
\hline 20.943554 & $5.890 \pm 0.049$ & $-2.5581 \pm 0.0083$ & $-0.003 \pm 0.114$ & $-1.614 \pm 0.058$ & $0.202 \pm 0.023$ & $9.62 \pm 1.10$ \\
\hline 20.946475 & $0.603 \pm 0.048$ & $2.1090 \pm 0.0792$ & & & & \\
\hline
\end{tabular}


about 45 in the spectral region of $\mathrm{H} \alpha$ and about 40 near $\mathrm{H} \beta$. The difficulties in determining the right continuum level limit the accuracy of our determinations, which are provided to offer some constraints on fundamental parameters, rather than as definite determinations. We stress that higher resolution, higher $\mathrm{S} / \mathrm{N}$ spectra are still needed.

The Balmer-line profiles are good indicators of effective temperature for A-type stars; thus, we have compared observed and synthetic profiles of the $\mathrm{H} \alpha$ and $\mathrm{H} \beta$ lines. The synthetic calculations of Balmer profiles were done using the SYNTH code by Piskunov (1992) and model atmospheres from Heiter et al. (2002) with solar metallicity (as determined by Anders \& Grevesse 1989). The source of the spectral line list was the Vienna Atomic Line Database (VALD; Kupka et al. 1999). Later analysis of metal lines demonstrated metal deficiency with respect to solar abundances, so we switched to working with low-metallicity models $([\mathrm{M} / \mathrm{H}]=-0.5)$. For $\mathrm{H} \alpha$ and $\mathrm{H} \beta$, the best fits for observed and synthetic profiles were obtained for this metallicity model, yielding $T_{\text {eff }}=7000$ and $6800 \mathrm{~K}$, respectively. Considering possible errors in continuum determination of several per cent, we estimate from the Balmer lines that $T_{\text {eff }}=7000 \pm 200 \mathrm{~K}$.

With our spectral material we cannot make reliable determinations of other parameters, namely $\log g$ and the microturbulence velocity $v_{\text {mic }}$. We calculated a number of synthetic spectra keeping $T_{\text {eff }}$ and one other parameter fixed (thus permitting the third variable to change), to find the best fit of the metal lines. After several iterations, we accepted a model atmosphere with $T_{\text {eff }}=7000 \mathrm{~K}, \log g=$ $3.6(\mathrm{cgs})$ and did calculations for microturbulence velocity resulting in $v_{\text {mic }}=2 \mathrm{~km} \mathrm{~s}^{-1}$. This $\log g$ value is only loosely constrained and we considered that the star shows HADS-star characteristics and is probably more evolved. However, the $\log g$ value obtained is lower than we expected, given that cool $\delta$ Sct stars tend to pulsate in low overtones (Breger \& Bregman 1975), and a low-overtone mode at $16 \mathrm{~d}^{-1}$ (the frequency of our highest amplitude mode) implies an evolutionary status much closer to the zero-age main sequence (ZAMS). We compared the spectrum with the spectrum of $\delta$ Sct itself, taken from the Elodie archive (http://atlas.obs-hp.fr/elodie/). The shapes of the $\mathrm{H} \beta$ profiles are very similar in these stars. However, the spectrum of $\delta$ Sct is richer in metal lines.

The weak spectral lines in KIC 11754974 are lost in the noise. In the averaged spectrum, we identified lines of $\mathrm{Fe}$ I, $\mathrm{Fe}$ II, Ti II, $\mathrm{Mg}$ I, Mg II and some others. For identification, we calculated a synthetic spectrum using our best-fitting parameters. From several lines including $\mathrm{Fe}$ II $\lambda 5018.440, \mathrm{Mg}$ I $\lambda 5172.684, \mathrm{Mg}$ I $\lambda 5183.604$, we determined a projected rotational velocity: $v \sin i=31 \pm 2 \mathrm{~km} \mathrm{~s}^{-1}$. A more cautious interpretation, taking into account more lines (with a poorer overall fit) is $v \sin i=25 \pm 7 \mathrm{~km} \mathrm{~s}^{-1}$ - given the low $\mathrm{S} / \mathrm{N}$ of our spectrum, we adopt the more cautious $v \sin i$ value.

Using several lines, we determined the abundances of iron: $\log N / N_{\text {tot }}=-5.8 \pm 0.3$ (while solar is -4.54 ), ${ }^{2}$ and for magnesium: $\log N / N_{\text {tot }}=-5.9 \pm 0.3$ (solar is -4.46 ). We note that fitting $\mathrm{H} \alpha$ and $\mathrm{H} \beta$ with $[\mathrm{M} / \mathrm{H}]=-1.0$ yielded negligible differences from the $[\mathrm{M} / \mathrm{H}]=-0.5$ models. Many other chemical elements in the spectra also show significant deficiencies but the lines are blended or below the detection threshold and cannot be tested. There is a problem in that that many lines of $\mathrm{Fe}$ I that are not visible in the observed spectra appear in synthetic ones and require yet lower abundances for best fitting than we obtained from available lines, the latter mostly belonging to $\mathrm{Fe}$ II. One must decrease the effective temperature to reduce such a disequilibrium. At least one strong
Table 3. Atmospheric parameters determined from spectroscopy.

\begin{tabular}{ccccc}
\hline $\begin{array}{c}T_{\text {eff }} \\
(\mathrm{K})\end{array}$ & $\begin{array}{c}\log g \\
(\mathrm{cgs})\end{array}$ & $\begin{array}{c}{[\mathrm{Fe} / \mathrm{H}]} \\
(\mathrm{dex})\end{array}$ & $\begin{array}{c}v \sin i \\
\left(\mathrm{~km} \mathrm{~s}^{-1}\right)\end{array}$ & $\begin{array}{c}v_{\text {mic }} \\
\left(\mathrm{km} \mathrm{s}^{-1}\right)\end{array}$ \\
\hline $7000 \pm 200$ & $3.6 \pm 0.3$ & $-0.5_{-0.5}^{+0.2}$ & $25 \pm 7$ & 2 \\
\hline
\end{tabular}

available line of $\mathrm{Fe}$ I $4404.750 \AA$ A shows similar abundances to the Fe II lines. This problem needs higher quality spectra for further analysis. Strömgren photometry is desirable for independent determinations of $T_{\text {eff }}$ and $\log g$ parameters. Our parameter determination from spectroscopy is given in Table 3 .

With these restrictions in mind, verification of the low metallicity was provided by a semi-automated routine for 476 lines from the VALD data base in the 5000-5200 $\AA$ wavelength range. The bestfitting $[\mathrm{M} / \mathrm{H}]$ value using $\chi^{2}$ minimization was $-0.58 \mathrm{dex}$, when the $\log g$ value was held at the determined value of 3.6. The $[\mathrm{M} / \mathrm{H}]$ value is not the $[\mathrm{Fe} / \mathrm{H}]$ value, but is close to it.

An independent check on our spectroscopic $T_{\text {eff }}$ can be obtained from the InfraRed Flux Method (IRFM) (Blackwell \& Shallis 1977). There is no sign of any interstellar Na D lines in the spectrum, so reddening is expected to be negligible. Broad-band APASS photometry from UCAC4 (Zacharias et al. 2012) and 2MASS were used to estimate the observed bolometric flux. The IRFM was then used to determine $T_{\text {eff }}=7110 \pm 150 \mathrm{~K}$, which is consistent with the $T_{\text {eff }}$ from the spectral analysis.

As another check, we also calculated a quartic polynomial fit in $\left(g^{\prime}-r^{\prime}\right)$ colour to calculate a temperature of $7170 \mathrm{~K}$, but the uncertainty on this value is large at about $\pm 250 \mathrm{~K}$ (Uytterhoeven et al. 2011).

We used the spectrum to discern the star's kinematics, finding a velocity shift of $-300 \mathrm{~km} \mathrm{~s}^{-1}$, in agreement with Balona \& Nemec (2012). We expect that the $16 \mathrm{~km} \mathrm{~s}^{-1}$ radial velocity variations inferred from the light curve (cf. Fig. 1) would be detectable from spectroscopic observations that were suitably spaced in time.

Underabundances of Fe-peak elements by factors of 10-100 are characteristic of the $\lambda$ Boo stars, which comprise only $\sim 2$ per cent of objects in the $\delta$ Sct spectral region (Paunzen et al. 2002). If this star is determined through a full abundance analysis to be of the $\lambda$ Boo class, it will be the most intensely photometrically studied $\lambda$ Boo star to date - only eight spectroscopic binary systems with suspected $\lambda$ Boo components are currently known (Paunzen et al. 2012). With our low-S/N spectrum, it is not possible to determine whether $\mathrm{C}, \mathrm{N}$ and $\mathrm{O}$ are normal, as in the $\lambda$ Boo stars, but many elements seem underabundant with respect to the Sun. The observed abundances are entirely consistent with the classification of KIC 11754974 as an SX Phe star by Balona \& Nemec (2012).

\section{PULSATION CHARACTERIZATION}

\subsection{The data and preliminaries}

In this paper, we analysed the SC Q6+Q7 light curves from the Kepler Asteroseismic Science Operations Center ${ }^{3}$ for the HADS star KIC 11754974, to which we make corrections to the times to account for the light-time effects associated with being in a binary system as mentioned in Section 2. We used the pre-search data conditioned data of the least-squares pipeline (PDC-LS), but made 


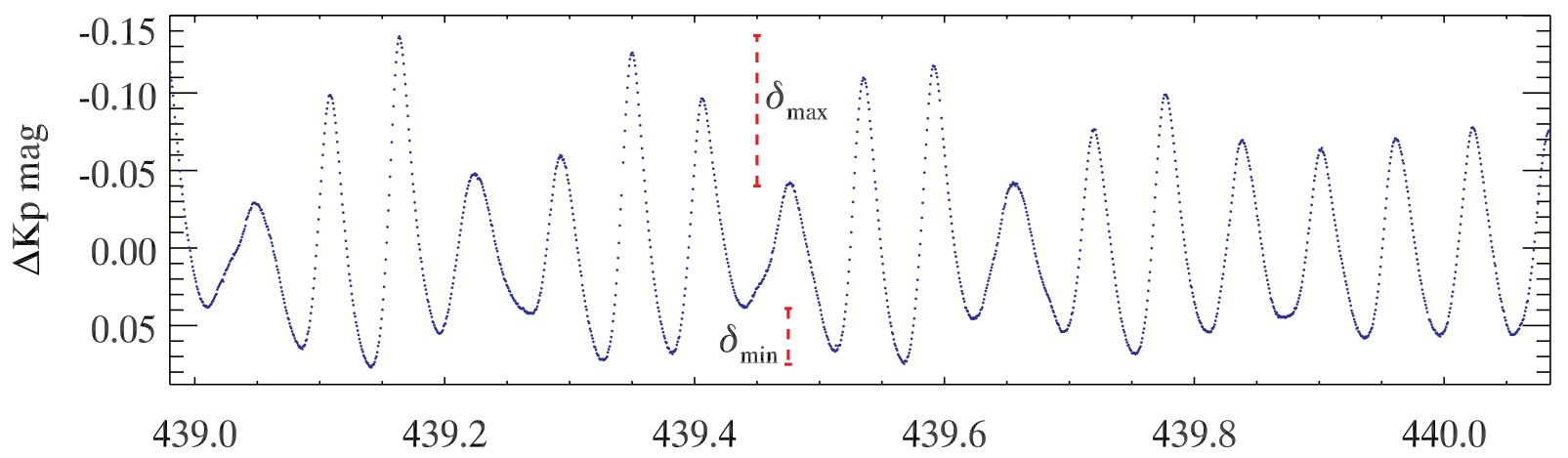

Time BJD-2455000

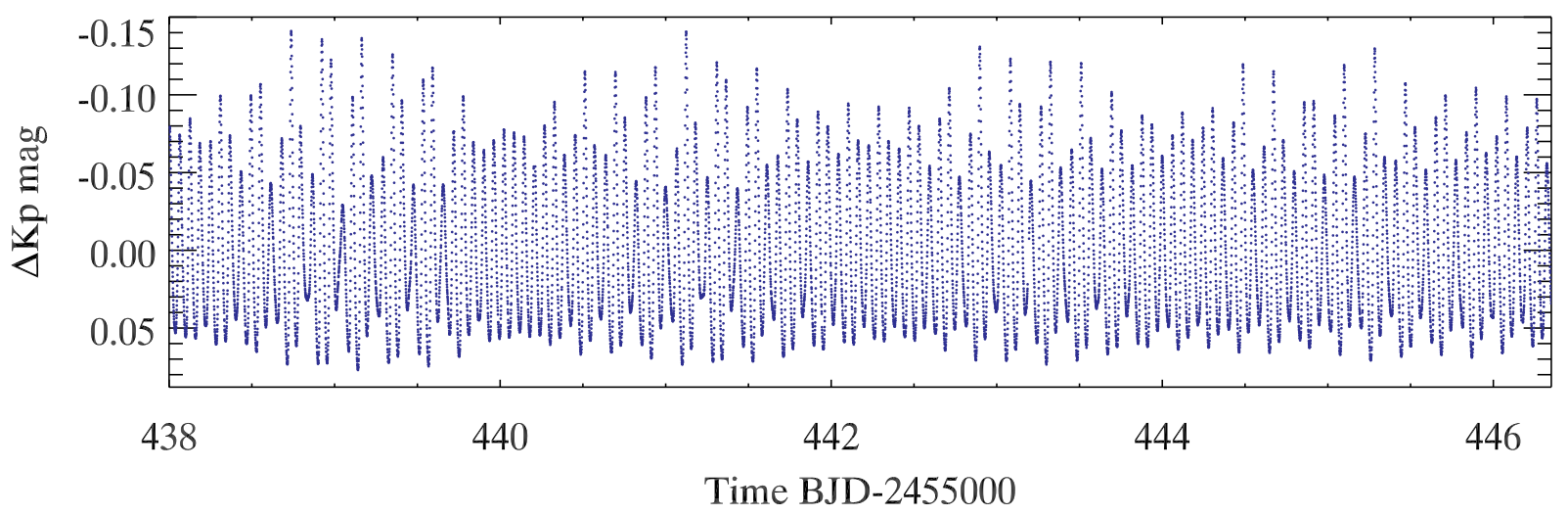

Figure 3. 1.1-d segment of the light curve of KIC 11754974, showing obvious beating. Flux maximum occurs over a greater range in brightness than does flux minimum, as indicated by the fact that $\delta_{\max }>\delta_{\min }$. The lower panel indicates this is not just localized to a short time segment.

checks with the Simple Aperture Photometry (SAP) data on which only basic calibration is performed in the data processing pipeline.

The PDC data are useful for their satisfactory removal of instrumental signals, including, but not limited to heating/cooling surrounding safe-mode events, cosmic ray events, and return to scientific operating focus and temperature after monthly downlinks (Kepler Data Characteristics Handbook ${ }^{4}$ ). The PDC data do not necessarily treat all jumps and outliers. For KIC 11754974, a manual check prior to analysis showed no clear outliers in need of removal; after fitting 166 frequencies, 103 'outliers' of the 256514 data points were manually discarded, 100 of which were at least $1 \mathrm{mmag}$ from the fit, and the independent frequencies were thereafter improved with non-linear least-squares fitting. While in a limited number of cases the PDC data modify some stellar variability, known cases are documented in the data release notes available on the Kepler Guest Observer website, ${ }^{5}$ and the benefits of using PDC data over SAP data outweigh the detriments in this case. Further discussion on the implications to asteroseismology of choosing which of the SAP or PDC data to use can be found in Murphy (2012a,b).

We also note that the variability of KIC 11754974 was already discovered from the All Sky Automated Survey observations (Pigulski et al. 2009, star \#143 in the catalogue). Due to the quality of the data, only the main mode was detected; we did not use those data in this investigation.

The data were imported into PERIOD04, for its useful graphical user interface, convenient least squares and discrete Fourier transform

\footnotetext{
${ }^{4}$ http://archive.stsci.edu/kepler/manuals/Data_Characteristics.pdf

${ }^{5}$ http://keplergo.arc.nasa.gov/Documentation.shtml
}

functions, and tools for frequency extraction with pre-whitening. A review of the functions of PERIOD04 has been published by the program's creators (Lenz \& Breger 2004), and a more comprehensive view of its capabilities can be found in the user guide (Lenz \& Breger 2005).

The corrected, edited Q6.1-Q7.3 data set contains 256411 points taken from (BJD) 2455372.439 to 2455552.558 (180.12 d), having a 97 per cent duty cycle.

\subsection{The light curve}

We extracted 166 frequencies that are an excellent fit to the light curve. We recalculated our fit both after outlier extraction, and after removing a cubic spline fit. The latter was done by calculating the average residuals at 2-d intervals, interpolating with a cubic spline, and subtracting the smoothed curve from the original data. Semiregular cycles (Fig. 3, lower panel) are evident on different time-scales as a result of beating between frequencies.

The peaks in brightness occur over a greater intensity range than the troughs (Fig. 3, upper panel). This kind of light-curve shape is typical for HADS stars and can be thought of as saturation of the driving mechanism (Balona et al. 2011). It has also been observed in many $\gamma$ Dor stars, and is common in RRab (RR Lyrae) variables. In particular, it is common in white dwarfs that show many combination frequencies in their pulsation spectra. Wu (2001) described how in white dwarfs, which pulsate in g modes, the convection turnover time is inferred from the phase of a combination frequency relative to its parents, and how the amplitude difference between the sums and differences in combination terms (e.g. $f_{1}+f_{2}$ and $f_{1}-f_{2}$ ) can 
give the thermal constant of the stellar convection zone at equilibrium. However, in an investigation into combination frequencies in $\delta$ Sct stars, Balona (2012) concluded that there is no information in the relative amplitude of a combination frequency that might be useful for mode identification in these p-mode pulsators.

\subsection{The region of independent frequencies}

In KIC 11754974, all of the high-amplitude independent frequencies are found in the range 16-25 $\mathrm{d}^{-1}$ (a full list of extracted frequencies with their corresponding amplitudes, along with identifications, is given in Table 4). Outside of this range, most frequencies detected can be explained as combination of dominant frequencies in the independently excited region. Such is also the case for the star $44 \mathrm{Tau}$, on which the literature is extensive. In KIC 11754974, independent peaks outside the $16-25 \mathrm{~d}^{-1}$ range have low amplitudes, typically of the order of $200 \mu \mathrm{mag}$.

In HADS stars, the period ratio of the two most dominant modes offers easy mode identification when that ratio is 0.77 (Poretti 2003, for example), as this is the theoretical period ratio of the first radial overtone mode (hereafter $F 1$ ) to the fundamental radial mode $(F 0)$. The period ratio can be plotted against the base-10 logarithm of the period of $F 0, \log P(F 0)$, in a so-called Petersen diagram, ${ }^{6}$ offering diagnostic information on the star. Petersen \& ChristensenDalsgaard (1996) discuss the diagrams in detail for double-mode HADS stars of different metallicities in their fig. 3. They showed that lower values of the $P(F 1) / P(F 0)$ ratio are found for metal-rich stars and stars closer to the ZAMS, but spectroscopy of KIC 11754974 indicates that this star has neither property. Hence, the period ratio of its two highest amplitude modes, $f_{1}$ and $f_{2}$, having $P\left(f_{2}\right) / P\left(f_{1}\right)=$ 0.7638 , is much below the anticipated value $(>0.770)$ from the metallicity-calibrated Petersen diagrams. We can thus argue that $f_{1}$ and $f_{2}$ are not the $F 0$ and $F 1$ modes. We also note that the highamplitude independent frequencies around $21 \mathrm{~d}^{-1}$ (Figs 4 and 5), particularly $f_{3}$ and $f_{4}$, could each potentially be the first radial overtone based on period ratios, though not all can be $\ell=0$ modes, and none exactly gives the anticipated ratio.

The high amplitude of $f_{1}$ alone does not necessarily imply it is the fundamental radial mode, either; in FG Vir whose pulsation amplitudes are comparable to this star, the fundamental radial mode was not found to be the highest in amplitude of the observed frequencies (Guzik, Bradley \& Templeton 2000; Breger et al. 2005). 4 CVn is another example (Breger, Davis \& Dukes 2008; Castanheira et al. 2008). The high amplitude of $f_{1}$ does have other consequences: its window pattern dominates the entire analysed spectrum, even far from its frequency of $16.345 \mathrm{~d}^{-1}$. Peaks arising solely from the spectral window of $f_{1}$ have amplitudes of 0.9 and $0.5 \mathrm{mmag}$ at 50 and $100 \mathrm{~d}^{-1}$, respectively. To make other peaks with lower amplitudes visible, Fig. 4 shows a schematic amplitude spectrum with all window patterns removed. The five highest amplitude peaks in the range $16-25 \mathrm{~d}^{-1}$ are all independent modes (note that the third and fourth highest peaks in that range are indistinguishable in Fig. 4). Other peaks with high amplitudes found outside this region are combinations of these frequencies.

Only once the five main independent modes are removed is it possible to see the high-frequency density in the Fourier transform.

\footnotetext{
${ }^{6}$ The diagrams were first discussed by Petersen (1978), but it was Art N. Cox who brought the term 'Petersen diagram' into general use through his conference talks and eventually in his papers.
}

Fig. 5 allows a clearer view of the lower amplitude peaks surrounding the main pulsation frequencies. In this frequency range, independent frequencies are common, and few frequencies appear to be combinations. The spacing of $0.036 \mathrm{~d}^{-1}$ between $f_{3}$ and $f_{4}$ is found repeated in combination frequencies all over the spectrum, with different coefficients of $f_{1}$ and $f_{2}$ added to them.

\subsection{Quintuplet}

The Fourier transform of the light curve of the star features a quintuplet, with a central frequency at $20.242 \mathrm{~d}^{-1}$, and two lower amplitude companions on each side with a mean separation of $0.218 \mathrm{~d}^{-1}$, shown in Fig. 6 (upper panel). We assume that the quintuplet is rotationally split. The exact morphology of the quintuplet depends on excitation and orientation, but unless one can safely assume that components are excited to the same amplitude, the amplitude ratios cannot be used to constrain the star's inclination. The separations are not perfectly identical between components, nor do we expect them to be for rotationally split quintuplets - exact frequency spacing is not expected unless there is frequency locking due to resonance (Buchler, Goupil \& Hansen 1997). The need for highdispersion spectroscopy or multicolour photometry is highlighted in this mode identification attempt - better spectroscopy can more tightly constrain both $v \sin i$ and the fundamental parameters required to improve the models (described in Section 5), allowing more certain mode identification.

In other case studies, peaks due to rotation have been found at low frequency with highly significant amplitudes (e.g. KIC 9700322; Breger et al. 2011; Guzik \& Breger 2011). Hence, we searched the Q6 and Q7 SC data for a peak at low frequency that might correspond to the rotation frequency. There is no evidence for a peak at the anticipated value, nor at half or double that value (cf. Balona 2011). In fact, there is no evidence for a peak due to rotation below $\sim 0.8 \mathrm{~d}^{-1}$, which has implications for our modelling discussion Section 5.3. To check that such a peak was not somehow removed by either our spline fit or the PDC pipeline, the SAP data were also searched for the same peak, but no peak was found.

Another quintuplet occurs at $\sim 36.59 \mathrm{~d}^{-1}$ (Fig. 6, lower panel), but this is a manifestation of the main quintuplet in combination with $f_{1}$. This is confirmed by inspecting the amplitudes of this second multiplet in light of the first - not only are they smaller, but they retain similar amplitude ratios between peaks within the multiplet. Similar quintuplets can be identified at $3.90 \mathrm{~d}^{-1}$, arising from $f_{\text {quintuplet }}-f_{1}$, and at $12.45 \mathrm{~d}^{-1}$ from $2 f_{1}-f_{\text {quintuplet }}$. The frequency distribution and the amplitudes of the quintuplets are shown in Fig. 7.

\subsection{Combination frequencies}

The frequencies in Table 4 have been extracted down to an amplitude level of $100 \mu \mathrm{mag}$ for the region $0-70 \mathrm{~d}^{-1}$, and to $20 \mu \mathrm{mag}$ for 70 $100 \mathrm{~d}^{-1}$ for closer examination of patterns. The region $70-100 \mathrm{~d}^{-1}$ shows the amplitude level at which pulsation-mode candidates can be identified. Kepler SC data allow high-frequency analysis, and the high precision of the satellite's photometry, along with its position outside of Earth's atmosphere, afford low noise levels $(<5 \mu \mathrm{mag}$ for the region $70-100 \mathrm{~d}^{-1}$ ). The combination of the two make the detection of high-order frequency combinations possible.

The tolerance criterion for each calculated combination frequency has been chosen as $0.001 \mathrm{~d}^{-1}$; to be identified as a combination, the calculated frequency must agree with the observed frequency within this value. This tolerance is strict by some definitions - for the data set analysed, $1 / T=0.0056 \mathrm{~d}^{-1}$ and the full 
Table 4. Multifrequency extraction and identification for KIC 11754972 . The absence of combinations between 16 and $25 \mathrm{~d}^{-1}$ is evident; the quintuplet and $f_{1}-f_{5}$ all lie in this region. Frequencies are only given an individual identification if they are of particular significance or form a combination. Errors on frequencies are generally smaller than $2 \times 10^{-4} \mathrm{~d}^{-1}$, and errors on amplitudes are all under $4 \mu \mathrm{mag}$. For $f_{1}-f_{5}$, from which combinations are calculated, the frequency uncertainty is $10^{-6} \mathrm{~d}^{-1}$.

\begin{tabular}{|c|c|c|c|c|c|c|c|c|}
\hline $\begin{array}{l}\text { Frequency } \\
\qquad\left(\mathrm{d}^{-1}\right)\end{array}$ & $\begin{array}{r}\text { Amplitude } \\
(\mu \mathrm{mag})\end{array}$ & ID & $\begin{array}{r}\text { Frequency } \\
\left(\mathrm{d}^{-1}\right)\end{array}$ & $\begin{array}{r}\text { Amplitude } \\
(\mu \mathrm{mag})\end{array}$ & ID & $\begin{array}{r}\text { Frequency } \\
\qquad\left(\mathrm{d}^{-1}\right)\end{array}$ & $\begin{array}{l}\text { Amplitude } \\
(\mu \mathrm{mag})\end{array}$ & ID \\
\hline 16.34474 & 63329 & $f_{1}$ & & & & & & \\
\hline 21.39898 & 15940 & $f_{2}$ & & & & & & \\
\hline 20.90740 & 11653 & $f_{3}$ & & & & & & \\
\hline 20.94355 & 8229 & $f_{4}$ & & & & & & \\
\hline 22.66020 & 2839 & $f_{5}$ & & & & & & \\
\hline 0.4554 & 270 & $=f_{2}-f_{4}$ & 20.4552 & 603 & $=f_{11}{ }^{*}$ & 42.7980 & 946 & $=2 f_{2}$ \\
\hline 0.4916 & 865 & $=f_{2}-f_{3}$ & 20.6552 & 422 & $=f_{12}{ }^{*}$ & 43.5676 & 122 & $=f_{3}+f_{5}$ \\
\hline 0.7723 & 202 & $=f_{7}-f_{1}$ & 21.0537 & 216 & & 44.0592 & 389 & $=f_{2}+f_{5}$ \\
\hline 1.0705 & 315 & $=f_{1}-f_{6}$ & 21.3973 & 257 & $\dagger$ & 44.4716 & 107 & $=4 f_{1}-f_{3}$ \\
\hline 1.2612 & 619 & $=f_{5}-f_{2}$ & 21.4282 & 274 & & 47.8522 & 103 & $=3 f_{2}-f_{1}$ \\
\hline 1.3804 & 178 & $=f_{2}-f_{9}$ & 21.7251 & 458 & $=f_{14}$ & 49.0342 & 2677 & $=3 f_{1}$ \\
\hline 1.7528 & 250 & $=f_{5}-f_{3}$ & 22.5325 & 270 & & 53.5969 & 1230 & $=2 f_{1}+f_{3}$ \\
\hline 3.4387 & 202 & $=f_{8}-f_{1}$ & 22.8592 & 107 & & 53.6330 & 828 & $=2 f_{1}+f_{4}$ \\
\hline 3.6738 & 291 & $=f_{9}-f_{1}$ & 22.8860 & 205 & & 54.0885 & 1466 & $=2 f_{1}+f_{2}$ \\
\hline 3.8976 & 334 & $=f_{10}-f_{1}$ & 23.7744 & 354 & $=f_{15}$ & 55.3497 & 235 & $=2 f_{1}+f_{5}$ \\
\hline 4.1040 & 141 & $=f_{13}-f_{1}$ & 24.3270 & 130 & & 58.1595 & 246 & $=f_{1}+2 f_{3}$ \\
\hline 4.1105 & 161 & $=f_{11}-f_{1}$ & 25.4701 & 257 & $=2 f_{3}-f_{1}$ & 58.1957 & 174 & $=f_{1}+f_{3}+f_{4}$ \\
\hline 4.3108 & 76 & $=f_{12}-f_{1}$ & 25.5062 & 229 & $=f_{3}+f_{4}-f_{1}$ & 58.6511 & 292 & $=f_{1}+f_{2}+f_{3}$ \\
\hline 4.5627 & 4625 & $=f_{3}-f_{1}$ & 25.6208 & 242 & & 58.6873 & 191 & $=f_{1}+f_{2}+f_{4}$ \\
\hline 4.5988 & 2234 & $=f_{4}-f_{1}$ & 25.9616 & 403 & $=f_{2}+f_{3}-f_{1}$ & 59.1427 & 705 & $=2 f_{2}+f_{1}$ \\
\hline 5.0542 & 4435 & $=f_{2}-f_{1}$ & 25.9961 & 280 & & 60.4039 & 165 & $=f_{1}+f_{2}+f_{5}$ \\
\hline 5.3804 & 119 & $=f_{14}-f_{1}$ & 26.2959 & 441 & $=f_{16}$ & 63.7054 & 152 & $=2 f_{2}+f_{3}$ \\
\hline 5.5458 & 118 & $=2 f_{2}-f_{1}-f_{3}$ & 26.4532 & 524 & $=2 f_{2}-f_{1}$ & 63.7415 & 126 & $=2 f_{2}+f_{4}$ \\
\hline 6.2363 & 139 & $=3 f_{1}-2 f_{2}$ & 26.5422 & 148 & & 64.1969 & 292 & $=3 f_{2}$ \\
\hline 6.3155 & 678 & $=f_{5}-f_{1}$ & 27.2590 & 105 & $=f_{4}+f_{5}-f_{1}$ & 65.3790 & 628 & $=4 f_{1}$ \\
\hline 6.7278 & 108 & $=3 f_{1}-f_{2}-f_{3}$ & 27.6352 & 481 & $=3 f_{1}-f_{2}$ & 69.9416 & 418 & $=3 f_{1}+f_{3}$ \\
\hline 9.1253 & 116 & $=2 f_{3}-2 f_{1}$ & 27.7144 & 172 & $=f_{2}+f_{5}-f_{1}$ & 69.9778 & 241 & $=3 f_{1}+f_{4}$ \\
\hline 9.1615 & 112 & $=f_{3}+f_{4}-2 f_{1}$ & 28.0907 & 238 & $=3 f_{1}-f_{4}$ & 70.4332 & 318 & $=3 f_{1}+f_{2}$ \\
\hline 9.6169 & 204 & $=f_{2}+f_{3}-2 f_{1}$ & 28.1268 & 426 & $=3 f_{1}-f_{3}$ & 71.6944 & 70 & $=3 f_{1}+f_{5}$ \\
\hline 10.0293 & 361 & $=2 f_{1}-f_{5}$ & 29.6549 & 393 & & 74.5043 & 94 & $=2 f_{1}+2 f_{3}$ \\
\hline 10.1085 & 233 & $=2 f_{2}-2 f_{1}$ & 29.6621 & 158 & & 74.5404 & 132 & $=2 f_{1}+f_{3}+f_{4}$ \\
\hline 11.2905 & 2066 & $=2 f_{1}-f_{2}$ & 30.5486 & 112 & $=2 f_{6}$ & 74.9959 & 201 & $=2 f_{1}+f_{2}+f_{3}$ \\
\hline 11.7459 & 1068 & $=2 f_{1}-f_{4}$ & 31.6190 & 196 & $=f_{1}+f_{6}$ & 75.0320 & 79 & $=2 f_{1}+f_{2}+f_{4}$ \\
\hline 11.7821 & 1844 & $=2 f_{1}-f_{3}$ & 32.1979 & 141 & $=2 f_{1}+f_{3}-f_{2}$ & 75.4874 & 271 & $=2 f_{1}+2 f_{2}$ \\
\hline 12.0343 & 41 & $=2 f_{1}-f_{12}$ & 32.6895 & 12311 & $=2 f_{1}$ & 76.7487 & 67 & $=2 f_{1}+f_{2}+f_{5}$ \\
\hline 12.2342 & 62 & $=2 f_{1}-f_{11}$ & 32.7252 & 112 & $=2 f_{1}+f_{4}-f_{3}$ & 80.0501 & 99 & $=2 f_{2}+f_{1}+f_{3}$ \\
\hline 12.4471 & 121 & $=2 f_{1}-f_{10}$ & 33.1811 & 148 & $=2 f_{1}+f_{2}-f_{3}$ & 80.0863 & 110 & $=f_{1}+2 f_{2}+f_{4}$ \\
\hline 12.6709 & 107 & $=2 f_{1}-f_{9}$ & 33.4618 & 272 & $=f_{1}+f_{7}$ & 80.5417 & 142 & $=3 f_{2}+f_{1}$ \\
\hline 12.9060 & 81 & $=2 f_{1}-f_{8}$ & 36.1282 & 175 & $=f_{1}+f_{8}$ & 81.7237 & 182 & $=5 f_{1}$ \\
\hline 14.5919 & 108 & $=f_{1}+f_{3}-f_{5}$ & 36.3633 & 216 & $=f_{1}+f_{9}$ & 81.8029 & 41 & $=f_{1}+2 f_{2}+f_{5}$ \\
\hline 15.0835 & 262 & $=f_{1}+f_{2}-f_{5}$ & 36.5871 & 270 & $=f_{1}+f_{10}$ & 85.5959 & 68 & $=4 f_{2}$ \\
\hline 15.2743 & 692 & $=f_{6}$ & 36.7935 & 112 & $=f_{1}+f_{13}$ & 86.2864 & 146 & $=4 f_{1}+f_{3}$ \\
\hline 15.8532 & 425 & $=f_{1}+f_{3}-f_{2}$ & 36.8000 & 136 & $=f_{1}+f_{11}$ & 86.3225 & 77 & $=4 f_{1}+f_{4}$ \\
\hline 15.8893 & 180 & $=f_{1}+f_{4}-f_{2}$ & 37.0000 & 74 & $=f_{1}+f_{12}$ & 86.7780 & 134 & $=4 f_{1}+f_{2}$ \\
\hline 16.3086 & 230 & $=f_{1}+f_{3}-f_{4}$ & 37.2410 & 192 & & 88.0392 & 29 & $=4 f_{1}+f_{5}$ \\
\hline 16.3618 & 155 & & 37.2521 & 4143 & $=f_{1}+f_{3}$ & 90.8490 & 46 & $=3 f_{1}+2 f_{3}$ \\
\hline 16.3807 & 285 & $=f_{1}+f_{4}-f_{3}$ & 37.2883 & 3158 & $=f_{1}+f_{4}$ & 90.8852 & 83 & $=3 f_{1}+f_{3}+f_{4}$ \\
\hline 16.7999 & 141 & $=f_{1}+f_{2}-f_{4}$ & 37.7437 & 5730 & $=f_{1}+f_{2}$ & 91.3406 & 120 & $=3 f_{1}+f_{2}+f_{3}$ \\
\hline 16.8363 & 369 & $=f_{1}+f_{2}-f_{3}$ & 37.9345 & 183 & $=f_{5}+f_{6}$ & 91.3768 & 32 & $=3 f_{1}+f_{2}+f_{4}$ \\
\hline 17.1170 & 871 & $=f_{7}$ & 38.0698 & 170 & $=f_{1}+f_{14}$ & 91.8322 & 97 & $=3 f_{1}+2 f_{2}$ \\
\hline 17.6060 & 212 & $=f_{1}+f_{5}-f_{2}$ & 39.0049 & 795 & $=f_{1}+f_{5}$ & 93.0934 & 38 & $=3 f_{1}+f_{2}+f_{5}$ \\
\hline 19.3224 & 424 & & 40.1192 & 146 & $=f_{1}+f_{1} 5$ & 95.9033 & 32 & $=2 f_{1}+f_{2}+2 f_{3}$ \\
\hline 19.7078 & 341 & & 40.6909 & 295 & $=f_{3}+f_{8}$ & 95.9394 & 28 & $=2 f_{1}+f_{2}+f_{3}+f_{4}$ \\
\hline 19.7835 & 636 & $=f_{8} *$ & 41.4176 & 128 & $=f_{2}+f_{9}$ & 96.3949 & 91 & $=2 f_{2}+2 f_{1}+f_{3}$ \\
\hline 19.8051 & 152 & & 41.8148 & 494 & $=2 f_{3}$ & 96.4310 & 42 & $=2 f_{1}+2 f_{2}+f_{4}$ \\
\hline 20.0186 & 814 & $=f_{9} *$ & 41.8509 & 760 & $=f_{4}+f_{3}$ & 96.8864 & 68 & $=3 f_{2}+2 f_{1}$ \\
\hline 20.2424 & 959 & $=f_{10}{ }^{*}$ & 42.3064 & 1139 & $=f_{2}+f_{3}$ & 98.0685 & 57 & $=6 f_{1}$ \\
\hline 20.2487 & 126 & & 42.3427 & 233 & $=f_{2}+f_{4}$ & 98.1476 & 27 & $=2 f_{1}+2 f_{2}+f_{5}$ \\
\hline 20.4487 & 508 & $=f_{13}$ & 42.6408 & 103 & $=f_{1}+f_{16}$ & & & \\
\hline
\end{tabular}




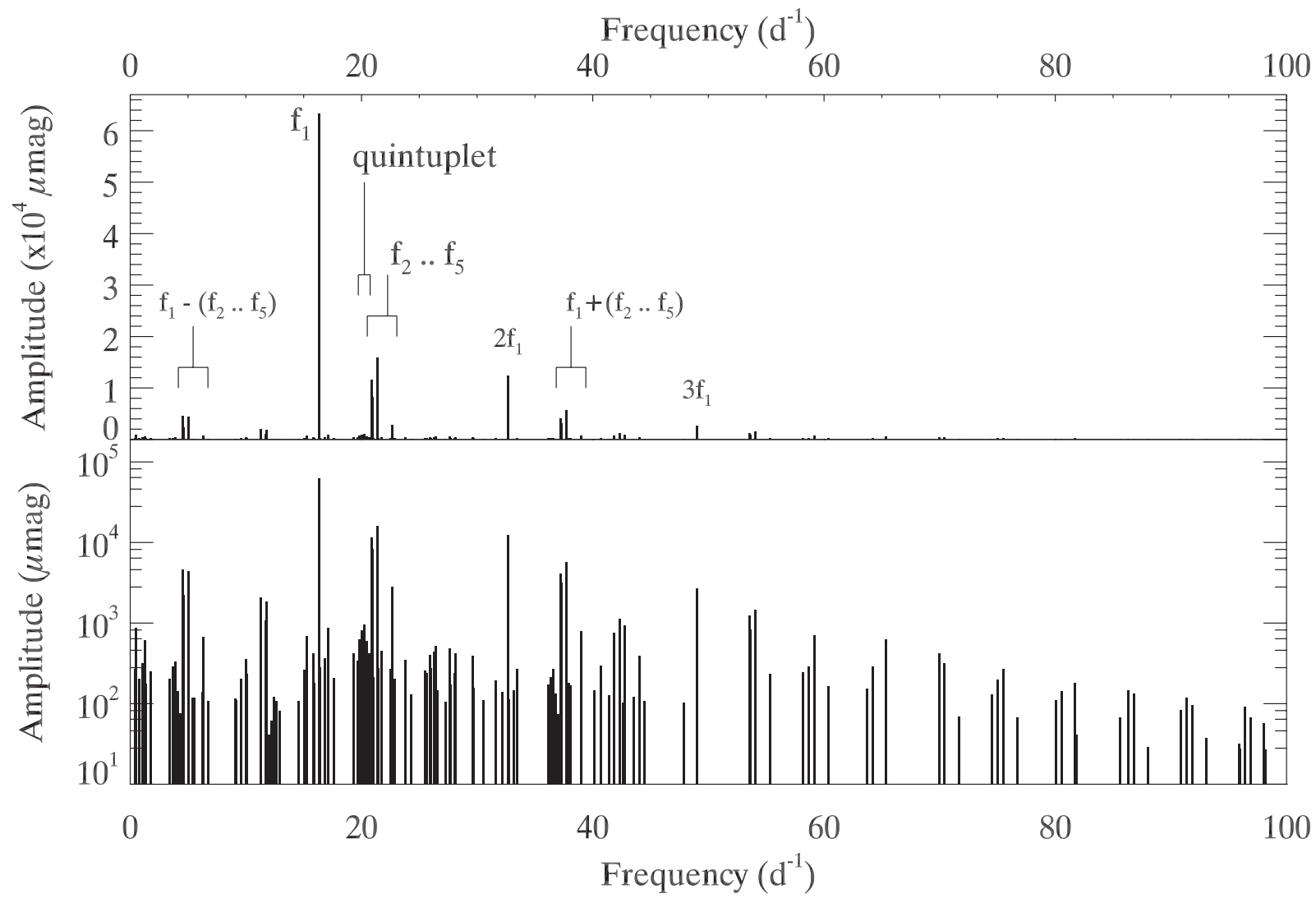

Figure 4. Upper panel: the schematic amplitude spectrum of KIC 11754974 from 0 to $100 \mathrm{~d}^{-1} \cdot f_{1}-f_{5}$ are the five highest amplitude peaks in the frequency region $16<f<25$, assigned in decreasing amplitude order. This region is magnified in Fig. 5. The $10^{4} \mu$ mag peak at $32.7 \mathrm{~d}^{-1}$ is $2 f_{1}$. Also labelled are the quintuplet (Section 4.4) and some combination frequencies (Section 4.5). $f_{4}$ is very close in frequency to $f_{3}$; the two are unresolvable in this figure, but $f_{4}$ has a higher frequency and the frequencies are resolved in the data. Lower panel: logarithmic version of the same plot. Note that these schematic diagrams show the frequencies listed in Table 4 only.

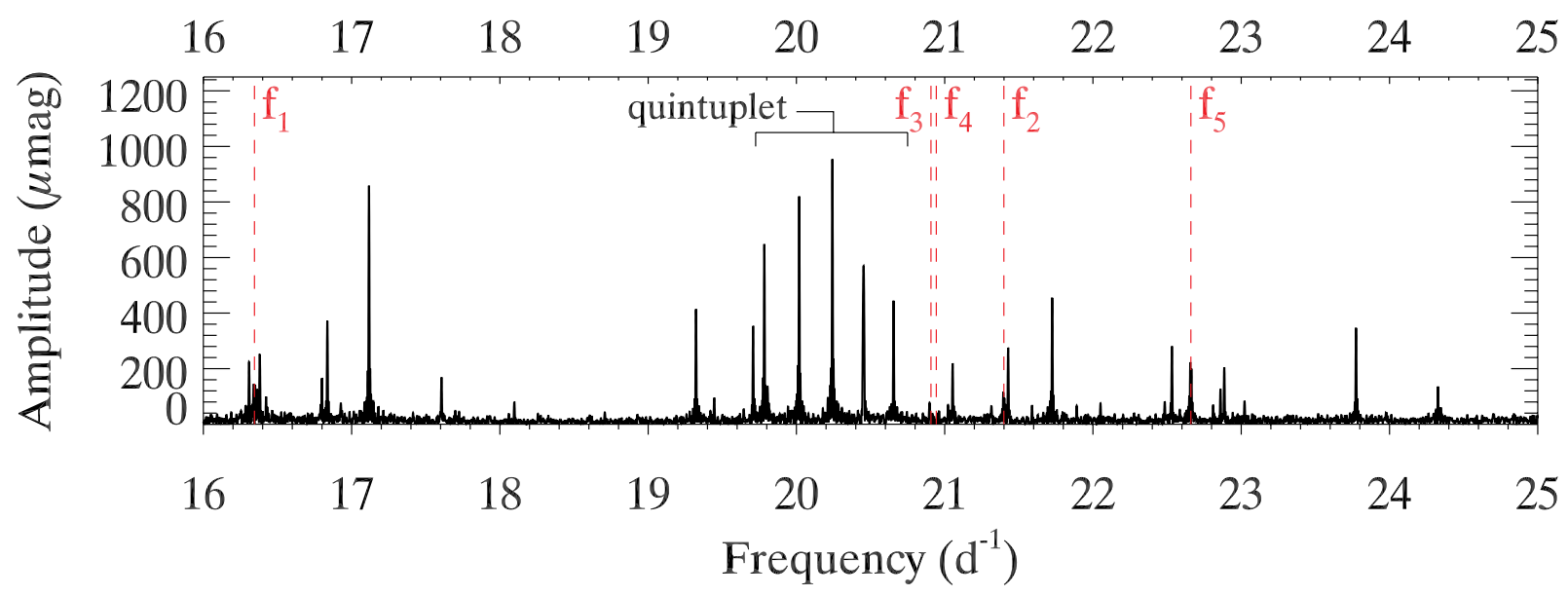

Figure 5. The amplitude spectrum of the combined Q6 and Q7 data set from 16 to $25 \mathrm{~d}^{-1}$ with $f_{1}-f_{5}$ pre-whitened. Without large amplitude modes dominating the scale, the large number of lower amplitude peaks becomes clear. The quintuplet centred at $20.243 \mathrm{~d}^{-1}$ is recognizable from its roughly equal spacings.

width of a peak at half-maximum (FWHM) is $0.0067 \mathrm{~d}$. A stricter tolerance reduces the probability of false positives for frequency combinations, and has been made necessary because of the high number of frequencies extracted.

In addition to meeting the tolerance criterion, the combination must also be physically sensible - high coefficients and many combining independent frequencies require increasingly unlikely interactions between modes. Hence, both the coefficients of the independent frequencies and the number of independent frequencies must be small. Sensible combinations typically have neither of those numbers greater than three, but no hard-and-fast rule was applied. In the region above $70 \mathrm{~d}^{-1}$, the coefficients have to be quite 


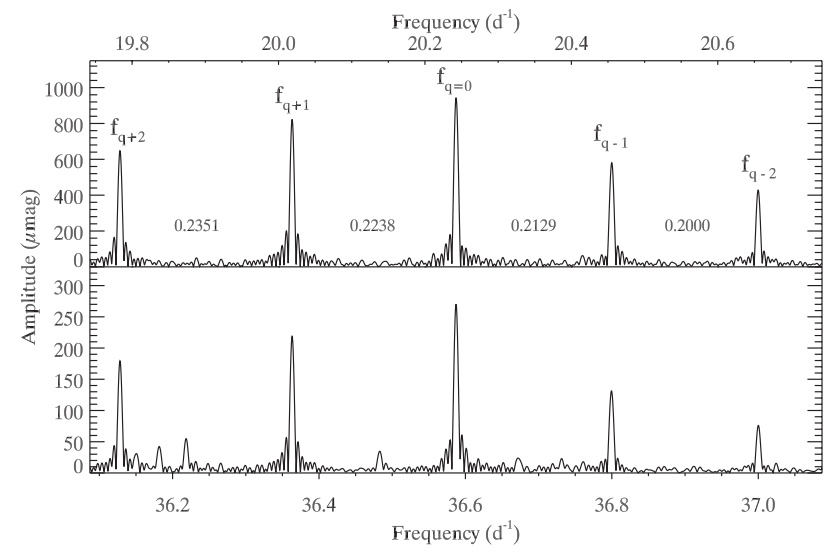

Figure 6. The main quintuplet at $20.242 \mathrm{~d}^{-1}$ (upper panel) and the quintuplet formed in combination with $f_{1}$ at $36.587 \mathrm{~d}^{-1}$ (lower panel). The spacings in the upper panel are equal to those in the lower one because the frequencies are formed in exact combinations with $f_{1}$ (but are only forced to be exact in the fitting - here, we present them as they are observed). Subscripted numbers represent the $m$-values of the mode. Least-squares uncertainties on the frequencies are $<10^{-4} \mathrm{~d}^{-1}$ in the bottom panel and $\ll 10^{-4} \mathrm{~d}^{-1}$ in the top panel.

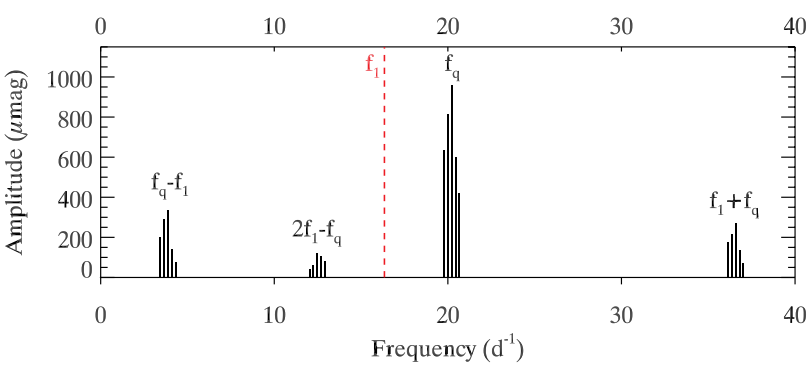

Figure 7. Schematic diagram of the many quintuplets in the data. The main quintuplet at $20.242 \mathrm{~d}^{-1}$ forms other quintuplets throughout the Fourier spectrum in combination with $f_{1}$. One can see the similar amplitude ratios of the quintuplets as the shape of the quintuplet is maintained in each combination.

high, especially since $f_{1}$ is only $16.345 \mathrm{~d}^{-1}$. We note that some of the remaining 'independent' frequencies without identifications in Table 4 can be explained with physically sensible combination frequencies if the tolerance is relaxed.

PERIOD04 is capable of testing for frequency combinations to a given tolerance criterion; such a method was employed by Breger et al. (2005) in their analysis of the star FG Vir, where their tolerance criterion was $0.001 \mathrm{~d}^{-1}$. We used the same tolerance criterion and ran simulations to determine the number of accidentally matched frequencies one can expect. Using the frequencies $f_{1}-f_{5}$, allowing coefficients between -1 and 6 , and allowing all five frequencies to be involved in the combinations simultaneously (i.e. there is much more freedom than in the combinations in Table 4), we find only one to two frequencies in the range $70-100 \mathrm{~d}^{-1}$ to be accidentally matched. Hence, almost all combinations presented in Table 4 are probably genuine. The two frequencies with the greatest uncertainties lie at 91.377 and $95.939 \mathrm{~d}^{-1}$, with errors of $0.001 \mathrm{~d}^{-1}$, which is the same variation as the combination frequency tolerance. These are the lowest amplitude frequencies extracted, and are the two prime candidates for mismatched frequencies.

Combination frequencies are even detected at beyond $100 \mathrm{~d}^{-1}$ (not shown). Particular caution has been made in identifying high
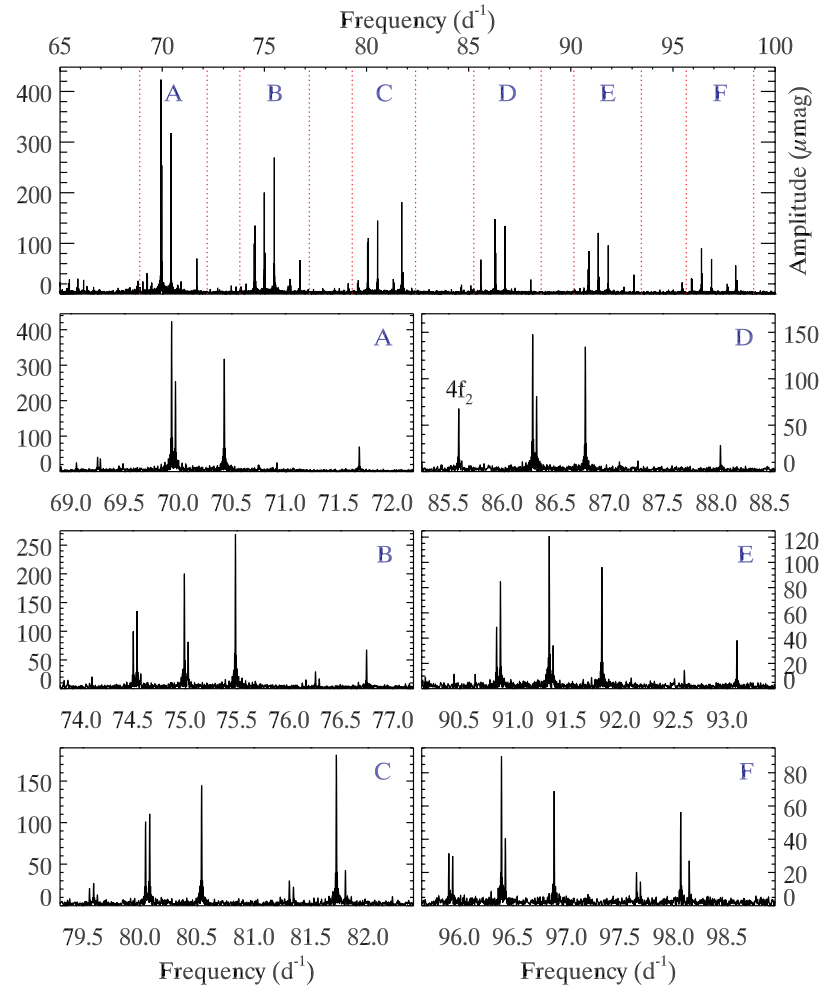

Figure 8. Repetitive patterns are caused by adding $f_{1}$ to other combinations of frequencies, hence panels $\mathrm{A}$ and $\mathrm{D}, \mathrm{B}$ and $\mathrm{E}$, and $\mathrm{C}$ and $\mathrm{F}$ are each separated in frequency by $f_{1}=16.345 \mathrm{~d}^{-1}$. The frequency range displayed by each is shown in the top panel. The first peak in panel $\mathrm{D}$ is $4 f_{2} ; 4 f_{2}-$ $f_{1}$ is too low in amplitude to be distinguishable from noise; hence, it is not seen in panel A. The cause of relative amplitude changes of neighbouring peaks following the addition of $f_{1}$ is unclear, but the absolute changes in amplitudes in adjacent panels (note the change in axis scale - units are $\mu \mathrm{mag}$ ) are a result of having a greater number of combination terms.

frequencies as combinations, especially because they often contain more than two independent frequencies and coefficients of 3, 4 or even higher. There is no doubt, however, that these are indeed combinations. Several patterns of frequencies repeat themselves, separated by $f_{1}$ (Fig. 8), in a way that could only happen dependently. The frequencies of these peaks are entirely calculable given the frequencies of the main independent modes, and at yet higher frequencies they recur with diminishing amplitudes until $\sim 140 \mathrm{~d}^{-1}$ at which point their amplitudes become indistinguishable from noise and the patterns become less discernible when peaks are missing.

\subsection{Validity of extracted frequencies}

One could continue to extract frequencies down to lower and lower amplitudes, but this begs the question of where to stop. $100 \mu \mathrm{mag}$ was chosen as a sensible limit, to extract the important frequencies for mode identification, and also for clarity: mode splittings become harder and harder to spot when the density of peaks increases, as happens when one lowers the extraction limit. On occasion, combination frequencies with amplitudes below $100 \mu \mathrm{mag}$ were extracted, such as when they are expected as part of a multiplet. There are additional complications to extracting frequencies to lower amplitudes. With a 180 -d data set, frequency resolution is $0.006 \mathrm{~d}^{-1}$. One cannot extract frequencies more closely spaced than this. Moreover, nothing is gained by continuing extraction to lower 


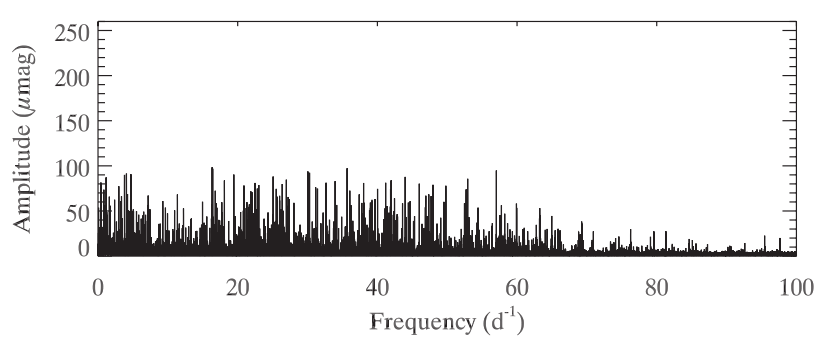

Figure 9. Residuals left behind after extraction of 166 frequencies, to amplitude limits $100 \mu \mathrm{mag}$ for $0 \leq f \leq 70 \mathrm{~d}^{-1}$, and $20 \mu \mathrm{mag}$ for $70<f \leq$ $100 \mathrm{~d}^{-1}$.

amplitudes - combination frequencies become less reliable as the risk of false positives rises sharply with the number of extracted frequencies, mode identification becomes more difficult when mode density increases, and the low-amplitude frequencies become 'noise' interspersed with the meaningful high-amplitude peaks. Fig. 9 shows the residuals left after pre-whitening all frequencies in Table 4.

The Kepler Input Catalogue (KIC; Brown et al. 2011) provides information on the amount of light contamination appearing in the data. The value of contamination for KIC 11754974 is 0.009 ; hence, less than 1 percent of light incident on the CCD pixels attributed to this star can be coming from background stars. As far as we can tell from looking at Kepler Full-Frame Images, 2MASS and DSS images, KIC 11754974 is safe from contamination. This makes negligible the possibility that a background star has a high-amplitude pulsation mode that is picked up and interpreted as a pulsation mode for KIC 11754974 . We know that the star is in a binary system, but with a star significantly less massive than the Sun, contributing little light and not expected to generate frequencies in the periodogram at the amplitudes and frequencies observed.

It may be possible to check whether a frequency is a part of a combination or is an independent frequency by examining the relationship between phase and frequency for the combination terms. In a recent paper on KIC 9700322, in which many combination frequencies were detected, Breger et al. (2011) found that the higher frequency modes have greater relative phases. The theory is based on that developed by Buchler et al. (1997) who described non-linear resonances mathematically, and Balona (2012) investigated further. A combination term with frequency $f=n_{\mathrm{j}} f_{\mathrm{i}}+n_{\mathrm{j}} f_{\mathrm{j}}$ should have a relative phase, $\phi_{\mathrm{r}}=\phi_{\mathrm{c}}-\left(n_{\mathrm{i}} \phi_{\mathrm{i}}+n_{\mathrm{j}} \phi_{\mathrm{j}}\right)$, where $\phi_{\mathrm{c}}$ is the phase calculated by least-squares fitting. We find that $\phi_{\mathrm{r}}$ decreases with frequency once phase folding about $2 \pi$ is taken into account - we shifted groups of points by multiples of $2 \pi$ in phase until they lay roughly in a straight line, then made $2 \pi$ corrections for individual points lying more than $\pi$ away from the fit in an iterative way. All combination frequencies listed in Table 4 are plotted in Fig. 10. Those 'combination frequencies' that do not fit the correlation between $\phi_{\mathrm{r}}$ and frequency in the figure might actually be independent frequencies, instead. On the other hand, perhaps the relationship is a purely mathematical construct. The points lying furthest from the fit are $3 f_{2}$ and $4 f_{2}$, which given the nature of the other combination frequencies in Table 4 would be expected to be present and with high $\mathrm{S} / \mathrm{N}$.

The correlation has been discussed by Balona (2012), who showed that for two interacting modes the phases of the harmonics of a given mode decrease linearly with increasing harmonic order (i.e. frequency). The observational evidence was not discussed in light of the precision with which frequencies, amplitudes and phases

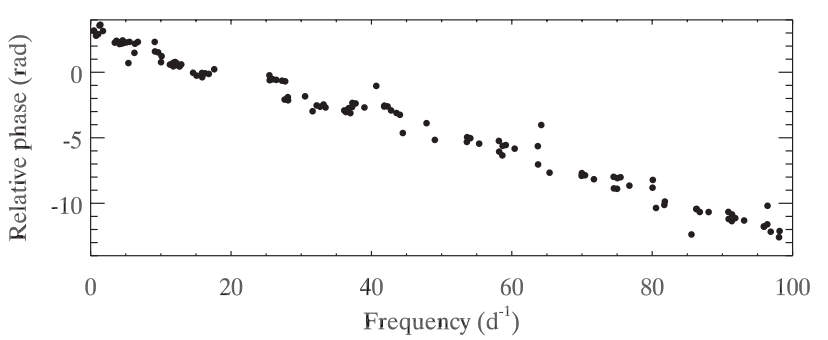

Figure 10. Relative phases are negatively correlated with frequency for the combination frequencies. Those points located far from the others might be interpreted as independent frequencies instead of combinations. Error bars for phases are shown, but are generally much smaller than the plot symbols. Phases and errors are the formal least-squares values and were calculated with respect to the mid-point of the combined Q6 and Q7 SC data set: BJD ${ }_{\text {UTC }} 2455462.49792$

can be determined with continuous space-based observations. The effect of such small error bars is to reduce the significance of the observational evidence substantially - the point with the median deviation lies over $27 \sigma$ away from the fit. The median was chosen because taking the mean is biased towards those points with minuscule relative phase errors, whose distance from the fit can be many hundred $\sigma$. The combinations giving rise to such small errors typically involve $f_{1}$ and $f_{2}$, the calculated phase errors of which are as low as $10^{-4} \mathrm{rad}$. This further supports a rejection of the hypothesis of any meaningful correlation, because combinations like $f_{1}+f_{2}$ are superiorly statistically significant in terms of $\mathrm{S} / \mathrm{N}$, and should consequently lend greater weight in this correlation. The reduced chi-squared parameter, $\chi_{\text {red }}^{2}$, is of the order of $10^{5}$, clearly indicating an inappropriate fit.

To establish whether the correlation need be rejected entirely, we ran a calculation whereby the frequency combinations and their phase errors were kept fixed, but instead of calculating phases from least squares we randomized them. We found that a correlation cannot be constructed by adding $\pm n 2 \pi$ to the random phases, so while it is clear that in the relative phases and frequencies of KIC 11754974 some correlation does exist, we conclude the correlation is neither tight nor useful.

\section{ASTEROSEISMIC MODELS}

KIC 11754974 lies near the red edge of the $\delta$ Sct instability strip. At these cooler temperatures, convection plays an important role. Indeed, without time-dependent convection (TDC) treatments, an actual red edge is not accurately predicted. Thus, the introduction of TDC models was an important step forward in the modelling of $\delta$ Sct stars (Dupret et al. 2004, 2005). Murphy et al. (2012) gave a successful first application of these models to an individual $\delta$ Sct star observed by Kepler.

Rotation plays a big role in the evolution and oscillations of $\delta$ Sct stars (Goupil et al. 2005; Rodríguez et al. 2006a,b; Poretti et al. 2011), even for KIC 11754974, which rotates slowly compared to the distribution of $\delta$ Sct star rotational velocities. Furthermore, asteroseismic analyses with rotating models can assist with mode identification through the effects of rotation on the mode couplings and frequency relationships (Suárez, Garrido \& Goupil 2006a; Suárez, Garrido \& Moya 2007).

No combination of both TDC and rotating models exists at present, so our modelling approach consists of the two methods applied separately. This section explains the details of the models used, and the methodology followed. 


\subsection{Description of the models}

Our TDC models use the same tools and selection method as that explained in detail in Murphy et al. (2012); the codes used are the Code Liégeois d'évolution stellaire (Scuflaire et al. 2008) for structure models and the MAD code (Dupret 2001) supplemented with Gabriel's treatment of TDC (Gabriel 1996; Grigahcène et al. 2005) for non-adiabatic non-radial oscillation calculations. Convection was treated using mixing-length theory (MLT; Böhm-Vitense 1958), and TDC runs throughout the evolution calculations.

In order to theoretically characterize KIC 11754974, pseudorotating seismic models (see Soufi, Goupil \& Dziembowski 1998; Suárez et al. 2002) of the star were built. These equilibrium models were computed with the evolutionary code CESAM (Morel 1997) using frozen convection (FC) and taking the strongest effects of rotation into account. The latter is done by including the spherically averaged contribution of the centrifugal acceleration, which is included by means of an effective gravity $g_{\text {eff }}=g-\mathcal{A}_{c}(r)$, where $g$ is the local gravity, $r$ is the radius and $\mathcal{A}_{c}(r)=\frac{2}{3} r \Omega^{2}(r)$ is the centrifugal acceleration of matter elements. The non-spherical components of the centrifugal acceleration are included in the adiabatic oscillation computations, but not in the equilibrium models (details in Suárez, Goupil \& Morel 2006b).

Since we have no information about the internal rotation profile of KIC 11754974 , i.e. about how the angular momentum is distributed in its interior, we have adopted the hypothesis of differential rotation caused by local conservation of the angular momentum (in the radiative zones) during the evolution of the star. Similar rotation profiles have been found when analysing the evolution of giant stars including rotationally induced mixing of chemical elements and transport of angular momentum (Maeder \& Meynet 2004). The physics of the equilibrium models has been chosen as adequate for intermediate-mass A-F stars, and was described in detail by Suárez et al. (2009).

Adiabatic oscillations were computed using the adiabatic oscillation code FILOu (Suárez 2002; Suárez \& Goupil 2008). This code provides theoretical adiabatic oscillations of a given equilibrium model corrected up to second order for the effects of rotation. These include near-degeneracy effects (mode coupling effects), which occur when two or more frequencies are close to each other. In addition, the perturbative description adopted takes radial variation of the angular velocity (differential rotation) into account in the oscillation equations. The oscillation spectra were calculated from frequencies around the fundamental radial mode (for each model) and the cut-off frequency. This allows the presence of low-order $g$ and $\mathrm{p}$ modes (mixed modes), which are generally present in $\delta$ Sct stars. Since non-radial oscillations are expected, modes were computed from degrees $\ell=0$ (radial) to $\ell=3$. Although the calculations are adiabatic, the effects of rotation alter the frequencies by an amount greater than the use of non-adiabatic codes.

\subsection{Methodology}

The low $T_{\text {eff }}$ of the target suggested TDC models would provide a more accurate first approach to the global properties of the star.
We selected the best-fitting model by perturbing the atmospheric parameters from Section 3 (Table 3) to obtain the best match with the observed oscillation frequencies, adjusting the TDC parameters (namely the convection efficiency) accordingly. The properties of the best TDC model are presented in Table 5.

It is important to stress that the TDC models do not include rotation. Our next step was therefore to calculate equivalent (in terms of global parameters) FC models, incorporating rotation. We considered that the error committed this way (i.e. TDC first) is much lower than through direct modelling with FC and rotation.

The modelling with rotating models involves building a grid, with masses ranging from 1.4 to $2.0 \mathrm{M}_{\odot}$ in steps of $0.1 \mathrm{M}_{\odot}$, a convective efficiency ranging from 0.5 (prescribed by Casas et al. 2006 for A-F stars) to 2.0 (which gave the best fit in TDC calculations) in steps of 0.1 , and an overshoot parameter $d_{\text {ov }}$ in the range of $[0,0.3]$ in steps of 0.05 . The surface rotational velocity of the models, $v_{\mathrm{eq}}$, ranged between 20 and $30 \mathrm{~km} \mathrm{~s}^{-1}$ offering enough dispersion to analyse different configurations of mass, metallicity and age. In principle, this gives splittings close to those observed for the quintuplet, but this was not constrained to be the case. In addition, it indirectly guarantees a dispersion in the inclination angle probed within the model grid. Metallicity was limited to $[\mathrm{Fe} / \mathrm{H}]=-0.5 \pm 0.1 \mathrm{dex}$, though metallicities closer to the solar values were investigated and offered a poorer match with the observed frequencies. This implies that the entire star is metal poor, and that we are not just observing a surface abundance anomaly, thus arguing against a $\lambda$ Boo classification (cf. debates in the literature about the interior metallicity of $\lambda$ Boo stars, e.g. Moya et al. 2010).

Model selection involves searching through the grid, minimizing the mean-square error function $\chi^{2}$. For each mode,

$\chi^{2}=\frac{1}{N} \sum_{i=1}^{N}\left(f_{\mathrm{obs}, i}-v_{\mathrm{th}, i}\right)^{2}$

is calculated, where $f_{\mathrm{obs}, i}$ and $v_{\mathrm{th}, i}$ represent the observed and theoretical frequencies respectively. The total number of observed frequencies is represented by $N$. Calculations are made considering the mass $M$, the rotational velocity $v_{\text {eq }}$, metallicity, $\alpha_{\mathrm{MLT}}, d_{\mathrm{ov}}$ and age as free parameters; pulsation amplitude information is not directly used. Importantly, all the frequencies are fitted simultaneously; assumptions like the 'strongest modes' being radial or quintuplet membership were not used as constraints for the models.

We did look separately for diagnostic frequency ratios in the data. We took models of three different masses and searched for ratios of $0.772 \pm 0.009$ (cf. discussion in Section 4.3) among the independent modes. Fig. 11 displays the four possible frequency ratios observed, none of which satisfies the $T_{\text {eff }}$ and (rather loose) $\log g$ uncertainties, or indicates a particularly promising identification for the first radial overtone. We stress this only reduces the likelihood that $F 1$ is present with F0; either could still be present without the other.

\subsection{Discussion of the models}

In general, we found that the models with the lowest $\chi^{2}$ were below $1.60 \mathrm{M}_{\odot}$. Moreover, those that matched either $F 0$ or $F 1$ to

Table 5. Properties of the best TDC model, obtained by perturbing the spectroscopic parameters and convection efficiency.

\begin{tabular}{lccccccccc}
\hline $\begin{array}{c}\text { Mass } \\
\left(\mathrm{M}_{\odot}\right)\end{array}$ & $\begin{array}{c}\text { Radius } \\
\left(\mathrm{R}_{\odot}\right)\end{array}$ & $\begin{array}{c}T_{\text {eff }} \\
(\mathrm{K})\end{array}$ & $\begin{array}{c}\text { Age } \\
(\mathrm{Myr})\end{array}$ & $\begin{array}{c}\log g \\
(\mathrm{cgs})\end{array}$ & $\log \left(L / \mathrm{L}_{\odot}\right)$ & $X$ & $Z$ & $\alpha_{\mathrm{MLT}}$ & $d_{\mathrm{ov}}$ \\
\hline 1.56 & 2.425 & 7110 & 1829 & 3.862 & 1.130 & 0.7465 & 0.0071 & 2.0 & 0.2 \\
\hline
\end{tabular}




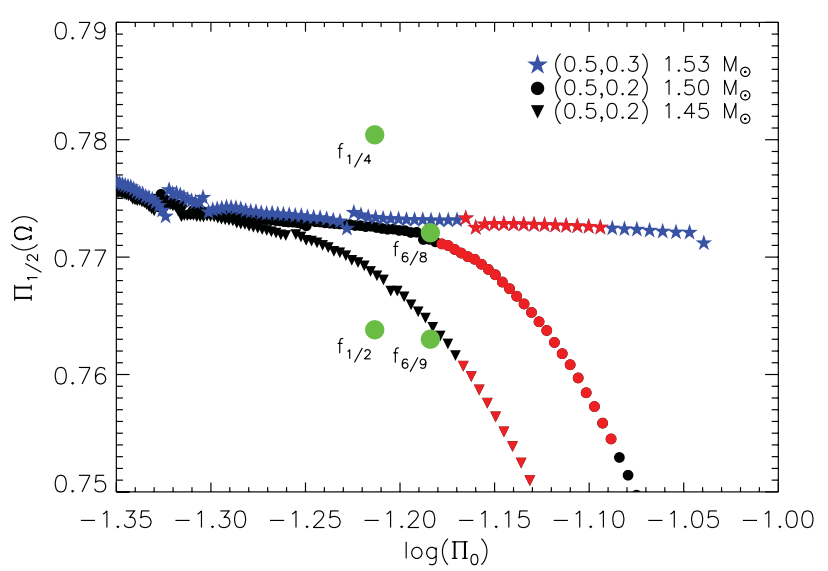

Figure 11. Rotational Petersen diagram covering models with 1.45$1.60 \mathrm{M}_{\odot}$. Coloured in red are the models that satisfy the $T_{\text {eff }}$ and $\log g$ uncertainties $(7000 \pm 200 \mathrm{~K} ; 3.6 \pm 0.5)$; we used a conservative $\log g$ uncertainty, because atmosphere models do not include rotation to calculate an effective gravity. Also plotted (green dots) are frequency ratios (e.g. $f_{1 / 4}$ indicates $f_{1} / f_{4}$ ) that fall within $0.772 \pm 0.009$ (see the text). Numbers in brackets in the legend denote the value of $\alpha_{\mathrm{MLT}}$ and the overshooting parameter, respectively.

the observed frequencies had masses in the range $1.50-1.53 \mathrm{M}_{\odot}$, which are very similar to the TDC-predicted masses. The best-fitting rotating model identifies $f_{1}$ as the fundamental radial mode, and the quintuplet as components of a mixed $(n, \ell)=(-1,3)$ mode. The parameters of this model are displayed in Table 6.

That the quintuplet is identified as a mixed mode has strong implications. It directly affects the Ledoux constant, $C_{L}$, depending on how much g-mode character such a mode has. For lower radial orders, $C_{L}$ can exceed 0.1 for $\ell=2$ modes. Therefore, we had to search a range of low frequencies to account for this factor in Section 4.4, but still no peak due to rotation was found up to twice the expected value (i.e. between 0.218 and $0.436 \mathrm{~d}^{-1}$ ).

The asymmetry of the quintuplet gives a proportionality constant, $D_{L}$, for the second-order effect (centrifugal force) of rotational splitting, which we can use as a consistency check. For moderately rotating $\delta$ Sct stars, oscillations can be expressed in terms of the perturbation theory as

$\omega=\omega_{0}+\left(C_{L}-1\right) m \Omega+D_{L} \frac{m^{2} \Omega^{2}}{\omega_{0}}$,

which describes the effects of rotation up to second order (adapted from Dziembowski \& Goode 1992), for a rotation frequency, $\Omega$, and observed and intrinsic (unperturbed) pulsation frequencies, $\omega$ and $\omega_{0}$, respectively. Under the assumption that the quintuplet is actually an $\ell=2$ mode, one gets $D_{L}=2.45$ in the case of $C_{L}=$ 0 , or $D_{L}=1.8$ for $C_{L}=0.12$. These are about the expected values for an $(n, \ell)=(2,2)$ mode. (See Dziembowski \& Goode 1992 for the example values.) The Q-value of an $(n, \ell)=(2,2)$ mode at the observed quintuplet frequencies is consistent with the assumption that the lowest independent frequency is near the radial fundamental mode.

Thus, we have the following scenario: the best rotating model indicates that $f_{1}$ is the fundamental radial mode, and inferences on the Q-value of the quintuplet if it is an $(n, \ell)=(2,2)$ mode, would agree with that identification for $f_{1}$. However, the model predicts that the quintuplet is part of an $\ell=3$ mode instead. This is only an apparent disagreement. First, that an $(n, \ell)=(2,2)$ mode can generate the observed frequency quintuplet does not make its identification as part of an $\ell=3$ septuplet impossible, as the expected rotational splitting asymmetry of such a mode is expected to be quite similar. Secondly, differential rotation in the radial direction is another possible explanation. It has been proven that the oscillations of low-order $\mathrm{p}$ and $\mathrm{g}$ modes are highly sensitive to variations of the rotation profile near the core (Suárez et al. 2006a). Further attempts at direct mode identification, through multicolour photometry, and further constraints for the models from high-S/N, high-resolution spectroscopy are required for further investigation.

\section{CONCLUSIONS}

KIC 11754974 is a cool, high-amplitude $\delta$ Sct star with a large number of combination frequencies that show a predictable pattern at high frequency. We discovered from the pulsations that the star is in a 343-d binary system; our seismic models show the primary (pulsating) member is $1.53 \mathrm{M}_{\odot}$, making the secondary $0.63 \mathrm{M}_{\odot}$ for a model-determined $i=47^{\circ}$. No direct contribution from the secondary is seen in the spectrum or the light curve.

Compared to most A-type stars, KIC 11754974 is a slow rotator, which is typical of HADS stars, and allowed us to construct perturbative rotating models. The models indicate the star is metal poor, in that solar-metallicity models were a poor fit to the observed frequencies. This agrees with literature classifications that this is a Population II (SX Phe) star, and argues against a surface abundance anomaly model like the Am stars or $\lambda$ Boo stars.

The location of this star on the rotating Petersen diagram argues against more than one of the high-amplitude $f_{1}, \ldots, f_{5}$ modes being radial. We cannot confirm whether the quintuplet found in the data is rotationally split - direct mode identification is required for this.

Combination frequencies are present all across the spectrum, arising from five independent modes that are all confined to a small frequency region. Outside the frequency region $16<f<$ $25 \mathrm{~d}^{-1}$ independent modes with amplitudes above $500 \mu \mathrm{mag}$ are non-existent, and most frequencies are combinations of some form.

The star has a lot of asteroseismic potential, being near the rededge of the $\delta$ Sct instability strip where the pulsation-convection interaction is under study, being a Population II star with precise and sensitive photometry, and being a high-amplitude pulsator for which mode interaction is also particularly important. Here, we have

Table 6. Characteristics of the best representative rotating model. The first six columns have their usual meanings, then headings are mean density, equatorial rotation velocity, the ratio of the equatorial rotation velocity to the keplerian orbital velocity at the stellar surface, inclination of the stellar rotation axis to the line of sight, fundamental radial mode frequency $f(F 0)$, first overtone radial mode frequency $f(F 1)$, the base-10 logarithm of the period of $F 0$, and the period ratio of $F 1$ and $F 0$. The model has metallicity $[\mathrm{Fe} / \mathrm{H}]=-0.52$, convective efficiency, $\alpha_{\mathrm{MLT}}=0.5$ and overshooting $d_{\mathrm{ov}}=0.3$. See Section 5.2 for model selection criteria

\begin{tabular}{cccccccccccc}
\hline $\begin{array}{c}\text { Mass } \\
\left(\mathrm{M}_{\odot}\right)\end{array}$ & $\begin{array}{c}\text { Radius } \\
\left(\mathrm{R}_{\odot}\right)\end{array}$ & $\begin{array}{c}T_{\text {eff }} \\
(\mathrm{K})\end{array}$ & $\begin{array}{c}\text { Age } \\
(\mathrm{Myr})\end{array}$ & $\begin{array}{c}\log g \\
(\mathrm{cgs})\end{array}$ & $\bar{\rho}$ & $\begin{array}{c}v_{\text {eq }} \\
\left(\mathrm{km} \mathrm{s}^{-1}\right)\end{array}$ & $\begin{array}{c}i \\
(\mathrm{deg})\end{array}$ & $\begin{array}{c}f(F 0) \\
\left(\mathrm{d}^{-1}\right)\end{array}$ & $\begin{array}{c}f(F 1) \\
\left(\mathrm{d}^{-1}\right)\end{array}$ & $\log P(F 0)$ & $P(F 1) / P(F 0)$ \\
\hline 1.53 & 1.764 & 7256 & 1465.93 & 4.129 & 0.392 & 34.18 & $47_{-15}^{+7}$ & 16.327 & 21.1 & -1.213 & 0.773 \\
\hline
\end{tabular}


scratched the surface with our models, but there is great potential to learn much more. We intend to study the star further, with multicolour photometry and higher resolution spectroscopy to identify the pulsation modes and constrain the atmospheric parameters. Despite being faint, the star is a promising target for learning about mode selection in these pulsators.

\section{ACKNOWLEDGEMENTS}

This paper includes data collected by the Kepler mission. Funding for the Kepler mission is provided by the NASA Science Mission directorate. The paper also includes observations made with the NOT, operated jointly by Denmark, Finland, Iceland, Norway and Sweden, on the island of La Palma at the Spanish Observatorio del Roque de los Muchachos of the Instituto de Astrofísica de Canarias (IAC). SJM would like to acknowledge the financial support of the STFC, and from the projects FCOMP-01-0124FEDER-009292 and PTDC/CTE-AST/098754/2008 under grant CAUP-09/2012-BI. AP acknowledges the support from the NCN grant No. 2011/03/B/ST9/02667. Some calculations have been carried out in Wrocław Centre for Networking and Supercomputing (http://www.wcss.wroc.pl), grant No. 219. JCS acknowledges support by Spanish National Research Plan through grants ESP201020982-C02-01 and AYA2010-12030-E. KU acknowledges financial support by the Spanish National Plan of R\&D for 2010, project AYA2010-17803. RS was supported by the János Bolyai Research Scholarship, the 'Lendület-2009 Young Researchers' Programme of the Hungarian Academy of Sciences, the HUMAN MB08C 81013 grant of the MAG Zrt, the Hungarian OTKA grant K83790 and the European Community's Seventh Framework Programme (FP7/2007-2013) under grant agreement no. 269194 (IRSES/ASK). AOT acknowledges support from Sonderforschungsbereich SFB 881 'The Milky Way System' (subproject A5) of the German Research Foundation (DFG). GH is thankful for support by the NCN grant 2011/01/B/ST9/05448.

\section{REFERENCES}

Anders E., Grevesse N., 1989, Geochim. Cosmochim. Acta, 53, 197

Balona L. A., 2011, MNRAS, 415, 1691

Balona L. A., 2012, MNRAS, 422, 1092

Balona L. A., Nemec J. M., 2012, MNRAS, 426, 2413

Balona L. A., Guzik J. A., Uytterhoeven K., Smith J. C., Tenenbaum P., Twicken J. D., 2011, MNRAS, 415, 3531

Blackwell D. E., Shallis M. J., 1977, MNRAS, 180, 177

Böhm-Vitense E., 1958, Z. Astrophys., 46, 108

Breger M., Bregman J. N., 1975, ApJ, 200, 343

Breger M. et al., 2005, A\&A, 435, 955

Breger M., Davis K. A., Dukes R. J., 2008, Commun. Asteroseismol., 153, 63

Breger M. et al., 2011, MNRAS, 414, 1721

Brown T. M., Latham D. W., Everett M. E., Esquerdo G. A., 2011, AJ, 142, 112

Buchler J. R., Goupil M., Hansen C. J., 1997, A\&A, 321, 159

Casas R., Suárez J. C., Moya A., Garrido R., 2006, A\&A, 455, 1019

Castanheira B. G., Breger M., Beck P. G., Elmasli A., Lenz P., Falcon R. E., 2008, Commun. Asteroseismol., 157, 124

Dupret M. A., 2001, A\&A, 366, 166

Dupret M.-A., Grigahcène A., Garrido R., Gabriel M., Scuflaire R., 2004, A\&A, 414, L17

Dupret M.-A., Grigahcène A., Garrido R., De Ridder J., Scuflaire R., Gabriel M., 2005, MNRAS, 361, 476

Dziembowski W. A., Goode P. R., 1992, ApJ, 394, 670
Eastman J., Siverd R., Gaudi B. S., 2010, PASP, 122, 935

Gabriel M., 1996, Bull. Astron. Soc. India, 24, 233

Goupil M.-J., Dupret M. A., Samadi R., Boehm T., Alecian E., Suarez J. C., Lebreton Y., Catala C., 2005, JA\&A, 26, 249

Grigahcène A., Dupret M.-A., Gabriel M., Garrido R., Scuflaire R., 2005, A\&A, 434, 1055

Guzik J. A., Breger M., 2011, Commun. Asteroseismol., 162, 62

Guzik J. A., Bradley P. A., Templeton M. R., 2000, in Breger M., Montgomery M., eds, ASP Conf. Ser. Vol. 210, Delta Scuti and Related Stars. Astron. Soc. Pac., San Francisco, p. 247

Heiter U. et al., 2002, A\&A, 392, 619

Koch D. G. et al., 2010, ApJ, 713, L79

Kupka F., Piskunov N., Ryabchikova T. A., Stempels H. C., Weiss W. W., 1999, A\&AS, 138, 119

Lenz P., Breger M., 2004, in Zverko J., Ziznovsky J., Adelman S. J., Weiss W. W., eds, Proc. IAU Symp. 224, The A-Star Puzzle. Cambridge Univ. Press, Cambridge, p. 786

Lenz P., Breger M., 2005, Commun. Asteroseismol., 146, 53

Maeder A., Meynet G., 2004, in Maeder A., Eenens P., eds, Proc. IAU Symp. 215, Stellar Rotation. Astron. Soc. Pac., San Francisco, p. 500

Morel P., 1997, A\&AS, 124, 597

Moya A., Amado P. J., Barrado D., Hernández A. G., Aberasturi M., Montesinos B., Aceituno F., 2010, MNRAS, 406, 566

Murphy S. J., 2012a, MNRAS, 422, 665

Murphy S. J., 2012b, Astron. Nachr., 333, 1057

Murphy S. J., Grigahcène A., Niemczura E., Kurtz D. W., Uytterhoeven K., 2012, MNRAS, 427, 1418

Murphy S. J., Shibahashi H., Kurtz D. W., 2013, MNRAS, 430, 2986

Nemec J., Mateo M., 1990, in Cacciari C., Clementini G., eds, ASP Conf. Ser. Vol. 11, Confrontation Between Stellar Pulsation and Evolution. Astron. Soc. Pac., San Francisco, p. 64

Olech A., Dziembowski W. A., Pamyatnykh A. A., Kaluzny J., Pych W., Schwarzenberg-Czerny A., Thompson I. B., 2005, MNRAS, 363, 40

Paunzen E., Iliev I. K., Kamp I., Barzova I. S., 2002, MNRAS, 336, 1030

Paunzen E., Heiter U., Fraga L., Pintado O., 2012, MNRAS, 419, 3604

Petersen J. O., 1978, A\&A, 62, 205

Petersen J. O., Christensen-Dalsgaard J., 1996, A\&A, 312, 463

Pigulski A., Pojmański G., Pilecki B., Szczygieł D. M., 2009, Acta Astron., 59,33

Piskunov N. E., 1992, in Glagolevskij Y. V., Romanyuk I. I., eds, Physics and Evolution of Stars: Stellar Magnetism. Nauka, St. Petersburg, p. 92

Pollacco D. L. et al., 2006, PASP, 118, 1407

Poretti E., 2003, A\&A, 409, 1031

Poretti E. et al., 2011, A\&A, 528, A147

Rodríguez E. et al., 2006a, A\&A, 450, 715

Rodríguez E. et al., 2006b, A\&A, 456, 261

Scuflaire R., Théado S., Montalbán J., Miglio A., Bourge P.-O., Godart M., Thoul A., Noels A., 2008, Ap\&SS, 316, 83

Shibahashi H., Kurtz D. W., 2012, MNRAS, 422, 738

Soufi F., Goupil M. J., Dziembowski W. A., 1998, A\&A, 334, 911

Suárez J. C., 2002, PhD thesis, Observatoire de Paris

Suárez J. C., Goupil M. J., 2008, Ap\&SS, 316, 155

Suárez J. C., Garrido R., Goupil M. J., 2006a, A\&A, 447, 649

Suárez J. C., Goupil M. J., Morel P., 2006b, A\&A, 449, 673

Suárez J. C., Garrido R., Moya A., 2007, A\&A, 474, 961

Suárez J.-C., Michel E., Pérez Hernández F., Lebreton Y., Li Z. P., Fox Machado L., 2002, A\&A, 390, 523

Suárez J. C., Moya A., Amado P. J., Martín-Ruiz S., Rodríguez-López C., Garrido R., 2009, ApJ, 690, 1401

Uytterhoeven K. et al., 2011, A\&A, 534, A125

Wu Y., 2001, MNRAS, 323, 248

Zacharias N., Finch C. T., Girard T. M., Henden A., Bartlett J. L., Monet D. G., Zacharias M. I., 2012, VizieR Online Data Catalog, 1322, 0

This paper has been typeset from a $\mathrm{T}_{\mathrm{E}} \mathrm{X} / \mathrm{L} \mathrm{T} \mathrm{E} \mathrm{X}$ file prepared by the author. 


\section{Chapter 6}

\section{Conclusions and Future Work}

\section{Abstract}

This chapter focusses on the main conclusions of the thesis and the future prospects thereof. More detailed conclusions can be found at the end of each chapter.

\subsection{Conclusions}

A large portion of this thesis is an investigation into the characteristics of Kepler data using Fourier transforms. It was shown that SC data offer greater precision than their LC counterparts, and were less noisy at low frequency under the PDC-LS pipeline. That pipeline also injected noise into about 15 per cent of light curves. Now, PDC-MAP is the pipeline in use, but does not perform as well as the PDC-LS pipeline did at removing instrumental trends. A new version of the PDC pipeline, "multi-scale MAP", has just been implemented as this thesis is being submitted.

Modification of otherwise equally spaced time stamps on Kepler data, to bring them into the Barycentric Dynamical Time (TDB) reference frame, causes Nyquist aliases to become multiplets separated by the orbital frequency of the satellite, while real frequencies remain as singlets in the Fourier transform. The requirements 


\section{CHAPTER 6}

are continuous data covering more than one Kepler orbit. This finding allows for hundreds of $\delta$ Sct stars to be studied using only LC data, i.e. without the need of precious SC resources to resolve Nyquist ambiguities - there aren't any Nyquist ambiguities.

An algorithm was developed to estimate the noise levels in Kepler data. Analysis of the noise levels was performed for stars of different KIC temperatures and magnitudes. Stars cooler than $7500 \mathrm{~K}$ were found to be noisier, on average, than hotter stars, implying granulation may persist on the surface of the cooler stars. As part of this analysis, the fraction of 'constant' stars between 7000 and $8500 \mathrm{~K}$ was determined to be 44 per cent. The definition of 'constant', as used here, is that no Fourier peak above $5 \mathrm{~d}^{-1}$ exceeds $50 \mu \mathrm{mag}$, thus a 'constant' star is a non- $\delta$ Sct star, in this context.

That so many stars in the $\delta$ Sct instability strip do not pulsate is intriguing. A suggested reason for this is chemical peculiarity - the stars do not pulsate because they are drained of helium in the driving zone. A sample of ten non- $\delta$ Sct stars were tested for peculiarity: six were chemically peculiar, one was not in the instability strip, and two were F1V $\gamma$ Dor stars (but not $\delta$ Sct stars). The one other target, at $\mathrm{A} 7 \mathrm{~V}$, is a non-pulsator right in the middle of the instability strip. At high dispersion one can see this is a double-lined spectroscopic binary, but with no obvious peculiarity at classification dispersion. The binarity complicates the pending abundance analysis, but it appears we have found a chemically normal, non- $\delta$ Sct star in the $\delta$ Sct instability strip. Furthermore, the stars with $\gamma$ Dor pulsations had milder or absent abundance anomalies, despite not showing $\delta$ Sct pulsation, indicating the $\gamma$ Dor phenomenon may interfere with the development of chemical peculiarities.

Models predict pulsation in evolved Am stars, which results in little turbulence as long as the velocity gradients within the star remain small. I argued that this implies pulsations in Am stars should be of low overtone and low degree. I also challenged 


\section{CHAPTER 6}

the number of Am stars that are observed to pulsate in the SuperWASP data, in that many of the Am stars in that SuperWASP sample may well be marginal Am stars, and STEREO data show substantially fewer non-magnetic stars to pulsate at an only slightly higher amplitude detection limit.

The physical process that is ultimately responsible for the development of chemical peculiarity is rotation. Mixing processes such as turbulence and meridional circulation are inefficient in slow rotators, allowing gravitational settling and radiative levitation to separate elements near the stellar surface. Rotation was extensively reviewed in this thesis. The braking mechanisms for the Am and magnetic Ap stars were discussed. The latter are strongly braked in the pre-main sequence phase, but there is no strong evidence for substantial braking on the main sequence. The main braking mechanism for Am stars is tidal braking in close binary systems, but there are show-stoppers - Am stars are also found in wide binaries, where braking is not efficient enough to produce the observed low $v \sin i$, and as single stars. An example of a single Am: star, KIC 3429637, was presented in Chapter 5. Although the development time-scale for Am peculiarities is short $\left(\sim 10^{6} \mathrm{yr}\right)$, it is longer than $10^{8} \mathrm{yr}$ for the Ap SrCrEu stars. There have been attempts in the literature to find the progenitors of the latter objects. An argument in favour of the 'sn' stars being Ap progenitors has been laid out.

Zorec \& Royer (2012) inferred that the rotational velocities of A stars accelerate for the first third of their main-sequence life. This counter-intuitive suggestion is explained as a redistribution of angular momentum; the surface regions of a star are more strongly braked on the pre-main sequence than the core, so that at the ZAMS the core rotates more quickly than the surface. This excess core angular momentum is transferred to the surface, accelerating the surface layers. I have reinvestigated older data of rotational velocities, namely those of Slettebak (1955), Wolff \& Simon (1997), and Erspamer \& North (2003), each of which was found to support the 


\section{CHAPTER 6}

acceleration scenario of Zorec \& Royer (2012).

In the Kepler era, many publications have focussed on broad, statistical overviews of the properties of A stars, particularly of pulsation. If progress is to be made, deeper case studies are required for individual objects, both in observations and modelling those observations. The jump in precision offered by space-based photometry facilitates this, but unification of models that treat important processes in the A stars is lagging. Separately, successful models of rotation, time-dependent convection and elemental mixing have been developed, but progress demands a united approach.

\subsection{Future Work}

The science presented in this thesis has focussed on four topics: Kepler data, rotation, chemical peculiarities and pulsation. In order to understand the link between the latter three physical processes at work, we can seek to maximally utilise the $K e$ pler data. One 'issue' with the Kepler data is that they are so good and so plentiful that one often does not know where to start. How does one sift through the exciting targets to find the really exciting ones - the ones that are the most astrophysically interesting and promising? From light curves or Fourier transforms alone, one cannot isolate, for instance, all of the chemically peculiar pulsators. It would be helpful to know in advance which stars have peculiar spectra before beginning a pulsation analysis. Hence it would be especially useful to obtain classification-dispersion spectra of A stars in the Kepler field, so that one can answer such questions as 'do the $\lambda$ Boo stars have a high incidence of pulsation?' This is something I plan to do: a systematic, classification-resolution survey to facilitate spectroscopic classification of probable A stars (according to KIC temperatures), in order to increase the utility of the Kepler data. Investigating the intriguing prospect of efficient pulsational driving in the $\lambda$ Boo stars will then easily follow. 


\section{CHAPTER 6}

In Chapter 3, the non-pulsating stars were investigated for chemical peculiarities. The stars used in the sample were limited to those that already had high-resolution spectra available, to enable abundance analyses to be carried out. It will be worthwhile extending this project to other non-pulsating stars believed to be in the $\delta$ Sct instability strip. Having a spectral type offers a rapid and reliable determination of peculiarity, and ultimately we would like well-constrained $T_{\text {eff }}$ and $\log g$ values from spectroscopy to ensure the stars are inside the instability strip, with high-resolution spectroscopy also allowing for abundance analyses. Lists of the most constant stars have been created, and I will write proposals to obtain more high-resolution spectra to continue the project. There are still many bright $(V<10)$, non-pulsating stars to study. An observing run for classification-dispersion spectra of fifty of these began at the end of 2013 May.

Many of the non-pulsating stars also showed peaks at low frequency. If these are attributable to rotation, this implies some sort of surface structure that rotates with the star. Objects with these properties include the $\alpha^{2}$ CVn stars - Ap stars with spots that produce light variations according to the orientation of the spots and the rotation axis with respect to the observer. The nature of the spectra obtained to determine $v \sin i$ allows these targets to be spectroscopically classified, and the selection of non-pulsating stars has created a subsample of Kepler A stars that have a high probability to be chemically peculiar. Essentially, this is an efficient method for finding Ap stars in Kepler data, though many of the 'rotational variables' have inferred rotational periods too short to be Ap. The aforementioned 50-star sample was selected to have low-frequency peaks, too.

In understanding the $\delta$ Sct stars, case studies of well-parametrized members of the class are in high demand. Modelling such targets and comparing these models with observations is the way to improve the physics of the models. Some astrophysically interesting objects for follow-up were presented in Chapter 4. These will be pursued 


\section{CHAPTER 6}

in due course, with multicolour photometry observing runs that began in 2013 May.

In Appendix A to Chapter 2, the Tycho 2 spectral type catalogue was used with Hipparcos parallaxes to obtain average absolute magnitudes at each temperature class for luminosity class $\mathrm{V}$ objects. Lack of uniformity arises using spectral types from multiple sources, so a reanalysis using a single source could be performed. However, this will further reduce the number of stars included to an undesirably low number. Instead of working on spectral types, the observations could be extended by working in $B-V$ colour. Tycho $B$ and $V$ photometry is precise, and related to Johnson photometry by $(B-V)_{J}=0.850\left(B_{T}-V_{T}\right)$. The number of stars is then limited to those with parallaxes rather than the smaller sample of those that have spectral types. This will allow a definition of the main-sequence like those done in the seventies by Crawford $(1975,1978,1979)$, especially when Gaia comes online.

The Tycho 2 data and other online catalogues also allow an efficient search for $\lambda$ Boo stars. One can select $B-V$ values, taking reddening into account, that correspond to A stars. Combining this with spectral type information or, even better, Strömgren photometry, one can generate a target list of A stars that are much more likely to be $\lambda$ Boo stars than 2 per cent - their fraction of field stars. This has already been done by Paunzen \& Gray (1997), but combining Tycho $B-V$ with other literature photometry and classification spectra is allowing a search for more $\lambda$ Boo stars down to $B=10$ that I am already involved in with Gray and others. Furthermore, the former technique is limited in that few surveys continue to observe stars in Strömgren filters. The future lies in Sloan photometry, but the filters do not have the resolution of the Strömgren ones, and thus equivalents of the $m_{1}$ and $c_{1}$ indices, which are useful for classification of peculiar stars, are not easy to replicate. Constructing and calibrating such indices with Sloan data would be of great use to the astronomy community but also very time-consuming. The resolution issue may be overcome by data from SkyMapper, which is a wide field survey telescope using 


\section{CHAPTER 6}

Sloan filters, plus an additional filter, $v_{s}$. The $v_{s}$ filter is a few hundred Ångströms wide and centred at the CaII K line, making it a very useful addition for seeking out chemically peculiar A stars.

Finally, the hypothesis that the 'sn' stars are proto-Ap stars can be evaluated by searching for magnetic fields in these objects. An observing proposal is being prepared to this end, from which we may finally learn about the nature of the Ap SrCrEu stars before the $\sim 200 \mathrm{Myr}$ (Abt, 2009) that it takes to develop their abundance anomalies. 


\section{Bibliography}

Abt H. A., 1961, ApJS, 6, 37

—, 1967, in Magnetic and Related Stars, R. C. Cameron, ed., pp. 173-+

- 1979, ApJ, 230, 485

—, 2004, in IAU Symposium, Vol. 215, Stellar Rotation, A. Maeder \& P. Eenens, ed., ASP, pp. $154-+$

一, 2009, AJ, 138, 28

Abt H. A., Boonyarak C., 2004, ApJ, 616, 562

Abt H. A., Hunter, Jr. J. H., 1962, ApJ, 136, 381

Abt H. A., Levato H., Grosso M., 2002, ApJ, 573, 359

Abt H. A., Levy S. G., 1978, ApJS, 36, 241

Abt H. A., Morgan W. W., 1976, ApJ, 205, 446

Abt H. A., Morrell N. I., 1995, ApJS, 99, 135

Abt H. A., Willmarth D., 2006, ApJS, 162, 207

Adelman S. J., 2004, in IAU Symposium, Vol. 224, The A-Star Puzzle, Zverko J., Ziznovsky J., Adelman S. J., Weiss W. W., eds., pp. 1-11

Aerts C., Christensen-Dalsgaard J., Kurtz D. W., 2010, Asteroseismology, Aerts, C., Christensen-Dalsgaard, J., \& Kurtz, D. W., ed. Springer

Aizenman M., Smeyers P., Weigert A., 1977, A\&A, 58, 41

Allen C. W., 1973, Astrophysical quantities. Athlone Press

Amado P. J., Moya A., Suárez J. C., Martín-Ruiz S., Garrido R., Rodríguez E., Catala C., Goupil M. J., 2004, MNRAS, 352, L11

Andreasen G. K., Hejlesen P. M., Petersen J. O., 1983, A\&A, 121, 241

Andrievsky S. M., 1997, A\&A, 321, 838

Antoci V., Breger M., Rodler F., Bischof K., Garrido R., 2007, A\&A, 463, 225 
Asplund M., Nordlund Å., Trampedach R., Allende Prieto C., Stein R. F., 2000, A\&A, 359,729

Babcock H. W., 1949, The Observatory, 69, 191

Baglin A., Breger M., Chevalier C., Hauck B., Le Contel J. M., Sareyan J. P., Valtier J. C., 1973, A\&A, 23, 221

Ballard S. et al., 2011, ApJ, 743, 200

Bally J., 2008, Overview of the Orion Complex, Reipurth B., ed., ASP, p. 459

Balona L. A., 2011, MNRAS, 415, 1691

—, 2012a, MNRAS, 422, 1092

—, 2012b, MNRAS, 3069

—, 2013, MNRAS, 431, 2240

Balona L. A. et al., 2010, MNRAS, 1549

Balona L. A., Dziembowski W. A., 1999, MNRAS, 309, 221

—, 2011, MNRAS, 417, 591

Balona L. A., Evers E. A., 1999, MNRAS, 302, 349

Balona L. A., Guzik J. A., Uytterhoeven K., Smith J. C., Tenenbaum P., Twicken J. D., 2011, MNRAS, 415, 3531

Balona L. A., Hearnshaw J. B., Koen C., Collier A., Machi I., Mkhosi M., Steenberg C., 1994, MNRAS, 267, 103

Balona L. A., Krisciunas K., Cousins A. W. J., 1994, MNRAS, 270, 905

Balona L. A., Nemec J. M., 2012, MNRAS, 426, 2413

Balona L. A., Stobie R. S., 1979, MNRAS, 189, 649

Barry D. C., 1970, ApJS, 19, 281

Bedding T. R., Kjeldsen H., Butler R. P., McCarthy C., Marcy G. W., O'Toole S. J., Tinney C. G., Wright J. T., 2004, ApJ, 614, 380

Bessell M. S., 1979, PASP, 91, 589

Blomme J. et al., 2010, ApJ, 713, L204

Boesgaard A. M., Friel E. D., 1990, ApJ, 351, 467

Bohlender D. A., Landstreet J. D., 1990, MNRAS, 247, 606

Böhm-Vitense E., 1958, ZAp, 46, 108

Borucki W. J. et al., 2010, Science, 327, 977 
—, 2011, ApJ, 736, 19

Braithwaite J., 2008, Contributions of the Astronomical Observatory Skalnate Pleso, 38, 179

Braithwaite J. et al., 2010, Highlights of Astronomy, 15, 161

Breger M., 1970, ApJ, 162, 597

—, 2000a, in Astronomical Society of the Pacific Conference Series, Vol. 203, IAU Colloq. 176: The Impact of Large-Scale Surveys on Pulsating Star Research, L. Szabados \& D. Kurtz, ed., ASP Conference Series, pp. 421-425

—, 2000b, in Astronomical Society of the Pacific Conference Series, Vol. 210, Delta Scuti and Related Stars, M. Breger \& M. Montgomery, ed., pp. 3-+

—, 2000c, Delta Scuti Star Newsletter, 14, 8

—, 2009, in American Institute of Physics Conference Series, Vol. 1170, American Institute of Physics Conference Series, J. A. Guzik \& P. A. Bradley, ed., pp. 410-414

Breger M. et al., 2011, MNRAS, 414, 1721

一, 2012, ApJ, 759, 62

一, 1999a, A\&A, 349, 225

Breger M., Kolenberg K., 2006, A\&A, 460, 167

Breger M., Lenz P., 2008, A\&A, 488, 643

Breger M. et al., 2005, A\&A, 435, 955

Breger M., Lenz P., Pamyatnykh A. A., 2009, MNRAS, 396, 291

Breger M., Pamyatnykh A. A., 2006, MNRAS, 368, 571

Breger M., Pamyatnykh A. A., Pikall H., Garrido R., 1999b, A\&A, 341, 151

Breger M. et al., 2004, A\&A, 419, 695

Buchler J. R., Goupil M., Hansen C. J., 1997, A\&A, 321, 159

Budaj J., 1996, A\&A, 313, 523

Buzasi D. L., 2000, in The Third MONS Workshop: Science Preparation and Target Selection, Teixeira T., Bedding T., eds., p. 9

Carrera R., Pancino E., 2011, A\&A, 535, A30

Casas R., Moya A., Suárez J. C., Martín-Ruíz S., Amado P. J., Rodríguez-López C., Garrido R., 2009, ApJ, 697, 522

Casas R., Suárez J. C., Moya A., Garrido R., 2006, A\&A, 455, 1019

Castanheira B. G., Breger M., Beck P. G., Elmasli A., Lenz P., Falcon R. E., 2008, Communications in Asteroseismology, 157, 124 
Castelli F., Kurucz R. L., 2004, ArXiv Astrophysics e-prints

Catanzaro G., Balona L. A., 2012, MNRAS, 421, 1222

Catanzaro G., Frasca A., Molenda-Żakowicz J., Marilli E., 2010, A\&A, 517, A3

Chambers J. E., 2010, ApJ, 724, 92

Charbonneau P., 1992, JRASC, 86, 31

—, 1993, in Astronomical Society of the Pacific Conference Series, Vol. 44, IAU Colloq. 138: Peculiar versus Normal Phenomena in A-type and Related Stars, Dworetsky M. M., Castelli F., Faraggiana R., eds., p. 474

Christiansen J. et al., 2012, Kepler Data Characteristics Handbook

Conti P. S., 1965, ApJ, 142, 1594

Coupry M. F., Burkhart C., 1992, A\&AS, 95, 41

Cousins A. W. J., 1992, The Observatory, 112, 53

—, 1994, The Observatory, 114, 51

Crawford D. L., 1975, AJ, 80, 955

-, 1978, AJ, 83, 48

-, 1979, AJ, 84, 1858

Daszyńska-Daszkiewicz J., Dziembowski W. A., Pamyatnykh A. A., 2003, A\&A, 407, 999

—, 2006, Mem. Soc. Astron. Italiana, 77, 113

Daszynska-Daszkiewicz J., Dziembowski W. A., Pamyatnykh A. A., Breger M., Zima W., 2004, in IAU Symposium, Vol. 224, The A-Star Puzzle, Zverko J., Ziznovsky J., Adelman S. J., Weiss W. W., eds., pp. 853-859

Daszyńska-Daszkiewicz J., Dziembowski W. A., Pamyatnykh A. A., Breger M., Zima W., Houdek G., 2005, A\&A, 438, 653

Daszyńska-Daszkiewicz J., Dziembowski W. A., Pamyatnykh A. A., Goupil M.-J., 2002, A\&A, 392, 151

de Meulenaer P., Carrier F., Miglio A., Bedding T. R., Campante T. L., Eggenberger P., Kjeldsen H., Montalbán J., 2010, A\&A, 523, A54

Debernardi Y., 2000, in IAU Symposium, Vol. 200, IAU Symposium on The Formation of Binary Stars, B. Reipurth \& H. Zinnecker, ed., ASP Conference Series, p. 161

Debernardi Y., Mermilliod J.-C., Carquillat J.-M., Ginestet N., 2000, A\&A, 354, 881

Deeming T. J., 1975, Ap\&SS, 36, 137

Degroote P. et al., 2011, A\&A, 536, A82

- 2009, A\&A, 506, 111 
Deutsch A. J., 1956, PASP, 68, 92

Dickens R. J., 1968, Astrophys. Lett., 1, 213

Dupret M., Grigahcène A., Garrido R., Gabriel M., Scuflaire R., 2005, A\&A, 435, 927

Dupret M.-A., Grigahcène A., Garrido R., Gabriel M., Scuflaire R., 2004, A\&A, 414, L17

Dziembowski W., 1977a, Acta Astron., 27, 203

—, 1977b, Acta Astron., 27, 95

Dziembowski W., Krolikowska M., 1985, Acta Astron., 35, 5

—, 1990, Acta Astron., 40, 19

Dziembowski W., Krolikowska M., Kosovichev A., 1988, Acta Astron., 38, 61

Dziembowski W. A., Goode P. R., 1992, ApJ, 394, 670

Eddington A. S., 1917, The Observatory, 40, 290

Erspamer D., North P., 2003, A\&A, 398, 1121

Faraggiana R., Bonifacio P., 1999, A\&A, 349, 521

-, 2005, A\&A, 436, 697

Faraggiana R., Bonifacio P., Caffau E., Gerbaldi M., Nonino M., 2004, A\&A, 425, 615

Fernie J. D., 1982, JRASC, 76, 319

Fitzgerald M. P., 1970, A\&A, 4, 234

Fossati L., Bagnulo S., Landstreet J., Wade G., Kochukhov O., Monier R., Weiss W., Gebran M., 2008a, A\&A, 483, 891

Fossati L., Bagnulo S., Monier R., Khan S. A., Kochukhov O., Landstreet J., Wade G., Weiss W., 2007, A\&A, 476, 911

Fossati L., Kolenberg K., Reegen P., Weiss W., 2008b, A\&A, 485, 257

García R. A. et al., 2011, MNRAS, 414, L6

García Hernández A. et al., 2009, A\&A, 506, 79

Garg A. et al., 2010, AJ, 140, 328

Garrido R., Garcia-Lobo E., Rodriguez E., 1990, A\&A, 234, 262

Gebran M., Monier R., 2007, in IAU Symposium, Vol. 239, Convection in Astrophysics, F. Kupka, I. Roxburgh \& K. Chan, ed., CUP, pp. 160-162

Gebran M., Monier R., Richard O., 2008, A\&A, 479, 189

Gebran M., Vick M., Monier R., Fossati L., 2010, A\&A, 523, A71 
Gerbaldi M., Faraggiana R., 2004, in Astronomical Society of the Pacific Conference Series, Vol. 318, Spectroscopically and Spatially Resolving the Components of the Close Binary

Stars, Hilditch R. W., Hensberge H., Pavlovski K., eds., pp. 312-315

Gerbaldi M., Faraggiana R., Lai O., 2003, A\&A, 412, 447

Gilliland R. L. et al., 2011, ApJS, 197, 6

—, 2010, ApJ, 713, L160

Glebocki R., Gnacinski P., Stawikowski A., 2000, Acta Astron., 50, 509

Gomez A. E., Grenier S., Jaschek M., Jaschek C., Heck A., 1981, A\&A, 93, 155

Gomez A. E., Luri X., Sabas V., Grenier S., Figueras F., North P., Torra J., Mennessier M. O., 1998, Contributions of the Astronomical Observatory Skalnate Pleso, 27, 171

González Hernández J. I., Delgado Mena E., Israelian G., Sousa S. G., Santos N. C., Udry S., 2012, in IAU Symposium, Vol. 282, IAU Symposium, Richards M. T., Hubeny I., eds., pp. $480-481$

González Hernández J. I., Israelian G., Santos N. C., Sousa S., Delgado-Mena E., Neves V., Udry S., 2010, ApJ, 720, 1592

Goupil M.-J., Dupret M. A., Samadi R., Boehm T., Alecian E., Suarez J. C., Lebreton Y., Catala C., 2005, Journal of Astrophysics and Astronomy, 26, 249

Gray D. F., Desikachary K., 1973, ApJ, 181, 523

Gray R. O., 1988, AJ, 95, 220

—, 1999, SPECTRUM: A stellar spectral synthesis program. Astrophysics Source Code Library

Gray R. O., Corbally, J. C., 2009, Stellar Spectral Classification. Princeton University Press

Gray R. O., Corbally C. J., 2002, AJ, 124, 989

Gray R. O., Garrison R. F., 1987, ApJS, 65, 581

—, 1989a, ApJS, 69, 301

-, 1989b, ApJS, 70, 623

Gray R. O., Graham P. W., Hoyt S. R., 2001, AJ, 121, 2159

Griffin R. E., Gray R. O., Corbally C. J., 2012, A\&A, 547, A8

Grigahcène A. et al., 2010, ApJ, 713, L192

Grigahcène A., Dupret M. A., Garrido R., Gabriel M., 2006, Mem. Soc. Astron. Italiana, 77,129

Guzik J. A., Bradley P. A., Templeton M. R., 2000, in Astronomical Society of the Pacific Conference Series, Vol. 210, Delta Scuti and Related Stars, Breger M., Montgomery M., eds., p. 247 
Guzik J. A., Breger M., 2011, Communications in Asteroseismology, 162, 62

Guzik J. A., Templeton M. R., Bradley P. A., 1998, in Astronomical Society of the Pacific Conference Series, Vol. 135, A Half Century of Stellar Pulsation Interpretation, Bradley P. A., Guzik J. A., eds., ASP Conference Series, p. 470

Handler G., 2005, Journal of Astrophysics and Astronomy, 26, 241

—, 2009, in American Institute of Physics Conference Series, Vol. 1170, American Institute of Physics Conference Series, Guzik J. A., Bradley P. A., eds., pp. 403-409

—, 2012, in Astronomical Society of the Pacific Conference Series, Vol. 462, Progress in Solar/Stellar Physics with Helio- and Asteroseismology, Shibahashi H., Takata M., Lynas-Gray A. E., eds., p. 111

Handler G. et al., 1997, MNRAS, 286, 303

Handler G., Shobbrook R. R., 2002, MNRAS, 333, 251

Hansen C. J., Cox J. P., Carroll B. W., 1978, ApJ, 226, 210

Hareter M., Fossati L., Weiss W., Suárez J. C., Uytterhoeven K., Rainer M., Poretti E., 2011, ApJ, 743, 153

Hareter M. et al., 2010, ArXiv e-prints

Hartoog M. R., 1977, ApJ, 212, 723

Hauck B., Mermilliod M., 1998, A\&AS, 129, 431

Hauck B., Slettebak A., 1983, A\&A, 127, 231

Heiter U., 2002, A\&A, 381, 959

Heiter U., Weiss W. W., Paunzen E., 2002, A\&A, 381, 971

Hilditch R. W., 2001, An Introduction to Close Binary Stars. CUP

Hill G. M., 1995, A\&A, 294, 536

Hill G. M., Landstreet J. D., 1993, A\&A, 276, 142

Hogg D. W., Bovy J., Lang D., 2010, ArXiv e-prints

Holman M. J., Murray N. W., 2005, Science, 307, 1288

Holmberg J., Nordström B., Andersen J., 2009, A\&A, 501, 941

Houk N., 1978, Michigan catalogue of two-dimensional spectral types for the HD stars. University of Michigan

—, 1982, Michigan Catalogue of Two-dimensional Spectral Types for the HD stars. Volume_3. Declinations $-40^{\circ}$ to $-26^{\circ}$. University of Michigan

Houk N., Cowley A. P., 1975, University of Michigan Catalogue of two-dimensional spectral types for the HD stars. Volume I. Declinations $-90_{-}$to $-53^{\circ}$. University of Michigan 
Houk N., Smith-Moore M., 1988, Michigan Catalogue of Two-dimensional Spectral Types for the HD Stars. Volume 4, Declinations $-26^{\circ}$ to $-12^{\circ}$. University of Michigan

Houk N., Swift C., 1999, Michigan catalogue of two-dimensional spectral types for the HD Stars ; vol. 5. University of Michigan

Huber D., Stello D., Bedding T. R., Chaplin W. J., Arentoft T., Quirion P.-O., Kjeldsen H., 2009, Communications in Asteroseismology, 160, 74

Hubrig S., North P., Mathys G., 2000, ApJ, 539, 352

Hubrig S., North P., Medici A., 2000, A\&A, 359, 306

Hubrig S., North P., Schöller M., 2007, Astronomische Nachrichten, 328, 475

Hubrig S., North P., Schöller M., Mathys G., 2006, Astronomische Nachrichten, 327, 289

Iliev I. K., Budaj J., Zverko J., Barzova I. S., Ziznovsky J., 1998, A\&AS, 128, 497

Iliev I. K., Paunzen E., Barzova I. S., Griffin R. F., Kamp I., Claret A., Koen C., 2002, A\&A, 381, 914

Irwin J., Bouvier J., 2009, in IAU Symposium, Vol. 258, IAU Symposium, Mamajek E. E., Soderblom D. R., Wyse R. F. G., eds., CUP, pp. 363-374

Jaschek C., Conde H., de Sierra A. C., 1964, Observatory Astronomical La Plata Series Astronomies, 28, 1

Jaschek C., Gomez A. E., 1998, A\&A, 330, 619

Jenkins J. M. et al., 2010, ApJ, 713, L120

Joshi S., Mary D. L., Martinez P., Kurtz D. W., Girish V., Seetha S., Sagar R., Ashoka B. N., 2006, A\&A, 455, 303

Kallinger T., Matthews J. M., 2010, ApJ, 711, L35

Kamp I., Paunzen E., 2002, MNRAS, 335, L45

Kaye A. B., Handler G., Krisciunas K., Poretti E., Zerbi F. M., 1999, PASP, 111, 840

Kennedy P. M., 1983, MK Classication Catalog Extension. Weston Creek: Mt. Stromlo \& Siding Spring Obs.

Kennelly E. J. et al., 1998, ApJ, 495, 440

King J. R., 1994, MNRAS, 269, 209

Koch D. G. et al., 2010, ApJ, 713, L79

Kochukhov O., Bagnulo S., 2006, A\&A, 450, 763

Kogure T., 1981, PASJ, 33, 399

Kouwenhoven M. B. N., Brown A. G. A., Zinnecker H., Kaper L., Portegies Zwart S. F., 2005, A\&A, 430, 137 
Kraft R. P., 1967, ApJ, 150, 551

Kraus A. L., Hillenbrand L. A., 2007, AJ, 134, 2340

Krisciunas K., 1993, in Bulletin of the American Astronomical Society, Vol. 25, American Astronomical Society Meeting Abstracts, p. 1422

Kumar P., Ao C. O., Quataert E. J., 1995, ApJ, 449, 294

Kurtz D. W., 1976, ApJS, 32, 651

—, 1978a, Information Bulletin on Variable Stars, 1436, 1

—, 1978b, ApJ, 221, 869

-, 1981, MNRAS, 196, 61

-, 1982, MNRAS, 200, 807

—, 1989, MNRAS, 238, 1077

-, 2000, in Astronomical Society of the Pacific Conference Series, Vol. 210, Delta Scuti and Related Stars, M. Breger \& M. Montgomery, ed., p. 287

Kurtz D. W., Breger M., Evans S. W., Sandmann W. H., 1976, ApJ, 207, 181

Kurtz D. W., Garrison R. F., Koen C., Hofmann G. F., Viranna N. B., 1995, MNRAS, 276,199

Landstreet J. D., Barker P. K., Bohlender D. A., Jewison M. S., 1989, ApJ, 344, 876

Landstreet J. D., Kupka F., Ford H. A., Officer T., Sigut T. A. A., Silaj J., Strasser S., Townshend A., 2009, A\&A, 503, 973

Landstreet J. D., Mathys G., 2000, A\&A, 359, 213

Lane M. C., 1981, PhD thesis, University of Toronto, Canada

Langer N., 2009, A\&A, 500, 133

Ledoux P., 1951, ApJ, 114, 373

Lee Y., Kim S. S., Shin J., Lee J., Jin H., 2008, PASJ, 60, 551

Lenz P., Daszyńska-Daszkiewicz J., Pamyatnykh A. A., Breger M., 2008a, Communications in Asteroseismology, 153, 40

Lenz P., Pamyatnykh A. A., Breger M., Antoci V., 2008b, A\&A, 478, 855

Lenz P., Pamyatnykh A. A., Zdravkov T., Breger M., 2010, A\&A, 509, A90

Leroy J. L., Bagnulo S., Landolfi M., Landi Degl'Innocenti E., 1994, A\&A, 284, 174

Li Y., 2000, ApJ, 538, 346

Lignières F., Georgeot B., 2009, A\&A, 500, 1173 
Mantegazza L., Poretti E., 2002, A\&A, 396, 911

Mantegazza L., Poretti E., Bossi M., 1994, A\&A, 287, 95

Marcy G. W., Benitz K. J., 1989, ApJ, 344, 441

Martinez P., Kurtz D. W., Hoffman M. J. H., van Wyk F., 1995, MNRAS, 276, 1435

Martínez-Galarza J. R., Kamp I., Su K. Y. L., Gáspár A., Rieke G., Mamajek E. E., 2009, ApJ, 694, 165

Mason B. D., Gies D. R., Hartkopf W. I., Bagnuolo, Jr. W. G., ten Brummelaar T., McAlister H. A., 1998, AJ, 115, 821

Mathys G., 2004, in IAU Symposium, Vol. 215, Stellar Rotation, Maeder A., Eenens P., eds., ASP, p. 270

Mathys G., Hubrig S., Landstreet J. D., Lanz T., Manfroid J., 1997, A\&AS, 123, 353

Mazeh T., Tamuz O., North P., 2006, Ap\&SS, 304, 343

McGahee C. E., 2010, Master's thesis, Appalachian State University

McNamara D. H., 1961, PASP, 73, 269

—, 2000, in Astronomical Society of the Pacific Conference Series, Vol. 210, Delta Scuti and Related Stars, M. Breger \& M. Montgomery, ed., pp. 373-+

McNamara D. H., Feltz, Jr. K. A., 1978, PASP, 90, 275

Meléndez J., Asplund M., Gustafsson B., Yong D., 2009, ApJ, 704, L66

Mermilliod J.-C., 1983, A\&A, 128, 362

Mermilliod J.-C., Mermilliod M., Hauck B., 1997, A\&AS, 124, 349

Michaud G., Charland Y., 1986, ApJ, 311, 326

Michaud G., Charland Y., Vauclair S., Vauclair G., 1976, ApJ, 210, 447

Michaud G., Tarasick D., Charland Y., Pelletier C., 1983, ApJ, 269, 239

Mittermayer P., Weiss W. W., 2003, A\&A, 407, 1097

Molenda-Żakowicz J. et al., 2010, Astronomische Nachrichten, 331, 981

—, 2009, in American Institute of Physics Conference Series, Vol. 1170, American Institute of Physics Conference Series, Guzik J. A., Bradley P. A., eds., pp. 531-534

Moss D., 1990, MNRAS, 244, 272

Mosser B., Aristidi E., 2007, PASP, 119, 127

Moya A., Rodríguez-López C., 2010, ApJ, 710, L7

Murphy S. J., 2012a, MNRAS, 422, 665 
—, 2012b, Astronomische Nachrichten, 333, 1057

Murphy S. J., Grigahcène A., Niemczura E., Kurtz D. W., Uytterhoeven K., 2012, MNRAS, 427, 1418

Murphy S. J. et al., 2013, MNRAS, 432, 2284

Murphy S. J., Shibahashi H., Kurtz D. W., 2013, MNRAS, 430, 2986

Nemec J., Mateo M., 1990, in Astronomical Society of the Pacific Conference Series, Vol. 11, Confrontation Between Stellar Pulsation and Evolution, C. Cacciari \& G. Clementini, ed., pp. 64-84

Nemec J. M., Mateo M., Burke M., Olszewski E. W., 1995, AJ, 110, 1186

Neugebauer G. et al., 1984, ApJ, 278, L1

North P., 1984, A\&A, 141, 328

,- 1985, A\&A, 148, 165

—, 1993, in Astronomical Society of the Pacific Conference Series, Vol. 44, IAU Colloq. 138: Peculiar versus Normal Phenomena in A-type and Related Stars, Dworetsky M. M., Castelli F., Faraggiana R., eds., ASP Conference Series, p. 577

- 1998, A\&A, 334, 181

North P., Berthet S., Lanz T., 1994, A\&A, 281, 775

North P., Ginestet N., Carquillat J.-M., Carrier F., Udry S., 1998, Contributions of the Astronomical Observatory Skalnate Pleso, 27, 179

North P., Jaschek C., Hauck B., Figueras F., Torra J., Kunzli M., 1997, in ESA Special Publication, Vol. 402, Hipparcos - Venice '97, Bonnet R. M., Høg E., Bernacca P. L., Emiliani L., Blaauw A., Turon C., Kovalevsky J., Lindegren L., Hassan H., Bouffard M., Strim B., Heger D., Perryman M. A. C., Woltjer L., eds., pp. 239-244

Ochsenbein F., Bauer P., Marcout J., 2000, A\&AS, 143, 23

O’Donoghue D. E., Warner B., 1982, MNRAS, 200, 563

Osaki Y., 1971, PASJ, 23, 485

Palla F., Stahler S. W., 2000, ApJ, 540, 255

Pamyatnykh A. A., 2000, in Astronomical Society of the Pacific Conference Series, Vol. 210, Delta Scuti and Related Stars, Breger M., Montgomery M., eds., p. 215

Paunzen E., 1997, A\&A, 326, L29

-, 2001, A\&A, 373, 633

Paunzen E., Duffee B., Heiter U., Kuschnig R., Weiss W. W., 2001, A\&A, 373, 625

Paunzen E., Gray R. O., 1997, A\&AS, 126, 407 
Paunzen E., Wraight K. T., Fossati L., Netopil M., White G. J., Bewsher D., 2013, MNRAS, 429, 119

Petersen J. O., 1978, A\&A, 62, 205

Petersen J. O., Christensen-Dalsgaard J., 1996, A\&A, 312, 463

Pinsonneault M. H., An D., Molenda-Żakowicz J., Chaplin W. J., Metcalfe T. S., Bruntt H., 2012, ApJS, 199, 30

Poretti E. et al., 2010, Astronomische Nachrichten, 331, 1049

- $2009, \mathrm{~A} \& \mathrm{~A}, 506,85$

-, 2011, A\&A, 528, A147

- 2005, A\&A, 440, 1097

Preston G. W., 1970, in IAU Colloq. 4: Stellar Rotation, Slettebak A., ed., Reidel Publishing Company, p. 254

Pych W., Kaluzny J., Krzeminski W., Schwarzenberg-Czerny A., Thompson I. B., 2001, A\&A, 367, 148

Ramírez I., Meléndez J., Asplund M., 2009, A\&A, 508, L17

Reese D. R., Prat V., Barban C., van 't Veer-Menneret C., MacGregor K. B., 2013, A\&A, 550, A77

Renson P., Manfroid J., 2009, A\&A, 498, 961

Richer J., Michaud G., Turcotte S., 2000, ApJ, 529, 338

Rieutord M., 1992, A\&A, 259, 581

Rodríguez E., Breger M., 2001, A\&A, 366, 178

Rodríguez E. et al., 2006, A\&A, 456, 261

Rodríguez E., López de Coca P., Costa V., Martín S., 1995, A\&A, 299, 108

Roxburgh I. W., 2004, A\&A, 428, 171

Royer F., 2009, in Lecture Notes in Physics, Berlin Springer Verlag, Vol. 765, The Rotation of Sun and Stars, pp. 207-230

Royer F., Gerbaldi M., Faraggiana R., Gómez A. E., 2002a, A\&A, 381, 105

Royer F., Grenier S., Baylac M.-O., Gómez A. E., Zorec J., 2002b, A\&A, 393, 897

Royer F., Zorec J., Gómez A. E., 2007, A\&A, 463, 671

Russell S. C., 1995, ApJ, 451, 747

Saio H., 1981, ApJ, 244, 299

一, 2005, MNRAS, 360, 1022 
Schmid V. S. et al., 2012, Astronomische Nachrichten, 333, 1080

Schwarzenberg-Czerny A., 2012, in IAU Symposium, Vol. 285, IAU Symposium, Griffin R. E. M., Hanisch R. J., Seaman R., eds., CUP, pp. 81-86

Shibahashi H., Kurtz D. W., 2012, MNRAS, 422, 738

Shore S. N., Brown D. N., Sonneborn G., 1987, AJ, 94, 737

Shorlin S. L. S., Wade G. A., Donati J.-F., Landstreet J. D., Petit P., Sigut T. A. A., Strasser S., 2002, A\&A, 392, 637

Shulyak D., Ryabchikova T., Kildiyarova R., Kochukhov O., 2010, A\&A, 520, A88

Sills A., Pinsonneault M. H., Terndrup D. M., 2000, ApJ, 534, 335

Slettebak A., 1954, ApJ, 119, 146

—, 1955, ApJ, 121, 653

Smalley B., 2004, in IAU Symposium, Vol. 224, The A-Star Puzzle, Zverko J., Ziznovsky J., Adelman S. J., Weiss W. W., eds., CUP, pp. 131-138

Smalley B., Dworetsky M. M., 1995, A\&A, 293, 446

Smalley B. et al., 2011, A\&A, 535, A3+

Smith J. C. et al., 2012, PASP, 124, 1000

Smith M. A., 1970, ApJ, 161, 1181

- 1971, A\&A, 11, 325

-, 1972, ApJ, 175, 765

—, 1973, ApJS, 25, 277

- 1974, ApJ, 189, 101

一, 1982, ApJ, 254, 242

Soszynski I. et al., 2008, Acta Astron., 58, 163

Stamford P. A., Watson R. D., 1981, Ap\&SS, 77, 131

Stateva I., Iliev I. K., Budaj J., 2012, MNRAS, 420, 1207

Stȩpień K., 2000, A\&A, 353, 227

Stello D. et al., 2009, ApJ, 700, 1589

Sterken C., Jaschek C., 1996, Light Curves of Variable Stars, A Pictorial Atlas. CUP

Stibbs D. W. N., 1950, MNRAS, 110, 395

Stobie R. S., Balona L. A., 1979, MNRAS, 189, 627 
Stumpe M. C. et al., 2012, PASP, 124, 985

Stütz C., Paunzen E., 2006, A\&A, 458, L17

Suárez J. C., Garrido R., Goupil M. J., 2006, A\&A, 447, 649

Suárez J. C., Garrido R., Moya A., 2007, A\&A, 474, 961

Takeda Y., Han I., Kang D.-I., Lee B.-C., Kim K.-M., 2008, Journal of Korean Astronomical Society, 41, 83

Tassoul J.-L., Tassoul M., 1992a, ApJ, 395, 259

Tassoul M., Tassoul J.-L., 1992b, ApJ, 395, 604

Théado S., Alecian G., LeBlanc F., Vauclair S., 2012, A\&A, 546, A100

Théado S., Vauclair S., Alecian G., Le Blanc F., 2009, ApJ, 704, 1262

Thompson S. E. et al., 2012, ApJ, 753, 86

Titus J., Morgan W. W., 1940, ApJ, 92, 256

Tkachenko A., Lehmann H., Smalley B., Debosscher J., Aerts C., 2012, MNRAS, 422, 2960

Townsend R. H. D., 2010, ApJS, 191, 247

Turcotte S., Charbonneau P., 1993, ApJ, 413, 376

Turcotte S., Richer J., Michaud G., Christensen-Dalsgaard J., 2000, A\&A, 360, 603

Uesugi A., Fukuda I., 1982, Catalogue of stellar rotational velocities (revised). Department Of Astronomy, Kyoto Univ

Uytterhoeven K. et al., 2011, A\&A, 534, A125

Varenne O., Monier R., 1999, A\&A, 351, 247

Vauclair G., 1976, A\&A, 50, 435

Vauclair S., 2008, in IAU Symposium, Vol. 252, IAU Symposium, Deng L., Chan K. L., eds., pp. $97-101$

Venn K. A., Lambert D. L., 1990, ApJ, 363, 234

Viskum M., Kjeldsen H., Bedding T. R., dall T. H., Baldry I. K., Bruntt H., Frandsen S., 1998, A\&A, 335, 549

Wade G. A., 1997, A\&A, 325, 1063

Walker G. et al., 2003, PASP, 115, 1023

Watson R. D., 1988, Ap\&SS, 140, 255

Weiss W. W., Paunzen E., 2000, in Astronomical Society of the Pacific Conference Series, Vol. 210, Delta Scuti and Related Stars, Breger M., Montgomery M., eds., p. 410 
Weiss W. W., Paunzen E., Pamyatnykh A., Mkrtichian D., 2000, in Astronomical Society of the Pacific Conference Series, Vol. 203, IAU Colloq. 176: The Impact of Large-Scale Surveys on Pulsating Star Research, Szabados L., Kurtz D., eds., pp. 492-493

Winget D. E. et al., 1991, ApJ, 378, 326

Witte M. G., Savonije G. J., 2001, A\&A, 366, 840

Wolff S., Simon T., 1997, PASP, 109, 759

Wolff S. C., 1967, ApJS, 15, 21

—, 1983, The A-type stars: problems and perspectives. NASA SP-463

Wright C. O., Egan M. P., Kraemer K. E., Price S. D., 2003, AJ, 125, 359

Zahn J.-P., 1975, A\&A, 41, 329

,- 1977, A\&A, 57, 383

Zahn J. P., 1984, in IAU Symposium, Vol. 105, Observational Tests of the Stellar Evolution Theory, Maeder A., Renzini A., eds., Reidel Publishing Company, p. 379

Zahn J.-P., 1992, A\&A, 265, 115

Zima W., Lehmann H., Stütz C., Ilyin I. V., Breger M., 2007, A\&A, 471, 237

Zima W. et al., 2006, A\&A, 455, 235

Zorec J., Royer F., 2012, A\&A, 537, A120

Zwintz K., 2008, ApJ, 673, 1088 